\title{
Synchronization, Neuronal Excitability, and Information Flow in Networks of Neuronal Oscillators
}

\author{
Dissertation
}

zur Erlangung des Doktorgrades

der Mathematisch-Naturwissenschaftlichen Fakultäten der Georg-August-Universität zu Göttingen

vorgelegt von

Christoph Kirst

aus Göttingen

Göttingen 2011 
D7

Referent: $\quad$ Prof. Dr. T. Geisel

Korreferent: Prof. Dr. R. Kree

Tag der mündlichen Prüfung: 28.09.2011 


\section{Contents}

$\begin{array}{lll}\text { I } & \text { Introduction } & 7\end{array}$

$\begin{array}{ll}\text { II Background } & 17\end{array}$

2 Principles of Theoretical and Experimental Neuroscience 18

2.1 Neuronal Morphology . . . . . . . . . . . . . . . . . . . . . . . . 18

2.2 Electrical Properties of Neurons . . . . . . . . . . . . . . . . . . . . 19

2.3 Action Potential Generation . . . . . . . . . . . . . . . . . . . 21

2.4 Conductance Based Neuron Models . . . . . . . . . . . . . . . . 23

2.5 Compartmental Neuron Models . . . . . . . . . . . . . . . . . 25

2.6 Simplified Models of Neuronal Activity . . . . . . . . . . . . . . . 26

2.7 Synapses and Neuronal Connectivity _. . . . . . . . . . . . . . . 29

2.8 The Dynamic Patch Clamp Technique . . . . . . . . . . . . . 33

3 Notions from Dynamical Systems and Bifurcation Theory 34

3.1 Unstable Attractors and Heteroclinic Networks . . . . . . . . . . . . . 34

3.2 Bifurcation Theory and Normal Forms . . . . . . . . . . . . . 35

3.3 A Bifurcation Dictionary . . . . . . . . . . . . . . 37

3.4 Phase Reduction and Averaging for Weakly Coupled Oscillators . . . . . 47

\section{Dynamics of Pulse Coupled Oscillators with Partial Reset 51}

4 Introduction

5 Networks of Pulse-Coupled Threshold Elements with Partial Reset $\quad 56$

5.1 Pulse-Coupled Threshold Elements . . . . . . . . . . . . . 56

5.2 Partial Reset for $\delta$-Pulse-Coupled Threshold Units . . . . . . . . 57

5.3 Why Partial Reset? . . . . . . . . . . . . . . . . . . . . 62

6 Partial Reset and Collective Network Dynamics - Sequential Desynchronization Transition $\quad 68$

6.1 Collective Network Dynamics - Numerical Results . . . . . . . . . . . 68

6.2 Strategy of the Analysis . . . . . . . . . . . . . . . . . 72

6.3 Notations: State Space, Firing and Return Map . . . . . . . . . . . 72

6.4 Existence and Stability of Asynchronous Periodic States in Meta-Oscillator

Networks . . . . . . . . . . . . . . . . . . . . . . . . . 73

6.5 Impact of Partial Reset on Intra-Cluster Stability . . . . . . . . . . . 77

6.6 Extensive Sequence of Desynchronizing Bifurcations - A Solvable Example 83 
6.7 Robustness of the Desynchronization Transition . . . . . . . . . . . . . 84

6.8 Cluster Desynchronization in Biophysical Models . . . . . . . . . . . . 87

7 From Networks of Unstable Attractors To Heteroclinic Switching 89

7.1 Network Dynamics - Unstable Attractors and

Heteroclinic Switching . . . . . . . . . . . . . . . . . . . . . 89

7.2 Bifurcation From Networks of Unstable Attractors To Heteroclinic Switching 92

8 Summary, Discussion and Outlook 106

\section{Leak-Induced Dynamic Excitability Transitions in Neurons}

9 Introduction

10 Leak-Induced Neuronal Excitability Transition in Model Neurons

10.1 Leak Conductance as a Bifurcation Parameter in Conductance-Based Neuron Models . . . . . . . . . . . . . . . . . . . . . . . . . . . 114

10.2 Leak-Induced Neuronal Excitability Transitions - Bifurcation Diagrams . . 116

10.3 Leak-Induced Neuronal Excitability Transitions - Mechanism and Analysis 132

10.4 Summary . . . . . . . . . . . . . . . . . . . . 145

11 Morphology, Effective Leak and Neuronal Excitability 146

11.1 Linear Shapes Integrate - Stellar Shapes Resonate . . . . . . . . . . . . . 146

11.2 Recovery of Type I Neuronal Excitability by Active Dendrites . . . . . . . 151

11.3 Neuronal Excitability and Morphology in Experiments . . . . . . . . . . . 152

11.4 Summary . . . . . . . . . . . . . . . . . . . 154

12 Leak Induced Neuronal Excitability Switches in Real Neurons 155

12.1 Experimental Methods . . . . . . . . . . . . . . . . 155

12.2 Leak Induced Neuronal Excitability Switches in DNLL and CA3 Neurons . 158

12.3 Leak Induced Transition From Integration to Resonance at Peri-Threshold in CA3 Pyramidal Cells . . . . . . . . . . . . . . . . . . 163

12.4 GABA Induced Transition From Integration to Resonance at Peri-Threshold in CA3 Pyramidal Cells . . . . . . . . . . . . . . . 166

12.5 Summary and Discussion . . . . . . . . . . . . . . . . 166

13 Dynamic Neuronal Excitability and Collective Network Dynamics 168

13.1 Leak-Induced Neuronal Excitability Transition and Phase Response . . . . 168

13.2 A Synchronization-Desynchronization Circuit . . . . . . . . . . . . 171

13.3 Summary . . . . . . . . . . . . . . . . . . . . . 172

14 Conclusion and Outlook $\quad 173$ 
15 Introduction

15.1 Neuronal Oscillations . . . . . . . . . . . . . . . . . . . . . 179

15.2 Measuring Information Flow . . . . . . . . . . . . . . . . . . 179

16 Local Control of Non-Local Information Flow in Networks of Coupled Phase Oscillators $\quad 182$

16.1 Model . . . . . . . . . . . . . . . . . . . . . . . . 182

16.2 Information Flow in Networks of Coupled Phase Oscillators . . . . . . . . . 183

16.3 Local Control of Information Flow in Hierarchical Networks of Phase

Oscillator . . . . . . . . . . . . . . . . . 194

16.4 Summary and Discussion . . . . . . . . . . . . . . . . . 203

17 Local Control of Non-Local Information Flow in Spiking Neuronal Networks

17.1 Model . . . . . . . . . . . . . . . . . . . . 206

17.2 Local Control of Non-Local Phase Information Flow in Spiking Networks . 208

17.3 Information Flow In Spike Patterns . . . . . . . . . . . . . . . . . 214

18 Summary, Discussion and Outlook $\quad 220$

18.1 Summary . . . . . . . . . . . . . . . . . . . . 220

18.2 Discussion and Outlook . . . . . . . . . . . . . . . . . . 221

18.3 Theoretical Challenges . . . . . . . . . . . . . . . . . . 224

\section{Conclusion and Outlook 225}

Appendix 233

A The Eneström-Kakeya Theorem 234

A.1 Spectral-Radius and Matrix-Norm . . . . . . . . . . . . . . . 234

A.2 Companion Matrices . . . . . . . . . . . . . . . . . . 234

A.3 The Eneström-Kakeya Theorem . . . . . . . . . . . . . . . . . . 234

B Rise Functions $\quad 236$

B.1 Rise Functions for Integrate-and-Fire Models . . . . . . . . . . . . . . . 236

B.2 Icpd and Dcpd Rise Functions . . . . . . . . . . . . . . . . . . 236

C Event-Sequence-Based Domain Analysis for $\mathcal{N}^{4} \quad 239$

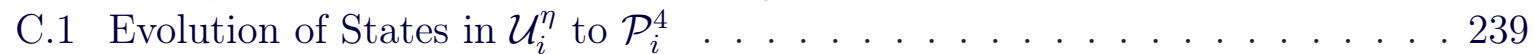

C.2 Event-Sequence-Based Domain and Parameter Analysis for $\mathcal{N}^{4}$. . . . . . . 241

D Conductance-Based Neuron Models $\quad 255$

D.1 The Hodgkin Huxley Model . . . . . . . . . . . . . . . . . . . . . 255

D.2 The Morris-Lecar Neuron Model . . . . . . . . . . . . . . . . . . 255

D.3 The Wang-Buzsaki Neuron Model . . . . . . . . . . . . . . . . . 256

D.4 The Fast Spiking Neuron Model by Erisir et al. . . . . . . . . . . . . . . 256

D.5 The Connor-Stevens and Rose-Hindmarsh Neuron Model . . . . . . . . . . 256 
D.6 The Simplified Traub-Miles Pyramidal Neuron Model . . . . . . . . . . . . 257

D.7 The Rinzel Neuron Model . . . . . . . . . . . . . . . . . . . . . . . 257

E Resonance and Impedance in Neuron Models $\quad 259$

F Normal Form Coefficients for the Degenerate Bogdanov-Takens Bifurcation

G Information Flow in the Ornstein Uhlenbeck Process 263

G.1 Stationary Joint Distribution _. . . . . . . . . . . . . 263

G.2 Delayed Mutual Information . . . . . . . . . . . . . . . . . 265

G.3 Delayed Transfer Entropy . . . . . . . . . . . . . . 265

H Information Flow in Hierarchical Networks of Phase Oscillators Near Synchronous Phase-Locked States

Bibliography

Nomenclature 


\section{Part I}

\section{Introduction}




\section{Chapter 1}

\section{Introduction}

Complex systems are fascinating. They are an over-abundant subject of the modern interdisciplinary sciences [18, 118, 418, 381, 285], including physics, biology, chemistry, cybernetics, economics, computer and social sciences. These systems consist of several components, interacting on an underlying network that frequently exhibit a complex and time varying structure $[401,283,60]$. The individual units are possibly very different and may change their properties in time and in response to the interactions with the other units. A natural question that arises is how the properties of individual units within such a complex system affect the global dynamical characteristics.

For instance, non-linear interactions among the units entail emergent collective behavior that is qualitatively different and may not be anticipated from the single unit dynamics. Examples include phase transitions in condensed matter physics [232], self-organization and pattern formation in biological and chemical systems [382, 153, 228, 286], as well as chaos [322], synchronization [298] and collective oscillations [407, 228] in excitable systems, as observed in the activity of nerve cells in neuronal networks $[128,53]$

The brain is a particularly complex system [219, 196] because both its structure and dynamics are distributed over a large range of spatial and temporal scales. These range from molecular sizes to the modular and hierarchical organization of brain regions, and from the diffusion time of ions through cell membranes to the time scales of years encountered in neuronal development, learning, and memory. A central question, not only in the neuro- and technological sciences, but also of high relevance in philosophy [266] and even entering economics [201] and social sciences [409] is how perception, memory, consciousness and behavior of humans and animals emerge from the collective neuronal dynamics in the brain.

Fundamental building blocks of the brain are excitable nerve cells - neurons - that generate stereotyped electrical pulses - action potentials or spikes. These are transmitted between neurons via synaptic connections that form a complex neuronal network. Action potentials are believed to be the primary signaling mechanism in nervous systems [196]. To understand how information is encoded in these signals and how information processing and ultimately behavior is reflected in the collective dynamics of neuronal networks is an important aspect of computational, theoretical and mathematical neurophysics [163, 304, 68,274 . One may further ask, how different characteristics of single nerve cell dynamics and network structure may be utilized for appropriate brain function, for example to control collective neuronal dynamics, information routing and processing.

Neuronal networks show a rich diversity of collective dynamics [264]. These include almost quiescent states of sparse neuronal activity in the cortex [337]; propagating ac- 
tivity waves, for example in the development of the visual system [413] or in connection with migraine [135]; and highly irregular and chaotic dynamics [343] in networks of balanced excitatory and inhibitory inputs [338, 402]. Also regular and rhythmic activity has been observed, for instance in central pattern generators involved in motor actions [253], during the daily sleep-wake cycle [115] and as collective oscillations over a broad range of frequencies $[26,374,53]$ for example in the hippocampus in connection with memory and navigation tasks [288, 275].

Synchronization is a frequently occurring collective phenomenon not only in neuronal networks but in complex systems in general [298]. Between individual neurons, synchronization manifests itself in the simultaneous generation of action potentials [264, 212], on larger spatial scales in collective oscillations of neuronal ensembles $[92,95,128,138,259$, 376]. In the latter case, individual neurons are often not synchronized spike to spike but instead share strong time-varying correlations that arise form the synaptic interactions within the network and lead to the macroscopic oscillations, observed for example in electroencephalographic recordings [26]. The oscillating groups themselves may synchronize or phase lock their rhythmic activity with other groups in the network not only locally [90, 138, 113, 412] but also across distant brain areas [330, 339].

The omnipresence of synchronized neuronal activity in the brain led to several explanations for its relevance in neuronal processing and coding. At the sensory stage synchronous inputs and more generally correlated signals trigger action potential generation in neurons specialized on coincidence detection [192], most notably in the auditory system [8]. At higher processing levels, synchronized spiking within a group of neurons may lead to stable propagation of this activity through a sequence of subgroups along embedded stronger feed-forward structures [77]. Also active conductances distributed on dendritic trees of cortical pyramidal neurons non-linearly amplify synchronous inputs $[161,300]$. Thus the degree of synchronization modulates neuronal gain [367] and facilitates the propagation of signals within and across neuronal populations [326, 393, 111]. It is hypothesized that synchronization of neuronal activity can effectively bind nerve cells that represent different information aspects of a single perceptual object. This feature binding $[398,399,141,140,340,342]$, i.e. the selective grouping of semantically related stimuli by synchrony, could provide a mechanism for dynamically linking neurons into cell assemblies [162, 94, 341] and thereby enlarging the representational and computational capacity of a neuronal network.

Instead of directly coding information into neuronal correlations, in a more dynamic picture $[326,393,111]$ synchronization and phase-locking among neuronal oscillations provides a basis for neuronal communication through coherence $[111,112]$ : Neurons in oscillatory groups not only synchronize their outgoing spikes which then become more effective in evoking postsynaptic responses [161,300], but also undergo rhythmic excitability fluctuations that periodically change their sensitivity to inputs [396, 49]. Thus, oscillations rhythmically open and close the neurons group's windows for communication and may provide a flexible mechanism for neuronal communication. Experimental evidence for this hypothesis comes from several studies [113, 330, 410, 412, 142, 408, 28]. In particular, in [412] it was shown that the mutual influence among neuronal groups depends crucially on the phase relationship between the oscillations.

Despite being beneficial for neuronal processing on the on hand, strong synchronous activity is associated with pathological effects on the other. For example it is thought to trigger epileptic seizures [259] and is associated with Parkinson disease [92]. The Parkinson resting tremor appears to be caused by a population of neurons located in the 
thalamus and the basal ganglia, which fire in a synchronized and intrinsically ryhtmic manner. This synchronized firing acts as a pacemaker for activating pre-motor areas and the motor cortex which in turn leads to the tremor with a similar frequency. Here it is important to understand the synchronization mechanisms in detail and to find possible mechanism to desynchronize these cells $[302,160]$.

Revealing fundamental principles and mechanisms underlying collective neuronal network dynamics and in particular synchronization is thus an important aspect of neuroscientific research. Moreover, regulatory mechanism in the brain typically act locally by changing the dynamical properties of individual neurons (e.g. due to neuro-modulators [397]) or their synaptic connections (e.g. due to neural plasticity [2]). Therefore a mechanistic knowledge of how local properties affect or even control the collective synchronization dynamics may provide helpful tools in understanding e.g. pathological synchronization and information transmission.

Since a single nerve cell already constitutes a complex system by itself [218] one of the challenging tasks is to find reasonable simplifications of the neuronal dynamics in order to enable the analysis on the network level. Therefore, physical and mathematical methods of abstraction and modeling are useful tools. The idea is to keep the units and their interactions as simple as possible [364], but at the same time to try to sustain and isolate the characteristic features underlying the dynamical phenomenon of interest $[1,215]$. Thereby mechanism underlying complex collective phenomena can be identified, but also important characteristics of the dynamics and relevant interference effects between several mechanisms may be overlooked. One thus has to analyze carefully which aspects of the dynamics can be neglected and which have to be kept.

A celebrated mathematical model for the ionic mechanisms underlying the initiation and propagation of action potentials are conductance-based models [167, 68], dating back to the seminal work of A. Hodgkin and A. Huxley [171]. This biophysical model neglects the detailed molecular dynamics ${ }^{1}$ and treats the cell membrane as an electrical capacitor and the voltage gated ion channels as non-linear electrical conductances. Action potentials are generated by an initial positive feedback of currents into the cell that raise the membrane potential which in turn increases the conductance for the inward currents. The further increase in the membrane potential activates slower outward currents and consequently decreases the membrane potential again.

Two main neuronal excitability types of spike generation have been identified [170]: While type I neurons show arbitrarily low periodic firing frequencies, type II neurons start firing with a frequency clearly distinct from zero. The neuronal excitability type strongly influences the collective network dynamics $[157,100,177]$ as well as the coding and information storage properties $[239,318,166]$. In particular, type I neurons have integrative properties and tend to desynchronize, while type II neurons have resonance properties and often undergo synchronization [316, 189]. Thus, given the strong interrelations between synchrony and neuronal processing discussed above, it is important to identifying factors and control mechanisms that influence the neuronal excitability type.

Several factors that determine neuronal excitability have been identified. They include ion channel properties, such as the maximal conductances, the activation curves and reversal potentials $[245,316,167]$ as well as the mixture and density of ion channels across the membrane [63, 68, 188]. Other factors are neuro-modulators [352], intrinsic dynamical states of the neuron such as up and down states [165] and in vivo vs. in vitro

\footnotetext{
${ }^{1}$ We note that on the molecular scale interesting interactions with chemical and genetic networks arise that contribute to a variety of regulatory mechanisms and are only partly understood so far.
} 
conditions [307]. However, despite the numerous numerical and experimental studies that relate neuronal morphology to firing patterns and response properties of neurons $[250,143,387,25,130]$, the impact of the dendritic structure on the intrinsic neuronal excitability type has not been studied so far.

Chapter 11 of this thesis is devoted to this question. We find that the morphological structure and the active ion channels of the dendritic tree have a crucial impact on the neuronal excitability type. For passive dendrites with arbitrary morphology we analytically determine the neuronal excitability type and show that linear extended shapes tend to integrative and desynchronizing type I excitability, while stellar shapes show resonant and synchronizing type II properties. Our analysis identifies a passive effective leak conductance as the underlying parameter that induces the excitability switch.

Previous experimental [165, 352, 306, 307, 359, 360] and theoretical [315, 316, 100, $117,189,188]$ work has identified type I and type II neuronal excitability and even in a single neuron model for different parameter sets [100]. Also there is a wealth of numerical bifurcation studies that investigate the dependence on the ion channel parameter $[109,316$, $147,100,117,379,353]$. In [306] it was shown that a combination of shunting inhibition and adaptation can lead to a neuronal excitability switch and a biophysical explanation was given. However, no detailed bifurcation study of how the leak conductance affects the neuronal dynamics exists and is not clear how neuronal excitability transitions are organized.

Part IV of this thesis addresses these two questions. Using multiple bifurcation theory, a combination of normal form and center manifold reduction and numerical continuation, we show analytically and numerically that leak-induced neuronal excitability transitions are organized by a degenerate Bogdanov-Takens-cusp bifurcation of codimension three [87]. We further give an intuitive explanation of the transition in terms of a non-linear equation for oscillatory dynamics - a Liénard equation - that arises via conjugation from a class of conductance based models.

Activation of inhibitory synapses provides shunting inhibition, that effectively imposes an additional leak conductance on the neuron. Using our results on leak induced type I to II transitions we therefore propose in part IV of this thesis that neuronal excitability and as consequence synchronization and resonance properties can be controlled dynamically via inhibitory synapses and study consequences for the collective network dynamics.

Experimentally, the change from type I and type II neuronal excitability has been found, induced for example by the application of neuron transmitter [352] or changing from in vivo to in vitro conditions [307]. Using the dynamic patch clamp method [281, 287] we experimentally study the effect of leak conductances on the neuronal excitability type in chapter 12 and confirm several predictions of our theory. We then show that the application of a neuro transmitter that activates the receptors of inhibitory synapses (GABA) is sufficient to induce a switch in the resonance and neuronal excitability properties. We conclude that neuronal excitability may be controlled dynamically via activation of inhibitory synapses.

As mentioned above, simplifications of neuronal dynamics are important to facilitate the analysis of collective network behavior. For periodic activity, found for example in individual neurons as the repeated generation of action potentials [64, 245] caused by supra-threshold stimuli or generated intrinsically (e.g. in pace-maker neurons [296, 269] or rhythmic pattern generators [253]) and in ensembles of neurons showing oscillations, the dynamics can be captured by a phase and amplitude variables. In situations where the limit cycle is strongly attracting the amplitudes decay fast towards the limit cycle and 
the description of the oscillators may be reduced to their phase variable and their phase response function that captures the change of the oscillator's phase in response to external stimuli or due to the interaction with other oscillators [228]. This reduction process leads to the general class of coupled phase oscillators, that not only serve as models for the dynamics of neuronal oscillator networks, but find wide applications in physics [5] and biology [407], in particular to study synchronization and phase locking phenomena [298].

The communication-through-coherence hypothesis inspired recent experimental and numerical studies $[412,48,28,112,23,408]$. They show that alternative phase shifts between different neurons or groups of neurons result in different information transfer properties between the groups. But how are these phase shifts controlled? Given that regulatory mechanisms in the brain act predominantly locally on the dynamical properties of individual neurons and their synaptic connections, one may further ask how local changes can contribute to the control of phase relations and information transfer.

In part IV of this thesis we study this question first analytically using coupled phase oscillators and then semi-analytically and numerically in more realistic networks of spiking model neurons. We quantify the information flow by analytically calculating the time delayed mutual information between oscillators in a phase locked state. Further, we present a theoretical framework to predict phase lag patterns within and between groups of oscillators in hierarchical networks. Combining both results we derive the non-local information flow between the groups as a function of structural and dynamical network parameter. We use our results to reveal how information transfer is controlled by the underlying physical connectivity and find that local changes of links or frequencies within a group can control the non-local information flow from and to this cluster. Via this mechanism a group may thus "tune" to sources of relevant information and specify receivers of its own information output. Interestingly, our analytics unravel that local changes in the strength of a single link can also remotely control the information transmission between two different physically unchanged oscillators. We finally also link our theoretical findings to more realistic hierarchical networks of spiking neurons exhibiting gamma oscillations (cf. also [23, 408]) and extend the study to information flow in spike patterns. We conclude this part of the thesis by hypothesizing that oscillations in hierarchical networks of neurons not only provide a basis for communication-through-coherence but also for local control mechanisms of this non-local communication.

A further model reduction from phase oscillators with continuous time coupling is possible, if synaptic time scales are short compared to intrinsic oscillation period of the neurons. In this situation the interactions may be described by the exchange of pulses $[248,174]$ : Whenever the phase variable of a certain unit crosses a trigger threshold the element sends a pulse to its connected units in the network where the pulses evoke a response in the phase of the receiving unit after a possible transmission delay. Because of their analytical tractability, pulse-coupled oscillator models are helpful tools to study collective dynamics of neuronal oscillators and in particular synchronization [296, 269, 229, $3,104,33,37,372,71]$. In [269] such a model was used to demonstrate that in homogeneous networks with excitatory all-to-all coupling biological oscillators always synchronize. A strong synchronizing mechanism in these networks is the simultaneous reception of a pulse by two initially non-synchronous oscillators which pushes both oscillators beyond their firing threshold, i.e provides supra-threshold excitation. The subsequent reset of their phases to the same value then synchronizes both units. Such a strong synchronizing reset, which is also insensitive to the strength of the supra-threshold excitation, is found in almost all previous studies using similar models with $\delta$-pulse-coupling [33, 104, 229, 
$333,371,66,71,174]$. A small number of studies uses complete conservation of the suprathreshold input which is added to the phases after the reset $[229,164,174,106]$. However, a systematic study on the influence of the reset on the collective network dynamics has not been performed so far. In particular, an open question is how synchronization in pulse-coupled networks depends on the reset mechanism.

In part III of this thesis we study these questions analytically. We introduce a partial reset into the general class of pulse-coupled threshold units and study its effect on the collective network dynamics. We first concentrate on interactions without transmission delay and find that the partial reset controls a sequential desynchronization transition: In globally coupled neurons an increase in the strength of the partial reset induces a sequence of bifurcations from states with large clusters of synchronously firing neurons, through states with smaller clusters to completely asynchronous spiking. This mechanism for neural desynchronization differs strongly from known mechanisms that are based, e.g., on heterogeneity, noise, or delayed feedback [389, 388, 71, 251, 302] and might be used in modified form to prevent pathological synchronization in neural systems like in Parkinson tremor or in epileptic seizures.

Pulse-coupled oscillator models with delayed interactions have intriguing mathematical and dynamical properties. For instance, networks of oscillators with global homogeneous delayed pulse-coupling may robustly exhibit unstable attractors [104, 105, 371, 372], i.e. invariant periodic orbits that have a non-zero measure basin of attraction (Milnor attractors $[267,268])$ but are locally unstable. In the presence of noise, these systems exhibit a dynamics akin to heteroclinic switching, a feature that may also have functional relevance for coding and computation in neural systems [155, 17, 238, 237]. In ref. [16] it was shown that invertible systems in general cannot have unstable attractors and that a saddle state can in principle be converted to an unstable attractor by locally adding a non-invertible dynamics onto the stable manifold. However, the potential relation of unstable attractors to heteroclinic cycles is not well understood and it is unknown whether and how unstable attractors may be created or destroyed via bifurcations.

In chapter 7 we provide analytical answers to these questions. In oscillator networks with delayed pulse coupling we find that the partial reset controls a transition from networks of unstable attractors to heteroclinic switching. We present a mathematical technique of event-sequence-based domain analysis and prove for a small network the existence of two unstable attractors that are completely enclosed by each other's basin volume if the reset mechanism is strong and non-invertible. We analytically show that upon continuously removing this local non-invertibility of the system, the two unstable attractors become a set of two non-attracting saddle states that are heteroclinically connected. This transition equally occurs from larger networks of unstable attractors to heteroclinic structures and constitutes a new type of bifurcation in dynamical systems.

This thesis is structured as follows (cf. also tab. 1.1). In part II, chapter 2 we provide the neurobiological background, introduce and discuss the neuron models of different levels of abstraction used in this thesis and show how they are related to each other by successive reduction steps. We further introduce the dynamic patch clamp technique used for our experiments. Chapter 3 provides the necessary mathematical background from dynamical systems, bifurcation, normal form, phase reduction and averaging theory relevant for this thesis.

In part III (chapters $4-8$ ) we introduce the novel concept of a partial reset into the general class of pulse-coupled systems and study its consequences for the collective dynamics if the individual units are oscillatory. In chapter 6 we concentrate on instan- 
taneous interactions and analytically prove that the partial reset controls a sequence of desynchronizing bifurcations. In chapter 7 we include interaction delays and analytically find that the partial reset controls a novel type of bifurcation from networks of unstable attractors to heteroclinic switching.

Part IV (chapters 9 - 14) is devoted to the theoretical and experimental study of leak-induced dynamic neuronal excitability transitions and its consequences for collective network dynamics. In chapter 10 we analytically and numerically study the bifurcations that organize neuronal excitability transitions and give an interpretation of the underlying mechanism by conjugating a neuron model to a Liénard type equation. We apply these results in chapter 11 to address the question how dendritic morphology influences neuronal excitability. In chapter 12 we investigate leak-induced neuronal excitability transitions experimentally using dynamic patch clamp experiments and confirmation our theoretical predictions. We further show evidence that synaptic activity is sufficient to dynamically induce neuronal excitability switches. In chapter 13 we then show how neuronal excitability can be switched dynamically to control synchronization properties of collective network dynamics.

In part $\mathrm{V}$ (chapters 15 - 18) we present a mechanism of how information flow between different groups of neurons can be controlled by local modifications within the groups only. In chapter 16 we derive for hierarchical networks of phase oscillators an analytical expressions for the information flow between clusters as a function of the local cluster properties. We use this theory to show how global information flow may be controlled locally. Chapter 17 confirms these finding in a more biophysical realistic network model of spiking neurons and extends the results to information transfer in spike patterns.

Parts III-V start with an introduction each and end with a discussion and outlook. The final chapter VI gives overall summarizing conclusions and future directions. 
Table 1.1: Thesis overview.

\begin{tabular}{|c|c|c|c|}
\hline & Part III & Part IV & Part V \\
\hline model & $\begin{array}{l}\text { networks of pulse-coupled } \\
\text { oscillators }\end{array}$ & $\begin{array}{l}\text { conductance based } \\
\text { neuron models }\end{array}$ & $\begin{array}{l}\text { hierarchical networks of } \\
\text { phase oscillators / } \\
\text { spiking neurons }\end{array}$ \\
\hline $\begin{array}{l}\text { oscillatory } \\
\text { dynamics }\end{array}$ & intrinsic oscillation & $\begin{array}{l}\text { bifurcations to periodic } \\
\text { spiking }\end{array}$ & $\begin{array}{l}\text { intrinsic oscillation / } \\
\text { pyramidal interneuron } \\
\text { gamma oscillations }\end{array}$ \\
\hline $\begin{array}{l}\text { essential } \\
\text { component } \\
\text { property }\end{array}$ & partial reset & leak / shunting inhibition & $\begin{array}{l}\text { local network structure / } \\
\text { frequencies / dynamical } \\
\text { states }\end{array}$ \\
\hline $\begin{array}{l}\text { collective } \\
\text { dynamics }\end{array}$ & $\begin{array}{l}\text { - synchrony / multistable } \\
\text { cluster states / } \\
\text { asynchrony } \\
\text { - unstable attractor } \\
\text { networks and } \\
\text { heteroclinic switching }\end{array}$ & $\begin{array}{l}\text { - synchrony / asynchrony } \\
\text { - bistable and partial } \\
\text { synchronization / } \\
\text { desynchronization }\end{array}$ & $\begin{array}{l}\text { - phase locking } \\
\text { - phase locked gamma } \\
\text { oscillations }\end{array}$ \\
\hline $\begin{array}{l}\text { main } \\
\text { results }\end{array}$ & $\begin{array}{l}\text { - introduced partial reset } \\
\text { in pulse-coupled oscilla- } \\
\text { tors } \\
\text { (chapter } 5) \\
\text { - proof for sequential } \\
\text { desynchronization of } \\
\text { multistable cluster } \\
\text { states induced by the } \\
\text { partial reset } \\
\text { (chapter } 6 \text { ) } \\
\text { - proof for a novel type of } \\
\text { bifurcation from unsta- } \\
\text { ble attractors to hetero- } \\
\text { clinic switching induced } \\
\text { by the partial reset } \\
\text { (chapter } 7 \text { ) }\end{array}$ & $\begin{array}{l}\text { - leak-induced neuronal } \\
\text { excitability transition } \\
\text { (chapters } 10-12 \text { ) } \\
\text { - numerical and analytic } \\
\text { proof that leak induced } \\
\text { neuronal excitability } \\
\text { switches are arranged } \\
\text { in a threefold transition } \\
\text { which itself is orga- } \\
\text { nized by a degenerate } \\
\text { Bogdanov-Takens bifur- } \\
\text { cation papter 10) } \\
\text { (chaptch clamp } \\
\text { - dynamic patch real } \\
\text { experiments with rearic } \\
\text { neurons confirm theory } \\
\text { and show dynamic } \\
\text { excitability control via } \\
\text { shunting inhibition } \\
\text { (chapter 12) } \\
\text { - dynamic excitability } \\
\text { controlled (bistable) } \\
\text { synchronization } \\
\text { desynchronization in } \\
\text { collective network dy- } \\
\text { namics } \\
\text { (chapter 13) }\end{array}$ & $\begin{array}{l}\text { - analytical theory for in- } \\
\text { formation flow in net- } \\
\text { works of phase oscilla- } \\
\text { tors with arbitrary net- } \\
\text { work topology } \\
\text { (chapter 16) } \\
\text { - local and remote control } \\
\text { mechanisms (infor- } \\
\text { mation flow tuning, } \\
\text { plasticity and combina- } \\
\text { torics) in hierarchical } \\
\text { phase oscillator net- } \\
\text { works } \\
\text { (chapter 16) } \\
\text { - phase information flow } \\
\text { in clustered networks } \\
\text { of spiking neurons un- } \\
\text { dergoing gamma oscilla- } \\
\text { tions and its local (re- } \\
\text { mote) control mecha- } \\
\text { nisms } \\
\text { (chapter 17) } \\
\text { - control mechanisms for } \\
\text { the flow of information } \\
\text { in spike patterns in clus- } \\
\text { tered spiking oscillatory } \\
\text { networks } \\
\text { (chapter 17) }\end{array}$ \\
\hline
\end{tabular}




\section{Part II}

\section{Background}




\section{Chapter 2}

\section{Principles of Theoretical and Experimental Neuroscience}

In this section the basic notions, concepts and models from neuroscience relevant for this thesis are introduced. We start by briefly introducing the reader to morphological, dynamical and functional properties of neurons and present a selection of neural models of different levels of abstraction. We briefly introduce synapses and neuronal connectivity and aspects of experimental neuroscience.

An in-depth treatment of the neurobiology and electrophysiology can be found for example in the textbooks [284], [336] and [196]. Textbooks on neuronal modeling and theoretical neuroscience are [68], [380] and [123].

\section{$2.1 \quad$ Neuronal Morphology}

Neurons are the elementary processing units in the central nervous system. They are highly specialized in generating electrical signals - action potentials (also called spikes) and transmitting them via synaptic connections to other neurons. Cortical brain matter consist of more than $10^{4}$ neurons per cubic millimeter; the human has approximately $10^{11}-10^{12}$ nerve cells and about $10^{14}$ synapses [196, 336, 284, 68]. Neurons receive on the order of $10^{4}$ inputs and make a similar number of connections to other neurons. Thus neurons are embedded into a large and complex network structure as already suggested from staining experiments done by S. Ramón y Cajal [311] in 1897 shown in fig. 2.1. In fig. 12.1 stained neurons from the experimental part of this thesis are shown.

The action potentials are stereotyped electrical signals generated by neurons, which encode and combine different modalities of information such as visual, auditory or somatosensory qualia within an uniform neuronal representation [284]. The origin and destination of these signals specify the information content, for example fibers in the optic nerve mainly carry visual information. Similarly the location and type of neurons within the neuronal network is related to their functions, which include tasks as diverse as receiving sensory information, initiating motor commands, processing information and memory. As a consequence of these specializations a broad variety of morphologically different types of neurons exists (cf. fig. 2.1).

Despite this diversity, almost all neurons consists of three parts: the dendrite, the soma and the axon (cf. fig. 2.1). The often widely arborized dendrites mark the beginning of the intracellular pathway. They receive most of the cell's synaptic input form other neurons and relay it to the cell body, the soma. The soma acts as a highly non-linear element, where 
(a)

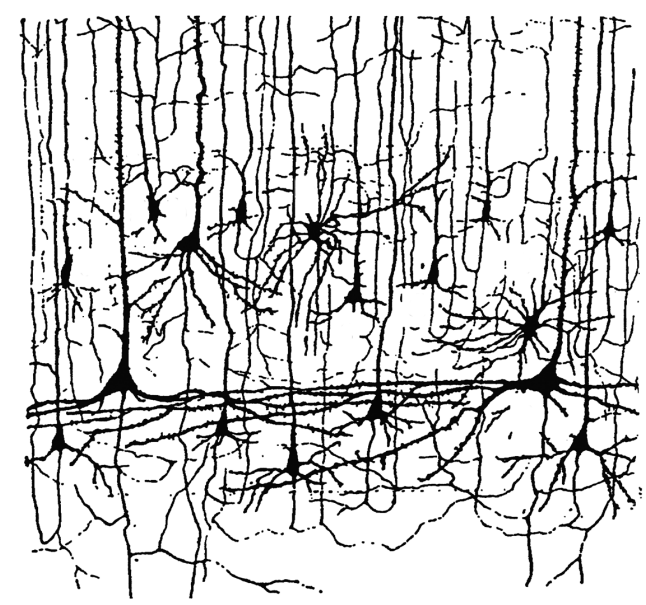

(b)

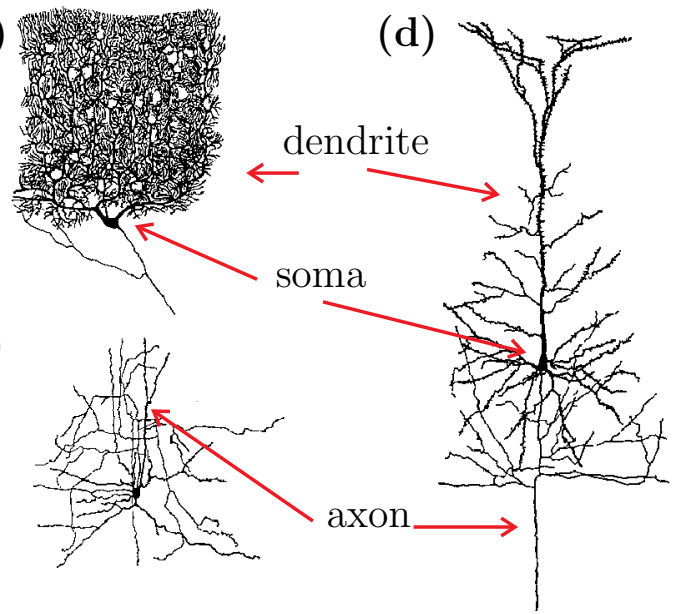

Figure 2.1. Morphology of Neurons. (a) Reproduction of a drawing by Ramón y Cajal showing a few neurons in the mammalian cortex [312]. Due to the Golgi staining method only a small fraction of neurons is visible, nevertheless the complex neural network structure is apparent. (b) Purkinje, (c) stellar and (d) pyramidal cell in cat cortex [311] consisting of three functional and morphological parts: a dendrite receiving the input signals from other cells, a soma integrating these inputs and an axon along which the output in form of an action potential is carried to other neurons.

inputs are integrated and action potentials are generated, which then propagate along the axons to the synapses. The synaptic connections transfer the presynaptic electrical signal to the dendrites or soma to the postsynaptic neuron (cf. sec. 2.7).

\section{$2.2 \quad$ Electrical Properties of Neurons}

The cell membrane of nerve cells is a lipid bilayer which separates the intracellular cytoplasm from the surrounding extracellular liquid $[68,196]$. The electrical signals of neurons consist of changes in the membrane potential $v$ defined as the difference between the electrical potential $v_{\text {intra }}$ of the intra- and extracellular space which is set to ground $v_{\text {extra }}=0 \mathrm{mV}$ :

$$
v=v_{\text {intra }}-v_{\text {extra }}
$$

The membrane is essentially impermeable to most charged ions and thus acts as an capacitor by separating the charges on the interior and exterior surface. The specific membrane capacitance is of the order of $c_{\mathrm{s}}=1 \frac{\mu \mathrm{F}}{\mathrm{cm}^{2}}$. Multiplication with the cell membrane area gives the total membrane capacity $c_{\mathrm{m}}$.

Embedded in the cell membrane there are ion channels or ion pumps, specific proteins structures which allow mainly sodium, potassium, and chloride ions to pass the membrane [167]. The channels make a contact between the intra- and extracellular media and allow ions to cross the membrane via diffusion. Usually ion channels are selective in that they allow only certain ion types to cross. Ion pumps consume energy to actively transport ions across the cell membrane.

For a typical neuron under resting conditions a fraction of channels for sodium, potassium and chloride are open. In this situation the $\mathrm{Na}^{+}$and $\mathrm{Cl}^{-}$concentrations are higher outside the cell, whereas the concentration of $\mathrm{K}^{+}$is higher inside. In thermal equilibrium there is no net current into or out of the cell, i.e. the difference in Gibbs free energy [232] vanishes. Thus the contribution to the free energy from the chemical potential originating 
in concentration gradients has to be canceled by an electrical potential difference. For a single ion species $x=\mathrm{K}, \mathrm{Na}, \mathrm{Cl}, \mathrm{Ca}, \ldots$ with concentrations $\left[x_{\text {extra }}\right]$ and $\left[x_{\text {intra }}\right]$ in extraand intracellular space, at a temperature $T$ this consideration yields the Nernst-equation $[380]$

$$
v_{x}=\frac{k_{\mathrm{B}} T}{q} \ln \left(\frac{\left[x_{\text {extra }}\right]}{\left[x_{\text {intra }}\right]}\right)
$$

where $k_{\mathrm{B}}$ is the Boltzmann constant and $v_{x}$ is the equilibrium potential for ion type $x$. Typically values for equilibrium potentials are $v_{\mathrm{K}}=+55 \mathrm{mV}, v_{\mathrm{Na}}=-77 \mathrm{mV}$ and $v_{\mathrm{Cl}}=-65 \mathrm{mV}$.

In neuronal cells more than a single ion species is present and the membrane potential in the steady state of the neuron, the resting potential $v_{\mathrm{r}}$, has to be determined from the Goldmann formula [380], a generalization of the Nernst equation 2.2.1, and lies between the equilibrium potentials of the participating ion species and typically takes values $v_{\mathrm{r}} \approx$ $-60 \mathrm{mV}$.

The equilibrium potentials are also called reversal potentials since for $v<v_{x}$ there is a net positive inward current $i_{x}$ of ions $x$, whereas for $v<v_{x}$ it changes sign. By convention, positive charges leaving the cell produce a positive membrane current and will hyperpolarize the cell by decreasing the membrane potential, conversely, positive charges entering the cell will depolarize it. Since the individual ion equilibrium potentials differ from the resting potential, there are total inward or outward net currents of the different ions. To maintain constant ion concentrations on either side ion pumps compensate these currents by moving the ions actively against their diffusive flow.

In linear approximation the membrane current $i_{x}$ of ion species $x$ is given by the Ohms law:

$$
i_{x}=\tilde{g}_{x}\left(v-v_{x}\right)
$$

where $\tilde{g}_{x}$ is the membrane conductance of the ion $x$ that can depend on the membrane potential and other neurophysiological parameters, such as the single ion channel conductance and the ion channel density that varies from a few to hundreds of channels in a square micrometer of membrane. Hyper- and depolarizations of the cell can also be induced by an external current $i_{\mathrm{e}}$ inserted via an electrode or through synaptic currents $i_{\text {syn }}$ (see also sec. 2.7). The chloride conductances are often combined with possible other conductances through the membrane into a single constant leak conductance $g_{\mathrm{L}}$ with leak reversal potential $v_{L}$ giving rise to a leak current $i_{\mathrm{L}}=g_{\mathrm{L}}\left(v-v_{\mathrm{L}}\right)$.

Combining all the described currents using Kirchhoff's laws and assuming that the ionic currents are all independent from each other this leads to the evolution equation for the membrane potential

$$
c_{\mathrm{m}} \frac{d}{d t} v=i_{\mathrm{e}}+i_{\mathrm{syn}}+i_{\mathrm{L}}+\sum_{x} i_{x}=i_{\mathrm{e}}+i_{\mathrm{syn}}+i_{\mathrm{L}}+i_{\text {ion }}
$$

where we defined $i_{\text {ion }}=\sum_{x} i_{x}$ as the total ion current. In using the total capacity $c_{\mathrm{m}}$ in this equation we assumed the neuron to be point-like. By replacing $c_{\mathrm{m}}$ by $c_{\mathrm{s}}$ and the the conductances by specific conductances per membrane area, eq. (2.2.2) yields specific currents per membrane area and eq. (2.2.3) becomes that of a patch of membrane and can be generalized to spatially extended neurons. For a passive membrane $i_{\text {ion }}=0$ and

$$
c_{\mathrm{m}} \frac{d}{d t} v=i_{\mathrm{e}}+i_{\mathrm{syn}}+g_{\mathrm{L}}\left(v_{\mathrm{L}}-v\right)
$$


(a)

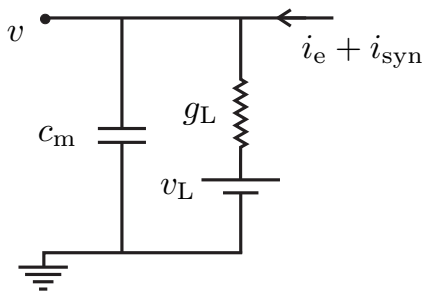

(b)

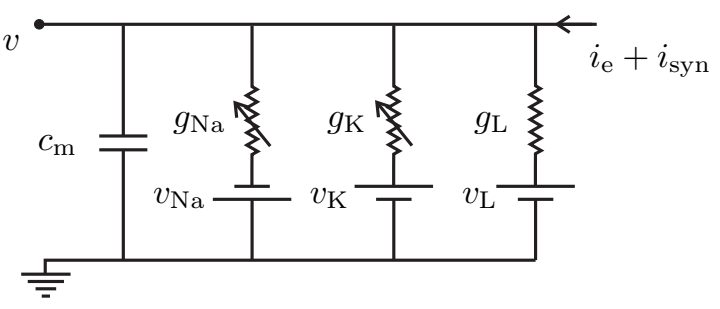

Figure 2.2. Equivalent electrical circuits for (a) a passive membrane (2.2.4) used e.g. in the leaky integrate-and-fire neuron eq. (2.6.4) and (b) for a active neuronal membrane (2.2.3), for example given by the cortical neuron model eq. (2.4.5).

The evolution equations above correspond to equivalent electric circuit diagrams shown in fig. 2.2. Here the potassium and sodium channels are conductances connected in series to a battery resembling the reversal potentials. Both conductances are drawn adjustable since they usually depend on the membrane potential rendering eq. (2.2.3) nonlinear and serve as the basis of the action potential generation discussed in the next section.

\subsection{Action Potential Generation}

Depolarization of the cell may cause a sudden onset of a short voltage pulse, the action potential. Action potentials are generated by nonlinearities associated with voltagedependent conductances.

A single channel has a voltage-dependent probability $P_{x}$ to be open. If the channel density is high $P_{x}$ can be identified with the actual fraction of open channels and thus the total conductivity may be written as $\tilde{g}_{x}(t)=g_{x} P_{x}(v(t))$ where $g_{x}$ is the maximal conductance when all channels are open.

Opening and closing of the channels usually involves conformational changes of more than one subunit of the channel molecule. The probability for such a subunit to be "open" is denoted by a single activation variable $m_{x}$. Some types of channels can undergo a further transition to an inactivated state in which the channel is blocked which is taken into account by an inactivation variable $h_{x}$ giving the probability that the channel (or one of its subunits) is not blocked. The variables $m_{x}$ and $h_{x}$ are also called gating variables which we denote by $a_{x, i}$. The total open probability is given by the product $P_{x}=m_{x}^{q_{x}} h_{x}^{p_{x}}$, or more generally by $P_{x}=\prod_{i} a_{x, i}^{q_{x, i}}$ where the exponents $q_{x}, p_{x}$ and $q_{x, i}$ are parameters related to the number of subunits relevant for opening or blocking the channel respectively. Thus, using (2.2.2), the active membrane current $i_{x}$ for ions of species $x$ can be written as

$$
i_{x}=g_{x} \prod_{i} a_{x, i}^{q_{x, i}}\left(v_{x}-v\right)
$$

Assuming that the transformational changes of an ion channel represented by a gating variable $a$ happen with voltage dependent rates $\alpha_{a}=\alpha_{a}(v)$ for opening and with $\beta_{a}=$ $\beta_{a}(v)$ for closing (or blocking) one obtains the master equation

$$
\frac{d}{d t} a=(1-a) \alpha_{a}-a \beta_{a}
$$

for their evolution. Equation (2.3.2) can be rewritten as

$$
\tau_{a}(v) \frac{d}{d t} a=a_{\infty}(v)-a
$$


(a)

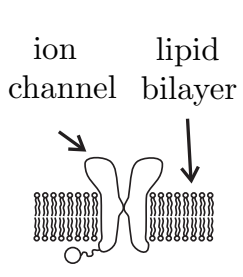

closed
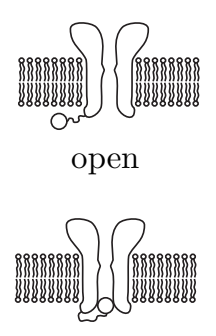

blocked (b)

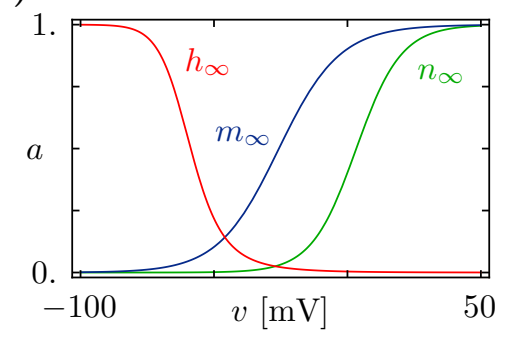

(c)

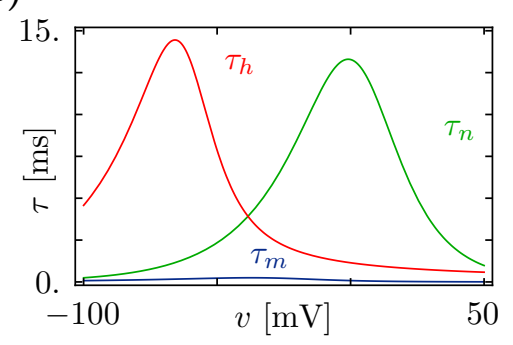

(d)

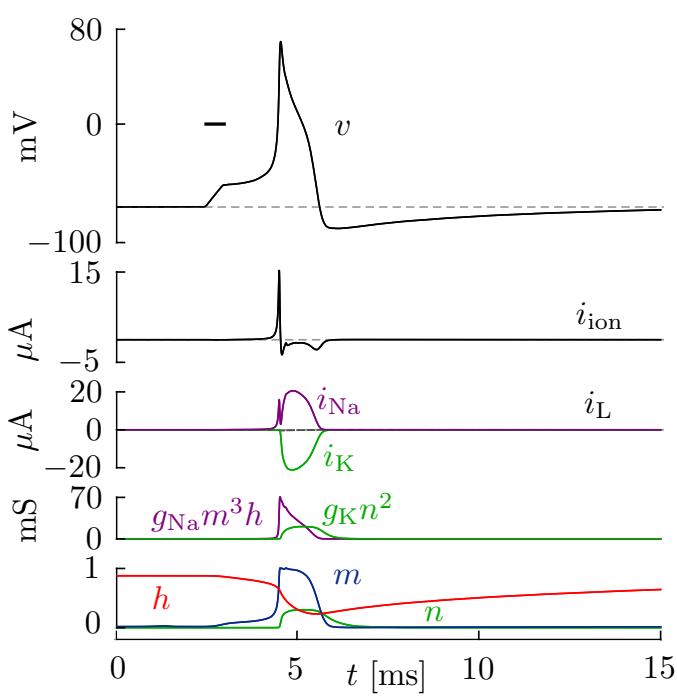

Figure 2.3. Action potential generation in conductance based neuron models. (a) Ion channels can be closed, open or blocked due to conformational changes. (b) time constants $\tau_{n}, \tau_{m}$ and $\tau_{h}$ and (b) steady-state activations $n_{\infty}, m_{\infty}$ and $h_{\infty}$ as a function of the membrane potential for the cortical neuron model in sec. 2.4.4. (d) action potential dynamics. The action potential in the membrane potential (top row) was evoked by a brief depolarizing current (horizontal bar). The second row shows the total ionic membrane current $i_{\text {ion }}$ composed of the sodium $i_{\mathrm{Na}}$ (purple), potassium $i_{\mathrm{K}}$ (green) and leak $i_{L}$ (gray) currents plotted in the row below. The channel conductances for $\mathrm{Na}^{+}$(purple) and $\mathrm{K}^{+}$(green) are shown in the second last row and are determined from the gating variables $m, h$ and $n$, eq. (2.4.5), plotted in the bottom row.

showing that for fixed $v$ the gating variables relax exponentially to a stationary value

$$
a_{\infty}(v)=\frac{\alpha_{a}(v)}{\alpha_{a}(v)+\beta_{a}(a)} \quad \text { with a time constant } \quad \tau_{a}(v)=\frac{1}{\alpha_{a}(a)+\beta_{a}(v)} .
$$

Equations (2.2.3), (2.3.1) and (2.3.3) define the class of conductance based neuron models. Note that for some channel types the current voltage relations might be more appropriately described by the Goldman-Hodgkin-Katz expression or a kinetic-barrier scheme [167] and the dynamics of the gating variables might involve a multistate Markov chain [68].

To understand the mechanism underlying an action potential it is convenient to look at the time evolution of the gating variables in the from of eq. (2.3.3) with voltage dependent asymptotic values $a_{\infty}(\mathrm{v})$ and relaxation times $\tau_{a}(v)$ shown for a cortical neuron model in fig. 2.3. Important for the onset of the action potential is the separation of timescales between the fast activation variable $m$ and the slower inactivation variable $h$. A depolarizing input will cause the fast variable $m$ to increase, resulting in an opening of the sodium channels. As a consequence positive sodium ions will flow into the cell and raise the membrane potential even further. If the initial depolarization is large enough this establishes a positive feedback responsible for the fast rise of the action potential (cf. fig. 2.3). Due to the high membrane potential the slower inactivation variable $h$ will decrease which shuts off the influx of $\mathrm{Na}^{+}$. On a similar timescale the potassium channels will open and $\mathrm{K}^{+}$ions will leave the cell decreasing the membrane potential further and even hyperpolarizing it until the potassium conductances close again and the resting potential is reached. The overall effect of these currents is a short action potential with a 
duration $\tau_{\text {ap }}$ in the order of $2-5 \mathrm{~ms}$ (cf. fig. 2.3).

The after-hyperpolarization that follows the action potential is one reason for the refractoriness of neurons. One distinguishes between an absolute refractory period in which it is impossible to elicit another spike and a relative refractory period where it is generally more difficult to cause a spike through excitatory inputs compared to the resting state. Another reason for neural refractoriness is the large number of open channels shortly after the potential peak which increases the membrane conductance so that depolarizing current pulses will decay more rapidly.

In the following we list several conductance based neuron models used in this thesis.

\subsection{Conductance Based Neuron Models}

\subsubsection{The Hodgkin Huxley Model}

Using recordings from the squid giant axon Nobel laureates Alan Hodgkin and Andrew Huxley [171] where the first to unravel the biophysical mechanisms behind the action potential generation. From experiments with the giant squid axon they determined the exponents for the gating variables, the voltage dependence of the transition rates as well as the reversal potentials and maximal conductances. The Hodgkin-Huxley model is given by

$$
c_{\mathrm{m}} \frac{d}{d t} v=i_{\mathrm{e}}+i_{\mathrm{syn}}+g_{\mathrm{L}}\left(v_{\mathrm{L}}-v\right)+g_{N a} m^{3} h\left(v_{N a}-v\right)+g_{K} n^{4}\left(v_{K}-v\right)
$$

with activation variables $m$ and $n$ for $\mathrm{Na}$ and $\mathrm{K}$ and sodium inactivation $h$ evolving according to (2.3.3) and transition rates and constants listed in appendix D.1.

Though the Hodgkin-Huxley model captures the essence of spike generation, it is a model for a squid axon which has electrophysiological properties that can be quite different from those of a cortical neuron [279].

\subsubsection{The Morris-Lecar Neuron Model}

The Morris-Lecar neuron model was introduced by Morris and Lecar [273] to describe the action potential generation in the barnacle giant muscle fiber from a series of experiments from 1969 to 1979. In contrast to the $\mathrm{HH}$ model, not sodium but calcium is responsible for the spike onset. Blocking either the $\mathrm{Ca}$ or $\mathrm{K}$ channels and changing the concentration of the ions in the external media lead to the following model,

$$
\frac{d}{d t} v=i_{\mathrm{e}}+i_{\mathrm{syn}}+g_{\mathrm{L}}\left(v_{\mathrm{L}}-v\right)+g_{\mathrm{Ca}} m\left(v_{\mathrm{Ca}}-v\right)+g_{\mathrm{K}} w\left(v_{\mathrm{K}}-v\right)
$$

with activation variables evolving according to (2.3.3)

$$
\begin{aligned}
m_{\infty}(v) & =\frac{1}{2}\left(1+\tanh \left(\frac{v-v_{1}}{v_{2}}\right)\right) & w_{\infty}(v) & =\frac{1}{2}\left(1+\tanh \left(\frac{v-v_{3}}{v_{4}}\right)\right) \\
\lambda_{m}(v) & =\tau_{m}^{-1}(v)=\Phi^{-1} \cosh \left(\frac{v-v_{1}}{2 v_{2}}\right) & \lambda_{w}(v) & =\tau_{m}^{-1}(v)=\Phi^{-1} \cosh \left(\frac{v-v_{3}}{v_{4}}\right)
\end{aligned}
$$

and no inactivation present. Parameters are given in appendix D.2. The form of the activation dynamics (2.4.3) can be explained thermodynamically in terms of statistical mechanics [218]. The activation dynamics for calcium, $m$, are fast and can therefore be 
substituted by the steady state value $m_{\infty}$ (cf. also sec. 2.6) to give the version of the Morris-Lecar model we use in this thesis:

$$
\begin{aligned}
c_{\mathrm{m}} \frac{d}{d t} v & =i_{\mathrm{e}}+i_{\mathrm{syn}}+g_{L}\left(v_{\mathrm{L}}-v\right)+g_{\mathrm{Na}} m_{\infty}(v)\left(v_{\mathrm{Na}}-v\right)+g_{\mathrm{K}} w\left(v_{\mathrm{K}}-v\right) \\
\frac{d}{d t} w & =\frac{1}{\tau_{w}(v)}\left(w_{\infty}(v)-w\right)
\end{aligned}
$$

The advantage of this model is that it is two dimensional which allows a representation of the phase portrait and of the trajectories in the phase plane and is therefore commonly used to study neuronal excitability from a dynamical systems point of view (cf. sec. 3.2). The Morris-Lecar model can be viewed as a hybrid between the realistic Hodgkin-Huxley and the abstract Fitzhugh-Nagumo model (cf. sec. 2.6.3). It is know that for different parameter the model exhibits either type I or type II neuronal excitability $[100,316]$ (cf. also chap. 9).

\subsubsection{Wang-Buzsaki Neuron Model}

The Wang-Buzsaki neuron is a conductance based model based on the Hodgkin-Huxley model (cf. sec. 2.4.1) but with adapted parameters to match the properties of cortical neurons [400]. It was used to study the mechanisms involved in the generation of gamma oscillations in the hippocampus by interneurons. The model has a three dimensional state space and is defined as the Hodgkin-Huxley equation (2.4.1) with $m$ substituted by $m_{\infty}$ and (2.3.2) for the gating variables with activation functions, time constants and standard parameter given in appendix D.3.

\subsubsection{The Fast Spiking Cortical Neuron Model by Erisir}

The neuron model by Erisir et al. [99] was introduced to study the role of Kv3.1-Kv3.2 ion channels for fast spiking neocortical interneurons with almost no adaptation. It is given by

$$
c_{\mathrm{m}} \frac{d}{d t} v=i_{\mathrm{e}}+i_{\mathrm{syn}}+g_{\mathrm{L}}\left(v_{\mathrm{L}}-v\right)+g_{\mathrm{Na}} m^{3} h\left(v_{\mathrm{Na}}-v\right)+g_{\mathrm{K}} n^{2}\left(v_{\mathrm{K}}-v\right)
$$

where $m$ and $h$ are the activation and inactivation variables of the sodium channels, and $n$ is the potassium activation that appears only quadratically in the equation. The gating variables evolve according to eq. (2.3.2) with experimentally determined rate functions given in the appendix D.4. The original model also includes a second slow potassium current representing a small fraction of the total membrane current and is responsible for weak adaptation effects of the spike rate [99, 123].

\subsubsection{The Connor-Stevens and Rose-Hindmarsh Model with A- type-current}

Besides the sodium and potassium conductances encountered in the above models there is a zoo of ion channels [167] modifying the properties of neuronal excitability. Connor and Stevens [61, 62, 63] included a second transient potassium A-channel (see also [280]) 
in their model with gating variables $a$ and $b$ to obtain:

$$
\begin{aligned}
c_{\mathrm{m}} \frac{d}{d t} v=i_{\mathrm{e}}+ & i_{\mathrm{syn}}+g_{\mathrm{L}}\left(v_{\mathrm{L}}-v\right) \\
& +g_{\mathrm{Na}} m^{3} h\left(v_{\mathrm{Na}}-v\right)+g_{\mathrm{K}} n^{4}\left(v_{\mathrm{K}}-v\right)+g_{\mathrm{A}} a^{3} b\left(v_{\mathrm{A}}-v\right)
\end{aligned}
$$

to fit the dynamics of molluscan neurons $[61,62]$ and later crab neurons [63]. The form of the steady state activation and time constants are given in appendix D.5. The A channel is activated at rest and contributes to an outward current of potassium channels. It slowly deactivates for increasing membrane potential and in this way delays the onset of spiking. Other effects, such as rebound spikes after release of hyperpolarizations are also attributed to the A-channel [323, 68].

The large phase space dimensionality of the Connor-Stevens model makes an analysis of the dynamics difficult. A systematic simplification of the model similar to the reduction process of the Hodgkin-Huxley equations in [220] was done by Rose and Hindmarsh [319, $320]$ to result in the Rose-Hindmarsh neuron model

$$
\begin{aligned}
c_{\mathrm{m}} \frac{d}{d t} v=i_{\mathrm{e}}+ & i_{\mathrm{syn}}+g_{\mathrm{L}}\left(v_{\mathrm{L}}-v\right) \\
& +g_{\mathrm{Na}} m_{\infty}^{3}\left[0.85-3\left(q-A * b_{\infty}(v)\right)\right]\left(v_{\mathrm{Na}}-v\right)+g_{\mathrm{K}} q\left(v_{\mathrm{K}}-v\right)
\end{aligned}
$$

with an effective activation variable $q$ that evolves also via eq. (2.3.3). The parameters and functions are given in appendix D.5.

\subsubsection{Reduced Traub-Miles Pyramidal Neuron Model}

A detailed neuron model for pyramidal neurons of the Hippocampus was developed by Traub and Miles [377, 375]. A simplified version of this model [375] which we employ in this thesis is given in appendix D.6 and was used to study synchronization in the presence of conduction delays [103]. Pyramidal neurons are larger cells that have a complex morphology and a distribution of ion channels within the membrane that depends on the precise location on the dendrite or axon. This allows for non-linear processing already in the dendritic part of the neuron $[309,261]$. To capture the dynamics the original TraubMiles model therefore divides the neuron in different compartments as discussed in the next section.

\subsection{Compartmental Neuron Models}

Neurons have an extended morphological structure (cf. fig. 2.1 and fig. 12.1). As a consequence the membrane potential can vary considerably when measured at different positions on the neuron, e.g. the potential at the dendrite usually differs from that at the soma. Also the ion channel densities can vary as a function of the location. Compartment models take into account these effects by subdividing the neuron into different resistively coupled components representing parts of the nerve cell with negligible variation in the membrane potential [309, 34] (cf. fig. 2.4).

The simplest multi-compartment model has two compartments consisting of a soma and a dendrite (cf. fig. 2.4). If the density of active ion channels in the dendrite is smaller than at the soma the dendritic membrane potential $v_{D}$ can be modeled as a passive leaky integrator (eq. 2.2.4), whereas the potential at the soma $v_{\mathrm{S}}$ is modeled using an active 
(a)

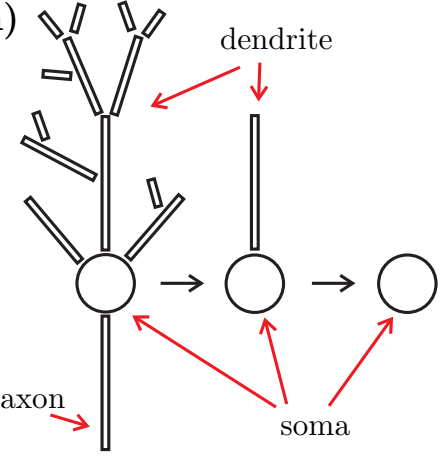

(b)

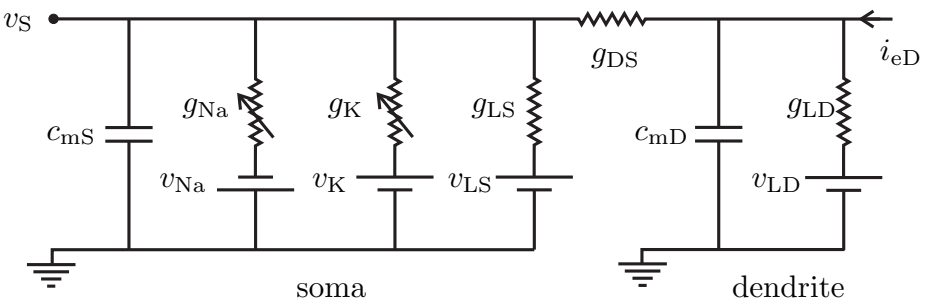

Figure 2.4. Compartmental neuron models. (a) a real neuron may be reduced to a multicompartment model which can consists of up to several hundreds of parts [34]. By choosing coarser and coarser partitions two compartment models with dendrite and soma and finally point models consisting only of a single somatic compartment are obtained. This simplification decreases the accuracy of the models but facilitates the study of neuronal excitability and collective effects in neuronal networks. (b) equivalent circuit for a two compartment model with active soma and passive dendrite (assuming $g_{\mathrm{DS}}=g_{\mathrm{SD}}$ ).

membrane (e.g. eq. 2.2.3). For two compartments that are coupled with a intra-cellular conductance $g_{\mathrm{DS}}$ one obtains the system of differential equations

$$
\begin{aligned}
c_{\mathrm{S}} \frac{d}{d t} v_{\mathrm{S}} & =i_{\text {tot, } \mathrm{S}}+g_{S D}\left(v_{\mathrm{D}}-v_{\mathrm{S}}\right) \\
c_{\mathrm{D}} \frac{d}{d t} v_{\mathrm{D}} & =i_{\mathrm{tot}, \mathrm{D}}+g_{\mathrm{DS}}\left(v_{\mathrm{S}}-v_{\mathrm{D}}\right)
\end{aligned}
$$

Note that, as compartment models arise as a space discretized version of an underlying space-continuous model, the coupling conductances $g_{\mathrm{DS}}$ and $g_{\mathrm{SD}}$ do not have to be the same [68].

The extended structure of neurons has important consequences for the integration of synaptic inputs. For example the location of synapses on the dendrite influences the synaptic efficiency of evoking action potentials: Proximal synapses, i.e. synapses closer to the soma, usually have a stronger effect on the somatic integration than distal ones [309, 310, 261]. Moreover, in the simple two compartment neuron above using a passive dendrite the action potential generation and subsequent reset of the membrane potential at the soma does not reset the dendritic membrane potential [38, 321]. Thus excitatory dendritic inputs triggering a spike at the soma can contribute additional excitatory currents during and after the reset of the soma thereby effectively resetting the neuron only partially (cf. part III).

\subsection{Simplified Models of Neuronal Activity}

Detailed conductance-based models provide a high biophysical realism and almost all parameters have a biophysical meaning. However, these models are generally not suitable for an analytical treatment, especially in the analytical study of collective network dynamics that often leads to better insights into the mechanisms behind emergent phenomena. Therefore we here introduce some systematic simplification methods that keep the main characteristics of neuronal dynamics but are reduced in state and parameter space dimension. 


\subsubsection{Reduction of a Cortical Neuron Model to Integrate-and- Fire Models}

Here we show how the cortical neuron by Erisir (cf. sec. 2.4.4) can be reduced to a non-linear integrate-and-fire model with a single variable using a reduction technique introduced in $[315,1,123]$.

In a first step we make use of the observation that reaching a large enough membrane potential is sufficient to trigger the generation of an action potential and that its shape is to first approximation insensitive to the way the threshold was reached [123]. We thus replace the short period of action potential generation and membrane potential reset involving complicated dynamical changes in the gating variables by a simple threshold process: Upon reaching a threshold potential $v_{\theta}$ the membrane is reset to a fixed value $v_{\mathrm{r}}$

$$
v\left(t^{-}\right) \geq v_{\theta} \Rightarrow v(t)=v_{\mathrm{r}}
$$

To model the absolute refractory period it is pinned to this values for a time $\tau_{\text {ap }}$ after which the integration of the membrane potential is started again (cf. fig. 2.5). Additionally the gating variables must also be reset to appropriate values.

In a second step we reduce the dimensionality of the system. Due to fact that the relaxation time $\tau_{\mathrm{m}}(v)$ of the activation variable $m$ towards the asymptotic value $m_{\infty}$ is small for all $v$ (cf. fig. 2.5) we can approximate $m(t) \approx m_{\infty}(v(t))$ reducing the model to three degrees of freedom. This reduction can mathematically be justified using Tikhonov's theorem [368]. From fig. 2.5 one observes further that the gating variables $n$ and $h$ vary little outside the action potential region and thus can be replaced by constant average

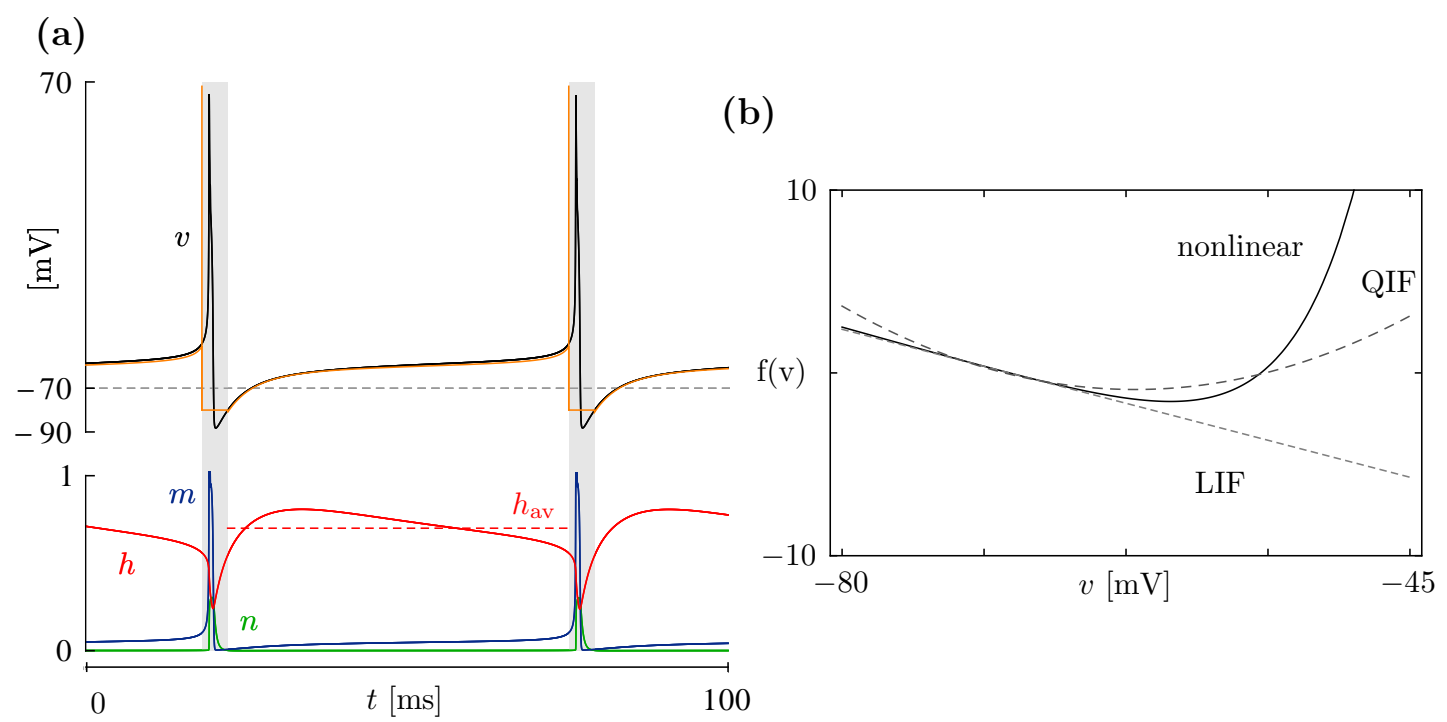

Figure 2.5. Simplification of neuronal models. (a) The neuron model eq. (2.4.5) has four variables, the membrane potential $v$, and the gating variables $m, n$ and $h$, and shows a periodic spiking when driven by an external current $i_{\mathrm{e}}=3.5$ (other parameters in appendix D.4). In a first step the action potential generation involving rapid changes in the state variables (gray shaded region) is substituted by a simpler threshold process with subsequent absolute refractory period. In a second step the fast variable $m$ is replaced by its asymptotic value $m_{\infty}$ and $h$ and $n$ are approximated by constant average values. This yields a non-linear IF model with membrane potential shown in orange. (b) The functions $f$ for the non-linear IF, the QIF and LIF model approximating the original model quadratically and linearly. 
values $h_{\mathrm{av}}$ and $n_{\mathrm{av}}$ (cf. [123]). We arrive at an equation for the membrane potential only

$$
c_{\mathrm{m}} \frac{d}{d t} v=i_{\mathrm{e}}+i_{\mathrm{syn}}+f(v)
$$

with a nonlinear function $f(v)=i_{\text {tot }}\left(v, m_{\infty}(v), h_{\text {av }}, n_{\text {av }}\right)$ that is plotted in fig. 2.5. For other neurons $f$ usually has different nonlinear shapes and the general model (2.6.2) accompanied with the reset condition (2.6.1) is know as the nonlinear integrate-and-fire neuron [3, 110, 123, 107].

When approximating $f$ by a quadratic function (cf. fig. 2.5) this results in the quadratic integrate-and-fire (QIF) neuron [235, 236, 154, 101]

$$
c_{\mathrm{m}} \frac{d}{d t} v=i_{\mathrm{e}}+i_{\mathrm{syn}}+g_{2}\left(v-v_{\mathrm{r}}\right)\left(v-v_{\mathrm{t}}\right)
$$

with a possible additional constant term of $f$ absorbed in $i_{\mathrm{e}}$. Approximating $f$ with a linear function leads to the leaky integrate-and-fire (LIF) model [234, 217, 380] with passive membrane time evolution eq. (2.2.4):

$$
c_{\mathrm{m}} \frac{d}{d t} v=i_{\mathrm{e}}+i_{\mathrm{syn}}+g_{\mathrm{L}}\left(v_{\mathrm{L}}-v\right) .
$$

Note that all these models have a strongly nonlinear reset (2.6.1).

In the following we list further simplified neuron models used in this thesis.

\subsubsection{Rinzel Model}

In the Rinzel model [315] no artificial threshold is introduced, but instead it is made use of the observation that in neuron models the $h$ and the $n$ activations evolve similar but reflected along the x-axes (cf. e.g. fig. 2.5), so that both $h$ and $n$ are expressed as a function of an effective activation $w$ :

$$
\begin{aligned}
c_{\mathrm{m}} \frac{d}{d t} v & =i_{\mathrm{e}}+i_{\mathrm{syn}}+g_{\mathrm{L}}\left(v_{\mathrm{L}}-v\right)+g_{\mathrm{Na}} m_{\infty}(v)^{3}(1-w)\left(v_{\mathrm{Na}}-v\right)+g_{\mathrm{K}}\left(\frac{w}{s}\right)^{2}\left(v_{\mathrm{K}}-v\right) \\
\frac{d}{d t} w & =\frac{1}{\tau_{w}(v)}\left(w_{\infty}(v)-w\right)
\end{aligned}
$$

with constants and functions given in appendix D.7.

\subsubsection{The Fitzhugh-Nagumo Model}

The abstract Fitzhugh-Nagumo model arises form a simplification of the neuronal dynamics similar to the steps shown above [108, 109], see also [278]. It is a widely used prototype for excitable systems in biology and chemistry. As a two dimensional system it is particularly useful for phase plane analysis and as its vector field is limited to cubic polynomials it is applicable to normal form theory (cf. sec. 3.2.1). It is given by

$$
\begin{aligned}
\frac{d}{d t} v & =i+\left(m_{1}-v\right)\left(m_{2}-v\right)\left(m_{3}-v\right)-w=i+q_{m}(v)-w \\
\frac{d}{d t} w & =l_{1}\left(v-l_{2} w\right)
\end{aligned}
$$

with $q_{m}(v)=\left(m_{1}-v\right)\left(m_{2}-v\right)\left(m_{3}-v\right)$ and standard parameter

$$
\kappa=\left(m_{1}, m_{2}, m_{3}, l_{1}, l_{2}\right)=\left(\frac{1}{3}, 1,0, \frac{1}{10}, 1\right)
$$

for which the system shows the dynamics of an relaxation oscillator [188]. 


\subsubsection{From the QIF to the Theta Neuron Model}

The theta neuron model $[101,177,151]$ is the result of a coordinate and state space transformation of the QIF neuron model. By an appropriate shift and rescaling of $v$ and $i$ in the QIF model eq. (2.6.3) we can assume without loss of generality that $c_{\mathrm{m}}=1, g_{2}=2$, $v_{\mathrm{r}}=0$ and $v_{t}=1$ to give the normalized QIF model

$$
\frac{d}{d t} v=2 v(v-1)+\tilde{i}
$$

where $\tilde{i}$ is the rescaled version of $i_{\mathrm{e}}+i_{\text {syn }}$. For $\tilde{i}>\frac{1}{2}$ the quadratic differential equation (2.6.7) explodes to infinity in finite time. In this way a spike can be defined by this event followed by a reset of the membrane potential to minus infinity

$$
v\left(t^{-}\right)=\infty \rightarrow v\left(t^{+}\right)=-\infty
$$

By identifying $\pm \infty$, the potential $v$ can be mapped onto a continuous phase $\theta$ on the unit circle $S^{1} \simeq \mathbb{R} / \mathbb{Z} \simeq[0,2 \pi] /(0 \sim 2 \pi)$ via the transformation

$$
\theta=2 \arctan (2 v-1)
$$

resulting in the theta neuron model

$$
\frac{d}{d t} \theta=1-\cos (\theta)+i(1+\cos \theta)
$$

with $\theta \in S^{1}$ and $i=\frac{\tilde{i}-1 / 2}{1 / 2}$. The theta neuron model can be viewed as the canonical model or the normal form of a saddle node on invariant cycle bifurcation (cf. sec. 3.2 below) $[101,177,176]$.

\subsection{Synapses and Neuronal Connectivity}

Synapses are sites of contact between the axon of a presynaptic neuron and the dendrite or soma of a postsynaptic neuron responsible for the transmission of signals between these cells. The most common type of synapse in vertebrates is the chemical synapse. Here the axon terminal comes very close to the postsynaptic dendrite leaving only a small synaptic cleft of roughly $40 \mathrm{~nm}$ between the neurons. Voltage transients due to the arrival of action potentials in the terminal open ion channels causing an influx of $\mathrm{Ca}^{+}$. This in turn forces vesicles to bind to the cell membrane and to release neurotransmitters into the cleft. The transmitters bind to receptors on the postsynaptic side causing postsynaptic ion channels to open (cf. fig. 2.6). The type of transmitter, the receptors and the channels determine whether the unidirectional interaction is excitatory or inhibitory. In the first case the membrane potential of the postsynaptic cell in the vicinity of the synapse is depolarized and called excitatory postsynaptic potential (EPSP), whereas in the latter case it is hyperpolarized and termed inhibitory postsynaptic potential (IPSP). Glutamate and AMPA receptors lead to EPSPs while GABA [226] and NMDA receptors mediate inhibition.

Using similar considerations as for the membrane currents through ion channels (cf. sec. 2.2, eq. (2.2.2)) the synaptic current is effectively modeled by a synaptic conductance $g_{\text {syn }}$ and a synaptic reversal potential $v_{\text {syn }}$ by

$$
i_{\mathrm{syn}}=g_{\mathrm{syn}}\left(v_{\mathrm{syn}}-v\right)
$$


(a)

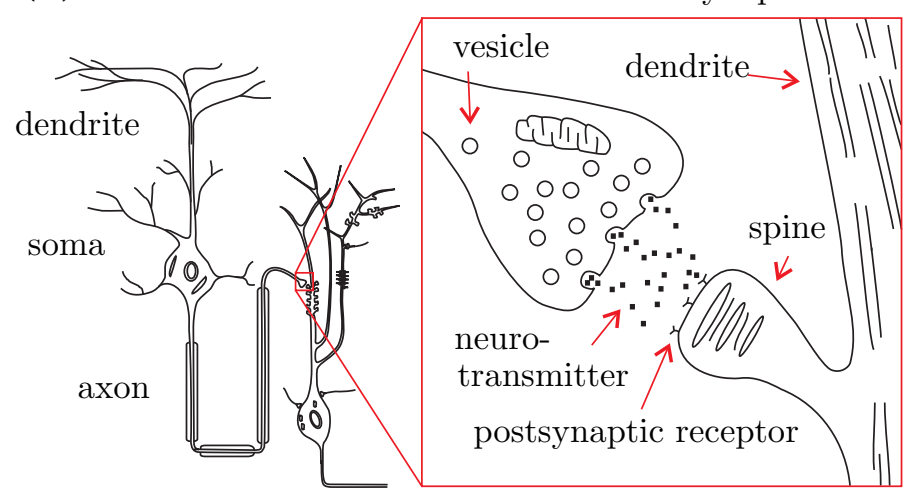

(b)

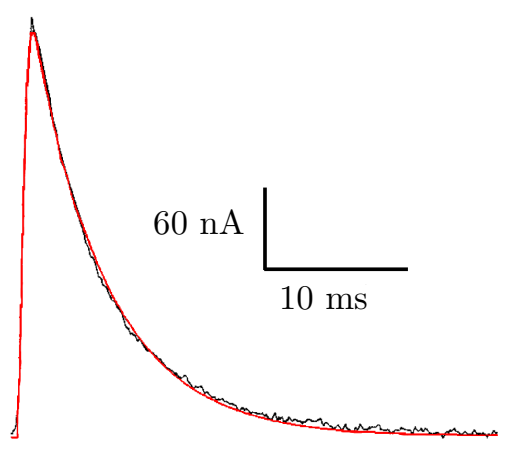

Figure 2.6. Synaptic interaction of neurons. (a) action potentials arriving at a chemical synapse cause the release of neurotransmitter in to the synaptic cleft. These bind to postsynaptic receptors which open ion channels thereby evoking a postsynaptic current. (b) fit of the alpha-function eq. (2.7.2) (red) to the average excitatory postsynaptic current (black) recorded from mossy fiber input to a CA3 pyramidal cell in a hippocampal slice preparation (adapted from [73]).

Excitatory reversal potentials typically are around $v_{\text {syn }} \approx 0 \mathrm{mV}$, inhibitory ones at $v_{\text {syn }} \approx$ $v_{\mathrm{r}} \approx-60 \mathrm{mV}$. The time evolution of synaptic conductances can be modeled in detail using Markov chains describing the morphological transitions from the closed to the open state of the channels in response to the transmitter release [68]. However, as can be seen from fig. 2.6, the synaptic conductance can be modeled to good approximation as $g_{\text {syn }}(t)=\bar{g}_{\text {syn }} \alpha(t)$ where $\bar{g}_{\text {syn }}$ is the maximal synaptic conductance and

$$
\alpha(t)=\Theta(t) \kappa\left(\exp \left(-\frac{t}{\tau_{\text {decay }}}\right)-\exp \left(-\frac{t}{\tau_{\text {rise }}}\right)\right)
$$

is called an alpha-function (cf. fig. 2.6). Here $\Theta(t)$ is the Heaviside step function

$$
\Theta(t)= \begin{cases}1 & t>0 \\ 0 & t<0\end{cases}
$$

and $\kappa$ a normalization constant chosen to set the peak value of $\alpha(t)$ to 1 . The parameter $\tau_{\mathrm{r}}$ and $\tau_{\mathrm{d}}$ are time constants determining the rise and decay of the postsynaptic potential yielding a typical time scale $\tau_{\text {syn }}$ for the synaptic interaction

$$
\tau_{\text {syn }}=\tau_{\text {rise }}+\tau_{\text {decay }}
$$

Typical time constants for fast excitatory synapses are $\tau_{\text {rise }} \approx 0.1 \mathrm{~ms}$ and $\tau_{\text {decay }} \approx 2-10 \mathrm{~ms}$. Inhibitory synapses typically have a longer decay time constant of around $\tau_{\text {decay }} \approx 10 \mathrm{~ms}$ [68].

The propagation of an action potential from the presynaptic soma to the synapse with a speed of roughly $0.4 \mathrm{~m} / \mathrm{s}$ causes transmission delays $\tau_{d}$ in the range of $0.1-10 \mathrm{~ms}$ [380]. Assuming linear additivity of the synaptic conductance, presynaptic spikes at times $t_{s}$, $s \in \mathbb{Z}$, result in a time dependent conductance

$$
g_{\mathrm{syn}}(t)=\bar{g}_{\mathrm{syn}} \sum_{s \in \mathbb{Z}} \alpha\left(t-t_{s}-\tau_{d}\right)
$$


This equation reflects the pulse-coupled nature of neurons interacting via chemical synapses, since only the discrete firing times $t_{s}$ of the presynaptic neuron enter the right hand side of eq. (2.7.5). Pulse-coupling can lead to interesting and counter intuitive collective dynamics [370, 371, 372, 16, 203] (see also below).

\subsubsection{Conductance Based Coupling in the Theta Neuron Model}

In part IV of this thesis we use networks of theta neurons coupled via conductance base synapses given by eq. (2.7.1) to investigate a mechanism for local control of information flow. The conductance based neuron model can be derived by inserting synaptic conductances into the QIF model eq. (2.6.7), setting

$$
\tilde{i}=\bar{i}+\sum_{j} g_{\mathrm{syn}, j}\left(v_{\mathrm{syn}, \mathrm{j}}-v\right)
$$

and employ the transformation (2.6.8) to the phase $\theta$ which result in the conductance base theta neuron model [31]:

$$
\frac{d}{d t} \theta=1-\cos (\theta)+\left(i+\sum_{j}\left(2 v_{\mathrm{syn}, j}-1\right) g_{\mathrm{syn}, j}(t)\right)(1+\cos (\theta))-\sum_{j} g_{\mathrm{syn}, \mathrm{j}}(t) \sin (\theta)
$$

\subsubsection{Current Based Synaptic Coupling}

Except during the short period of an action potential the membrane potential $v$ is much smaller than the synaptic reversal potential $v_{\text {syn }}$ for excitatory synapses and thus the driving potential $v_{\text {syn }}-v$ for the synaptic current may be approximated as constant. In this situation the constant $\kappa$ in (2.7.2) is chosen to normalize the total charge transferred into the postsynaptic neuron, i.e. $\int_{-\infty}^{\infty} \alpha(t) \mathrm{d} t=1$. The synaptic current then becomes

$$
i_{\mathrm{syn}}(t)=\varepsilon \sum_{s \in \mathbb{Z}} \alpha\left(t-t_{s}-\tau_{\mathrm{d}}\right)
$$

where $\varepsilon$ is the total charge transferred in a single synaptic event. For very fast synaptic interactions, i.e. $\tau_{\text {rise }} \rightarrow 0$ and $\tau_{\text {decay }} \rightarrow 0$ this current may be approximated by a sum of Dirac $\delta$-functions [44]

$$
i_{\text {syn }}(t)=\varepsilon \sum_{s \in \mathbb{Z}} \delta\left(t-t_{s}-\tau_{\mathrm{d}}\right)
$$

Usually a single neuron receives synaptic inputs from many other neurons. Due to different locations of the synapses on the dendrite and different synapse types the postsynaptic currents of neuron can vary and hence not all synapses evoke the same response in the postsynaptic neuron and are said to have different synaptic efficiency.

\subsubsection{Synaptic Shunting and Leak}

For inhibitory synapses the reversal potential is close to the resting membrane potential which is also often close to the leak reversal potential [196]. Thus an increase in inhibitory synaptic activity effectively increased the leak conductance. As the synaptic activity is typically on a time scale larger then the time scale of an action potential this shunting [88] can effectively change the constant leak conductance to a higher value. We will employ this fact in part IV of this thesis. 


\subsubsection{Neuronal Connectivity}

Neurons in the central nervous system are part of a larger network structure in which each neuron receives synaptic inputs from and makes synaptic contacts to other neurons. In fig. 2.7 the synaptic connections of neurons in the nervous system of the nematode Caenorhabditis Elegans [6] are shown. One observes that each neuron only makes connections to a small fraction of the total number of neurons. Such a network structure is said to have a sparse connectivity and is also found in other neuronal networks [36, 347,6]. In contrast there are parts of nervous tissues where neurons have a very high interconnectivity [195] and are close to an all-to-all coupled network where each neuron makes synaptic contacts to all other neurons in the network (cf. fig. 2.7).

Neuronal network connectivity is multi-scale. On the largest scale, the brain is organized into anatomically different modular regions each responsible for a certain information processing tasks [196]. On smaller scales neuronal structure is found to be organized in cortical columns [180], i.e.vertical aggregates of cells in the layered structure of cerebral cortex with similar response properties or receptive fields sharing long range interconnections. On a cellular scale, experimental studies [344] show that the statistics of synaptic connectivity may be viewed as a skeleton of strong connections with an over representation of recurrently connected motives embedded in a sea of weaker connections. In ref. [294] it was shown that in neocortex, pyramidal neurons cluster in to several interlaced groups of a few dozen neurons.

\subsubsection{Modeling Neuronal Networks with Directed Graphs}

A neural network is best described by a directed weighted graph [58, 78, 32], which consists of $N$ vertexes $i \in\{1,2, \ldots, N\}$ representing the single neurons and directed links $j \rightarrow i$ between them representing a synaptic connection from neuron $j$ to neuron $i$. To each link $j \rightarrow i$ we associate a weight $\varepsilon_{i j}$ rendering the synaptic efficiency. If there is no link

(a)

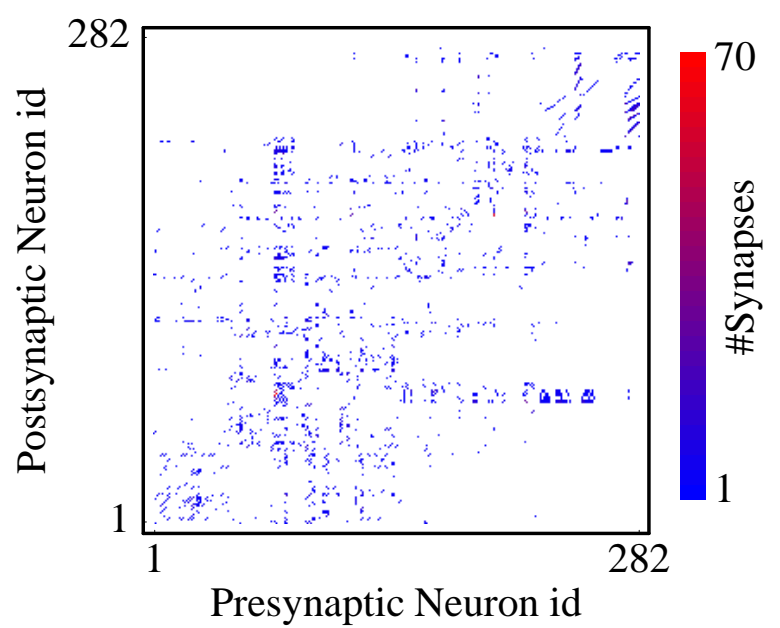

(b)

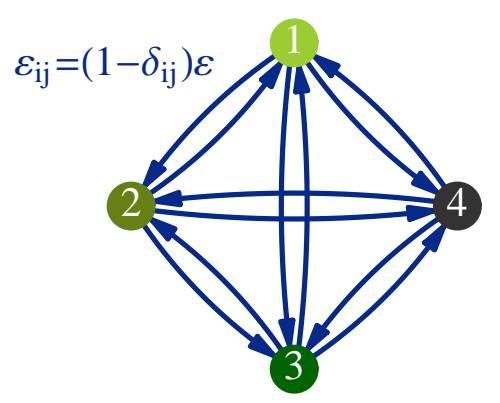

Figure 2.7. (a) Synaptic connectivity of neurons in the nervous system of the nematode Caenorhabditis Elegans containing roughly 300 neurons and 7000 synapses [6]. (b) Graph of a small $(N=4)$ homogeneous network with all-to-all coupling but no self-interactions $\varepsilon_{i j}=$ $\varepsilon\left(1-\delta_{i j}\right)$. 
between neuron $j$ to $i$ we set $\varepsilon_{i j}=0$. The $N \times N$ matrix $\varepsilon_{i j}$ is called the adjacency matrix of the network.

Most of this study is concerned with simple homogeneous all-to-all coupled networks (cf. fig. 2.7) with adjacency matrix given by

$$
\varepsilon_{i j}=\varepsilon\left(1-\delta_{i j}\right)
$$

where the Kronecker symbol $\delta_{i j}$, taking the value 1 for $i=j$ and zero otherwise, was introduced to exclude self-coupling of neurons.

\subsection{The Dynamic Patch Clamp Technique}

Most of the above neuroscientific knowledge is the result of electrophysiological experiments. Often the patch clamp technique [281, 287] is used to record the electrical activity of a neuron (cf. fig. 2.8). In contrast to recent optical methods [270], this method is invasive but yields a much higher time resolution to resolve the dynamics of single action potentials. There are three sub-types of patch clamp experiments we will use in the experimental part of this thesis:
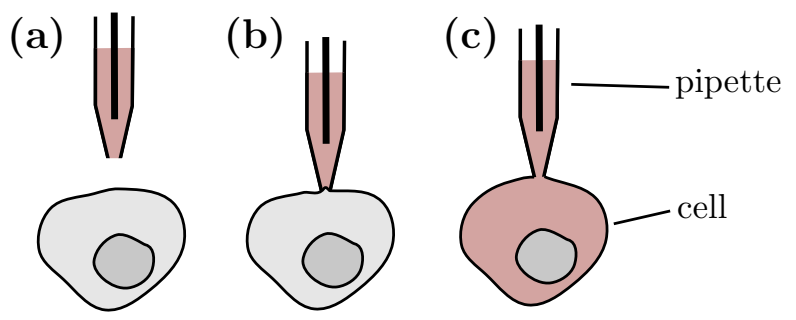

Figure 2.8. Patch-clamp recording technique. (a) to record the electrical activity from a cell an electrode is positioned via an micro-manipulator close to the cell (b) when the electrode is on the cell membrane the pressure in the electrode is lowered to obtain a high resistance between between the external medium and the inner part of the electrode (giga-seal). This constitutes the cell-attached configuration. By further lowering the pressure in the electrode or by electrical stimulation the membrane patch within the electrode can be removed leading to (c) the wholecell recording configuration.

Voltage-Clamp: The neuron membrane voltage $v$ is held constant during this type of experiment by regulating the current $i_{\mathrm{e}}$ through the patch-electrode. Measuring the currents give rise to data on the voltage dependence of the currents. If a steady state is reached the function $i_{\mathrm{e}}(v)$ is know as the $i-v$-curve.

Current-Clamp: In this type of experiment a predefined external current $i_{\mathrm{e}}$ is injected via the patch-electrode and the trans-membrane voltage $v$ is measured.

Dynamic-Clamp: Dynamic clamp experiments are patch-clamp experiments [334, 72], where the voltage is recorded form the neuron and used to compute an current $i_{\mathrm{e}}$ that is injected back into the neuron in real time. In this way a closed biological-artificial loop is obtained. This technique can be used to study the effects of different ion channels on neuronal dynamics. In part IV of this thesis we use an artificial leak conductance to study its effect on neuronal excitability. 


\section{Chapter 3}

\section{Notions from Dynamical Systems and Bifurcation Theory}

In this chapter we present the background from dynamical systems theory relevant for this thesis. An in depth treatment of the classical theory can be found in $[199,145]$. Hybrid dynamical systems are described in [129] and non-smooth dynamical systems in [76].

\subsection{Unstable Attractors and Heteroclinic Networks}

In this thesis we consider dynamical systems described by a set of states $\mathcal{X}$ together with a semi flow $\mathcal{F}^{t}: \mathcal{X} \rightarrow \mathcal{X}$ that satisfies $\mathcal{F}^{t} \circ \mathcal{F}^{s}=\mathcal{F}^{t+s}$ for $s, t \geq 0$ and $\mathcal{F}^{0}(x)=x$. Here $t$ is either continuous or discrete time. We assume that the state space $\mathcal{X}$ is a metric space with Lebesgue measure $\lambda(\cdot)$.

In part IV we encounter dynamical systems describe by an ordinary differential equation of the form

$$
\frac{d}{d t} x=f(x)
$$

where $x \in \mathbb{R}^{N}$ is the state of the system in the state space $\mathbb{R}^{N}$ and $f(x) \in T_{x} \mathbb{R}^{N}$ it's smooth vector field on the tangent bundle $T \mathbb{R}^{N}=\mathbb{R}^{N}$. For vectors $p, q \in \mathbb{R}^{N}$ the Jacobian $D f$ and Hessian $D^{2} f$ are defined by

$$
D f(x ; p):=D f(x) p:=\sum_{j} \frac{\partial f_{i}}{\partial x_{j}}(x) p_{j} \text { and } D^{2} f(x ; p, q):=\sum_{j, k} \frac{\partial^{2} f_{i}}{\partial x_{j} \partial x_{k}}(x) p_{j} q_{k} .
$$

In part III we consider hybrid dynamical systems where a continuous flow is interrupted as discrete points in time and a map is applied.

The forward orbit $\mathcal{O}(x)$ is the set of states that are encountered during the time evolution of a dynamical system starting at $x$, i.e.

$$
\mathcal{O}(x)=\left\{\mathcal{F}^{t}(x) \mid t \geq 0\right\}
$$

For a fixed point $x, \mathcal{O}(x)=\{x\}$. For a periodic point $x$ there exists a $T>0$ such that $\mathcal{F}^{T+t}(x)=\mathcal{F}^{t}(x)$ and $\mathcal{O}(x)$ is a periodic orbit. A set $\mathcal{A}$ is invariant if $\mathcal{F}^{t}(\mathcal{A}) \subset \mathcal{A}$ for all $t \geq 0$. 
Definition 3.1.1. A heteroclinic connection $\mathcal{H}_{12}$ is a collection of orbits that connects two invariant sets $\mathcal{A}_{1}$ and $\mathcal{A}_{2}$ in a saddle-sink connection, i.e. for any open neighbourhoods $\mathcal{U}_{i} \supset \mathcal{A}_{i}, \cup_{t \geq 0} \mathcal{F}^{t}\left(\mathcal{H}_{12} \cap \mathcal{U}_{1}\right) \cap \mathcal{U}_{2} \neq \emptyset$ and $\mathcal{H}_{12} \cap \mathcal{U}_{2}$ is invariant. A heteroclinic network is a graph with vertices being invariant sets $\mathcal{A}_{i}, i \in\{1, \ldots, n\}$ and directed edges $\mathcal{A}_{i} \rightarrow \mathcal{A}_{j}$ if there is a heteroclinic connection $\mathcal{H}_{i j}$ between them.

The invariant $\omega$-limit set for a point $x$ is defined by

$$
\omega(x)=\bigcap_{t \geq 0} \overline{\left\{\mathcal{F}^{s}(x) \mid s \geq t\right\}}
$$

The basin of attraction $\mathcal{B}(\mathcal{A})$ of a invariant set $\mathcal{A}$ consists of all states in state space that eventually stay in $\mathcal{A}$, i.e.

$$
\mathcal{B}(\mathcal{A})=\{x \in \mathcal{X} \mid \omega(x) \subset \mathcal{A}\}
$$

A Milnor attractor for $\mathcal{F}^{t}$ is then an compact invariant set $\mathcal{A}$ with positive measure basin $\lambda(\mathcal{B}(\mathcal{A}))>0$ and such that an proper invariant subset has a basin with stricitly smaller measure $[267,268]$.

For a subset $\mathcal{U} \subset \mathcal{X}$ we define the lingering set as consisting of points in $\mathcal{U}$ which do not leave $\mathcal{U}$ in the future

$$
\mathcal{L}(\mathcal{U})=\left\{x \in \mathcal{U} \mid \mathcal{F}^{t}(x) \in \mathcal{U} \text { for all } t>0\right\}
$$

Definition 3.1.2. (unstable attractor)[16]. A Milnor attractor $\mathcal{A}$ is an unstable attractor if there is a neighbourhood $\mathcal{U}$ of $\mathcal{A}$ such that $\lambda(\mathcal{L}(\mathcal{U}))=0$

Thus an unstable attractor has a basin with positive measure but in its neighbourhood almost all trajectories must leave this neighbourhood. Thus one unstable attractor can lie within the basin of attraction of a second unstable attractor.

Definition 3.1.3. A set of unstable attractors $\mathcal{A}_{i}, i \in\{1, \ldots, n\}$ forms a network of unstable attractors given by the graph with vertices $\mathcal{A}_{i}$ and directed edges from $\mathcal{A}_{i}$ to $\mathcal{A}_{j}$ if and only if for any neighbourhood $\mathcal{U}_{i}$ of $\mathcal{A}_{i}$ we have

$$
\lambda\left(\mathcal{B}\left(\mathcal{A}_{j}\right) \cap \mathcal{U}_{i}\right)>0
$$

Thus there is a link between two attractors $\mathcal{A}_{i} \rightarrow \mathcal{A}_{j}$ if every neighborhood of $\mathcal{A}_{i}$ contains a positive basin volume of $\mathcal{A}_{j}$. The definition extends to networks consisting of unstable as well as stable attractors.

\subsection{Bifurcation Theory and Normal Forms}

The use of bifurcation theory in this thesis is twofold: On the one hand we discover a new type of bifurcation for a hybrid dynamical system in part III. On the other hand bifurcation theory for smooth dynamical systems is used in part IV of this thesis to study neuronal excitability. Therefore, in this section the notions from bifurcation theory relevant for this thesis are introduced. A detailed introduction to the subject can be found in [355], an in-depth treatment of the subject can be found in the text books $[145,230,133,134]$. Systematic treatments of versal deformations and normal form theory are $[13,276,327,133,134]$. Aspects of bifurcation theory for continuous but non-smooth dynamical systems are studied in [76]. 
Definition 3.2.1. (Bifurcation) For a dynamical system that depends on certain parameters $\mu$ in a $d$-dimensional parameter space, the bifurcation set $\Sigma$ is the subset of parameter values for which there is no neighborhood in parameter space such that the phase portraits of the corresponding dynamical system within this neighborhood are all topologically equivalent. Thus, when passing a bifurcation point, the phase portrait changes qualitatively.

This is the general concept we need in part III of this thesis to identify a novel type of bifurcation in hybrid systems.

For part IV we need results from bifurcation theory for dynamical systems defined via ordinary differential equations. A nullcline is the set in state space at which one specific component of the vector field $f$ in (3.1.1) vanishes. At intersections of all nullclines the vector field vanishes identically and the corresponding point in state space is a fixed point.

The co-dimension of a bifurcation is defined as the minimal dimension of the parameter space in which the bifurcation occurs in a persistent way, i.e. it cannot be removed by a arbitrarily small deformation of the vector field and thus is a generic phenomenon. A bifurcation-diagram is a map that assigns to each point in the complement of $\Sigma$ the corresponding representative of the phase portrait. A bifurcation is local if the changes in the phase portrait are confined to a small neighborhood, and global if the changes also involve larger invariant sets such as limit cycles or homoclinic and heteroclinic connections.

For parameter dimension $d=1$ a generic bifurcation set consists of points of codimension one. In one dimensional bifurcation diagrams we therefore include a meaningful coordinate of the state space showing the values for fixed points or the minima and maxima attained in the case of the existence of periodic orbits. For parameter dimension $d=2$ generic bifurcation sets are lines or points corresponding to co-dimension one and two bifurcations respectively which we visualize in the plane. For a three dimensional parameter space $(d=3)$ generic bifurcation sets are surfaces, lines or points corresponding to co-dimension one, two and three bifurcations, respectively. We visualize these in a three-dimensional plot.

\subsubsection{Normal Forms and Unfoldings}

Classification of dynamical systems is an important part of dynamical systems theory [199, 276]. For example two dynamical systems $A$ and $B$ are topologically equivalent, if there exists a homeomorphism sending $A$-orbits to $B$-orbits, i.e. the phase portraits are qualitatively the same and can be grouped into a single equivalence class. Normal form theory aims at finding the 'simplest' representative of such a class. Both, the equivalence relation, as well as the definition of 'simplest' can vary depending on the questions one is interested in.

Conjugacy as an equivalence relation is defined by the transformation of a vector field $f$ under a coordinate change $s$ given by

$$
s^{*} f(x):=D s(x)^{-1} f(s(x))
$$

where $D s(x)$ is the Jacobian of the transformation $s$. For local dynamical systems, i.e. defined by Taylor expansions of the vector field around a certain point $x_{0}$ the definition of simplest is usually taken to be the largest number of monomials in the expansion that can be removed without changing the equivalence class. The simplest normal form result is the Hartman-Grobman theorem that assures that for a hyperbolic point, where the eigenvalues 
of the Jabian have non-zero real part, the vector field is conjugated to a linear flow. In general removable terms can be determined by the homological equation [276]. For fixed points with degeneracies, e.g. zero eigenvalues of the Jacobian also called singular points, only non-resonant terms can be removed and the normal form becomes non-linear.

Typically the vector field $f$ also depends on parameters that deform the vector field. An unfolding [13] of a normal form is the simplest system into which all systems close to the original system can be transformed. This can be achieved by the introduction of additional terms into the normal form that depend on a number of unfolding parameters $\mu$. Using parameter dependent coordinate transformations the unfolding parameters become functions of the original parameters of the vector field $f$.

An example for an unfolding of a normal form is given in eq. (3.3.1) for the degenerate Bogdanov-Takens point described in sec. 3.3.3 and encountered in the second part of this thesis. In the following we provide a brief description of all bifurcations encountered in this thesis.

\subsection{A Bifurcation Dictionary}

In this section a number of bifurcations relevant for this thesis are introduced. A detailed mathematical treatment of all the bifurcations listed below can be found in [87, 145, 230].

Throughout this thesis we associate to each bifurcation a specific color in the bifurca-

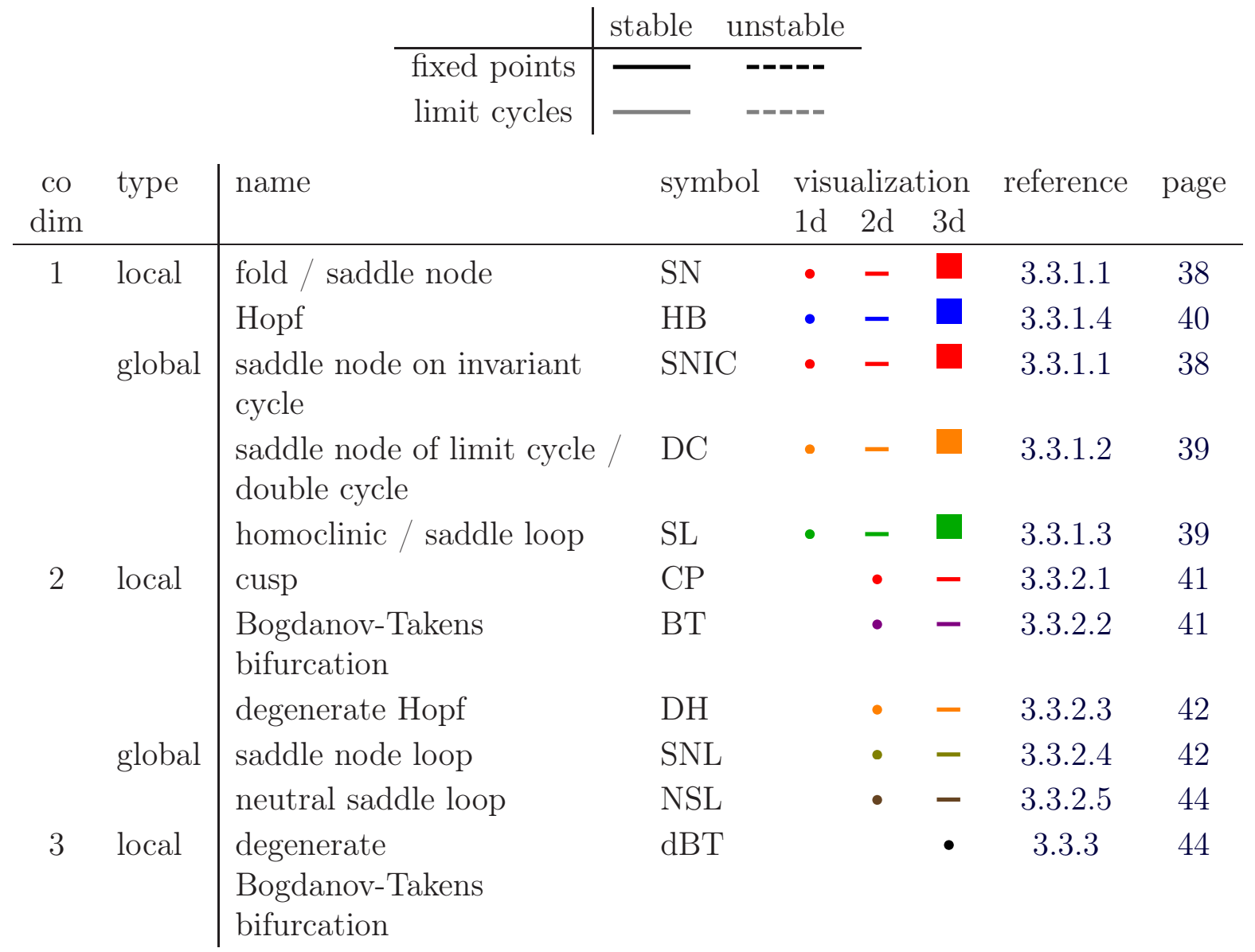

Table 3.1: List of bifurcations encountered in this thesis and their color code used in all bifurcation diagrams. 
tion diagrams with color-code given in table 3.1. The different bifurcations we encounter in our study are described below in the context of the two-dimensional Morris-Lecar neuron model (2.4.2) as its phase portraits can be visualized easily. Most of the bifurcations found in the context of neuron models are also described in [316, 177, 188].

Here, however, we use different bifurcation parameters in the figures as they not only serve for this introduction but also constitute results of part IV of this thesis.

\subsubsection{Codimension-1 Bifurcations}

\subsubsection{Saddle Node or Fold Bifurcation and Saddle Node on Invariant Cycle Bifurcation}

In a fold or saddle node (SN) bifurcation two fixed points of a vector field coalesce and vanish. In a saddle node on invariant cycle (SNIC) bifurcation the two fixed points lie
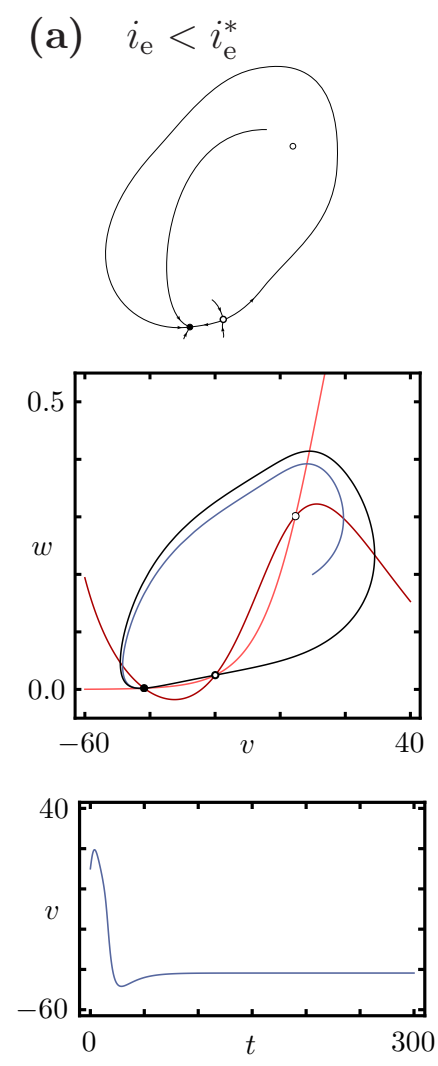

(b) $i_{\mathrm{e}}=i_{\mathrm{e}}^{*}$
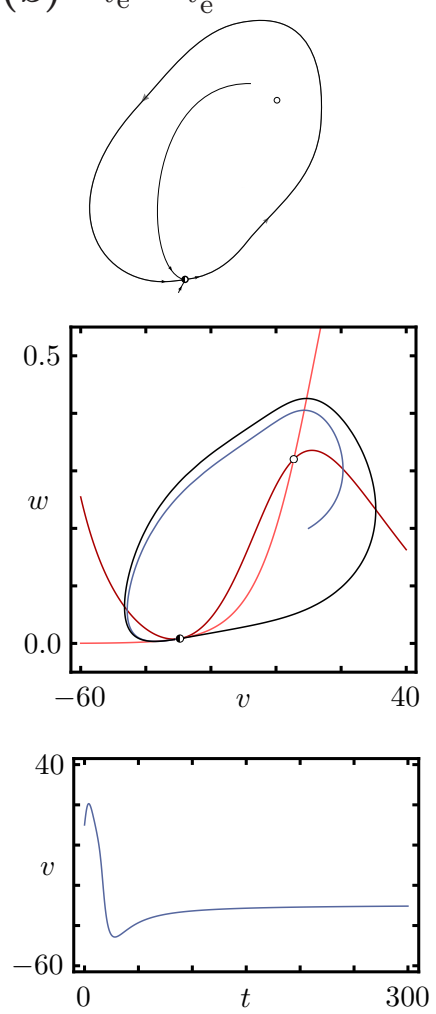

(c) $i_{\mathrm{e}}>i_{\mathrm{e}}^{*}$
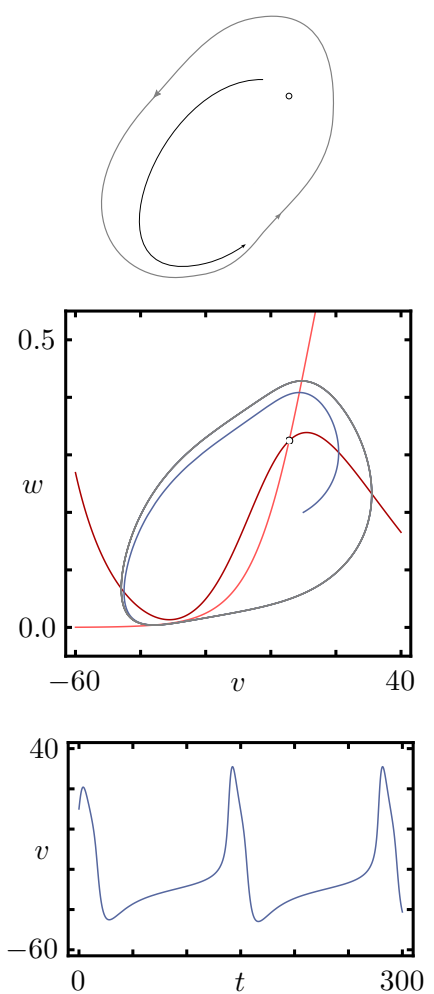

Figure 3.1. Saddle node on limit cycle (SNIC) bifurcation in the Morris-Lecar neuron model (2.4.2) (parameter as in appendix D.2) with bifurcation diagram in fig. 10.1. Top row shows sketches of the phase portrait. Second row shows actual phase portraits with nullclines (red), stable fixed points (solid dots), unstable fixed points (open dots) saddle nodes (half open dots), and sample trajectories (blue) with membrane potential evolution shown in the third row. (a) for input currents $i_{\mathrm{e}}$ below the bifurcation value $i_{\mathrm{e}}^{*}$ there are three fixed points. The unstable manifolds of the saddle approach the stable fixed point (black). Increasing $i_{\mathrm{e}}$ moves the $v$ nullcline (dark red) up so that (b) at the bifurcation value $i_{\mathrm{e}}=i_{\mathrm{e}}^{*}$ the stable fixed point and the saddle coalesce on an invariant orbit (black). (c) for $i_{\mathrm{e}}>i_{\mathrm{e}}^{*}$ the two fixed disappear leaving the system with a stable limit cycle around an unstable fixed point. Note that the dynamics on the limit cycle is slow close to the point where the fixed points disappeared due to the vincinity to the nullclines. 
on an invariant cycle and their disappearance leads to the creation of a limit cycle. This bifurcation is illustrated for the Morris-Lecar neuron model (cf. sec. 2.4.4) in fig. 3.1. A corresponding bifurcation diagram can be found in fig. 10.1 .

\subsubsection{Double Limit Cycle Bifurcation}

In a double limit cycle (DC) or saddle node of limit cycle bifurcation two periodic orbits coalesce and disappear. An example is shown in figure 3.1 with corresponding bifurcation diagram in figure 10.4 .

(a) $\quad i_{\mathrm{e}}<i_{\mathrm{e}}^{*}$
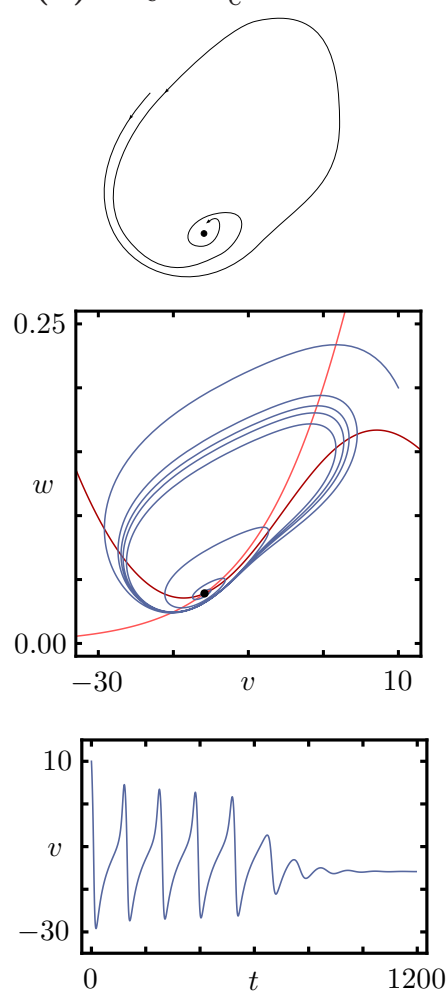

(b)
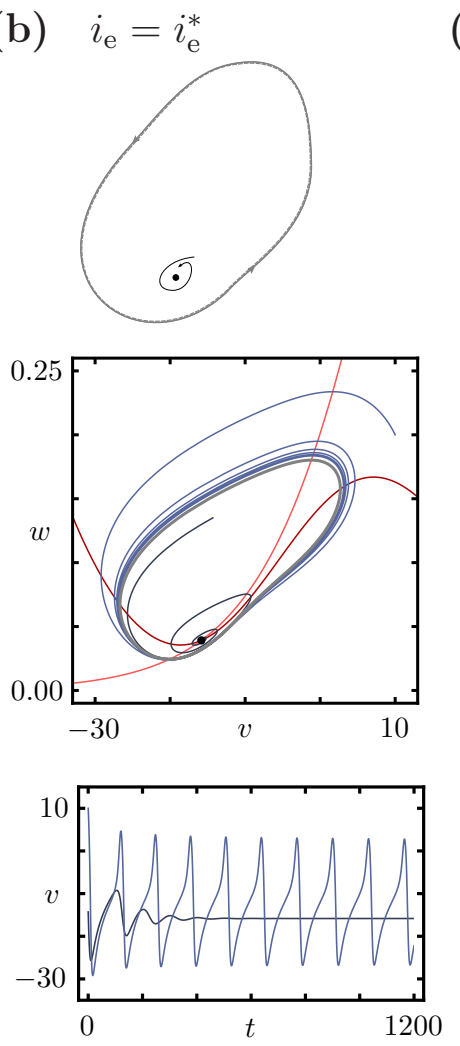

(c) $i_{\mathrm{e}}>i_{\mathrm{e}}^{*}$
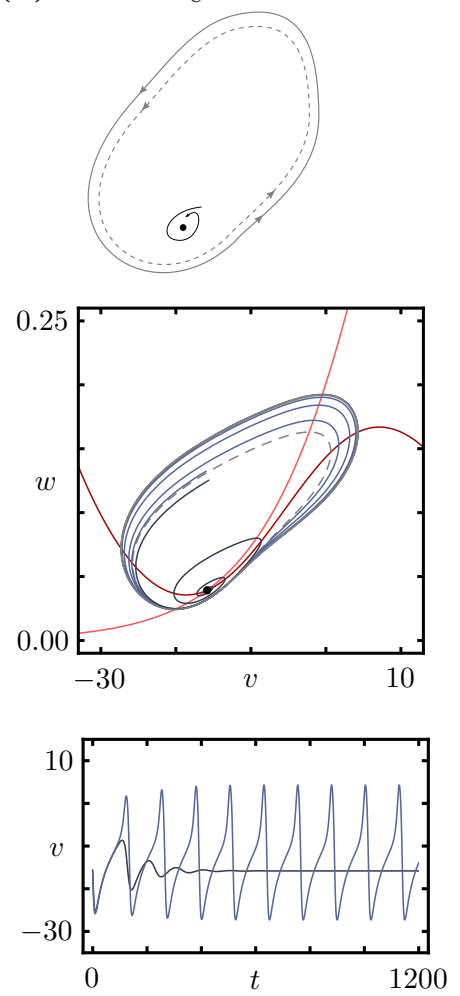

Figure 3.2. Double limit cycle (DC) bifurcation in the Morris-Lecar neuron model. Figure syntax as in 3.1. At the bifurcation value $i_{\mathrm{e}}^{*}$ a stable and unstable limit cycle (gray dashed line) are generated simultaneously. In this model after the bifurcation the unstable periodic orbit separates the basin of attraction of the stable limit cycle from the one of the coexisting stable fixed point.

\subsubsection{Homoclinic Bifurcation}

At a homoclinic or saddle loop bifurcation (SL) the stable and unstable manifolds of a saddle node form a homoclinic orbit that gives rise to the birth of a limit cycle. In neuron models one discriminates [188] between a big homoclinic bifurcation that gives rise to a large stable limit cycle surrounding the fixed points and reflecting periodic spiking dynamics (figs. 3.3, 10.2) and a small homoclinic saddle node loop that generates a unstable limit cycle around the stable fixed point (figs. 3.4, 10.3). 
(a) $i_{\mathrm{e}}<i_{\mathrm{e}}^{*}$
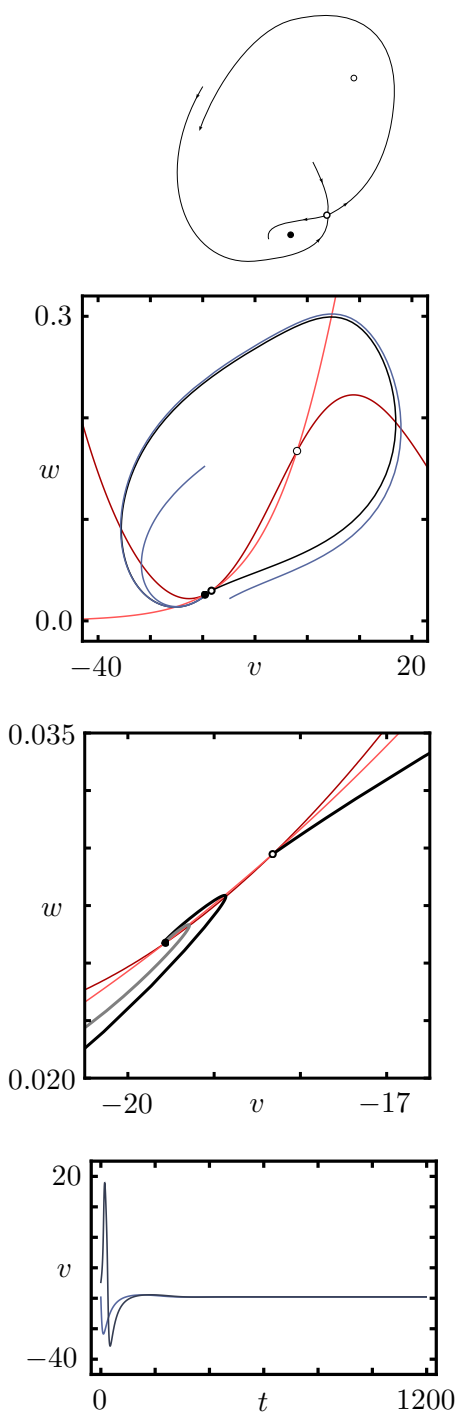

(b) $i_{\mathrm{e}}=i_{\mathrm{e}}^{*}$
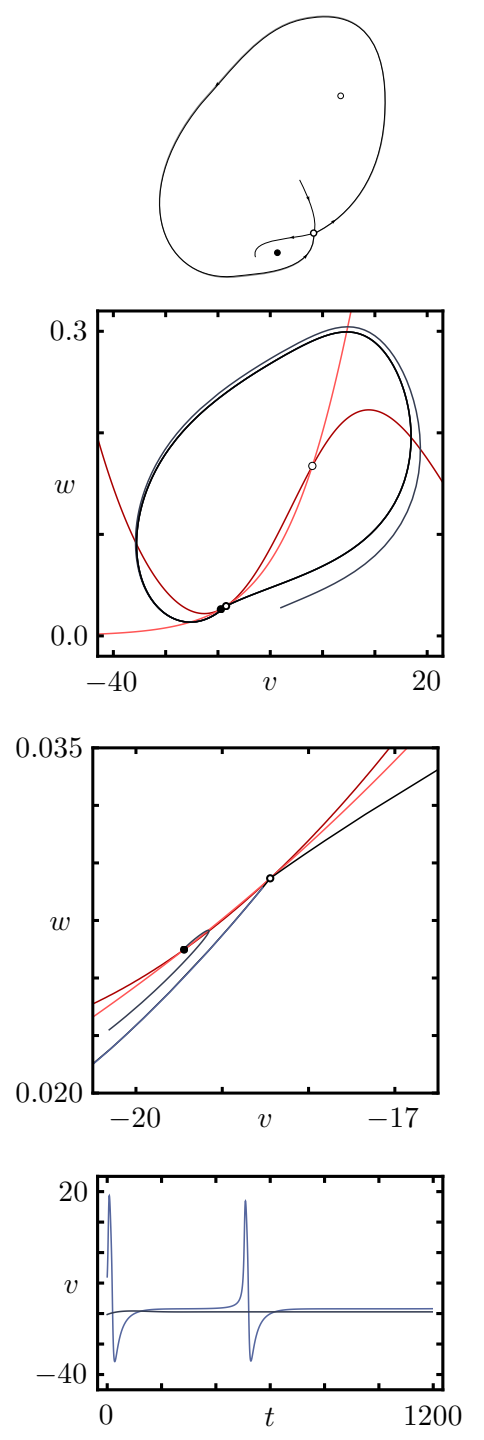

(c) $i_{\mathrm{e}}>i_{\mathrm{e}}^{*}$
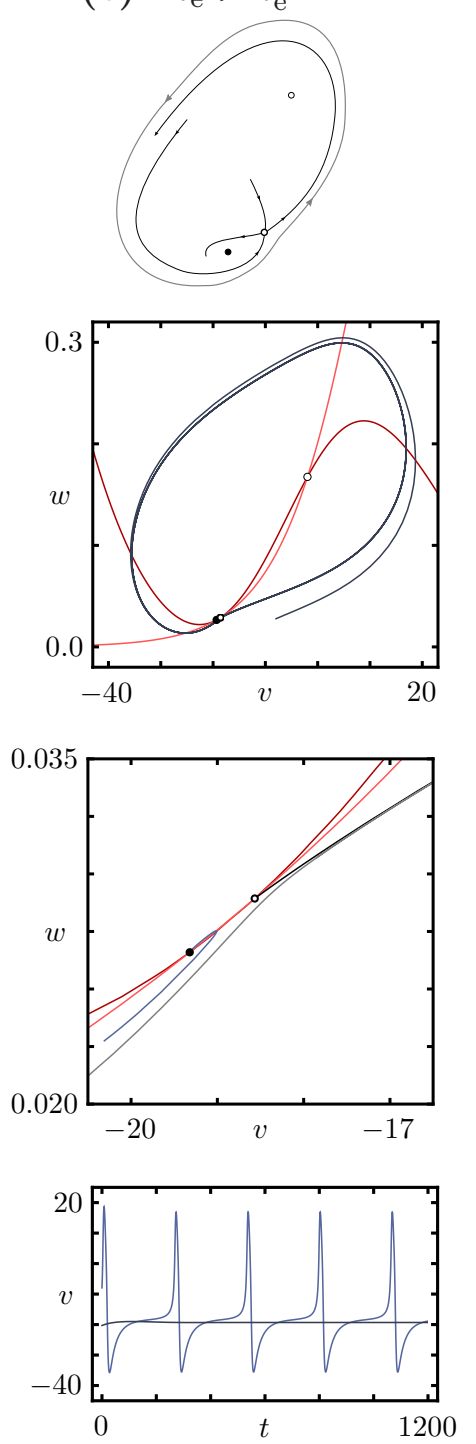

Figure 3.3. Big homoclinic or saddle loop (SL) bifurcation in the Morris-Lecar neuron model (2.4.2). Upper three rows show the phase portraits and a zoom into the relevant area using figure syntax as in 3.1, bottom row shows the corresponding spiking dynamics of the shown trajectories (blue). The bifurcation diagram is shown in fig. 10.2. (a) below the critical input currents the unstable manifold (black) of the saddle node ends up in the stable fixed point. (b) at the critical value the unstable manifold approaches the saddle and merges with the stable manifold to form a homoclinic loop (black). (c) above the critical value the homoclinic orbit has split off the saddle node to form a stable periodic orbit (gray). Note that the dynamics near the saddle are arbitrary slow so that the periodicity can become arbitrarily large by decreasing the bifurcation parameter closer and closer to the critical value.

\subsubsection{Hopf Bifurcation}

In a Hopf $(H B)$ bifurcation a limit cycle emerges from a focus or vice versa. Mathematically, it is characterized by two complex conjugate eigenvalues of the Jacobian crossing the imaginary axis with nonzero imaginary part. A Hopf bifurcation for the Morris-Lecar neuron model is illustrated in figure 3.5 and 10.5. 
(a) $i_{\mathrm{e}}<i_{\mathrm{e}}^{*}$
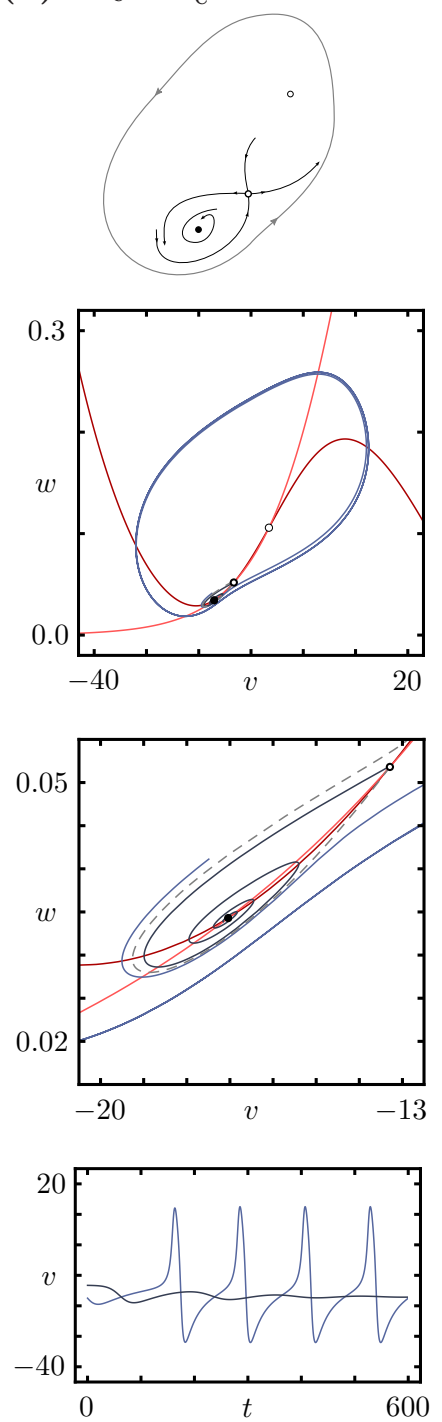

(b) $i_{\mathrm{e}}=i_{\mathrm{e}}^{*}$
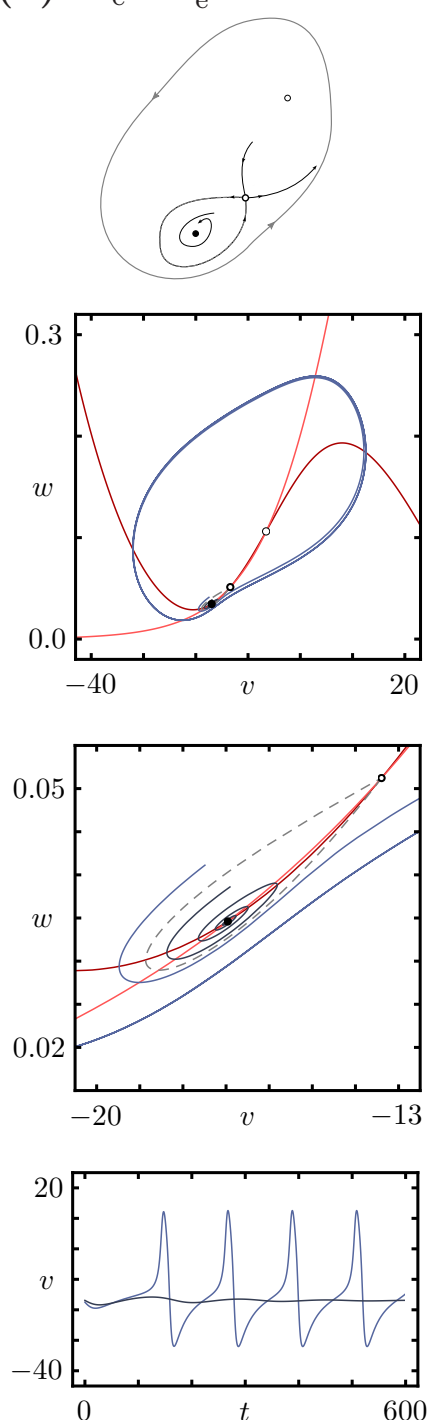

(c) $i_{\mathrm{e}}>i_{\mathrm{e}}^{*}$
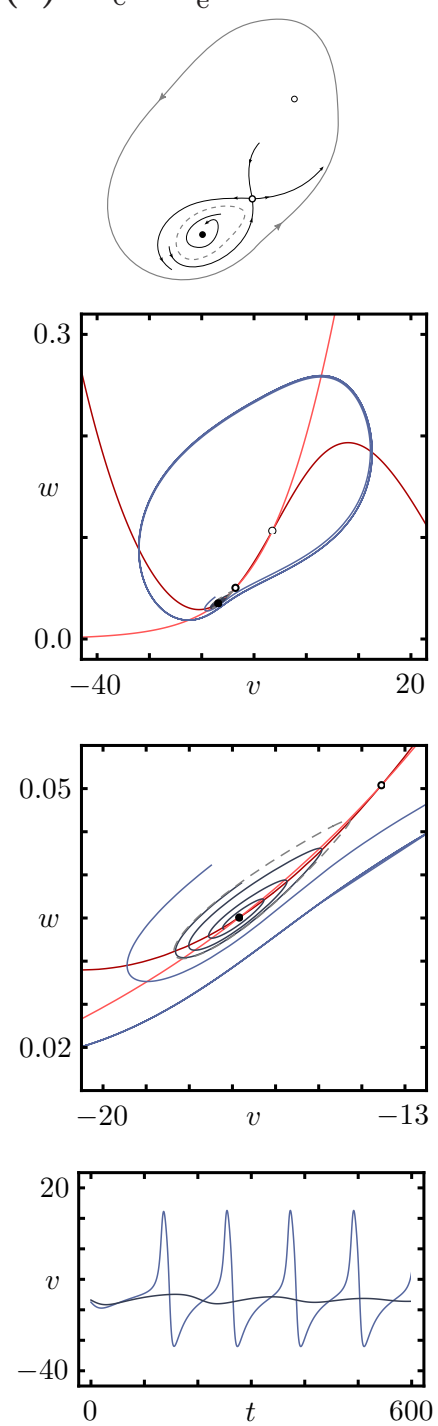

Figure 3.4. Small homoclinic or saddle loop (SL) bifurcation in the Morris-Lecar neuron model (2.4.2) with figure syntax and colors as in 3.1 but with and additional zoom of the relevant phase space in the third row. The bifurcation diagram is shown in fig. 10.3. (a) below the critical input currents the stable manifold of the saddle node (dashed) winds around the stable fixed point and passes above the saddle. (b) at the critical value the stable manifold approaches the saddle node again and forms a homoclinic loop (dashed). (c) above the critical value the homoclinic orbit has split of the saddle node to form a unstable periodic orbit (gray, dashed).

\subsubsection{Codimension-2 bifurcations}

\subsubsection{Cusp bifurcation}

At a cusp (CP) bifurcation two fold bifurcations coalesce.

\subsubsection{Bogdanov Takens bifurcation and Organizing Centers}

A fixed point $x_{\mathrm{BT}}$ of a dynamical system is a Bogdanov-Takens (BT) point if the Jacobian matrix $D f\left(x_{\mathrm{BT}}\right)$ has a two-dimensional invariant subspace on which $D f\left(x_{\mathrm{BT}}\right)$ is nilpotent, i.e. it can be transformed by a linear coordinate transformation into Jordan normal-form. 
(a) $i_{\mathrm{e}}<i_{\mathrm{e}}^{*}$
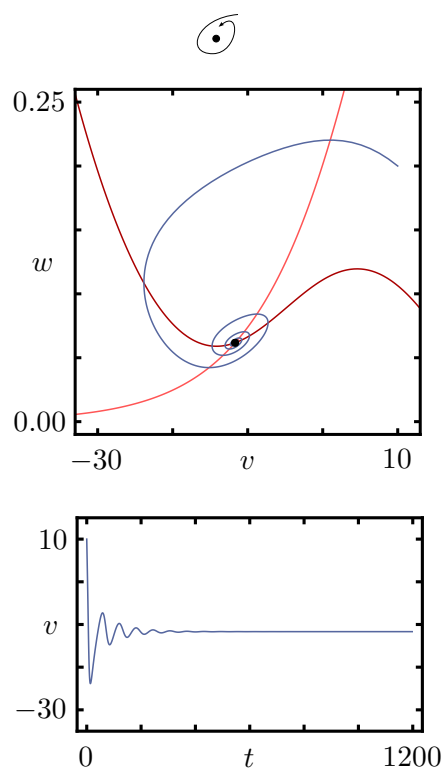

(b) $i_{\mathrm{e}}>i_{\mathrm{e}}^{*}$
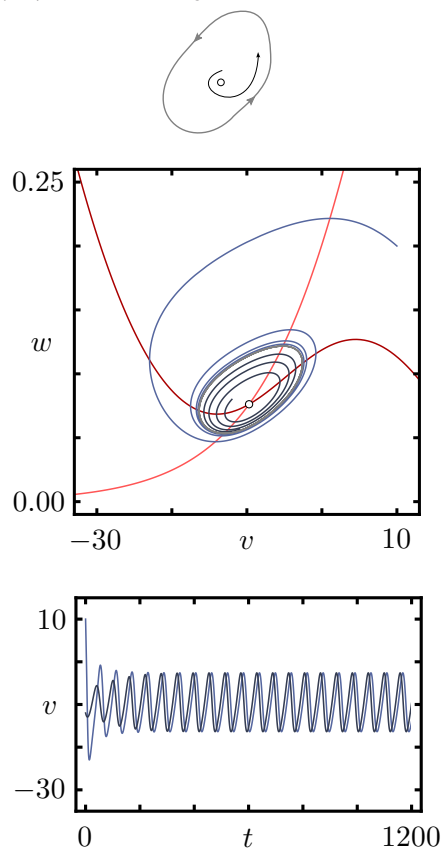

Figure 3.5. Supercritical Hopf (HB) bifurcation in the Morris-Lecar neuron model (2.4.2) and parameter as in fig. 10.5. The bifurcation diagram is shown in fig. 10.5. In a Hopf bifurcation a limit cycle grows out from a focus as a parameter is changed; here form below (a) to above (b) the critical input current $i_{\mathrm{e}}^{*}$. Figure conventions as is fig. 3.1.

For a two dimensional system we have

$$
D f\left(x_{\mathrm{BT}}\right) \sim\left(\begin{array}{ll}
0 & 1 \\
0 & 0
\end{array}\right)
$$

where $\sim$ denotes conjugacy (cf. sec. 36). The generic unfolding of this singularity has been studied in the seminal work by Takens [358, 42] and also by Bogdanov [29]. In their work they determine the unfolding of this singularity and prove that the BT point acts as an organizing center, in the way that its existence entails the existence of three codimension one bifurcation lines emanating form the BT point: a SN, a SL and a HB curve as illustrated in figure 3.6. In particular the local BT point organizes the existence of a global homoclinic bifurcation. This phenomenon is a part of multiple bifurcation theory $[144,145]$ which is concerned with bifurcation points of higher co-dimension that organize the bifurcation diagrams in lower co-dimensions.

\subsubsection{Degenerate Hopf bifurcation}

At a degenerate Hopf (DH) bifurcation, the Hopf bifurcation interacts with a double limit cycle bifurcation in which an stable and and unstable orbit are generated simultaneously from a fixed point.

\subsubsection{Saddle Node Loop}

In a saddle node loop (SNL) bifurcation [328] a homoclinic orbit and a fold bifurcation occur simultaneously, in which the homoclinic orbit is attached to the fold in its noncentral manifold. From this point a saddle node, a homoclinic and a saddle node on 


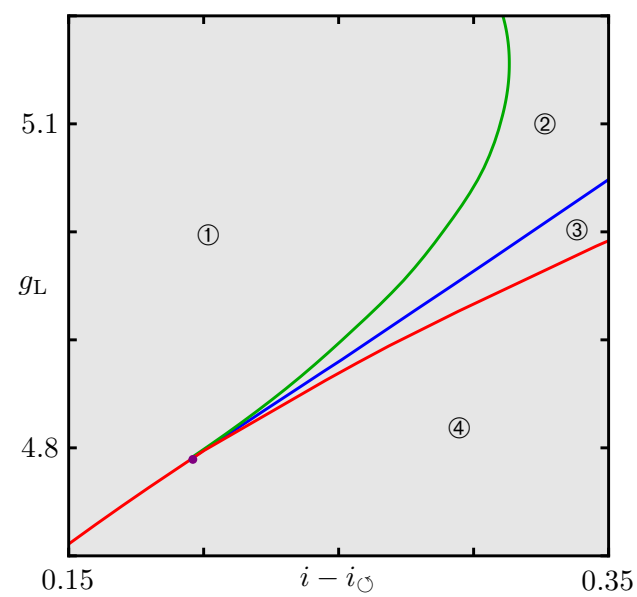

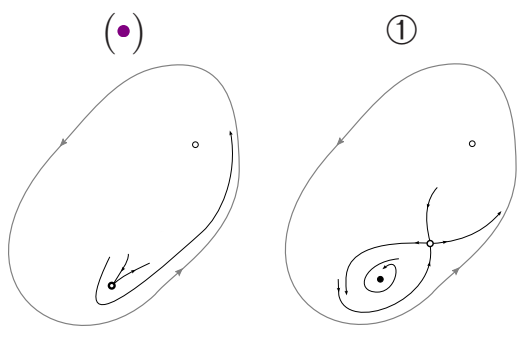

(3)

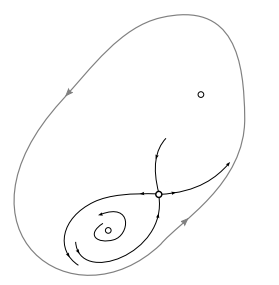

(2)

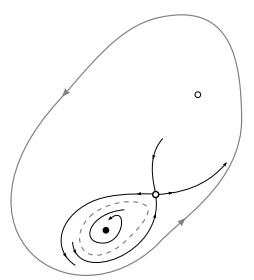

(4)

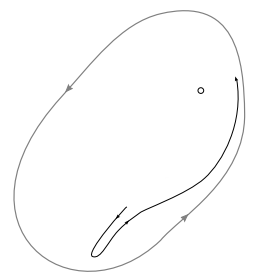

Figure 3.6. Bogdanov Takens (BT) bifurcation in the Morris-Lecar neuron model. Bifurcation diagram in the $\left(i_{\mathrm{e}}, g_{\mathrm{L}}\right)$-parameter plane and corresponding phase portraits around the co-dimension-2 BT bifurcation point $(\bullet)$ which organizes the SL $(-)$, the SN $(-)$ and the HB (-) bifurcations of co-dimension-1. Note that the surrounding large stable limit cycle as well as the third upper most unstable fixed point are not involved in the bifurcations but drawn here for later reference as this is a typical situation in which a BT point is encountered in neuronal models (cf. part IV).

invariant cycle bifurcation emanate as illustrated in fig. 3.7. In neuron models it acts as an organizing center for type I neuronal excitability [177] by organizing the saddle node on invariant cycle and homoclinic bifurcations, both leading to limit cycles with arbitrarily large period.

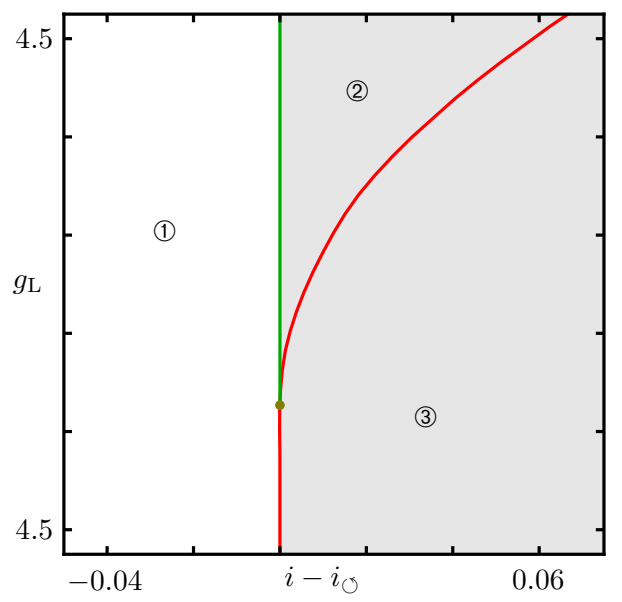

$(\bullet)$

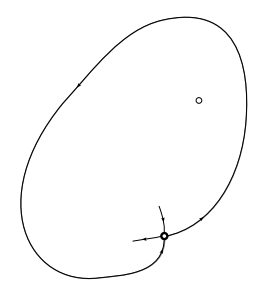

(2)

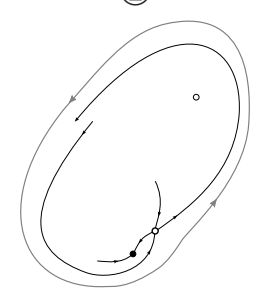

(1)

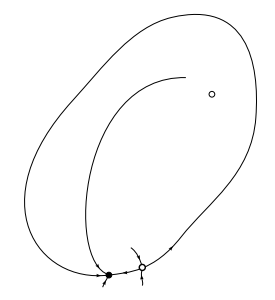

(3)

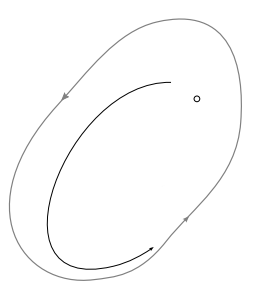

Figure 3.7. Saddle node loop bifurcation in the Morris-Lecar neuron model. Bifurcation diagram in the $\left(i_{\mathrm{e}}, g_{\mathrm{L}}\right)$-parameter plane and corresponding phase portraits around the co-dimension $2 \mathrm{SNL}$ point $(\bullet)$ which organizes the SL $(-)$, the SN (-, above $\bullet$ ) and the SNIC (-, below $\bullet$ ) bifurcations of co-dimension-1. 


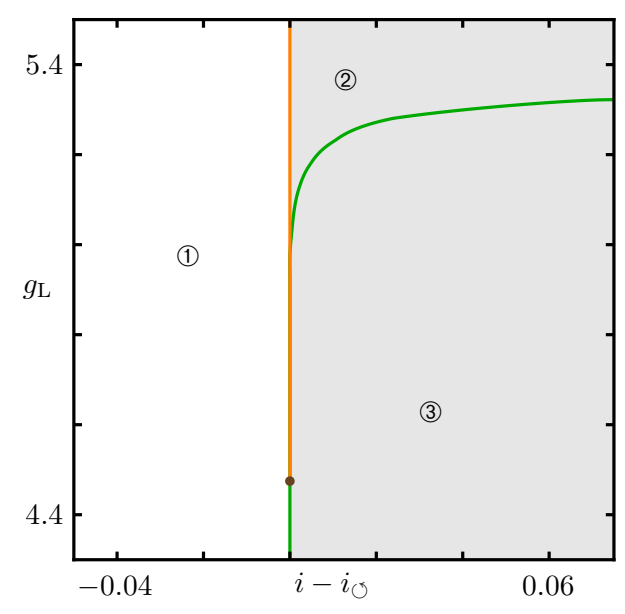

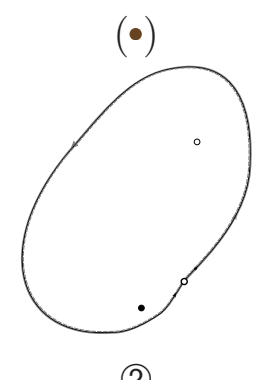

(2)

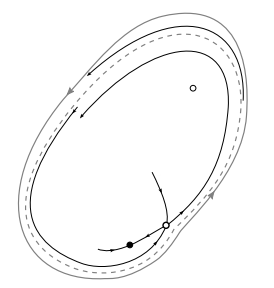

(1)

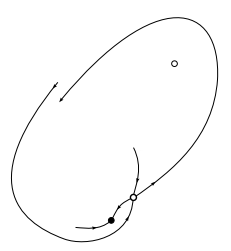

(3)

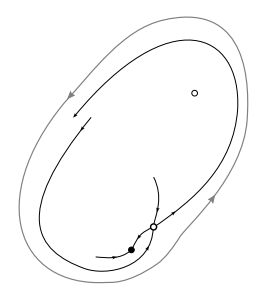

(-) above

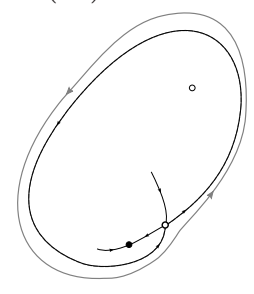

(-) below

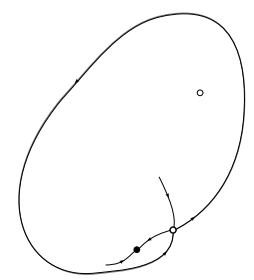

Figure 3.8. Neutral saddle loop (NSL) bifurcation in the Morris-Lecar neuron model. Bifurcation diagram in the $\left(i_{\mathrm{e}}, g_{\mathrm{L}}\right)$-parameter plane and corresponding phase portraits around the co-dimension-2 NSL point $(\bullet)$ which organizes the SL $(-)$ and the DC (-) bifurcations of codimension-1. Note that the stability of the invariant cycle at the SL bifurcation changes from stable below to unstable above the NSL bifurcation point. At the bifurcation point it has neutral stability.

\subsubsection{Neutral Saddle Loop}

In a neutral saddle loop (NSL) bifurcation the invariant cycle created via a double limit cycle bifurcation contains a fixed point forming a homoclinic loop. From the neutral saddle node loop bifurcation two homoclinic and a double limit cycle bifurcation lines emerge. In the homoclinic bifurcation at one side of the NSL point a stable limit cycle is created while in on the other an unstable limit cycle emerges form a homoclinic loop. At the bifurcation the homoclinic loop has neutral stability. We will argue in part IV of this thesis that this point acts as a organizing center for type I to type II neuronal excitability transitions. A bifurcation diagram is sketched in figure 3.8 .

\subsubsection{Co-dimension-3 Degenerate Bogdanov-Takens Bifurcation}

At a degenerate Bogdanov-Takens (dBT) or Bogdanov-Takens-cusp bifurcation a cusp point interacts with a Bogdanov-Takens point [86, 87, 85]. Three topological subclasses have been identified: a saddle, an elliptic and a focus dBT point. Its normal form is conjectured $^{1}$ to be of the form.

$$
\frac{d}{d t}\left(\begin{array}{l}
x \\
y
\end{array}\right)=\left(\begin{array}{c}
y \\
\mu_{1}+\mu_{2} x-x^{3}+y\left(\mu_{3}+\rho x-x^{2}\right)
\end{array}\right)=f_{\mathrm{dBT}}(x, y)
$$

with unfolding parameters $\mu_{i}$ and with a topological parameter $\rho$ that for the focus type takes the values $0<\rho<2 \sqrt{2}$ and does not alter the bifurcation diagram topologically. Figure 3.9 shows the three dimensional bifurcation set, which we constructed semianalytically with the methods described in sec. 3.3.4 for the dBT bifurcation of focus

\footnotetext{
${ }^{1}$ Note that [87] provides no complete proof for the unfolding of the dBT points and thus strictly speaking the unfolding of the bifurcation point in (3.3.1) can only be conjectured. This points out the limits of the tools established so far to study complex bifurcations.
} 


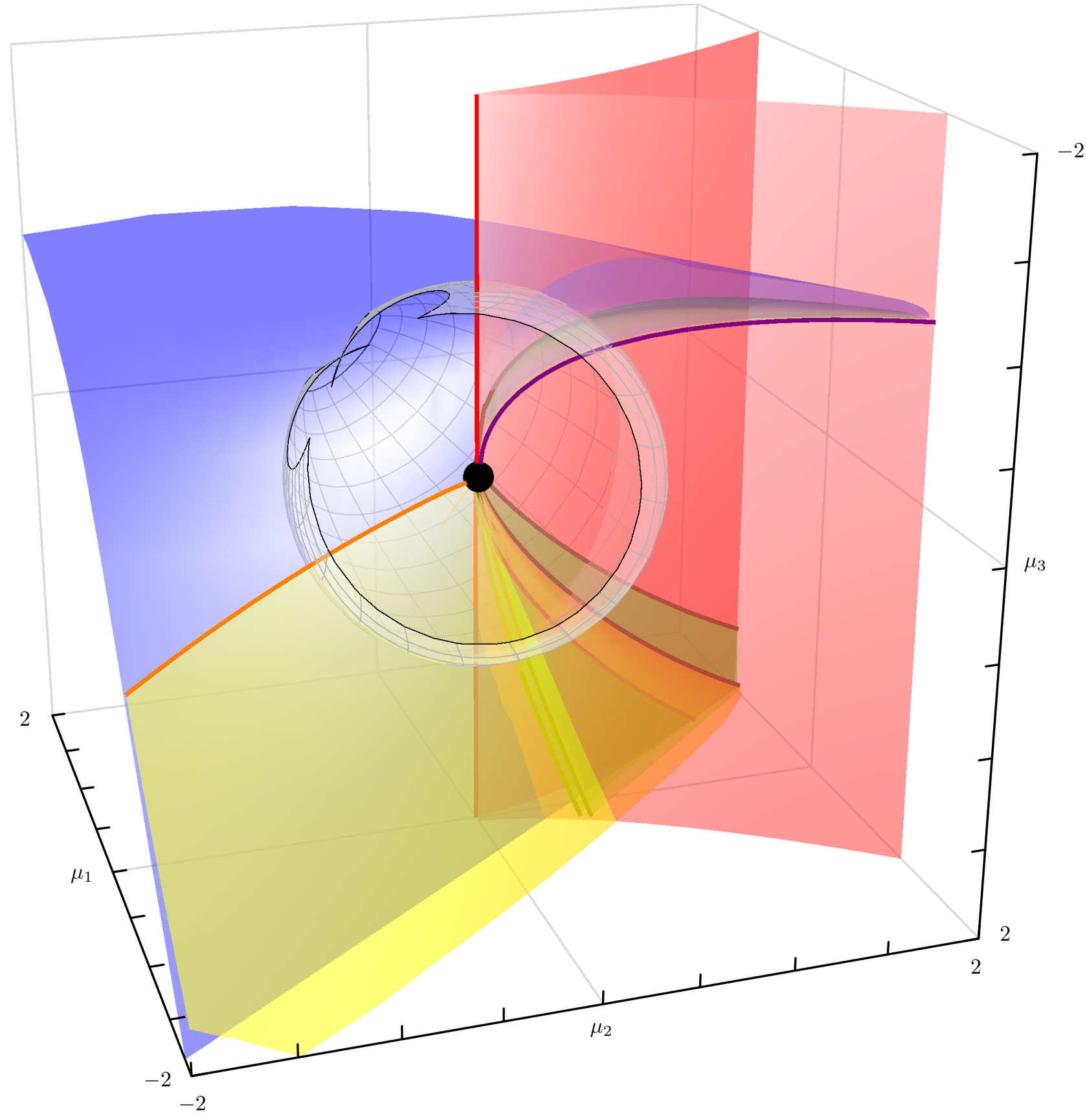

Figure 3.9. Bifurcation diagram of the degenerate Bogdanov-Takens-cusp (dBT) bifurcation in $\left(\mu_{1}, \mu_{2}, \mu_{3}\right)$-parameter space. The colors encode the bifurcation surface or line using the color code form 3.1. The diagram is a topological cone with tip being the dBT point $(\bullet)$. The white sphere shows the coordinate system used in fig. 3.10 to visualize the base of this cone.

type and cannot be found in the literature. The dBT bifurcation point in the center of the diagram organizes the bifurcations of co-dimension one (planes) and two (lines). The diagram has a conic structure with an intersection of a sphere centered around the dBT point as shown in fig. 3.10. 
(a)

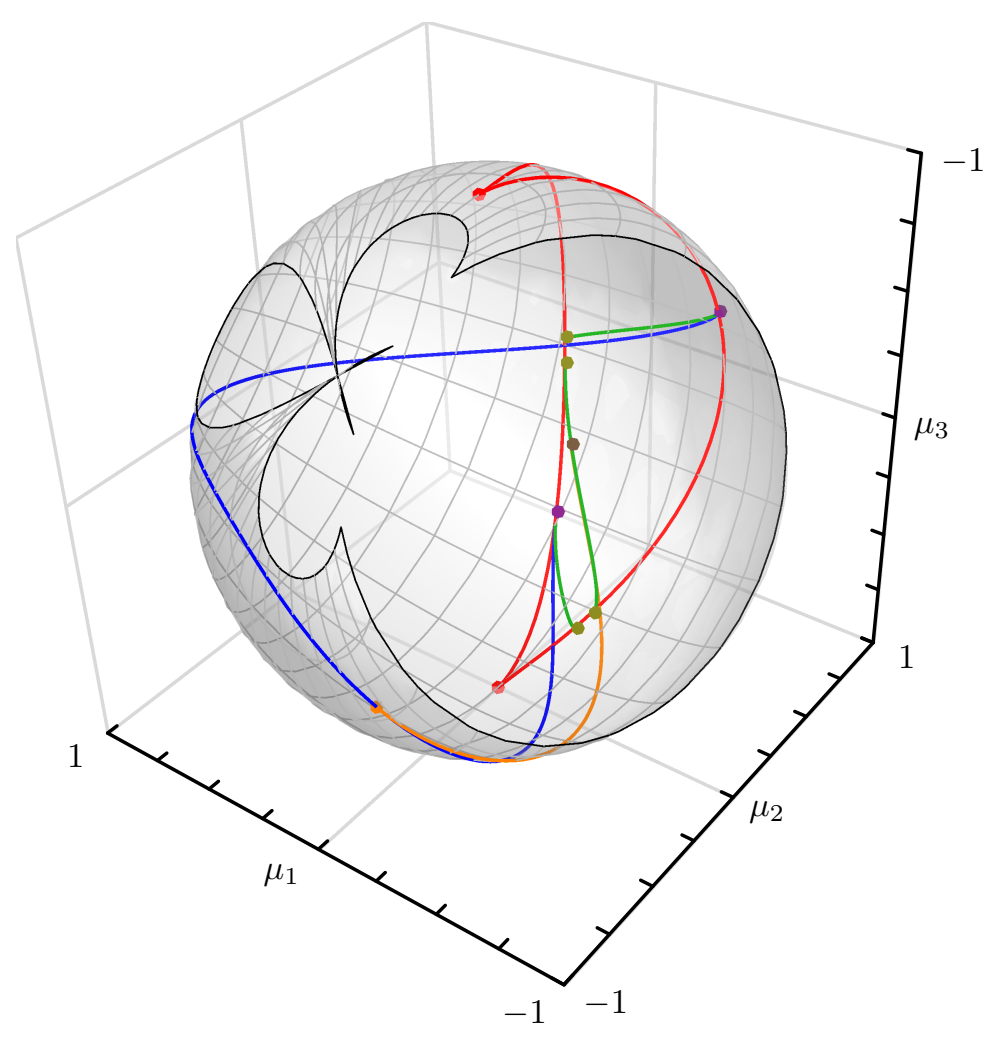

(b)

(c)
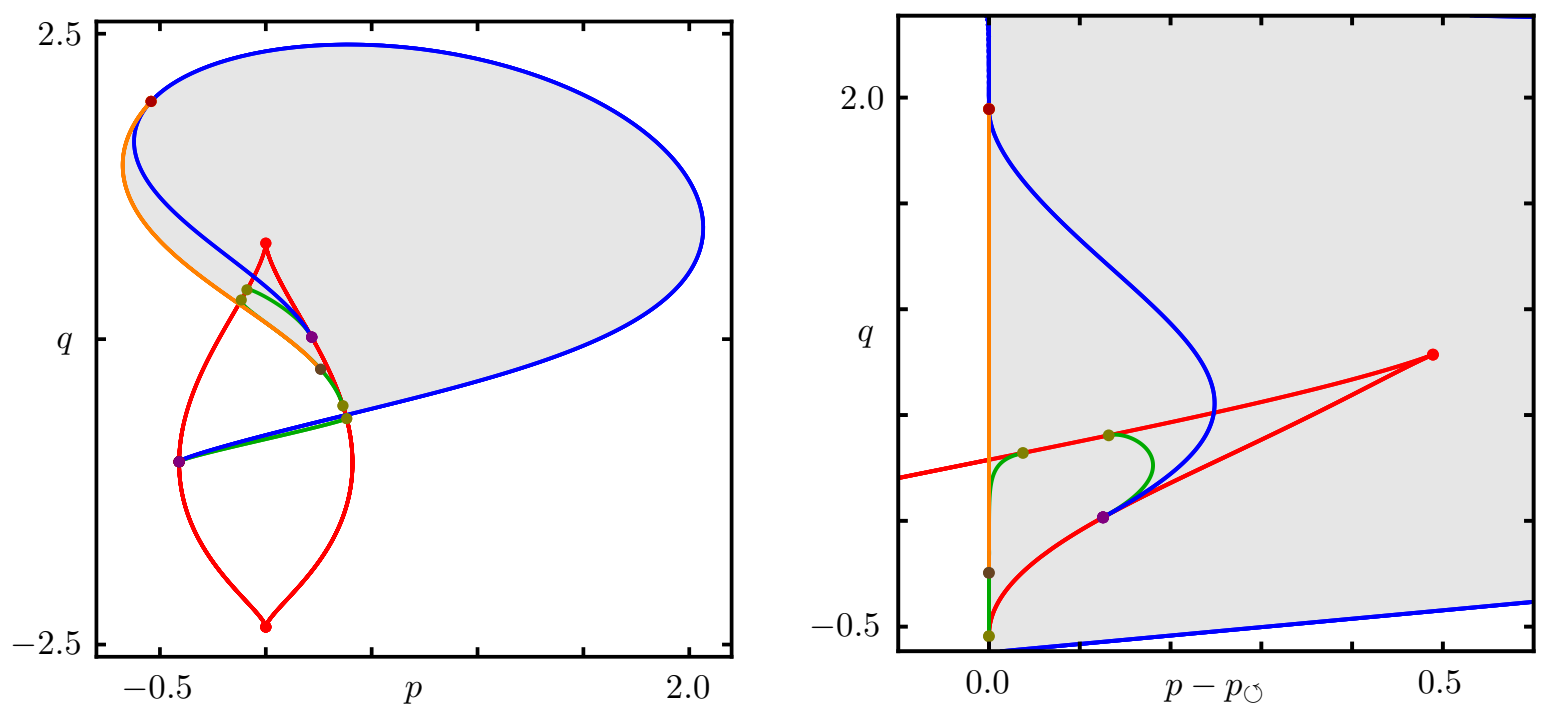

Figure 3.10. Cone-basis of the degenerate Bogdanov-Takens bifurcation. (a) Show is the coordinate system as in fig. 3.9 with the intersection of the bifurcation surfaces here shown as lines and bifurcation lines here shown as dots using the color code 3.1. In deformed stereographic projection onto the plane with coordinates $(p, q)$ this yields the (b) 2D-bifurcation diagram of the cone-basis of the dBT bifurcation. (c) zoom and shift of the $p$ coordinate by $p_{\circlearrowleft}=p_{\circlearrowleft}(q)$ so that the generation of a stable limit cycle via one of the shown bifurcations is at zero. Note that there is an ordinary Bogdanov-Takens point as well as a cusp, a neutral saddle loop and three saddle node loop bifurcations. 


\subsubsection{Numerical Calculation of Bifurcation Sets}

In this thesis we locate saddle nodes by solving the defining equations either analytically or using the newton method to locate zeros of certain functions numerically. Hopf points are located by solving for simultaneous zeros of the determinant and the determinant of a bi-alternate product of the Jacobian matrix as described in [136, 224]. Cusp points are located by solving for singular Hessians in direction of a zero-eigenvector. All nonlocal bifurcations are located using the numerical continuation software AUTO [81, 82] with HomCont [57] based on the basic algorithms described in [79, 80]. In essence, these methods work by constructing conditions for the dynamical system under consideration, that vanish at the points of interest (e.g. in the simplest case these are the fixed point conditions). These conditions depend on the bifurcation parameter and starting at a point for which these conditions are fulfilled, points in the vicinity of the initial point that also fulfill the conditions are located by varying the parameters using numerically techniques such as pseudo-arclength methods [136, 224].

For this thesis we developed a software-interface between AUTO and Wolfram's Mathematica [205] which allows automated code generation of AUTO equations and constant files for arbitrary systems of ordinary differential equations. It makes use of the symbolic manipulation power of Mathematica to calculate Jacobians and several parameter derivatives. It is part of a dynamical systems tool box for Mathematica [206] for the study and organization of dynamical systems defined by an ordinary differential equation, providing a large ensemble of tools for the investigation of dynamical systems, including the detection of fixed points, periodic orbits and nullclines, solving the adjoint equations and phase response calculations.

\subsection{Phase Reduction and Averaging for Weakly Coupled Oscillators}

When driven by a sufficiently strong constant external current many neuron models generate periodic spiking dynamics (cf. fig. 3.11) and constitute a neuronal oscillator with a stable limit cycle and period $T$. If synaptic interaction between the neurons is weak, phase reduction and averaging methods [228, 407, 327, 102] can be used to analytically investigate the collective dynamics of such neuronal oscillators. Here we introduce these concepts as we make use of them in all parts of this thesis.

\subsubsection{Phase Reduction and Phase Response Curves}

Consider a dynamical system given by

$$
\frac{d}{d t} x=f(x)
$$

with $x \in \mathbb{R}^{d}$ that has a stable limit cycle solution

$$
x_{\circlearrowleft}(t)=x_{\circlearrowleft}(t+T)
$$

with period $T$. This limit cycle may be parametrized by a phase variable $\phi \in S^{1}=$ $[0,1] /(0 \sim 1)$ that increases monotonically in time such that 


$$
\frac{d}{d t} \phi=\frac{1}{T}=\omega
$$

This phase description can be extended to the basin of attraction of the stable limit cycle by assigning to each point $x_{0}$ a scalar phase $\phi\left(x_{0}\right)$ such that $\lim _{t \rightarrow \infty} \phi(x(t))-(\omega t+\phi)=0$ for the solution $x(t)$ of (3.4.1) with $x(0)=x_{0} \cdot{ }^{2}$ By definition

$$
\begin{aligned}
\frac{d}{d t} \phi(x) & =\omega \\
& =\nabla_{x} \phi(x) \cdot \dot{x}=\nabla_{x} \phi(x) \cdot f(x)
\end{aligned}
$$

Introducing a small perturbation $p$ of order $\varepsilon$ into the vector field, i.e. $f(x) \rightarrow f(x)+$ $\varepsilon p(x)$ in (3.4.1) one obtains

$$
\begin{aligned}
\frac{d}{d t} \phi & =\omega+\varepsilon \nabla_{x} \phi(x) \cdot p(x, t) \\
& \approx \omega+\varepsilon \nabla_{x} \phi\left(x_{\circlearrowleft}(\phi)\right) \cdot p\left(x_{\circlearrowleft}(\phi), t\right)
\end{aligned}
$$

where in the second step we have neglected contributions of order $\mathcal{O}\left(\varepsilon^{2}\right)$ by inserting the limit cycle solution (3.4.2) to obtain a closed form expression for the effect of the perturbation in the direction of $\phi$. It shows that the phase response vector or phase response curve (PRC)

$$
z(\phi)=\nabla_{x} \phi\left(x_{\circlearrowleft}(\phi)\right)
$$

determines the oscillators response in the phase direction to a perturbation $p$ applied at $\phi$. The systems' time evolution thus has been reduced to a one dimensional phase model

$$
\frac{d}{d t} \phi=\omega+\varepsilon z(\phi) \cdot p\left(x_{\circlearrowleft}(\phi), t\right)
$$

in which the fast decaying amplitudes transverse to the limit cycle have been removed. Differentiating equation (3.4.4) with respect to the phase shows that the phase response can be calculated by solving the adjoint equation

$$
\frac{d}{d \phi} z(\phi)=-D f^{T}\left(x_{\circlearrowleft}(\phi)\right) z(\phi)
$$

with initial condition

$$
z(0) f\left(x_{\circlearrowleft}(0)\right)=\omega
$$

and further $d-1$ periodic boundary conditions. Here $D f$ is the Jacobian matrix of the vector field $f$.

In experimental praxis one can use a small pulse of strength $\varepsilon$ applied to the oscillation at phase $\phi$ into a phase space direction $e_{i}$ to determine the PRC. After this perturbation the dynamics will relax back to its periodic firing but with a phase shift $\delta \phi$ with respect to the unperturbed motion (cf. fig. 3.11) that depends on the perturbation strength $\varepsilon$, the direction $\mathrm{e} \in \mathbb{R}^{d}$ and perturbation time $\phi T$ within the limit cycle. We define this to be the non-infinitesimal phase response curve

$$
\mathrm{z}(\phi, \varepsilon \mathrm{e}):=\delta \phi(\phi, \varepsilon \mathrm{e})
$$

from which the infinitesimal phase response is then obtained via

$$
\mathrm{e} \cdot \mathrm{z}(\phi)=\lim _{\varepsilon \rightarrow 0} \frac{1}{\varepsilon} \mathrm{z}(\phi, \varepsilon \mathrm{e})
$$

\footnotetext{
${ }^{2}$ In mathematical terms the orbit has to be stable and normally hyperbolic so that there is a neighborhood of the limit cycle which is invariantly foliated by isochrons, i.e. stable submanifolds to which a constant phase value $\phi$ can be assigned such that eq. (3.4.3) holds [145, 177].
} 


\subsubsection{Averaging of Weakly Coupled Oscillators}

The phase response reduction can be used to obtain a simplified evolution equation for weakly coupled oscillators. Here the coupling plays the role of the perturbation in the previous section. Consider a system of $N$ weakly coupled oscillators given by

$$
\frac{d}{d t} x_{i}=f_{i}\left(x_{i}\right)+\varepsilon \sum_{j} g_{i j}\left(x_{i}, x_{j}\right)
$$

where $x_{i} \in \mathbb{R}^{d_{i}}$ is the state vector of each individual oscillator $i \in\{1, \ldots, N\}, f_{i}$ it's local dynamics, $g_{i j}$ the two-point coupling function from oscillator $j$ to $i$, and $\varepsilon$ the overall coupling strength. We assume that for the uncoupled case, $\varepsilon=0$, each individual system $i$ has a stable limit cycle solution $x_{i, \circlearrowleft}(t)$ with period $T_{i}$ and frequencies $\omega_{i}=\frac{1}{T_{i}}$ that differ only by $\mathcal{O}(\varepsilon)$, i.e. $\omega_{i}=\omega+\varepsilon \delta \omega_{i}$. Using the phase reduction method from above (3.4.6) we obtain the $N$-dimensional system

$$
\frac{d}{d t} \phi_{i}=\omega+\varepsilon\left(\delta \omega_{i}+\sum_{j} z_{i}\left(\phi_{i}\right) \cdot g_{i j}\left(x_{i, \circlearrowleft}\left(\phi_{i}\right), x_{j, \circlearrowleft}\left(\phi_{j}\right)\right)\right)
$$

A further reduction of the system may be achieved by taking into account that for small $\varepsilon \ll \omega$ in (3.4.11) the interaction of the system may be averaged over one period to obtain

$$
\frac{d}{d t} \phi_{i}=\omega+\varepsilon\left(\delta \omega_{i}+\sum_{j} \gamma_{i j}\left(\phi_{i}-\phi_{j}\right)\right)
$$

with the effective coupling

$$
\gamma_{i j}\left(\phi_{i}-\phi_{j}\right)=\int_{0}^{1} z_{i}\left(\phi_{i}+t\right) g_{i j}\left(x_{i, \circlearrowleft}\left(\phi_{i}+t\right), x_{j, \circlearrowleft}\left(\phi_{j}+t\right)\right) d t
$$

which only depends on the phase differences as the functions under the integral are periodic in $\phi$. The precise mathematical justification for this averaging step comes from normal form theory (cf. sec. 3.2.1) for periodic vector fields [327].

Equation (3.4.12) is the general form of the celebrated Kuramoto system [228, 5], the 'standard model' to study synchronization in coupled oscillators. For $\gamma_{i j}(\phi)=k_{i j} \sin (\phi)$ it becomes the phase part of the discrete network Ginzburg-Landau equation [125] that describes the dynamics of coupled oscillators close to a multiple Hopf bifurcation [177] (cf. sec. 3.3.1.4).

Consider two identical oscillators with phase difference $\Delta \phi=\phi_{1}-\phi_{2}$ then

$$
\frac{d}{d t} \Delta \phi=\gamma(\Delta \phi)-\gamma(-\Delta \phi):=\bar{\gamma}(\Delta \phi)
$$

and phase locked states $\Delta \phi_{0}$ are given by the zeros of $\bar{\gamma}$ which are linearly stable if $\bar{\gamma}^{\prime}\left(\Delta \phi_{0}\right)<0$. The oscillators are phase synchronous if $\Delta \phi_{0}=0$.

\subsubsection{Phase Response in Neuronal Models}

Activation of a single synapse typically produces post synaptic potentials of the order of $\approx 1 \mathrm{mV}$ which may be considered as a weak perturbation [177] and thus the above theory may be applied to interacting neurons. 
For systems close to a bifurcation in which a stable limit cycle is created, e.g. corresponding to the onset of spiking, the phase response curve may be derived analytically $[100,335]$. For example a neuronal oscillator close to a saddle node on limit cycle bifurcation (cf. fig. 3.1) with normal form given by the theta neuron model in sec. 2.6.4 equation (2.6.9) compared with (3.4.6) shows that the phase response is given by $z(\phi)=1+\cos (\phi)$, which is non-negative. Thus for this class of models excitatory perturbations $\varepsilon>0$ always advance the phase. In contrast for a Hopf or a double limit cycle bifurcation the PRC may be derived to be $z(\phi)=\sin (\phi)$ giving rise to both negative and positive parts. Thus an excitatory perturbation may advance or delay the phase (cf. fig. 3.11). Experimentally both types of phase response curve have been observed [314, 313, 116, 352].

The reduced phase description of neuron models is useful in the study the collective dynamics of neuronal networks $[156,157]$ and optimal information coding in neurons $[239,329]$.

(a)

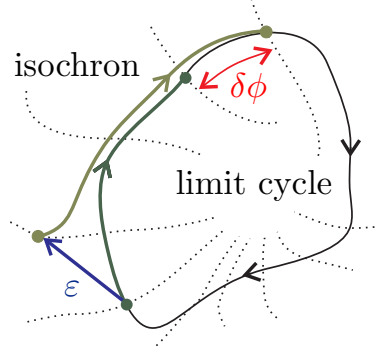

(b)

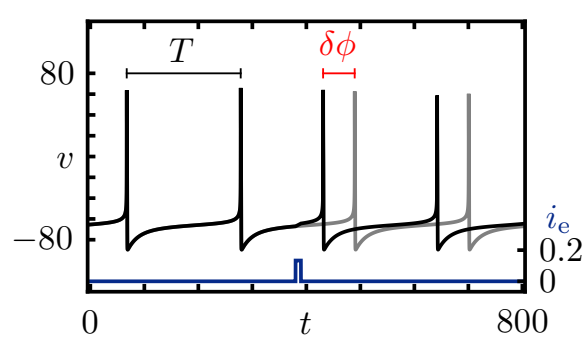

(c)

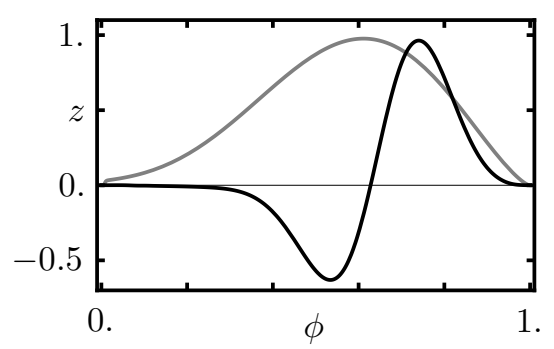

Figure 3.11. Phase response in neuronal models. (a) For a system with a stable limit cycle a small perturbation $\varepsilon$ will return back to its periodic motion but with a phase shift $\delta \phi$. The dotted lines show isochrons, i.e. points which asymptotically collapse onto the same phase on the limit cycle for $t \rightarrow \infty$. (b) When driven by a constant external input $i_{\mathrm{e}}$ the coritcal neuron model by Erisir (sec. 2.4.4) shows periodic spiking with period $T$ (gray). Adding a small pulse at a phase $\phi$ advances the phase by $\delta \phi$. (c) phase response curve $z(\phi)$ for the Erisir model (cf. sec. 2.4.4) (gray) is purely positive while for the Hodgkin-Huxley model (cf. sec. 2.4.1) (black) it has both signs. 


\section{Part III}

\section{Dynamics of Pulse Coupled Oscillators with Partial Reset}


In this part of the thesis we propose a model of pulse-coupled threshold units with partial reset and study the collective network dynamics. We first motivate a partial reset in pulse-coupled units from a theoretical and neuroscientific perspective. We then concentrate on instantaneous couplings and numerically find and analytically proof that the partial reset induces a sequence of cluster destabilization bifurcations that cause a desynchronization transition. We then study effects of the partial reset in networks with delayed interactions and give an analytical prove for a novel type of bifurcation from networks of unstable attractors to heteroclinic switching.

Most of the results in this part are published in [210, 213, 211]. 


\section{Chapter 4}

\section{Introduction}

Networks of pulse-coupled units serve as paradigmatic models for a wide range of physical and biological systems as different as cardiac pacemaker tissue, plate tectonics in earthquakes, chirping crickets, flashing fireflies and neurons in the brain [47, 289, 33, 248, 269, 378]. In such systems, units interact by sending and receiving pulses at discrete times that interrupt the otherwise smooth time evolution. These pulses may be sound signals, electric and electromagnetic activations as well as packets of mechanically released stress. Pulses are generated once the state of a unit crosses a certain threshold value (e.g. the mechanical stress of a tectonic plate becomes sufficiently large or the voltage across a nerve cell membrane becomes sufficiently high); thereafter the state of the sending unit is reset.

Synchronization of oscillators is one of the most prevalent collective dynamics in pulsecoupled systems $[269,104,105,37,39,157,124,369]$. Often not all units are synchronized but form clusters consisting of synchronized sub-groups of units which in turn are phaselocked to other clusters [104, 371, 263, 20, 157, 155, 244, 297, 299].

In neuronal networks synchronization and clustering of pulses constitute potential mechanisms for effective feature binding. In this paradigm, different information aspects of the same object represented by activity of different nerve cells are pooled together by temporal correlations and in particular due to synchronous firing [398, 341]. However, strong synchronized firing of nerve cells can also be detrimental: synchrony is prominent during epileptic seizures [259] and observed in the basal ganglia during Parkinson tremor [92]. Here mechanisms for desynchronizing neural activity are desirable [362, 251, 160].

To study key mechanisms that are underlying (de)synchronization, e.g. in biological neural networks, analytical tractable models of pulse-coupled oscillator are helpful tools $[296,269,229,3,104,37,372,71]$. Here the rise of the state variable of a free oscillatory unit towards the threshold, the unit's rise function characterizes the sub-threshold dynamics. If, after reception of a pulse, the state variable of the unit stays below threshold it is said to receive sub-threshold input, whereas excitation above the threshold is suprathreshold. Mirollo and Strogatz [269] showed that biological oscillators always synchronize their firing in homogeneous networks with excitatory all-to-all coupling if the rise function has a concave shape. The synchronization mechanism they find has two parts: (i) effective decrease of phase differences of units due to sub-threshold inputs and (ii) instant synchronization due to supra-threshold inputs and subsequent reset to a fixed value.

In general, supra-threshold excitation and a subsequent reset is a dominant mechanism for synchronization of pulse-coupled oscillators because input pulses that force non-synchronized units to cross threshold at nearby times are reset to the same value 
leaving the units in the same state or in very similar states afterwards. Although this reset mechanism plays a crucial role in the synchronization process and the coordination of pulse generation times, its implications for the collective network dynamics has not been investigated systematically so far: most existing model studies reset the units with supra-threshold inputs to a fixed value independent of the strength of supra-threshold excitation $[33,104,229,333,371,66,71,174]$. This results in a complete loss of information about the prior state of the units and makes the dynamics non-invertible. Some other studies consider the opposite extreme: a complete conservation of supra-threshold inputs during pulse sending and reset $[174,37]$. Here we aim at closing this gap by presenting and analyzing a model with a partial reset where the reset value (and thus the loss of information about the prior state and the strength of supra-threshold excitation) can be varied systematically .

Motivation for our model comes from neuroscience. The brain processes information in networks of neurons, which interact by sending and receiving electrical pulses called action potentials or spikes. The response of a neuron to incoming signals strongly depends on whether or not it has just sent a spike itself. After the initiation of a spike the membrane potential at the cell body (soma) is reset towards some potential and the response to further synaptic input is reduced due to the refractoriness of the neuron $[68,196]$. The dendritic part of the neuron where incoming signals are integrated, is affected only indirectly by this reset due to intra-neuronal interactions [332, 321, 38].

Several multi-compartment models have been proposed, in which different parts of a single neuron interact to characterize this effect [332]. For instance, in a two-compartment model [38] of coupled dendrite and soma, the membrane potential at the soma is reset after spike emission while the dendritic dynamics is affected only by the resistive coupling from the soma to the dendrite. This accounts for the fact that in several kinds of neurons residual charge remains on the dendrite (following the somatic reset) and is then transferred to the soma $[321,250]$. Thus the dynamics of the individual neurons is modified which severely affects the collective capabilities of networks of such neurons.

Here we propose a simple neuron model which captures this response to residual input charges following spike emission in form of the partial reset and at the same time allows an analytical study of the collective network dynamics. To reveal the basic mechanisms underlying the collective dynamics of networks of such neurons we focus on globally and homogeneously coupled neurons. Despite their simplicity these networks already exhibit a rich variety of dynamics that is controlled by the partial reset. In particular we find and show analytically that for a broad class of neurons there is a desynchronization transition in the network dynamics determined by a sequence of bifurcations: For a strong dissipative partial resets with a strong loss of supra-threshold inputs the fully synchronous state coexists with a variety of cluster states consisting of differently sized groups of synchronously firing neurons (cf. [198, 297]). With increasing partial reset strength states with synchronized clusters of a certain size and larger become unstable in a sequence of bifurcations. First the fully synchronous state loses stability followed by smaller and smaller clusters until finally for conservative resets only an asynchronous state is left. We investigate the main mechanisms generating this sequence of bifurcations analytically and give an intuitive explanation. We also discuss key consequences of this desynchronization mechanisms for biophysically more detailed systems.

The concepts of attractor and stability are at the core of dynamical systems theory [199] because attractivity and stability determine the long term behaviour and often the typical properties of a system. Attraction and stability which may change via bifurcations 
are thus fundamental to modeling in all of science and engineering. For systems with smooth and invertible flows these concepts have long been studied and are well understood, allowing classifications of dynamical systems and their bifurcations, for example by using topological equivalence and normal forms.

Dynamical systems with non-smooth or non-invertible flows, such as hybrid [43], Fillipov [76] or pulse coupled systems are far less understood although they model a variety of natural phenomena, ranging from the mechanics of stick-slip motion through the switching dynamics of electrical circuits to the generation of earthquakes [289, 69, 269, 164, 104, $105,333,370,371,372]$. These systems often have intriguing mathematical and dynamical properties. For instance, pulse-coupled oscillator models with delayed interactions may robustly exhibit unstable attractors $[104,105,371,372]$, i.e. invariant periodic orbits that have a non-zero measure basin of attraction (Milnor attractors $[267,268]$ ) but are locally unstable. In the presence of noise, these systems exhibit a dynamics akin to heteroclinic switching, a feature that may also have functional relevance for coding and computation in neural systems [155, 17, 238, 237]. In ref. [16] it was shown that invertible systems in general cannot have unstable attractors and that a saddle state can in principle be converted to an unstable attractor by locally adding a non-invertible dynamics onto the stable manifold. However, the potential relation of unstable attractors to heteroclinic cycles is not well understood and it is unknown whether and how unstable attractors may be created or destroyed via bifurcations.

In chapter 7 of the thesis we study partial reset models with delay and numerically and analytically show the existence of two unstable attractors that are enclosed by the basin of attraction of each other. We explain this counter-intuitive phenomenon: Changing the partial reset continuously lifts a local non-invertibility of a system with two unstable attractors and creates a standard heteroclinic two-cycle. This transition constitutes a new type of bifurcation in hybrid dynamical systems. 


\section{Chapter 5}

\section{Networks of Pulse-Coupled Threshold Elements with Partial Reset}

In this chapter we propose a general class of pulse-coupled threshold units with partial reset and thereafter focus on units that oscillate intrinsically. Finally, motivation for and a discussion on the validity of the partial reset models is given from a neuroscientific perspective.

\subsection{Pulse-Coupled Threshold Elements}

Chapter 2.6 shows how the dynamics of single neurons can be approximated by single variable threshold elements defined by eq. (2.6.1) and eq. (2.6.2). In chapter 2.7 we saw how synaptic interactions between neurons can be described via pulse-coupling, eq. $(2.7 .5)$.

We consider $N$ threshold elements, which at time $t$ are characterized by a single real state variable $u_{i}(t)$ with $i \in\{1,2, \ldots, N\}$. In the absence of interactions the state variables evolve freely according to the differential equation

$$
\frac{d}{d t} u_{i}=F\left(u_{i}\right)
$$

with a smooth function $F: \mathbb{R} \rightarrow \mathbb{R}$ specifying the intrinsic dynamics of the units. The free dynamics are endowed with an additional nonlinear reset upon reaching a fixed threshold $\theta$ from below

$$
u_{i}\left(t^{-}\right)=\theta \quad \Rightarrow \quad u_{i}(t)=\rho
$$

where $\rho<\theta$ is the reset value and we used the notation $u_{i}\left(t^{ \pm}\right)=\lim _{s \searrow 0} u_{i}(t \pm s)$. By an appropriate shift and rescaling of the state variable and its dynamics we set $\rho=0$ and $\theta=1$ without loss of generality.

The units are pulse-coupled, i.e. the interactions only depend on the discrete time events at times $t_{s, i}, s \in \mathbb{Z}$, when a neuron $i$ crosses the threshold, i.e. $u_{i}\left(t_{s, i}\right) \geq \theta$. The network dynamics is then governed by the system:

$$
\frac{d}{d t} u_{i}=F\left(u_{i}\right)+Q\left(u_{i}\right) P_{i}(t) \quad i \in\{1,2, \ldots, N\}
$$

together with the reset (5.1.2). Here

$$
P_{i}(t)=\sum_{j=1}^{N} \sum_{s \in \mathbb{Z}} \varepsilon_{i j} K_{i j}\left(t-t_{s, i}\right)
$$


where $\varepsilon_{i j}$ is the connection weight from neuron $j$ to $i$ (cf. sec. 2.7.4). The $K_{i j}(t)$ are causal $\left(K_{i j}(t)=0\right.$ for $\left.t<0\right)$ and normalized $\left(\int_{0}^{\infty} K_{i j}(t) \mathrm{d} t=1\right)$ kernels characterizing, together with the state dependent coupling function $Q$ the unit's response to a pulse at time $t=0$. The multiplicative form of the pulse coupling $Q\left(u_{i}\right) P_{i}(t)$ is motivated by eq. (2.7.1). In writing (5.1.4) we have assumed linear additivity of the interactions which is a good approximation at least for weak coupling (but cf. [264]).

Under weak conditions (e.g. $Q(u)>0$ for all $\rho \leq u \leq \theta$, see [3]) it is possible to simplify eq. (5.1.3) to a constant coupling term $Q\left(u_{i}\right)=$ const. via the transformation

$$
\hat{u}_{i}(t)=\frac{1}{M} \int_{\rho}^{u_{i}(t)} \frac{1}{Q(u)} \mathrm{d} u \quad M=\int_{\rho}^{\theta} \frac{1}{Q(u)} \mathrm{d} u
$$

which yields

$$
\frac{d}{d t} \hat{u}_{i}=\hat{F}\left(\hat{u}_{i}\right)+\frac{1}{M} P_{i}(t) \quad \hat{F}\left(\hat{u}_{i}\right)=\frac{1}{M} \frac{F\left(u_{i}\left(\hat{u}_{i}\right)\right)}{Q\left(u_{i}\left(\hat{u}_{i}\right)\right)}
$$

where $u_{i}\left(\tilde{u}_{i}\right)$ is determined by solving eq. (5.1.5) for $u_{i}$.

Thus we may assume the following form of the dynamics

$$
\frac{d}{d t} u_{i}=F\left(u_{i}\right)+P_{i}(t) \quad i \in\{1,2, \ldots, N\}
$$

where we have omitted the hats and absorbed the constant $\frac{1}{M}$ into the synaptic weights $\varepsilon_{i j}$

\subsection{Partial Reset for $\delta$-Pulse-Coupled Threshold Units}

For $\delta$-pulse-coupled threshold elements the response kernels $K_{i j}$ in eq. (5.1.4) are Dirac $\delta$-functions [44]

$$
K_{i j}(t)=\delta\left(t-\tau_{i j}\right)
$$

with $\tau_{\mathrm{ij}} \geq 0$ representing a possible time delay for pulse transmission. In writing eq. (5.2.1) we assume that the duration of the response of a unit $i$ to an incoming pulse is sufficiently small compared to the time scale $\tau_{\mathrm{m}}=\mathcal{O}\left(\frac{1}{\max _{\rho \leq u \leq \theta}|F(u)|}\right)$ of the variations in the state variable $u_{i}$. For neurons this corresponds to the situation where the time scale $\tau_{\text {syn }}$ of the synaptic interactions (cf. eq. (2.7.7)) is much smaller than $\tau_{m}$, i.e. $\tau_{\text {syn }} \ll \tau_{\text {m }}$.

Systems with $\delta$-pulse-coupling are hybrid dynamical system [129] where the free continuous dynamics (5.1.1) is interrupted by discrete time dynamics at times $t_{s, i}$ due to a pulse generating or spiking event $s_{i}$ of unit $i$ and at times $t_{r, i, j}$ by the pulse reception events $r_{i, j}$ of unit $j$ of a pulse generated by unit $i$.

\subsubsection{Supra-Threshold Excitation and Absorption Rule}

Using (5.2.1) in eq. (5.1.7), a pulse from unit $i$ received by unit $j$ at time $t_{r, i, j}$ causes a change in the potential $u_{j}$ given by

$$
u_{j}\left(t_{r, i, j}\right)=u_{j}\left(t_{r, i, j}^{-}\right)+\varepsilon_{i j}
$$

If

$$
u_{j}\left(t_{r, i, j}^{-}\right) \geq \theta-\varepsilon_{i j}
$$


a unit $j$ crosses the threshold $\theta$ due to a pulse from neuron $i$ and is said to receive suprathreshold input. Since in this situation the unit crosses the threshold from below it fires a pulse itself and has to be reset.

Previous models usually reset these units in the same way as if they reached the threshold without this recurrent input, also referred to as the absorption rule (e.g. [269])

$$
u_{i}(t) \geq \theta \quad \Rightarrow \quad u_{i}\left(t^{+}\right)=\rho
$$

where the total supra-threshold input is lost. As a consequence two or more units initially in different states $u_{i}$ and simultaneously receiving supra-threshold inputs will all be reset to the same value $\rho$, making the absorption rule a strong instant synchronizing element of the network dynamics.

An alternative considered in previous studies $[229,174]$ is total input conservation,

$$
u_{i}(t) \geq \theta \quad \Rightarrow \quad u_{i}\left(t^{+}\right)=\rho+\left(u_{i}(t)-\theta\right)
$$

i.e. the total supra-threshold input charge $\zeta=u_{i}(t)-\theta$ is added to the potential $\rho$ after the reset.

\subsubsection{Partial Reset}

Here we propose a more general model where the reset value is given by a partial reset function $R(\zeta)$ that depends on the supra-threshold input charge $\zeta=u_{i}(t)-\theta$,

$$
u_{i}(t) \geq \theta \quad \Rightarrow \quad u_{i}\left(t^{+}\right)=\rho+R\left(u_{i}(t)-\theta\right)
$$

We assume that supra-threshold inputs only have excitatory effects and thus define:

Definition 5.2.1. A function $R: \mathbb{R} \rightarrow \mathbb{R}$ which is monotonically increasing and satisfies $R(0)=0$ is called a partial reset function.

For a linear partial response we set

$$
R_{c}(\zeta)=c \zeta
$$

with the remaining fraction $0 \leq c \leq 1$ of supra-threshold input charge after the reset. For $c=0$ we recover the absorption rule (5.2.4) while $c=1$ corresponds to total charge conservation (5.2.5).

Motivation for this extension comes from neural networks. Neurons consist of functionally different compartments, including the dendrite and the soma. While synaptic input currents are collected at the dendrite, the electrical pulses are generated at the soma. Additional charges not used to excite a spike may stay on the dendrite and contribute to the membrane potential after reset at the soma. Due to intra-neuronal interactions and the reset at the soma a part of this supra-threshold input charge may be lost:

Definition 5.2.2. A partial reset function $R$ is said to be neuronal, if $0 \leq R(\zeta) \leq \zeta$ for all $\zeta \geq 0$.

We note that the partial reset is expansive if $|\phi-\psi|<|R(\phi)-R(\psi)|$ for all $\phi$ and $\psi$, which is in particular the case if $\left|R^{\prime}(\phi)\right|>1$ for all $\phi$. 


\subsubsection{The Avalanche Process}

For instantaneous interactions, $\tau_{i j}=0$, a pulse generated by unit $j$ may lift other units above threshold simultaneously. These then generate a pulse on their own and may push further units above threshold, etc. This leads to an avalanche of pulses ${ }^{1}$ (cf. fig. 5.1): Units reaching the threshold at time $t$ due to the free time evolution define the triggering set

$$
\Theta^{(0)}=\left\{j \mid u_{j}\left(t^{-}\right)=\theta\right\}
$$

The units $j \in \Theta^{(0)}$ generate spikes which are instantaneously received by all the connected units $i$ in the network. In response, their potentials are updated according to

$$
u_{i}^{(1)}:=u_{i}\left(t^{-}\right)+\sum_{j \in \Theta_{0}} \varepsilon_{i j}
$$

The initial pulse may trigger certain other units $k \in \Theta^{(1)}=\left\{k \mid u_{k}\left(t^{-}\right)<\theta \leq u_{k}^{(1)}\right\}$ to spike, etc. This process continues $n \leq N$ steps until no new unit crosses the threshold. At each step $m \in\{2,3, \ldots, n\}$ the potentials are updated according to

$$
u_{i}^{(m+1)}:=u_{i}^{(m)}+\sum_{j \in \Theta_{m}} \varepsilon_{i j}
$$

where

$$
\Theta^{(m)}=\left\{k \mid u_{k}^{(m-1)}<\theta \leq u_{k}^{(m)}\right\}
$$

The potentials immediately after the avalanche $\Theta=\bigcup_{q=0}^{n} \Theta^{(q)}$ of size $a=|\Theta|$ are obtained via

$$
u_{i}\left(t^{+}\right)= \begin{cases}u_{i}\left(t^{-}\right)+\sum_{j \in \Theta} \varepsilon_{i j} & i \notin \Theta \\ \rho+R\left(u_{i}\left(t^{-}\right)+\sum_{j \in \Theta} \varepsilon_{i j}-\theta\right) & i \in \Theta\end{cases}
$$

using the partial reset $R$ for units having received supra-threshold inputs. ${ }^{2}$

The avalanche process and subsequent partial reset are illustrated in fig. 5.1. In chap. 5.3 we argue how these processes can be derived from underlying neuronal mechanism and their time scale relations.

For non-zero partial reset functions potential differences of oscillators involved in a single avalanche will in general not be fully synchronized after the reset. Thus despite the fact that units are generating pulses simultaneously they can have different phases afterwards. We therefore distinguish between phase synchrony where units have identical phases and the weaker condition of pulse synchrony which corresponds to simultaneous firing only but allows differences in the phases. When examining the system with a higher time resolution phase synchronized units will stay synchronized whereas pulse synchronized units fire within a short time interval.

\footnotetext{
${ }^{1}$ We note that avalanches of neuronal activity are observed in cortical networks [24] and studied in connection with self-organized criticality [242].

${ }^{2}$ Note that there is an ambiguity in fixing the precise order of potential updates and resets during an avalanche. Our choice is motivated by neuroscience for the situation where the time scale $\tau_{\text {ap }}$ of the action potential (and subsequent reset) is much faster than the time scale $\tau_{\text {syn }}$ of the synaptic input currents. These in turn should be faster than the time scale $\tau_{\text {ref }}$ of the mechanism reducing the supra-threshold input, e.g. the refractory period. Our model (5.2.11) then is the limit where all these time scales become small compared to the time scale $\tau_{\mathrm{m}}$ of the intrinsic interaction-free dynamics. See also sec. 5.3.1.
} 
(a)

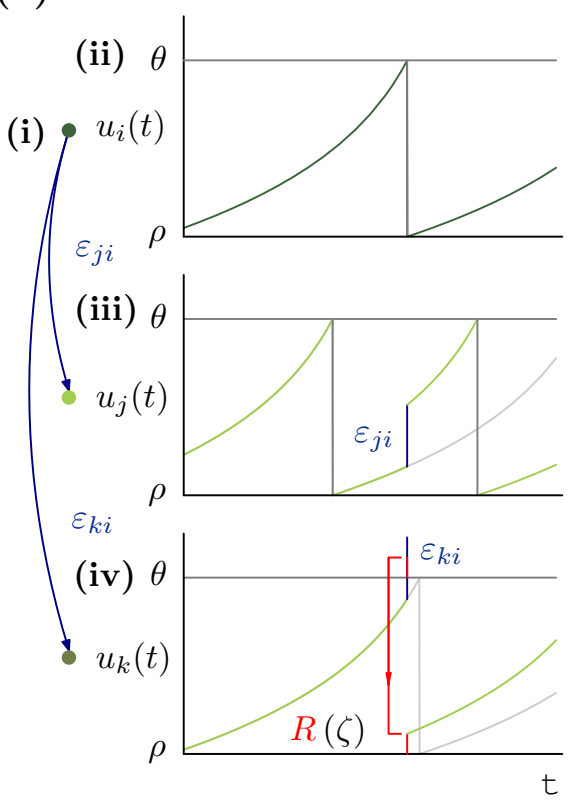

(b)

(i)

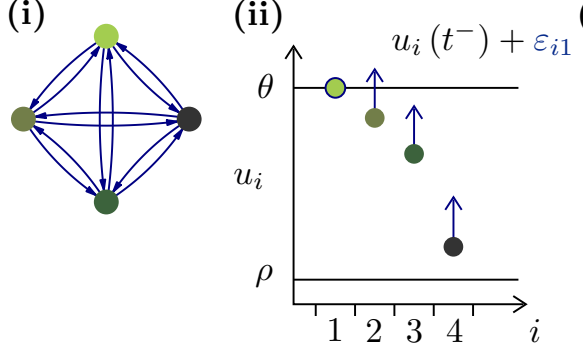

(iii) $u_{i}^{(1)}\left(t^{-}\right)+\varepsilon_{i 2}$
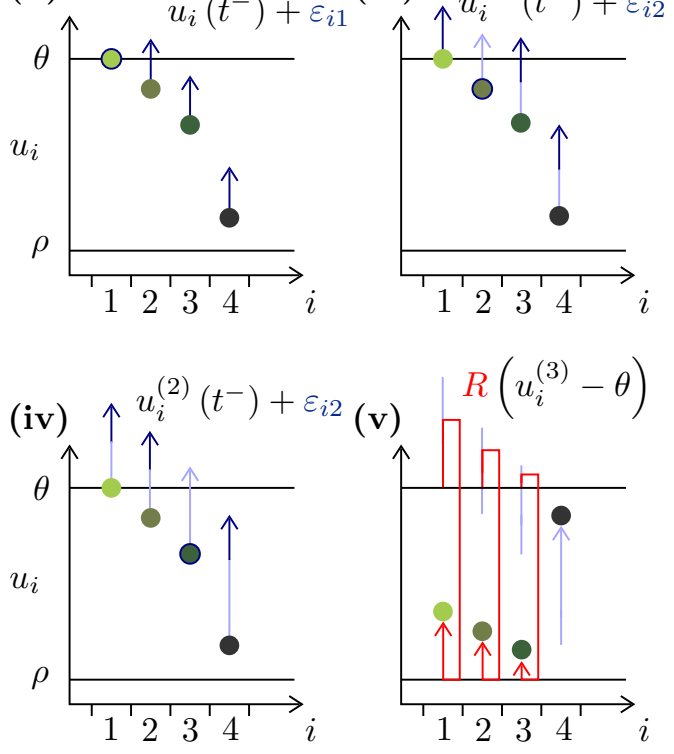

Figure 5.1. Model dynamics for instantaneous interactions. (a) sample traces of three units with (i) network connectivity $\varepsilon_{j i}=\varepsilon_{k i}>0$. (ii) At time $t=t_{1}$ unit $i$ reaches the threshold $\theta$ and its membrane potential is reset to $\rho$. It generates a pulse which is send to the units $j$ and $k$. (iii) unit $j$ receives the pulse and its membrane potential is increased to $u_{j}\left(t_{1}^{-}\right)+\varepsilon_{j i}<\theta$, the pulse is sub-threshold. (iv) unit $k$ receives a supra-threshold pulse, $u_{k}\left(t_{1}^{-}\right)+\varepsilon_{k j} \geq \theta$, its membrane potential is reset to $R(\zeta)=R\left(u_{j}\left(t_{1}^{-}\right)+\varepsilon_{k j}-\theta\right)$ using the partial reset function $R$. (b) sample avalanche process with $\Theta=\{1,2,3\}$ and $n=3$ in a (i) $N=4$ all-to-all network $\varepsilon_{i j}=\left(1-\delta_{i j}\right) \varepsilon$. (ii) unit $i=1$ reaches the threshold $\Theta^{(0)}=\{1\}$ and sends a pulse to the other units (arrows). Their potentials are updated to $u_{i}^{(1)}=u_{i}\left(t^{-}\right)+\varepsilon_{i 1}$, causing unit $i=2$ to generate a pulse, $\Theta^{(1)}=\{2\}$. (iii) the pulse is received by the other units yielding a potential $u_{i}^{(2)}=u_{i}^{(1)}+\varepsilon_{i 2}$ which brings unit $i=3$ above threshold $\Theta^{(2)}=\{3\}$. (iv) the potentials become $u_{i}^{(3)}=u_{i}^{(2)}+\varepsilon_{i 3}$ and no further unit crosses the threshold, $\Theta^{(3)}=\emptyset$. ( $\left.\mathbf{v}\right)$ the avalanche stops and units that received supra-threshold input are reset to $u_{i}(t)=\rho+R\left(u_{i}^{(3)}-\theta\right)$.

\subsubsection{Phase Representation of Pulse-Coupled Oscillators with Par- tial Reset}

In the remainder of this chapter we will concentrate on units with strictly positive $F>0$ in (5.1.1). Then the individual units become oscillatory as the strictly monotonically increasing trajectory $u_{i}(t)$ of a unit $i$ starting at $u_{i}(0)=0$ reaches the threshold after a time $T$ and is reset to zero again. By an appropriate rescaling of time we set $T=1$. Defining a phase like coordinate (cf. [269]) $\phi_{i} \in S^{1}=\mathbb{R} / \mathbb{Z}$ via

$$
\phi_{i}(t)=U^{-1}\left(u_{i}(t)\right):=\int_{0}^{u_{i}(t)} \frac{1}{F(u)} \mathrm{d} u
$$

the interaction free dynamics simplify to

$$
\frac{d}{d t} \phi_{i}(t)=1
$$

By definition $U^{-1}$ is strictly monotonically increasing and has a strictly monotonically increasing inverse $U$. By our choice of normalization they obey $U^{-1}(0)=0=U(0)$ 
and $U^{-1}(1)=1=U(1)$. Note that the function $U(\phi)$ captures the intrinsic rise of the membrane potential towards the threshold and hence for the phases of the individual units.

Definition 5.2.3. A smooth function $U:[0, \infty) \rightarrow[0, \infty)$ is called a rise function if it is strictly monotonic increasing $U^{\prime}>0$ and is normalized to $U(0)=0$ and $U(1)=1$.

Definition 5.2.4. Given a rise function $U$ and a partial reset function $R$ we define for $\varepsilon \geq 0$ the (sub-threshold) interaction function $H_{\varepsilon}:\left[0, U^{-1}(\theta-\varepsilon)\right) \rightarrow S^{1}$ by

$$
H_{\varepsilon}(\phi):=H(\phi, \varepsilon):=U^{-1}(U(\phi)+\varepsilon)
$$

and the supra-threshold interaction function $J_{\varepsilon}:\left[U^{-1}(\theta-\varepsilon), \infty\right) \rightarrow S^{1}$ by

$$
J_{\varepsilon}(\phi):=J(\phi, \varepsilon):=U^{-1}(R(U(\phi)+\varepsilon-\theta))
$$

The pulse-coupling in the potential representation eq. (5.2.11) after an avalanche $\Theta$ at time $t$ then carries over to the phase picture as

$$
\phi_{i}\left(t^{+}\right)= \begin{cases}H\left(\phi_{i}\left(t^{-}\right), \sum_{j \in \Theta} \varepsilon_{i j}\right) & i \notin \Theta \\ J\left(\phi_{i}\left(t^{-}\right), \sum_{j \in \Theta} \varepsilon_{i j}\right) & i \in \Theta\end{cases}
$$

We remark that $H_{\varepsilon}^{-1}=H_{-\varepsilon}$.

\subsubsection{Interaction Function and Phase Response Models}

The interaction function $H$ in (5.2.14) is related to the non-infinitesimal phase response curve (3.4.9) via

$$
\mathrm{z}(\phi, \varepsilon)=H(\phi, \varepsilon)-\phi
$$

Thus the rise function $U$ may be also interpreted in a more general setting as defining a phase response of a unit via eq. (5.2.14). From eq. (5.2.17) the infinitesimal phase response (3.4.5) is given by

$$
\mathrm{z}(\phi)=\lim _{\varepsilon \rightarrow 0} \frac{1}{\varepsilon}(H(\phi, \varepsilon)-\phi)=\frac{1}{\frac{d}{d \phi} U(\phi)}
$$

\subsubsection{Rise Functions and Equivalence to Other Models}

The integrate-and-fire neuron models introduced in chapter 2.6 show periodic firing for sufficiently large input currents $i_{\mathrm{e}}$. For current based synapses $i_{\mathrm{syn}}(t)=P(t)$ these models are of the form (5.1.7) and their rise functions are easily determined from eq. (5.2.12). For example for the leak integrate and fire model (2.6.4) we obtain

$$
U_{\mathrm{LIF}}(\phi)=v_{\text {eq }}\left(1-e^{-g_{1} T_{\mathrm{LIF}} \phi}\right)
$$

where $T_{\mathrm{LIF}}=-\frac{1}{g_{1}} \ln \left(1-v_{1}\right)$ is the non-normalized firing period of the non-interacting neuron and $v_{\mathrm{eq}}=\frac{i_{\mathrm{e}}}{g_{l}}+v_{\mathrm{l}}>1$ the equilibrium potential reached asymptotically if there were no threshold and reset.

For conductance based synaptic currents $i_{\text {syn }}=g_{\text {syn }}\left(v_{\text {syn }}-u\right) P(t)$ we first have to transform the system into the form (5.1.7) according to eq. (5.1.5). The general result is, 


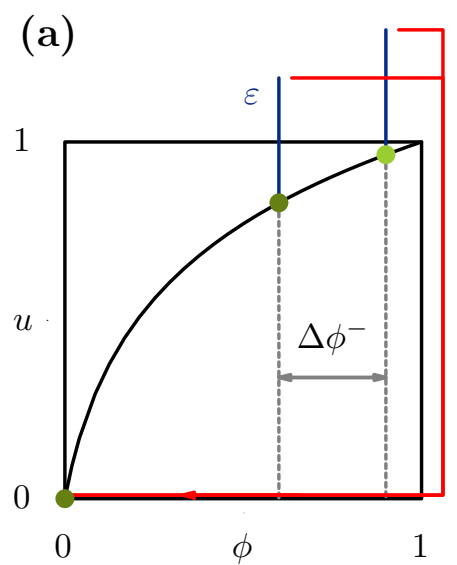

(b)

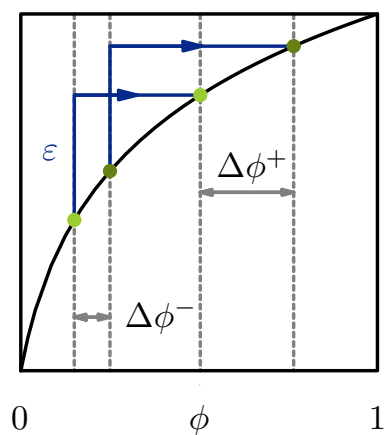

(c)

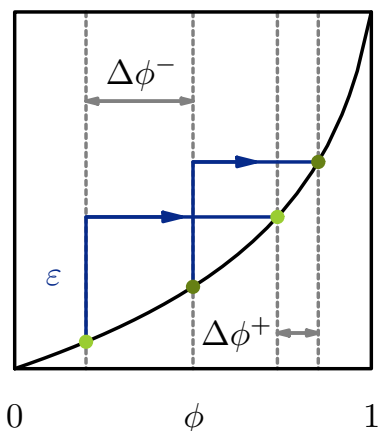

Figure 5.2. Synchronizing and desynchronizing effects in pulse coupled-oscillator models. (a) synchronization due to the absorption rule. A phase difference $\Delta \phi^{-}>0$ of two oscillators just before the simultaneous arrival of a supra-threshold input pulse is reduced to $\Delta \phi^{+}=0$. (b-c) Depending on the curvature of the rise function $U(\phi)$ the phase difference $\Delta \phi^{-}$is expanded $\Delta \phi^{+}>\Delta \phi^{-}$or contracted $\Delta \phi^{+}<\Delta \phi^{-}$on the simultaneous arrival of an excitatory pulse of strength $\varepsilon$.

that if the rise function $U$ for current based synapses is is known the conductance based rise function $U^{\mathrm{CB}}$ is given as

$$
U^{\mathrm{CB}}(\phi)=\frac{\ln \left(1-v_{\mathrm{syn}}^{-1} U(\phi)\right)}{\ln \left(1-v_{\mathrm{syn}}^{-1}\right)}
$$

Rise functions for several integrate-and-fire models are derived in appendix B. Depending on the IF model and coupling type convex, concave and sigmoidal shapes are possible (cf. tab. B.1). Figure 5.2 shows how the curvature of the rise function synchronizes or desynchronizes the phases of two units receiving simultaneous sub-threshold inputs.

For analytical investigations it is helpful to use a rise function originally introduced in [269] and given by

$$
U_{b}(\phi)=\frac{1}{b} \ln \left(1+\left(e^{b}-1\right) \phi\right)
$$

where $b$ parametrizes the curvature of $U$. For $b>0$ the function is concave $\left(U^{\prime \prime}<0\right)$ whereas for $b<0$ it is convex $\left(U^{\prime \prime}>0\right)$. In the limit $b \rightarrow 0$ it becomes linear $U_{b=0}(\phi)=\phi$. $U_{b}$ has the important property that it yields an affine interaction function:

$$
H_{b}(\phi, \varepsilon)=e^{b \varepsilon} \phi+\frac{e^{b \varepsilon}-1}{e^{b}-1}
$$

for $b \neq 0$ and $H_{b=0}(\phi, \varepsilon)=\phi+\varepsilon$

\subsection{Why Partial Reset?}

In 1964 C.F. Stevens [351] already made a comment on the reset of a neuronal threshold model [122] driven by synaptic input:

"Many neurophysiologists would have some additional reservations [...], the most serious of which is probably the following: The model assumes that the 
neuron's membrane potential is reset following each spike [...]. In physiological terms this implies that the nerve impulse destroys all remaining postsynaptic potentials."

Here we show how the partial reset can be used to model these "remaining postsynaptic potentials" in pulse-coupled elements.

\subsubsection{Time Scale Considerations}

\subsubsection{Time Scales in Neuronal Dynamics}

Neuronal dynamics involve several mechanisms operating on different time scales [284] (cf. also chap. 2). When approximating these dynamics with $\delta$-pulse coupled threshold elements there are five relevant time scales involved:

1. $\tau_{\mathrm{m}}$, the time scale of variations of the sub-threshold membrane potential due to the intrinsic dynamics (cf. chap. 5.2),

2. $\tau_{\text {syn }}$, the time scale synaptic interactions (cf. chap. 2.7),

3. $\tau_{\text {ap }}$, the time scale for action potential generation and absolute refractoriness (cf. chap. 2.3),

4. $\tau_{\text {ref }}$, the time scale for the relative refractory period (cf. chap. 2.3) and

5. $\tau_{\mathrm{d}}$, the time scale for transmission delays of spikes.

\subsubsection{Validity of $\delta$-Pulse-Coupled Threshold Models}

Modeling neuronal dynamics via $\delta$-pulse-coupled threshold elements (cf. chap. 5.2) both the synaptic interaction and the action potential generation with subsequent reset of the membrane potential are assumed to be fast compared to the sub-threshold membrane potential dynamics, i.e.

$$
\tau_{\mathrm{m}} \gg \tau_{\mathrm{syn}} \quad \text { and } \quad \tau_{\mathrm{m}} \gg \tau_{\mathrm{ap}} .
$$

Additionally we can assume

$$
\tau_{\mathrm{m}} \gg \tau_{\text {ref }}
$$

without loss of generality since otherwise the change of the response properties of the neuron to synaptic inputs due to the refractoriness can be modeled by adjusting the function $Q$ in eq. (5.1.3).

In a $\delta$-pulse-coupled model the Dirac-pulses exchanged between units (cf. (5.2.1)) represent the total charge transferred between pre- and postsynaptic neuron during the synaptic interaction. Thus the integrated remaining synaptic input current after action potential generation of the neuron is the supra-threshold input charge $\zeta$ in the pulsecoupled model.

\subsubsection{Partial Reset and Time Scale Relations for $\tau_{\text {syn }}, \tau_{\text {ap }}$ and $\tau_{\text {ref }}$}

The above conditions (5.3.1) and (5.3.2) leave the relations among $\tau_{\text {syn }}, \tau_{\text {ap }}$ and $\tau_{\text {ref }}$ undetermined. However, as shown in the following three examples (cf. fig. 5.3), the precise relations among these time scales determine the response of a neuron to the "remaining 


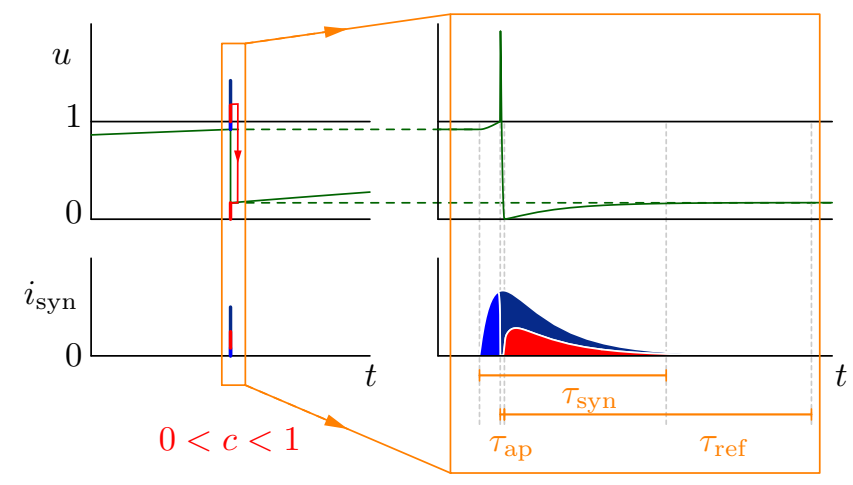

Figure 5.3. Partial reset for time scales $\tau_{\text {ap }} \ll \tau_{\text {syn }} \ll \tau_{\text {ref. }}$ Graph on the left shows the dynamics of a $\delta$-pulse-coupled threshold element receiving supra-threshold input and its subsequent partial reset approximating the time resolved neuronal dynamics shown on the right. Green traces show normalized membrane potential $u$, blue bar represents a Dirac $\delta$-input pulse resembling the integrated synaptic input current $i_{\text {syn }}$ of duration $\tau_{\text {syn }}$ on the right. The fraction of the integrated current, i.e. the charge, which lifts the membrane potential up to the firing threshold, is represented in light blue. Due to action potential generation and refractory period of the neuron further synaptic input, represented in dark blue, is effectively reduced to a charge represented in red. Assuming that the relative refractoriness reduces the input current on average by a constant factor $c$, a partial reset $R_{c}$, eq. (5.2.7) with $0<c<1$ best resembles the detailed neuronal dynamics.

postsynaptic potentials" after spike generation. If an accurate approximation of these neuronal dynamics with a $\delta$-pulse-coupled model is desired, this imposes restrictions on the reset of a threshold unit receiving supra-threshold inputs, i.e. the partial reset function.

In the first example we assume that for the neurons under consideration the synaptic interaction is much faster than the action potential generation:

$$
\tau_{\text {syn }} \ll \tau_{\text {ap }} .
$$

If the synaptic input is strong enough, the neuron is excited beyond the threshold and generates an action potential. In the absolute refractory period $\tau_{\text {ap }}$ it is completely insensitive to any remaining synaptic inputs that only last for the shorter time $\tau_{\text {syn }}$ and hence has no effect on the neuron. Since this current has no effect on the neuron a valid choice for the partial reset function when modeling these detailed dynamics with a $\delta$-pulse-coupled is $R=R_{c=0} \equiv 0$, i.e. the absorption rule (cf. eq. (5.2.4)) where all supra-threshold inputs are lost.

In a second scenario we assume

$$
\tau_{\text {syn }} \gg \tau_{\text {ap }} \text { and } \tau_{\text {syn }} \gg \tau_{\text {ref }} .
$$

In this situation the effect of action potential generation and refractory period of the neuron on remaining synaptic input currents is negligible. Thus when approximating the neuronal dynamics in this regime with $\delta$-pulse-coupled threshold model, almost all suprathreshold input charges will contribute to the membrane potential after reset and a partial reset function close to $R=\mathrm{id}=\mathrm{R}_{\mathrm{c}=1}$, i.e. total charge conservation (cf. eq. (5.2.5)), best resembles the underlying dynamics.

The third example is concerned with time scales obeying the relations

$$
\tau_{\text {syn }} \gg \tau_{\text {ap }} \quad \text { and } \quad \tau_{\text {syn }} \ll \tau_{\text {ref }} \text {. }
$$


and is illustrated in fig. 5.3. Here if a neuron is forced to fire by synaptic inputs, remaining input currents are not affected due the short absolute refractoriness but due to the longer relative refractory period. In the relative refractory period of the neuron still a large number of ion channels are open as a remainder of the action potential generation and thus the membrane conductivity is increased. As a consequence synaptic input charges will leave the cell without contributing to the membrane potential integration. Usually the relative refractoriness of neurons varies in time, but for simplicity we here assume that on average the synaptic inputs are reduced by a constant factor $0 \leq r \leq 1$. We can take into account this effect in $\delta$-pulse-coupled models by using a partial reset with reset function $R=R_{c}$ using $c=r$.

Using the same arguments, validity of the avalanche mechanism in sec. 5.2.3 is obtained for neuronal systems where the transmission delay $\tau_{\mathrm{d}}$ is effectively zero, i.e. $\tau_{\mathrm{d}} \ll \tau_{\mathrm{m}}$ and the time scale relations (5.3.5) for the neurons are satisfied. Additionally it has to be assumed that the maximal duration $\tau_{\mathrm{a}}$ of an avalanche involving all $N$ neurons in the network obeys

$$
\tau_{\mathrm{a}}:=N\left(\tau_{\text {syn }}+\tau_{\mathrm{d}}\right) \leq \tau_{\text {ref }} \ll \tau_{\mathrm{m}}
$$

\subsubsection{Spike Time Response}

A sufficiently large tonic stimulus $i_{\mathrm{e}}$ causes periodic spiking in many neuron models (cf. chap. 2 and also part IV), i.e. they become a neuronal oscillator. The phase reduction method (sec. 3.4) then reduces such oscillators to a phase variable and a phase response function $z$ that characterizes the change of the phase due to inputs. In particular, the non-infinitesimal phase response $z(\phi, \varepsilon)(\mathrm{cf}$. (3.4.9) and also (5.2.17)) gives the asymptotic phase change due to a stimulus of strength $\varepsilon$ applied at a phase $\phi$.

In this description the precise effect of the stimulus on the following spike times is not accounted for. In particular supra-threshold synaptic inputs shortly before the spike generation of the neuron, not only cause the neuron to fire earlier but also shorten the following inter spike interval (ISI). To capture how total phase response is distributed over the individual ISIs we introduce a spike time response (STR): Let $T$ denote the unperturbed ISI and write $T_{i}, i \in\{0,1,2, \ldots\}$ for the $i^{\text {th }}$ ISI after the perturbation, with $T_{0}$ being the ISI in which the perturbation of strength $\varepsilon$ is applied at phase $\phi$. Then the $i^{\text {th }}$ spike time response curve $z_{\mathrm{sp}}^{(i)}$ is defined as

$$
z_{\mathrm{sp}}^{(i)}(\phi, \varepsilon):=\frac{T-T_{i}}{T}
$$

From this the phase response is recovered via

$$
\mathrm{z}(\phi, \varepsilon)=\sum_{\mathrm{i}=0}^{\infty} \mathrm{z}_{\mathrm{sp}}^{(\mathrm{i})}(\phi, \varepsilon) .
$$

This is illustrated in fig. 5.4a,b for the cortical neuron model eq. (2.4.5): A brief input conductance a a later stage of the ISI not only shortens this one $T_{0}<T$ but also the next $T_{1}<T$. This effect is captured by the $z_{\mathrm{sp}}^{(1)}(\phi, \varepsilon)$ attaining non-zero values close before spiking of the neuron. For higher orders $i \geq 2$ we find $z_{\mathrm{sp}}^{(i)}(\phi, \varepsilon)=0$.

We can model this effect by a non-zero partial reset in a $\delta$-pulse coupled oscillator model. This is shown in fig. 5.4c,d where a good approximation to the spike time response 

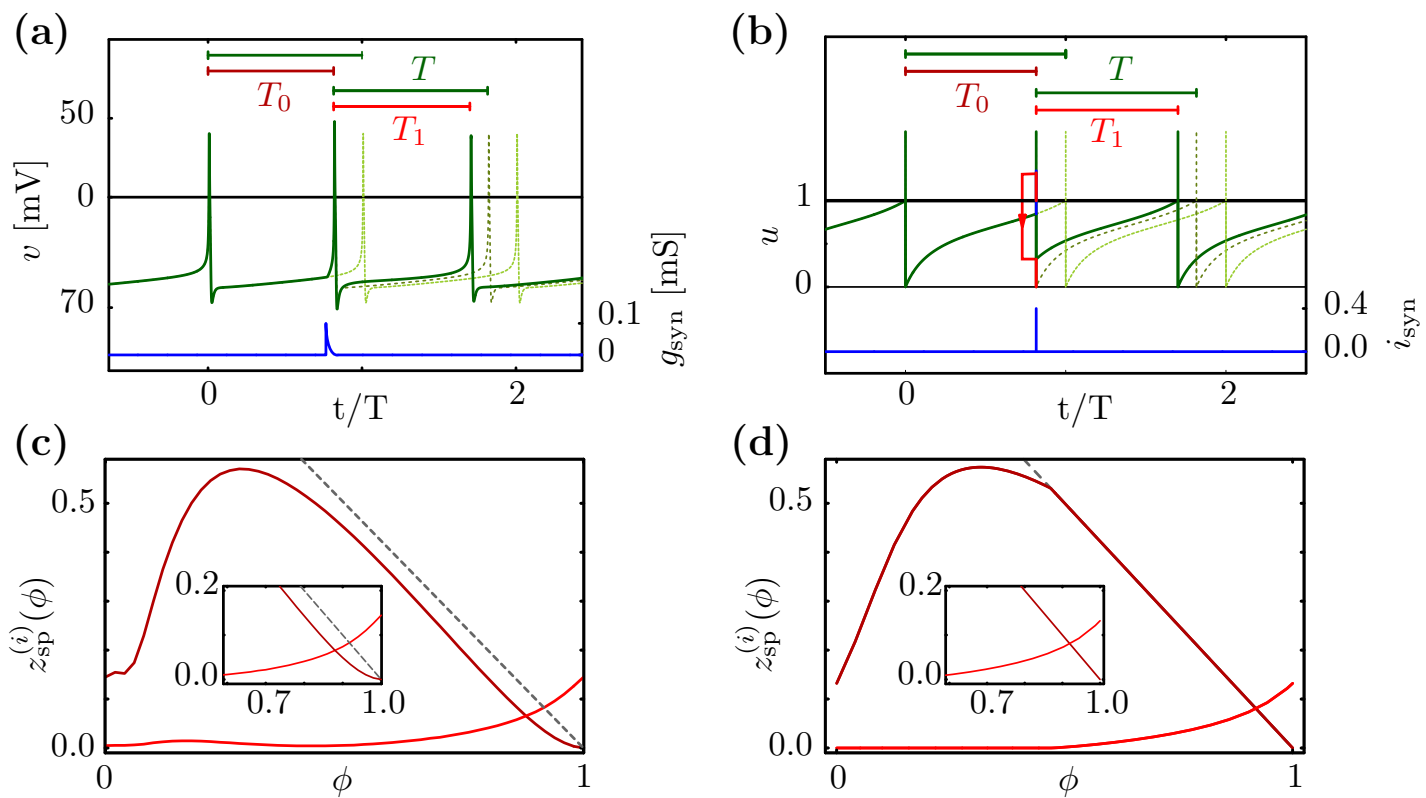

Figure 5.4. Spike time response and partial reset. (a) membrane potential for the cortical neuron model (2.4.5) with $i_{e}=5 \mathrm{nA}$ (other parameter as in appendix D.4) shows periodic spiking with $T \approx 65 \mathrm{~ms}$. A brief synaptic pulse into the dendrite of duration $1.5 \mathrm{~ms}$ and strength $g_{\text {syn }}=0.1 \mathrm{mS}$ forces the neuron to fire and shortens the actual ISI to $T_{0} \approx 53 \mathrm{~ms}$. In addition also the next ISI is shorter $T_{1} \approx 57 \mathrm{~ms}$. (c) spike time response functions $\mathrm{z}_{\mathrm{sp}}^{(0)}$ (bordeaux) and $\mathrm{z}_{\mathrm{Sp}}^{(1)}$ (red) for the cortical neuron model. The dashed line $(1-\phi)$ indicates instantaneous firing after stimulus presentation. All responses $\mathrm{z}_{\mathrm{sp}}^{(\mathrm{i})} i \geq 2$ are zero. (b) as in (a) using a pulse coupled oscillator model with partial reset $R(\zeta)=0.4\left(\frac{\zeta}{0.4}\right)^{1.3}$ and QIF rise function (B.1.3) $(\alpha=1.3$, $\left.\beta=-0.7, v_{\mathrm{syn}}=2\right)$. (d) as in (b) for the QIF model in (c) with $\varepsilon=0.4$ showing similar spike time response functions to the cortical neuron model. Note that with absorption rule $R \equiv 0$ the curve $\mathrm{z}_{\mathrm{sp}}^{(1)} \equiv 0$ would not capture the effect.

curves of the cortical neuron model is obtained using a non-zero, non-linear partial reset. In general for a partial reset $R$ and rise function $U$ we have the identity

$$
z_{\mathrm{sp}}^{(1)}(\phi, \varepsilon)=U^{-1}(R(U(\phi)+\varepsilon-\theta))
$$

if the pulse is supra-threshold, i.e. $U(\phi)+\varepsilon \geq \theta$. Moreover, the neuron fires instantaneously due to this input and hence $z_{\mathrm{sp}}^{(0)}(\phi, \varepsilon)=1-\phi$ in this case. Note that in fig. $5.4 \mathrm{~b}$ the cortical neuron model does not fire instantaneously because the activation rates for of the ionic currents are finite.

We also observed [204] non-zero $z_{\mathrm{sp}}^{(1)}(\phi, \varepsilon)$ in two compartmental models of an active soma and a passive dendrite as in eq. 2.5.1. Here only the soma is directly repolarized after spike generation and remaining input charges on the dendrite contribute to the integration of the membrane potential in the next cycle. This is also observed in more biophysically detailed compartmental models $[250,291]$ and captured in simpler two compartment integrate-and-fire neurons by resetting the somatic membrane after threshold crossings only [38, 321]. 


\subsubsection{Theoretical Aspects}

From the viewpoint of dynamical systems theory the analytical study of pulse-coupled oscillator systems and their variations is an interesting topic in itself: The majority of dynamical systems studied mathematically exhibit continuous interactions and most of the theoretical framework and concepts that have been found to analyze, describe and classify dynamical systems are based on the assumption of continuity or even differentiability [199]. Networks of $\delta$-pulse-coupled units, which are dynamical systems with temporally discrete and discontinuous interactions, are far less studied. Thus detailed analytical studies of these systems may also help to develop general theoretical concepts in this area. For example in pulse-coupled oscillator networks an event-based analysis has proven to be useful $[269,333,16]$. In chapter 7 we extend this technique to an event-sequence-based domain analysis.

Beside these general considerations, an open question in the theory of pulse-coupled threshold elements is how the reset influences the collective network dynamics and in particular synchronization. For example, after simultaneous supra-threshold excitation, the absorption rule (5.2.4) has a strong synchronizing effect as shown in fig. 5.2. Via a partial reset the response to supra-threshold inputs can be varied systematically and implications for the collective network dynamics can be studied analytically. Furthermore, an absorption rule introduces a non-invertibility into the pulse-coupled oscillator systems as phases after an absorptive reset do not carry any information about their previous state. In contrast, via a bijective partial reset function all information about the state prior reset is kept and the system becomes invertible, at least locally. Consequences for the dynamics of this aspect are subject to chapter 7 . 


\section{Chapter 6}

\section{Partial Reset and Collective Network Dynamics - Sequential Desynchronization Transition}

In this chapter we study the impact of the partial reset mechanisms on the collective network dynamics of pulse coupled oscillators when the interactions are instantaneous. We concentrate on homogeneous networks. For concave rise functions we find synchronization if the partial reset is not expanding and otherwise irregular dynamics. For convex and sigmodial rise functions the partial reset controls a sequential desynchronization transition: An increase in the strength of the partial response induces a sequence of bifurcations from states with large clusters of synchronously firing neurons, through states with smaller clusters to completely asynchronous spiking. We also discuss key consequences of this desynchronization mechanisms for biophysically more detailed systems.

\subsection{Collective Network Dynamics - Numerical Results}

To identify the effects of the partial reset on the collective network dynamics we here first focus on homogeneous networks without interaction delays, $\tau_{i j}=0$, consisting of $N$ units with all-to-all coupling and without self-interaction, i.e.

$$
\varepsilon_{i j}=\left(1-\delta_{i j}\right) \varepsilon
$$

$i, j \in\{1,2, \ldots, N\}$. We impose the condition $\sum_{j} \varepsilon_{i j}=(N-1) \varepsilon<\theta-\rho=1$ to avoid self-sustained avalanches of infinite size.

\subsubsection{Concave Rise Functions}

We first briefly focus on networks consisting of oscillators with a strictly concave rise function.

$$
\frac{d^{2}}{d \phi^{2}} U(\phi)<0
$$

resembling for example the dynamics of a LIF neuron eq. (5.2.18) or a conductance based LIF neuron eq. (B.1.1) for parameter values $v_{\mathrm{syn}}>v_{\mathrm{eq}}$ (cf. tab. B.1). We considered these types of networks in detail in our earlier work [204].

Figure 6.1 shows a simulation of such a network using a strictly neuronal partial reset with dynamics that converge towards a state of synchronous firing which is stable against 
(a)

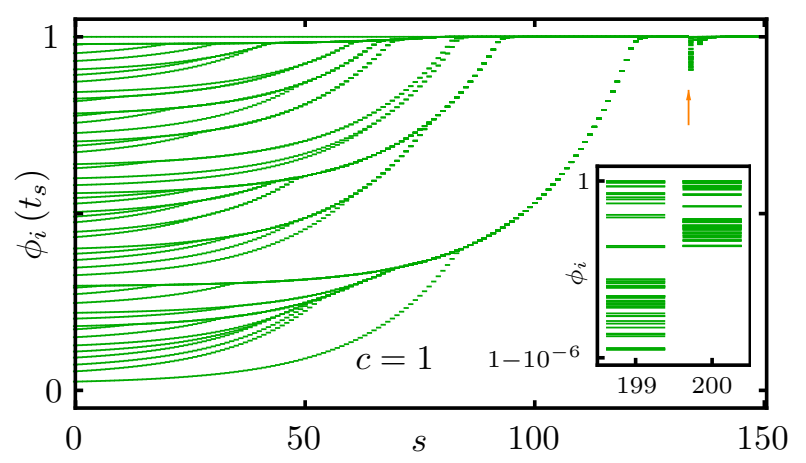

(b)

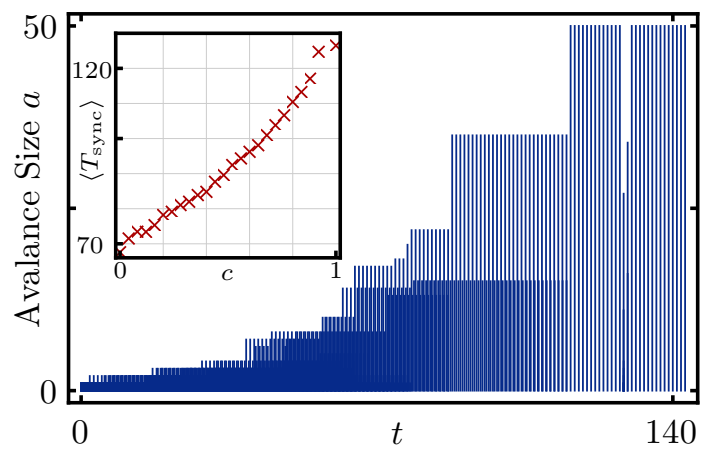

Figure 6.1. Synchronization in homogeneous networks with concave rise function and strictly neuronal partial reset. All-to-all coupled network $(\varepsilon=0.001)$ of $N=50$ oscillators with rise function $U_{b=1}$ and strictly neuronal partial reset $R(\zeta)=\zeta$. (a) oscillator phases $\phi_{i}\left(t_{s}^{-}\right)$just before the $s^{\text {th }}$ spike of oscillator $i=1$ at times $t_{s}$. The system forms larger and larger groups of synchronously firing neurons until finally a state of synchronous firing is reached, which is stable against small perturbations (orange arrow). Inset: Although synchronous firing is reached the phases are not fully synchronized but the maximal phase difference converges exponentially fast towards zero. (b) avalanche sizes vs. time for the same dynamics: after a transient neurons fire synchronously in a single avalanche of size $N=100$. The inset shows the average times $\left\langle T_{\text {sync }}\right\rangle$ to reach synchronized firing for different values of the reset strength $c$ (average over 100 simulations starting from random uniformly distributed initial phases).

small perturbations. Here larger and larger groups of synchronized neurons (clusters) from until finally the system reaches the synchronized state. Numerically, we observe stable synchrony for a variety of rise functions which are concave and partial resets that are strictly neuronal. In particular, for the linear partial reset function $R_{c}$ we observe synchronization if $c$ is chosen in $[0,1]$ as shown for $c=1$ in fig. 6.1. The transient time $T_{\text {syn }}$ needed to reach synchronous firing starting from random initial conditions depends on the precise form of the partial reset. For $R_{c}$ it scales almost linear with $c$ (cf. inset fig. $6.1 b)$. We note that during synchronized firing the precise phases values are typically not fully synchronized as illustrated in the inset of fig. 6.1a. However, phase differences decay exponentially and phase synchrony is reached asymptotically.

For a partial reset that is expanding synchronous firing is usually not reached. Instead more complex periodically firing states are observed as shown in fig. 6.2. However when increasing the concavity of the rise function sufficiently the network again reaches the state of synchronous firing.

The analysis of Mirollo and Strogatz [269] shows that when the rise function is concave $\left(U^{\prime \prime}<0\right)$ and the absorption rule is used $(R \equiv 0$ but with a different avalanche process $)$ synchronization from almost all initial conditions is achieved. In fact, in our earlier work [204] we gave a mathematical proof that their result can be generalized to the partial reset model used here and any partial reset function $R$ that is non-expansive (e.g. $R^{\prime} \leq 1$ ).

For expansive $R$ the stability of the synchronized stated depends strongly on the expansion rate of the partial reset, as shown in fig. 6.2. The synchronous firing state is reached if the sub-threshold synchronization process compensates for the expansion of the partial reset. If the expansion rate gets to strong the larger clusters get unstable and we observe irregular spiking as shown in fig. 6.2. We also gave a detailed mathematical analysis of this transition in [204] which showed that it is the result of an mutual inter- 


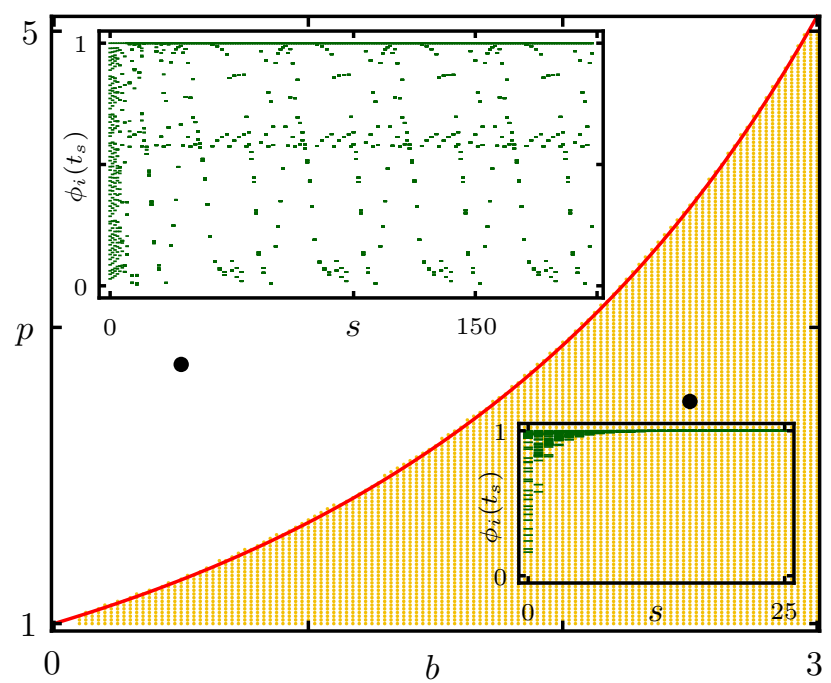

Figure 6.2. From synchronization to irregular dynamics in networks with concave rise functions and expanding partial reset. Dynamics and phase diagram of a homogeneous network of $N=50$ oscillators with coupling strength $\varepsilon=0.01$, rise function $U_{\mathrm{b}}$ and partial reset $R_{p}=R_{p}(\zeta)=$ $(N-1) \varepsilon\left(\frac{\zeta}{(N-1) \varepsilon}\right)^{p}$ with expansion parameter $p$. The insets show that the dynamics either converges to the synchronized state (lower right $b=2.5, p=2.5$, phases $\phi_{i}\left(t_{s}^{-}\right)$as in fig. 6.1) or to irregular spiking (upper left, $b=0.5, p=2.75$ ). The main plot shows beige dots at parameter values $p \in\{1,1.025, \ldots 5\}$ and $b \in\{0,0.025, \ldots, 3\}$ where synchronized firing is reached after $10^{6}$ spikes in 100 simulations starting from random initial conditions. The solid red line is the analytical result obtained in our earlier work [204] that is solvable for the rise function $U_{b}$ and given by $p=\frac{1}{\ln \left(\frac{N-2}{N-1}\right)} \ln \left(\frac{\ln \left(e^{b(N-1) \varepsilon}+e^{b(1-\varepsilon)}-e^{b}\right)}{b(N-1) \varepsilon}\right)$. Black dots show the parameter values for the insets.

action between the synchronization process due to the sub-threshold dynamics as in fig. 6.1 and the desynchronization due to an expanding partial reset.

\subsubsection{Convex Rise Functions: A Sequence of Desynchronizing Bi- furcations}

In this section we concentrate on convex rise functions $U$, i.e.

$$
\frac{d^{2}}{d \phi^{2}} U(\phi)>0
$$

This property holds for a large class of conductance based leaky-integrate-and-fire (LIF) neurons and a class of quadratic-integrate-and-fire (QIF) neurons (cf. appendix B). Studying convex rise functions is further motivated by the fact that for these rise functions we already observe a rich diversity of collective network dynamics with a strong dependence on the partial reset $R$. However, our results also apply to more general rise functions and in particular to sigmoidal shapes (cf. sec. 6.7) as often found for neurons [100, 110].

Systematic numerical investigations indicate a strong dependence of the network dynamics on the partial reset $R$. In particular, we find synchronous states, cluster states, asynchronous states and a sequential desynchronization of clusters when increasing the partial reset strength, e.g. by increasing the parameter $c$ when using $R=R_{c}$. 
(a)

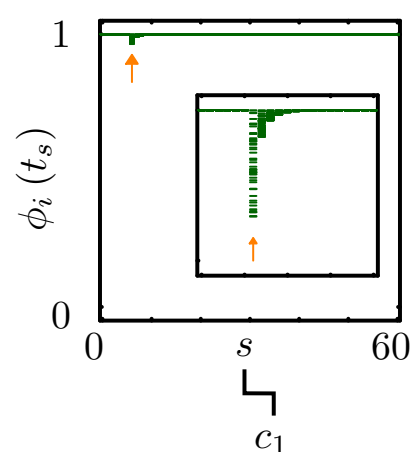

(b)

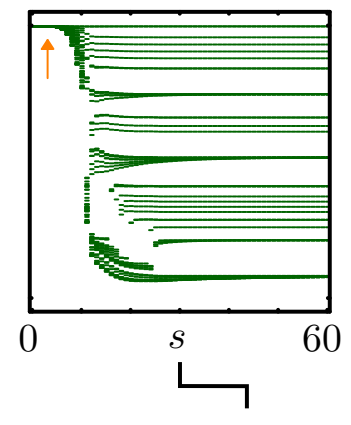

(c)

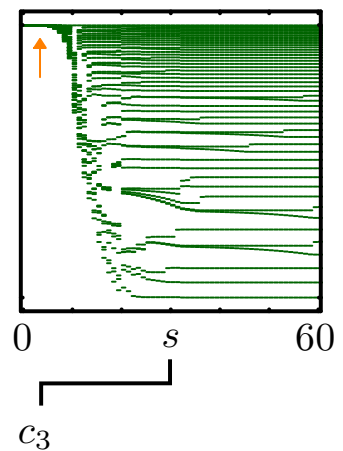

(d)

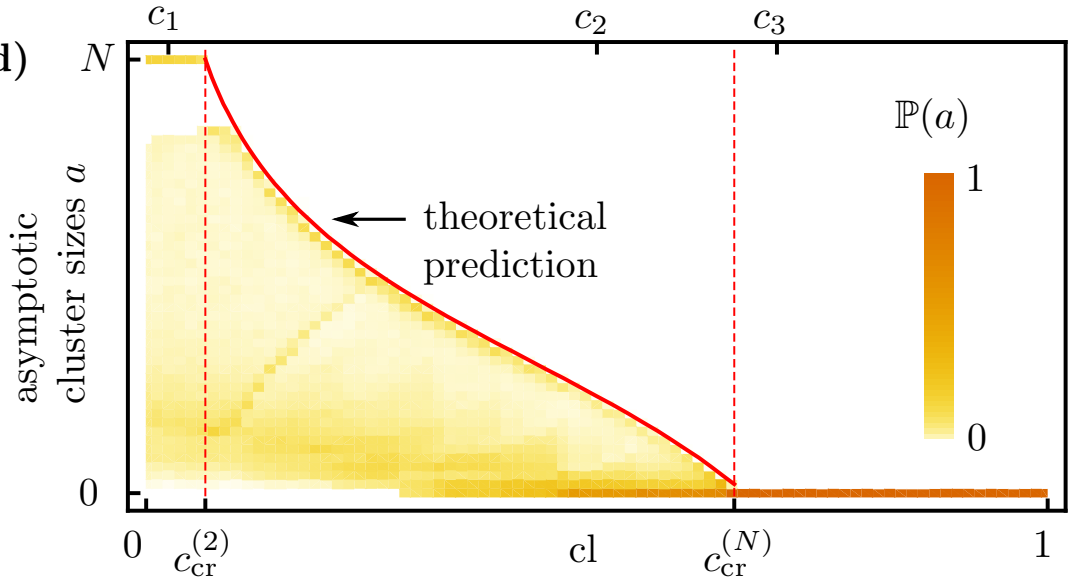

Figure 6.3. Sequential desynchronization transition in a network of $N=50$ neurons $\left(U=U_{b}\right.$, $b=-3, \varepsilon=0.0175)$. The phases $\phi_{i}$ of all neurons are plotted against the $s$-th spike of a reference neuron. Starting from a synchronous state and perturbing at $s=5$, the phase dynamics are shown for (a) $c_{1}=0.025<c_{\mathrm{cr}}^{(N)}$ (inset: magnification), (b) $c_{2}=0.5 \in\left(c_{\mathrm{cr}}^{(N)}, c_{\mathrm{cr}}^{(2)}\right)$ and (c) $c_{3}=$ $0.7>c_{\mathrm{cr}}^{(2)}$. (d) probability $\mathbb{P}(a)$ of observed cluster sizes $a$ in the asymptotic dynamics of 1500 simulations for each $c \in\{0,0.0125, \ldots, 1\}$ starting from random phases uniformly distributed in $[0,1)$. red line: exact theoretical prediction (6.6.1) above which clusters are unstable. The interval $\left(c_{\mathrm{cr}}^{(N)}, c_{\mathrm{cr}}^{(2)}\right)$ constitutes the sequential transition region. The gap in the probability distribution for small $c$ and large cluster sizes is explained by lemma 6.4.2.

Starting in the synchronized state and then applying a small perturbation to the phases we observe that the synchronized state is stable for sufficiently small $c$ (fig. 6.3a). When increasing the partial reset strength the synchronized state becomes unstable and we observe smaller clusters in the asymptotic network dynamics (fig. 6.3b) where the final cluster state depends on the precise form of the perturbation. The maximally observed cluster sizes depend on the value of $c$ (fig. 6.3d). For sufficiently large $c$ only the asynchronous splay state, i.e. a state with maximal cluster size $a=1$, is observed (fig. $6.3 \mathrm{c})$.

Starting from random initial conditions we find that for sufficiently small $c$ the synchronous state coexists with a variety of cluster states and the asynchronous state. Increasing $c$, the states involving larger clusters become unstable until finally all random initial conditions lead to the asynchronous state.

What is the origin of this rich repertoire of dynamics and which mechanisms control the observed transition of sequential desynchronizing bifurcations? To answer these questions, we analytically investigate the existence and stability of periodic states involving clusters 
of arbitrary sizes $a \leq N$. The following analysis reveals that the sequence of bifurcations is controlled by two effects: sub-threshold inputs that are always synchronizing and suprathreshold inputs that are either synchronizing or desynchronizing depending on the partial reset strength.

\subsection{Strategy of the Analysis}

We split up our analysis of the dynamics into two parts. First we assume that all avalanches are invariant, i.e. the given clusters do not decay into smaller sub-clusters. This assumption allows to group all oscillators firing in a single avalanche together into a single "meta-oscillator" with increased firing strength and an effective self-interaction. The analysis of the homogeneous all-to-all network $\left(\varepsilon_{i j}=\left(1-\delta_{i j}\right) \varepsilon\right)$ of $N$ oscillators with avalanche sizes $a_{s}, s \in\{1,2, \ldots, m\}, \sum_{s} a_{s}=N$ then reduces to analyzing a network of $m$ meta-oscillators with coupling strengths

$$
\varepsilon_{i j}=\left(1-\delta_{i j}\right) \varepsilon_{i}+\delta_{i j} \varepsilon_{i i}
$$

and $\varepsilon_{i}=a_{i} \varepsilon, \varepsilon_{i i}=\left(a_{i}-1\right) \varepsilon$. Thus we employ symmetry and reduce the analysis of cluster states to the dynamics of the corresponding quotient network [132].

In a second step we derive conditions under which an avalanche of a certain size will indeed not decay into smaller groups.

\subsection{Notations: State Space, Firing and Return Map}

A state of a network of $N$ pulse-coupled oscillators is completely specified by a phase vector

$$
\Phi=\left(\phi_{1}, \phi_{2}, \ldots, \phi_{N}\right) \in \mathcal{X}=\mathbb{T}^{N}=\underbrace{S^{1} \times \cdots \times S^{1}}_{N \text { times }}
$$

where $\phi_{i} \in S^{1}=\mathbb{R} / \mathbb{Z}$ are the phases of the individual units. Since the time evolution in between avalanches is a pure phase shift (5.2.13) it is convenient to consider a Poincare section $\mathcal{P}$ of $\mathbb{T}^{N}$ with states just before the firing of one or more oscillators, i.e.

$$
\mathcal{P}=\left\{\Phi \in \mathbb{T}^{N} \mid \exists j \in\{1, \ldots, N\}, \phi_{j}=1\right\} .
$$

It is convenient to relabel the oscillators after each avalanche such that $1=\phi_{1} \geq \phi_{2} \geq$ $\ldots \phi_{N-1} \geq \phi_{N}>0$. To specify the state of the network completely the permutation $\pi^{-1}$ used for relabeling of the oscillators is remembered. The largest phase $\phi_{1}=1$ thus belongs to the oscillator $i=\pi(1)$, the second largest $\phi_{2}$ to $i=\pi(2)$, etc. Thus an equivalent description of the state space $\mathcal{P}$ is given by

$$
\mathcal{P}^{p}=\left\{\left(\left(\phi_{2}, \ldots, \phi_{N}\right), \pi\right) \in S^{N-1} \times S_{N} \mid 1 \geq \phi_{2} \geq \ldots \phi_{N-1} \geq \phi_{N} \geq 0\right\}
$$

Here $S_{N}$ is the group of all permutations of $N$ elements. We use the convention that all index labels $i$ are taken modulo the network size $N$, e.g. labels $i$ and $i+N$ denote the same oscillator.

The Poincare map of the network dynamics for the Poincare section $\mathcal{P}$ is the firingmap $\mathbf{K}$ that maps the state $\Phi \in \mathcal{P}$ of the network just before the $s$-th firing time $t_{s}$ of an avalanche to the state just before the next avalanche that occurs at time $t_{s+1}$ :

$$
\mathbf{K}\left(\Phi\left(t_{s}^{-}\right)\right)=\Phi\left(t_{s+1}^{-}\right) \in \mathcal{P}
$$


Having determined the next avalanche $\Theta$ from a state $\Phi \in \mathcal{P}$, the map $\mathbf{K}$ is a composition of the avalanche map (5.2.16) and a subsequent shift of all phases by $\sigma$ to a state in $\mathcal{P}$. Note that the firing map is fully determined by the pair $(\Theta, \sigma)$ which is a function of $\Phi$. We denote a phase shift of size $\sigma$ by

$$
S(\phi, \sigma):=S_{\sigma}(\phi):=\phi+\sigma
$$

The equivalent firing map acting on the state space $\mathcal{P}^{p}$ is denoted by $\mathbf{K}^{p}$. For the phase part we write

$$
\mathbf{K}_{\Phi}^{p}\left(\left(\psi_{2}^{(0)}, \ldots, \psi_{N}^{(0)}\right), \pi^{(0)}\right)=\left(\psi_{2}^{(1)}, \ldots, \psi_{N}^{(1)}\right) .
$$

To track the network dynamics we consider a mapping of the state just before a fixed reference oscillator $k$ fires in an avalanche at time $t_{r}$ to the state just before this oscillator fires again at $t_{r+1}$ :

$$
\mathbf{M}\left(\Phi\left(t_{r}^{-}\right)\right)=\Phi\left(t_{r+1}^{-}\right)
$$

$\mathbf{M}$ is called the return-map and is the Poincaré return map of the system on the section $\left\{\Phi \in \mathcal{P} \mid \phi_{k}=1\right\}$. Again the equivalent return map acting on $\mathcal{P}^{p}$ is denoted by $\mathbf{M}^{p}$. The number $m$ of avalanches occurring in the application of the return map is a function of the initial phase vector $\Phi=\Phi\left(t_{r}^{-}\right)$and thus the return map $\mathbf{M}$ is a composition of $m$ firing maps $\mathbf{K}$. Hence $\mathbf{M}$ is completely specified by an ordered firing sequence

$$
\mathcal{F}=\mathcal{F}(\Phi)=\left\{\left(\Theta_{s}, \sigma_{s}\right)\right\}_{s=1}^{m}
$$

where the pairs $\left(\Theta_{s}, \sigma_{s}\right)$ specify the avalanche set $\Theta_{s}$ and subsequent shift $\sigma_{s}$ of the $s$-th firing map.

Given a firing sequence (6.3.7), we set $a_{s}=\left|\Theta_{s}\right|$ and in the case of homogeneous networks with coupling (6.1.1), $\varepsilon_{s}=a_{s} \varepsilon$. A composition of shift and interaction maps is denoted as

$$
\bigodot_{s=1}^{m}\left(S_{\sigma_{s}} \circ H_{\varepsilon_{s}}\right)(\phi):=S_{\sigma_{m}} \circ H_{\varepsilon_{m}} \circ S_{\sigma_{m-1}} \circ H_{\varepsilon_{m-1}} \ldots \circ S_{\sigma_{2}} \circ H_{\varepsilon_{2}} \circ S_{\sigma_{1}} \circ H_{\varepsilon_{1}}(\phi)
$$

\subsection{Existence and Stability of Asynchronous Periodic States in Meta-Oscillator Networks}

Definition 6.4.1. An asynchronous periodic state of a network of $N$ pulse-coupled oscillators is a state $\Phi \in \mathcal{P}$ which is invariant under the return map, i.e. $\mathbf{M}(\Phi)=\Phi$, and with avalanche sizes $a_{s}=1, s \in\{1,2, \ldots, N\}$, i.e. each oscillator generates a pulse separately.

Initially assume that all clusters stay forward invariant, i.e. do not decay in to smaller sub-clusters during the network dynamics (cf. sec. 6.2) and thus consider networks of metaoscillators with effective coupling matrix (6.2.1). A periodic cluster state in the original model thus becomes an periodic asynchronous state in the reduced effective meta-oscillator network. In the following we derive conditions for the existence of the asynchronous state and its stability in a meta-network.

Lemma 6.4.2. Consider a network (5.2.13)-(5.2.16) of $N$ oscillators with pulse coupling matrix (6.2.1) and neuronal partial reset. Let $\Sigma=\left(\sigma_{1}, \ldots, \sigma_{N}\right) \in \mathbb{R}^{N}$ and define $\mathbf{L}$ : $\mathbb{R}^{N} \times S^{1} \rightarrow \mathbb{R}^{N}$ by

$$
\mathbf{L}_{i}(\Sigma, \phi):=\bigodot_{s=i+1}^{N+i-1}\left(S_{\sigma_{s}} \circ H_{\varepsilon_{s}}\right) \circ S_{\sigma_{i}} \circ J_{\varepsilon_{i i}}(\phi)
$$


for $i \in\{1,2, \ldots, N\}$. Then the asynchronous state exists if and only if there is a solution $\Sigma^{*} \in \mathbb{R}^{N}$ to the equation

$$
\mathbf{L}(\Sigma, 1)=(1,1, \ldots, 1)
$$

that satisfies $\sigma_{r}^{*}>0$ for all $r \in\{1,2, \ldots, N\}$.

Proof. Assume there is a solution $\Sigma^{*}, \sigma_{i}^{*}>0$. Set

$$
\phi_{1}^{*}=1, \quad \phi_{i}^{*}=\left(H_{\varepsilon_{1}}^{-1} \circ S_{\sigma_{1}^{*}}^{-1}\right) \circ\left(H_{\varepsilon_{2}}^{-1} \circ S_{\sigma_{2}^{*}}^{-1}\right) \circ \cdots \circ\left(H_{\varepsilon_{i-1}}^{-1} \circ S_{\sigma_{i-1}^{*}}^{-1}\right)
$$

for $i \in\{2 \ldots, N\}$. Using that $\Sigma^{*}$ is a solution to (6.4.1) we have $\bigodot_{r=1}^{N-1}\left(S_{\sigma_{r}^{*}} \circ H_{\varepsilon_{r}}\right) \circ$ $S_{\sigma_{N}^{*}} \circ J_{\varepsilon_{N N}}(1)=1$ and $\phi_{N}^{*}=S_{\sigma_{N}^{*}} \circ J_{\varepsilon_{N N}}(1)>0$ since $\sigma_{N}^{*}>0$. Further using $\varepsilon_{i}>0$ and $\sigma_{r}^{*}>0$ the phases are ordered according to $\phi_{1}^{*}=1>\phi_{2}^{*}>\cdots>\phi_{N}^{*}>0$ and $\Phi^{*}=\left(\phi_{1}^{*}, \phi_{2}^{*}, \ldots, \phi_{N}^{*}\right) \in \mathcal{P}$.

Starting from the state $\Phi^{*}$ the first pulse of oscillator $i=1$ results in potentials $u_{1}^{(1)}=R\left(\varepsilon_{11}\right), u_{i}^{(1)}=U\left(\phi_{i}^{*}\right)+\varepsilon_{1}, i \in\{2,3, \ldots, N\}$. Since $R\left(\varepsilon_{i i}\right) \leq \varepsilon_{i i} \leq \varepsilon_{i}<\varepsilon_{i}+U(\phi)$ for all $\phi>0$ and $H_{\varepsilon_{i}}(\phi)<H_{\varepsilon_{i}}(\psi)$ for $\phi<\psi$ we have $u_{1}^{(1)}<u_{N}^{(1)}<u_{N-1}^{(1)}<\cdots<u_{2}^{(1)}$. Further

$$
u_{2}^{(1)}=U\left(\phi_{2}^{*}\right)+\varepsilon_{1}=U\left(\left(H_{\varepsilon_{1}}^{-1} \circ S_{\sigma_{1}^{*}}^{-1}\right)(1)\right)+\varepsilon_{1}=U\left(1-\sigma_{1}^{*}\right)<1
$$

as $\sigma_{1}^{*}>0$. Thus oscillator $i=1$ fires without triggering any further oscillators yielding an avalanche set $\Theta_{1}=\{1\}$. In addition the oscillators have to be shifted by $\sigma_{1}^{*}$ to obtain $\phi_{2}=1$. Thus the first pair in the firing sequence is $\left(\{1\}, \sigma_{1}^{*}\right)$. Applying the same arguments to the new phases $\Phi^{*(1)}=\mathbf{K}\left(\Phi^{*}\right)$ yields $\left(\{2\}, \sigma_{2}^{*}\right)$ for the second pair. Repeating these steps $N$ times one obtains a firing sequence

$$
\mathcal{F}\left(\Phi^{*}\right)=\left\{\left(\{r\}, \sigma_{r}^{*}\right)\right\}_{r=1}^{N}
$$

Thus

$$
\begin{aligned}
\mathbf{M}_{i}\left(\Phi^{*}\right) & =\bigodot_{r=i+1}^{N}\left(S_{\sigma_{r}^{*}} \circ H_{\varepsilon_{r}}\right) \circ S_{\sigma_{i}^{*}} J_{\varepsilon_{i i}} \circ \bigodot_{r=1}^{i-1}\left(S_{\sigma_{r}^{*}} \circ H_{\varepsilon_{r}}\right)\left(\phi_{i}^{*}\right) \\
& =\bigodot_{r=i+1}^{N}\left(S_{\sigma_{r}^{*}} \circ H_{\varepsilon_{r}}\right) \circ S_{\sigma_{i}^{*}} J_{\varepsilon_{i i}}(1) \\
& =\left(H_{\varepsilon_{1}}^{-1} \circ S_{\sigma_{1}^{*}}^{-1}\right) \circ\left(H_{\varepsilon_{2}}^{-1} \circ S_{\sigma_{2}^{*}}^{-1}\right) \circ \cdots \circ\left(H_{\varepsilon_{i-1}}^{-1} \circ S_{\sigma_{i-1}^{*}}^{-1}\right) \\
& =\phi_{i}^{*}
\end{aligned}
$$

using (6.4.1) in the third row. Hence $\mathbf{M}\left(\Phi^{*}\right)=\Phi^{*}$ and the asynchronous state $\Phi^{*}$ is invariant under the return map.

Conversely a periodic asynchronous state yields a solution to (6.4.1), since each oscillator fires separately and thus there are a phase shifts $\sigma_{i}>0$ after each pulse generation of the oscillators $i \in\{1,2, \ldots, N\}$. Invariance of the periodic asynchronous state then shows that in fact $\Sigma=\left(\sigma_{1}, \sigma_{2}, \ldots, \sigma_{N}\right)$ is a solution to (6.4.1). Hence there is no periodic asynchronous state if the solution does not exist. If there is a solution with $\sigma_{i}^{*} \leq 0$, let $s$ be the smallest index such that $\sigma_{s}^{*} \leq 0$. Starting in the state $\Phi^{*}$ the first firing of oscillator 
$i=s$ will cause oscillator $i=s+1$ to fire in the same avalanche since its potential at this point is given by

$$
\begin{aligned}
u_{s+1}^{(1)} & =U\left[\bigodot_{r=1}^{s-1}\left(S_{\sigma_{r}^{*}} \circ H_{\varepsilon_{r}}\right)\left(\phi_{s+1}^{*}\right)\right]+\varepsilon_{s} \\
& =U\left[H_{\varepsilon_{s}}^{-1} \circ S_{\sigma_{s}^{*}}^{-1}(1)\right]+\varepsilon_{s}=U\left(1-\sigma_{s}^{*}\right) \geq 1
\end{aligned}
$$

i.e. $\{s, s+1\} \subset \Theta_{s}$ and the system is not in a periodic asynchronous state.

Corollary 6.4.3. In a network (5.2.13)-(5.2.16) of $N$ oscillators with homogeneous allto-all coupling matrix (6.1.1) an asynchronous (splay) state exists.

Proof. Let

$$
L(\sigma):=\bigodot_{s=1}^{N-1}\left(S_{\sigma} \circ H_{\varepsilon}\right) \circ S_{\sigma} \circ J_{0}(1)
$$

Now since $L(0)=U^{-1}(\varepsilon(N-1))<1$ and $\frac{\partial}{\partial \sigma} L(\sigma) \geq 1$ the intermediate value theorem ensures the existence of a $\sigma^{*}>0$ satisfying $L\left(\sigma^{*}\right)=1$. $\Sigma^{*}=\left(\sigma^{*}, \ldots, \sigma^{*}\right)$ is a solution to (6.4.1). As $\varepsilon_{i i}=0$, no oscillator receives supra-threshold input in the asynchronous state, i.e. $\zeta=0$, and this result is independent of the partial reset function $R$ as $R(0)=0$ (cf. definition 5.2.1).

In fig. 6.3 we observe no cluster states involving avalanches of size 43 to 49 . This is precisely because (6.4.1) has no solutions when setting $\varepsilon_{i}=a_{i} \varepsilon, \varepsilon_{i i}=\left(a_{i}-1\right) \varepsilon$ for $a_{1} \in\{43,44, \ldots, 49\}$ and any further $0<a_{i} \in \mathbb{N}, i \geq 1$ and $m$ such that $\sum_{s=1}^{m} a_{s}=50$.

Note that lemma 6.4.3 holds for any rise function $U$. If there are $q$ different positive solutions to (6.4.1) there coexist $q$ different periodic asynchronous states. A convex $U$ ensures that the solution is unique because $\mathbf{L}(\Sigma, 1)$ then becomes invertible for all $\Sigma \in \mathbb{R}^{N}$.

A further consequence of the convexity is that given the existence of an asynchronous state in a meta-oscillator network it is linearly stable as the following theorem shows:

Theorem 6.4.4. Consider a network (5.2.13)-(5.2.16) of $N$ oscillators with pulse coupling matrix (6.2.1) and neuronal partial reset. If a periodic asynchronous state exists it is linearly stable.

Proof. Existence of the asynchronous state $\left(\Phi^{*}, \mathrm{id}\right) \in \mathcal{P}^{p}$ with $\Phi^{*}=\left(\phi_{2}^{*}, \ldots, \phi_{N}^{*}\right)$ implies invariance under the return map $\mathbf{M}^{p}$,

$$
\mathbf{M}^{p}\left(\Phi^{*}, \mathrm{id}\right)=\left(\Phi^{*}, \mathrm{id}\right)
$$

For the intermediate states we set

$$
\left(\Phi^{(s)}, \pi^{(\mathrm{s})}\right):=\left(\mathbf{K}^{p}\right)^{s}\left(\Phi^{*}, \mathrm{id}\right) \quad s \in\{0,1,2, \ldots, N\}
$$

If oscillator $i$ generates a pulse all oscillators $j \neq i$ receive the same input $\varepsilon_{i}$ and oscillator $i$ receives an input $\varepsilon_{i i} \leq \varepsilon_{i}$. Hence, using $R(\zeta) \leq \zeta$, we find that the oscillators do not change their firing order and $\pi^{(s)}$ is a cyclic permutation to the left $\pi^{(s)}(i)=i-s$.

We show that the asynchronous state is linearly stable: Adding a perturbation $\Delta^{(0)}=$ $\left(\delta_{1}^{(0)}, \ldots, \delta_{N-1}^{(0)}\right)$ to the asynchronous state such that initially the phases are given by

$$
\Psi^{(0)}:=\left(\phi_{1}^{(0)}, \ldots, \phi_{N-1}^{(0)}\right)=\Phi^{*}+\Delta^{(0)}
$$


We take the perturbation to be sufficiently small such that the oscillators still fire asynchronously, i.e. the avalanches are of size $a_{s}=1$ and the order of the events is preserved. In the following, terms of order $\mathcal{O}\left(\left(\Delta^{(0)}\right)^{2}\right)$ are neglected which we indicate by a dot above the equality sign $(\doteq)$. After $s$ firing events the phases are

$$
\Psi^{(s)}=\mathbf{K}_{\Phi}^{p}\left(\Psi^{(s-1)}, \pi^{(s-1)}\right) \doteq \mathbf{K}_{\Phi}^{p}\left(\Phi^{(s-1)}, \pi^{(r-1)}\right)+\Delta^{(s)}=\Phi^{(s)}+\Delta^{(s)}
$$

where

$$
\Delta^{(s)}=\mathbf{A}^{(s)} \Delta^{(s-1)}
$$

is the phase perturbation before the next firing and $\mathbf{A}^{(s)}$ is the Jacobian matrix of $\mathbf{K}_{\Phi}^{p}$ at $\left(\Phi^{(s-1)}, \pi^{(s-1)}\right)$

$$
A_{i j}^{(s)}=\frac{d \mathbf{K}_{i}^{p}}{d \phi_{j}}\left(\Phi^{(s-1)}, \pi^{(s-1)}\right) .
$$

Setting $\sigma=1-H\left(\psi_{2}, \varepsilon_{\pi(1)}\right)$ the phase part of the firing-map for $N \geq 3$ is

$$
\boldsymbol{K}_{\Phi}^{p}(\Psi, \pi)=\left(\begin{array}{c}
H\left(\psi_{3}, \varepsilon_{\pi(1)}\right)+\sigma \\
H\left(\psi_{4}, \varepsilon_{\pi(1)}\right)+\sigma \\
\cdots \\
H\left(\psi_{N}, \varepsilon_{\pi(1)}\right)+\sigma \\
J\left(1, \varepsilon_{\pi(1) \pi(1)}\right)+\sigma
\end{array}\right)^{T}
$$

Inserting (6.4.4) into (6.4.3) gives

$$
\mathbf{A}^{(s)}=\left(\begin{array}{ccccc}
-a_{2}^{(s)} & a_{3}^{(s)} & 0 & \ldots & 0 \\
-a_{2}^{(s)} & 0 & a_{4}^{(s)} & \ddots & \vdots \\
\vdots & \vdots & \ddots & \ddots & 0 \\
-a_{2}^{(s)} & 0 & \ldots & 0 & a_{N}^{(s)} \\
-a_{2}^{(s)} & 0 & \ldots & 0 & 0
\end{array}\right)
$$

with

$$
a_{i}^{(s)}=\frac{d}{d \phi} H_{\varepsilon_{s}}\left(\phi_{i}^{(s-1)}\right)=\frac{U^{\prime}\left(\phi_{i}^{(s-1)}\right)}{U^{\prime}\left(H_{\varepsilon_{s}}\left(\phi_{i}^{(s-1)}\right)\right)}
$$

Since $\varepsilon_{j}>0$ it follows that $H_{\varepsilon_{j}}(\phi)=U^{-1}\left(U(\phi)+\varepsilon_{j}\right)>\phi$. Thus $a_{i}^{(s)}<1$ since $U$ is convex. Also $U^{\prime}>0$ and hence

$$
0<a_{i}^{(s)}<1
$$

Now the Eneström-Kakeya theorem (cf. appendix A and [178]) applied to the matrix $\mathbf{A}^{(s)}$ shows that with these properties the spectral radius $\rho\left(\mathbf{A}^{(s)}\right)$ of $\mathbf{A}^{(s)}$ satisfies

$$
\rho\left(\mathbf{A}^{(s)}\right) \leq r^{(s)}=\max _{i \in\{1, \ldots, N-1\}} a_{i}^{(s)}<1
$$

Thus

$$
\left\|\Delta^{(n N)}\right\|=\left\|\left(\prod_{r=1}^{N} \mathbf{A}^{(s)}\right)^{n} \Delta^{(0)}\right\| \leq \prod_{r=1}^{N} \rho\left(\mathbf{A}^{(s)}\right)^{n}\left\|\Delta^{(0)}\right\| \rightarrow 0 \quad \text { as } n \rightarrow \infty
$$

and the asynchronous state is linearly stable. For $N=2, \rho\left(\mathbf{A}^{(s)}\right)=a_{2}<1$. 
(a)

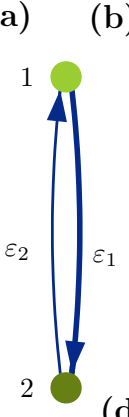

(d)

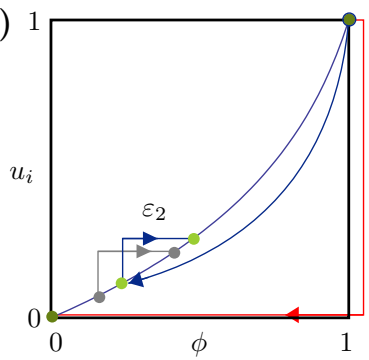

(c)
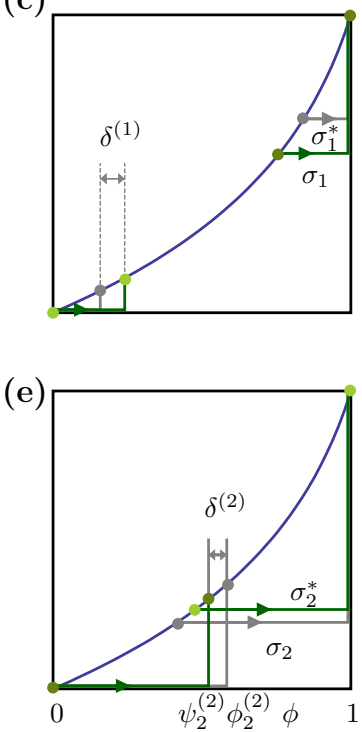

Figure 6.4. Stability of the asynchronous state (theorem 6.4.4). (a) graph of a network of $N=2$ oscillators with connectivity $\varepsilon_{i j}=\left(1-\delta_{i j}\right) \varepsilon_{j}$ with $0<\varepsilon_{2}<\varepsilon_{1}$. (b) firing of oscillator $i=1$. For the oscillator $i=2$ with initial phase $\psi_{2}^{(0)}=\phi_{2}^{(0)}+\delta^{(0)}$ smaller than in the invariant asynchronous state $\phi_{2}^{(0)}$ (gray) the input advances the phase $\psi_{2}^{(0)}$ more in comparison with the advance of $\phi_{2}^{(0)}$ in the asynchronous state due to the convexity of the rise function $U$. (c) after the interaction a subsequent shift completes the firing map K. In total the derivation from the asynchronous state $\delta^{(1)}$ has become smaller. (d) firing of oscillator $i=2$. Phases which are perturbed to larger values than the asynchronous state are less advanced by inputs due to convexity of the rise function. (d) in total the return map $\mathbf{M}$ decreases the phase perturbations $\left|\delta^{(2)}\right|<\left|\delta^{(0)}\right|$. These stabilizing dynamics of the asynchronous state due to the convexity of the rise function generalizes to larger networks as proven in theorem 6.4.4.

This result is illustrated in fig. 6.4: Due to the convexity of the rise function oscillators perturbed to larger (smaller) phases compared to the asynchronous state are less (more) advanced by input pulses pulling the perturbed phases back to the invariant asynchronous dynamics.

Combining corollary 6.4 .3 and theorem 6.4 .4 we obtain:

Corollary 6.4.5. In a network (5.2.13)-(5.2.16) of $N$ oscillators with homogeneous allto-all coupling matrix (6.1.1), neuronal partial reset $R$ and convex rise function $U$ the periodic asynchronous (splay) state exists and is linearly stable.

\subsection{Impact of Partial Reset on Intra-Cluster Stability}

In the state of synchronous firing all units in an all-to-all coupled network receive a supra-threshold input pulse of strength $(N-1) \varepsilon$ suggesting a rather strong influence of the partial reset $R$ onto the network dynamics. Indeed, as shown in fig. 6.3 for the partial reset $R_{c}$ one observes a sequential destabilization of clusters starting at large cluster sizes when increasing the reset strength $c$. In this subsection we study this behavior analytically and explain the observed transition. The strategy is to focus on a single cluster of size $a_{1}$ and derive general conditions which ensure the stability of this cluster under the return map. As the return map depends on the firing sequence $\mathcal{F}$ we use best- and worst-case 
scenarios to obtain bounds for the stability of cluster states. We introduce a property of a rise function that allows to estimate the worst and best-case return maps by simpler return maps that do not depend on all details of the firing sequence $\mathcal{F}$. For a special class of rise functions we find that a full analytical treatment is possible.

Definition 6.5.1. A firing sequence $\mathcal{F}$ is admissible if there is a state $\Phi \in \mathcal{S}$ which has firing sequence $\mathcal{F}=\mathcal{F}(\Phi)$. It is further called trigger invariant if for the oscillators $i \in \Theta_{1}^{(0)}=\left\{j \in\{1,2, \ldots, N\} \mid \phi_{j}=1\right\}$ triggering the first avalanche of the state $\Phi=$ $\left(\phi_{1}, \ldots, \phi_{N}\right)$ (cf. (5.2.8)) the return map satisfies $\mathbf{M}_{i}(\Phi)=1$. Thus for a trigger invariant firing sequence $\mathcal{F}$ with $m$ intermediate avalanches $\Theta_{1}^{(0)} \subset \Theta_{m+1}^{(0)}$. The set of all trigger invariant firing sequences is denoted by $\mathcal{T}$. The subset of $\mathcal{F} \in \mathcal{T}$ with initial avalanche size $a_{1}=\left|\Theta_{1}\right|$ is denoted by $\mathcal{T}_{a_{1}}$.

Let us focus on a single avalanche of size $a_{1}$ in the network dynamics. To ensure that all units in this avalanche fire together again after the return map is applied all units in this avalanche which were triggered to fire by $a \in\left\{1,2, \ldots, a_{1}-1\right\}$ preceding spikes i.e. with phases in

$$
I_{a}^{T}=\left[U^{-1}(1-a \varepsilon), 1\right]
$$

have to be triggered again after applying the return map. Given a firing sequence $\mathcal{F}=$ $\left\{\left(\varepsilon_{r}, \sigma_{r}\right)\right\}_{r=1}^{m}$ the return map for oscillators $i \in \Theta_{1}$ in the first avalanche is given by

$$
M_{\mathcal{F}}(\phi)=\bigodot_{r=2}^{m}\left(S_{\sigma_{r}} \circ H_{\varepsilon_{r}}\right) \circ S_{\sigma_{1}} \circ J_{\varepsilon_{1}}(\phi)
$$

Hence the conditions

$$
\mathcal{M}_{\mathcal{F}}\left(I_{a}^{T}\right) \subset I_{a}^{T}
$$

for all $a \in\left\{1, \ldots, a_{1}-1\right\}$ and all admissible firing sequences $\mathcal{F} \in \mathcal{T}_{a_{1}}$ ensure a cluster of size $a_{1}$ to not split up under return. By finding the most synchronizing and most desynchronizing firing sequences $\mathcal{F} \in \mathcal{T}_{a_{1}}$, i.e. the best- and worst-case scenarios these conditions yield upper and lower bounds for the stability of a cluster of size $a_{1}$ under the return map:

Lemma 6.5.2. Consider a network (5.2.13)-(5.2.16) of $N$ oscillators with homogeneous all-to-all coupling matrix (6.1.1).

Set

$$
w_{a}^{a_{1}}=\inf _{\mathcal{F} \in \mathcal{T}_{a_{1}}} M_{\mathcal{F}}\left(U^{-1}(1-a \varepsilon)\right)
$$

and

$$
b_{a}^{a_{1}}=\sup _{\mathcal{F} \in \mathcal{T}_{a_{1}}} M_{\mathcal{F}}\left(U^{-1}(1-a \varepsilon)\right)
$$

Then the conditions

$$
w_{a}^{a_{1}} \geq U^{-1}(1-a \varepsilon)
$$

for $a \in\left\{1,2, \ldots, a_{1}-1\right\}$ are sufficient and

$$
b_{a}^{a_{1}} \geq U^{-1}(1-a \varepsilon)
$$

are necessary for a cluster of size $a_{1}$ to be invariant under return. 
(a)

(d)
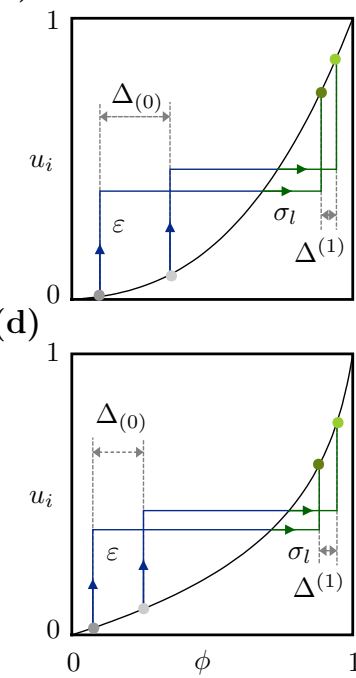

(b)

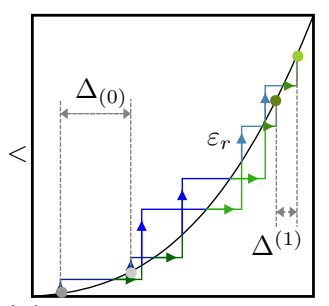

(e)

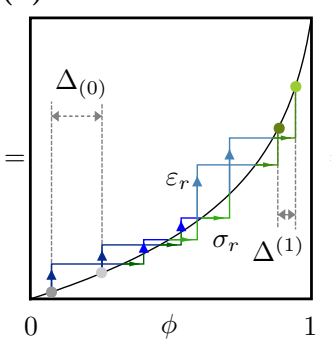

(c)

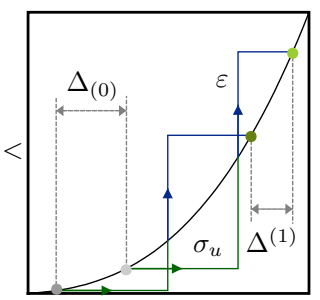

(f)

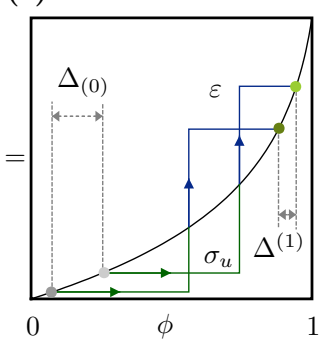

Figure 6.5. Rise functions with increasing change (icpd) and no change of phase differences. (a-c) icpd rise function. An initial phase difference $\Delta^{(0)}$ changes to $\Delta^{(1)}$ after applying a combination of interaction maps $H_{\varepsilon_{r}}$ (blue) of total strength $\varepsilon=\sum_{r=1}^{m} \varepsilon_{r}$ and shifts $S_{\sigma_{r}}$ (green) such that the final maximal phase values are identical. (a) for icpd rise functions the difference $\Delta^{(1)}$ is the smallest when the interaction is applied in total before the shifts, i.e. $H_{\varepsilon} \circ S_{\sigma_{l}}$ and (c) largest when applied after the shifts $S_{\sigma_{u}} \circ H_{\varepsilon}$. (b) All other maps $\bigodot_{s=1}^{m}\left(H_{\varepsilon_{s}} \circ S_{\sigma_{s}}\right)$ produce phase differences which lie in between these extremal values (cf. lemma 6.5.4). (d-f) the rise function $U_{b}$ is icpd and dcpd, i.e. the phase difference $\Delta^{(1)}$ is independent of the order in which the interactions and shifts are applied.

Proof. $\frac{\partial}{\partial \phi} M_{\mathcal{F}}(\phi)>0$ and thus conditions (6.5.1) are equivalent to

$$
\mathcal{M}_{\mathcal{F}}\left(U^{-1}(1-a \varepsilon)\right) \geq U^{-1}(1-a \varepsilon)
$$

for $a \in\left\{1,2, \ldots, a_{1}-1\right\}$ and all admissible $\mathcal{F} \in \mathcal{T}_{a_{1}}$.

Finding the $w_{a}^{a_{1}}$ and $b_{a}^{a_{1}}$ for general $U$ and $R$ can be done numerically using optimization techniques. However, there are two classes of rise functions (cf. definition 6.5.3 below) which allow further analytical investigation as the effect of their worst- and bestcase return maps can be estimated. Most of the commonly used rise functions, as e.g. the rise function of the LIF neuron or the conductance based LIF neuron fall into one of these classes (cf. appendix B).

The idea is to study the change of phase differences due to the application of the interaction function. Two oscillators initially at phases $\phi$ and $\phi+\Delta \phi$ receiving a pulse of strength $\varepsilon$ will have a new phase difference

$$
\Delta H(\phi, \Delta \phi, \varepsilon):=H_{\varepsilon}(\phi+\Delta \phi)-H_{\varepsilon}(\phi)
$$

where the domain of $\Delta H$ is given by

$$
\mathcal{D}:=\left\{(\phi, \Delta \phi, \varepsilon) \mid 0 \leq \varepsilon \leq 1,0 \leq \phi \leq 1,0 \leq \Delta \phi \leq U^{-1}(1-\varepsilon)-\phi\right\} .
$$

Definition 6.5.3. A rise function $U$ is increasing the change of phase differences (icpd) iff

$$
\frac{\partial}{\partial \phi} \Delta H(\phi, \Delta \phi, \varepsilon) \geq 0 \quad \text { for all }(\phi, \Delta \phi, \varepsilon) \in \mathcal{D}
$$


Conversely, it is decreasing the change of phase differences (dcpd) iff

$$
\frac{\partial}{\partial \phi} \Delta H(\phi, \Delta \phi, \varepsilon) \leq 0 \quad \text { for all }(\phi, \Delta \phi, \varepsilon) \in \mathcal{D}
$$

As shown in appendix B.2 the icpd (dcpd) property is related to the third derivative of $U$. If the rise function is icpd or dcpd the change in phase differences after application of the return map can be bounded as show in the following lemma and illustrated in fig. $6.5 \mathrm{a}-\mathrm{c}$ for icpd rise functions.

Lemma 6.5.4. Let $\varepsilon_{r}, \sigma_{r} \geq 0, r \in\{1,2, \ldots, m\}, \varepsilon=\sum_{r=1}^{m} \varepsilon_{r}, \sigma_{l} \geq 0$. Choose a $\sigma_{u} \geq 0$ such that

$$
\bigodot_{r=1}^{m}\left(S_{\sigma_{r}} \circ H_{\varepsilon_{r}}\right)(\phi) \leq H_{\varepsilon} \circ S_{\sigma_{u}}(\phi) .
$$

and let $\phi \geq \psi$. Then for an icpd rise function $U$

$$
\begin{array}{r}
S_{\sigma_{l}} \circ H_{\varepsilon}(\phi)-S_{\sigma_{l}} \circ H_{\varepsilon}(\psi) \leq \bigodot_{r=1}^{m}\left(S_{\sigma_{r}} \circ H_{\varepsilon_{r}}\right)(\phi)-\bigodot_{r=1}^{m}\left(S_{\sigma_{r}} \circ H_{\varepsilon_{r}}\right)(\psi) \\
\leq H_{\varepsilon} \circ S_{\sigma_{u}}(\phi)-H_{\varepsilon} \circ S_{\sigma_{u}}(\psi)
\end{array}
$$

For $U$ that is dcpd (6.5.7) holds replacing $\leq$ with $\geq$.

Proof. Consider icpd rise functions first: To show the first inequality of eq. (6.5.7) we use induction on $m$. The statement is clearly true for $m=1$. Assume it is true for $m \geq 1$ then

$$
\begin{aligned}
S_{\sigma_{l}} \circ H_{\varepsilon}(\phi)-S_{\sigma_{l}} \circ H_{\varepsilon}(\psi) \\
=H_{\varepsilon_{m+1}} \circ H_{\varepsilon-\varepsilon_{m+1}}(\phi)-H_{\varepsilon_{m+1}} \circ H_{\varepsilon-\varepsilon_{m+1}}(\psi) \\
=\Delta H\left(H_{\varepsilon-\varepsilon_{m+1}}(\psi), H_{\varepsilon-\varepsilon_{m+1}}(\phi)-H_{\varepsilon-\varepsilon_{m+1}}(\psi), \varepsilon_{m+1}\right) \\
\leq \Delta H\left(H_{\varepsilon-\varepsilon_{m+1}}(\psi), \bigodot_{r=1}^{m}\left(S_{\sigma_{r}} \circ H_{\varepsilon_{r}}\right)(\phi)-\bigodot_{r=1}^{m}\left(S_{\sigma_{r}} \circ H_{\varepsilon_{r}}\right)(\psi), \varepsilon_{m+1}\right) \\
\leq \Delta H\left(\bigodot_{r=1}^{m}\left(S_{\sigma_{r}} \circ H_{\varepsilon_{r}}\right)(\psi), \bigodot_{r=1}^{m}\left(S_{\sigma_{r}} \circ H_{\varepsilon_{r}}\right)(\phi)-\bigodot_{r=1}^{m}\left(S_{\sigma_{r}} \circ H_{\varepsilon_{r}}\right)(\psi), \varepsilon_{m+1}\right) \\
=\bigodot_{r=1}^{m+1}\left(S_{\sigma_{r}} \circ H_{\varepsilon_{r}}\right)(\phi)-\bigodot_{r=1}^{m+1}\left(S_{\sigma_{r}} \circ H_{\varepsilon_{r}}\right)(\psi)
\end{aligned}
$$

where we used the induction hypothesis and $\frac{\partial}{\partial \Delta \phi} \Delta H>0$ (cf. (6.5.4)) in the third, and in the fourth again the icpd property and the fact that $H_{\varepsilon-\varepsilon_{m+1}}(\psi) \leq \bigodot_{r=1}^{m}\left(S_{\sigma_{r}} \circ H_{\varepsilon_{r}}\right)$ if $\sum_{r=1}^{m+1} \varepsilon_{r}=\varepsilon, \sigma_{i} \geq 0$. Substituting $\leq$ with $\geq$ we obtain the result for dcpd rise functions.

For the second inequality we also use induction over $m$. The statement is trivially true for $m=1$. Let it be true for $m \geq 1$ and let $\sigma_{u} \geq 0$ such that $\bigodot_{r=1}^{m+1}\left(S_{\sigma_{r}} \circ H_{\varepsilon_{r}}\right)(\phi) \leq$ 
$H_{\varepsilon} \circ S_{\sigma_{u}}(\phi)$. Then

$$
\begin{aligned}
H_{\varepsilon} \circ S_{\sigma_{u}}(\phi)-H_{\varepsilon} \circ S_{\sigma_{u}}(\psi) & \\
& =H_{\varepsilon_{m+1}} \circ H_{\varepsilon-\varepsilon_{m+1}} \circ S_{\sigma_{u}}(\phi)-H_{\varepsilon_{m+1}} \circ H_{\varepsilon-\varepsilon_{m+1}} \circ S_{\sigma_{u}}(\psi) \\
& =\Delta H\left(H_{\varepsilon-\varepsilon_{m+1}} \circ S_{\sigma_{u}}(\psi), H_{\varepsilon-\varepsilon_{m+1}} \circ S_{\sigma_{u}}(\phi)-H_{\varepsilon-\varepsilon_{m+1}} \circ S_{\sigma_{u}}(\psi), \varepsilon_{m+1}\right) \\
& \geq \Delta H\left(H_{\varepsilon-\varepsilon_{m+1}} \circ S_{\sigma_{u}}(\psi), \bigodot_{r=1}^{m}\left(S_{\sigma_{r}} \circ H_{\varepsilon_{r}}\right)(\phi)-\bigodot_{r=1}^{m}\left(S_{\sigma_{r}} \circ H_{\varepsilon_{r}}\right)(\psi), \varepsilon_{m+1}\right) \\
& \geq \Delta H\left(\bigodot_{r=1}^{m}\left(S_{\sigma_{r}} \circ H_{\varepsilon_{r}}\right)(\psi), \bigodot_{r=1}^{m}\left(S_{\sigma_{r}} \circ H_{\varepsilon_{r}}\right)(\phi)-\bigodot_{r=1}^{m}\left(S_{\sigma_{r}} \circ H_{\varepsilon_{r}}\right)(\psi), \varepsilon_{m+1}\right) \\
& =\bigodot_{r=1}^{m+1}\left(S_{\sigma_{r}} \circ H_{\varepsilon_{r}}\right)(\phi)-\bigodot_{r=1}^{m+1}\left(S_{\sigma_{r}} \circ H_{\varepsilon_{r}}\right)(\psi)
\end{aligned}
$$

where in the third row we used the implication

$$
\bigodot_{s=1}^{m+1}\left(S_{\sigma_{s}} \circ H_{\varepsilon_{s}}\right)(\phi) \leq H_{\varepsilon} \circ S_{\sigma_{u}}(\phi) \Rightarrow \bigodot_{s=1}^{m}\left(S_{\sigma_{s}} \circ H_{\varepsilon_{s}}\right)(\phi) \leq H_{\varepsilon-\varepsilon_{m+1}} \circ S_{\sigma_{u}}(\phi)
$$

to apply the induction hypothesis. In the fourth row we again used $\frac{\partial}{\partial \Delta \phi} \Delta H>0$, eq. (6.5.8) and the icpd property. Substituting $\geq$ with $\leq$ we obtain the result for dcpd rise functions.

Using the previous result we can estimate the effect of worst and best case return maps on avalanches of a certain size and thus determine bounds on the network parameters which ensure invariance or decay of states that involve these cluster sizes:

Theorem 6.5.5. Consider a homogeneous excitatory all-to-all network of $N$ pulse-coupled oscillators evolving according to (5.2.13)-(5.2.16) with neuronal partial reset $R$.

For icpd rise functions $U$ the conditions

$$
\begin{array}{r}
U^{-1}\left(R\left(\left(a_{1}-1\right) \varepsilon\right)\right)-U^{-1}\left(R\left(\left(a_{1}-1\right) \varepsilon-a \varepsilon\right)\right) \leq \\
U\left(1-\left(N-a_{1}\right) \varepsilon\right)-U^{-1}\left(1-\left(N-a_{1}\right) \varepsilon-a \varepsilon\right)
\end{array}
$$

for all $a \in\left\{1,2, \ldots, a_{1}-1\right\}$ are sufficient to ensure the invariance of an $a_{1}$-avalanche under return. Necessary conditions are

$$
\begin{gathered}
U^{-1}\left(R\left(\left(a_{1}-1\right) \varepsilon\right)+\left(N-a_{1}\right) \varepsilon\right)-U^{-1}\left(R\left(\left(a_{1}-1\right) \varepsilon-a \varepsilon\right)+\left(N-a_{1}\right) \varepsilon\right) \\
\leq 1-U^{-1}(1-a \varepsilon) .
\end{gathered}
$$

Likewise for dcpd rise functions $U$ sufficient conditions are (6.5.10) and necessary conditions (6.5.9) for an $a_{1}$-avalanche to not split up under return.

Proof. To prove the theorem we use lemma 6.5.4 and find for an icpd rise function and $\mathcal{F} \in \mathcal{T}_{a_{1}}$

$$
\begin{aligned}
M_{\mathcal{F}}(1) & -M_{\mathcal{F}}\left(U^{-1}(1-a \varepsilon)\right) \\
& =\bigodot_{r=2}^{m}\left(S_{\sigma_{r}} \circ H_{\varepsilon_{r}}\right)\left(S_{\sigma_{1}} \circ J_{\varepsilon_{1}}(1)\right)-\bigodot_{r=2}^{m}\left(S_{\sigma_{r}} \circ H_{\varepsilon_{r}}\right)\left(S_{\sigma_{1}} \circ J_{\varepsilon_{1}}\left(U^{-1}(1-a \varepsilon)\right)\right) \\
& \leq H_{\left(N-a_{1}\right) \varepsilon} \circ S_{\sigma_{u}} \circ J_{\varepsilon_{1}}(1)-H_{\left(N-a_{1}\right) \varepsilon} \circ S_{\sigma_{u}} \circ J_{\varepsilon_{1}}\left(U^{-1}(1-a \varepsilon)\right) \\
& =1-H_{\left(N-a_{1}\right) \varepsilon} \circ S_{u} \circ J_{\varepsilon_{1}}\left(U^{-1}(1-a \varepsilon)\right)
\end{aligned}
$$


if we choose

$$
\sigma_{u}=U^{-1}\left(1-\left(N-a_{1}\right) \varepsilon\right)-U^{-1}\left(R\left(a_{1}-1\right) \varepsilon\right)
$$

and thus $w_{a}^{a_{1}}=H_{\left(N-a_{1}\right) \varepsilon} \circ S_{\sigma_{u}} \circ J_{\varepsilon_{1}}\left(U^{-1}(1-a \varepsilon)\right)$ in (6.5.2) yielding conditions (6.5.9). Similarly we find for (6.5.3), $b_{a}^{a_{1}}=1-H_{\left(N-a_{1}\right) \varepsilon} \circ J_{\varepsilon_{1}}(1)+H_{\left(N-a_{1}\right) \varepsilon} \circ J_{\varepsilon_{1}}\left(U^{-1}(1-a \varepsilon)\right)$ which yields the necessary conditions (6.5.10). For dcpd rise functions the expressions for $w_{a}^{a_{1}}$ and $b_{a}^{a_{1}}$ are interchanged.

We used theorem 6.4.4 to determine for a convex LIF rise function $U_{\mathrm{LIF}}^{\mathrm{CB}}$ (cf. B.1.1 eq. (B.1.3)) and linear partial reset $R_{c}$ the regime where avalanches of different sizes become unstable under return. The most strict condition in (6.5.9) is that for $a=1$ which yields an implicit equation for the lower bounds on the critical $c$ values below which the invariance of $a_{1}$-avalanches is ensured. The upper bound is obtained by (6.5.10) also using $a=1$. Both bounds are plotted in fig. 6.6 and are in good agreement with the numerical data.

Near the lower transition point $c_{\text {crit }}^{(N)}$ the system shows aperiodic behavior when starting close to the synchronous state. A possible explanation for this dynamics is the competition of two counteracting mechanisms: (i) Large avalanches become unstable under return and thus tend to desynchronize the phases which results in a split of the avalanche into smaller stable avalanches. (ii) The solution to equation (6.4.1) for these asynchronously firing smaller clusters involves $\sigma_{r}^{*} \leq 0$, i.e. the smaller avalanches tend to absorb each other and resynchronize the system yielding again larger unstable avalanches. Note that here irregular dynamics arise via a mechanism that differs from network heterogeneity [71] or using balanced excitatory and inhibitory interactions [390].
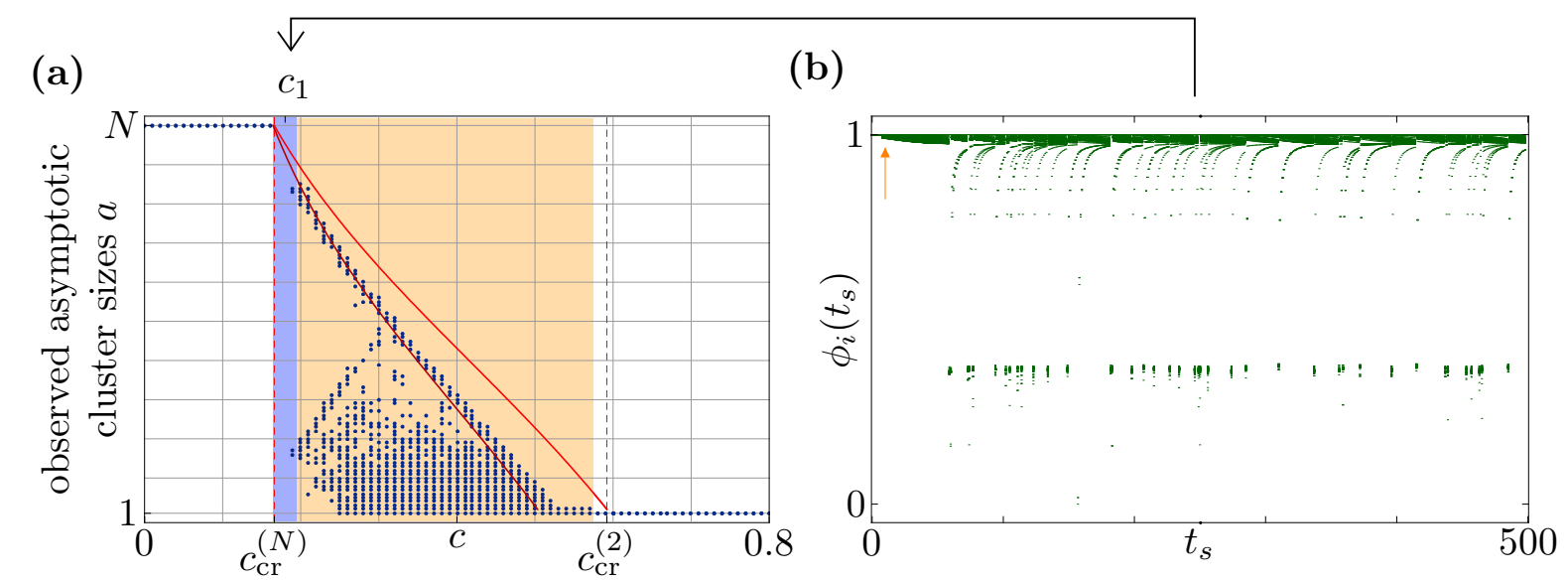

Figure 6.6. Sequential desynchronization in a network $(N=100)$ with icpd rise function $U_{\text {LIF }}^{\mathrm{CB}}$ $\left(E_{\text {eq }}=1.1, E_{\mathrm{syn}}=3\right)$ and linear partial reset $R_{c}$. (a) observed cluster sizes (dots) of periodic states after a transient time $t=10000$. For each $c$ value 100 simulations were started in the synchronous state with a small perturbation added. The upper line shows the bounds on $a$ obtained from (6.5.10) in theorem 6.5.5 above which $a$-clusters are unstable. The lower line is the bound obtained via (6.5.9) below which $a$-clusters are ensured to be stable. The shaded area marks the transition region where states other than the synchronous and asynchronous state are observed. In the blue region we find no periodic asymptotic dynamics. The dashed lines show the theoretical bounds for the transition region. (b) aperiodic dynamics for $c_{1}=0.18$. 


\subsection{Extensive Sequence of Desynchronizing Bifurcations - A Solvable Example}

Figure 6.5d-f illustrates that the rise function $U_{b}$ is both icpd and dcpd. In fact,

$$
\Delta H_{b}(\phi, \Delta \phi, \varepsilon):=H_{b}(\phi+\Delta \phi, \varepsilon)-H_{b}(\phi, \varepsilon)=\Delta \phi e^{b \varepsilon}
$$

is independent of $\phi$ and hence

$$
\frac{\partial}{\partial \phi} \Delta H_{b}(\phi, \Delta \phi, \varepsilon)=0
$$

Thus for $U_{b}$ equality holds in (6.5.7) and the best- and worst-case return maps become identical. This property allows us to obtain exact analytical results.

Proposition 6.6.1. Consider a homogeneous excitatory all-to-all network of $N$ pulsecoupled oscillators evolving according to (5.2.13)-(5.2.16) with convex rise function $U_{b}$ $(b<0)$ and neuronal partial reset $R_{c}$.

Then for each $2 \leq a \leq N$ there exist a critical reset strength $c_{\mathrm{cr}}^{(a)}$ such that for all $c>c_{\mathrm{cr}}^{(a)}$ avalanches of size greater or equal to a are unstable under return and avalanches of size smaller than a are stable. For $c \leq c_{\mathrm{cr}}^{(N)}$ all avalanches are stable under return. The critical reset strengths are determined from the equation

$$
e^{b\left(1-\left[(N-a)+c_{\mathrm{cr}}^{(a)}(a-1)\right] \varepsilon\right)}=\frac{\left(e^{-b c_{\mathrm{cr}}^{(a)} \varepsilon}-1\right)}{\left(e^{-b \varepsilon}-1\right)}
$$

and satisfy $0<c_{\mathrm{cr}}^{(N)}<c_{\mathrm{cr}}^{(N-1)}<\cdots<c_{\mathrm{cr}}^{(2)}<1$.

Proof. Since $U_{b}$ is icpd and dcpd, equality holds in (6.5.7), i.e. for $\mathcal{F} \in \mathcal{T}_{a_{1}}$

$$
\Delta M_{\mathcal{F}}(\Delta \phi):=1-M_{\mathcal{F}}(1-\Delta \phi)=1-S_{\sigma_{l}} \circ H_{\left(N-a_{1}\right) \varepsilon} \circ J_{a_{1} \varepsilon}(1-\Delta \phi)
$$

Thus the return map for the phase differences only depends on the avalanche size $a_{1}$ and is independent of the precise form of the other avalanches $a_{i}, i>1$ and intermediate shifts $\sigma_{i}$. Explicitly

$$
\Delta M_{\mathcal{F}}(\Delta \phi)=\frac{e^{b \varepsilon\left(N-a_{1}+c\left(a_{1}-1\right)\right)}}{1-e^{b}}\left(e^{-b c}\left(e^{b}+\left(1-e^{b}\right) \Delta \phi\right)^{c}-1\right)
$$

for all $\mathcal{F} \in \mathcal{T}_{a_{1}}$. A straight forward calculation shows that $\Delta M_{\mathcal{F}}$ has the properties

$$
\Delta M_{\mathcal{F}}(0)=0 \quad, \quad \frac{d}{d \Delta \phi} \Delta M_{\mathcal{F}}(\Delta \phi) \geq 0 \quad \text { and } \quad \frac{d^{2}}{d \Delta \phi^{2}} \Delta M_{\mathcal{F}}(\Delta \phi) \leq 0
$$

Thus if the condition

$$
\Delta M_{\mathcal{F}}\left(1-U^{-1}(1-\varepsilon)\right) \leq 1-U^{-1}(1-\varepsilon)
$$

is met all other conditions for $1 \leq a<a_{1}$ in (6.5.9) are also satisfied. On the other hand almost all perturbations will cause the avalanche to be triggered by a single oscillator. Thus if condition (6.6.4) is not satisfied, i.e. $\Delta M_{\mathcal{F}}(\Delta \phi)>\Delta \phi$ for all $\Delta \phi \geq U^{-1}(1-\varepsilon)-1$ the avalanche will split up after a finite number of iterations of the return map. Thus 
(6.6.4) is a necessary and sufficient condition for stability of an $a$-cluster under the return map. We are interested in the critical strengths $c_{\text {crit }}^{(a)}$ for which an $a$-cluster becomes unstable and hence we use equality in (6.6.4) and basic algebra to obtain the implicit expressions (6.6.1) for the $c_{\mathrm{cr}}^{(a)}$.

Since we have assumed $(N-1) \varepsilon<1, b<0$ and $c \in[0,1]$ we see that the left hand side of (6.6.1) lies in the interval $(0,1)$ and decreases monotonically with increasing $c$. The right hand side is 0 for $c=0$ and increases monotonically with $c$ until it becomes 1 for $c=1$. Thus by continuity for all $2 \leq a \leq N$ there always exist a solution $0<c_{\mathrm{cr}}^{(a)}<1$ to this equation. Note that the special case $a=2$ is explicitly solvable for $c_{\mathrm{cr}}^{(2)}$ and yields

$$
c_{\mathrm{cr}}^{(2)}=\frac{1}{b \varepsilon} \log \left(1+e^{-b(N-2) \varepsilon+b}\left(1-e^{-b \varepsilon}\right)\right)
$$

For fixed $0 \leq c<1$ the left hand side of (6.6.1) is strict monotonically decreasing as $a$ increases whereas the left hand side is independent of $a$, thus $0<c_{\mathrm{cr}}^{(N)}<c_{\mathrm{cr}}^{(N-1)}<\cdots<$ $c_{\mathrm{cr}}^{(2)}<1$.

The theoretical prediction (6.6.1) for the desynchronization transition is plotted in fig. 6.3 and is in excellent agreement with the numerically observed transition.

Remark 6.6.2. Note that (6.6.1) involves all relevant network parameter. In particular, choosing $b \rightarrow-\infty$ in equation (6.6.5) shows that $c_{\mathrm{cr}}^{(2)}$ can be made arbitrarily small. This implies that the entire sequence of desynchronizing bifurcations may occur over an arbitrary small interval $\left[c_{\mathrm{cr}}^{(N)}, c_{\mathrm{cr}}^{(2)}\right]$.

Remark 6.6.3. We also remark that the number of bifurcation points in this sequence is $N-1$. At each bifurcation point $c_{\mathrm{cr}}^{(a)}$ all periodic states with at least one cluster of size $a$ and all other cluster sizes less or equal to $a$, i.e. an extensive combinatorial number of states, becomes unstable simultaneously.

The mechanism underlying the desynchronization transition are opposing synchronization and desynchronization dynamics in the network as illustrated in fig. 6.7: (a) due to the convexity of the rise function sub-threshold inputs are always synchronizing and stabilize the avalanche, whereas depending on the strength of the partial reset supra-threshold inputs in an avalanche can either (b) synchronize or (c) desynchronize the phases. Thus for a weak partial reset (e.g. $R_{c}$ with $c \approx 0$ ) states with large avalanches are stable. When the partial reset is stronger it desynchronizes the cluster and, depending on the avalanche size, it may outweigh the synchronization effect due to sub-threshold inputs. Larger avalanches receive less synchronizing sub-threshold input from other oscillators and simultaneously produce a larger supra-threshold input than smaller ones. Thus they lose invariance under return first when increasing the partial reset strength.

\subsection{Robustness of the Desynchronization Transition}

The desynchronization transition is robust against structural perturbations in the coupling matrix and the rise function $U$.

\subsubsection{Coupling Strength Inhomogeneity}

With respect to perturbations in the coupling matrix $\varepsilon_{i j}$ numerical experiments show that the transition is observed when using coupling strengths from a uniform distribution on 
(a)

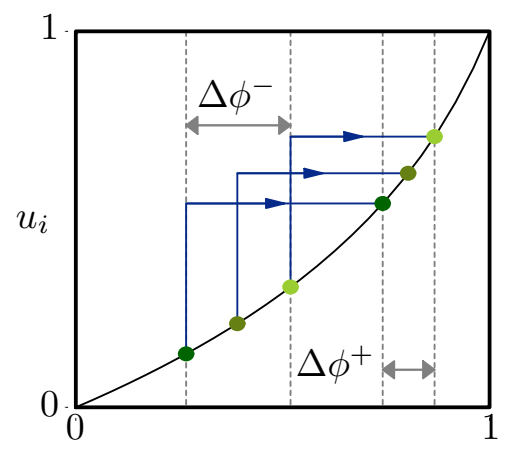

(b)

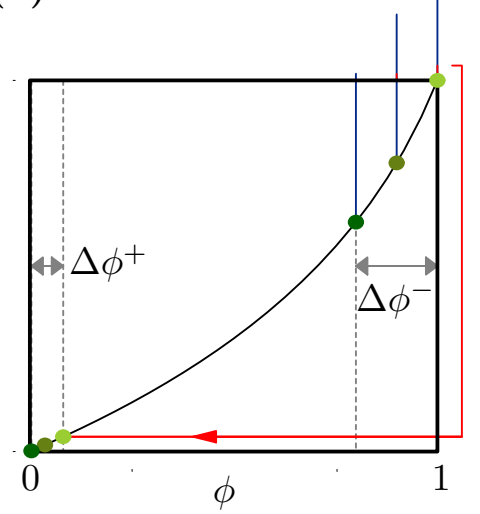

(c)

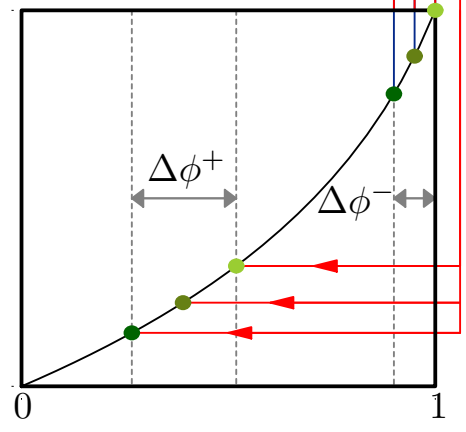

Figure 6.7. Synchronization and desynchronization of avalanches in networks with convex rise function and partial reset. (a) sub-threshold inputs synchronize the oscillators. The phase difference of a cluster before pulse reception $\Delta \phi^{+}$is decreased to $\Delta \phi^{-}$afterwards, i.e. $\Delta \phi^{+}<$ $\Delta \phi^{-}$. (a) weak partial reset (e.g. $c \approx 0$ for $R_{c}$ ) synchronize phase differences: $\Delta \phi^{+}<\Delta \phi^{-}$. (b) due to the convexity of the rise function a strong partial reset $(c \approx 1)$ expands the phase differences $\Delta \phi^{+}>\Delta \phi^{-}$. Clusters lose stability if the mechanism in (c) becomes dominant over the stabilizing effect in (a).

an interval $\left[\varepsilon_{\min }, \varepsilon_{\max }\right]$ for a interval length $\Delta \varepsilon=\varepsilon_{\max }-\varepsilon_{\min }$ as large as $20 \%$ of the average coupling strength $\bar{\varepsilon}=\left(\varepsilon_{\max }-\varepsilon_{\min }\right) / 2$. When $\Delta \varepsilon$ becomes larger usually complex spike patterns and non-periodic states are observed.

The coupling inhomogeneity destabilizes clusters since also sub-threshold inputs of different strengths desynchronize units initially at the same phase. In fact, already the lower bound $c_{\text {crit }}^{(a)}$ obtained for homogeneous networks via theorem 6.5.5 using the coupling strength $\bar{\varepsilon}$ over-estimates the stability of the clusters. The regime where we observe aperiodic dynamics becomes larger in comparison to homogeneous networks with the same average coupling strength. This is due to clusters with asymptotic phases which are close to an absorption (i.e. where $\sigma_{i}^{*} \approx 0$ for some $i$ ). A perturbation in the coupling now enables the absorption and the restless competition between desynchronization and synchronization (cf. sec. 6.5) induces the aperiodic dynamics.

\subsubsection{Sigmoidal Rise Functions}

Typically rise functions in biological or physical systems are neither purely concave nor purely convex. In particular intrinsic neuronal dynamics are often best described with a sigmoidal rise function. The quadratic-integrate-and-fire or exponential-integrate-andfire neuron $[101,110]$ (cf. also appendix B) constitute major examples. In networks with sigmoidal rise functions a combination of the effects inherent to concave and convex rise functions influences the network dynamics: Synchronization of units to larger clusters due to the concave part (cf. [269, 212]) and stabilization of states with asynchronously firing clusters due to the convex part (cf. theorem 6.4.4). Numerical studies show that for strictly neuronal partial resets and rise functions with dominant concave part synchronized firing of oscillators in the asymptotic state is typically found. In contrast, if the convex part is larger it is more likely to find clusters of smaller sizes and the asynchronous state. Indeed, for general rise functions $U$ we still obtain the stability matrix $\mathbf{A}$ in (6.4.5) but 
(a)

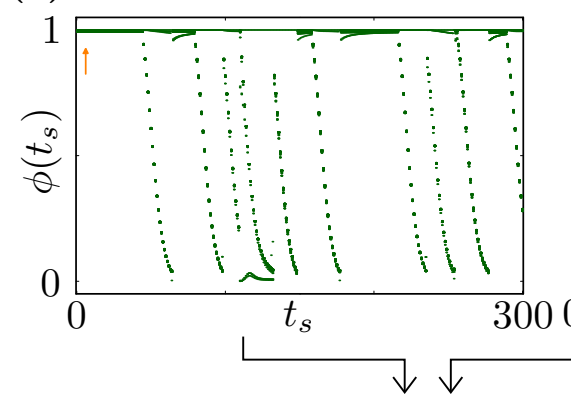

$$
\text { (d) }
$$

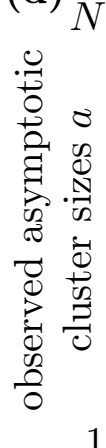

(b)

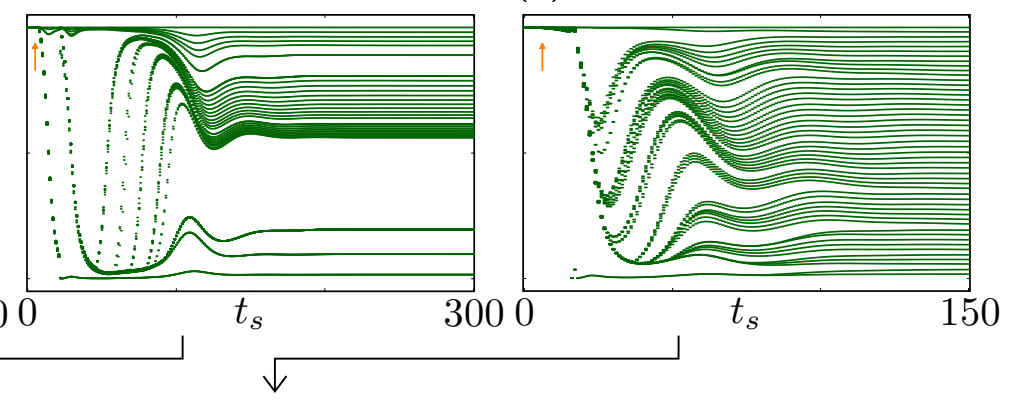

(c) (e)

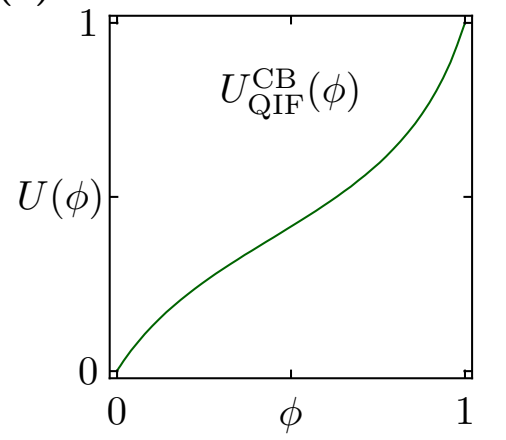

Figure 6.8. Sequential desynchronization transition in networks of neural oscillators with a sigmoidal rise function. Shown are the dynamics of a homogeneous network $(N=100, \varepsilon=$ $0.002)$ with linear partial reset $R_{c}$ and (e) sigmoidal rise function $U_{\mathrm{LIF}}^{\mathrm{CB}}\left(E_{\mathrm{syn}}=2, \alpha=-1\right.$, $\beta=1$ ). Starting with synchrony and inducing a small perturbation (arrow) the network shows (a) aperiodic dynamics for $c=c_{1}=0.45$, (b) clustering for $c=c_{2}=0.46$ and (c) asynchronous dynamics for $c=c_{3}=0.54$. Note the damped oscillations of the phase which do not appear for purely convex rise functions (cf. fig. 6.6). (d) cluster sizes of periodic states observed in the dynamics at $t=5000$ starting from 200 perturbed synchronous states for each value of $c$. Shaded area marks the transition region with states other than solely synchronous or asynchronous. The blue shaded are marks occurrence of aperiodic dynamics.

the non-zero entries (6.4.6) can become larger than 1 in the regime where $U$ is concave. Thus if the concave part becomes dominant the eigenvalues are no longer bounded by 1 and asynchronous clusters states become unstable.

In fig. 6.8 a desynchronization transition for the sigmoidal rise function $U_{\mathrm{QIF}}^{\mathrm{CB}}$ and linear partial reset $R_{c}$ is shown. In the synchronous state oscillators do not receive any intermediate sub-threshold pulses between successive firing and the return map for an oscillator with phase $\phi$ can be written as

$$
M_{\{\{1, \ldots, N\}, \sigma\}}(\phi)=U^{-1}(R(U(\phi)+(N-1) \varepsilon-1))+1-U^{-1}(R((N-1) \varepsilon))
$$

for any partial reset $R$ and any rise function $U$. After a perturbation the avalanche is typically triggered by a single unit and thus the synchronous state becomes unstable if $M_{\{\{1, \ldots, N\}, \sigma\}}(\phi)<\phi$ for all $\phi \in\left[1-U^{-1}(1-\varepsilon), 1\right]$ which yields the condition (6.5.9) for $a_{1}=N$. This can be used to determine the onset of a desynchronization transition in the general case as shown in fig. 6.8 (dashed line). The stability of smaller avalanches $a_{1}<N$ can still be estimated with the help of theorem 6.5.5 if the rise function is dcpd but not necessarily convex. Conditions for the sigmoidal rise functions $U_{\mathrm{QIF}}$ and $U_{\mathrm{QIF}}^{\mathrm{CB}}$ to be dcpd 
are given in appendix B.

Desynchronization due to a partial reset has three components: Translation of phase differences into potential differences via the rise function $U$, the relative change of potential differences due to the partial reset $R$ after supra-threshold excitation and back-translation of this potential difference into phase differences via $U^{-1}$ (cf. fig. $6.7 \mathrm{c}$ ). For convex rise functions the slope in the reset zone $I^{R}=\left[0, U^{-1}(R((N-1) \varepsilon))\right]$ is always smaller than in the supra-threshold zone $I^{T}=\left[U^{-1}(1-(N-1) \varepsilon), 1\right]$. As a consequence the phase differences in $I^{T}$ are translated via $U$ to larger potential differences and the potential differences after reset become larger phase differences during the back translation $U^{-1}$. This causes an effective phase desynchronization even for partial resets that are nonexpansive as depicted in fig. 6.7c.

For general rise functions and non-expansive partial resets the destabilization of a cluster state due to a partial reset thus can only occur if the slopes in $I^{T}$ are sufficiently larger than in $I^{R}$. In fact if this ratio becomes to small the transition may not be observed completely for non-expansive partial reset, e.g. for $R_{c}$ in the range $c \in[0,1]$ and can be shifted to partial resets that have to be expansive (e.g. for $c>1$ ).

Finally note that, in contrast to convex rise functions, for sigmoidal rise functions we always observe "damped oscillations" in the Poincaré phase plots fig. 6.8b,c. The amplitudes of these oscillations become larger when the slope of the rise function at the point of inflection becomes smaller. We therefore attribute these oscillations to subthreshold inputs received by oscillators near the inflection point of the rise function.

\subsection{Cluster Desynchronization in Biophysical Models}

In this section we briefly discuss relations of the simple partial reset model to more realistic neuron models.

We already discussed that time scale relations among the different neuronal processes that have to be satisfied in order for our simple model to give a good approximation to the dynamics (cf. sec. 5.3.1). Interestingly, in networks of more biological realistic model neurons we find similar desynchronization transitions if these time scale relations are satisfied. For example, we considered quadratic integrate-and-fire neurons (cf. eq. (2.6.3)) driven by a constant external current such that the spiking period was $\tau_{\mathrm{m}}=$ $\tau_{\text {osc }}=100 \mathrm{~ms}$ and coupled synapses (eq. (2.7.2)) with a time extension of $\tau_{\text {syn }} \approx \tau_{\mathrm{d}}=$ $10 \mathrm{~ms}$. To model the refractoriness we included an additional after-hyperpolarizing current $i_{\text {ahp }}=g_{\text {ahp }}\left(v_{\mathrm{K}}-v\right)[235]$ that becomes active after spiking of a neuron and decays with time constant of $\tau_{\text {ref }}=\tau_{\text {ahp }}=30 \mathrm{~ms}$. In this situation the times scale relations (5.3.1), (5.3.2) and (5.3.5) are all satisfied. When decreasing the maximal conductance $g_{\text {ahb }}$ we numerically observe a desynchronization transition very similar to the one observed in fig. 6.8. As an decrease of $g_{\text {ahp }}$ lowers the relative refractoriness of the QIF neurons and therefore raises the response to synaptic inputs shortly after action potential generation this model may be regarded as a temporally extended version of the infinitely fast partial reset model.

Moreover, we also numerically observed similar transitions in networks of conductance based neuron models [99] (cf. sec. 2.4.4) when the synaptic time scale was increased but stayed in the regime in which the partial reset model is valid. Here the dynamics typically did not converge to periodic states but showed clustering of neurons, with maximal cluster sizes that decayed with increasing synaptic time scale. This transition may as well be explained by a partial reset effect when considering fig. 5.4 where the spike time responses 
(a)

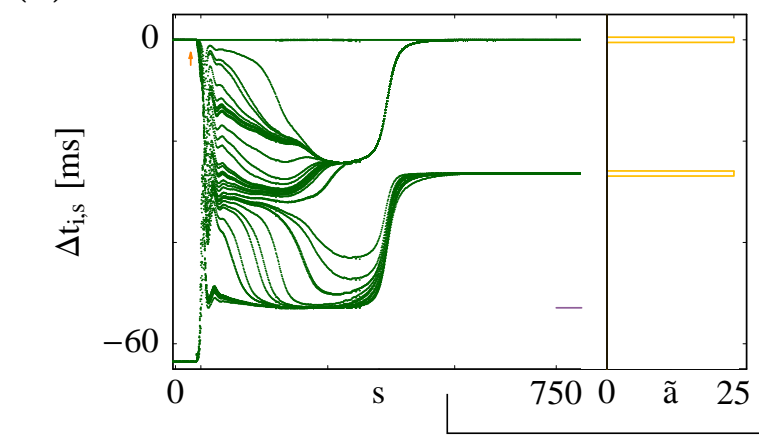

(c)

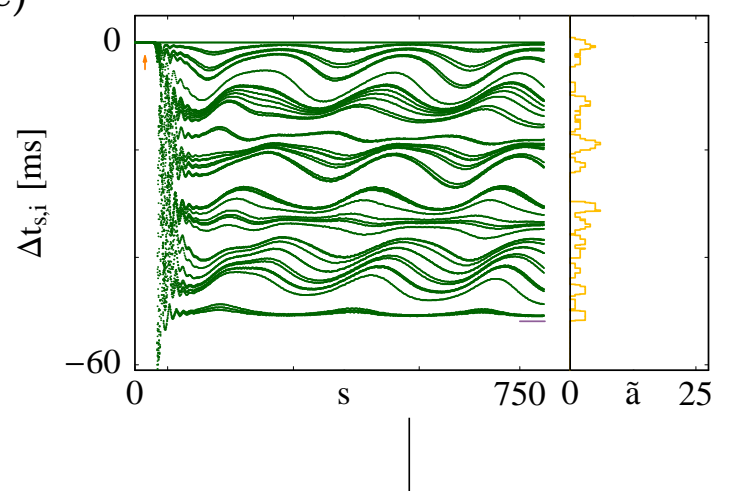

(b)

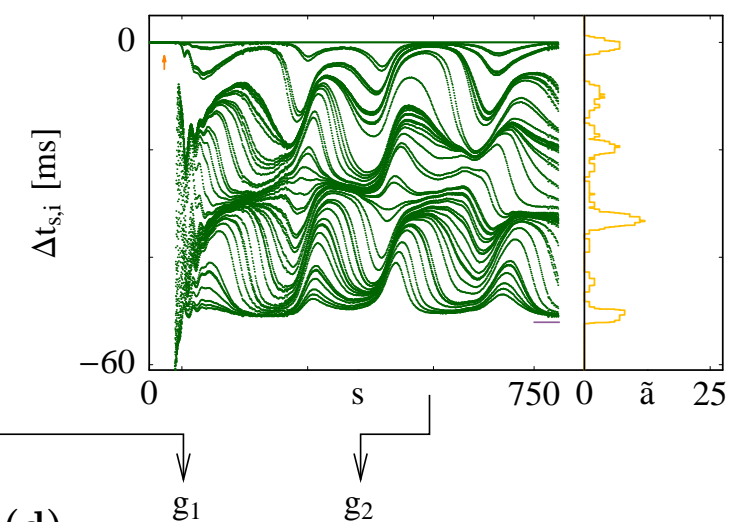

(d)

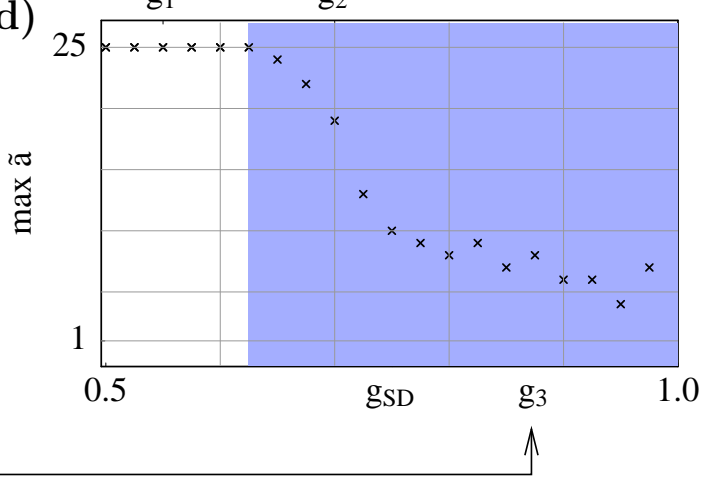

Figure 6.9. Clustering in a homogeneous all-to-all coupled network of $N=50$ two compartment cortical neurons. Neuron model as in section 2.4.4 and appendix D.4 with passive dendrite (cf. (2.4.5), (2.5.1), (2.2.4), (11.1.2) with $\left.g_{\mathrm{LD}}=-70 \mathrm{mV}, g_{\mathrm{DS}}=0.2 \mathrm{mS}\right)$. (a-c) stroboscopic spike time plots of the network dynamics for the different values of the somatic dendritic coupling $g_{\mathrm{DS}}: g_{1}=0.55, g_{2}=0.7$ and $g_{3}=0.875$. In (a) convergence of the dynamics towards a periodic state of two alternate firing clusters with $\tilde{a}=25$ neurons each is observed whereas in (b) and (c) the dynamics do not converge but show variable differently sized groups of nearly synchronously firing units. Orange histograms show that number $\tilde{a}(\Delta t)$ of neurons firing in the time interval $\left[t_{1, s}+\Delta t-\delta t / 2, t_{1, s}+\Delta t+\delta t / 2\right]$ with $\delta t=1 \mathrm{~ms}$ at $s=750$. The violet line indicates the time difference between $t_{i, 249}-t_{i, 250}$. (d) maximal cluster sizes $\max _{\Delta t} \tilde{a}(\Delta t)$, $\Delta t \in\{200,201, \ldots, 1000\}$ in 100 simulations for each $g_{\mathrm{DS}} \in\{0.5,0.525, \ldots 1.0\}$ show a decrease with increasing $g_{\mathrm{DS}}$. Blue shading indicates no convergence to a periodically firing state after $s=25000$ spikes.

for the same conductance based model are shown. Here already a short input current shortly before firing not only shortened the time to the next spike but also the following inter spike interval. Increasing the timescale of this pulse decreases the the next interspike-interval even further and in this way increases the partial reset function when this kind of dynamics is modeled by pulse-coupling. A similar effect is observed when adding a passive dendrite (cf. sec. 2.5, eq. (2.5.1)) and injecting the synaptic currents into the dendrite. The low-pass filtering property of passive dendrites then broadens the synaptic pulse and causes a similar effect as when directly increasing the synaptic time scale. An example is shown in fig. 6.9. However, the partial reset model can only give some insight into the transition observed in these two compartment cortical neurons and further studies are necessary for a detailed understanding (cf. also chapter 11). 


\section{Chapter 7}

\section{From Networks of Unstable Attractors To Heteroclinic Switching}

In this section we study the impact of the partial reset on phase oscillator networks with delayed pulse-coupling. We focus on homogeneous networks and purely excitatory interactions. We first give a precise definition of the model and then present numerical evidence for a transition from a network of unstable attractors (cf. def. 3.1.3) to heteroclinic switching dynamics (cf. def. 3.1.1) when the partial reset strength is increased from zero. We then introduce the method of event-based domain analysis to prove the existence of this novel type of bifurcation and reveal its underlying changes in phase space.

\subsection{Network Dynamics - Unstable Attractors and Heteroclinic Switching}

In this section we present numerical evidence for a bifurcation from a network of unstable attractors to heteroclinic switching dynamics when the partial reset strength is increased from zero.

We focus on homogeneous networks of pulse coupled phase oscillators as described in chapter 5.1 with interaction $\varepsilon_{i j}=\varepsilon\left(1-\delta_{i j}\right)$ and homogeneous delays $\tau_{i j}=0$. For the numerical simulations we use interaction functions $H$ and $J$ as defined in (5.2.14) and (5.2.15) with a rise function $U_{b}$, eq. (5.2.20) and partial reset $R_{c}(\zeta)=c \zeta$, eq. (5.2.7). Note that $J$ is invertible for all $c \neq 0$ and non-invertible for $c=0$. Our analysis below shows that the studied phenomena are insensitive against structural perturbations in the parameter $\tau, \varepsilon$ and the rise function $U$. We therefore concentrate on the influence of the partial reset strength $c \in[0,1]$ on the collective network dynamics.

For non-invertible resets $(c=0)$ the above system exhibits unstable attractors in form of periodic orbits $\mathcal{O}_{i}$ in a large fraction of parameter space and for different network sizes $N[371,372,16]$. The smallest system in which we observed unstable attractors has $N=4$ units. Curiously, numerical simulations, e.g. fig. 7.1, indicate that such a system exhibits two unstable attractors each of which is fully enclosed by the basin volume of the other attractor.

If we remove the local non-invertibility $(c>0)$, the dynamics changes qualitatively as shown in fig. 7.2: The two periodic orbits $\mathcal{O}_{i}$ still exist. However, starting in a state near one of the $\mathcal{O}_{i}$ leads to trajectories with perpetual switching between both. The switching times increase exponentially with the number of switches (cf. fig. 7.2b) indicating that 
(a)

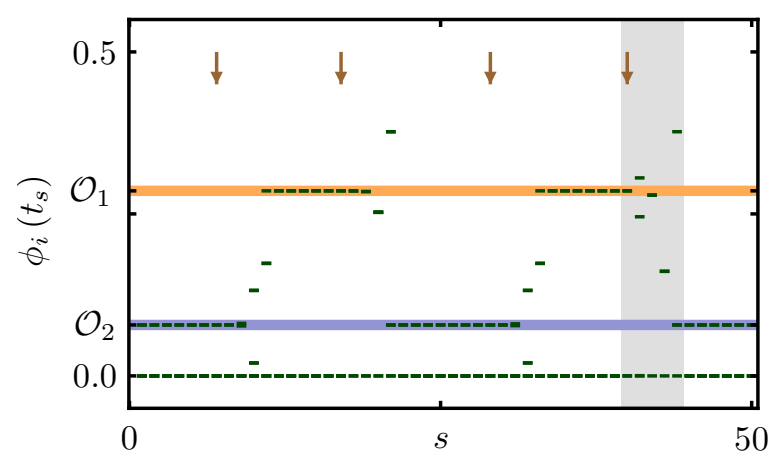

(b)

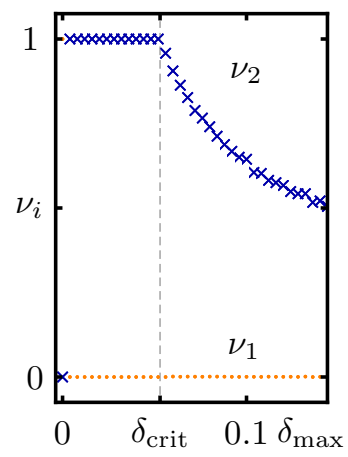

Figure 7.1. Full reset $(c=0)$ : Two unstable attractors enclosed by the basins of each other. (a) phases $\phi_{i}\left(t_{s}\right)$ (dots) of all units at just after after spike generation events at times $t_{s}$ of a reference unit $i=1$. Lines indicate the phases on an invariant orbit $\mathcal{O}_{1}$ (orange) and $\mathcal{O}_{2}$ (blue). Arrows mark times of small phase perturbations which induce switches from $\mathcal{O}_{1}$ to $\mathcal{O}_{2}$ or vice versa. The shaded area highlights a switch from $\mathcal{O}_{1}$ to $\mathcal{O}_{2}$ that is shown in detail in fig. 7.6. (b) fraction $\nu_{i}$ of 5000 trajectories reaching the periodic orbit $\mathcal{O}_{i}(\bullet: i=1, \times: i=2)$ starting from random phases distributed uniformly in a box of side width $2 \delta_{\max }$ centered around a state $a_{1}$ on the orbit $\mathcal{O}_{1}$. For $0<\delta<\delta_{\text {crit }} \approx 0.05$ all trajectories reach the orbit $\mathcal{O}_{2}\left(\nu_{2}=1\right)$, indicating that $\mathcal{O}_{1}$ is enclosed by the basin volume of $\mathcal{O}_{2}$ and in particular that $\mathcal{O}_{1}$ is an unstable attractor. Parameter values are $\varepsilon=0.23, \tau=0.02$, and $b=4.2$ for which the model exhibits short switching times between periodic orbits which simplifies the analysis in sec. 7.2.

these dynamics originate from an orbit near a heteroclinic two-cycle. Furthermore the switching times diverge as $c \rightarrow 0$ (cf. fig. 7.2b), suggesting the transition to a network of unstable attractors at $c=0$.

Recall the definition 3.2.1 for a bifurcation set as the set of parameter values at which the dynamics change qualitatively. Applying this definition to the transition observed here we conclude that there is a bifurcation from networks of unstable-attractors to het-

(a)

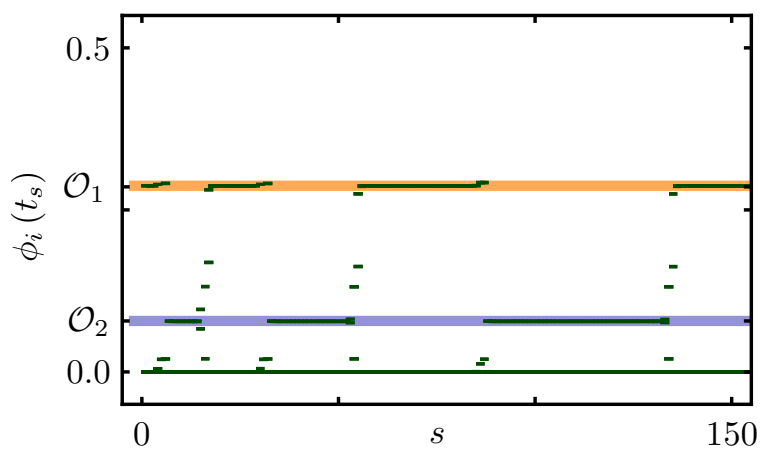

(b)

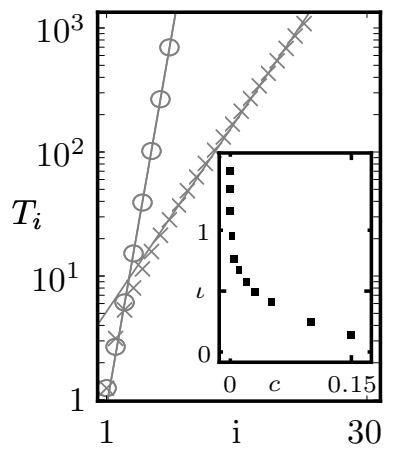

Figure 7.2. Partial reset $(c>0)$ : Heteroclinic switching. (a) phases $\phi_{i}\left(t_{s}\right)$ (dots) as in fig. 7.1a for $c=0.05$. The invariant periodic orbits $\mathcal{O}_{i}$, being unstable attractors at $c=0$, still exist for $c>0$ (orange and blue line). Starting in a state near $\mathcal{O}_{1}$ leads to repeated switching between the two states. (b) switching times $T_{i}$ until the $i$-th switch (o: $c=0.1, \times: c=0.01$ ) increase exponentially with $k$, indicating that the dynamics evolve near a heteroclinic cycle between the invariant states. Inset: Fitting $T_{i}=\gamma e^{i i}$ to the switching times for several values of $c$ we find a divergence of $\iota$ as $c \rightarrow 0$. 
(a)

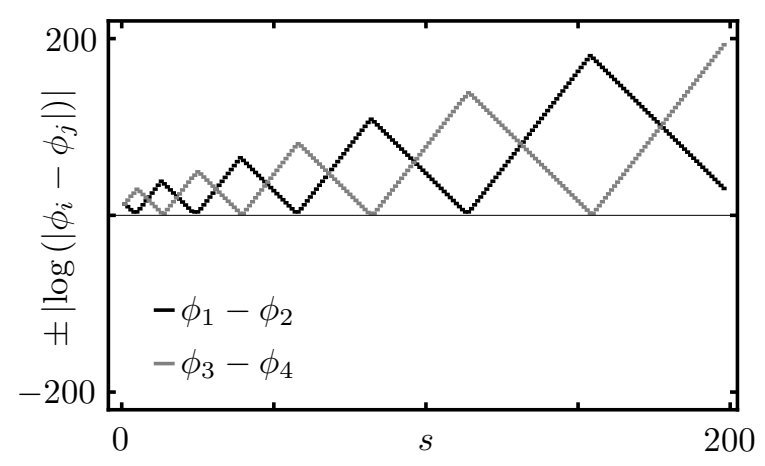

(b)

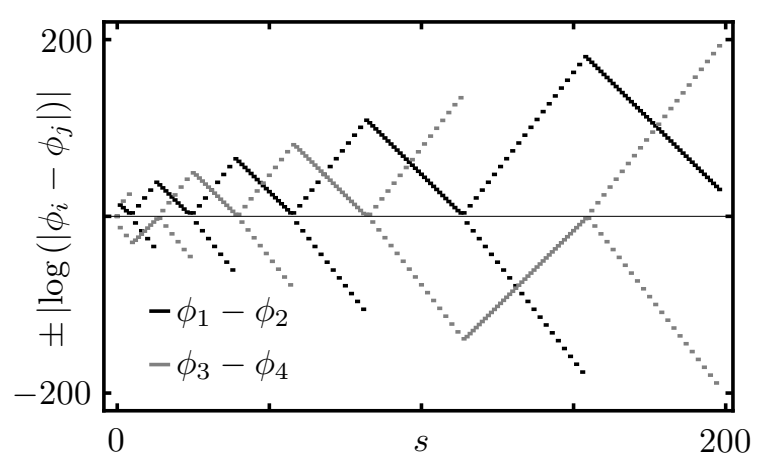

Figure 7.3. Order preservation and reversal in the heteroclinic switching dynamics. Network as in fig. 7.1. Phase differences sign $\left(\phi_{i}-\phi_{j}\right)|\ln | \phi_{i}-\phi_{j}||$ for $(i, j)=(1,2)$ (black) and $(i, j)=(3,4)$ (gray) at spike times $t_{s}$ of oscillator $i=1$ for (a) $c=0.05>0$ and (b) $c=-0.05<0$. Switching times are visible as kinks. At these points one phase difference is large and starts to decrease while the other is small and starts to increase, reflecting the contraction and expanding dynamics near a saddle point. If the second difference becomes to large a switch is caused and the situation reverses. The dynamics is order preserving for $c>0$, i.e. always $\phi_{1}>\phi_{2}$ and $\phi_{3}>\phi_{4}$ while for $c<0$ the order is reversed after each cycle in the expanding part. This is also visible in the return map $R_{i}^{\uparrow}$, eq. (7.2.22) derived in sec. 7.2.

eroclinic switching at the parameter value $c=0$. We note, that for $c<0$ we also observe heteroclinic switching dynamics where the order of the oscillators is not preserved (cf. fig. 7.3). Thus the unstable attractor network dynamics arise only at the singular bifurcation value $c=0$. In total, we therefore observe a bifurcation from order-preserving to non-order preserving heteroclinic switching dynamics through a network-of unstable attractors.

The transition is neither restricted to small network sizes nor to the small network structure of the two unstable attractors for non-invertible partial resets. In fact, large networks of unstable attractors are found in networks of pulse-coupled oscillators for

(a)

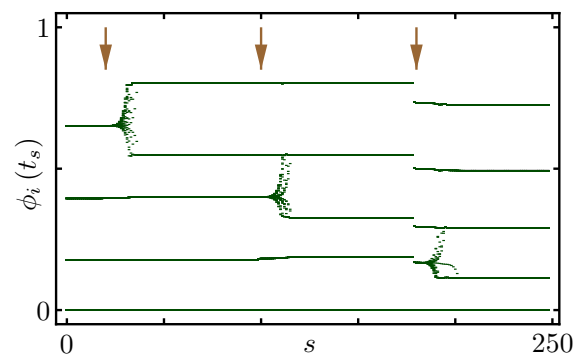

(b)

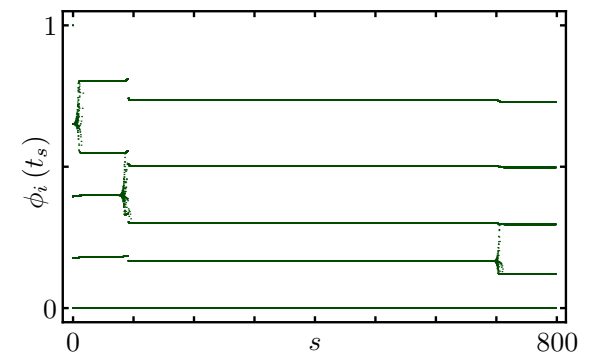

Figure 7.4. From networks of unstable attractors to heteroclinic switching in large networks. $N=100$ pulse coupled oscillators. (a) non-invertible dynamics $(c=0)$ : A 4 cluster state switches to a 5 cluster state after a small perturbation to the phases is applied at $t_{s}=20$ (arrow). Further switches to other 5 cluster states are induced by perturbations at $t_{s}=100$ and $t_{s}=180$. (b) locally invertible dynamics $(c=0.2)$ : free network evolution after a small perturbation is applied to the 4 cluster state in (a) in the beginning. Switching dynamics with exponential growing switching times are observed. Parameter used are $b=3, \tau=0.15$ and $\varepsilon=0.02$. 
$c=0$ in a large fraction of parameter space (cf. e.g. [371] and fig. 7.4). For example, in the $N=100$ network used in fig. 7.4 the second attractor consist of five clusters of synchronized neurons with sizes $(21,21,21,16,21)$. After a small perturbation the cluster of size 21 firing just after the cluster of size 16 desynchronizes and the system rearranges to another invariant 5 cluster state with cluster sizes $(21,21,16,21,21)$ which again is an unstable attractor. The number of such five cluster states is $\frac{100 !}{(21 !)^{4} 16 !} \approx 10^{65}$ and each attractor is surrounded by the basins of at least $\frac{21 !}{5 ! 16 !} \approx 10^{4}$ other unstable attractors. In such a system the precise form of the perturbation determines to which attractor the system will evolve next.

For $c>0$, there is a transition from the network of unstable attractors to complex heteroclinic structures with switching dynamics as shown in fig. 7.4. The switches along the orbit now strongly depend on the initial conditions. Moreover, for small $c$ switches only occur between states for which we observed a switch in the network of unstable attractors induced by arbitrarily small perturbations.

We also observe these transitions in systems with reduced symmetry, for example in systems with a sub-symmetry of the full permutation symmetry of the oscillators. In heterogeneous networks we did not find these transitions so far. This observation is related to fact that in non-hybrid dynamical systems heteroclinic structures only exist robustly in systems with symmetry [146, 225].

\subsection{Bifurcation From Networks of Unstable Attractors To Heteroclinic Switching}

In this section we give an analytical proof for the existence and robustness of the bifurcation from networks of unstable attractor networks to heteroclinic switching observed numerically in the previous section. Our main result of this section can be stated as follows:

Theorem 7.2.1. In homogeneous networks of oscillators with delayed pulse coupling and zero partial reset two cycles of unstable attractors exist robustly and bifurcate to heteroclinic two cycles when the partial reset strength is increased continuously.

The section is structured as follows: We first introduce the notations and state space structure for pulse coupled oscillator networks with delay and then generally introduce the method of even-sequence-based domain analysis. We then specify a small network for which we prove the above theorem: We first show the existence of two periodic orbits and derive a three dimensional representation for Poincare sections in state space near these orbits. Using the the event-sequence-based domain analysis we show for zero partial reset the existence of two unstable attractors that are completely enclosed in the basins of attraction of each other, i.e. form a two cycle of unstable attractors. We then extend the results to non-zero partial resets and show that the two unstable attractors become saddle states in a heteroclinic two cycle.

\subsubsection{State Space and Event Description for Pulse-Coupled Oscillators with Delayed Interactions}

Pulse coupled oscillator system as described in chapter 5.1 with delayed interactions formally have an infinite dimensional state space. We here give a precise definition of this 
space and a description of the dynamics in terms of events including the partial reset. We focus on networks with excitatory couplings $\varepsilon_{i j} \geq 0$ and homogeneous delays $\tau_{i j}=\tau>0$.

We first define the state space similar to $[16,265]$. A state $x \in \mathcal{X}$ of the network is fully described by the actual phases $\phi_{i} \in[0,1), i \in\{1, \ldots N\}$ and the pulse generation times $\sigma_{i, k} \in[0, \infty)$ of the $k^{\text {th }}$-last pulse of oscillator $i$. Thus the state space is given by

$$
\mathcal{X}:=[0,1)^{N} \times[0, \infty)^{\mathbb{N}} \subset \mathbb{R}^{\mathbb{N}}
$$

with states

$$
x=\left(\left(\phi_{i}\right)_{i \in\{1, \ldots N\}},\left(\sigma_{i, k}\right)_{i \in\{1 \ldots, N\}, k \in \mathbb{N}}\right) \in \mathcal{X}
$$

Recall, that we write $f\left(t^{-}\right)=\lim _{s / t} f(s)$. The hybrid time evolution of a state $x$ is then given by a continuous part, consisting of an uniform increase of all variables according to $(5.2 .13)$, i.e.

$$
\frac{d}{d t} \phi_{i}=1 \quad \frac{d}{d t} \sigma_{i, k}=1
$$

interrupted at discrete times by the following pulse generation and receiving events:

1. $\left(s_{i_{1}}, \ldots, s_{i_{k}}\right)$ : simultaneous pulse generation of $k$ oscillators $i \in\left\{i_{1}, \ldots i_{k}\right\}$ at time $t$ defined by

$$
\begin{aligned}
& \phi_{i}\left(t^{-}\right)=1 \text { for } i \in\left\{i_{1}, \ldots i_{k}\right\} \\
& \phi_{i}\left(t^{-}\right)<1 \text { for } i \in\left\{i_{1}, \ldots i_{k}\right\}
\end{aligned}
$$

For all $i \in\left\{i_{1}, \ldots i_{k}\right\}$ the phase are reset to $\phi_{i}(t)=0$ and the spike times are updated according to

$$
\sigma_{i, k+1}(t)=\sigma_{i, k}\left(t^{-}\right) \quad k \in \mathbb{N} \quad \text { and } \sigma_{i, k}(t)=0
$$

2. $\left(r_{j_{1}}, \ldots, r_{j_{k}}\right)$ : simultaneous reception of $k$ pulses generated by oscillators $j \in\left\{j_{1}, \ldots j_{k}\right\}$ at time $t$ defined by $\sigma_{j, k_{j}}\left(t^{-}\right)=\tau$ for some $k_{j} \in \mathbb{N}$. The phases are updated according to the interaction function $H$ (cf. e.g. (5.2.14)) as

$$
\phi_{i}(t)=H\left(\phi_{i}\left(t^{-}\right), \sum_{l=1}^{k} \varepsilon_{i j_{l}}\right)
$$

where the conditions

$$
H\left(\phi_{i}\left(t^{-}\right), \sum_{l=1}^{k} \varepsilon_{i j_{l}}\right)<1 \quad \text { for all } i \in\{1, \ldots, N\}
$$

apply. We further update the spike times of the received pulses $j \in\left\{j_{1}, \ldots j_{k}\right\}$ by

$$
\sigma_{j, k_{j}}(t)=\sigma_{j, k_{j}}\left(t^{-}\right)+1
$$

as their precise value will not influence the future evolution.

3. $\left(r_{j_{1}}, \ldots, r_{j_{k}}, s_{i_{1}}, \ldots s_{i_{l}}\right)$ : simultaneous reception of pulses generated by oscillators $j \in\left\{j_{1}, \ldots j_{k}\right\}$ at time $t$ defined by $\sigma_{j, k_{j}}\left(t^{-}\right)=\tau$ for some $k_{j} \in \mathbb{N}$ and spike generation of oscillators $i \in\left\{i_{1}, \ldots i_{l}\right\}$ receiving supra-threshold input. The phases 
are updated according to the interaction function $H$ and partial reset function $J$ (cf. e.g. (5.2.15))

$$
\phi_{i}(t)= \begin{cases}H\left(\phi_{i}\left(t^{-}\right), \sum_{l=1}^{k} \varepsilon_{i j_{l}}\right) & i \notin\left\{i_{1}, \ldots i_{l}\right\} \\ J\left(\phi_{i}\left(t^{-}\right), \sum_{l=1}^{k} \varepsilon_{i j_{l}}\right) & i \in\left\{i_{1}, \ldots i_{l}\right\}\end{cases}
$$

where the conditions

$$
\begin{array}{ll}
H\left(\phi_{i}\left(t^{-}\right), \sum_{l=1}^{k} \varepsilon_{i j_{l}}\right)<1 & \text { for all } i \notin\left\{i_{1}, \ldots i_{l}\right\} \\
H\left(\phi_{i}\left(t^{-}\right), \sum_{l=1}^{k} \varepsilon_{i j_{l}}\right) \geq 1 & \text { for all } i \in\left\{i_{1}, \ldots i_{l}\right\}
\end{array}
$$

apply. The spike times $\sigma_{i, k_{j}}$ for $j \in\left\{j_{1}, \ldots j_{k}\right\}$ are update according to (7.2.5) and for the spiking oscillators $i \in\left\{i_{1}, \ldots i_{l}\right\}$ via (7.2.3).

We assume that $J_{\varepsilon}(\phi)<1$ for all $\phi \in[0,1]$ and $\varepsilon \leq \hat{\varepsilon}$ where the maximal interaction strength $\hat{\varepsilon}$ is defined as

$$
\hat{\varepsilon}:=\max _{i} \sum_{j} \varepsilon_{i j}<1
$$

which excludes self excitations, i.e. ensures $\left\{i_{1}, \ldots i_{l}\right\} \cap\left\{j_{1}, \ldots j_{k}\right\}=\emptyset$ in the third event. Then the the above dynamics are well defined and give rise to a semi flow $\mathcal{F}^{t}$ on $\mathcal{X}$. We note that similar to [265] we added a jump to the spike times at pulse reception in (7.2.5). This does not influence the future dynamics but ensures that, when using the Euclidean distance, states in a small neighborhood close to pulse reception will not have additional spikes in the delay lines. Thus adding non-received spikes into the delay lines is rendered in this way as a large perturbation.

We denote the forward orbit of a state $x \in \mathcal{X}$ generated by this flow as

$$
\mathcal{O}(x)=\left\{\mathcal{F}^{t}(x) \mid t \geq 0\right\}
$$

The time evolution of the system is determined by the constant rise of the phases towards the pulse generation-threshold and interactions that also increase the phase or lift the unit above threshold. Thus for a state $x \in \mathcal{X}$ there exists a finite time $t^{(1)}(x)<\infty$ at which a discrete interaction event $e^{(1)}(x)$ is generated by the flow $\mathcal{F}^{t}$. Repeating this argument there is an infinite sequence of such events $e^{(k)}(x)$ at times $t^{(k)}(x), k \in \mathbb{N}$. We define the set of all admissible events by

$$
\mathcal{E}=\bigcup_{x \in \mathcal{X}}\left\{e^{(l)}(x)\right\}_{l \in \mathbb{N}}
$$

and an event based map

$$
F^{(l)}: \mathcal{X} \rightarrow \mathcal{X}, \quad x \mapsto \mathcal{F}^{t^{(l)}}(x)
$$

that sends a state $x$ to the state just after the $l^{\text {th }}$ event $e^{(l)}(x)$ has taken place.

We further denote by $\kappa_{i}(x)$ the smallest $k$ such that $\sigma_{i, k+1}>\tau$, i.e. the number of pulses in the delay lines not received yet and define the subspace of states with at most $k$ traveling pulses in the delay lines by

$$
\mathcal{X}_{k}^{N}=\left\{x \in \mathcal{X}: \kappa_{i}(x) \leq k \text { for all } i \in\{1, \ldots, N\}\right\} \subset\left([0,1) \times[0, \infty)^{k}\right)^{N} \subset \mathbb{R}^{(k+1) N}
$$


which is a finite dimensional space. Under weak conditions it can be shown that the dynamics often evolve towards a finite dimensional state in $\mathcal{X}_{k}^{N}$ and that for $k$ sufficiently large these spaces are invariant under the flow [16]. We endow these spaces with the induced topology of open sets in $\mathbb{R}^{(k+1) N}$, i.e. a set $\mathcal{U} \subset \mathcal{X}_{k}^{N}$ is open if there is a open set $U \subset \mathbb{R}^{(k+1) N}$ such that $\mathcal{U}=U \cap \mathcal{X}_{k}^{N}$ and choose the Lebesgue measure $\lambda$ on the Borel $\sigma$-algebra. The open sets and the measure are induced by any norm in $\mathbb{R}^{(k+1) N}$, we here choose the maximum norm, denoted by $\|\cdot\|$.

\subsubsection{Event-Sequence-Based Domain Analysis}

In this section we describe the general technique of a event-sequence based domain and parameter analysis which we will use to prove the existence of the bifurcation from networks of unstable attractors to heteroclinic switching and to investigate the underlying phase space structure. The idea is to derive conditions on the system's states (and parameters) that give rise to a predefined event sequence.

More concretely, given an predefined finite event sequence

$$
E=\left(e_{1}, \ldots, e_{k}\right)
$$

with $e_{i} \in \mathcal{E}$ we define the domain $\operatorname{Dom}(E) \subset \mathcal{X}$ to be the set of states $x \in \mathcal{X}$ that give rise to the event sequence $E$, i.e.

$$
\operatorname{Dom}(E)=\left\{x \in \mathcal{X} \mid e^{(l)}(x)=e_{l}, l \in\{1, \ldots,|E|\}\right\}
$$

For a given a set $\mathcal{U} \subset \mathcal{X}$ then event-sequence based domain analysis then aims at determining the set $\mathcal{U} \cap \mathcal{D}(E)$ via the following steps:

1. find a suitable parametrization for all states of the set $\mathcal{U} \subset \mathcal{X}$ under consideration

2. formally derive the pseudo event maps $\tilde{F}^{(l)}, l \in\{1, \ldots, k\}$ for all the states $x \in \mathcal{U}$ assuming the event sequence (7.2.7)

3. collect all conditions on the states $x \in \mathcal{U}$ that have to be satisfied so that the pseudo event map coincides with the actual event map, i.e $\tilde{F}^{(l)}(x)=F^{(l)}(x)$.

4. reduce the conditions to the strongest conditions which then define $\operatorname{Dom}(E) \cap \mathcal{U}$.

We note that the first step is facilitated by the fact that in the pulse-coupled oscillator systems of sec. 7.2.1 all states often evolve towards a finite dimensional subspace in finite time [16] or that there are finite dimensional invariant subspaces. The second step is then most conveniently done using event-based analysis tables $[371,16]$ : Here the initial finial dimensional state $x \in \mathcal{X}_{k}^{N}$ is listed together with the event sequence and the results of the maps $\tilde{F}^{(l)}(x)$. An example is shown in tab. 7.1. At each step in this table conditions arise for the pseudo map $\tilde{F}^{(l)}$ to be valid: First, no other events should occur in the dynamics between the predefined events $e_{l}$. Second for the different types of events the conditions (7.2.2) for spike generation, (7.2.4) for sub-threshold and (7.2.6) for supra-threshold pulse reception. Typically these conditions can be reduced to a smaller subset from which all constraints follow. Moreover, also constraints on unspecified parameter may arise which have to be satisfied in order for the system to have a particular event sequence. This approach can be used for inverse engineering of pulse-coupled systems that show certain sequences. For infinite periodic sequences this was done in $[263,262]$ to determine network structures that exhibit such sequences. 
Here we concentrate on the domain of such sequences and use this non-local analysis to prove the existence of a novel type of bifurcation from networks of unstable attractors to heteroclinic switching. Besides this first application, this technique has further applications in estimating the domain size of certain sequences in order to estimate their probability of occurrence and their stability against noise. The algorithmic nature of the method also makes it suitable for computer based analysis and proofs using symbolic computation software.

\subsubsection{Non-Local Non-Linear Bifurcation Analysis in a Small Network}

In this section we give a proof of theorem 7.2.1. We first introduce a small network $\mathcal{N}^{4}$ of $N=4$ oscillators. Then we determine two periodic orbits in this system for which we show that they are unstable attractors in the basin of each other. We then show that this structure bifurcates to a heteroclinic two cycle. Most of the technical calculations are deferred to appendix C.

\subsubsection{Definition of the Network $\mathcal{N}^{4}$}

In this section we specify the network used in the analysis. We consider a homogeneous network $\mathcal{N}^{4}$ of $N=4$ oscillators with homogeneous coupling strengths $\varepsilon_{i j}=\varepsilon\left(1-\delta_{i j}\right)$ and interaction delay $\tau$ as in section 7.2.1.

We assume a general smooth sub-threshold interaction function $H:[0, \infty) \rightarrow[0, \infty)$ with the following properties

$$
\frac{\partial}{\partial \phi} H(\phi, \varepsilon)>0 \quad \text { and } \quad \frac{\partial}{\partial \varepsilon} H(\phi, \varepsilon)>0
$$

which are satisfied if $H$ originates from a pulsed-coupled system as in eq. (5.2.14). We further assume that the reception of a pulse before a time shift $\sigma \geq 0$ increases the phase less than in the reversed situation, i.e.

$$
\sigma+H_{\varepsilon}(\phi)<H_{\varepsilon}(\phi+\sigma)
$$

for all $\phi>0$. If $H$ is defined via a rise function $U$ that is concave this property is satisfied.

For the partial reset function $J=J^{(c)}$ we assume a smooth dependence on a parameter $c$ so that for $c=0, J^{(0)}(\phi, \varepsilon) \equiv 0$ and for $c \neq 0$

$$
\frac{\partial}{\partial \phi} J^{(c)}(\phi, \varepsilon)>0 \quad \text { and } \quad \frac{\partial}{\partial \varepsilon} J^{(c)}(\phi, \varepsilon)>0
$$

We usually suppress the dependence on $c$ in the calculations and write $J_{\varepsilon}>0$ to indicate a parameter $c>0$. We further assume

$$
J(\phi, \varepsilon) \leq H(0, \varepsilon)<1
$$

An particular example for $J$ is given in (5.2.15) with $R=R_{c}$ (5.2.7). We note that for a given $\eta>0$ we can obtain

$$
\max _{\phi[0,1]} \max _{\varepsilon \in[0, \hat{\varepsilon}]}|J(\phi, \varepsilon)|<\eta
$$


by choosing $c$ sufficiently small. For notational convenience we define

$$
\begin{aligned}
H_{\varepsilon, \tau}(\phi): & =H_{\varepsilon} \circ S_{\tau}(\phi)=H_{\varepsilon}(\phi+\tau) \\
J_{\varepsilon, \tau}(\phi): & =J_{\varepsilon} \circ S_{\tau}(\phi)=J_{\varepsilon}(\phi+\tau)
\end{aligned}
$$

and

$$
\gamma_{1}(\phi)=1-H_{2 \varepsilon, \tau} \circ S_{\phi} \circ H_{\varepsilon, \tau}(0)
$$

We assume the following conditions on the interaction function $H$ and the network parameter $\varepsilon$ and $\tau$ to facilitate the analysis

$$
\begin{aligned}
H_{\varepsilon, \tau} \circ H_{\varepsilon, \tau} \circ H_{\varepsilon, \tau}(0) & <1<H_{2 \varepsilon, \tau} \circ H_{\varepsilon, \tau}(\tau) \\
S_{\tau} \circ H_{\varepsilon, \tau} \circ H_{\varepsilon, \tau}(\tau) & <1<H_{\varepsilon, \tau} \circ H_{2 \varepsilon, \tau} \circ H_{\varepsilon, \tau}(0) \\
H_{2 \varepsilon, \tau}\left(\tau+1-H_{\varepsilon, \tau}^{3}(0)\right) & <1<H_{\varepsilon, \tau}\left(H_{\varepsilon, \tau}(\tau)+1-H_{\varepsilon, \tau}^{3}(0)\right) \\
H_{\varepsilon, \tau}\left(H_{\varepsilon, \tau}(0)+1-H_{2 \varepsilon, \tau} \circ H_{\varepsilon, \tau}(0)\right) & <1<H_{2 \varepsilon, \tau}\left(H_{\varepsilon, \tau}(0)+1-H_{2 \varepsilon, \tau} \circ H_{\varepsilon, \tau}(0)\right)
\end{aligned}
$$

These conditions imply

$$
\begin{aligned}
\gamma_{1}(0) & >0 \\
\gamma_{1}\left(\gamma_{1}(0)\right) & <0
\end{aligned}
$$

Figure 7.7 shows that all these conditions define an open non empty set in parameter space when choosing a rise function $U_{b}$ (cf. eq. (5.2.20)). Or results however are not restricted to this choice of rise function.

\subsubsection{Existence of Periodic Orbits $\mathcal{O}_{i}$}

In this section we show the existence of two periodic orbits whose local stability and non-local attractivity properties strongly depend on the partial reset as we will see below.

Lemma 7.2.2. In the network $\mathcal{N}^{4}$ defined in section 7.2.3.1 the states $o_{i} \in \mathcal{X}_{1}^{4}$ defined by

$$
o_{1}=((0,0, \alpha, \alpha),(0,0, T-\tau, T-\tau)), \quad o_{2}=((\alpha, \alpha, 0,0),(T-\tau, T-\tau, 0,0))
$$

where $\alpha$ is determined implicitly by

$$
\alpha:=H_{\varepsilon, \tau} \circ J_{2 \varepsilon, \tau}(\alpha)+1-H_{2 \varepsilon, \tau} \circ H_{\varepsilon, \tau}(0)
$$

give rise to two periodic orbits $\mathcal{O}_{i}=\mathcal{O}\left(o_{i}\right), i=1,2$ with period $T=2 \tau+\gamma_{1}(0)$.

Proof. The state $o_{2}$ is obtained from $o_{1}$ via the exchange of oscillators $(1,2) \leftrightarrow(3,4)$ and it thus suffices to prove the existence for $\mathcal{O}_{1}$. We set $O_{i}$ to be the first three events in the event sequence of $o_{i}$ and claim:

$$
O_{1}=\left(s_{1}, s_{2}\right)\left(r_{1}, r_{2}, s_{3}, s_{4}\right)\left(r_{3}, r_{4}\right)
$$

We use event-sequence based domain and parameter analysis to verify this. The event table for the sequence $O_{1}$ starting in $o_{1}$ is shown in tab. 7.1. To obtain periodicity of $\mathcal{O}\left(o_{1}\right)$ the first and last row have to be identical, resulting in eq. (7.2.17). For the table 
(a)

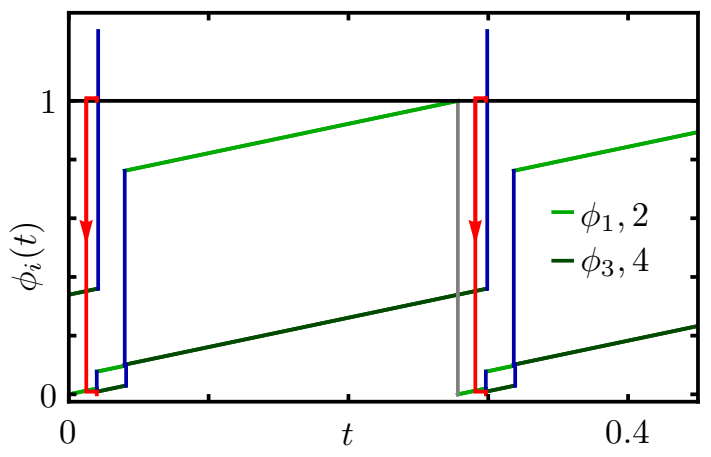

(b)

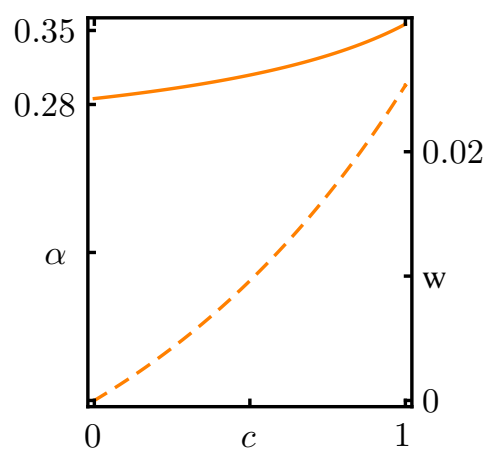

Figure 7.5. Periodic orbit $\mathcal{O}_{1}$ of $\mathcal{N}^{4}$. (a) oscillator phases $\phi_{i}$ (green) as a function of time $t$ starting at $t=0$ in the state $o_{1}(7.2 .16)$ with the event $\left(s_{1}, s_{2}\right)$. The pulses from oscillator $i=1,2$ are received after a delay time $\tau$ inducing the supra-threshold event $\left(r_{1}, r_{2}, s_{3}, s_{4}\right)$ with partial reset of oscillators $i=3,4$ (red arrow). At $t=2 \tau$ their pulses are received in the event $\left(r_{3}, r_{4}\right)$ with only sub-threshold excitation (blue). The cycle repeats at $t=2 \tau+\gamma_{1}(0)$ with the the event $\left(s_{1}, s_{2}\right)$ (cf. also tab. 7.1). (b) phase difference $\alpha$, eq. (7.2.17) (solid line) and width $w$, eq. (7.2.24), of the image $\mathcal{C}_{i}^{\prime}$ depend continuously on the partial reset strength $c$.

to be valid, we derive the condition $0<\alpha<1$ from the event $\left(s_{1}, s_{2}\right)$. This is true as $\gamma_{1}(0) \leq \alpha$ and $0<\gamma_{1}(0)$ by parameter condition (7.2.14). Further we assumed

$$
\alpha \leq H_{\varepsilon, \tau} \circ H_{2 \varepsilon}(0)+\gamma_{1}(0)<H_{\varepsilon, \tau} \circ H_{2 \varepsilon, \tau}(0)+\gamma_{1}(0)=1
$$

where in the first step we used (7.2.11) and in the second (7.2.8). From the event $\left(r_{1}, r_{2}, s_{3}, s_{4}\right)$ we obtain $H_{\varepsilon}(\tau)<1$ and $H_{2 \varepsilon}(\alpha+\tau) \geq 1$. The first inequality follows from $\gamma_{1}(0)>0$, the second one from

$$
H_{2 \varepsilon}(\alpha+\tau) \geq H_{2 \varepsilon}\left(\tau+H_{\varepsilon}(\tau)+\gamma_{1}(0)\right)=1-\gamma_{1}\left(\gamma_{1}(0)\right)>1
$$

where the last inequality is obtained from (7.2.15). Finally, from the event $\left(r_{3}, r_{4}\right)$ we get $H_{2 \varepsilon}\left(H_{\varepsilon}(\tau)+\tau\right)<1$ and $H_{\varepsilon}\left(J_{2 \varepsilon}(\tau+\alpha)+\tau\right)<1$ which both follow form (7.2.11) and $\gamma_{1}(0)>0$..

The orbit $\mathcal{O}_{1}$ is illustrated in fig. 7.5.

\begin{tabular}{cccccc}
\hline event & time & $\phi_{1}, \phi_{2}$ & $\sigma_{1}, \sigma_{2}$ & $\phi_{3}, \phi_{4}$ & $\sigma_{3}, \sigma_{4}$ \\
\hline \hline$s_{1}, s_{2}$ & 0 & $1 \rightarrow 0$ & 0 & $\alpha$ & - \\
\hline$r_{1}, r_{2}, s_{3}, s_{4}$ & $\tau$ & $\phi_{1,1}:=H_{\varepsilon}(\tau)$ & $\tau \rightarrow-$ & $\phi_{3,1}:=J_{2 \varepsilon}(\alpha+\tau)$ & 0 \\
\hline$r_{3}, r_{4}$ & $2 \tau$ & $\phi_{1,2}:=H_{2 \varepsilon}\left(\phi_{1,1}+\tau\right)$ & - & $\phi_{3,2}:=H_{\varepsilon}\left(\phi_{3,1}+\tau\right)$ & $\tau \rightarrow-$ \\
\hline$s_{1}, s_{2}$ & $2 \tau+1-\phi_{1,2}$ & $1 \rightarrow 0$ & 0 & $\phi_{3,2}+1-\phi_{1,2}$ & - \\
\hline
\end{tabular}

Table 7.1: Event table for the invariant periodic orbit $\mathcal{O}_{1}$. As the precise values of $\sigma_{i, 1}:=\sigma_{i}>\tau$ do not influence the future dynamics we indicate such values with a - . 


\subsubsection{Local Finite Dimensional Poincare Representations of the State Space}

In this section we derive local representations of the state space and show that they represent Poincare sections for the dynamics near the periodic orbits $\mathcal{O}_{i}, i \in\{1,2\}$

Note that the orbit $\mathcal{O}_{i}$ consists of three straight lines in state space (cf. fig. 7.5). We consider the open sets $\mathcal{U}_{i} \subset \mathcal{X}_{1}^{4}$ for $i \in\{1,2\}$

$$
\mathcal{U}_{i}^{\eta}=\left\{x \in \mathcal{X}_{1}^{4} \mid\|x-y\|<\eta, y \in \mathcal{O}_{i}\right\} \cap\left[[0,1)^{4} \times([0, \tau) \cup(\tau+1-\eta, \infty))^{4}\right]
$$

where $\eta$ is chosen sufficiently small and we explicitly excluded the interval $[\tau, 1+\tau-\eta]$ in the pulse generation times $\sigma_{i, 1}$ which according to (7.2.5) is not part of any orbit. Hence this set is a open neighbourhood of the orbit $\mathcal{O}_{i} \subset \mathcal{U}_{i}$ with positive measure $\lambda\left(\mathcal{U}_{i}\right)>0$.

Remark 7.2.3. Taking the product of this set with $(1, \infty)^{N(k-1)}$ we obtain an open neighbourhood $\mathcal{U}_{i, k} \subset \mathcal{X}_{k}^{4}$ of $\mathcal{O}_{i}$ of positive measure in any finite dimensional subspace $\mathcal{X}$.

We further define the sets

$$
\mathcal{P}_{1}=\left\{\left(\delta_{2}, \delta_{3}, \delta_{4}\right)|| \delta_{2} \mid<,-\alpha<\delta_{3}, \delta_{4}<1-\alpha-\tau\right\} \subset \mathbb{R}^{3}
$$

and similarly $\mathcal{P}_{2}$ by exchanging the indices $1 \leftrightarrow 3$ and $2 \leftrightarrow 4$. We define the set valued map

$$
P_{1}:\left(\delta_{2}, \delta_{3}, \delta_{4}\right) \mapsto \begin{cases}\left(\left(0, \delta_{2}, \delta_{3}, \delta_{4}\right),\{0\} \times(1+\tau, \infty)^{3}\right) & \delta_{2}>0 \\ \left(\left(0,0, \delta_{3}, \delta_{4}\right),\{0\}^{2} \times(1+\tau, \infty)^{2}\right) & \delta_{2}=0 \\ \left(\left(\delta_{2}, 0, \delta_{3}, \delta_{4}\right),(1+\tau, \infty) \times\{0\} \times(1+\tau, \infty)^{2}\right) & \delta_{2}>0\end{cases}
$$

and

$$
\mathcal{P}_{1}^{4}=\bigcup_{\delta \in \mathcal{P}_{1}} P_{1}(\delta) \subset \mathcal{X}_{1}^{4}
$$

I.e. for states in $\mathcal{P}_{1}^{4}$ one or both oscillators $i \in\{1,2\}$ just have reached the threshold due to their intrinsic rotation and where reset. Note that due to the restriction of the $\delta \in \mathcal{P}_{1}$ these are not all states in $\mathcal{X}_{1}^{4}$ of this type. We similarly define $\mathcal{P}_{2}^{4}$ via a map $P_{2}$. Note that we have $o_{i} \in P_{i}(0)$, i.e. the periodic orbits $\mathcal{O}_{i}$ are represented by $\delta=0$ in $\mathcal{P}_{i}$

Lemma 7.2.4. There is a $\eta>0$ so that for each $x \in \mathcal{U}_{i}^{\eta}$ there is a finite time $t(x)$ such that $\mathcal{F}^{t(x)}(x) \in \mathcal{P}_{i}^{4}$, i.e. the $\mathcal{P}_{i}^{4}$ are Poincare sections of the flow near the orbits $\mathcal{O}_{i}$. Via the maps $P_{i}$ these Poincare sections have three dimensional representations $\mathcal{P}_{i}$.

Proof. For $\eta$ sufficiently small the neighbourhood $\mathcal{U}_{i}^{\eta}$ consists of three connected components around the three segments of the orbit $\mathcal{O}_{i}(\mathrm{cf}$. tab. 7.1). The evolution of these states are derived in appendix C.1. The states evolve according to tab. C.1 and C.2 together with a uniform phase shift in the third component where all pulses have been received. This shows that these states cross $\mathcal{P}_{i}^{4}$ in finite time. As received pulses do not influence the future dynamics we may identify states that have the same phases and unreceived pulses. In $\mathcal{P}_{i}^{4}$ this results in equivalence classes given by the images of $P_{i}$.

Via this result we can reduce the study of the dynamics near the orbits $\mathcal{O}_{i}$ to the spaces $\mathcal{P}_{i}^{4}$ and their return maps, or the induced maps on $\mathcal{P}_{i}$. We even go one step further and also consider transient maps between these two spaces in order to capture the non-local dynamics of the network. We first concentrate on zero partial reset. 


\subsubsection{A Two Cycle of Unstable Attractors}

Throughout this section we focus on zero partial reset functions $J_{\varepsilon}=0$. We fix $\eta>0$ as in lemma 7.2.4 and write $\mathcal{U}_{i}=\mathcal{U}_{i}^{\eta}$. Denoting the basin of attraction of $\mathcal{O}_{i}$ by $\mathcal{B}\left(\mathcal{O}_{i}\right)$ we will show the following:

Proposition 7.2.5. Under the conditions (7.2.13) and $J_{\varepsilon}=0$ the open positive measure neighbourhoods $\mathcal{U}_{i}$ of the periodic orbits $\mathcal{O}_{i}$ satisfy

$$
\lambda\left(\mathcal{U}_{1} \cap \mathcal{B}\left(\mathcal{O}_{2}\right)\right)=\lambda\left(\mathcal{U}_{1}\right) \quad \lambda\left(\mathcal{U}_{2} \cap \mathcal{B}\left(\mathcal{O}_{1}\right)\right)=\lambda\left(\mathcal{U}_{2}\right)
$$

i.e. the two orbits $\mathcal{O}_{i}$ are unstable attractors forming a two cycle (cf. .def. (3.1.3)).

Note that via remark 7.2.3 this also holds for any finite dimensional restriction of the dynamics to $\mathcal{X}_{k}^{4}, k \in \mathbb{N}$.

In order to show (7.2.21) we use event-sequence-based domain analysis (cf. sec. 7.2.2) to determine the domains of several event sequences in the Poincare sets $\mathcal{P}_{i}^{4}$ (or $\mathcal{P}_{i}$ ), $i \in\{1,2\}$. In particular, we consider the event sequences of several return maps $R_{i}^{z}$ and $Q_{i}^{z}, z \in\{\uparrow, a, b\}$ from subsets of $\mathcal{P}_{i}$ back to $\mathcal{P}_{i}$ and transient maps $T_{i j}^{z}, z \in\{a, b, c, d\}$ from a subset in $\mathcal{P}_{i}$ to $\mathcal{P}_{j}, i \neq j$. If $X_{i}$ is one of these maps starting in $\mathcal{P}_{i}$ with event sequence $E\left(X_{i}\right)$ we define its domain by

$$
\mathcal{D}\left(X_{i}\right):=\operatorname{Dom}\left(E\left(X_{i}\right)\right) \cap \mathcal{P}_{i}^{4}
$$

We also write $\mathcal{D}\left(X_{i}\right)$ for the representation of this set in $\mathcal{P}_{i}$.

The event sequences and the technical domain analysis for all maps is given in appendix C.2.1 - C.2.3. Some of the domains are visualized in fig. 7.6. Also the trajectory that led to a switch from a perturbed state near $\mathcal{O}_{1}$ to $\mathcal{O}_{2}$ as indicated in fig. 7.1 is drawn in this three dimensional representation of the state space.

We first focus on the maps $R_{i}^{\uparrow}$ and show that they have an expanding property:

Lemma 7.2.6. For all states $x \in \mathcal{D}\left(R_{i}^{\uparrow}\right)$ there is a $t(x)<\infty$ such that $\mathcal{F}^{t(x)}(x) \notin$ $\mathcal{D}\left(R_{i}^{\uparrow}\right)$.

Proof. Consider the map $R_{1}^{\uparrow}$ analyzed in sec. C.2.1.1. In particular, application of the map to $\delta^{(0)} \in \mathcal{D}_{1}\left(R_{1}^{\uparrow}\right)$ gives

$$
\begin{aligned}
& \delta_{2}^{(1)}=\operatorname{sign}\left(\delta_{2}^{(0)}\right)\left[H_{2 \varepsilon, \tau} \circ H_{\varepsilon, \tau}\left(\left|\delta_{2}^{(0)}\right|\right)-H_{2 \varepsilon, \tau+\left|\delta_{2}^{(0)}\right|} \circ H_{\varepsilon, \tau}\left(-\left|\delta_{2}^{(0)}\right|\right)\right] \\
& \delta_{3}^{(1)}=H_{\varepsilon, \tau} \circ J_{\varepsilon, \mid \delta_{2}^{(0)}} \circ H_{\varepsilon, \tau}\left(\alpha+\delta_{3}^{(0)}\right)+1-H_{2 \varepsilon, \tau} \circ H_{\varepsilon, \tau}\left(\left|\delta_{2}^{(0)}\right|\right)-\alpha \\
& \delta_{4}^{(1)}=H_{\varepsilon, \tau} \circ J_{\varepsilon, \mid \delta_{2}^{(0)}} \circ H_{\varepsilon, \tau}\left(\alpha+\delta_{4}^{(0)}\right)+1-H_{2 \varepsilon, \tau} \circ H_{\varepsilon, \tau}\left(\left|\delta_{2}^{(0)}\right|\right)-\alpha
\end{aligned}
$$

and thus for with $\delta_{2}^{(0)} \geq \eta>0$ we obtain

$$
\begin{aligned}
\left|\delta_{2}^{(1)}\right| & =H_{2 \varepsilon, \tau} \circ H_{\varepsilon, \tau}\left(\left|\delta_{2}^{(0)}\right|\right)-H_{2 \varepsilon, \tau} \circ S_{\left|\delta_{2}^{(0)}\right|} \circ H_{\varepsilon, \tau}\left(-\left|\delta_{2}^{(0)}\right|\right) \\
& >H_{\varepsilon, \tau}\left(\left|\delta_{2}^{(0)}\right|\right)-S_{\left|\delta_{2}^{(0)}\right|} \circ H_{\varepsilon, \tau}\left(-\left|\delta_{2}^{(0)}\right|\right) \\
& >H_{\varepsilon, \tau}\left(\left|\delta_{2}^{(0)}\right|\right)-H_{\varepsilon, \tau}(0) \\
& >(1+\Delta)\left|\delta_{2}^{(0)}\right|
\end{aligned}
$$


where $\Delta=\min _{\phi}\left(H_{\varepsilon, \tau}^{\prime}(\phi)\right) \eta>0$. As for any $\delta^{(0)} \in \mathcal{D}\left(R_{1}^{\uparrow}\right)$ we can find $\eta>0$ such that $\delta_{2}^{(0)} \geq \eta>0$ it follows by repeated application of this argument that there is and $n \in \mathbb{N}$ such that the $n^{\text {th }}$ iterate satisfies $\delta_{2}^{(n)}>\tau$, i.e. $\delta^{(n)} \notin \mathcal{D}\left(R_{1}^{\uparrow}\right)$. A similar argument holds for $R_{2}^{\uparrow}$.

Defining the lower dimensional synchronization manifolds $\mathcal{S}_{1}=\left\{\delta \in \mathcal{P}_{1} \mid \delta_{2}=0\right\}$ and $\mathcal{S}_{2}=\left\{\delta \in \mathcal{P}_{2} \mid \delta_{4}=0\right\}$ we observe that the periodic orbit $\mathcal{O}_{i}$ represented by $o_{i} \in \mathcal{P}_{i}^{4}$ or $\delta=0$ in $\mathcal{P}_{i}$ is enclosed by the set $\mathcal{D}\left(R_{i}^{\uparrow}\right) \cup \mathcal{S}_{i}$ (cf. the domain definition (C.2.3)). Thus the previous lemma shows that the orbits $\mathcal{O}_{i}$ are unstable. We now show that they are also unstable attractors by inspection of the the time evolution of states in the sets $\mathcal{D}\left(R_{i}^{\uparrow}\right)$.

Lemma 7.2.7. For $J_{\varepsilon}=0$, a state in $\mathcal{D}\left(R_{i}^{\uparrow}\right)$ is eventually mapped to a state on the orbit $\mathcal{O}_{j}, i, j \in\{1,2\}, i \neq j$, i.e. $\mathcal{D}\left(R_{i}^{\uparrow}\right) \subset \mathcal{B}\left(\mathcal{O}_{j}\right)$

Proof. The image $\mathcal{C}_{i}=R_{i}^{\uparrow}\left(\mathcal{D}\left(R_{i}^{\uparrow}\right)\right)$ for $J_{\varepsilon}=0$ is a line in $\mathcal{P}_{i}$ due to the supra threshold synchronization of two oscillators (cf. C.2.2). It is contained in the set $\cup_{z \in\{a, b, c\}} \mathcal{D}\left(T_{i}^{z}\right) \cup$ $\mathcal{D}\left(R_{i}^{\uparrow}\right)$ as can be verified using the domain definitions (C.2.12), (C.2.15) and (C.2.18) together with the explicit expression for the map $R_{i}^{\uparrow}$ in (C.2.2) (cf. also fig. 7.6). Lemma 7.2.6 then shows that all states in $\mathcal{D}\left(R_{i}^{\uparrow}\right)$ eventually leave $\mathcal{D}\left(R_{i}^{\uparrow}\right)$ and are mapped to states in $\mathcal{C}_{i} \backslash \mathcal{D}\left(R_{i}^{\uparrow}\right)$. Application of the transient maps $T_{i}^{a}$ to these states then result in the sets $\mathcal{A}_{i}=\cup_{z \in\{a, b, c\}} T_{i}^{z}\left(\mathcal{D}\left(T_{i}^{z}\right) \cap \mathcal{C}_{i}\right)$ which are part of the synchronization manifolds, i.e. $\mathcal{A}_{i} \subset \mathcal{S}_{j}$. Closer inspection of the the set $\mathcal{A}_{i}$ and the domains of the maps $Q_{i}^{z}$, $z \in\{\uparrow, a, b\}$ given by (C.2.3), (C.2.6) and (C.2.9) when setting $\delta_{2}=0$ then shows that

$$
\mathcal{A}_{i} \subset \bigcup_{z \in\{\uparrow, a, b\}} \mathcal{D}\left(Q_{j}^{z}\right)
$$

To complete the proof we finally show that states in $\mathcal{D}\left(Q_{j}^{z}\right)$ are mapped to $o_{j}$ in finite time. First consider $Q_{j}^{\uparrow}$ that has the same event sequence as a single period of the orbit $\mathcal{O}_{j}$. In particular after one application of this map and using $J_{\varepsilon}=0$ all states in $\mathcal{D}\left(Q_{j}^{\uparrow}\right)$ are mapped to $o_{j}$ via (C.2.2). Lemma (C.2.4) and lemma (C.2.5) then show that states in $\mathcal{D}\left(Q_{j}^{z}\right), z \in\{a, b\}$ are mapped to $\mathcal{D}\left(Q_{j}^{\uparrow}\right)$ in a single step and thus via a further step also to $o_{j}$.

We note that the maps $Q_{j}^{z}$ as return maps could give rise to further periodic orbits, in particular in $\mathcal{D}\left(Q_{j}^{b}\right)$ we find a periodic orbit if the parameter condition (7.2.13) is not satisfied. In this case not all transients from $\mathcal{D}\left(R_{i}^{\uparrow}\right)$ end up in the orbit $\mathcal{O}_{i}$ but also in the periodic orbit in $\mathcal{D}\left(Q_{j}^{b}\right)$.

We can now proof proposition 7.2.5:

Proof. The union $\mathcal{D}\left(R_{i}^{\uparrow}\right) \cup \mathcal{D}\left(Q_{i}^{\uparrow}\right)$ via (C.2.3) encloses the periodic orbit represented by $\delta=0$ in $\mathcal{P}_{i}$. We thus choose $\mathcal{U}_{i}$ small enough to lie within this union. As $\lambda\left(\mathcal{D}\left(Q_{i}^{\uparrow}\right)\right)=0$ and $\mathcal{D}\left(R_{i}^{\uparrow}\right)$ is mapped to $\mathcal{O}_{j}$ the claim (7.2.21) follows. 
(a)

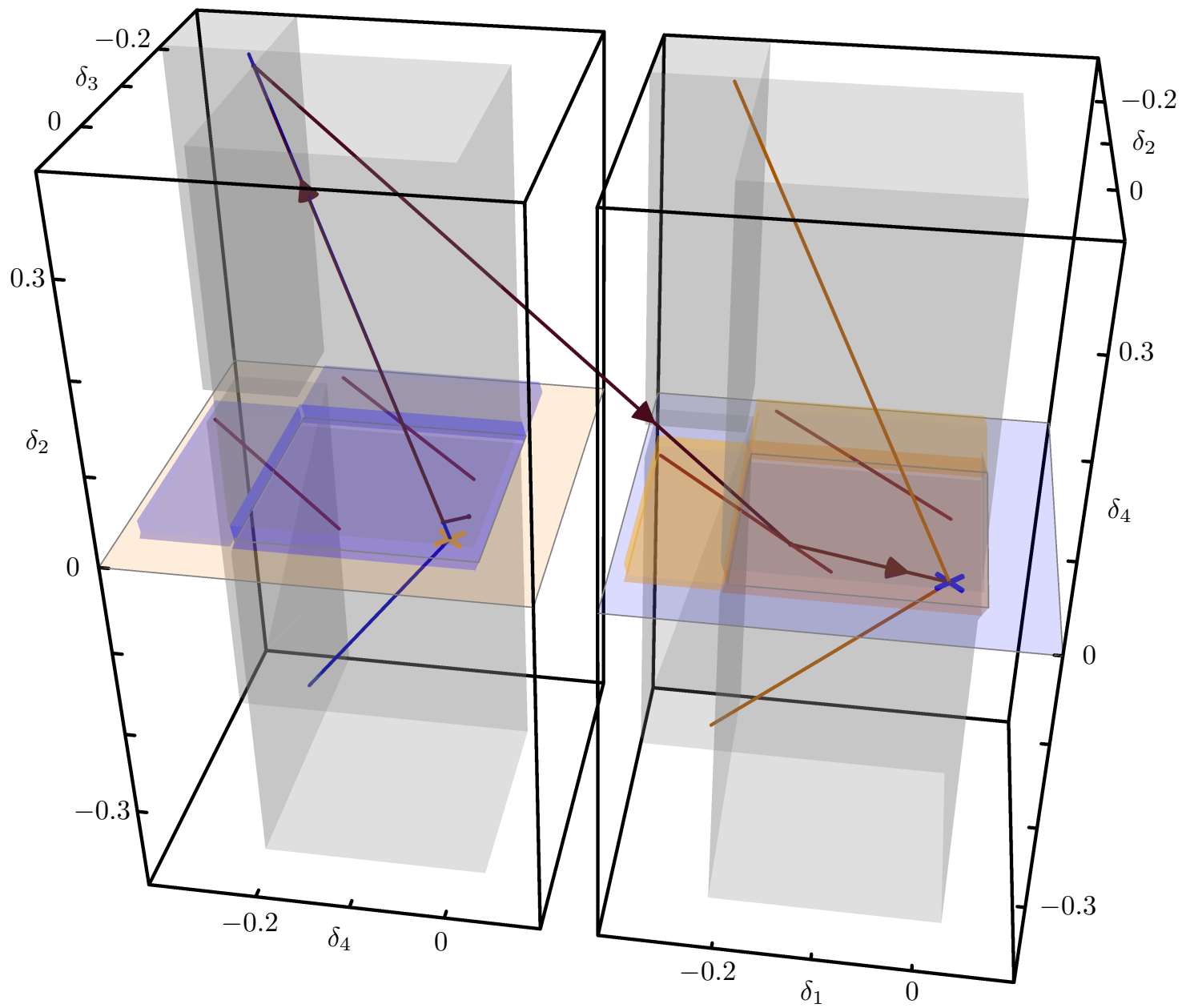

(b)

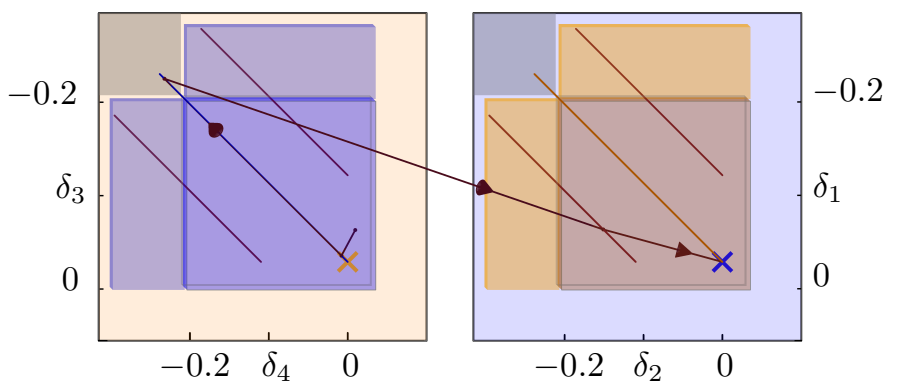

(c)

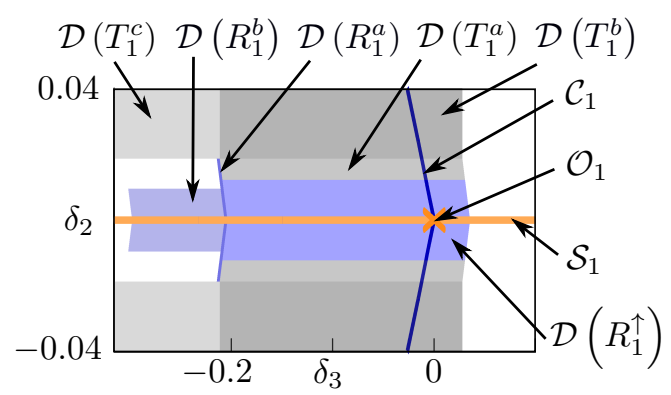

Figure 7.6. Structure of the three-dimensional reduced state space for the network in fig. 7.1 with $c=0$, illustrating that the $\mathcal{O}_{i}$ are unstable attractors enclosed by the basins of each other. (a) representations $\mathcal{P}_{i}$ in a neighbourhood of $a_{1} \in \mathcal{P}_{1}$ (orange cross) and $a_{2} \in \mathcal{P}_{2}$ (blue cross) with the stable manifolds $\mathcal{S}_{i}$ (left - orange plane, right - blue plane), domains of the return maps $R_{i}^{\uparrow}$ and $R_{i}^{a}$ (left blue, right orange) and transient maps $T_{i}^{a}, T_{i}^{b}$ and $T_{i}^{c}$ (gray). Also shown are the images $\mathcal{C}_{i}=R_{i}^{\uparrow}\left(\mathcal{D}\left(R_{i}^{\uparrow}\right)\right)$ as lines (blue left, orange right) and the image of this line under the transient maps $T_{i}^{z}, z \in\{a, b, c\}$ (brown lines). Trajectories starting in $\mathcal{D}\left(R_{i}^{\uparrow}\right)$ (close to $\mathcal{O}_{1}$ ) lead to a switch to $\mathcal{O}_{2}$. The bordeaux line shows the trajectory of the marked switch in fig. 7.1a. (b) Projection of $\mathcal{P}_{1}$ onto the $\delta_{2}-\delta_{3}$ plane and (c) onto the $\delta_{3}-\delta_{4}$ plane, illustrating that, except for the lower dimensional subset $\mathcal{S}_{1}$, the attractor $O_{1}$ is enclosed by $\mathcal{D}\left(R_{i}^{\uparrow}\right)$, i.e. there is a full measure neighbourhood of $\mathcal{O}_{1}$ which belongs to $\mathcal{B}\left(\mathcal{O}_{2}\right)$. 


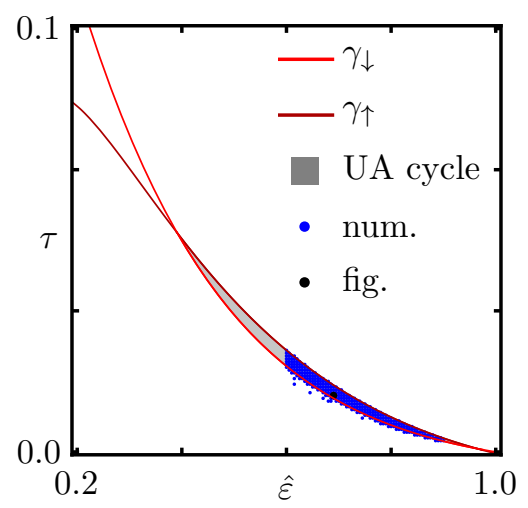

Figure 7.7. Robustness of the unstable attractor cycle and bifurcation to heteroclinic switching. Results are for the network used in fig. 7.1 and 7.2. Parameters in this network are the curvature $b$ of the rise function $U_{b}$ defining $H$, the total interaction strength $\hat{\varepsilon}$ and the interaction delay $\tau$. The area between the curves (gray) is the open set in $(\hat{\varepsilon}, \tau)$-parameter space $(b=4.2)$ in which a two cycle of unstable attractors exists and bifurcates to a heteroclinic cycle when the partial reset is increased from zero. The red lines show the boundaries determined by the conditions $\gamma_{\uparrow}: H_{2 \varepsilon, \tau}\left(\tau+1-H_{\varepsilon, \tau}^{3}(0)\right)>1$ and $\gamma_{\downarrow}: H_{2 \varepsilon, \tau} \circ H_{\varepsilon, \tau}(\tau)>1$ in eq. (7.2.13) which for this particular choice of $H$ define the region. For $\hat{\varepsilon} \in\{0.6,0.602, \ldots 1.0\}$ and $\tau \in\{0,0.001, \ldots 0.2\}$ we determined the existence of a two cycle of unstable attractors as in fig. 7.1b. Blue dots show parameters where the estimated basin size $\delta_{\max }>0$. They are in full agreement with our theoretical result. The black dot indicates the parameter values used for fig. 7.1-7.3. Note that there are blue dots outside the predicted region indicating that there are more two cycles of unstable attractors. Indeed, there are further transient maps between the spaces $\mathcal{P}_{i}$ that induce a switch from one attractor to the other which we did not include in our analysis. In the lower part there also is a region where dots occur irregularly. Here a third unstable attractor given by a periodic orbit of the map $Q_{i}^{b}$ exists that partially absorbs the transients, i.e. in this region the network of unstable attractors is larger than a two cycle.

Figure 7.7 shows that the conditions (7.2.13) define an open neighbourhood in the $(\hat{\varepsilon}, \tau)$-parameter space using a the rise function $U_{b}$ for fixed $b=4.2$. As the conditions depend smoothly on $b$ we also obtain an open set in the full parameter space $(b, \hat{\varepsilon}, \tau)$. Thus the occurrence of a two cycle of unstable attractors is a robust phenomenon in these types of models.

\subsubsection{Bifurcation To a Heteroclinic Two Cycle}

In this section we proof that the two cycle of unstable attractors becomes a heteroclinic two cycle if the partial reset is increased from zero. In particular we take $J_{\varepsilon}>0$, i.e. $c>0$ throughout this section.

We assume that the parameter $c$ is sufficiently small so that (7.2.12) holds, i.e.

$$
\left|J_{\varepsilon}(\phi)\right|<\eta \text { for all } \phi \geq 0, \varepsilon \geq 0
$$

for some $\eta>0$ which we specify during the following analysis.

Lemma 7.2.2 shows that the periodic orbits $\mathcal{O}_{i}$ still exists for $J_{\varepsilon}>0$ only the phase difference $\alpha$ changes continuously with $J_{\varepsilon}$ (cf. also fig. 7.5b). We further note that the domain boundaries of all return and transient maps derived in appendix C.2.1 - C.2.3 do not depend on $J_{\varepsilon}$. For example, the domain $\mathcal{D}\left(R_{i}^{\uparrow}\right)$ defined via (C.2.3) does not depend 
on $J_{\varepsilon}$. So the state space structure remains as illustrated in fig. 7.6. Moreover, as eq. (7.2.22) shows, the expansion property of $R_{i}^{\uparrow}$ (lemma 7.2.6) does not depend on $J_{\varepsilon}$ and hence the orbits remain unstable.

However, since $J_{\varepsilon}$ becomes invertible for $c>0$, according to (7.2.22) a phase difference $\left|\delta_{3}-\delta_{4}\right|$ shrinks under the return map $R_{1}^{\uparrow}$, but does not collapse to zero as for $c=0$; hence the images $\mathcal{C}_{i}^{\prime}=R_{i}^{\uparrow}\left(\mathcal{D}\left(R_{i}^{\uparrow}\right)\right)$ do not collapsed onto a line but stay three-dimensional. They consists of tubes around the original lines $\mathcal{C}_{1}$ with a square cross-section of side width

$$
w(c)=H_{\varepsilon, \tau} \circ J_{\varepsilon}^{(c)}(1)-H_{\varepsilon, \tau} \circ J_{\varepsilon}^{(c)}(0)
$$

shown in fig. (7.5)b.

Lemma 7.2.8. For sufficiently small $\eta>0$ in (7.2.23) the image $\mathcal{C}_{i}^{\prime}$ is mapped to a neighbourhood of $\mathcal{O}_{j}, i, j \in\{1,2\}$ and $i \neq j$.

Proof. As the domain boundaries do not change with $J_{\varepsilon}$, lemma 7.2 .7 shows that the image is almost contained in $\cup_{z \in\{a, b, c\}} \mathcal{D}\left(T_{i}^{z}\right) \cup \mathcal{D}\left(R_{i}^{\uparrow}\right)$ except near the boundaries of $T_{i}^{b}$ and $T_{i}^{c}$ that only meet in a line. We therefore include a fourth transient map $T_{i}^{d}$ (cf. (C.2.20)) with a domain (C.2.21) that fills this gap. The expansion property of $R_{i}^{\uparrow}$ ensures that all states are eventually mapped to the set $\mathcal{C}_{i}^{\prime} \backslash \mathcal{D}\left(R_{i}^{\uparrow}\right)$. As before, application of the maps $T_{i}^{z}$ to this set results in a set $\mathcal{A}_{i}^{\prime} \subset \mathcal{P}_{j}$ centered around the image $\mathcal{A}_{i}$ for $J_{\varepsilon}=0$ but now extending into all $\delta$-directions. Because of continuity of the maps this extension can be made arbitrarily small by a sufficiently small $\eta$. In particular, we can chose it such that that it is contained in the domains of the maps $R_{j}^{z}$ and $Q_{j}^{z}$ with $z \in\{\uparrow, a, b\}$. This can be verified using the explicit expression for the domains (C.2.3), (C.2.6) and (C.2.9). In lemma 7.2.7 we showed that states in $\mathcal{D}\left(Q_{i}^{z}\right)$ are mapped to the periodic orbit $o_{i}$ for $J_{\varepsilon}=0$. Now observe, that the maps $Q_{1}^{z}$ arise from the $R_{i}^{z}$ via the continuous limit $\delta_{2} \rightarrow 0$ (or $\delta_{4} \rightarrow 0$ ). Thus, via continuity of the maps we conclude that $\mathcal{A}_{i}^{\prime}$ is mapped to a neighbourhood of $o_{j}$ in $\mathcal{D}\left(R_{j}^{\uparrow}\right)$

We note that this explains the observed switching phenomena for nonzero partial resets as observed in fig. 7.2: States near $\mathcal{O}_{1}$ are mapped to a neighbourhood of $\mathcal{O}_{2}$ and then back near $\mathcal{O}_{1}$ and so forth. We remark further that states in $\mathcal{D}\left(Q_{i}^{\uparrow}\right) \subset \mathcal{S}_{i}$ still reach the point $o_{i}$ asymptotically. Thus the sets $\mathcal{D}\left(Q_{i}^{\uparrow}\right)$ are parts of a stable manifold of the orbits $\mathcal{O}_{i}$ in this hybrid dynamical system. Furthermore, the states in $\mathcal{D}\left(R_{1}^{\uparrow}\right)$ with $\delta_{3}=\delta_{4}$ together with their images are mapped to $\mathcal{D}\left(Q_{i}^{\uparrow}\right)$ and thus form a heteroclinic connection from $\mathcal{O}_{1}$ to $\mathcal{O}_{2}$. By a similar argument a further heteroclinic connection from $\mathcal{O}_{2}$ to $\mathcal{O}_{1}$ is obtained. We thus conclude:

Corollary 7.2.9. For $J_{\varepsilon}>0$ sufficiently small the periodic orbits $\mathcal{O}_{1}$ to $\mathcal{O}_{2}$ are saddle states on a heteroclinic cycle.

Together with the result in 7.2.5 this shows that in the network $\mathcal{N}^{4}$ a two cycle of unstable attractors for $J_{\varepsilon}=0$ continuously bifurcates to a heteroclinic two-cycle for $J_{\varepsilon}>0$ and thus proves theorem 7.2.1.

Corollary 7.2.9 only depends on the smallness assumption on $J_{\varepsilon}$ but not on its precise form and not on $H$ and the other parameter $\varepsilon$ and $\tau$ as long as the conditions (7.2.13) 
are satisfied. Figure 7.7 then shows that there is an open set of parameters in which the bifurcation occurs robustly.

The underlying mechanism relies on the interplay of the local instability (lemma 7.2.6) and the parameter $c$ dependent contraction of state space volume induced by the reset as visible in eq. (7.2.24), implying the same transition in larger systems as observed in fig. 7.4 as well as in larger networks of unstable attractors. Recall from chapter 3 that in these networks there is a link between two attractors $\mathcal{O}_{i} \rightarrow \mathcal{O}_{j}$ if every neighborhood of $\mathcal{O}_{i}$ contains a positive basin volume of $\mathcal{O}_{j}$. Based on our analytical result for the transition in two cycles of unstable attractors and numerical observations (fig. 7.4), we conjecture that in larger networks of unstable attractors each link is replaced by a heteroclinic connection when lifting the non-local invertibility. 


\section{Chapter 8}

\section{Summary, Discussion and Outlook}

In summary, we proposed a model of pulse-coupled threshold units with partial reset an intrinsic response property of the local units - and studied its impact on the collective network dynamics.

For instantaneous pulse-coupling this partial reset acts as a desynchronization mechanism in the collective network dynamics. It causes an extensive sequence of desynchronizing bifurcations of cluster states in networks of pulse-coupled oscillators with convex rise function. This sequential desynchronization transition is robust against structural perturbations in the coupling strength and variations of the local subthreshold dynamics. We found similar transitions in biologically more realistic neuron models and briefly discussed relations to partial reset mechanisms.

For delayed pulse interaction we proved analytically that the partial reset induces a robust transition from networks of unstable attractors to heteroclinic switching. This constitutes a new type of global bifurcation in dynamical systems and establishes the first known bifurcation of unstable attractors.

Previous studies have not particularly focused on the collective implications of partial or graded resets. In network models with pulses that are extended in time typically a full conservation of the input is considered [378, 389, 154]. Models with instantaneous responses to inputs consider fully dissipative reset $(R(\zeta) \equiv 0$ in our model) $[269,124,33$, 333, 370, 369], fully conservative reset $(R(\zeta)=\zeta)[37,39]$ as well as both extremes [174] without discussing particular consequences of the reset mechanism. Here we closed this gap and showed that in fact the reset mechanism influences the synchronization processes.

Partial reset in pulse-coupled oscillators keeps the collective network dynamics analytically tractable and at the same time describes additional, physically or biologically relevant dynamical features of local units. In neurons, for instance, synaptic inputs are collected in the dendrite and then transmitted to the cell body (soma). At the soma the integration of the membrane potential takes place and spikes are generated. Remaining input charges on the dendrite not used to trigger a spike at the soma may therefore contribute to the potential after somatic reset [83, 321, 38].

Such features are effectively modeled by the simple partial reset introduced here. In particular, spike time response curves (that may be obtained for any tonically firing neuron $[295,314,116])$ encode the shortening of the inter-spike intervals (ISI) following an excitatory input at different phases of the neural oscillation. An excitatory stimulus that causes the neuron to spike will maximally shorten the ISI in which the stimulus is applied. Additionally, the second ISI that follows is typically affected as well, e.g. due to compartmental effects. Exactly this shortening of the second ISI is characterized by 
appropriately choosing a partial reset function in our simplified system (cf. fig. 5.4).

The desynchronization due to the partial reset, i.e. due to local processing of suprathreshold input, differs strongly from that induced by previously known mechanisms based on, e.g. heterogeneity, noise, or delayed feedback [389, 388, 251, 214, 301, 71]. Possibly, this desynchronization mechanism may also be helpful in modified form to prevent synchronization in neural activity such as in Parkinson tremor or in epileptic seizures $[362,160]$.

We considered a partial reset mechanism for supra-threshold inputs due to excitatory couplings. For inhibitory couplings one can define a lower threshold [70] below which inhibitory inputs becomes less effective, i.e. a partial inhibition. In models of neurons, for instance, this could characterize shunting inhibition [9]. If two units simultaneously receive inhibitory inputs below a lower threshold, a zero partial inhibition, i.e. setting the state of the units to a fixed lower value, is strongly synchronizing in analogy to a full reset after supra-threshold excitation. Our findings suggest that similar to a partial reset a less synchronizing non-zero partial inhibition may also have a strong influence on the collective network dynamics. Our partial reset model might also find applications in studying network dynamics of neurons with post-inhibitory rebound [317]. These neurons get more excitable when hyperpolarized by inhibitory inputs, e.g. due to the opening of slowly inactivating calcium channels. After the release from sufficient strong inhibition the neurons generate a spike and thereafter may still exhibit stronger excitability. In our simple model this enhanced excitability then could be modeled using a partial reset mechanism for inhibition.

In biologically more detailed neuronal network models both excitatory and inhibitory couplings as well as complex network topologies play important roles in generating irregular [390] and synchronized spiking dynamics [4]. It would therefore be an interesting task to study the impact of partial resets in such networks.

To deal with dynamical effects due to the partial reset in networks with delayed pulsecoupling, we developed a novel non-local nonlinear method of event-sequence based domain analysis. We used this method to give a full analytic proof for the existence of a novel bifurcation from unstable attractor networks to hetero-clinic switching in a small network. For local non-invertible dynamics, i.e. zero partial reset, we first showed the robust existence of the intriguing phenomenon of two unstable attractors that are fully enclosed by each other's basin volume. Continuously removing the local non-invertibility by increasing the partial reset strength induced the bifurcation to a heteroclinic cycle. We numerically showed that larger networks of unstable attractors equally show this transition to more complex heteroclinic structures.

Our results show that this bifurcation occurs upon continuously removing the noninvertibility of the system, whereas both the non-invertible $(c=0)$ and the locally invertible $(c>0)$ system exhibit equally discontinuous interactions. This explicitly demonstrates that the local non-invertibility and not the discontinuity is responsible for the creation of unstable attractors in pulse coupled oscillator networks. For $(c<0)$ we showed a similar transition to heteroclinic switching where the ordering of the oscillators is not preserved, (cf. also [203]). Thus the unstable attractor network dynamics arise at the singular bifurcation value $c=0$ and in total there is a bifurcation from nonorder-preserving to order preserving heteroclinic switching dynamics through a network-of unstable attractors.

The continuity of the bifurcation has theoretical and practical consequences: For instance, one may investigate features of a system exhibiting heteroclinic switching $[67,303$, 
(a)

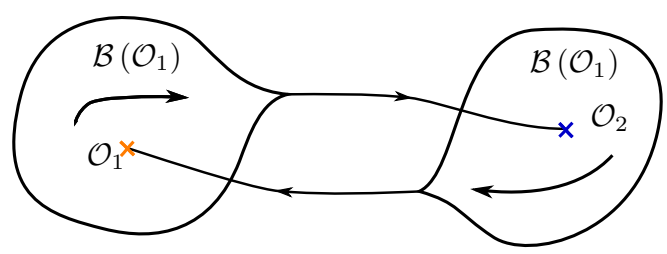

(b)

(i)

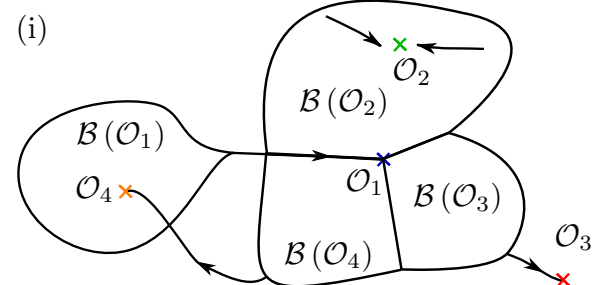

(ii)

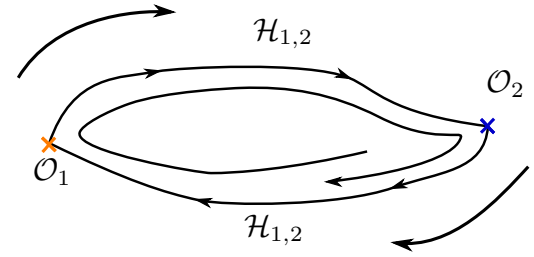

(ii)

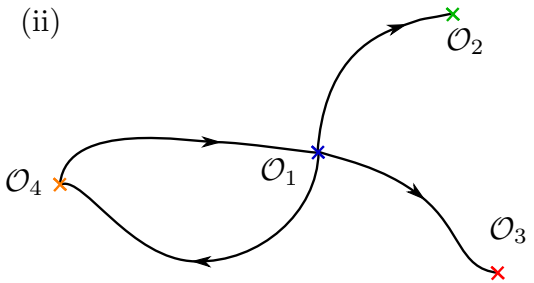

(c)

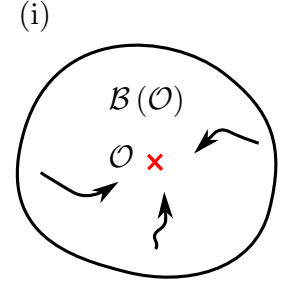

(ii)

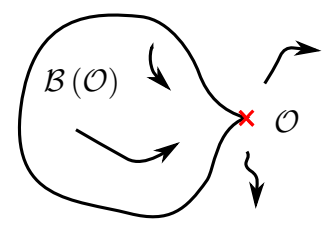

(iii)

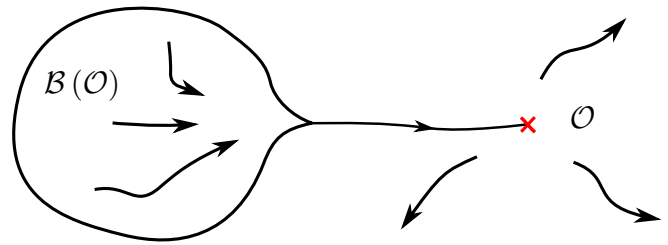

Figure 8.1. Bifurcations from and to unstable attractors (UA). Sketches of bifurcations in state spaces. Attractors are marked as $\mathcal{O}_{i}$, basins of attraction as $\mathcal{B}\left(\mathcal{O}_{i}\right)$. (a) bifurcation from a two cycle of UAs (i) to a heteroclinic two cycle (ii) as observed and proved analytically in sec. 7.2. (b) bifurcation of an attractor network involving stable as well as unstable attractors (i) to a heteroclinic network (ii). (c) creation of an unstable attractor (iii) from a stable attractor (i) .

$258,156,222,15]$ by studying its limiting counterpart with unstable attractors. Furthermore, this may help designing systems with specific heteroclinic structure, for instance in artificial neural networks, and guide our understanding of time series of switching phenomena in nature, cf. [17]. The associated limiting systems with unstable attractors may not only be analytically accessible, also numerical simulations can be performed in a more controlled way because typical problems with simulations of heteroclinic switching, e.g. exponentially increasing switching times and exponentially decreasing distances to saddles, do not occur if the heteroclinic switching is replaced by networks of unstable attractors.

In continuous time dynamical systems heteroclinic cycles exist robustly in systems with symmetry [146, 225]. We here observed an analog phenomenon in a hybrid dynamical system. The transition from unstable attractor networks to heteroclinic cycles robustly exists in systems with lower symmetry but we did not observe the occurrence of unstable attractor networks and heteroclinic switching dynamics in systems with inhomogeneous network structure, i.e. where all permutation symmetries of the oscillators are broken. However, small symmetry breaking perturbations to the network structure induce transitions from unstable attractor networks (or heteroclinic switching dynamics for no zero partial reset) to periodic dynamics. This is in analogy to homoclinic and heteroclinic bifurcations in continuous time dynamical systems where for example periodic orbits are generated from homoclinic orbits (cf. e.g. sec. 3.2). An interesting application of our analysis therefore would be to investigate how such bifurcations are organized and what kind of periodic or even more complex dynamics, such as chaos, can be generated from an unstable attractor network. 
(a)

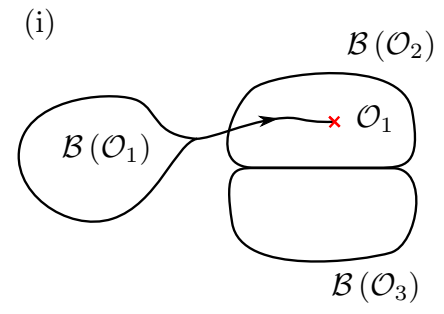

(b)

(i) (ii)

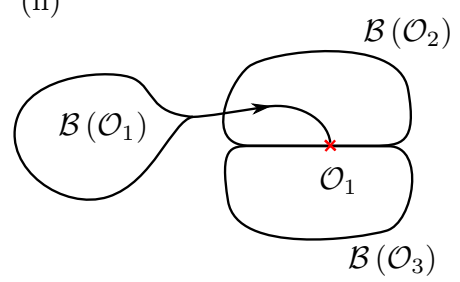

(ii)

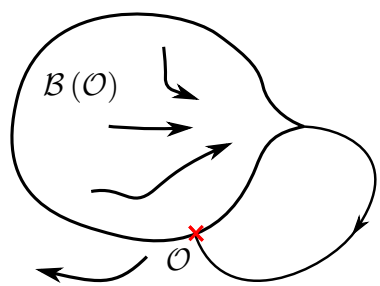

(iii)

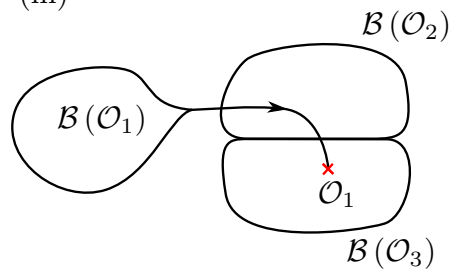

(iii)
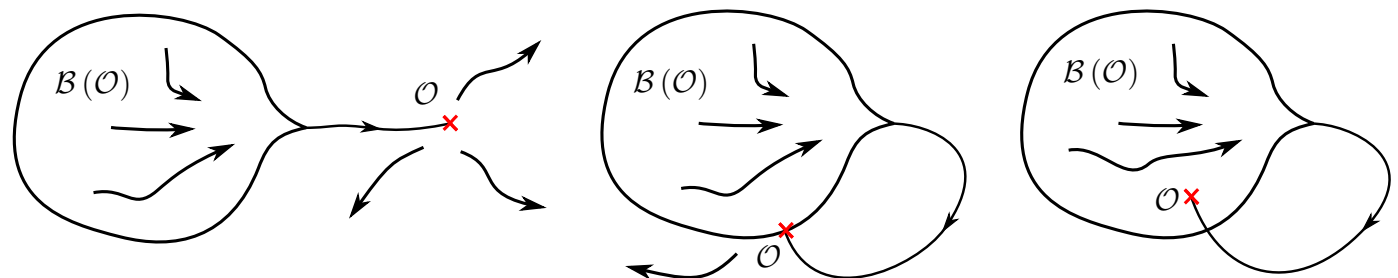

Figure 8.2. Basin bifurcations of unstable attractors. Sketches of bifurcations in state spaces. Attractors are marked as $\mathcal{O}_{i}$, basins of attraction as $\mathcal{B}\left(\mathcal{O}_{i}\right)$. (a) basin bifurcation of an unstable attractor $\mathcal{O}_{i}$ changing its location from basin $\mathcal{B}\left(\mathcal{O}_{2}\right)$ (i) to $\mathcal{B}\left(\mathcal{O}_{3}\right)$ (ii). (b) bifurcation of an unstable attractor $\mathcal{O}_{i}$ moving into its own basin of attraction.

For the mathematical investigation of the bifurcation from unstable attractor to heteroclinic networks we developed an event-sequence based domain analysis that provides a tool for the study of the dynamics and state space structure of pulse-coupled systems. The analysis is non-linear and non-local. Despite the high technicality, it's algorithmic nature makes it optimally suitable for computer based analysis and proofs using symbolic computation software. We used this tool for a global bifurcation analysis, but it finds more applications in the study of pulse-coupled systems, for example in estimating the domain size of predefined event sequences and to predict their probability of occurrence and their stability against noise. A potentially interesting application could be to better understand the flux-tube state space structure found in networks of pulse-coupled integrate-and-fire neurons [271]: Here two states initially separated by a small distance converge onto the same trajectory, i.e. the dynamics are stable with respect to small perturbations. However, if larger perturbations are introduced an exponential separation is observed reflecting chaotic aspects of the dynamics. An detailed analysis of the state space structure using event-sequence based domain analysis for several trajectories might help to unravel the underlying mechanisms.

We here, for the first time, fully analyzed a bifurcation involving unstable attractors in a hybrid system. This opens a new field of bifurcation theory for unstable attractors. In fig. 8.1 we have sketched some other possible bifurcations for the creation and destruction of unstable attractors. The bifurcation from unstable attractor networks to heteroclinic structures as analyzed in this chapter is depicted in (a) and may also occur in larger and more general attractor networks involving both stable and unstable attractors (b). In (c) the creation of an unstable attractor form a stable attractor is shown. A different possible class of bifurcations involves the change of the unstable attractor network instead of the unstable attractor itself. Two examples are shown in fig. 8.2. Here an unstable attractor changes its location from one basin of attraction to another. This interesting field offers many promising opportunities for future research, not only mathematically interesting but also with applications to physical systems. 


\section{Part IV}

\section{Leak-Induced Dynamic Excitability Transitions in Neurons}




\section{Chapter 9}

\section{Introduction}

Neuronal excitability characterizes the dynamics of a neuron to generate an action potential. It is one of the core ingredients of neuronal dynamics and strongly influences the collective network dynamics $[157,100,177]$ as well as coding and information storage properties [239, 318, 166, 329]. A first classification of neuronal excitability of periodically spiking neurons $[64,245]$ into two classes goes back to Hodgkin [170] and is based on earlier work on cancer pagurus axons [14] and nerves of frogs and squids [41]:

- Type I neurons show arbitrarily low periodic firing frequencies and thus can maintain spiking with arbitrarily large inter spike intervals. There is a smooth dependence of the firing frequency on the input current over a broad range of frequencies.

- Type II neurons start firing with a frequency clearly distinct from zero and cannot maintain periodic firing below a certain frequency. The dependence of the firing frequency on the input current is weak.

Whereas type I dynamics show an all or none stereotyped spike and are associated with integration properties of the inputs, type II excitability is associated with graded spike amplitudes and resonance $[316,183]$. Type I neurons tend to desynchronize, while type II neuron often undergo synchronization [316, 189]. Thus, given the strong interrelations between synchrony and neuronal processing [140, 398, 113, 141, 341, 340, 342] it is important to identifying factors and control mechanisms that influence the neuronal excitability type.

Several factors that determine neuronal excitability have been identified [316, 305]. They include ion channel properties, such as the maximal conductances, the activation curves and reversal potentials $[245,316,167]$ as well as the mixture and density of ion channels across the membrane $[63,68,188]$. Experimentally it has been shown that the neuronal excitability type of neurons can also be influenced by neuro modulators [352], the intrinsic states of the neuron such as up and down states [165] and in vivo vs. in vitro conditions [307]. However, despite the numerous numerical and experimental studies that relate neuronal morphology to firing patterns and response properties of neurons $[250,143,387,25,130]$, the impact of the dendritic structure on the intrinsic neuronal excitability type has not been subject to a systematic investigation so far. In chapter 11 we study this question and find that the morphological structure and the density of active ion channels on the dendritic tree have a crucial impact on the neuronal excitability type. For passive dendrites with arbitrary morphology we show that linear extended shapes tend to type I excitability, while stellar shapes show resonant type II properties. Our analysis 
identifies a passive effective leak conductance imposed onto the soma as the underlying parameter that induces the excitability switch.

An excitable system in dynamical systems terminology is characterized by a stable fixed point close to a large amplitude trajectory (pseudo-orbit) or an stable limit cycle, indicating that the system is close to a bifurcation. Indeed, the neuronal excitability type can been linked to the underlying bifurcation that generates a stable limit cycle $[100,335,189]$. In particular, in two dimensional state space the co-dimension-1 saddle node on invariant cycle and homoclinic bifurcations will show type I excitability as close to the bifurcation the dynamics on the limit cycle gets arbitrarily slow in the region where the fixed points are generated (cf. also sec. 3.3.1.1 and 3.3.1.3). For type II excitability the underlying co-dimension-1 bifurcations are the double limit cycle and Hopf bifurcations both having oscillatory nature (cf. also sec. 3.3.1.2 and 3.3.1.4).

Some of these bifurcations imply the coexistence of a stable fixed point together with a stable limit cycle. This implies that the onset of spiking due to destabilization of the fixed point followed by a jump onto the stable limit cycle may start with a different frequency than the offset of spiking due to the destabilization of the limit cycle. This hysteresis effect is accounted for by a more detailed classifications scheme discriminating the neuronal excitability types for on- and offset of spiking [189, 137].

Bifurcation diagrams in different parameters have been studied for several neuron models, including the Hodgkin-Huxley [147], the Morris-Lecar [316, 100, 117, 379], the ConnorStevens [100], the Rose-Hindmarsh [353], the Traub-Miles [375], the Wilson-Cowan [177] and the Fitzhugh-Nagumo [109] neuron models. Also different neuronal excitability types in the same model for different sets of parameter have been identified (e.g. [100, 117]). However, the leak conductance itself, which we identify as an effective parameter that controls a switch in neuronal excitability in morphological extended neurons, has not been subject to a systematic bifurcation analysis before. In particular, open questions are in which neurons leak-induced switches between the neuronal excitability types are possible, how these transitions are organized and what kind of intermediate dynamics arise. Further, given that synaptic shunting inhibition imposes an effective leak conductance onto the neuron, one can further ask, how this may be utilized to dynamically control neuronal excitability and ultimately the collective neuronal network dynamics.

In this part of the thesis we theoretically and experimentally study these questions. In chapter 10 we perform a systematic numerical and analytical bifurcation analysis and find in a large number of neurons, ranging from fast spiking inter neurons to pyramidal cells $[273,319,320,168,400,99,61,109,315,103,406]$, that the strength of the leak conductance controls a switch in neuronal excitability from type I to type II. We show that this switch is via a threefold transition and always accompanied by a region of a bistable coexistence of resting and periodic firing and a further transition from integration to resonance at peri-threshold membrane potentials. We show that this structure can be explained by a degenerate Bogdanov-Takens-cusp (dBT) bifurcation [87] of codimension three acting as an organizing center. Using a combination of normal form theory and center manifold reduction, we then analytically prove that all conductance based neuron models with type I neuronal excitability have a dBT point and thus are capable of switching their neuronal excitability by an increase in leak conductance. On the contrary, we show that by a decrease in leak conductance type II neurons cannot be switch to type I neurons in general. For a class of neuron models we derive an equivalence to a Liénard equation and use it to give an interpretation of the neuronal excitability switch in terms of non-linear acceleration and dampening forces acting on a moving massive particle. 
In chapter 11 we apply our results to spatially extended neuron models. Taking model neurons with the same surface area, total dendritic branch length, and underlying biophysics, we vary the pattern of dendritic branching and change the number of primary dendrites that attach directly to the soma. We find that a change from a long, linearly extended configuration of a single primary dendrite (akin to the apical shaft in layer 5 pyramidal cells in neocortex) to starlike shapes with a profusion of proximal, primary dendrites (akin to stellate cells) induces a transition from type I to type II behavior and explain this by a change of the effective leak imposed onto the soma. By analyzing data from experiments in which the dendritic tree can be pinched temporarily [25] we also find a neuronal excitability transition fully consistent with our theoretical findings .

In chapter 12 we use the dynamic patch clamp technique $[281,287]$ to control the leak conductance artificially in real neurons and experimentally confirm the main predictions of our general theory. We find leak-induced neuronal excitability switches, a region of bistability and a transition at peri-threshold resonance. Application of an inhibitory synaptic transmitter likewise induces this transition. We therefore conclude that in principle neuronal excitability can be controlled dynamically via shunting inhibition.

In chapter 13 we study several consequences of dynamic leak-induced neuronal excitability switches for the control of collective network dynamics. As the neuronal excitability is connected to synchronization properties of weakly coupled neurons $[316,100$, $184,177]$ we conclude that regulating neuronal excitability via changes in leak conductance provides an effective mechanism to dynamically control the collective synchronization properties of neurons. We demonstrate this in a small circuit. In larger networks consisting of neurons with different neuronal excitability types we show that the subgroup of type II neurons synchronizes while the type I neurons remain asynchronous. We conclude, that dynamic switching of neuronal excitability may thus serve as a mechanism for dynamic grouping of neurons with a wide range of applications in neuronal coding $[162,398,138,111]$. We further show that in the region of bistability complex dynamics emerge for stronger couplings in homogeneous networks: For smaller leak conductances, stronger pulses arising via synchronization induce silencing of neurons, while for larger leak values the desynchronization causes irregular dynamics due to switching between resting and spiking of the individual neurons.

We finally summarize and discuss our results in chapter 14 . 


\section{Chapter 10}

\section{Leak-Induced Neuronal Excitability Transition in Model Neurons}

In this chapter we show that the leak conductance controls a switch in neuronal excitability in a large number of neuron models $[273,319,320,168,400,99,61,109,315,103,406]$. We start by motivating the choice of a leak conductance as a bifurcation parameter and then systematically study the bifurcations underlying leak-induced neuronal excitability transitions using numerical continuation in section 10.2, as well as analytical multiple bifurcation and normal form theory in section 10.3 .

\subsection{Leak Conductance as a Bifurcation Parameter in Conductance-Based Neuron Models}

type we consider general conductance based neuron models as introduced in sections 2.2 and 2.4 of the functional form

$$
\begin{aligned}
c_{\mathrm{m}} \frac{d}{d t} v & =i_{\mathrm{e}}+g_{\mathrm{L}}\left(v_{\mathrm{L}}-v\right)+i_{\mathrm{a}}(v, a) \\
\frac{d}{d t} a_{j} & =\frac{1}{\tau_{j}(v)}\left(a_{\infty, j}(v)-a_{j}\right)
\end{aligned}
$$

where $v$ is the membrane potential, $c_{\mathrm{m}}$ the capacity, $i_{\mathrm{e}}$ the external input current, $g_{\mathrm{L}}$ and $v_{\mathrm{L}}$ the conductance and reversal potential of the leak current. The active ion currents are given by

$$
i_{\mathrm{a}}(v, a)=\sum_{k} g_{k} a_{i_{k, 1}}^{l_{k, 1}} \ldots a_{i_{k, p_{k}}}^{l_{k, p_{k}}} a_{\infty, j_{k, 1}}^{m_{k, 1}} \ldots a_{\infty, j_{k, q_{k}}}^{m_{k, q_{k}}}\left(v_{k}-v\right)
$$

and depend on the maximal conductances $g_{\mathrm{k}}$ and reversal potentials $v_{j}$ as well as the activation variables $a=\left(a_{2}, a_{2}, \ldots, a_{N}\right)^{T}$ with steady state activations $a_{\infty, j}$ and time constants $\tau_{j}$, Note that this formula is a generalization of (2.3.1) that also covers neuron models for which certain fast activation variables have been replaced by their steady state value $a_{\infty, j}$ that may depend on $v$.

To study the bifurcations involved in the onset of spiking a canonical choice for a single bifurcation parameter is the input current $i_{\mathrm{e}}$ : Due to an increase of his parameter, a stable resting state of the neuron typically becomes unstable and the neurons starts to generate spikes. To study the impact on the leak current onto neuronal excitability we 
further concentrate with out loss of generality on the leak conductance $g_{\mathrm{L}}$ as a change in the leak reversal potential $v_{\mathrm{L}}$ may be absorbed into a change in the input current $i_{\mathrm{e}}$. Our study is motivated by the following observations and facts:

1. Despite the large number of theoretical and numerical bifurcation studies on neuronal models in the literature, the leak current, and in particular the leak conductance $g_{\mathrm{L}}$ has not been subject to a bifurcation analysis before. A reason for this might be, that in the construction of neuronal models the leak current is the last ingredient introduced to fit e.g. the resting potential and has therefore been payed less attention.

2. As mentioned in the introduction and as we will show in chapter 11, the leak conductance can be systematically related to morphological properties in compartmental neuronal models.

3. Akin to the external input current, $g_{\mathrm{L}}$ is a universal parameter present in almost all neuron models and therefore a comparison between bifurcation diagrams of different neuron models is possible.

4. The leak equally well describes the conductance of a shunting inhibitory synapse with a time scale $\tau_{\text {syn }}$ much slower than the time scale $\tau_{\text {ap }}$ of action potential generation. More generally, a situation in which both slow (compared to $\tau_{\text {ap }}$ ) synaptic excitatory and inhibitory conductance's are present can be mapped one-to-one to effective parameters $i_{\mathrm{e}}^{\prime}$ and $g_{\mathrm{L}}^{\prime}$ via the relation

$$
i_{\mathrm{e}}+g_{\mathrm{L}}\left(v_{\mathrm{L}}-v\right)=i_{\mathrm{e}}^{\prime}+g_{\mathrm{L}}^{\prime}\left(v_{\mathrm{L}}^{\prime}-v\right)+g_{\mathrm{ex}}\left(v_{\mathrm{ex}}-v\right)+g_{\mathrm{in}}\left(v_{\mathrm{ex}}-v\right)
$$

which gives

$$
\begin{aligned}
i_{\mathrm{e}} & =i_{\mathrm{e}}^{\prime}+g_{\mathrm{L}}^{\prime} v_{\mathrm{L}}^{\prime}+g_{\mathrm{ex}} v_{\mathrm{ex}}+g_{\mathrm{in}} v_{\mathrm{in}}-g_{\mathrm{L}} v_{\mathrm{L}} \\
g_{\mathrm{L}} & =g_{\mathrm{L}}^{\prime}+g_{\mathrm{ex}}+g_{\mathrm{in}} .
\end{aligned}
$$

5. The parameter $g_{\mathrm{L}}$ appears linearly in the equations of motion, which facilitates an analytical treatment (see sec. 10.3.2).

In the next section we give a detailed numerical study of the bifurcations encountered by varying the leak conductance $g_{\mathrm{L}}$. We first consider bifurcation diagrams in the single parameter $i_{\mathrm{e}}$ for different values of the leak and then consider the two parameter $\left(i_{\mathrm{e}}, g_{\mathrm{L}}\right)$ as well as three parameter diagrams $\left(i_{\mathrm{e}}, g_{\mathrm{L}}, c_{\mathrm{m}}\right)$ including the capacity $c_{\mathrm{m}}$.

Similarly to the leak conductance, the capacity is also present in all conductance-based neuron models. In writing the general membrane evolution equation as

$$
\begin{aligned}
\frac{d}{d t} v & =\frac{1}{c_{\mathrm{m}}}\left(i_{\mathrm{e}}+g_{\mathrm{L}}\left(v_{\mathrm{L}}-v\right)+i_{\text {ion }}(v, a)\right) \\
& =\tilde{i}_{\mathrm{e}}+\tilde{g}_{\mathrm{L}}\left(v_{\mathrm{L}}-v\right)+\alpha i_{\text {ion }}(v, a)
\end{aligned}
$$

one observes that the inverse capacity $\alpha=\frac{1}{c_{\mathrm{m}}}$ controls the overall strength of the active ion currents with respect to the relative leak $\tilde{g}_{\mathrm{L}}=\frac{g_{\mathrm{L}}}{c_{\mathrm{m}}}$ and external current $\tilde{i}_{\mathrm{e}}=\frac{i_{\mathrm{e}}}{c_{\mathrm{m}}}$. 
(a)

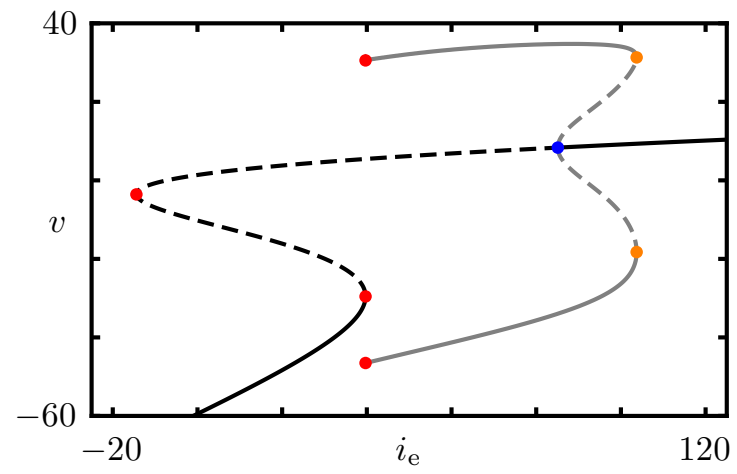

(b)

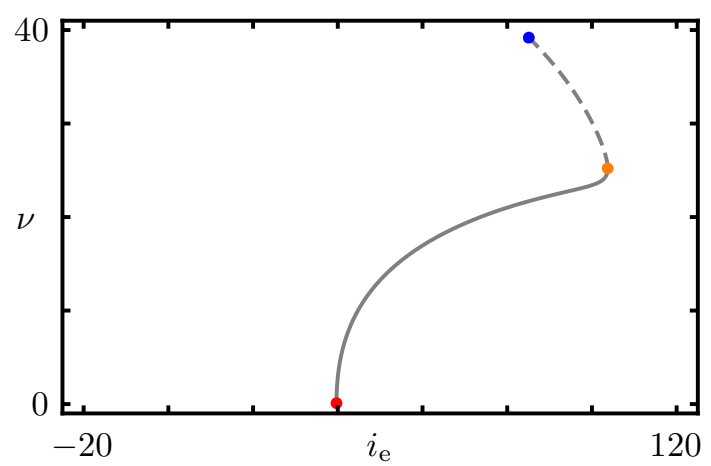

Figure 10.1. Saddle node on limit cycle bifurcation in the Morris-Lecar neuron 2.4.4 determines spike onset for small leak conductances $\left(g_{\mathrm{L}}=2\right.$, cf. also fig. 3.2 for the corresponding phase portraits). (a) $i_{\mathrm{e}}$-bifurcation diagram with membrane potential values $v$ of the fixed points (stable: - - unstable: -----) and maximal and minimal amplitudes of periodic orbits (stable: - , unstable: -----). There are two saddle node bifurcations $(\bullet)$ of the fixed points, the second one at $i_{\mathrm{e}}^{*} \approx 39.69$ being on an invariant cycle giving rise to a stable periodic orbit. A second unstable limit cycle is created in a Hopf bifurcation $(\bullet)$ and coalesces with the stable orbit in a double cycle bifurcation $(\bullet)$. (b) frequency of the periodic orbits as a function of the external input current $i_{\mathrm{e}}$ which starts to rise continuously form zero at $i_{\mathrm{e}}^{*}$ indicating type I neuronal excitability.

\subsection{Leak-Induced Neuronal Excitability Transitions - Bifurcation Diagrams}

In this section we study how the leak conductance affects the neuronal excitability type by determining the bifurcations to period spiking using the semi-analytical and numerical methods as described in section 3.3.4.

All bifurcations encountered here are introduced in detail in section 3.2. Their abbreviations together with the color code used in all the bifurcation diagrams are listed in tab. 3.1.

\subsubsection{Leak-Induced Neuronal Excitability Transition in the Morris- Lecar Model Neuron}

In this section we study how neuronal excitability is influenced by the leak conductance in the Morris-Lecar (ML) model neuron [273] described in sec. 2.4.2. We start with $i_{\mathrm{e}^{-}}$ bifurcation diagrams for different but fixed values of the leak conductance $g_{\mathrm{L}}$ and then summarize the results in a $\left(i_{\mathrm{e}}, g_{\mathrm{L}}\right)$-bifurcation diagram.

\subsubsection{One Parameter Bifurcation Diagrams}

The ML model has the advantage of being two dimensional which facilitates the visualization of the phase portraits. For each bifurcation diagram in this section the phase-portraits are shown in sec. 3.2 using exactly the same parameter values.

Figure 10.1a shows the bifurcation diagram with corresponding phase plane portraits in fig. 3.2 for the original parameter values of the ML model with a small leak conductance 
(a)

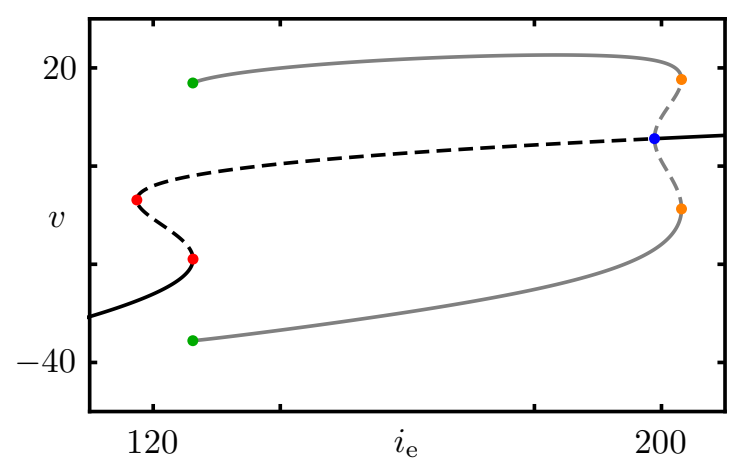

(c)

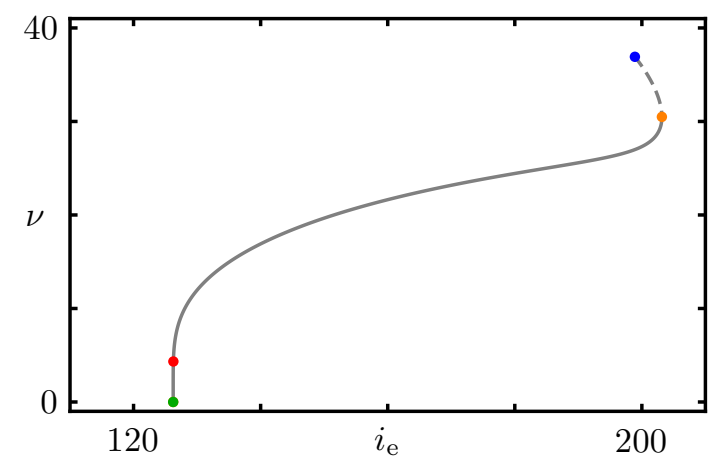

(b)

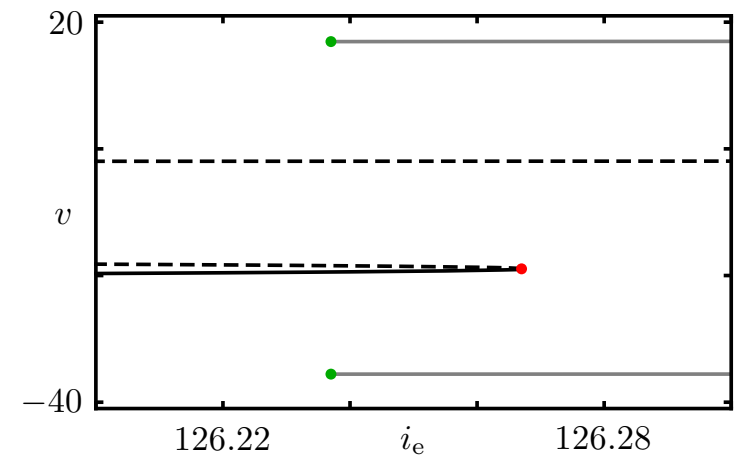

(d)

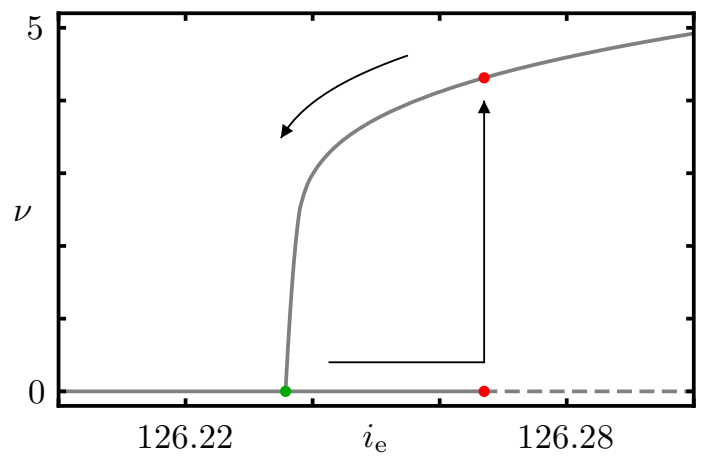

Figure 10.2. Homoclinic bifurcation in the Morri-Lecar model 2.4.4 determines spike onset for small to intermediate values of the leak $\left(g_{\mathrm{L}}=4.4\right)$. (a) $i_{\mathrm{e}}$-bifurcation diagram (syntax as in fig. 10.1) with corresponding phase portraits shown in fig. 3.3. The limit cycle is created at $i_{\mathrm{e}}^{*} \approx 126.267$ via a homoclinic bifurcation $(\bullet)$. (b) zoom of (a) close to $i_{\mathrm{e}}^{*}$. The homoclinic bifurcation happens before the saddle node bifurcation of the fixed point $(\bullet)$ implying a small interval in which a stable limit cycle and a stable fixed point coexist. (c,d) frequency of the periodic spiking as a function of the external input current $i_{\mathrm{e}}$ starts to rise continuously form zero at $i_{\mathrm{e}}^{*}$ indicating type I neuronal excitability. Due to the bistability there is a hysteresis effect: Increasing the input current from below the system remains on the fixed point and only when the $\mathrm{SN}(\bullet)$ is passed the system starts spiking with a non zero frequency. Decreasing the input current from above the system remains on the periodic orbit with arbitrarily small frequencies.

$\left(g_{\mathrm{L}}=2\right)^{1}$. The onset of periodic spiking is via a saddle node on limit cycle bifurcation (SNIC), in accordance with previous results [316, 100, 117, 379]. As derived in [100, 335], the neuron's spike frequency $\nu$ starts continuously form zero with $\nu\left(i_{\mathrm{e}}\right) \propto \sqrt{i_{\mathrm{e}}}$ for $i_{\mathrm{e}} \geq i_{\mathrm{e}}^{*}$ (cf. fig. 10.1b). In total this shows that the ML neuron has type I neuronal excitability for small values of the leak.

Increasing the leak to $g_{\mathrm{L}}=4.4$ the bifurcation scenario changes and onset of periodic spiking is via a big homoclinic bifurcation as shown in fig. 10.2 and 3.3. In this regime the creation of the limit cycle no longer coincides with the saddle node (SN) bifurcation and there is a small region of bistability. This further implies that there is a hysteresis effect: Increasing the input current from below the system remains on the stable fixed

\footnotetext{
${ }^{1}$ Here and in the following we will not indicate the physical units for the parameters $g_{\mathrm{L}}, i_{\mathrm{e}}$ and $c_{\mathrm{m}}$ as depending on the interpretation of the model (10.1.1) these can be both specific parameters normalized to a certain membrane surface area or the total conductance, input current and capacity for a point neuron model (cf. also sec. 2.2).
} 
(a)

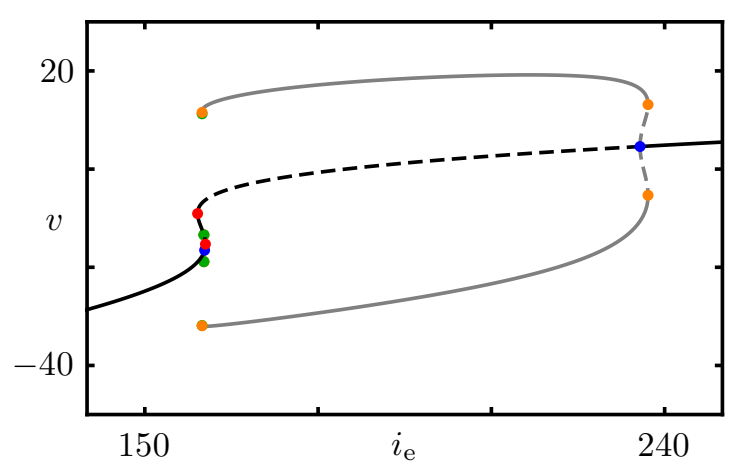

(c)

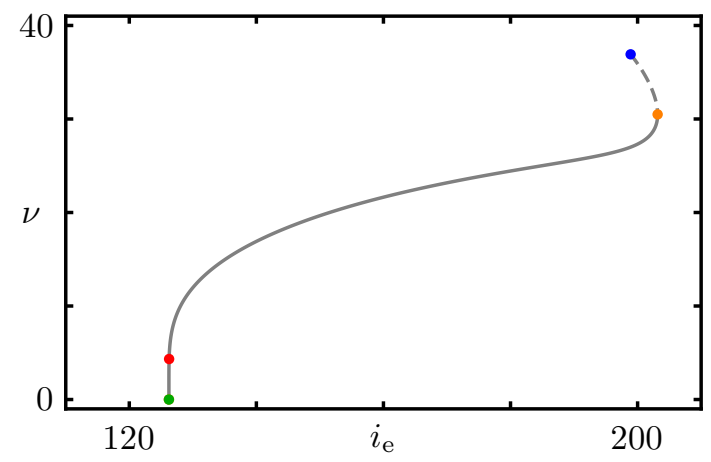

(b)

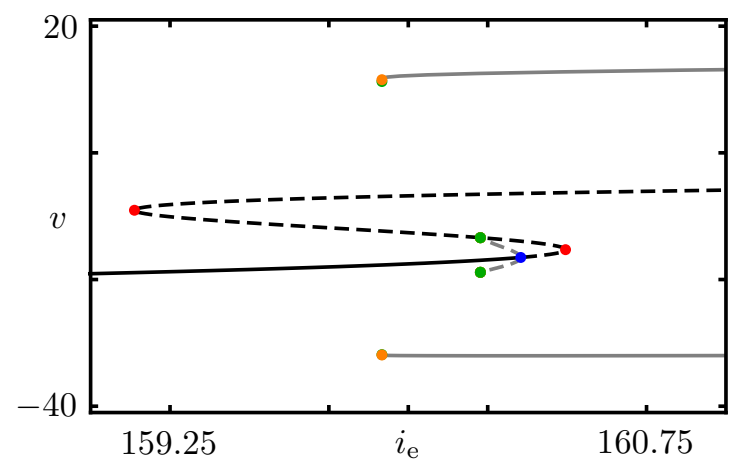

(d)

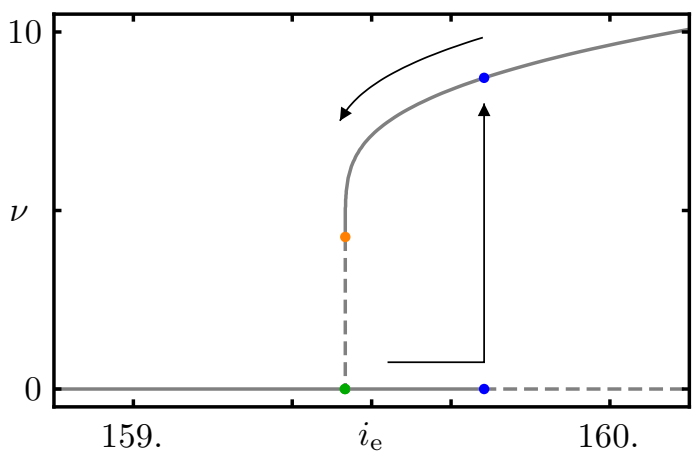

Figure 10.3. Saddle-node and small homoclinic bifurcation determines spike onset and subthreshold resonance in the Morri-Lecar model 2.4.4 for intermediate leak conductances $\left(g_{\mathrm{L}}=5.2\right)$. $(\mathbf{a}, \mathbf{b}) i_{\mathrm{e}}$-bifurcation diagram (syntax as in fig. 10.1). The stable and an unstable limit cycle is created at $i_{\mathrm{e}}^{*} \approx 160.35$ via a double limit cycle bifurcation $(\mathrm{DC})(\bullet)$. The unstable limit cycle vanishes via a big homoclinic bifurcation. In addition there is a small homoclinic bifurcation in which a small unstable limit cycle is created that shrinks towards the fixed point and destabilizes it via a Hopf bifurcation $(\bullet$ ) indicating a non-zero sub-threshold resonance frequency (cf. also fig. 3.4 for the phase portraits). (c,d) frequency of the periodic orbit as a function of $i_{\mathrm{e}}$. Increasing the external input form below the fixed point gets destabilized via the Hopf bifurcation $(\bullet)$ and jumps onto the stable periodic orbit with a non-zero frequency while due a decrease from above the frequency jumps discontinuously to zero at the DC bifurcation $(\bullet)$. Both jumps indicate type II behavior.

point and only jumps onto the stable limit cycle with a non-zero frequency when the stability of the fixed point is lost due to the SN bifurcation. On the contrary, decreasing the external current form above the system stays on the limit cycle with arbitrarily small frequencies. Thus the system is no longer purely type I but already shows aspects of type II excitability.

Note that for a limit cycle close to a homoclinic bifurcation the dynamics near the saddle point are slow and dominate the overall period. Close to the saddle the dynamics can be linearized yielding exponential decay in the stable direction and exponential separation form the saddle in the unstable direction. The distance of the limit cycle form the saddle scales linearly with the bifurcation parameter [145]. Taken together we obtain the frequency scaling $\nu\left(i_{\mathrm{e}}\right) \propto-\frac{1}{\log \left(i_{\mathrm{e}}-i_{\mathrm{e}}^{*}\right)}$.

Increasing the leak even further to $g_{\mathrm{L}}=5.2$ the bifurcation diagram changes again in two aspects (cf. fig. 10.3 and 3.4): First, the generation of the stable limit cycle is 
(a)

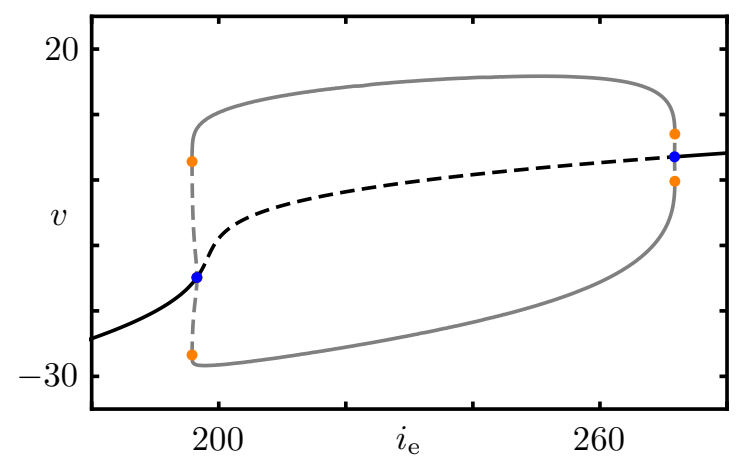

(c)

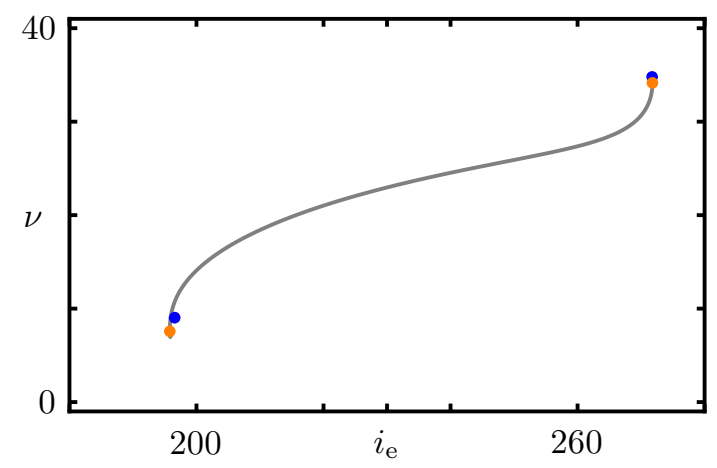

(b)

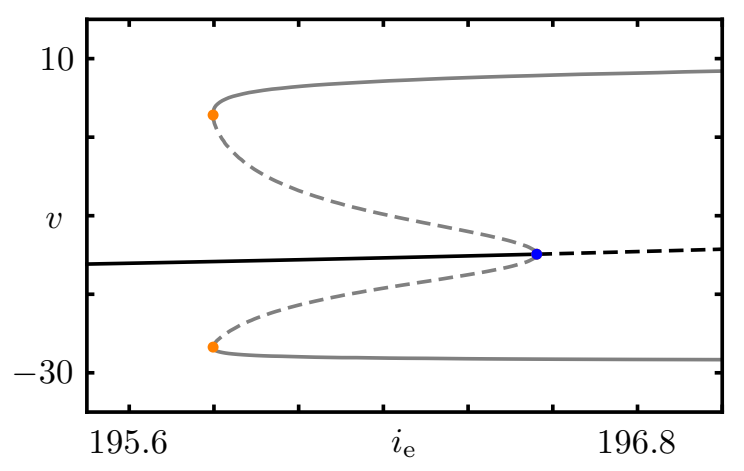

(d)

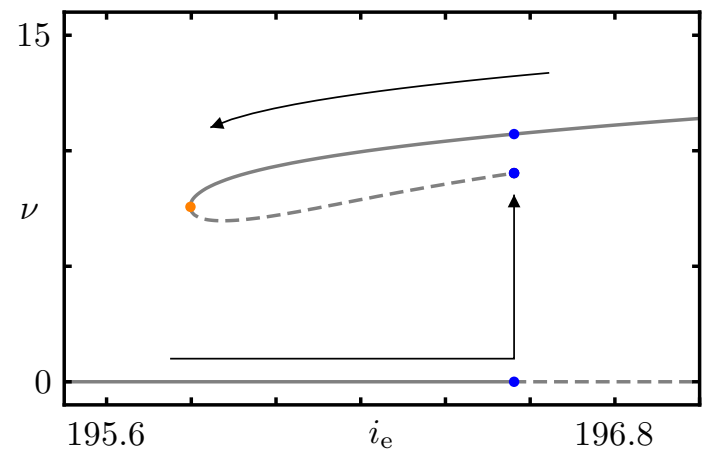

Figure 10.4. Double limit cycle bifurcation in the Morri-Lecar model 2.4.4 determines spike onset for intermediate to large leak conductances $\left(g_{\mathrm{L}}=6.0\right)$. $(\mathbf{a}, \mathbf{b}) i_{\mathrm{e}}$-bifurcation diagram (syntax as in fig. 10.1). The limit cycle is created at $i_{\mathrm{e}}^{*} \approx 195.798$ via a DC bifurcation (॰) with corresponding phase portraits shown in fig. 3.2. (c,d) Frequency of the periodic orbit as a function of the external input current $i_{\mathrm{e}}$ as in fig. 10.3 without the big unstable homoclinic bifurcation. Again there are different frequencies for spike on- and offset due to bistability.

now via a double limit cycle bifurcation, i.e. the system starts to spike with a non-zero frequency, indicating type II excitability. Second the destabilization of the fixed point is no longer via a SN bifurcation but due to a Hopf bifurcation involving a small unstable periodic orbit that is created via a small homoclinic bifurcation (cf. also fig. 3.4). This has two consequences: First, as fig. 10.3d shows, there is again a hysteresis effect, but now both the onset of periodic spiking as well as the offset of spiking when decreasing the current occur at non-zero frequencies. Second, the destabilization of the fixed point via a Hopf-bifurcation just before the onset of spiking implies that the fixed point has a attained non-zero resonance frequency which we refer to as peri-threshold resonance.

For even larger leak conductances $\left(g_{\mathrm{L}}=6.0\right)$ the small homoclinic bifurcation vanishes and one is left only with a DC bifurcation for the onset of spiking and a Hopf bifurcation for the destabilization of the fixed point as shown in fig. 10.4 and 3.2. Thus again, the system is type II with different on- and offset spiking frequencies and shows peri-threshold resonance.

Finally, for very large leak conductances $\left(g_{\mathrm{L}}=8\right)$ the the onset of periodic oscillations is via a Hopf bifurcation with an identical on- and offset frequency as the bistability is no longer present (cf. fig. 10.5 and 3.5). Additionally, the amplitude of the limit cycle increases continuously form zero.

In summary, we have shown that in the ML neuron, an increase in the leak conductance 
(a)

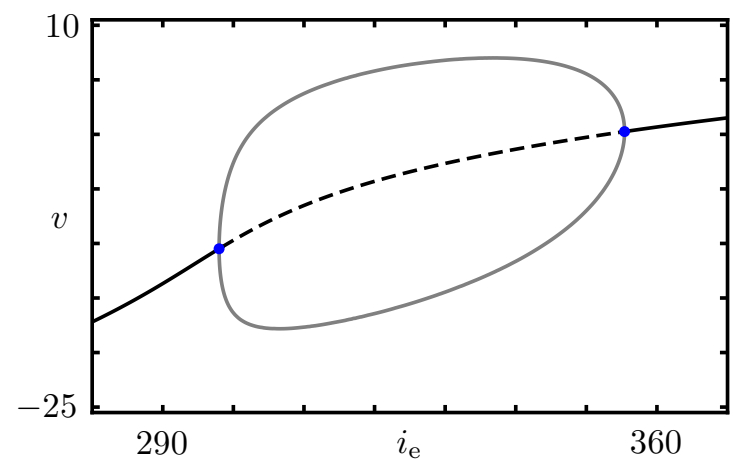

(b)

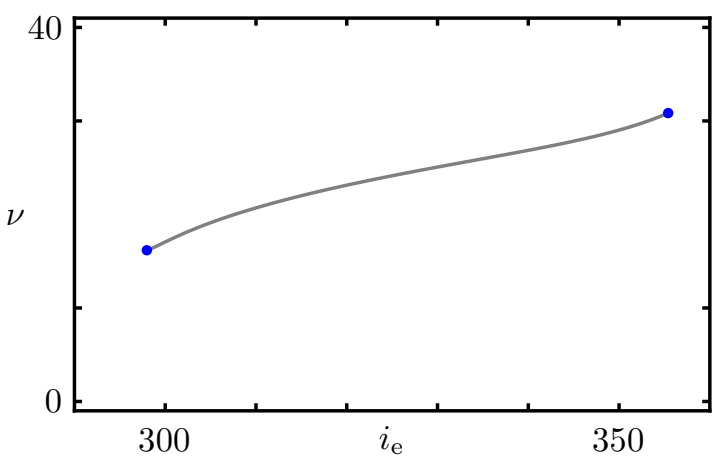

Figure 10.5. Hopf bifurcation in the Morri-Lecar model determines spike onset large leak conductances $\left(g_{\mathrm{L}}=8.0\right)$. (a) $i_{\mathrm{e}}$-bifurcation diagram with notation as in fig. 10.1. The limit cycle is created at $i_{\mathrm{e}}^{*} \approx 297.9$ via a Hopf bifurcation $(\bullet)$ with corresponding phase portraits shown in fig. 3.5. (b) the frequency of the periodic orbit as a function of the external input current $i_{\mathrm{e}}$. There is no region of bistability.

induces a transition from type I to type II neuronal excitability. Interestingly, we here encountered all possible generic co-dimensions 1 bifurcations that lead to a creation of a stable limit cycle in the plane $[145,335]$.

\subsubsection{The Threefold Organization of the Neuronal Excitability Transition}

The results form the previous section show, that leak induces a switch form type I to type II neuronal excitability. To get a better understanding of the transition we constructed a full $\left(i_{\mathrm{e}}, g_{\mathrm{L}}\right)$-bifurcation diagram shown in fig. 10.6. The one-parameter bifurcation diagrams in figs. 10.1-10.5 found above are indicated by dashed lines of constant $g_{\mathrm{L}}$ values.

Figure 10.6c shows that for the spike onset the transition in neuronal excitability from type I to type II happens in three steps:

1. The generation of the stable limit cycle via a SNIC bifurcation (region $\mathrm{I}_{\mathrm{a}}$ ) changes to a big homoclinic bifurcation (region $\mathrm{I}_{\mathrm{b}}$ ). This transition is organized by the saddlenode-loop bifurcation (cf. fig. 3.3.2.4). It implies the creation of a region with coexistence of a stable fixed point and stable limit cycle. This in turn implies the hysteresis effect in the on and offset spiking frequencies for leak conductances above the SNL point as observed in fig. 10.2. The neuron in this regime displays type II neuronal excitability for the spike onset and type I excitability for the offset. Further the frequency dependence of the limit cycle on $i_{\mathrm{e}}$ close after the spike onset switches form a square root scaling for a SNIC bifurcation [100] to an inverse logarithmic one.

2. Spike onset via the homoclinic bifurcation (region $\mathrm{I}_{\mathrm{b}}$ ) switches to the onset via a double limit cycle bifurcation (region $\mathrm{II}_{\mathrm{a}}$ ). This switch is organized by a neutral saddle loop bifurcation (cf. also fig. 3.8). The bistable region is not affected but above the NSL point the hysteresis effect shows type II behaviour for both on and offset of spiking. This is the main step in the transition form type I to type II neuronal excitability.

3. In a third step the DC bifurcation (region $\mathrm{II}_{\mathrm{a}}$ ) switches to a Hopf bifurcation (region 
(a)

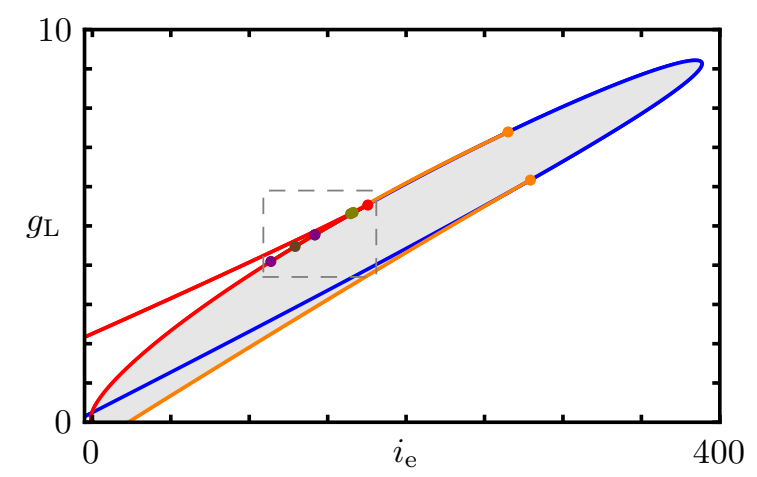

(b)

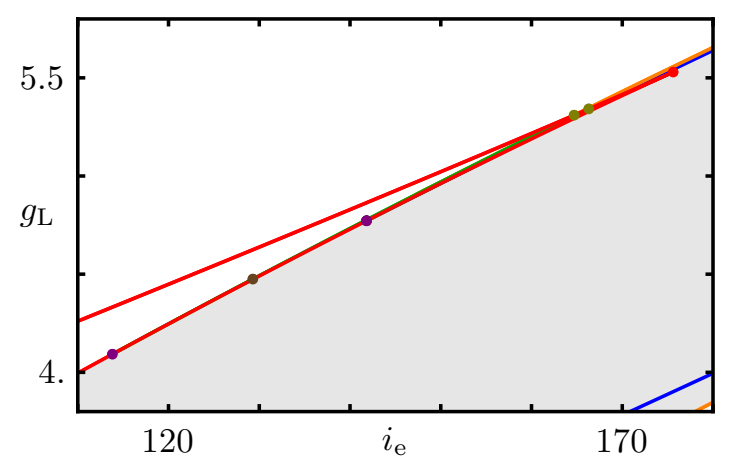

(c)

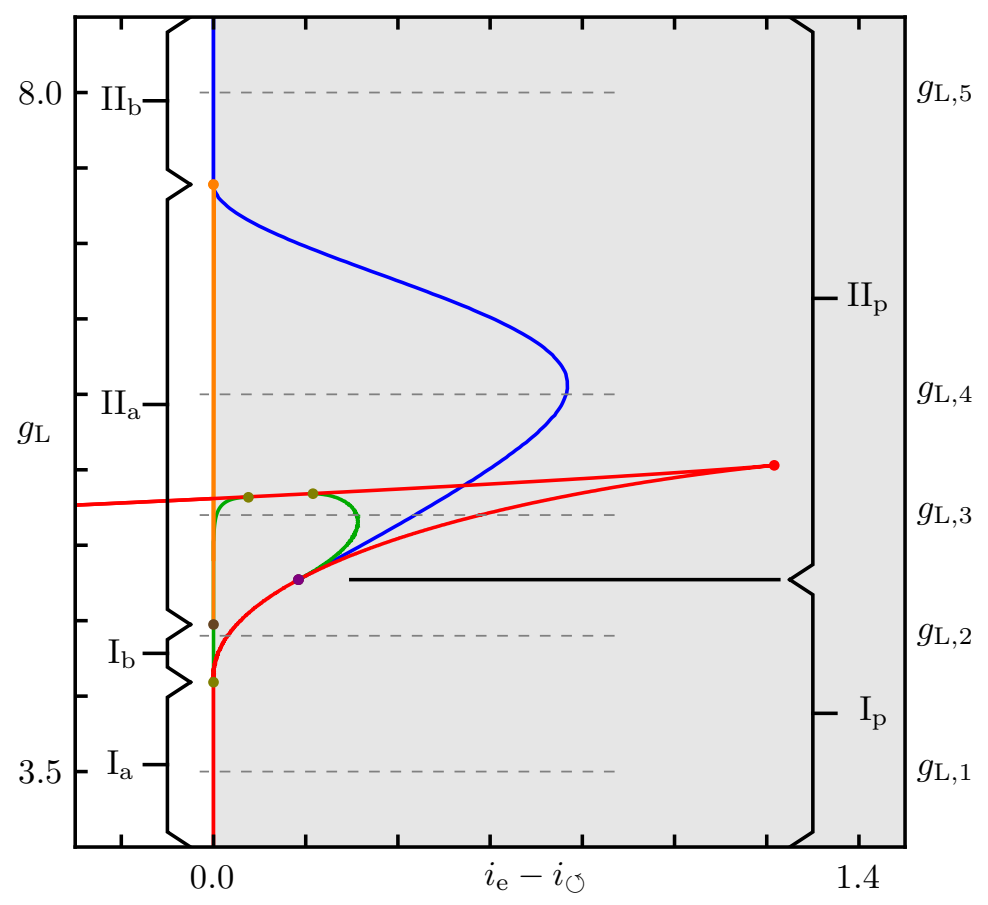

Figure 10.6. Neuronal excitability transition in the ML neuron. Bifurcation diagrams in $\left(i_{\mathrm{e}}, g_{\mathrm{L}}\right)$ parameter space are shown using the model eq. 2.4.4 standard parameters given in D.2. In particular, there are SN and SNIC (-), Hopf (-), DC (-) and homoclinic (-) bifurcations (for notations and color codes cf. also tab. 3.1). In addition we observe the following co-dimension-2 bifurcations: Bogdanov-Takens $(\bullet)$, cusp $(\bullet)$, degenerate Hopf $(\bullet)$, saddle node loop $(\bullet)$ and neutral saddle node $(\bullet)$. The area in which a stable periodic orbit exists is indicated with a gray shading $(\square)$. (a) complete bifurcation diagram in $\left(i_{\mathrm{e}}, g_{1}\right)$-parameter space. (b) zoom into the type I to type II transition area (indicated by a dashed box in (a)). (c) same as is (a) but shifting the input current $i_{\mathrm{e}}$ by $i_{\circlearrowleft}=i_{\circlearrowleft}\left(g_{\mathrm{L}}\right)$ such that a stable periodic orbit is generated at $i_{\mathrm{e}}-i_{\circlearrowleft}=0$ to better resolve the fine-structure of the bifurcation diagram. Dashed lines indicate the values of the leak used for the one-dimensional bifurcation diagrams shown in fig. 10.1-10.5. The neuronal excitability transition is organized in three steps via a saddle node loop $(\bullet)$, a neutral saddle node $(\bullet)$ and a degenerate Hopf (•) bifurcation. This leads to four different regions for the generation of a stable limit cycle: The type I regions $\mathrm{I}_{\mathrm{a}}$ (SNIC), $\mathrm{I}_{\mathrm{b}}$ (homoclinic) and the type II regions $\mathrm{II}_{\mathrm{a}}$ (DC) and $\mathrm{II}_{\mathrm{b}}$ (Hopf). The transition is accompanied with a transition at peri-thershold at the BT point $(\bullet)$ due to a change in the destabilization of the fixed point via a SN bifurcation in the $\mathrm{I}_{\mathrm{p}}$ region to a subcritical Hopf bifurcation in $\mathrm{II}_{\mathrm{p}}$. 
$\mathrm{II}_{\mathrm{b}}$ ) organized by a degenerate Hopf $(\mathrm{DH})$ bifurcation. Above the $\mathrm{DH}$ point limit cycle amplitudes grow continuously from zero and the bistability is lost. The on and offset spiking frequencies become identical.

The transition above is accompanied by a transition to peri-threshold resonance. Here the destabilization of the fixed point via a $\mathrm{SN}$ bifurcation (region $\mathrm{I}_{\mathrm{p}}$ ) changes to a subcritcal Hopf bifurcation (region $\mathrm{II}_{\mathrm{p}}$ ) which is organized by an ordinary Bogdanov-Takens bifurcation (cf. fig. 3.6).

We proceed by investigating whether this transition can also be found in models for cortical neurons and of higher state space dimensions, such as the Wang-Buzsaki neuron.

(a)

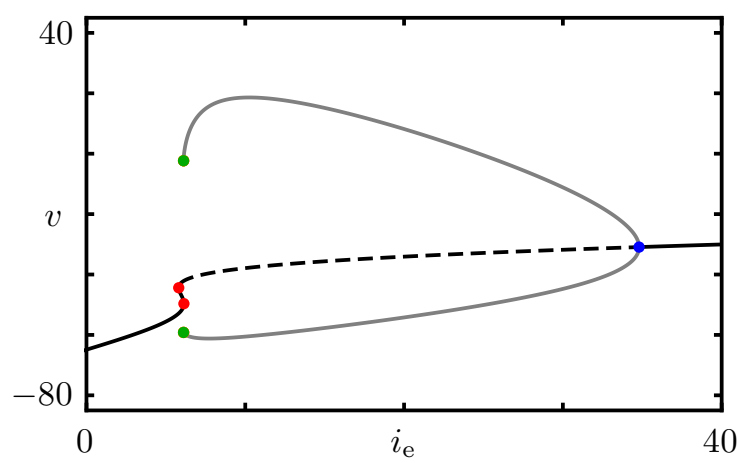

(c)

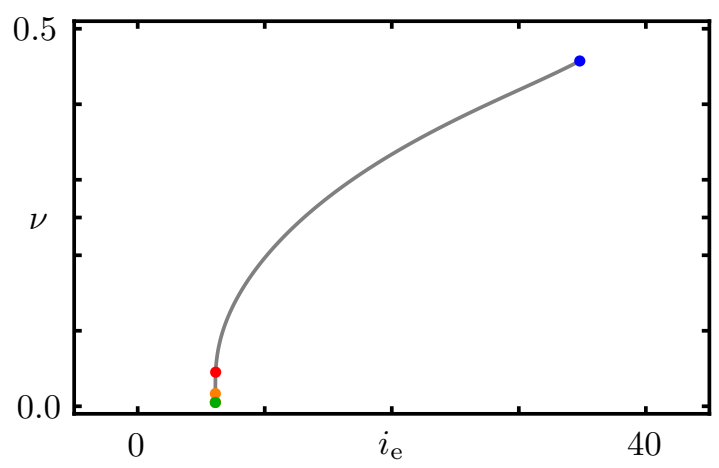

(b)

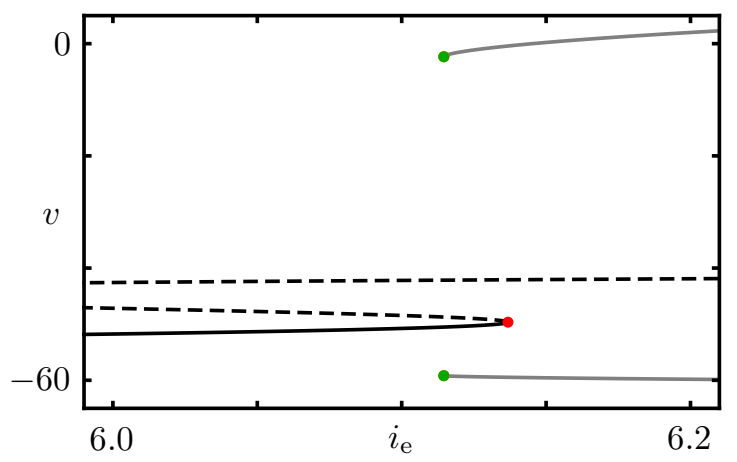

(d)

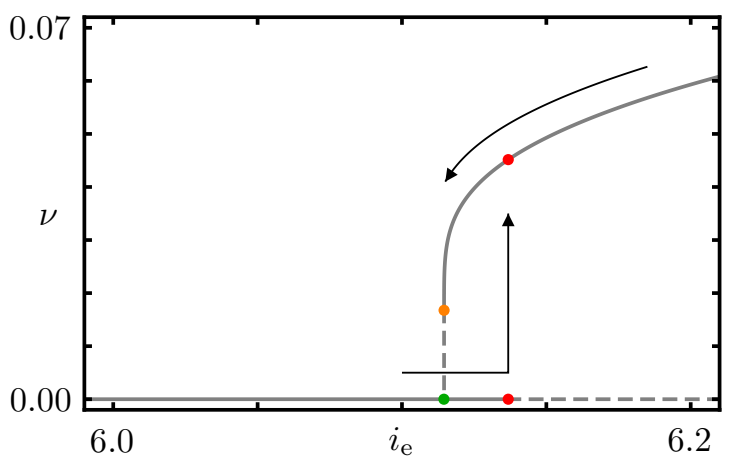

Figure 10.7. Double limit cycle and saddle node bifurcation determine spike onset in the WangBuzsaki model (sec. 2.4.3) for $g_{\mathrm{L}}=0.65$ and parameter as in appendix D.3. (a,b) bifurcation diagram showing that a stable limit cycle is generated via a DC bifurcation $(\bullet)$. The unstable orbit vanishes at a homoclinic $(\bullet)$ bifurcation, the stable cycle at a Hopf point $(\bullet)$. The zoom (b) shows that there is a region where the stable limit cycle coexists with a stable fixed point that vanishes via a SN bifurcation $(\bullet)$. (c,d) frequency of periodic orbit as a function of the external input current $i_{\mathrm{e}}$. Increasing $i_{\mathrm{e}}$ the neuron starts firing when the fixed point vanishes via the fold $(\bullet)$. Decreasing the input the neuron can maintain periodic firing only up to a finite frequency determined by the DC bifurcation (०). The frequency of the unstable periodic orbit is shown (dashed) which terminates in a homoclinic bifurcation $(\bullet)$. 


\subsubsection{Leak Induced Neuronal Excitability Transition in the Wang- Buszaki Neuron}

In this section we study the effect of leak conductance changes on the bifurcation scenarios for a cortical neuron model, the Wang-Buzsaki (WB) model [400] described in section 2.4.3. We use the original parameters as listed in appendix D.3.

For small leak conductances $\left(g_{\mathrm{L}}=0.55\right)$ the WB neuron shows a SNIC bifurcation for the onset of periodic firing similar to the one observed in fig. 10.1. It therefore has type I neuronal excitability. In contrast to the ML neuron model the termination of the periodic spiking due to the conductance block at large input currents is here via a Hopf bifurcation.

For $g_{\mathrm{L}}=0.65$ the situation changes as shown in fig. 10.7. Creation of the limit cycle is now via a DC bifurcation. The unstable orbit vanishes via a big unstable homoclinic bifurcation. There is a region of a stable limit cycle coexisting with a stable fixed point that leads via the hysteresis effect to different on and offset spike frequencies which are both non-zero. Therefore the neuron is type II. In contrast to the situation in fig. 10.2 for the ML model the fixed point gets still unstable via a saddle node bifurcation and hence there is no peri-threshold resonance.

Increasing the leak further to $g_{\mathrm{L}}=0.718$ we obtain the bifurcation diagram of fig. 10.8. Here the limit cycle is also generated via an DC bifurcation, the unstable orbit, however, vanishes via an SNIC bifurcation. Additionally the neuron shows peri-threshold resonance as the stable fixed point which coexists with the stable limit cycle becomes unstable via a Hopf bifurcation. Spike on- and offset have different frequencies but are both type II.

For even larger values of the leak $g_{\mathrm{L}}=0.8$ the system shows a DC bifurcation followed by a subcritical Hopf bifurcation as in the ML model.

In summary, an increase in the leak conductance induces a transition similar to the one observed in the ML neuron (sec. 10.2.1). We resolved the fine structure of this transition, via a two parameter bifurcation diagram shown in fig. 10.9. The organization of the transition is as in fig. 10.6 via the three codimension two pints SNL, NSL and $\mathrm{DH}$ accompanied by a transition to peri-threshold resonance via the BT point (cf. sec. 10.2.1.2).

In total, this shows, that the leak induced transition from type I to type II is also present in cortical neurons with higher state space dimension. The high similarity between the bifurcation diagrams in the ML and WB model suggest to study further neuron models to check for a general principle underlying leak-induced type I to type II transitions.

\subsubsection{Prevalence of Leak-Induced Excitability Transitions in Type I Model Neurons}

In this section we study the bifurcation diagrams for various neuron models developed for different types of neurons in different regions of the brain and also consider models with additional A-type current. In all these models we find that the transition in neuronal excitability generally follows the scheme described in detail in section 10.2.1.2.

We start the bifurcation analysis directly in the $\left(i_{\mathrm{e}}, g_{\mathrm{L}}\right)$-plane. The bifurcation diagram for the fast spiking neuron model by Erisir et al. (sec. 2.4.4) is shown in fig. 10.10, for the reduced Traub-Miles pyramidal neuron (sec. 2.4.6) in fig. 10.11 and for the RoseHindmarsh neuron model with A-type current (sec. 2.4.5) in fig. 10.12. Together with 
(a)

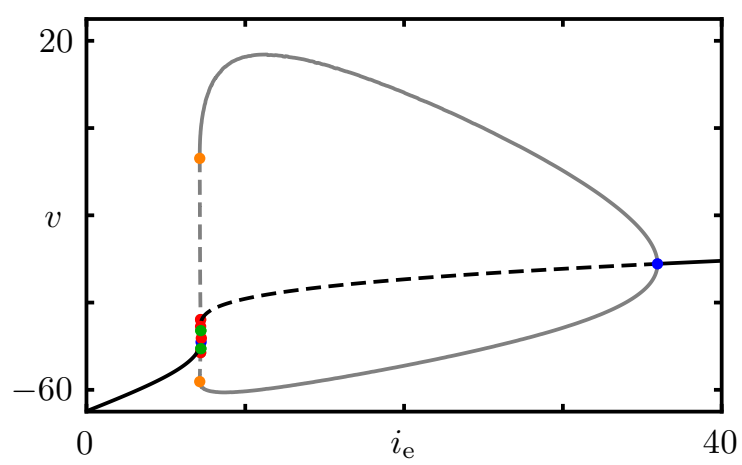

(c)

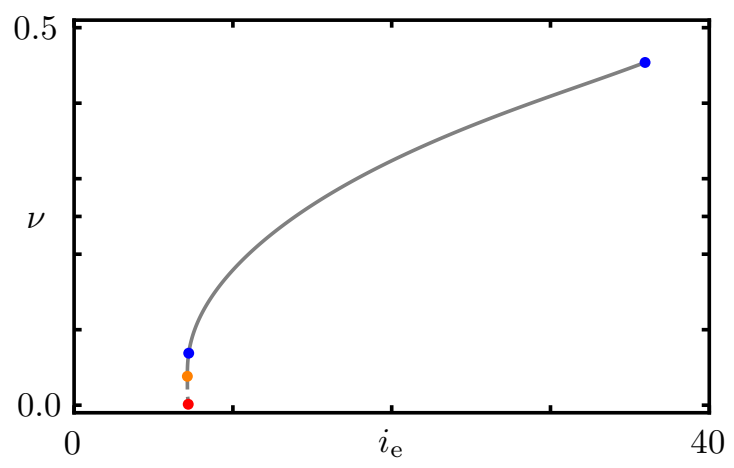

(b)

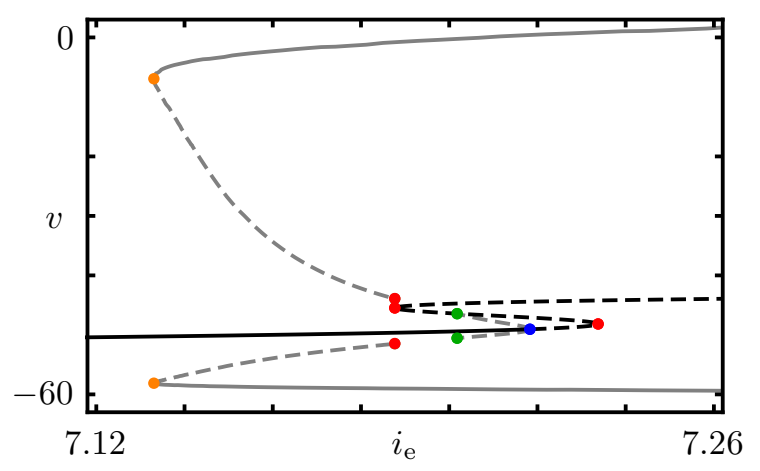

(d)

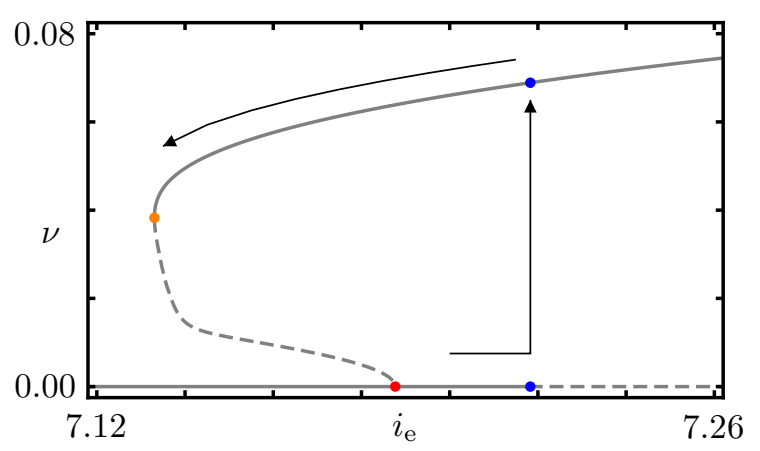

Figure 10.8. Double limit cycle and Hopf bifurcation determine spike onset in the WangBuzsaki model for $g_{\mathrm{L}}=0.718$. $(\mathbf{a}, \mathbf{b})$ bifurcation diagram as in fig. 10.7 with the difference that the unstable periodic orbit terminates in a SNIC bifurcation $(\bullet)$ and a second unstable limit cycle is generated via a small homoclinic bifurcation $(\bullet)$ which coalesces with the fixed point at a HB point $(\bullet)$. (c,d) Frequency $\nu$ of periodic orbit as a function of the external input current $i_{\mathrm{e}}$. On $(\bullet)$ and offset $(\bullet)$ of spiking happen with different non-zero frequencies.

the diagrams for the ML and WB neurons in figs. 10.6 and 10.9 all exhibit a transition from type I to type II neuronal excitability organized in three steps via the SNL, the NLS and $\mathrm{DH}$ bifurcation as described in detail in sec. 10.2.1.2. This transition involves the creation of a region where a stable limit cycle coexists with a stable fixed point, resulting in a mismatches for on- and offset frequencies of periodic spiking within the transition region. Further more, this transition is always accompanied by a switch from integration to resonance at peri-threshold, organized via the BT point. We also observe this behavior in the Rinzel model (cf. sec. 2.6.2) and the somatic compartment of the Mainen-Senjowski neuron [250].

Note, that all these models have in common, that they are intrinsically type I neurons. The prevalence of this neuronal excitability transition in type I neurons suggest a common underlying principle which we unravel in the next section.

\subsubsection{A Degenerate Bogdanov-Takens Bifurcation Organizes Neuronal Excitability Transitions}

In the previous section we have seen that the three-step transition from type I to type II neuronal excitability is always organized by the same co-dimension two bifurcations: 
(a)

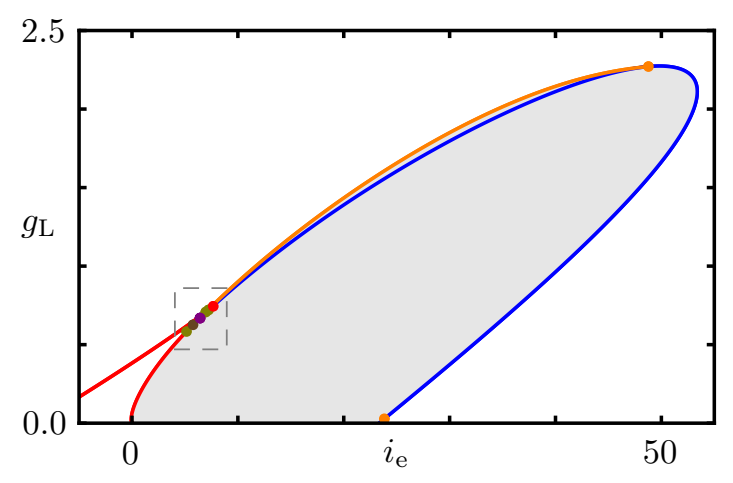

(b)

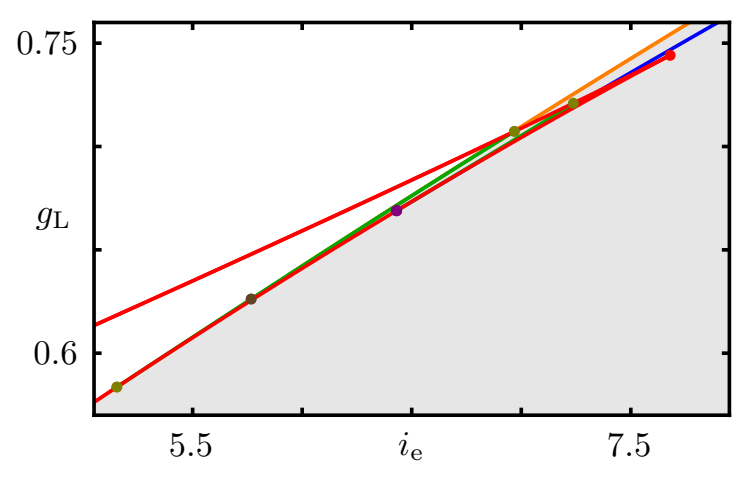

(c)

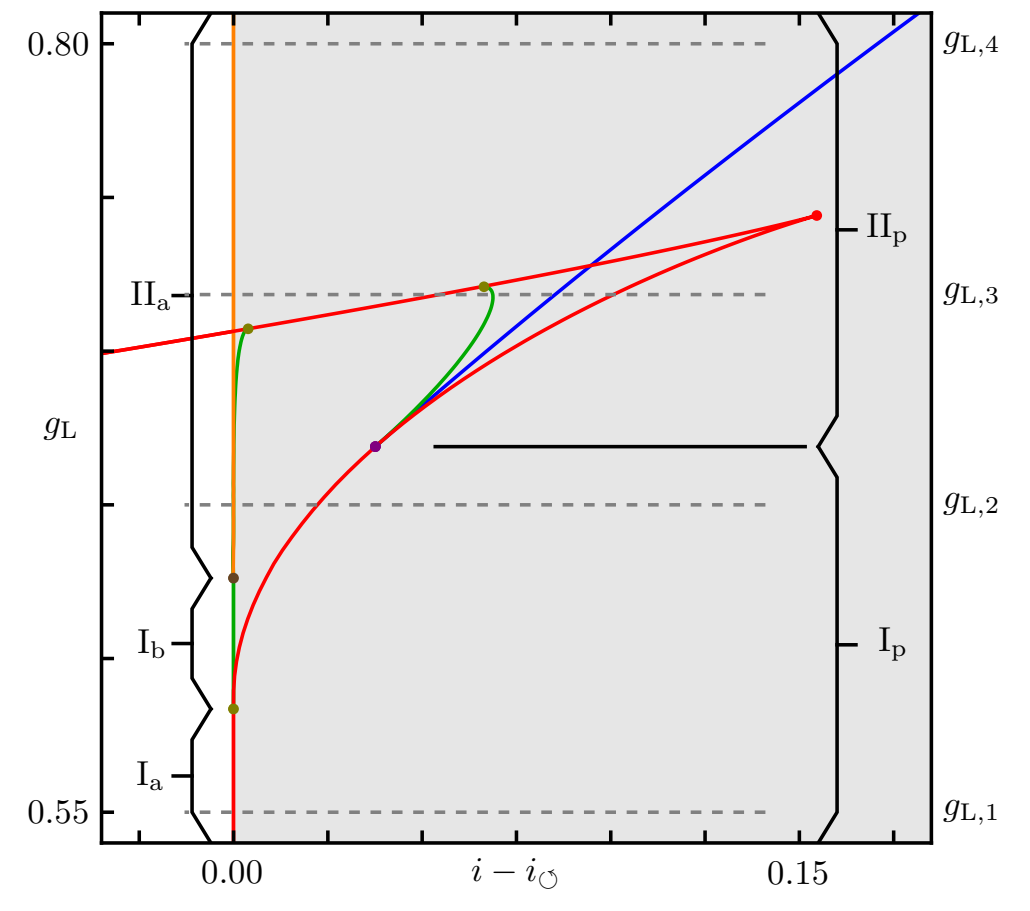

Figure 10.9. Neuronal excitability transition in the Wang-Buzsaki neuron. Bifurcation diagrams in $\left(i_{\mathrm{e}}, g_{1}\right)$-parameter space are shown for the model in sec. 2.4.3 and remaining parameter as in appendix D.3. Lines show the co-dimension-1 bifurcations: SN and SNIC (-), Hopf (-), DC (-) and homoclinic (-) bifurcations. In addition we observe Bogdanov-Takens $(\bullet)$, cusp $(\bullet)$, degenerate Hopf $(\bullet)$, saddle node loop $(\bullet)$ and neutral saddle node $(\bullet)$ bifurcations of co-dimension2. Existence of a stable periodic orbit is indicated with a gray shading ( $\square$ ). (a) complete bifurcation diagram $\left(i_{\mathrm{e}}, g_{\mathrm{l}}\right)$-parameter space. (b) zoom into the area where the transition from type I to type II appears (indicated by a dashed box in (a)). (c) same as is (a) but shifting the input current $i_{\mathrm{e}}$ by $i_{\circlearrowleft}=i_{\circlearrowleft}\left(g_{\mathrm{L}}\right)$ such that a stable periodic orbit is generated at $i_{\mathrm{e}}-i_{\circlearrowleft}=0$ to resolve the precise structure of the bifurcation diagram. Dashed lines indicate the values of the leak used for the one-dimensional bifurcation diagrams described in the text and in figs. 10.7 and 10.8. Also the different excitability regions as described in sec. 10.2.1.2 are indicated.

A SNL, a NSL and a DH point (cf. sec. 10.2.1.2). Using the idea of multiple bifurcation theory in which bifurcations of lower co-dimension are organized by higher degenerate bifurcations of larger co-dimension [144, 276], one can ask the question whether there is a higher degenerate bifurcation point that organizes the existence of the SNL the NSL and 


\section{DH bifurcations.}

To answer this question, observe that in all $\left(i_{\mathrm{e}}, g_{\mathrm{L}}\right)$-bifurcation diagrams there exists a cusp point. Moreover, the transition in neural excitability is always accompanied by a transition from integration to resonance at peri-threshold organized by a Bogdanov-Takens point. In $[85,87,86]$ it is shown that these two co-dimension-2 bifurcations can interact in a co-dimension-3 degenerate Bogdanov-Takens-cusp (dBT) bifurcation as introduced in sec. 3.3.3. The bifurcation diagram for the unfolding of the dBT point of focus type is shown in figs. 3.9 and 3.10. It can bee seen that the dBT point organizes the two BT and CP bifurcation lines and in addition also three SNL, a NSL and a DH bifurcation line,

(a)

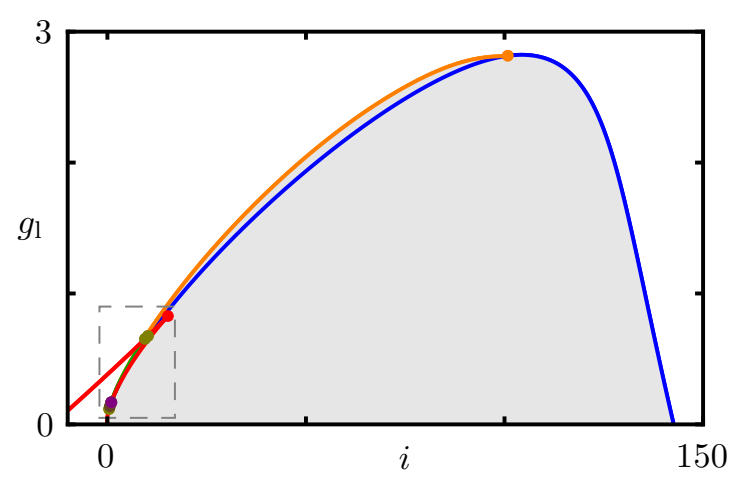

(b)

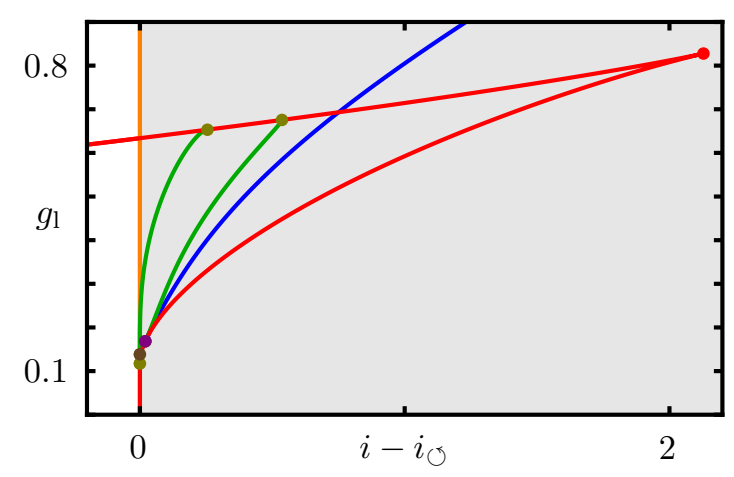

Figure 10.10. Bifurcation diagram for the fast spiking inter-neuron model by Erisir et al. in the $\left(i_{\mathrm{e}}, g_{\mathrm{L}}\right)$-plane. Model and parameters as in sec. 2.4.4 and appendix D.4. Figure syntax as in fig. 10.9 and tab. 3.1. (a) full bifurcation diagram showing topologically the same structure as for the WB model in fig. 10.9. Box indicates transition region shown in (b). (b) zoomed and shifted diagram showing the fine structure of the transition from type I to II excitability. As in the ML and WB model the SNL $(\bullet)$, NSL $(\bullet)$ and DH $(\bullet)$ points organize the transition. Further the existence of the BT point organizes the transition to peri-threshold resonance.

(a)

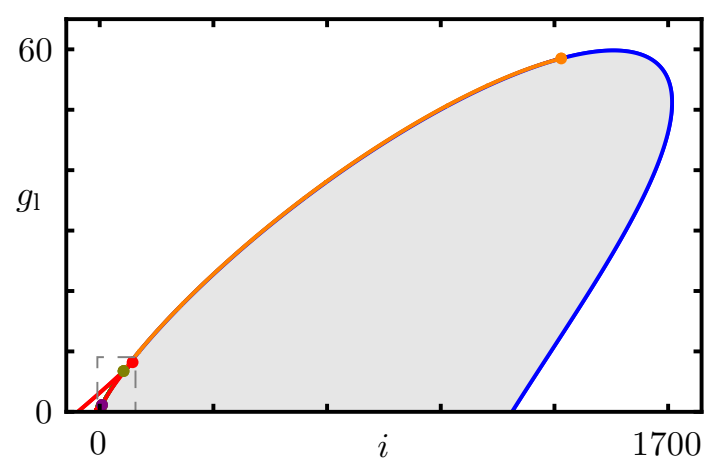

(b)

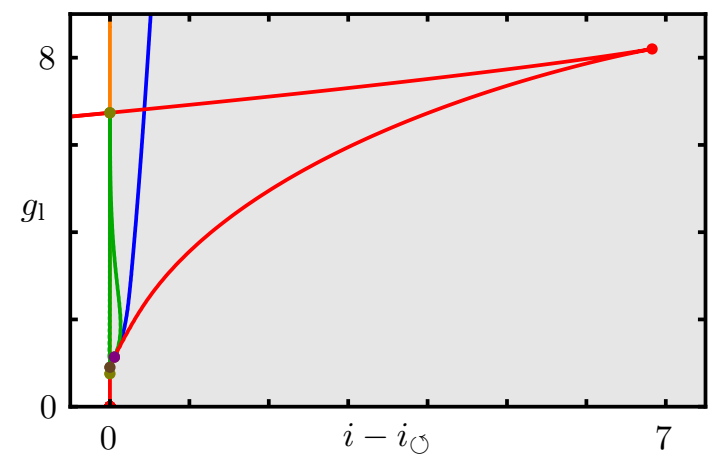

Figure 10.11. Bifurcation diagram for the reduced Traub-Miles pyramidal neuron model 2.4.6 in the $\left(i_{\mathrm{e}}, g_{\mathrm{L}}\right)$-plane. Model and parameters as in sec. 2.4.6 and appendix D.6. Figure color syntax as in fig. 10.9 and tab. 3.1. (a) full bifurcation diagram. Box indicates transition region shown in (b). (b) zoomed and shifted diagram showing the fine-structure of the transition from type

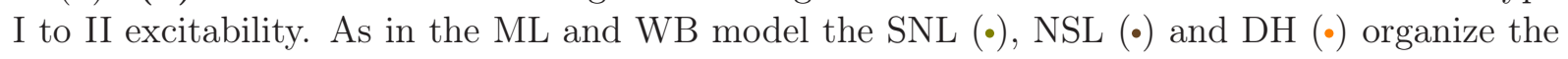
transition form type I to type II excitability and the BT point the transition to peri-threshold resonance. 
(a)

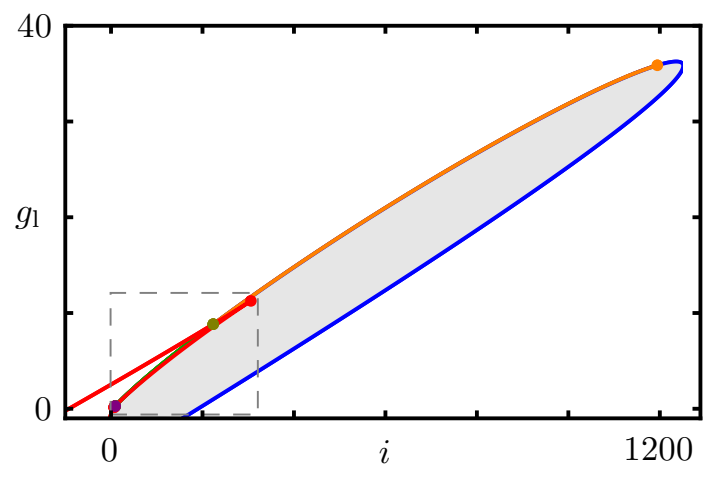

(b)

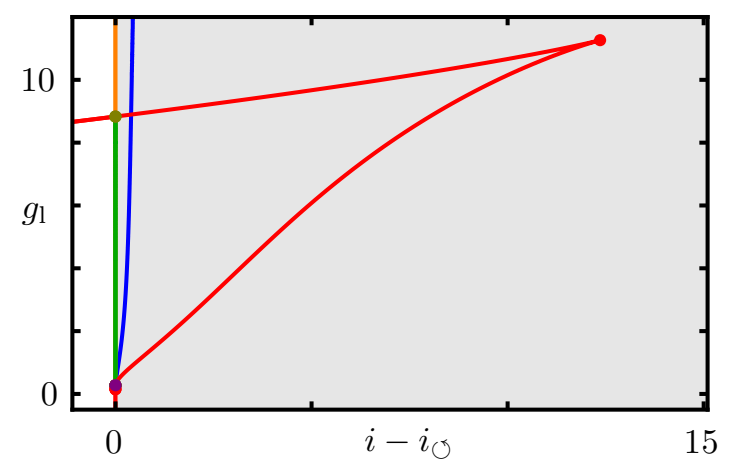

Figure 10.12. Bifurcation diagram for the Rose-Hindmarsh neuron model with A-type current in the $\left(i_{\mathrm{e}}, g_{\mathrm{L}}\right)$-plane. Model and parameter as in sec. 2.4.5 and appendix D.5. Figure syntax as in fig. 10.9 and tab. 3.1. (a) full bifurcation diagram showing topologically the same structure as for the WB model in fig. 10.9. Box indicates transition region shown in (b). (b) zoomed and shifted diagram showing the fine-structure of the transition from type I to II excitability. As in the WB model the SNL $(\bullet)$, NSL $(\bullet)$ and DH $(\bullet)$ organize the transition and the BT point the transition to peri-threshold resonance.

making the $\mathrm{dBT}$ bifurcation a potential candidate for an organizing center of type I to type II transitions.

To check this hypothesis we have calculated a three dimensional bifurcation diagram for the Wang-Buzsaki model using the capacity $c_{\mathrm{m}}$ as a third bifurcation parameter. The resulting three-dimensional bifurcation diagram is shown in fig. 10.13 and with a zoom and rotation in fig. 10.14. They confirm the existence of a dBT point. Furthermore, one observes that the bifurcation diagram forms a topological cone with base shown in fig. 10.15 which by comparison with fig. 3.10 shows that the point is a degenerate BT point of focus type. Figures 10.13 and 10.14 demonstrate, that the dBT point is located above the plane $c_{\mathrm{m}}=1$, (the original capacity of the WB model) and organizes the structure of the $\left(i_{\mathrm{e}}, g_{L}\right)$-bifurcation diagram shown in fig. 10.9 .

Note the striking similarity between the rotated and zoomed WB bifurcation diagram in fig. 10.14 to the diagram shown in fig. 3.9. Especially, the SN bifurcation surface has a very similar shape. One reason for this similarity is the choice of $c_{\mathrm{m}}$ as the third bifurcation parameter: As it multiplies the the vector field in $v$ direction the position of the fold points is not affected by $c_{\mathrm{m}}$ and therefore the SN bifurcation lines do not depend on $c_{\mathrm{m}}$.

In [87] it is conjectured that every system close to a dBT point of focus type is equivalent (but not necessarily conjugated) to an unfolding of the normal form of a dBT of focus type given by (3.3.1), i.e. is of the form

$$
\begin{aligned}
& \frac{d}{d t} x=y \\
& \frac{d}{d t} y=\mu_{1}+\mu_{2} x-x^{3}+y\left(\mu_{3}+\rho x-x^{2}\right)
\end{aligned}
$$

with unfolding parameter $\mu_{i}, i=1,2,3$ (cf. also sec. 3.2.1 and 3.3.3). Comparing the bifurcation diagrams for the normal form in fig. 3.9 and for the WB neuron in fig. 10.14 one observes that $\mu_{3}$ is mainly controlled by $c_{\mathrm{m}}$, while $\mu_{2}$ and $\mu_{1}$ are controlled by a 


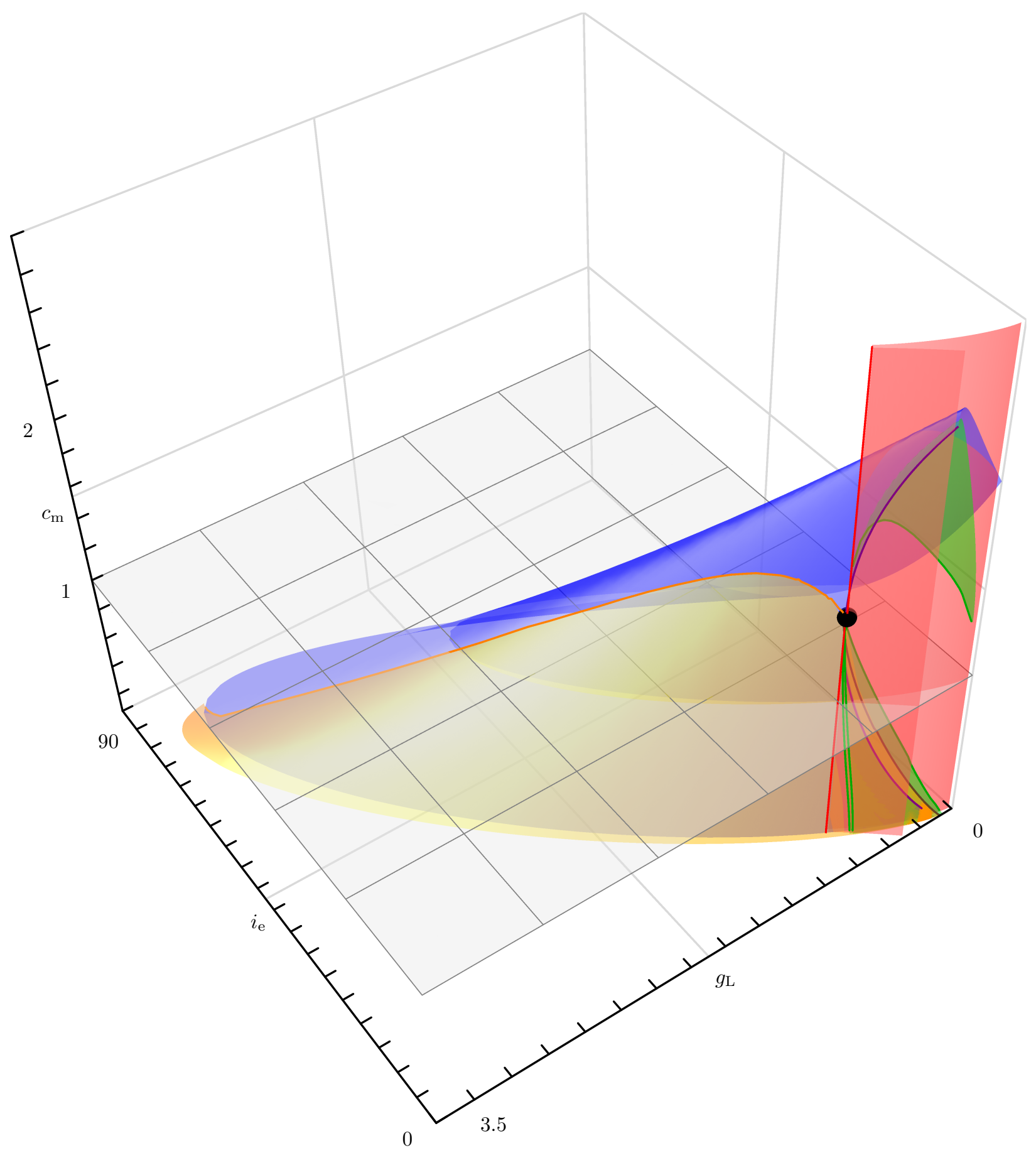

Figure 10.13. Neuronal excitability transitions are organized by a degenerate Bogdanov-Takenscusp (dBT) bifurcation. Three parameter $\left(i_{\mathrm{e}}, g_{\mathrm{L}}, c_{\mathrm{m}}\right)$-bifurcation diagram for the Wang Buzsaki model in sec. 2.4.3 with standard parameter as in appendix D.3. The dBT point $(\bullet)$ of codimension-3 organizes the surrounding co-dimension two (lines) and one (surfaces) bifurcations. The co-dimension-2 bifurcations lines are: cusp (-), BT (-), NSL (-), SNL (-) and DH (-).

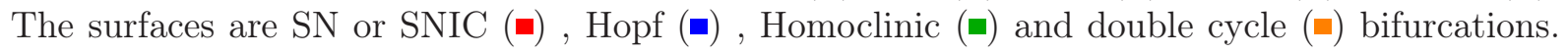
The gray shaded plane shows the section of the bifurcation diagram as shown in fig. 10.9.

combination of $i_{\mathrm{e}}$ and $g_{\mathrm{L}}$. We confirm these findings in an analytical treatment of the FHN model in section 10.3.4.

To conclude, we have found numerically that an increase in leak conductance induces 


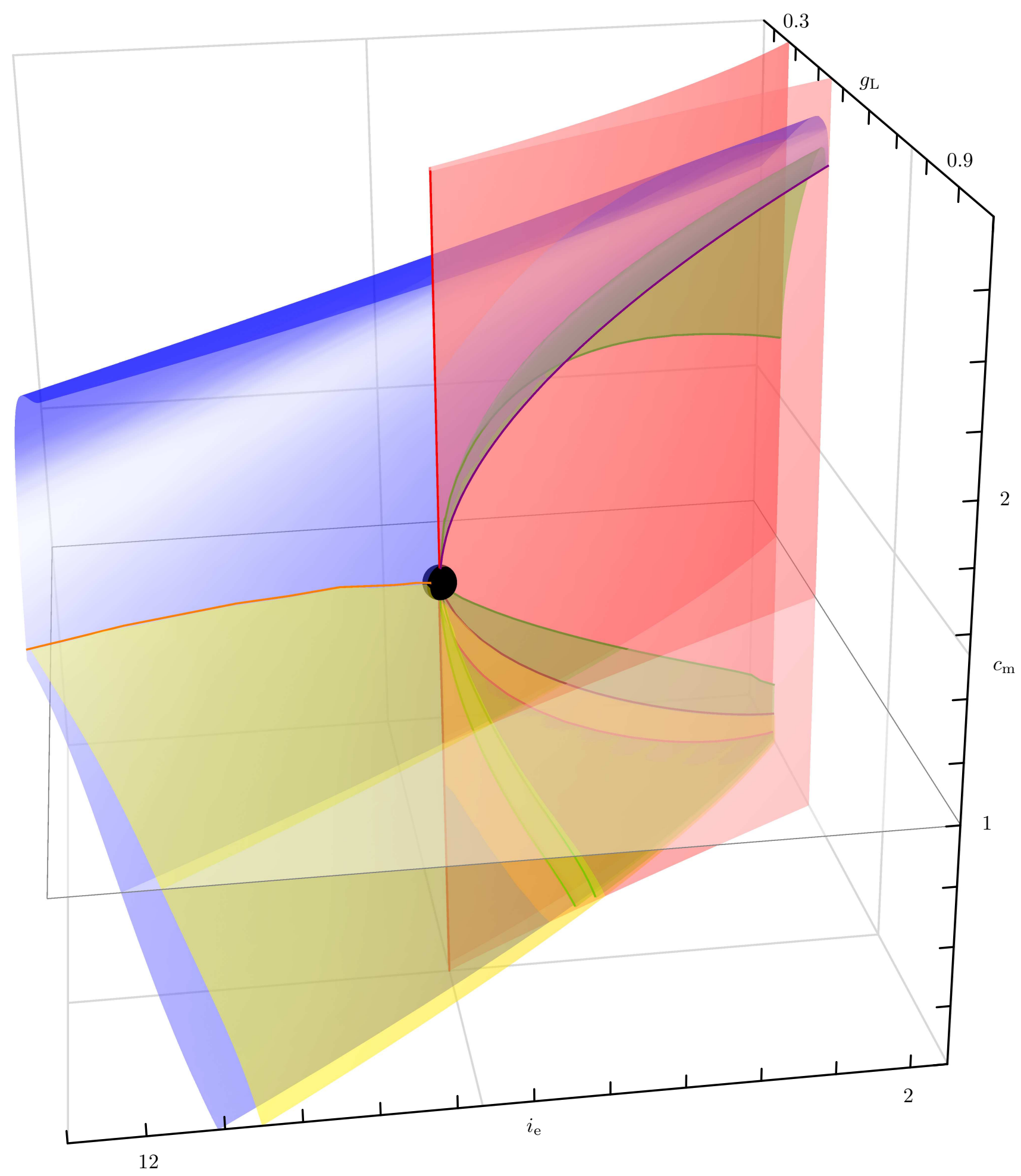

Figure 10.14. Neuronal excitability transitions are organized by a degenerate Bogdanov-Takenscusp (dBT) bifurcation. Shown is the three parameter $\left(i_{\mathrm{e}}, g_{\mathrm{L}}, c_{\mathrm{m}}\right)$-bifurcation diagram for the Wang Buzsaki model 2.4.3 as in fig. 10.13 but rotated and zoomed onto the degenerate BT point (•)In this representation the diagram is strikingly similar to the diagram of the unfolding of the normal form of the $\mathrm{dBT}$ point as shown in fig. 3.9, indicating that the $\mathrm{dBT}$ point organizes the transition from type I to type II neuronal excitability. 
(a)

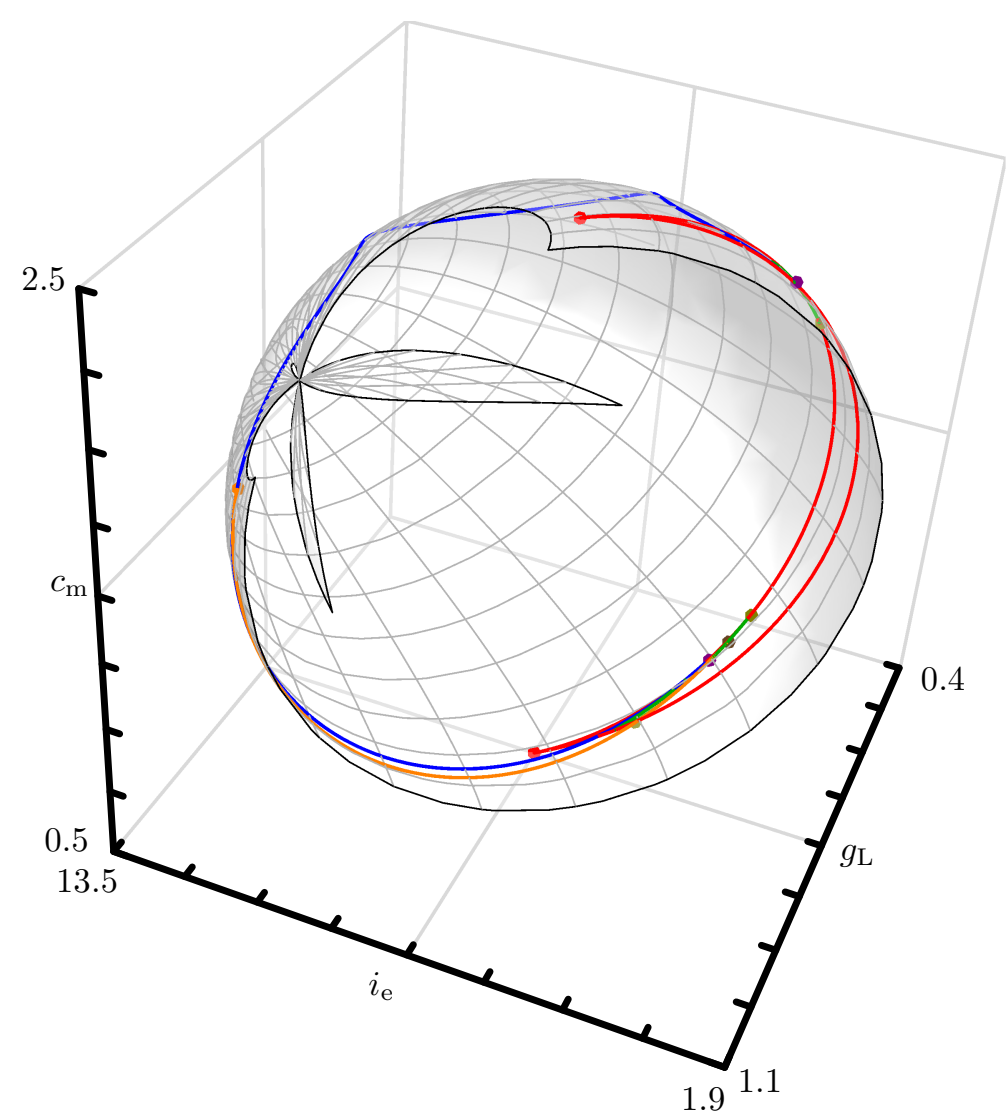

(b)

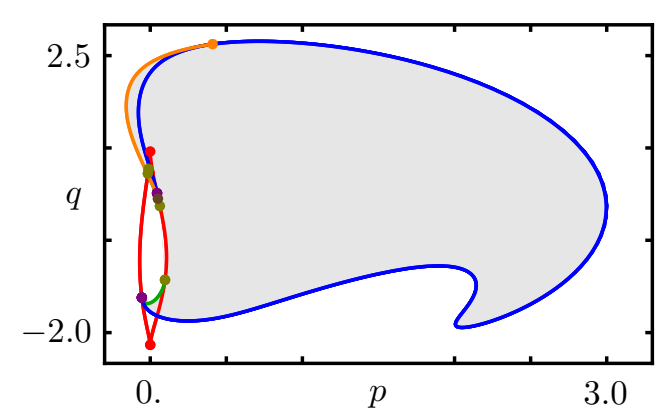

(c)

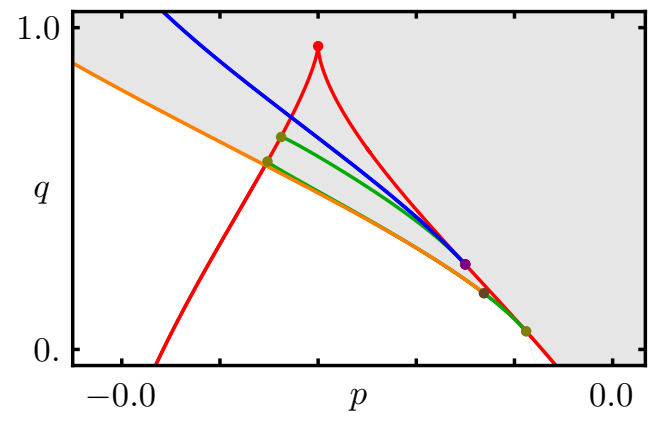

Figure 10.15. Cone structure of the $\left(i_{\mathrm{e}}, g_{\mathrm{L}}, c_{\mathrm{m}}\right)$-bifurcation diagram for the WB neuron model as in fig. 10.13 and 10.14. (a) Show are the intersections of the bifurcation surfaces and lines (color syntax given in fig. 10.13, cf. also tab. 3.1) of the bifurcation diagram in fig. 10.14 with an ellipsoid centered at the dBT point and coordinate system $(p, q)$ used in (c,b). (b) The bifurcation diagram on the ellipsoid in a $2 \mathrm{D}$ coordinate system showing the basis of the cone structure. (c) zoom into the lower area of the ellipsoid showing the type I to type II transition region.

a switch from type I to type II neuronal excitability which is organized by a degenerate Bogdanov-Takens-cusp bifurcation for the Wang-Buzsaki model. Given the strong similarity of the two parameter bifurcation diagrams for the different neuron models found in sec. 10.2.3 we conclude that the leak induced transition is generally organized by a degenerate Bogdanov-Takens bifurcation point of focus type in these type I neuron models. Indeed, in sec. 10.3.2 we will give an analytically proof that every type I conductance 
based neuron model has a dBT point of focus type in the $\left(i_{\mathrm{e}}, g_{L}, c_{\mathrm{m}}\right)$ parameter space that can serve as an organizing center for the observed neuronal excitability transitions.

\subsubsection{Leak Induced Neural Excitability Transition in Type II Neuron Models}

In the previous section 10.2 .3 we showed that generally a type I neuron can be switched to type II via a three-fold transition organized by a degenerate Bogdanov-Takens point. Thus starting with an initially larger leak conductance in one of the above models that gives rise to type II excitability it is clear that they can be switched back to type I. But what about other type II neurons?

A classical type II neuron is the Hodgkin-Huxley model $[172,171]$ introduced in sec. 2.4.1. The bifurcation diagram for this model in the $\left(i_{\mathrm{e}}, g_{\mathrm{L}}\right)$-plane is shown in fig. 10.16. For small positive values of the leak conductance periodic spiking is generated via a saddle-node of limit cycle bifurcation and the stable fixed point becomes unstable via a subcritical Hopf bifurcation. In total this gives rise two non-zero frequencies for on- and offset of spiking. The Hopf-bifurcation of the fixed point also indicates peri-threshold resonance.

When increasing the leak we find a DH point in which the DC line ends and which organizes the transition of limit cycle generation from DC to a Hopf bifurcation, similar to the third part of the transition discussed in sec. 10.2.1.2. However, the DC curve is folded twice and we observe a bifurcation to an invariant torus, a period doubling bifurcation as well as a twisted saddle loop (not shown). The latter two bifurcations have also been observed in $\left(i_{\mathrm{e}}, g_{\mathrm{K}}\right)$ parameter space in ref. [147]. As the neuron is already type II in this regime, we defer the study of the finestructure of these bifurcations involving mainly unstable periodic orbits to future work and instead concentrate on a possible switch to

(a)

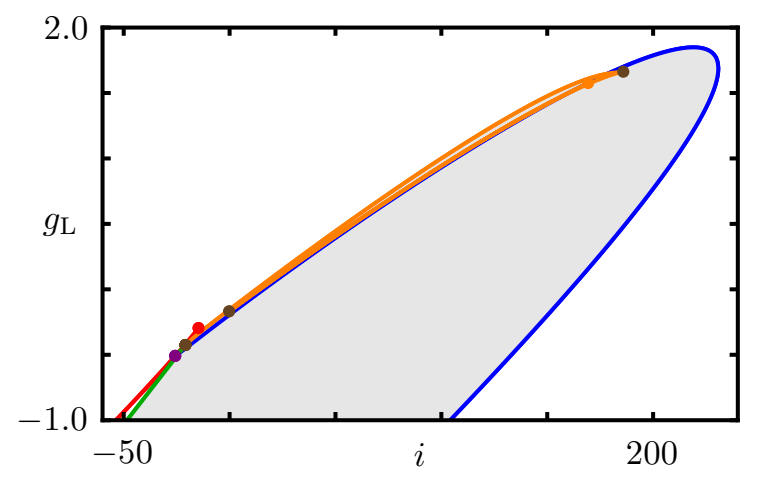

(b)

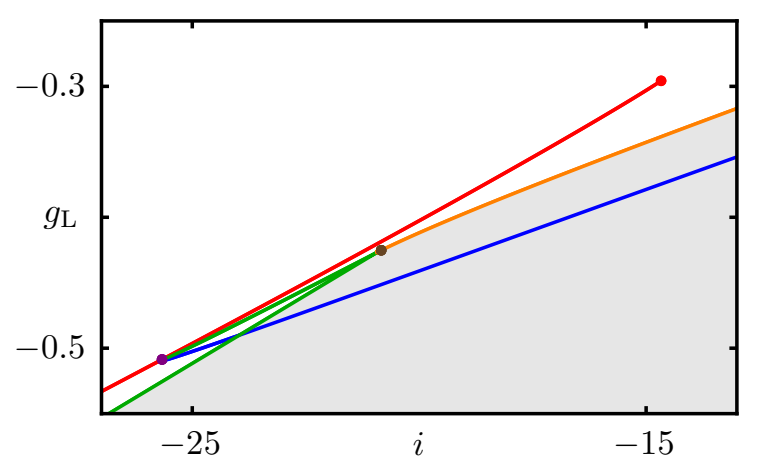

Figure 10.16. Not all type II neurons can be switched to type I neurons. Bifurcation diagrams of the Hodgkin-Huxley model as in sec. 2.4.1 and appendix D.1. (a) bifurcation diagram in the $\left(i_{\mathrm{e}}, g_{\mathrm{L}}\right)$-plane. For positive leak the neuron is type II as a limit cycle is generated via a DC bifurcation $(-)$. An increase of the leak leads to a switch of the limit cycle generation by a Hopf bifurcation (-) organized by a DH point $(\bullet)$. Not shown are a period doubling bifurcation and bifurcations to an invariant torus in the type II regime. (b) zoom into the a region of the diagram with negative leak values. Remnants of the transition for type I neuron as are observed, in particular a cusp $(\bullet)$, a BT point $(\bullet)$ and an NSL point $(\bullet)$. However, the dynamics in this region becomes unstable. See text for details. 
type I by a subtraction of leak conductance.

Decreasing the leak conductance to zero does not change the DC-Hopf bifurcation scenario (cf. fig. 10.16) and we thus conclude that for non-negative leak values the $\mathrm{HH}$ model cannot be switched to type I. Despite the fact that a negative leak conductance lacks biophysical plausibility, we decrease the leak to negative values to obtain further insight into type I - II transitions. At large enough negative values the bifurcation structure changes. In particular we find that the Hopf-bifurcation line ends in a BT point on a SN curve that is created in a cusp. Furthermore the DC bifurcation line ends at an NSL point on the homoclinic bifurcation line that starts at the BT point. The creation of a stable limit cycle via the DC bifurcation is switched at the NSL point implying a switch from type II to type I excitability akin to step (2) in sec. 10.2.1.2. Hence, we encounter remnants of the bifurcation diagram observed previously for type I neuron models, consistent with a dBT point still acting in the background. However, there is only single SN line emanating form the cusp. The reason for this is that the negative leak destabilizes the dynamics and the stable fixed point moves to infinity as the leak approaches the cusp point.

In summary, in the $\mathrm{HH}$ model a switch form type II dynamics to type I neuronal excitability is not possible by reduction in the leak conductance. Parts of the transitions structure observed for type I neurons remains, but in general a switch to type I is not possible due to the destabilization of the dynamics by negative leak conductances.

\subsection{Leak-Induced Neuronal Excitability Transitions - Mechanism and Analysis}

In this section we theoretically analyze neuronal excitability transitions using multiple bifurcation theory and normal form theory. To prepare the analysis, we first give an explanation of the biophysical mechanism underlying the neuronal excitability switch and then proceed with the general theory. By transforming a class of neuron models into a Liénard equation we further give a different interpretation of the mechanism in terms of non-linear accelerating and dampening forces. Finally we consider higher degenerate organizing centers for neuronal excitability .

\subsubsection{Biophysical Mechanism Underlying the Neuronal Excitability Switch}

In this section we discuss the biophysical mechanism underlying the transition from type I to type II neuronal excitability based on arguments in [316, 305] and obtain the critical leak conductance at the NSL point, the main step in the neuronal excitability transition.

To generate an action potential a positive feedback mechanism is required (cf. sec. 2.3 ). If the steady state $i$-v-curve $i_{\infty}(v)$ has a positive slope (negative resistance) for some values of the membrane potential this feedback mechanism works on arbitrarily slow time scales and the neuron can sustain spiking with arbitrarily large inter-spike-intervals.

On the contrary, if the steady state $i$ - $v$-curve has only negative slope the positive feedback required for spike generation can only be established if the fast depolarizing ionic currents $i_{\text {inst }}$ (e.g. the sodium current $i_{\mathrm{Na}}$ ) overcome the negative feedback of the slower delayed rectifiers, e.g $i_{\mathrm{K}}$. Thus, in this situation the spike process cannot become arbitrarily slow and the neuron exhibits type II neuronal excitability. 
(a) Type I

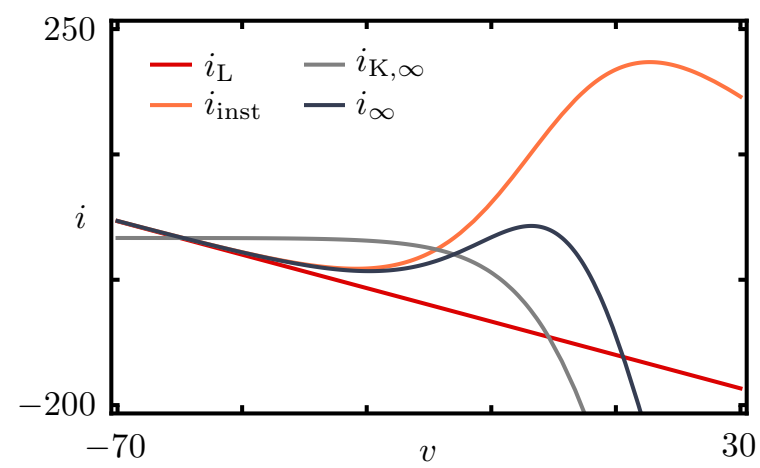

(b)

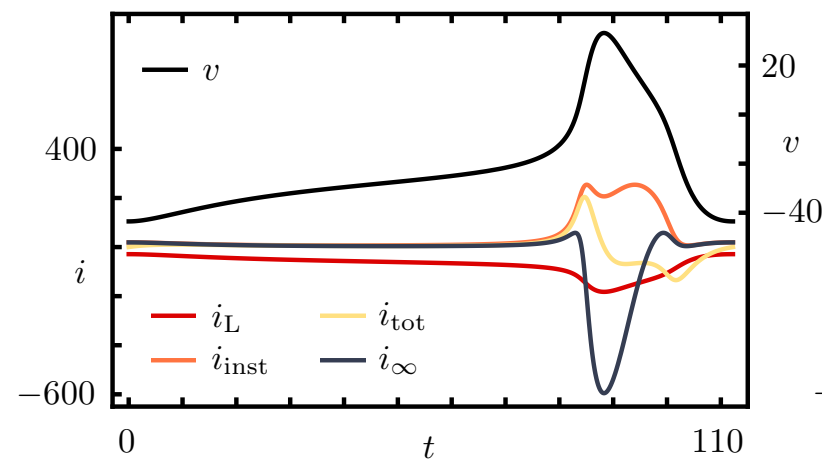

(c) Type II

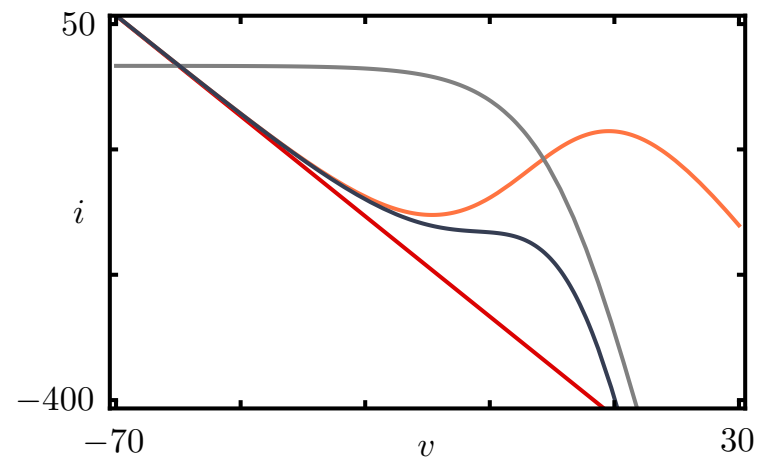

(d)

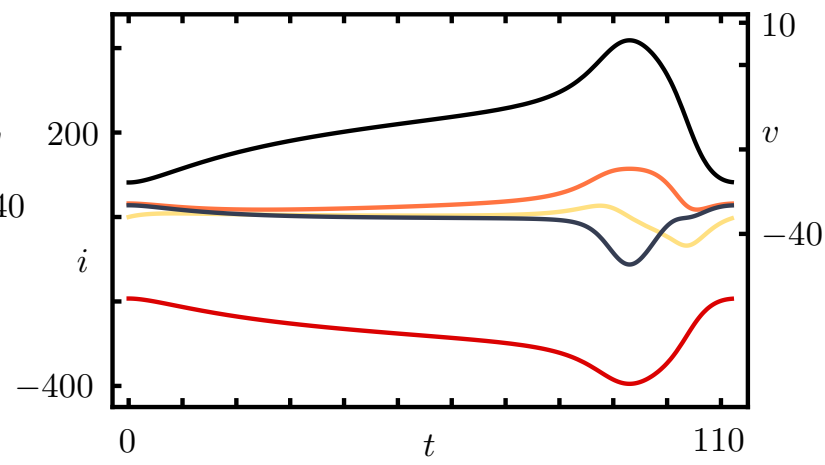

Figure 10.17. Biophysical mechanism of neuronal excitability switches via an increase in leak conductance. Curves are for the Morris-Lecar model, eq. 2.4.4 with standard parameter in appendix D.2 leading to type I excitability for $g_{\mathrm{L}}=2.0(\mathrm{a}, \mathrm{b})$ and to type II for $g_{\mathrm{L}}=6.0(\mathrm{c}, \mathrm{d})$. The external input current for (b) is $i_{\mathrm{e}}=43.5$ and for (d) $i_{\mathrm{e}}=196.0$ resulting in similar spike frequencies. (a,c) $i$ - $v$-curves for the leak current $i_{\mathrm{L}}$, the instantaneous current $i_{\text {inst }}=i_{\mathrm{L}}+i_{\mathrm{Na}}$, the steady state potassium current $i_{\mathrm{K}, \infty}=g_{\mathrm{K}} w_{\infty}(v)\left(v_{\mathrm{K}}-v\right)$ and the steady state current $i_{\infty}$ (eq. (10.3.1)). (b,d) Voltage and currents at periodic spiking. Note the positive slope of the total current $i_{\text {tot }}$ in both figures indicating active spiking dynamics. See text for details.

Now, changing the leak conductance $g_{\mathrm{L}}$ results in different negative slopes of the instantaneous leak current $i_{\mathrm{L}}(v)=g_{\mathrm{L}}\left(v_{\mathrm{L}}-v\right)$. As the steady state current $i_{\infty}$ is composed of the leak and the active steady state currents $i_{a, \infty}$,

$$
i_{\infty}=i_{\mathrm{e}}+i_{\mathrm{L}}+i_{\mathrm{a}, \infty}
$$

an increase in the leak conductance decreases the slope of the steady state $i$ - $v$-curve. Thus for a type I neuron that has positive slope of $i_{a, \infty}$ in some region of potential values $v$ the condition

$$
g_{\mathrm{L}}>g_{\mathrm{L}, 0}=\max _{v} \frac{d}{d v} i_{\mathrm{a}, \infty}
$$

is sufficient for $i_{\infty}$ to only have negative slope. As a result the neuron switches to a type II neuron if (10.3.2) is fulfilled. The process is illustrated in fig. 10.17. In the previous sections we have shown that the actual transition process from type I to type II is more complex and accompanied by a sequence of bifurcations. In particular there is a NSL point that organizes the second step in the transition, at which the existence of limit 
cycles with arbitrarily large period cease to exist. Thus eq. (10.3.2) gives the critical value for the leak to be at the NSL point.

Having obtained this first analytical result from biophysical reasoning, we proceed to a full analytical treatment in the next sections.

\subsubsection{Existence of degenerate Bogdanov-Takens Bifurcations in Conductance-Based Neuron Models}

In this chapter we study the neuronal excitability transition induced by leak changes analytically using a combination of a center-manifold reduction and normal form reduction technique $[231,276]$ together with multiple bifurcation theory $[144,145]$.

There are three main ingredients that facilitate an analytical treatment: First, the observation that the topological structure of the $\left(i_{\mathrm{e}}, g_{\mathrm{L}}\right)$-bifurcation diagrams computed for the various type I neuron models in sections 10.2.1.2 and 10.2.3 are the same and display properties akin to the degenerate Bogdanov-Takens bifurcation of focus type [87] introduced and discussed in sec. 3.3.3. Second, the fact that the bifurcation parameters $i_{\mathrm{e}}$ and $g_{\mathrm{L}}$ as well as a third parameter, the capacity $c_{\mathrm{m}}$, are general parameter that appear in the vector fields of all conductance based neuron models, with $i_{\mathrm{e}}$ and $g_{\mathrm{L}}$ appearing as coefficients of the constant and first order terms in $v$ only. Third, in conductance based neuron models the dynamics of the gating variables are coupled solely over the the membrane potential. These facts allow to prove the following theorem:

Theorem. Every conductance based type I neuron of the form (10.1.1) has a degenerate Bogdanov-Takens point of focus type in the $\left(i_{\mathrm{e}}, g_{\mathrm{L}}, c_{\mathrm{m}}\right)$-parameter space.

Proof. We make the biophysically plausible assumption, that all conductances $g_{k}$ in (10.1.2) are positive, that the steady state activations are bounded and positive,

$$
a_{j, \infty}(v) \in[0,1] \quad \text { for all } v \in \mathbb{R}
$$

and become sufficiently flat in the limits $v \rightarrow \pm \infty$

$$
\lim _{v \rightarrow \pm \infty} v^{l} \partial_{v}^{n} a_{j, \infty}(v)=0 \quad n \in\{1,2\}, \quad l \in\{0,1\}
$$

By introducing new parameters

$$
\alpha=\frac{1}{c_{\mathrm{m}}} \quad i=\frac{i_{\mathrm{e}}+g_{\mathrm{L}} v_{\mathrm{L}}}{c_{\mathrm{m}}} \quad \text { and } \quad g_{\mathrm{l}}=\frac{g_{\mathrm{L}}}{\mathrm{c}_{\mathrm{m}}}
$$

we rewrite the model (10.1.1) as

$$
\frac{d}{d t} x=\frac{d}{d t}\left(\begin{array}{c}
v \\
a
\end{array}\right)=\left(\begin{array}{c}
i-g_{1} v+\alpha i_{\mathrm{a}}(v, a) \\
\frac{1}{\tau}\left(a_{\infty}-a\right)
\end{array}\right)=\left(\begin{array}{c}
f_{v}(v, a) \\
f_{a}(v, a)
\end{array}\right)=f(v, a)=f(x)
$$

where we used the notation

$$
\frac{1}{\tau}=\operatorname{diag}\left(\frac{1}{\tau_{2}}, \ldots, \frac{1}{\tau_{N}}\right), \quad a_{\infty}(v)=\left(a_{2, \infty}(v), \ldots, a_{N, \infty}(v)\right)^{T}
$$

and the convention that component wise multiplication of two vectors $a$ and $b$ is denoted by $a b=\left(a_{1} b_{1}, \ldots, a_{N} b_{N}\right)$. To indicate a scalar product we explicitly include the transpose, i.e. $a^{T} b=\sum_{i} a_{i} b_{i}$. 
The idea of the proof is to show that one can always adjust the three parameters $\kappa=$ $\left(i, g_{1}, \alpha\right)$ in such a way that the system is at a codimension three dBT point at parameter values $\kappa_{\mathrm{dBT}}=\kappa_{0}=\left(i_{0}, g_{1,0}, \alpha_{0}\right)$ and some point in state space $x_{\mathrm{dBT}}=x_{0}=\left(v_{0}, a_{j, 0}\right)$. In order to show this, we will consider step by step all defining conditions for a dBT point in the following and show that they can be solved.

A dBT point is a fixed point and thus, setting the right hand side of (10.3.6) to zero the fixed point values $a_{j, 0}$ for the activation variables are uniquely determined by

$$
a_{j, 0}=a_{j, \infty}\left(v_{0}\right)
$$

given the steady state voltage $v_{0}$. Instead of solving for the membrane potential $v_{0}$ to satisfy the remaining fixed point equation obtained from (10.3.6) we use the observation that this equation can uniquely be solved for $i_{0}$ given the fixed point $x_{0}$ and parameters $g_{1,0}$ and $\alpha_{0}$ as:

$$
i_{0}=-g_{1,0} v_{0}-\alpha_{0} i_{\mathrm{a}}\left(v_{0}, a_{\infty}\left(v_{0}\right)\right)
$$

We define the steady state current reached when voltage-clamping the model at membrane potential $v$, i.e. the steady state $i$-v-curve (cf. sec. 2.8) as

$$
i_{\infty}(v):=f_{v}\left(v, a_{j, \infty}(v)\right)
$$

Besids being a fixed point, an ordinary Bogdanov-Takens point [358, 29] is characterized by a zero eigenvalue of algebraic multiplicity two (and geometric multiplicity 1), i.e. by a nilpotent Jacobian for the reduced dynamical system within a center manifold. Thus, for such a point $x_{0}$ we must demand the existence of four generalized eigenvectors $q_{0}, q_{1}, p_{0}$, $p_{1}$ of $A=\left.D f\right|_{x_{0}}$, the Jacobian at $x_{0}$, such that

$$
A q_{1}=q_{0} \quad A q_{0}=0 \quad A^{T} p_{1}=0 \quad A^{T} p_{1}=0 \quad p_{i}^{T} q_{j}=\delta_{i j}
$$

Note the effective exchange of the indices when exchanging $q$ and $p$ (and $A \leftrightarrow A^{T}$ ).

We now use the fact that the dynamics of the activation variables $a_{j}$ in a conductance based neuron model only couple via the membrane potential $v$. This is reflected in the special structure of $D f$ having a block diagonal matrix on the lower right:

$$
D f=\left(\begin{array}{cccc}
\partial_{v} f_{v} & \partial_{a_{2}} f_{v} & \ldots & \partial_{a_{N}} f_{v} \\
\partial_{v} f_{a_{2}} & -\frac{1}{\tau_{1}} & & 0 \\
\vdots & & \ddots & \\
\partial_{v} f_{a_{N-1}} & 0 & & -\frac{1}{\tau_{N-1}}
\end{array}\right)=\left(\begin{array}{cc}
\partial_{v} f_{v} & \partial_{a} f_{v}^{T} \\
\partial_{v} f_{a} & -\frac{1}{\tau}
\end{array}\right)
$$

We can thus solve the equations (10.3.10) using eq. (10.3.11) for all components $p_{j, i}$ and $q_{j, i}, j \in\{0,1\}$ and $i \geq 2$ to obtain the $q_{j}$ and $p_{j}$ in the form

$$
\begin{array}{ll}
q_{0}=q_{0,1}\left(\begin{array}{c}
1 \\
\tau \partial_{v} f_{a}
\end{array}\right) & p_{1}=p_{1,1}\left(\begin{array}{c}
1 \\
\tau \partial_{a} f_{v}
\end{array}\right) \\
q_{1}=\left(\begin{array}{c}
q_{1,1} \\
\left(q_{1,1}-q_{0,1} \tau\right) \tau \partial_{v} f_{a}
\end{array}\right) & p_{0}=\left(\begin{array}{c}
p_{0,1} \\
\left(p_{0,1}-p_{1,1} \tau\right) \tau \partial_{a} f_{v}
\end{array}\right)
\end{array}
$$

The remaining equations for the first component $i=1$ then become

$$
\begin{aligned}
q_{0,1}\left(\partial_{v} f_{v}+\partial_{a} f_{v}^{T}\left(\tau \partial_{v} f_{a}\right)\right) & =0 \\
q_{1,1}\left(\partial_{v} f_{v}+\partial_{a} f_{v}^{T}\left(\tau \partial_{v} f_{a}\right)\right)-q_{0,1}\left(1+\left(\tau \partial_{a} f_{v}\right)^{T}\left(\tau \partial_{v} f_{a}\right)\right) & =0 \\
p_{1,1}\left(\partial_{v} f_{v}+\partial_{v} f_{a}^{T}\left(\tau \partial_{a} f_{v}\right)\right) & =0 \\
p_{0,1}\left(\partial_{v} f_{v}+\partial_{a} f_{v}^{T}\left(\tau \partial_{v} f_{a}\right)\right)-p_{1,1}\left(1+\left(\tau \partial_{a} f_{v}\right)^{T}\left(\tau \partial_{v} f_{a}\right)\right) & =0
\end{aligned}
$$


As the generalized eigenvectors should be non-zero, we must have $q_{0,1} \neq 0 \neq p_{1,1}$ and thus the above linear system can only be solved if the determinant of the coefficients for $q_{j, 1}$ and $p_{j, 1}$ vanish. This condition is equivalent to the following two equations

$$
\begin{aligned}
\partial_{v} f_{v}+\partial_{a} f_{v}^{T}\left(\tau \partial_{v} f_{a}\right) & =0 \\
\left(1+\left(\tau \partial_{a} f_{v}\right)^{T}\left(\tau \partial_{v} f_{a}\right)\right) & =0
\end{aligned}
$$

These equations render the fixed point $x_{0}$ to be an ordinary Bogdanov-Takens point (cf. also sec. 3.3.2.2). Using (10.3.6) and

$$
\partial_{v} f_{a}=\frac{1}{\tau}\left(\partial_{v} a_{\infty}-\frac{\partial_{v} \tau}{\tau}\left(a_{\infty}-a\right)\right) \quad \text { and thus }\left.\quad \partial_{v} f_{a}\right|_{x_{0}}=\left.\frac{1}{\tau} \partial_{v} a_{\infty}\right|_{x_{0}}
$$

as $a=a_{\infty}$ at $x_{0}$ via the fixed point condition (10.3.7). Hence the first BT point condition (10.3.13) is equivalent to

$$
\left.\frac{d}{d v} i_{\infty}\right|_{x_{0}}=0
$$

Note that this equation is also equivalent to the condition that the determinant at $x_{0}$ given by

$$
\left.\operatorname{det}(D f)\right|_{x_{0}}=\left.\frac{(-1)^{N-1}}{\prod_{j} \tau_{j}}\left(\partial_{v} f_{v}+\sum_{j} \partial_{v} a_{j, \infty} \partial_{a_{j}} f_{v}\right)\right|_{x_{0}}=\left.\frac{(-1)^{N-1}}{\prod_{j} \tau_{j}} \frac{d}{d v} i_{\infty}\right|_{x_{0}}
$$

evaluates to zero indicating the position of a fold point in general. Note further, that the fold condition (10.3.15) is an equation linear in the two parameters $g_{1}$ and $\alpha$ and can be solved uniquely for $g_{1}$ to give

$$
g_{1,0}=\left.\alpha_{0} \frac{d}{d v} i_{\mathrm{a}}\left(v, a_{\infty}(v)\right)\right|_{v_{0}}=\left.\alpha_{0} \frac{d}{d v} i_{\mathrm{a}, \infty}(v)\right|_{v_{0}}
$$

The next step is to solve the second BT point condition (10.3.14) for the parameter $\alpha$ which gives

$$
\alpha_{0}^{-1}=-\left(\partial_{a} i_{\mathrm{a}}\left(v_{0}, a_{\infty}\left(v_{0}\right)\right)^{T} \tau \partial_{v} a_{\infty}\left(v_{0}\right)\right)
$$

Note that for a two dimensional system this condition becomes $\left.\operatorname{tr}(D f)\right|_{x_{0}}=0$ and together with (10.3.16) this again shows that these are the conditions for a ordinary BT point. Using this information the second equation for a $2 \mathrm{~d}$ system then takes the form

$$
\alpha_{0}^{2 \mathrm{~d}}=\left(\tau_{2} \partial_{v} i_{\mathrm{a}}\right)^{-1}\left(1+\tau_{2} g_{1,0}\right)
$$

Finally, we calculate the equation that forces the BT point to be degenerate. A BT point is degenerate, if, in a two dimensional center manifold with coordinates $\left(w_{0}, w_{1}\right)$, the vector field $f$ up to order $\mathcal{O}^{2}\left(w_{0}, w_{1}\right)$, also called the 2-jet [276], $J_{2} f$ of $f$, has the following form

$$
J_{2} f \sim w_{1} \frac{\partial}{\partial w_{0}}+\left(\gamma w_{0}^{2}+\beta w_{0} w_{1}\right) \frac{\partial}{\partial w_{1}}
$$

with the degeneracy condition being either $\gamma \neq 0, \beta=0$ or $\gamma=0, \beta \neq 0[87,86,85]$. A degenerate BT point of focus type falls into the second class. We therefore now show that we can tune the fixed point value $v_{0}$ such that $\gamma=0$. 
The normal form coefficient $\gamma$ can be calculated using center manifold theory and imposing the Fredholm solvability conditions [231] (also cf. appendix F for a complete derivation of this non-standard result). This yields an expression for $\gamma$ in terms of the generalized eigenvectors $q$ and $p$ as

$$
\gamma=\frac{1}{2} p_{1}^{T} D^{2} f\left(x_{0}, q_{0}, q_{0}\right)
$$

where $D^{2} f$ is the Hessian quadratic form of the vector field $f$ at $x$ defined in eq. (3.1.2). Using the expressions (10.3.12) for the generalized eigenvectors we obtain

$$
\begin{aligned}
\frac{1}{q_{0,1}^{2}} D^{2} f_{v}\left(q_{0}, q_{0}\right) & =\partial_{v}^{2} f_{v}+2\left(\partial_{a} \partial_{v} f_{v}\right)^{T}\left(\tau \partial_{v} f_{a}\right)+\partial_{a}^{2} f_{v}\left(\tau \partial_{v} f_{a}, \tau \partial_{v} f_{a}\right) \\
\frac{1}{q_{0,1}^{2}} D^{2} f_{a}\left(q_{0}, q_{0}\right) & =\partial_{v}^{2} f_{a}+2\left(\partial_{v} \partial_{a} f_{a}\right)\left(\tau \partial_{v} f_{a}\right)+\partial_{a}^{2} f_{a}\left(\tau \partial_{v} f_{a}, \tau \partial_{v} f_{a}\right) \\
& =\partial_{v}^{2} f_{a}+2\left(\frac{\partial_{v} \tau}{\tau^{2}}\right)\left(\tau \partial_{v} f_{a}\right)
\end{aligned}
$$

where in the second equation we used $\partial_{a} f_{a}=-\frac{1}{\tau}$ and $\partial_{a}\left(-\frac{1}{\tau}\right)=0$. Thus

$$
\begin{aligned}
\frac{2}{p_{1,1} q_{0,1}^{2}} \gamma= & \partial_{v}^{2} f_{v}+2\left(\partial_{a} \partial_{v} f_{v}\right)^{T}\left(\tau \partial_{v} f_{a}\right)+\partial_{a}^{2} f_{v}\left(\tau \partial_{v} f_{a}, \tau \partial_{v} f_{a}\right) \\
& +\left(\partial_{a} f_{v}\right)^{T}\left(\tau \partial_{v}^{2} f_{a}\right)+2\left(\tau \partial_{a} f_{v}\right)^{T}\left(\frac{\partial_{v} \tau}{\tau^{2}}\right)\left(\tau \partial_{v} f_{a}\right)
\end{aligned}
$$

Using

$$
\left.\partial_{v}^{2} f_{a}\right|_{x_{0}}=\frac{1}{\tau} \partial_{v}^{2} a_{\infty}-\left.2 \frac{\partial_{v} \tau}{\tau^{2}} \partial_{v} a_{\infty}\right|_{x_{0}}
$$

we obtain

$$
\begin{aligned}
\left.\frac{2}{p_{1,1} q_{0,1}^{2}} \gamma\right|_{x_{0}} & =\partial_{v}^{2} f_{v}+2\left(\partial_{v} \partial_{a} f_{v}\right)^{T} \partial_{v} a_{\infty}+\partial_{a}^{2} f_{v}\left(\partial_{v} a_{\infty}, \partial_{v} a_{\infty}\right)+\left.\left(\partial_{a} f_{v}\right)^{T} \partial_{v}^{2} a_{\infty}\right|_{x_{0}} \\
& =\left.\frac{d^{2}}{d v^{2}} i_{\infty}\right|_{x_{0}}
\end{aligned}
$$

The degeneracy condition for the BT point thus becomes

$$
\left.\frac{d^{2}}{d v^{2}} i_{\infty}\right|_{x_{0}}=0
$$

Now we use the second ingredient, namely that the $i$ and $g_{1}$ are coefficients of the constant and linear part of the vector field $f$ so that $\frac{d^{2}}{d v^{2}} i_{\infty}$ does not depend on $i$ and $g_{1}$. It further is proportional to $\alpha \neq 0$, so that the condition can be solved for $v_{0}$ independently of the choice of the parameters $\left(i, g_{\mathrm{L}}, \alpha\right)$.

To show the existence of a solution of (10.3.19) we use the precondition that the neuron has type I excitability and is capable of generating periodic spiking activity with arbitrarily large inter-spike-intervals. This implies that there is a voltage regime with positive feedback in the adiabatic limit, i.e. the steady state $i$-v-curve $i_{\infty}$ must have a positive slope for some voltage $v_{+} \in \mathbb{R}$ (cf. also sec. 10.17). We thus have for the non-tuned initial parameter set

$$
\frac{d}{d v} i_{\infty}\left(v_{+}\right)>0
$$


Observe that $i_{\infty}$ has the form $i_{\infty}=q(v)-p(v) v$ with $q(v)$ and $p(v)$ being polynomials in the variables $a_{j, \infty}(v)$ only. As the steady state activations are bounded and positive (10.3.3) and become flat for $v \rightarrow \pm \infty$ (10.3.4) we have for $n \in\{1,2\}, l \in\{0,1\}$

$$
\lim _{v \rightarrow \pm \infty} v^{l} \partial_{v}^{n} q(v)=\lim _{v \rightarrow \pm \infty} v^{l} \partial_{v}^{n} p(v) \rightarrow 0
$$

and thus $i_{\infty}$ approaches a straight line for $v \rightarrow \pm \infty$. Hence

$$
\lim _{v \rightarrow \pm \infty} \frac{d^{2}}{d v^{2}} i_{\infty}(v)=0
$$

Furthermore, as all maximal conductances and activation variables are positive we have

$$
p(v) \geq g_{1}>0
$$

and thus

$$
\lim _{v \rightarrow \pm \infty} \frac{d}{d v} i_{\infty}(v) \leq-g_{1}
$$

Combining equations (10.3.20) and (10.3.22) it follows that $\partial_{v} i_{\infty}$ must have at least one local maximum and hence there is a $v_{0} \in \mathbb{R}$ such that $\left.\frac{d^{2}}{d v^{2}} i_{\infty}(v)\right|_{v_{0}}=0$. Note here, that if we take $v_{0}$ at the maximum, eq. (10.3.17), expressed in the in original parameter, becomes eq. (10.3.2) derived in sec. 10.17 from biophysical considerations.

Moreover, combining (10.3.20) with (10.3.17) shows that choosing $v_{0}$ at such a maximum we have

$$
\operatorname{sign}\left(\mathrm{g}_{1,0}\right)=\operatorname{sign}\left(\alpha_{0}\right)
$$

Finally, given $v_{0}$ that solves the degeneracy condition (10.3.19) the equations (10.3.8), (10.3.17) and (10.3.18) show that there exists a parameter set $\left(i_{0}, g_{l, 0}, \alpha_{0}\right)$ such that $x_{0}$ is a degenerate BT point.

This shows the existence of a dBT point in all type I conductance based neuron models. In principle, one can calculate the remaining normal form coefficients analytically using the method in [231] (cf. also appendix F) to construct conditions for the dBT point to be of focus type. We use a simpler approach to show that the dBT point is of focus type by employing the following two facts: For $v \rightarrow \pm \infty$ the leak conductance $g_{\mathrm{L}}$ dominates the dynamics as the activation variables become flat. Using (10.3.23) implies via (10.3.5) that

$$
\operatorname{sign}\left(g_{\mathrm{L}, 0}\right)=\operatorname{sign}\left(g_{1,0}\right) \operatorname{sign}\left(c_{\mathrm{m}}\right)=\operatorname{sign}\left(g_{1,0}\right) \operatorname{sign}\left(\alpha_{0}\right)>0
$$

and hence $g_{\mathrm{L}, 0}>0$ and the overall dynamics is bounded. In [87] it is shown that in the bifurcation diagrams for the degenerate BT points there are always unbounded dynamics in the the saddle and the elliptic case, leaving only the focus type point with bonded dynamics as a consistent alternative.

Note also, that we had to demand type I excitability in order to ensure the existence of a dBT point of focus type due to a positive value for $g_{\mathrm{L}, 0}$ via (10.3.24) and (10.3.23). For type II neurons a dBT may still exist, however, one cannot exclude negative values of $g_{\mathrm{L}, 0}$ leading to unbounded dynamics and to either an elliptic or saddle dBT point.

Note, that in general there can be more than one solution to eq. (10.3.19). For the neuron models studied above we typically find two solutions where the second one corresponds to a minimum of $\frac{d}{d v} i_{\infty}$. In this case $g_{1,0}$ has the opposite sing of $\alpha_{0}$, rendering 
the leak conductance $g_{\mathrm{L}, 0}$ negative, which due to the unbounded dynamics again indicates less biophysical relevance of this point.

Note further that the existence of other degenerate bifurcation points closer to the original parameter plane may alter the fine structure of the bifurcation diagram. For example, in the original Connor-Stevens model, eq. (2.4.6), we observe that in the transition from type I to type II the $\left(i_{\mathrm{e}}, g_{\mathrm{L}}\right)$-bifurcation diagram shows additional signatures of a nearby swallow tail bifurcation (cf. [145] for a definition) as there are two additional cusp points leading in some parts of the $\left(i_{\mathrm{e}}, g_{\mathrm{L}}\right)$-parameter space to the existence of five fixed points of which four are unstable. However the three-fold transition from type I to type II excitability as discussed earlier in sec. 10.2.1.2 remains present.

To summarize, we have analytically shown the existence of dBT points in all conductance based type I neuron models. The existence of such a point of focus type implies the existence of the three organizing centers of co-dimension two, a NSL a SNL and a DH bifurcation that we observed in the transition from type I to type II neuronal excitability as well as a BT bifurcation that organizes the transition observed in peri-threshold resonance. We found that the presence of additional degenerate points can alter the fine-structure of the transition but leave the overall organization of the transition intact. Combining these facts together with the numerical results for the various neurons considered above, we conclude that the structure of the transition from type I to type II neuronal excitability is generally organized by a degenerate Bogdanov-Takens point in the background.

\subsubsection{Neuronal Excitability, Morris-Lecar Type Neuron Models and Liénard's Equation}

We have seen above that type I neuron models have a dBT point of focus type. The dBT point has an unfolding given by eq. (10.2.1). This unfolding is versal [87] in the sense that every two dimensional system (or a reduced system on a two dimensional center manifold) that depends on three parameters (e.g. $\left.\kappa=\left(i_{\mathrm{e}}, g_{\mathrm{L}}, c_{\mathrm{m}}\right)\right)$ and is close to a dBT point can be transformed ${ }^{2}$ into the unfolding (10.2.1), whereby the unfolding parameter $\mu_{i}$ become functions of the original parameter $\kappa$.

Further more the unfolding eq. (10.2.1) can be directly transformed into a single second order differential equation of the form

$$
\ddot{x}=F(x)+\dot{x} G(x)
$$

This is a Liénard type equation originally introduced to study oscillating electrical circuits [243]. The physical interpretation of a Liénard equation is that of a particle of mass 1 at position $x$ with space dependent accelerating force $F(x)$ and dampening $-G(x)$. Alternatively on can think of an electrical current $x$ in an LRC-circuit with non-linear resistance and inductivity. A special case of a Liénard type equation is the van der Pol oscillator [385]. From a mathematical point of view the Liénard equation is interesting since one can formulate precise conditions for the existence and number of limit cycles [84]. Combining this observation and the versality of the unfolding with theorem (10.3.2) shows:

Corollary 10.3.1. Every two dimensional type I conductance based neuron of the form (10.1.1) can be transformed into a Liénard equation.

\footnotetext{
${ }^{2}$ For the dBT point the equivalence relation is defined via $C^{\infty}$-fiber equivalence [87], an even weaker form of equivalence introduced in (3.2.1)
} 
The general theory only asserts the existence of such a transformation [87, 276, 134]. The goal of this section is to derive this transformation for a sub-class of neuron models analytically.

We will consider two dimensional neuron models of the form

$$
f_{\kappa}(x, y)=\left(\begin{array}{c}
a_{\kappa}(x)+y b_{\kappa}(x) \\
c_{\kappa}(x)+y d_{\kappa}(x)
\end{array}\right)
$$

where $a, b, c$ and $d$ are arbitrary smooth functions for some $n \in \mathbb{N}$ and the subscript $\kappa$ denotes an arbitrary parameter dependence. For example, the Morris-Lecar neuron (2.4.2) is a special case of (10.3.26) when setting $(x, y)=(v, w)$ and

$$
\begin{array}{ll}
a_{\kappa}(v)=\frac{1}{c_{\mathrm{m}}}\left(i+g_{\mathrm{L}}\left(v_{\mathrm{L}}-v\right)+g_{\mathrm{Na}} m_{\infty}(v)\left(v_{\mathrm{Na}}-v\right)\right) & b_{\kappa}(v)=\frac{1}{c_{\mathrm{m}}} g_{\mathrm{K}}\left(v_{\mathrm{K}}-v\right) \\
c_{\kappa}(v)=\frac{1}{\tau_{w}(v)} w_{\infty}(v) & d_{\kappa}(v)=-\frac{1}{\tau_{w}(v)}
\end{array}
$$

We therefore also refer to this class as Morris-Lecar type neurons.

Our goal is to transform (10.3.26) in to (10.3.25). A naive approach using the transformation $x \rightarrow x, y \rightarrow a_{\kappa}(x)+y b_{\kappa}(x)$ induces (via eq. (3.2.1)) a term proportional to $y^{2}$ in the second component of the transformed vector field and thus does not yield a Liénard equation. However, we can employ normal form theory to solve this problem. It suggests (via the homological equations [276]) to remove non-resonant terms of order $y^{2}$ and higher in the second component of the vector field by introducing terms of order $x^{2}$ and higher in the coordinate transformation for $x[358,145]$. We thus consider the more general transformation

$$
x \rightarrow u_{\kappa}(x), y \rightarrow r_{\kappa}(x)+s_{\kappa}(x) y
$$

It results in the transformed vector field

$$
g_{\kappa}(x, y)=\left(\begin{array}{c}
\frac{a_{\kappa}\left(u_{\kappa}(x)\right)+b_{\kappa}\left(u_{\kappa}(x)\right)\left(r_{\kappa}(x)+s_{\kappa}(x) y\right)}{u_{\kappa}^{\prime}(x)} \\
\frac{c_{\kappa}\left(u_{\kappa}(x)\right)+d_{\kappa}\left(u_{\kappa}(x)\right)\left(r_{\kappa}(x)+s_{\kappa}(x) y\right)}{s_{\kappa}(x)}-\frac{\left(a_{\kappa}\left(u_{\kappa}(x)\right)+b_{\kappa}\left(u_{\kappa}(x)\right)\left(r_{\kappa}(x)+s_{\kappa}(x) y\right)\right)\left(r_{\kappa}^{\prime}(x)+s_{\kappa}^{\prime \prime}(x) y\right)}{s_{\kappa}(x) u_{\kappa}^{\prime}(x)}
\end{array}\right)
$$

where ' denotes the derivative with respect to $x$. To obtain $y$ in the first component of the vector field we must have

$$
r_{\kappa}(x)=-\frac{a_{\kappa}\left(u_{\kappa}(x)\right)}{b_{\kappa}\left(u_{\kappa}(x)\right)} \quad \text { and } \quad s_{\kappa}(x)=\frac{u_{\kappa}^{\prime}(x)}{b_{\mu}\left(u_{\kappa}(x)\right)} .
$$

As for the naive approach, the second component of the vector field is quadratic in $y$ due to the term

$$
y^{2} \frac{b_{\kappa}\left(u_{\kappa}(x)\right) s_{\kappa}^{\prime}(x)}{u_{\kappa}^{\prime}(x)}
$$

unless we determine $u_{\kappa}, s_{\kappa}$ and $r_{\kappa}$ in such a way to make it vanish. Using (10.3.29) this requirement becomes

$$
\frac{b_{\kappa}^{\prime}\left(u_{\kappa}(x)\right) u_{\kappa}^{\prime}(x)^{2}-b_{\kappa}\left(u_{\kappa}(x)\right) u_{\kappa}^{\prime}(x)}{b_{\kappa}\left(u_{\kappa}(x)\right) u_{\kappa}^{\prime}(x)}=\frac{d}{d x} \log \frac{b_{\kappa}\left(u_{\kappa}(x)\right)}{u_{\kappa}^{\prime}(x)}=0
$$

which gives a differential equation for the transformation $u_{\kappa}$ of the form

$$
u_{\kappa}^{\prime}(x)=C_{\kappa, 1} b_{\kappa}\left(u_{\kappa}(x)\right)
$$


with some integration constant $C_{\kappa, 1}>0$ that may depend on $\kappa$. The general solution to this equation is

$$
u_{\kappa}(x)=t_{\kappa}^{-1}\left(C_{\kappa, 1} x+C_{\kappa, 0}\right) \quad \text { with } \quad t_{k}(x)=\int_{1}^{x} \frac{1}{b_{\kappa}(\tilde{x})} d \tilde{x}
$$

with another integration constant $C_{\kappa, 0}$. By demanding $u_{\kappa}(0)=0$ and $u_{\kappa}^{\prime}(0)=1$ to obtain a near identity transformation we find $C_{\kappa, 0}=1$ and $C_{\kappa, 1}=\frac{1}{b_{\kappa}(0)}$. Now, choosing the transformations according to (10.3.29) and (10.3.30) we obtain the following general result of this section:

Proposition 10.3.2. A Morris-Lecar type neuron of the form (10.3.26) is conjugated to a Liénard equation of the form (10.3.25)

$$
\ddot{x}=F_{\kappa}(x)+\dot{x} G_{\kappa}(x)
$$

with

$$
\begin{aligned}
F_{\kappa}(x) & =\frac{b_{\kappa}(0)}{b_{\kappa}\left(u_{\kappa}(x)\right)}\left(c_{\kappa}\left(u_{\kappa}(x)\right) b_{\kappa}\left(u_{\kappa}(x)\right)-a_{\kappa}\left(u_{\kappa}(x)\right) d_{\kappa}\left(u_{\kappa}(x)\right)\right) \\
G_{\kappa}(x) & =d_{\kappa}\left(u_{\kappa}(x)\right)+a_{\kappa}^{\prime}\left(u_{\kappa}(x)\right)-\frac{a_{\kappa}\left(u_{\kappa}(x)\right) b_{\kappa}^{\prime}\left(u_{\kappa}(x)\right)}{b_{\kappa}\left(u_{\kappa}(x)\right)}
\end{aligned}
$$

Note, that this form can be used as a starting point to calculate the unfolding parameter $\mu_{i}$ in the dBT normal form (10.2.1). However, these steps involve removing further non-resonant terms by applying the Weierstrass preparation theorem [345, 87] and can be done only for a truncated expansion of the vector field in the variables and relevant parameters. We refer the reader to the next section 10.3.4, where this procedure is applied to the Fitz-Hugh-Nagumo model (2.6.6).

For the original Morris-Lecar neuron (10.3.27) $b_{k}(x)$ is linear in $x$, so that we can solve (10.3.30) to explicitly obtain

$$
v=u_{\kappa}(x)=v_{\mathrm{K}}\left(1-\exp \left(-\frac{x}{v_{\mathrm{K}}}\right)\right)
$$

and thus

$$
\begin{aligned}
F_{\kappa}(x) & =\frac{1}{c_{\mathrm{m}} \tau_{w}(v)} e^{\frac{x}{v_{\mathrm{K}}}} i_{\infty}(v) \\
G_{\mu}(x) & =\frac{1}{c_{\mathrm{m}}}\left(-\frac{c_{\mathrm{m}}}{\tau_{w}(v)}-i_{\text {inst }}^{\prime}(v)+\frac{1}{v_{k}} e^{\frac{x}{v_{\mathrm{K}}}} i_{\text {inst }}(v)\right)
\end{aligned}
$$

for the Liénard equation (10.3.25) where $i_{\infty}(v)=i_{\text {inst }}(v)+g_{\mathrm{K}} w_{\infty}(v)\left(v_{\mathrm{K}}-v\right)$ and $i_{\text {inst }}(v)=$ $i_{\mathrm{e}}+g_{\mathrm{L}}\left(v_{\mathrm{L}}-v\right)+g_{\mathrm{Na}} m_{\infty}(v)\left(v_{\mathrm{Na}}-v\right)$ are the steady state and instantaneous currents as discussed in sec. 10.17 .

We thus obtained a conjugation between ML type neurons and the Liénard equation (10.3.25). This provides a novel interpretation of neuronal dynamics in terms of a particle of mass 1 at position $x$ subject to a position dependent acceleration force $F$ and dampening $-G$ as depicted in fig. 10.18. Moreover, besides the biophysical interpretation (cf. sec. 10.3.1) we obtain a second explanation of the mechanisms underlying type I to type II neuronal excitability transition: For type I dynamics $F$ has a region with positive slope 
(a)

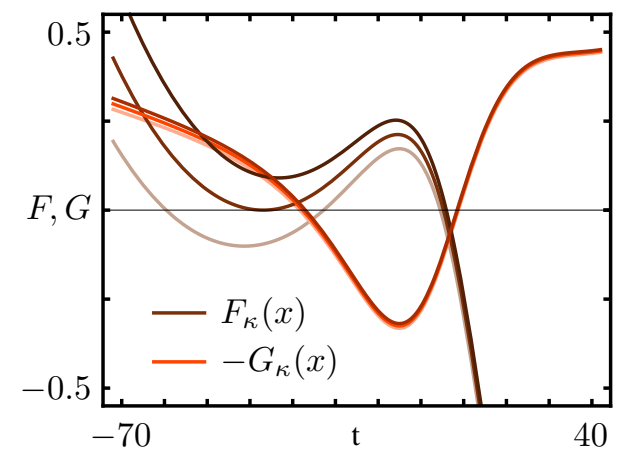

(c)

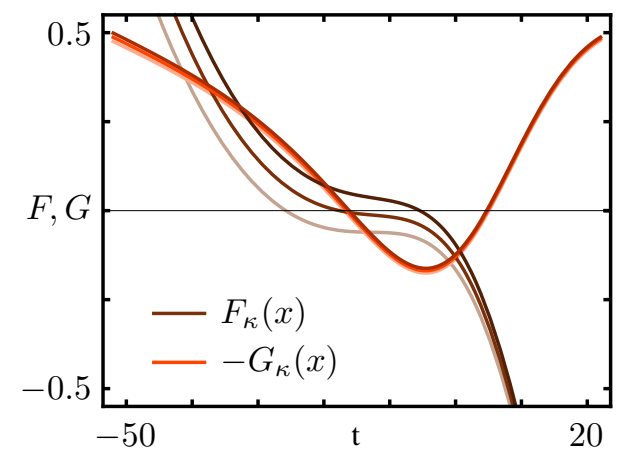

(b)

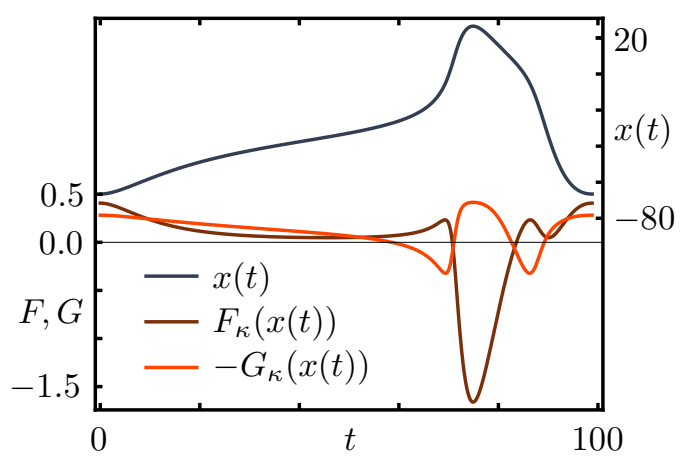

(d)

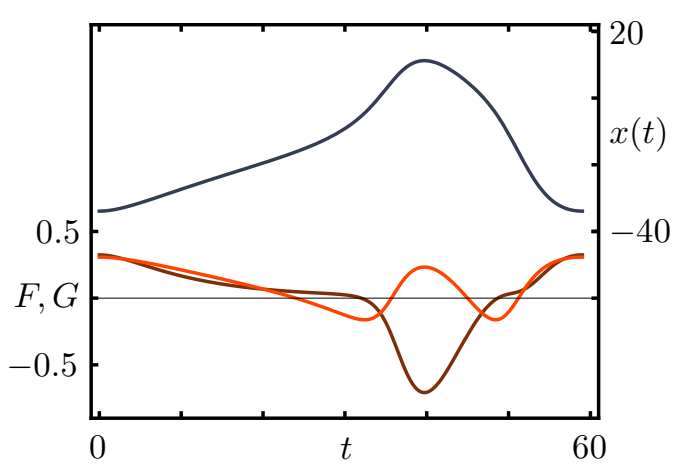

Figure 10.18. Neuronal Excitability transition in the Morris-Lecar Neuron in Liénard form (10.3.31) and (10.3.32) using standard parameter. (a) Acceleration force $F_{\kappa}$ and dampening $-G_{\kappa}$ as a function of position $x=v_{\mathrm{K}} \log \left(\frac{v_{\mathrm{K}}}{v_{\mathrm{K}}-v}\right)$ for $g_{\mathrm{L}}=2.0$, below ( $i_{\mathrm{e}}=40$, lighter color), at $\left(i_{\mathrm{e}}=45\right)$, and above threshold $\left(i_{\mathrm{e}}=50\right.$, darker color $)$. The type I excitability is present by a region of positive slope in the accelerating force that leads to a saddle node bifurcation at onset of spiking. (b) One period of the spiking activity for the parameters $g_{\mathrm{L}}=2.0, i_{\mathrm{e}}=50$ above threshold. Shown are the time dependence of the transformed potential $x$, the accelerating force $F_{\kappa}$ and the dampening $-G_{\kappa}$ as a function of time $t$. In the early phase of the cycle $x$ is in the range where $F_{\kappa}$ and $-G_{\kappa}$ are of the same sign and similar in absolute value so that the increase in $x$ is slow. At $x \approx-30$ the dampening gets negative while the acceleration increases resulting in the fast rise of $x$ at action potential onset. The situation reverts for large $x \approx 10$ leading to the repolarization. (c,d) same as in $(\mathrm{a}, \mathrm{b})$ with $g_{\mathrm{L}}=6.0, i_{\mathrm{e}}=186,196,206$. While the dampening has not changed qualitatively in comparison with $(a, b)$ the acceleration force now does monotonically decrease. The type II neuronal excitability at threshold in this picture can be explained by the observation that a positive acceleration needed to generate a spike is only possible via the negative part of the dampening. This demands a non-zero velocity which in turn implies that a non-zero spike frequency is required for periodic spiking.

and a minimum. An increase in the input current mainly shifts $F$ upwards and thus induces a saddle node bifurcation at the point where the minimum touches the $\mathrm{x}$-axis. For input currents just above this point the acceleration force is arbitrarily small and as for slow velocities the dampening term can be ignored the periodic spike generation can be arbitrarily slow. In contrast, for type II dynamics $F$ is monotonically decaying. In this situation a positive acceleration needed to generate a spike is only achievable via the negative dampening term. For the dampening to become effective a non-zero velocity is required which in turn implies that a non-zero spike frequency is required for periodic 
spiking (cf. fig. 10.18).

In the next sec. 10.3.4 we apply the transformation in proposition 10.3.2 in the first step when calculating the dBT unfolding for the Fitzthugh-Nagumo model.

\subsubsection{Degenerate Bogdanov-Takens Points of Higher Codimen- sion in Neuronal Models}

So far we have focused on degenerate Bogdanov-Takens-cusp points with co-dimension-3. From the viewpoint of multiple bifurcation theory higher degenerate points organize the bifurcation diagrams of smaller co-dimension. It is therefore reasonable to ask if higher degenerate bifurcation points in neuron models exists and what consequences for the dynamics one can infer from such findings. Note however, that this approach is limited by the fact that not much about bifurcation points of larger co-dimensions and especially in higher dimensional state spaces are know [233, 405]. In this section we identify a degenerate Bogdanov-Takens point of co-dimension-4 in the Fitzthugh-Nagumo model (cf. sec. 2.6.3). This point has an unfolding conjugated to a cubic Linéard equation.

We employ the fact that the Fitzhugh-Nagumo model (2.6.6) is an abstract neuron model that has a polynomial vector field given by the equations

$$
\begin{aligned}
c_{\mathrm{m}} \frac{d}{d t} v & =i_{\mathrm{e}}+i_{\mathrm{L}}(v)+i_{\mathrm{a}}(v)-w \\
\frac{d}{d t} w & =l_{1}\left(v-l_{2} w\right)
\end{aligned}
$$

with $i_{a}(v)=\left(m_{1}-v\right)\left(m_{2}-v\right)\left(m_{3}-v\right)$ and we have added an explicit leak current $i_{\mathrm{L}}=g_{\mathrm{L}}\left(v_{\mathrm{L}}-v\right)$ and a capacity $c_{\mathrm{m}}$ in the first equation. Note that this model is of Morris-Lecar type (10.3.26) with

$$
\begin{array}{rlrl}
a_{\kappa}(v) & =\frac{1}{c_{\mathrm{m}}}\left(i_{\mathrm{e}}+g_{\mathrm{L}}\left(v_{\mathrm{L}}-v\right)+i_{\mathrm{a}}(v)\right) & b_{\kappa}(v) & =-\frac{1}{c_{\mathrm{m}}} \\
c_{\kappa}(v) & =l_{1} v & d_{\kappa}(v)=-l_{1} l_{2}
\end{array}
$$

for $\kappa=\left(i_{\mathrm{e}}, g_{\mathrm{L}}, v_{\mathrm{L}}, m_{1}, m_{2}, m_{3}, l_{1}, l_{2}\right)$. Using the results form the previous sec. 10.3.3 we find that the transformations

$$
v \rightarrow x \quad w \rightarrow i_{\mathrm{e}}+i_{\mathrm{L}}(x)+i_{\mathrm{a}}(x)-c_{\mathrm{m}} y
$$

result in the vector field

$$
f(x, y)=\left(\begin{array}{c}
y \\
-\frac{l_{1}}{c_{\mathrm{m}}}\left(x-l_{2}\left(i_{\mathrm{e}}+i_{\mathrm{L}}(x)+i_{\mathrm{a}}(x)-c_{\mathrm{m}} y\right)\right)+\frac{1}{c_{\mathrm{m}}} y\left(i_{\mathrm{a}}^{\prime}(x)-g_{\mathrm{L}}\right)
\end{array}\right)
$$

of Liénard type. Inserting the definition of $i_{\mathrm{a}}$ and $i_{\mathrm{L}}$ shows that the vector field contains only monomials in $x$ and $y$ that are present in the unfolding of the normal form (10.2.1) of the dBT point plus an additional term in the $\frac{\partial}{\partial y}$ component of the vector field proportional to $x^{2}$. Using normal form theory for polynomial functions [133, 134, 276] it follows that we can remove this term by an appropriate coordinate shift and a rescaling:

$$
x \rightarrow \frac{1}{\sqrt{l_{1} l_{2}}} x+\frac{1}{3}\left(m_{1}+m_{2}+m_{3}\right) \quad y \rightarrow \frac{1}{\sqrt{l_{1} l_{2}}} y
$$


By a second rescaling step including time ${ }^{3}$ given by

$$
x \rightarrow \sqrt{c_{\mathrm{m}}} \frac{l_{1} l_{2}}{3} x \quad y \rightarrow \frac{l_{1}^{2} l_{2}^{2}}{9 \sqrt{c_{\mathrm{m}}}} y \quad t \rightarrow \frac{3 c_{\mathrm{m}}}{l_{1} l_{2}} t
$$

we can further simultaneously normalize the coefficients of $x^{3} \frac{\partial}{\partial y}$ and $x^{2} y \frac{\partial}{\partial y}$ to -1 yielding in total the normal form (10.2.1),

$$
f_{\mathrm{dBT}}=\left(\begin{array}{c}
y \\
\mu_{1}+\mu_{2} x-x^{3}+\left(\mu_{3}+\rho x-x^{2}\right) y
\end{array}\right)
$$

with unfolding parameters expressed in terms of the original parameters as

$$
\begin{aligned}
\mu_{1} & =\frac{1}{\left(c_{\mathrm{m}} l_{1}\right)^{\frac{3}{2}} l_{2}^{\frac{5}{2}}}\left[l_{2}\left(\left[27\left(i_{\mathrm{e}}+g_{\mathrm{L}} v_{\mathrm{L}}\right)-9 m_{\Sigma} g_{\mathrm{L}}+m_{\Pi}\right]\right)-9 m_{\Sigma}\right] \\
\mu_{2} & =\frac{3}{c_{\mathrm{m}} l_{1} l_{2}^{2}}\left[l_{2}\left(m_{\Delta}-3 g_{\mathrm{L}}\right)-3\right] \\
\mu_{3} & =\frac{1}{c_{\mathrm{m}} l_{1} l_{2}}\left(m_{\Delta}-3 g_{\mathrm{L}}\right)-3
\end{aligned}
$$

with

$$
\begin{aligned}
& m_{\Sigma}=\left(m_{1}+m_{2}+m_{3}\right) \quad m_{\Delta}=m_{1}^{2}+m_{2}^{2}+m_{3}^{2}-m_{1} m_{2}-m_{1} m_{3}-m_{2} m_{3} \\
& m_{\Pi}=\left(-2 m_{1}+m_{2}+m_{3}\right)\left(m_{1}-2 m_{2}+m_{3}\right)\left(m_{1}+m_{2}-2 m_{3}\right)
\end{aligned}
$$

and, interestingly, with a topological parameter $\rho=0$. This means that at $\mu_{i}=0, i \in$ $\{1,2,3\}$, i.e at the dBT point, there is an additional degeneracy and thus the codimension of the $\mathrm{dBT}$ point is 4 and not 3 . We thus have encountered a degenerate Bogdanov-Takens point of codimension 4 in the Fitzhugh-Nagumo neuron model, the main result of this section.

The transformation of the FHN model into the normal form (10.3.35) also shows that the FHN neuron is equivalent to a general cubic Liénard equation (10.3.25) with $F_{\mu}$ and $G_{\mu}$ being polynomials of degree 2 and 3 respectively. In [202] the authors conjecture ${ }^{4}$ the four dimensional $\left(\mu_{1}, \mu_{2}, \mu_{3}, \mu_{4}\right)$-bifurcation diagram for these types of systems using $\mu_{4}=\rho$. The FHN model thus may be viewed as a three-dimensional slice through this four dimensional bifurcation diagram at fixed $\mu_{4}=0$.

In addition to all the features encountered in the dBT point of codimension three the four dimensional bifurcation diagram in [202] also involves a cupsodial loop [85, 86] and a limit cycle of multiplicity four bifurcation, both predicting the coexistence of two stable periodic orbits for some region in $\mu$-parameter space. This observation might be useful for studying neuronal excitability in systems that show coexisting sub-threshold oscillations [183] and periodic spiking as well as possible interactions between both mechanisms. Besides generating regular spikes, pyramidal neurons in the hippocampus also show spikelets $[197,96]$, a small amplitude version of a spike. The exact origin of these spiklets is not clear [96]. One possibility is that they are part of the intrinsic neural dynamics. In this case a simple model description is lacking. Taking into account the occurrence of two stable limit cycles in the FHG model in the form (10.3.35) for appropriate values of

\footnotetext{
${ }^{3}$ This is not a conjugacy but a $C^{\infty}$-equivalence (cf. sec. 3.2.1)

${ }^{4}$ The results are partly derived analytically, the final result is conjectured in [202] using consistency arguments for the bifurcation diagram.
} 
$\rho>0$ this observation might help in the construction of a simple model description for the spikelet phenomenon.

Finally note, when using that the parameter satisfy $l_{i}>0$ and $c_{\mathrm{m}}>0$ for a biophysical meaningful model, (10.3.36) yields that $\mu_{1}$ is proportional to the input current $i_{\mathrm{e}}$. Further the resting potential in the FHN model is at $v_{\mathrm{r}}=0$ so that we have for $v_{\mathrm{L}} \approx v_{\mathrm{r}}$ and $m_{\Sigma}>0$ that $\mu_{1}$ is also proportional to $-g_{\mathrm{L}} \cdot \mu_{2}$ is controlled by $g_{\mathrm{L}} \cdot \mu_{1}$ and $\mu_{2}$ only scale with $c_{\mathrm{m}}$ while $\mu_{3}$ can changes sign due to an increase in $c_{\mathrm{m}}$. These findings are fully consistent with the observations for the WB model in sec. 10.2.4 and fig. 10.14.

To summarize, we have analytically transformed the FHN neuron model to a cubic Liénard equation and thereby proved the existence of a degenerate Bogdanov-Takens point of codimension 4 in this model. The unfolding of such a point includes regions in parameter space with the coexistence of two stable limit cycles that might serve as a starting point to construct simple models for studying the influence of the leak conductance on interactions between sub-threshold oscillations and action-potential generation as well as on intrinsic spikelet dynamics.

\subsection{Summary}

In this chapter we have found that an increase in leak conductance induces as switch in neuronal excitability for a large number of type I neurons, including the models presented in $[273,319,320,168,400,99,61,109,315,103,406]$. For type II neurons we showed that decreasing the leak does not always lead to switch back to type I as the dynamics can become unstable. We showed that the transition is organized by three steps as described in section 10.2.1.2 and organized by a degenerate Bogdanov-Takens-cusp bifurcation.

We then proved that such a degenerate Bogdanov-Takens-cusp point exists in all type I neuron models and concluded that leak induced neuronal excitability switches are generally organized by such a point. We derived a conjugation of Morris-Lecar type neuron models to a Liénard equation and used this to give an interpretation of the excitability switch in terms of accelerating and dampening forces. For an abstract neuron model we found the existence of an even higher degenerate Bogdanov-Takens point that organizes neuronal excitability and discussed possible applications for the study of the leak conductance in interactions between sub-threshold and spiking dynamics. 


\section{Chapter 11}

\section{Morphology, Effective Leak and Neuronal Excitability}

In the previous chapter we have found that a change in leak can induce a switch in neuronal excitability type I to type II behavior. In this chapter we apply these results to predict effects of different neuronal morphologies on neuronal excitability.

We consider $N$-compartment models (cf. sec. 2.5) where each compartment $k \in$ $\{1, \ldots, N\}$ is described by a trans-membrane voltage $v_{k}$ and a set of gating variables $\left\{a_{k, j}\right\}_{j=1}^{n_{k}}$ which are conductively coupled via the matrix $g_{k l}$, representing the tree structure of the neuronal morphology with a somatic root at $k=1$. The system evolves according to

$$
c_{\mathrm{m}, k} \frac{d}{d t} v_{k}=\sum_{l} g_{k l}\left(v_{l}-v_{k}\right)+i_{\mathrm{tot}, k}\left(v_{k},\left\{a_{k, j}\right\}\right)
$$

together with the evolution equations of the gating variables $a_{k, j}$ as in (2.3.3). Here $i_{\text {tot }, k}=i_{\mathrm{e}, k}+i_{\mathrm{L}, k}+i_{\mathrm{a}, k}$ is the total current for the compartment $k$ composed of the external, the leak and the ionic currents.

\subsection{Linear Shapes Integrate - Stellar Shapes Resonate}

In this section we consider compartment models of neurons consisting of an active somatic and $N_{\mathrm{D}}=N-1$ passive dendritic compartments, i.e. $i_{\mathrm{a}, k}=0$ for $k \geq 2$. We investigate how the dendritic morphology, i.e., the precise arrangement of the passive compartments influences the neuronal excitability type.

We find that changing the morphology from a single linear dendrite to stellar like shapes induces a switch from type I to type II excitability akin to the transition observed in sec. 10.2.1.2. This is shown in fig. 11.1 where we considered $N_{\mathrm{D}}=5$ dendritic compartments attached to a soma modeled by type I Morris-Lecar equations (2.4.4). We fix all parameters and calculate for different arrangement of the dendritic compartments (first column in fig. 11.1) the bifurcation diagrams for the canonical parameter $i_{\mathrm{e}}$ (second column) and corresponding spike frequencies (third column). For a single linear extended dendritic tree the system shows a SNIC bifurcation with type I neuronal excitability. For a more compact but still single dendritic tree the limit cycle is created by a homoclinic bifurcation, showing a small hysteresis effect due to bistability, but still having type I excitability. For two primary dendrites attached to the soma the stable limit cycle is generated via a DC bifurcation and the neuron has switched to type II excitability. For 
(a)
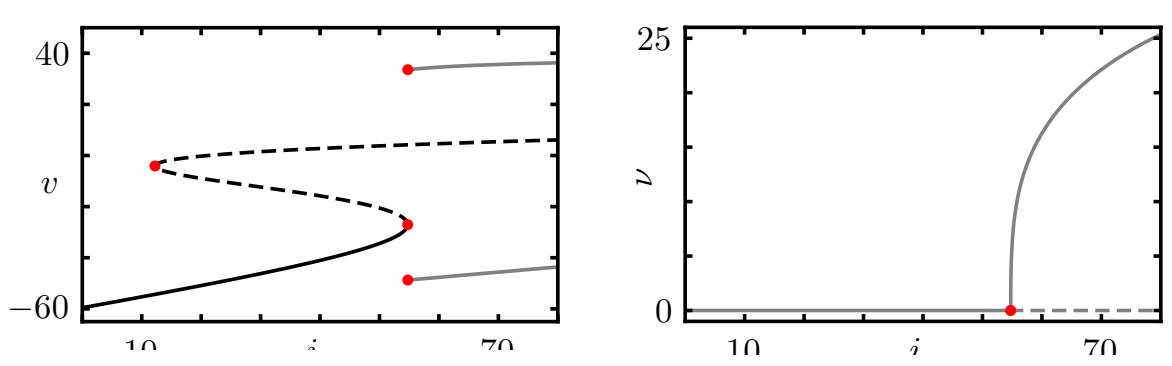

(b)
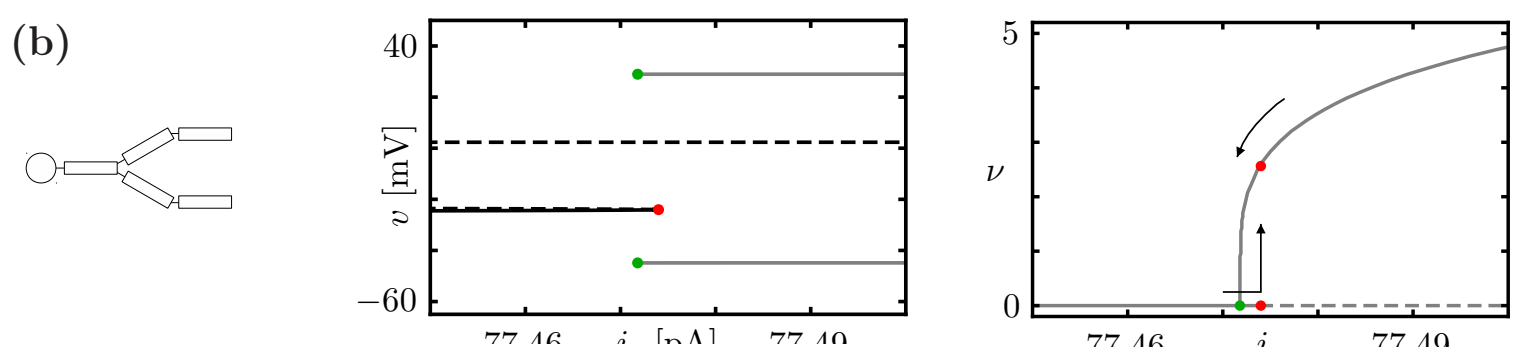

(c)
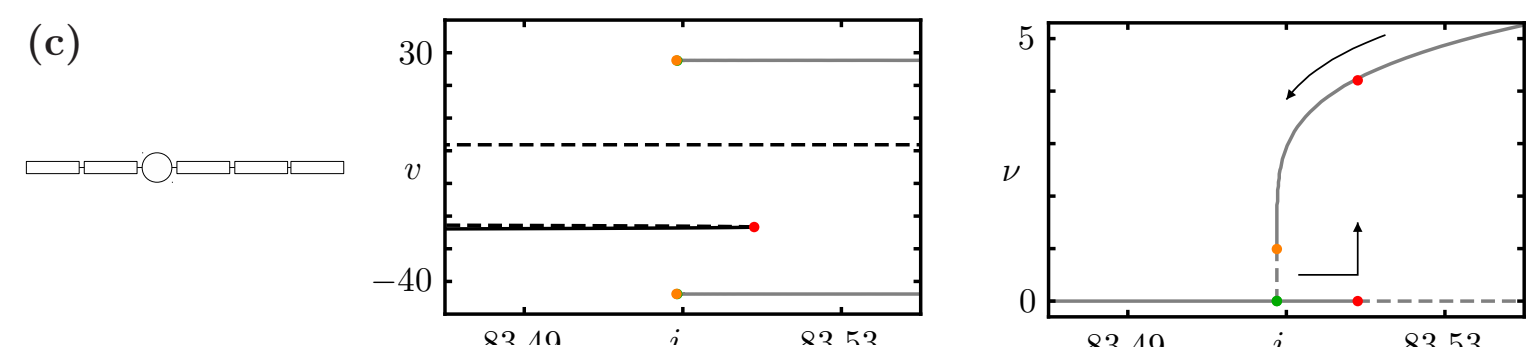

(d)
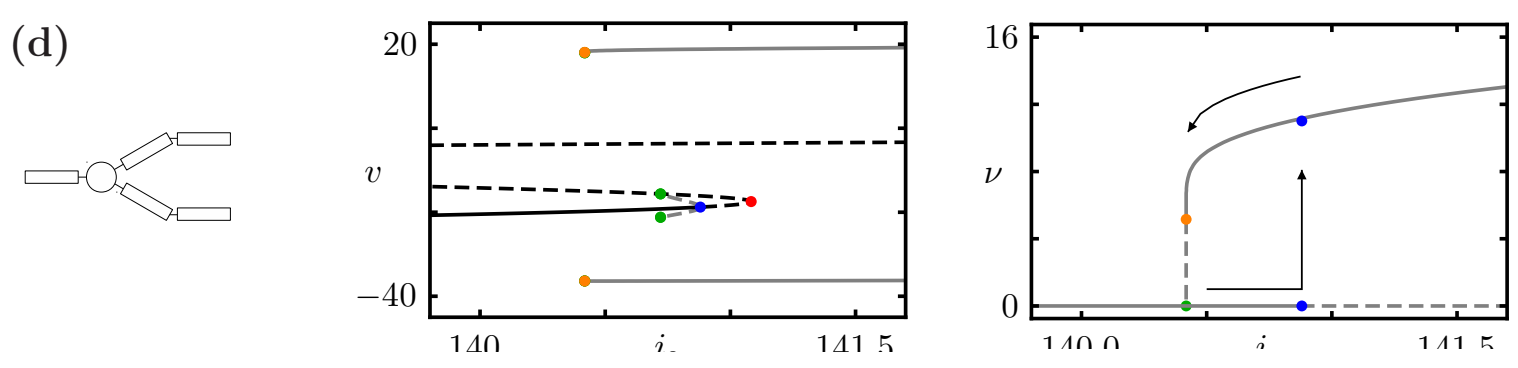

(e)
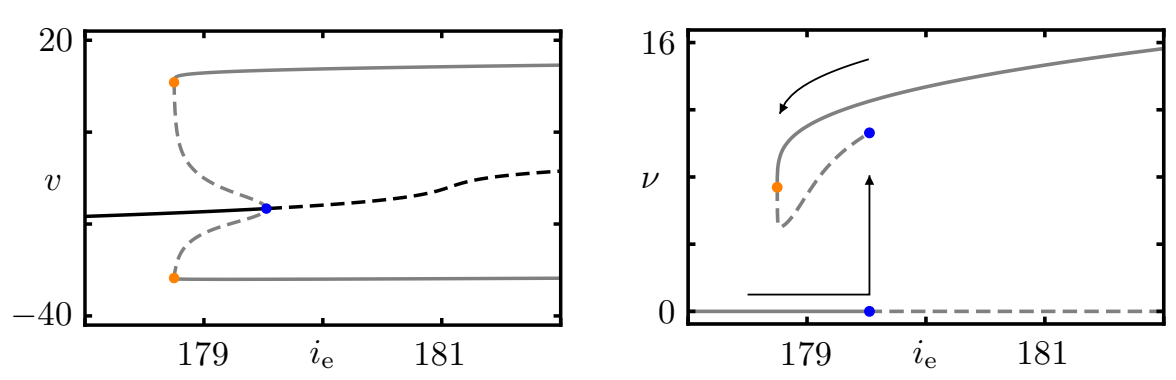

Figure 11.1. Neuronal morphology determines neuronal excitability. $i_{\mathrm{e}}$-bifurcation diagrams (second column, colors as in tab. 3.1) and frequency of the stable periodic orbit (third column) for a compartmental neuron model, consisting of an active soma with Morris-Lecar type I dynamics, sec. (2.4.2), eq. (2.4.4), and $N_{\mathrm{D}}=5$ differently arranged passive dendritic compartments (first column). Changing the morphology induces a transition of spike onset bifurcations from (a) SNIC $(\bullet)$, to (b) homoclinic $(\bullet)$ to $(\mathbf{c}, \mathbf{d})$ DC $(\bullet)$ accompanied by a change of the destabilization of the fixed point from a (c) fold $(\bullet)$ to a (d) Hopf $(\bullet)$ bifurcation. The hysteresis effect for spike on and off set is indicated by arrows in the frequency plots.

For all morphologies we use identical biophysical parameters as given in appendix D.2 for the soma $\left(g_{\mathrm{L}, S}=0.5, c_{\mathrm{m}, S}=10\right)$ and for the dendritic compartments $c_{\mathrm{m}, \mathrm{D}}=1, g_{\mathrm{L}, D}=1.0$, $v_{\mathrm{L}, \mathrm{D}}=v_{\mathrm{L}, \mathrm{S}}$ and $i_{\mathrm{e}, \mathrm{D}}=0$. For linked compartments the coupling conductance is $g_{\mathrm{I}}=6.5$. 
three primary dendrites we additionally observe a small homoclinic and Hopf bifurcation indicating a transition in peri-threshold resonance. Finally, for the stellar shape we observe classical type II behaviour with a DC and a Hopf bifurcation. This transition is very similar to the neuronal excitability transition observed in the previous sections.

This transition can be understood by considering the effective leak a passive dendrite imposes on the soma. To see this, we consider the system (11.0.1) with a notation separating the dendritic membrane potentials $v_{\mathrm{D}}=\left(v_{2}, \ldots v_{N}\right)^{T}$ form the somatic one $v_{S}=v_{1}$ and setting

$$
\begin{aligned}
g_{\mathrm{LD}}^{d} & =\operatorname{diag}\left(g_{\mathrm{L}, 2}, \ldots g_{\mathrm{L}, N}\right) & g_{D} & =\left(g_{i j}\right)_{i, j=2}^{N} \\
g_{\mathrm{DS}}^{T} & =\left(g_{2,1}, \ldots g_{N, 1}\right) & g_{\mathrm{SD}}^{T} & =\left(g_{1,2}, \ldots, g_{1, N}\right)
\end{aligned}
$$

where $g_{\mathrm{D}}$ is conductive coupling matrix between the dendritic compartments, and $g_{\mathrm{DS}}$ is a $N-1$ dimensional vector representing the coupling from the soma to the dendrite and $g_{\mathrm{SD}}$ the coupling from the dendrite to the soma. For convenience, we also define the diagonal matrices $c_{\mathrm{mD}}=\operatorname{diag}\left(c_{\mathrm{m}, 2}, \ldots, c_{\mathrm{m}, N}\right)$ and $g_{\mathrm{DS}}^{\mathrm{d}}=\operatorname{diag}\left(g_{\mathrm{DS}}\right)$ and $N_{\mathrm{D}}$ dimensional vectors $v_{\mathrm{LD}}^{T}=\left(v_{\mathrm{L}, 2}, \ldots, v_{\mathrm{L}, N}\right), 1_{\mathrm{D}}^{T}=(1,1, \ldots, 1)$. The system (11.0.1) with passive dendrite may then be written as

$$
\begin{aligned}
c_{\mathrm{mS}} \frac{d}{d t} v_{\mathrm{S}} & =i_{\mathrm{e}, \mathrm{S}}+g_{\mathrm{LS}}\left(v_{\mathrm{LS}}-v_{\mathrm{S}}\right)+i_{\mathrm{a}, \mathrm{S}}\left(v_{\mathrm{S}},\left\{a_{\mathrm{S}, j}\right\}\right)+g_{\mathrm{SD}}^{T}\left(v_{\mathrm{D}}-1_{\mathrm{D}} v_{\mathrm{S}}\right) \\
c_{\mathrm{mD}} \frac{d}{d t} v_{\mathrm{D}} & =i_{\mathrm{e}, \mathrm{D}}+g_{\mathrm{LD}}\left(v_{\mathrm{LD}}-v_{\mathrm{D}}\right)+g_{\mathrm{DS}}^{d}\left(1_{\mathrm{D}} v_{\mathrm{S}}-v_{\mathrm{D}}\right)+g_{\mathrm{D}} v_{\mathrm{D}} .
\end{aligned}
$$

From a dynamical point of view the leak conductance leads to an instantaneous current. For electrotonically compact neurons [68] the compensation of membrane potential differences between different compartments is fast. Thus, in this situation, we can enslave $[153,152]$ the dynamics of the dendritic compartments to the dynamics of the soma by substituting the steady state currents of the dendrites into the equation for the soma.

Given a somatic membrane potential $v_{\mathrm{S}}$ the steady state of the dendritic compartments $v_{\mathrm{D}}^{*}=v_{\mathrm{D}}^{*}\left(v_{\mathrm{S}}\right)$ is determined by a vanishing vector field $\frac{d}{d t} v_{\mathrm{D}}=0$ and using eq. (11.1.2) we can solve this for $v_{\mathrm{D}}^{*}$ to obtain:

$$
v_{\mathrm{D}}^{*}=\left(g_{\mathrm{LD}}^{d}+g_{\mathrm{DS}}^{d}-g_{\mathrm{D}}\right)^{-1}\left(i_{\mathrm{D}}+g_{\mathrm{LD}}^{d} e_{\mathrm{LD}}+g_{\mathrm{DS}}^{d} 1_{\mathrm{D}} v_{\mathrm{S}}\right)
$$

This results in the enslaved system

$$
\frac{d}{d t} v_{\mathrm{S}}=i_{\mathrm{e}}^{\mathrm{eff}}+g_{\mathrm{L}}^{\mathrm{eff}}\left(v_{\mathrm{L}, \mathrm{S}}-v_{\mathrm{S}}\right)+i_{\mathrm{a}, \mathrm{S}}\left(v_{\mathrm{S}},\left\{a_{\mathrm{S}, j}\right\}\right)
$$

with effective leak conductance $g_{\mathrm{L}, \mathrm{S}}^{\mathrm{eff}}$ and input current given by

$$
\begin{aligned}
g_{\mathrm{L}}^{\mathrm{eff}} & =g_{\mathrm{S}, \mathrm{L}}+g_{\mathrm{SD}}^{T}\left(1_{\mathrm{D}}-\left(g_{\mathrm{LD}}^{d}+g_{\mathrm{DS}}^{d}-g_{\mathrm{D}}\right)^{-1} g_{\mathrm{DS}}\right) \\
i_{\mathrm{e}}^{\mathrm{eff}} & =i_{\mathrm{S}}+g_{\mathrm{SD}}^{T}\left(\left(g_{\mathrm{LD}}^{d}+g_{\mathrm{DS}}^{d}-g_{\mathrm{D}}\right)^{-1}\left(i_{\mathrm{D}}+g_{\mathrm{LD}}^{d} e_{\mathrm{LD}}+g_{\mathrm{DS}}^{d} e_{\mathrm{LS}}\right)-1_{\mathrm{D}} e_{L S}\right)
\end{aligned}
$$

Thus, under the assumption of a fast compensation of potential differences between the soma and the dendritic compartments the morphologically extended neuron can be modeled as a point neuron with an effective leak given in (11.1.4). This derivation shows that changes in the dendritic topology result in changes in the effective leak and furthermore, that input currents into the dendritic tree are converted into effective input currents at the soma. In fig. 11.2 we have plotted the effective leak conductances $g_{\mathrm{L}}^{\text {eff }}$ given by eq. 


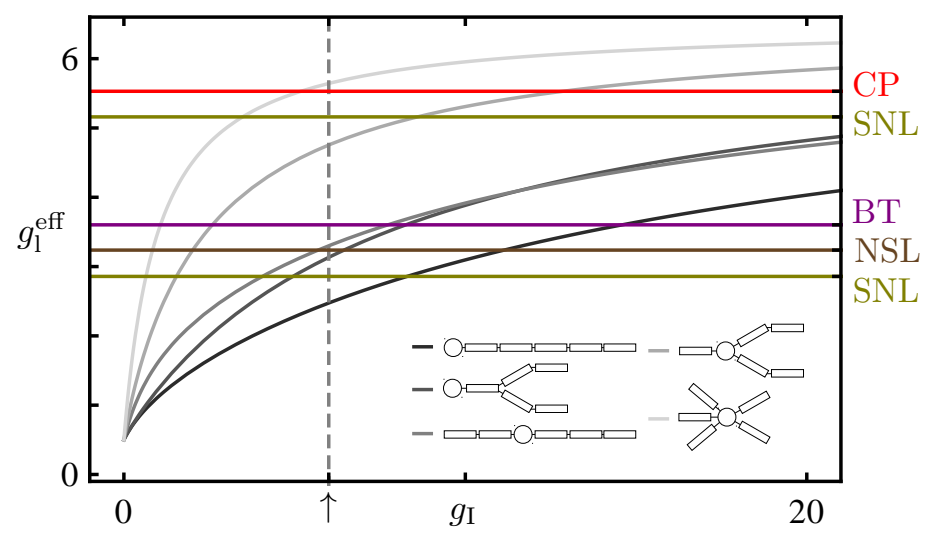

Figure 11.2. Dendritic morphology determines the effective leak conductance. Plotted is the effective leak conductance (11.1.4) for different dendritic arrangements as a function of the intercompartmental coupling $g_{\mathrm{I}}$. The arrow indicates the value for $g_{\mathrm{I}}$ used in fig. 11.3. The lines represent the values of the leak conductances for the effective model (11.1.3) to be at the codimension two bifurcation points indicated on the right and shown in fig. 11.3.

(11.1.4) as a function of the strength of the intracellular resistance $g_{\mathrm{I}}$ between the linked compartments for the dendritic topologies considered in fig. 11.1. For $g_{\mathrm{I}}=0$ the effective leak reduces to the somatic leak $g_{\mathrm{L}}^{\text {eff }}=g_{\mathrm{L}, S}$. For $g_{\mathrm{I}}>0$, one observes that stellar like arrangements of the dendrites result in a larger effective leak than extended linear dendrites. This effect is illustrated on the right half of fig. 11.3 and has the following explanation: The leak current through a dendritic compartment that is directly attached to the soma is high as there is only a small inter-compartmental coupling resistance. For a stellar cell with many primary dendrites these currents thus sum up to a large total effective leak. On the contrary, for a linear dendrite, the contribution to the total leak from currents through distal compartments is small as they have to pass several intracellular resistances.

As the reduced effective system (11.1.3) corresponds to a single compartment neuron model, we can use the results on leak-induced neuronal excitability transitions studied in sec. 10.2.1.2 to explain the transition here caused by changing the morphological shape of the dendrite. Figure 11.3 shows the bifurcation diagram for the original single compartment model together with the effective leak conductances given by eq. (11.1.4) for the multi-compartment models used in fig. 11.1. Comparison with the one dimensional bifurcation diagrams in fig. 11.1 gives good agreement of the encountered bifurcation scenarios. Thus, the two-dimensional bifurcation diagram computed once, can be used to predict the bifurcation scenario for any dendritic morphology that satisfies the assumption of electrotonic compactness. In particular using (10.3.2) or (10.3.17) the neuron is predicted to have type I neuronal excitability if

$$
g_{\mathrm{L}}^{\mathrm{eff}} \leq g_{\mathrm{L}, 0}=\max _{v} \frac{d}{d v} i_{\mathrm{a}, \infty}(v)
$$

and type II otherwise.

We derived the effective leak from the assumption of a dendritic tree with small capacity compared to the somatic capacity. However, the above calculations are the same for a neuron in an equilibrium state and thus the fold bifurcation lines for the fixed points are not affected by this assumption. Numerical investigation show that for the Morris-Lecar model the conclusions stay valid, if the dendritic capacity is of the order of the somatic capacity. For electrotonically large dendritic trees, the dynamics may become more com- 


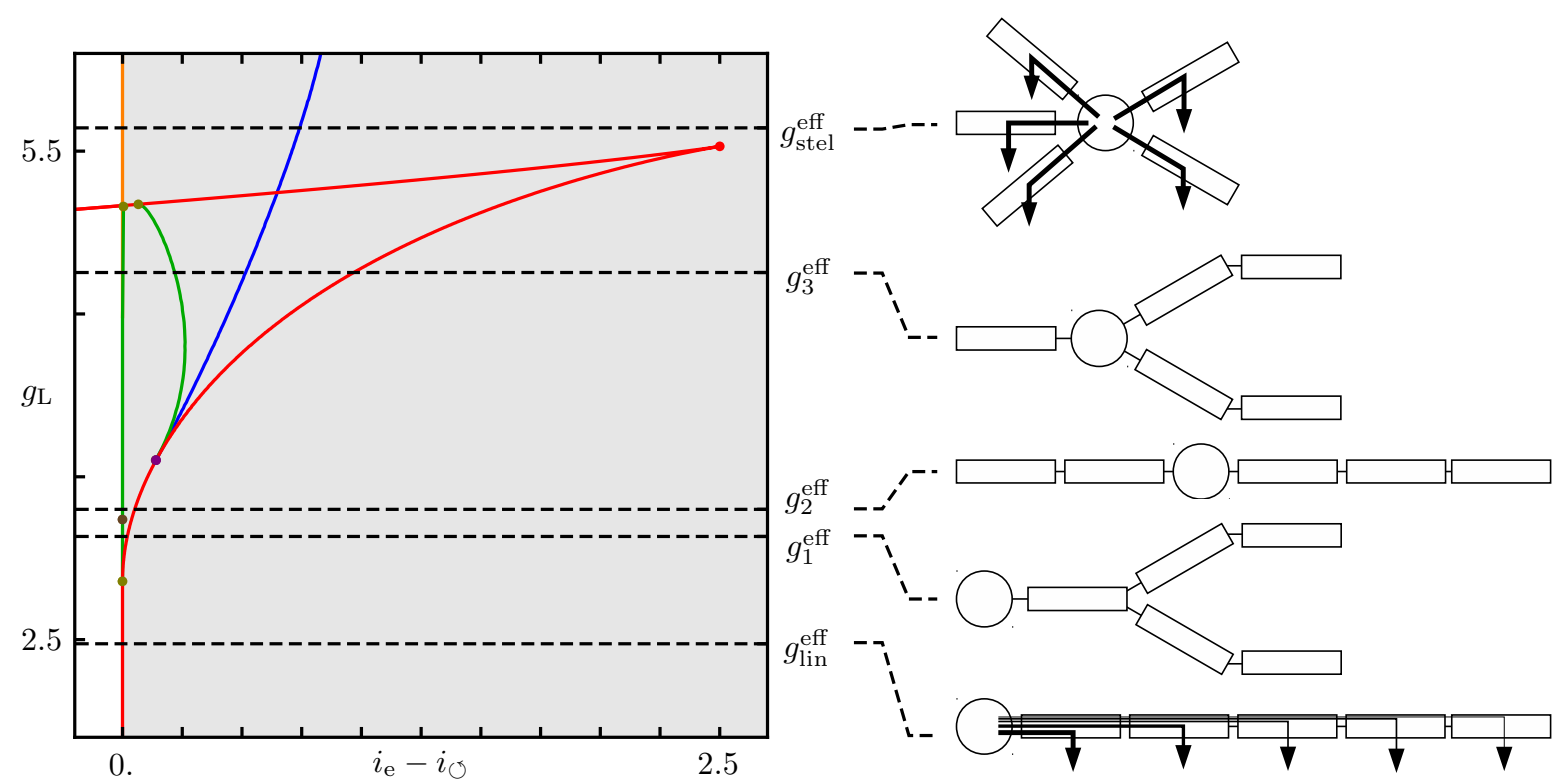

Figure 11.3. Dendritic morphology determines neuronal excitability. Bifurcation diagram for the Morris-Lecar neuron model in the $\left(i_{\mathrm{e}}-i_{\circlearrowleft}, g_{\mathrm{L}}\right)$-parameter space with standard parameter D. 2 but reduced capacity $c_{\mathrm{m}}=10$. Dashed lines indicate the effective leak conductances for the compartmental models of fig. 11.1 with topological arrangement of the dendritic tree as indicated on the right and calculated by eq. (11.1.4). Comparison with fig, 11.1 yields excellent agreement for the encountered bifurcation scenarios determining the neuronal excitability.

Arrows in the morphological representations of the dendrite on the right indicate the mechanism resulting in different leaks: For the stellar like cell (top) the leak currents escaping through the dendritic compartments only "feel" a single intracellular resistance due to the direct coupling to the soma. For the linear extended shapes the leak currents to more distal compartments are reduced as they have to pass intracellular resistances of several compartments.

plex due to back- and forth-propagation of spikes on the dendrites and active properties of their membranes. However, our conclusions is still useful when concentrating on the local dynamics close to the action potential generation site, i.e. the local neuronal excitability

(a)

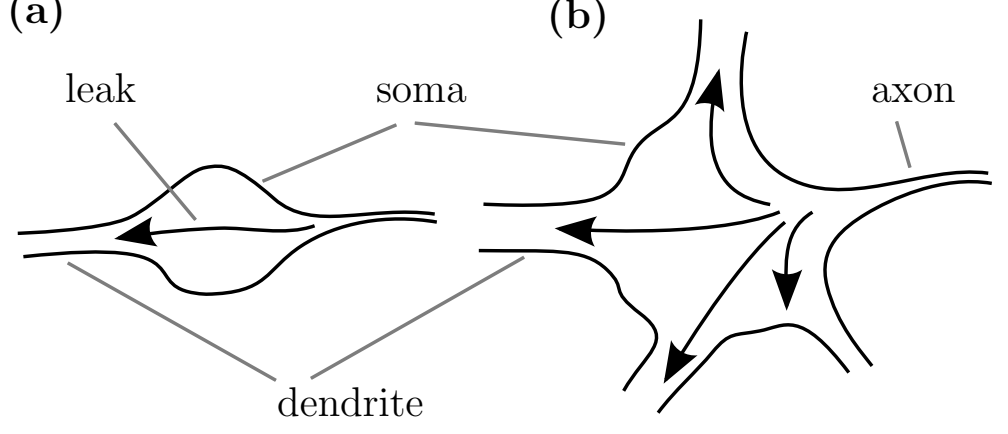

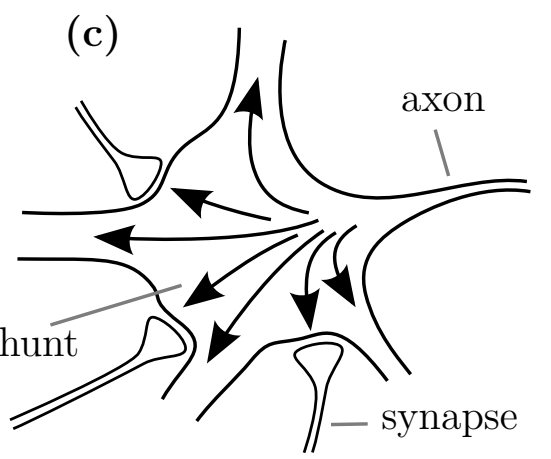

Figure 11.4. Local leak currents depend on morphology and synaptic shunting. Sketch of possible mechanism changing the effective leak felt at the action potential generation site and thereby influencing the neuronal excitability. (a) a single primary dendrite only contributes little to the effective leak at the action potential generation site on the axon. (b) many primary dendrites e.g. as observed in stellar cells and (b) shunting due to active inhibitory synapses can increase this effective leak conductance. 
(cf. fig. 11.4). For such a site the effective leak is co-determined by the detailed morphology and ion-channel properties of the soma together with the number, diameter and electronic properties of the primary dendrites with a small electrotonic distance. Furthermore, activation of nearby inhibitory synapses may change the effective leak dynamically due to shunting inhibition. We study such dynamic effects due to synaptic shunting in chapter 13 and experimentally in section 12.4 .

In section 10.2.1.2 we saw that the transition in neuronal excitability induced by changes in the leak conductance is accompanied by a switch from integration to resonance at peri-threshold, with an increase of the frequencies for higher values of the leak. Thus, combining this fact with the results from this section, we conclude that dendritic morphology also influences the resonance properties of the cell. While linear extended shapes of the dendrite tend to integration, stellar morphology can induce resonate properties at peri-threshold.

\subsection{Recovery of Type I Neuronal Excitability by Active Dendrites}

In the previous section we saw that passive dendritic trees, and in particular stellar like shapes can switch a type I soma to a type II neuron. Here we show that active dendritic conductances on the dendrite can compensate for this and switch the neuron back to type I.

We consider compartmental neuron models of the form (11.0.1) with $N_{\mathrm{D}}$ primary dendritic compartments with a fraction $\alpha$ of the active somatic channels. Figure 11.5 shows a bifurcation diagram for an electrotonic compact neuron with $N_{\mathrm{D}}=2$. For $\alpha=0$ we have a passive dendritic tree and the neuron shows type II excitability. Increasing $\alpha$ we observe a transition to type I dynamics in the reverse order to the transition observed when switching form type I to type II by increasing the leak. Thus by introducing active conductances onto the dendrite, the neuron can be switched back to type I neuronal excitability.

Note however, that similar restrictions apply as in the previous section, i.e. if the electrotonic compactness is lost more complex dynamics will be observed [250]. But again

(a)

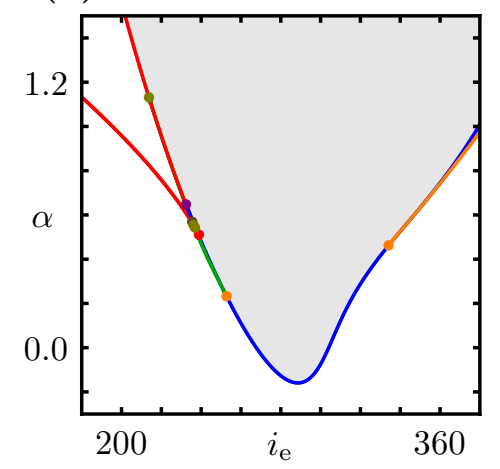

(b)

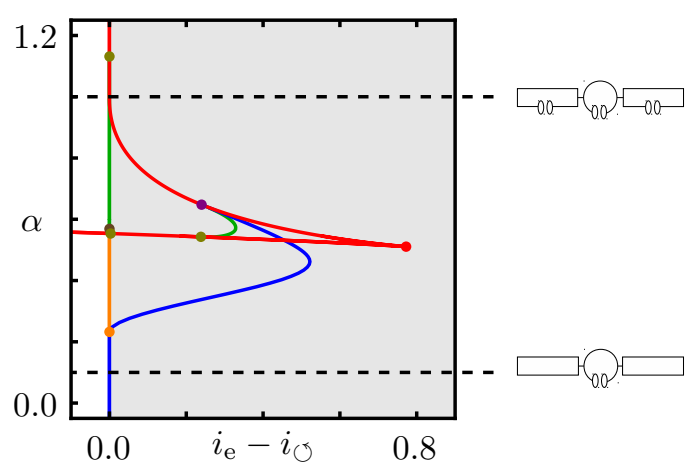

Figure 11.5. Active dendritic conductances recover type I neuronal excitability. Bifurcation diagrams for a three compartment Morris-Lecar neuron model with fraction $\alpha$ of active dendritic ion-channels. 
the local neuronal excitability at the action potential generation site can be influenced by the local distribution of ion channels on the primary dendrites.

\subsection{Neuronal Excitability and Morphology in Experiments}

In this section we show experimental evidence for the above reasoning that neuronal morphology can induce neuronal excitability switches. In firing rate data from dendritic pinching experiments [25], we find a switch in neuronal excitability form type II to type I of Purkinje cells consistent with our theoretical predictions. For pyramidal neurons we do not observed a switch as onset frequencies decrease little due to pinching for these cells.

One method to study the influence of the dendritic tree onto the spiking dynamics of the soma and axon is by targeted dendrotomy [25], In these type of experiments the dendritic tree can be occluded or amputated form the soma by pinching it adiabatically between two pipettes. Using this method, in ref. [25] it is shown that the dendrite of cerebellar Purkinje and cortical layer 5 Pyramidal neurons of rat acts as a passive electric load if the excitation is weak excitation. For stronger excitation its active properties contribute to burst firing and enhance excitability. Effects on neural excitability type are not considered in their study. ${ }^{1}$

We therefore analyzed firing data from these dendrotomy experiments with emphasis on how the neuronal excitability type of the soma is affected by occluding the dendrite. The result is shown in fig. 11.6. For both cell types, the input current threshold for firing is lowered when occluding the dendrite, confirming that the dendritic tree increases the leak conductance [25]. Moreover, Prukinje cells only have a single, large dendrite and thus occluding it removes all the effective leak form the soma. The $\nu-i_{\mathrm{e}}$-curve increases non-continuously from zero when the dendrite is present, whereas if it is pinched, we do not detect a significant frequency offset (fig. 11.6). We thus conclude that the Purkinje cells switch their excitability type form II to I when the dendrite is occluded. This finding is fully consistent with our theoretical results from sections 10.2.1.2 and 11.1.

For layer 5 Pyramidal neurons we observe a change in the effective leak at the soma when pinching the dendrite due to a left shift of the $\nu-i_{\mathrm{e}}$-curve . However, we do not observe a switch in neuronal excitability. In particular, we find that cells show a weak form of type II neuronal excitability with an offset frequency around $5 \mathrm{~Hz}$, which either decreases or does not differ significantly when the dendrite is occluded. Pyramidal neurons have multiple primary dendrites, and in the experiments only the apical dendrite is pinched, whereas the basal part remains intact. Despite the larger number of primary dendrites, the only weak type II neuronal excitability for the pyramidal cells can be partly explained by the recovery mechanism of type I neuronal excitability (cf. sec. 11.2) due to their active dendritic conductances [89, 357, 247]. The absence of a switch to type I excitability may be either due to the fact that the reduction in leak conductance by occluding only the apical dendrite is to small or the soma is intrinsically type II (cf. sec. 10.2.5).

Note, that in [126] it is shown, that resonance properties of stellate cells in medial

\footnotetext{
${ }^{1}$ In fact in [25] a detailed model of a Purkinje cell is used to predict the input-frequency relationship for high frequencies. However it fails to match the data at low frequencies. The model shows type I excitability for the control and type II for the pinched situation. In contrast, the data shows the opposite (cf. fig. 3 in $[25]$ ).
} 
(a)

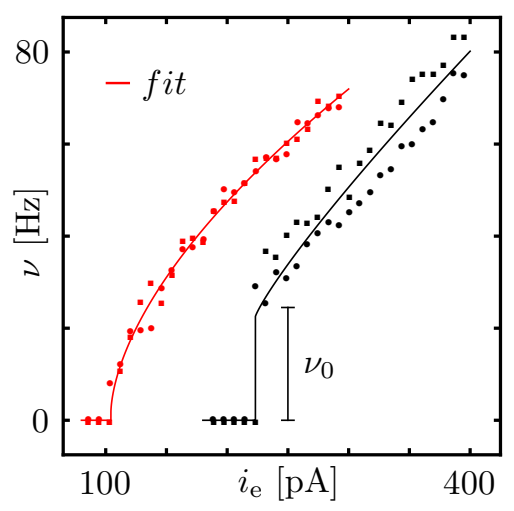

(c)

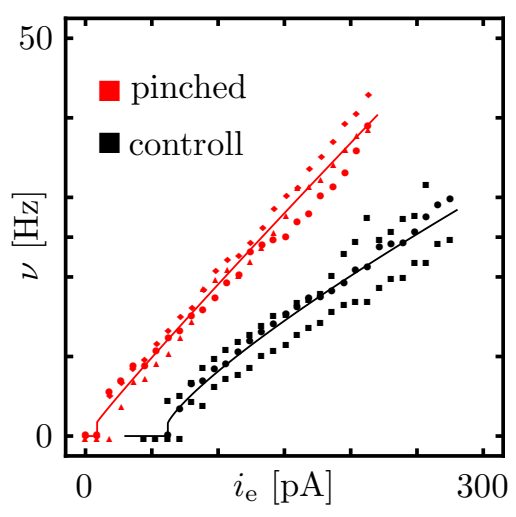

(b)

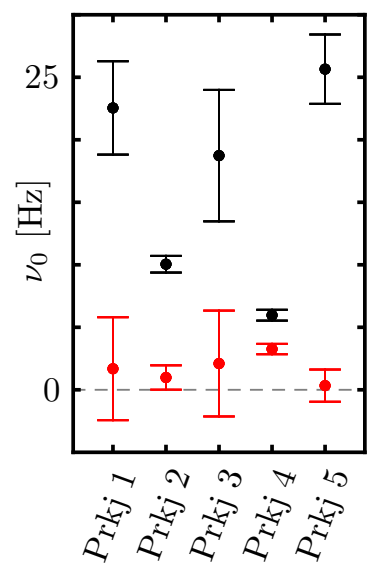

(d)

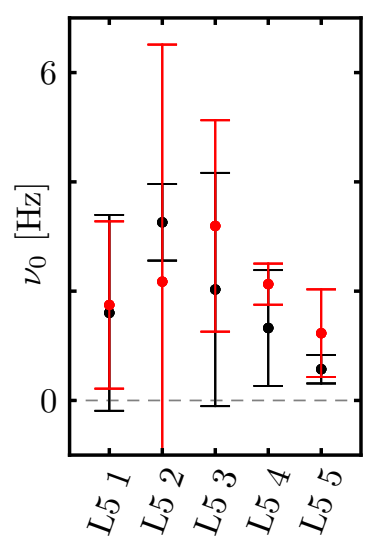

Figure 11.6. Firing rate and onset frequencies for rat Purkinje and pyramidal neurons under dentrotomy. (a) spike frequency $\nu$ vs. input current $i_{\mathrm{e}}$ for a rat cerebellar Purkinje cell for an intact (black) or a pinched (red) dendrite. Curves show a increase in the threshold current for spiking, as well as an increase in the onset frequency $\nu_{0}$. Lines show best fits of the form $\nu=c_{1}\left(i_{\mathrm{e}}-c_{2}\right)^{c_{3}}+\nu_{0}$, for $i_{\mathrm{e}}>c_{2}$ and zero otherwise for parameters $c_{i}$ and $v_{0}$. (b) fitted onset frequency $\nu_{0}$ obtained as in (a) for all Purkinje in control (red) and pinched (black) situation. All cells shown an increase in onset frequency which differs from zero only for cell 4 . (c) $\nu-i_{\mathrm{e}}$-curve as in (a) but for layer 5 cortical Pyramidal neurons shows no significant change in the onset frequency $v_{0}$ between control and occluded dentrite. (d) offset frequencies as in (b) do not show a significant change. Raw data courtesy of J. Bekkers (cf. [25] for methods of data acquisition).

entorhinal cortex of rat vary systematically along the dorsal ventral axis from lower to higher frequencies. In [119] it is further shown that the leak conductance increases along this axis as a result of a gradient in leak and HCN channels, as well as an increase in the number of primary dendrites. Moreover, in [10] it is shown that at peri-threshold this resonance does not require a special ion channel configuration but can be attributed to the sodium channels alone. Taken together, these findings are fully consistent with our findings of a change in resonance frequencies due to an increase in leak as discussed in section 12 .

To summarize, analyzing data from targeted dendrotomy experiments revealed strong evidence, that a switch from type I to type II neuronal excitability can be induced by an effective leak conductance imposed onto the soma by the passive load of a dendritic tree as predicted in section 11 . 


\subsection{Summary}

In this chapter we have studied how neuronal morphology influences the neuronal excitability type. We showed that for electrotonically compact neurons a passive dendritic tree imposes an effective leak onto the soma and thus influences the neuronal excitability type via the same mechanism found in chapter 10. We argued that for larger neurons the local neuronal excitability type close to the action potential generation site is still affected by effective leak conductances determined by the detailed morphology and ion-channel properties of the soma together with the number, diameter and electronic properties of the primary dendrites. By analyzing experimental data we showed that in Purkinje cells a switch from type I to type II neuronal excitability is caused by the passive load of the dendritic tree. 


\section{Chapter 12}

\section{Leak Induced Neuronal Excitability Switches in Real Neurons}

In this section we study the neuronal excitability transition induced by a change in leak conductance experimentally ${ }^{1}$. Using dynamic patch clamp recordings to change the leak conductance artificially we find a switch form type I to type II neurons. We confirm the main features of the transition predicted by the degenerate Bogdanov-Takens-cusp bifurcation scenario in chapter 10. We further show that shunting conductances activated by $\mathrm{GABA}^{2}$ are sufficient to induce a neuronal excitability switch. This implies that neuronal excitability may be controlled dynamically by neuronal activity.

\subsection{Experimental Methods}

(a)

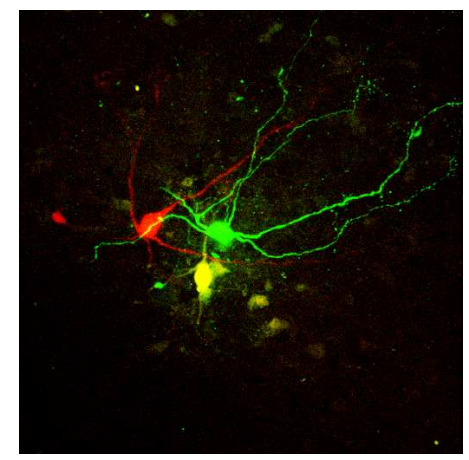

(b)

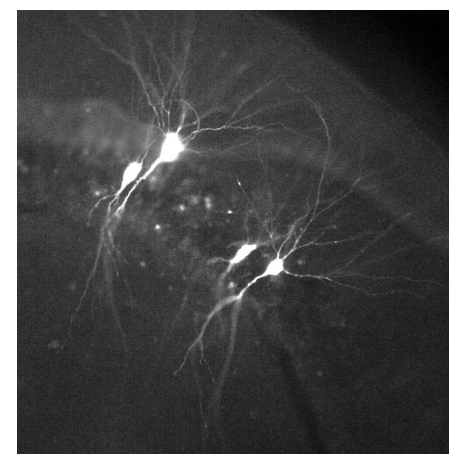

Figure 12.1. Confocal images of gerbil neurons studied experimentally. (a) projection from a stack of confocal images oft three neurons in the DNLL stained with Alexa488 (green), Alexa568 (red) or both (yellow). (b) picture of hippocampal pyramidal neurons and layer structure taken with an Axio imager fluorescence microscope. Both pictures taken after fixating slices and mounting them on slides.

To study the neuronal excitability switch experimentally we use the dynamic patch clamp method [281, 287] (cf. sec. 2.8) to control the leak conductance artificially. We focus on neurons in the dorsal nucleus of the lateral lemniscus (DNLL) and pyramidal cells in the

\footnotetext{
${ }^{1}$ Experiments where performed in collaboration with Julian Ammer, Dr. Felix Felmy, Dr. Martin Stemmler and Prof. Dr. Andreas Herz at the LMU Munich, Germany.

${ }^{2} \gamma$-amino-butyric-acid
} 
CA3 area of the hippocampus in Mongolian gerbils as shown in fig. 12.1. The GABAergic DNLL is part of the binaural system and receives projections from many lower brainstem nuclei and projects to the contralateral DNLL as well as the ipsi- and contralateral inferior colliculs of the midbrain [19]. It has a possible role in the precedence effect but its precise function is not clear. The CA3 area of the hippocampus is involved in learning mechanisms [91] and spatial navigation [288].

\subsubsection{Slice preparation}

All experiments complied with institutional guidelines, national and regional laws. Slices were prepared from Mongolian gerbils (Meriones uniguiculatus) of postnatal day (P) 10 to 18. Animals were decapitated and brains were removed in dissection solution containing (in mM) 50 sucrose, $25 \mathrm{NaCl}, 25 \mathrm{NaHCO} 3,2.5 \mathrm{KCl}, 1.25 \mathrm{NaH} 2 \mathrm{PO} 4,3 \mathrm{MgCl} 2,0.1 \mathrm{CaCl} 2$, 25 glucose, 0.4 ascorbic acid, 3 myo-inositol and 2 Na-pyruvate (pH 7.4 when bubbled with $95 \% \mathrm{O} 2$ and 5\% CO2). After removal of the brain either $200 \mu \mathrm{m}$ thick transverse slices containing the DNLL (P10-11) or $300 \mu \mathrm{m}$ thick horizontal slices containing the Hippocampus (P16-18) were taken with a VT1200S vibratome (Leica, Wetzlar, Germany). Slices were incubated in extracellular recording solution (same as dissection solution but with $125 \mu \mathrm{M} \mathrm{NaCl}$, no sucrose, $2 \mathrm{mM} \mathrm{CaCl} 2$ and $1 \mathrm{mM} \mathrm{MgCl} 2$ ) at $36^{\circ} \mathrm{C}$ for 45 minutes, bubbled with $5 \% \mathrm{CO}_{2}$ and $95 \% \mathrm{O}_{2}$.

\subsubsection{Electrophysiology}

After incubation slices were transferred to a recording chamber attached to a microscope (BX50WI, Olympus, Hamburg, Germany) equipped with gradient contrast illumination (Luigs and Neumann, Ratingen, Germany) and continuously perfused with extracellular solution. All recordings were carried out at near physiological temperature $\left(34-36{ }^{\circ} \mathrm{C}\right)$. Cells were visualized and imaged with a TILL Photonics system (Gräfelfing, Germany) composed of an Imago CCD-camera, a Poly-IV monochromator, and its control unit. All recordings were performed in current-clamp mode using an EPC10/2 amplifier (HEKA Elektronik, Lambrecht, Germany). Data were acquired at $50 \mathrm{kHz}$ and filtered at $3 \mathrm{kHz}$. The bridge balance was set to $100 \%$ after estimation of the access resistance and was monitored repeatedly during recordings. The internal recording solution consisted of (in mM): $145 \mathrm{~K}$-gluconate, $5 \mathrm{KCl}, 15$ HEPES, 2 Mg-ATP, 2 K-ATP, 0.3 Na2-GTP, 7.5 Na2-Phospocreatine, 5 K-EGTA (pH 7.2). $100 \mu \mathrm{M}$ Alexa 488 or 568 were added to the internal solution to control for cell type and location. To change the resting leak conductance during recordings, a constant conductance with a reversal potential equal to the neurons resting potential was applied with an analogue conductance amplifier (SM-1, Cambridge Conductance, Royston, UK). For some recordings, GABA (500 $\mu \mathrm{M}$ in HEPES) was applied via a puff electrode with continuous low pressure controlled by a picospritzer (Picospritzer III, Science Products, Hofheim, Germany). Glycinergic and Glutamatergic synaptic inputs were blocked with $0.5 \mu \mathrm{M}$ Strychnine, $20 \mu \mathrm{M}$ DNQX, $10 \mu \mathrm{M}$ R-CPP and GABAergic inputs were blocked with $10 \mu \mathrm{M}$ SR95531 in all experiments except when GABA was used as an agonist.

\subsubsection{Data Analysis}

Data obtained in the measurements were analyzed using a self-written software package [208] for Mathematica 8.0, Wolfram Inc. 
(a)

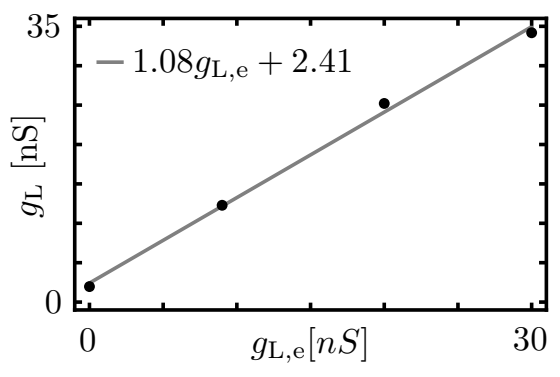

(b)

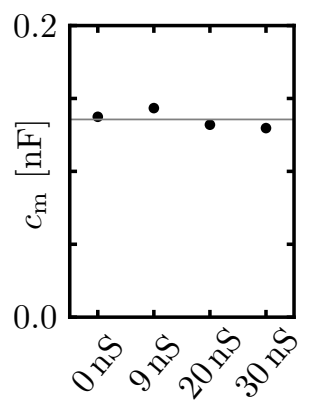

Figure 12.2. Measured leak $g_{\mathrm{L}}$ and capacitance $c_{\mathrm{m}}$ as a function of applied leak $g_{\mathrm{L}, \mathrm{e}}$ for the DNLL neuron in fig. 12.6. (a) $g_{\mathrm{L}}$ scales linearly with $g_{\mathrm{L}, e}$. gray line: linear fit with $R^{2}=0.996$. (b) capacitance does not change for the four different externally applied leak conductances.

The leak was estimated from small negative step currents of amplitude $\delta i_{\mathrm{e}}$ and $0.5 \mathrm{~s}$ duration introduced into the neuron. The average of 50 such traces was taken and an exponential decay $v \propto \exp \left(-\frac{t}{\tau_{\mathrm{L}}}\right)$ of this trace starting from the stimulus onset at voltage $v_{0}$ to the minimum voltage $v_{\min }$ was fitted. The leak was then estimated via $g_{\mathrm{L}}=\frac{\delta i_{\mathrm{e}}}{v_{\min }-v_{0}}$. The capacitance $c_{\mathrm{m}}$ was estimated from the fitted membrane time constant $\tau_{\mathrm{L}}$ using the relation ship $c_{\mathrm{m}}=\tau_{\mathrm{L}} g_{\mathrm{L}}$.

To check for validity of changing the leak conductance via the dynamic clamp method we determined the relation ship between the imposed leak $g_{\mathrm{L}, e}$ and the measured leak $g_{\mathrm{L}}$. For all measured neurons this relation was linear with slopes close to 1 as shown for a DNNL neuron in fig. 12.2a. Further more in all experiments the capacitance stayed constant for different externally applied leak conductances as shown in fig. 12.2b.

Membrane voltage data from depolarizing step currents of $1 \mathrm{~s}$ duration were used to determine the $\nu$ - $i_{\mathrm{e}}$-curve and the area of bistability. To ensure a good resolution around the firing threshold, the current threshold was estimated with an automated procedure and the current amplitude was increased from sub- to supra-threshold in very fine increments $(1-20 \mathrm{pA})$. Peaks where determined using the peak detection algorithm in [373]. Spikes where identified by having a peak amplitude $>50 \mathrm{mV}$. The onset of periodic spiking was determined by spikes throughout the duration of the stimulation with subsequent inter spike interval (ISI) variability of less than $50 \%$. Bistability was pre-detected by a coefficient of variation (CV) of the inter spike intervals being 1.5 times larger than the $\mathrm{CV}$ of the peaks and then by visual inspection of the traces. Spike frequency was determined by the average ISI, in case of bistable dynamics by the average inter peak interval.

The spike onset frequency $\nu_{0}$ was determined by fitting the curve

$$
\nu=\Theta\left(i_{\mathrm{e}}-c_{2}\right)\left[c_{1}\left(i_{\mathrm{e}}-c_{2}\right)^{c_{3}}+v_{0}\right]
$$

with parameters $c_{i}, i \in\{1,2,3\}$ and $\nu_{0}$ to the $\nu$ - $i_{\mathrm{e}}$-curve data. Here $\Theta$ is the Heaviside step function, eq. (2.7.3). Errors where estimated using the parameter errors of this fit.

Resonance frequencies where estimated by inducing a zap stimulus [150, 184, 308, 185] with a time dependent instantaneous frequency $\nu(t)$ of the form

$$
\operatorname{Zap}(t)=a \sin \left(2 \pi \int_{0}^{t} \nu\left(t^{\prime}\right) d t^{\prime}\right)+i_{\text {off }}
$$

We used both, a linear increase of the frequency $\nu(t)=\alpha t$ with Zap $(t)=a \sin \left(\pi \alpha t^{2}\right)+i_{\text {offf }}$ and a similar expression for the linear decrease as a control with a frequency range form 
$0-25 \mathrm{~Hz}$ in $t_{\mathrm{d}}=30 \mathrm{~s}$. The amplitude of the zap currents was adjusted for each sweep to give a membrane voltage deflection of $\pm 5 \mathrm{mV}$. The constant offset current $i_{\text {off }}$ was adjusted to make the cell be either just below or above spike threshold. Spikes where determined as in the $\nu$ - $i_{\mathrm{e}}$-curves.

To measure the impedance we used eq. (E.2), i.e. the ratio of the Fourier transformations of the voltage signal divided by the stimulus. The frequency resolution is determined by the recording time $t_{d}$ to be $\frac{1}{t_{d}}$. The general form of the impedance for a conductance based neuron model is derived in appendix E, eq. (E.1), showing that the impedance takes the form of a parallel RCL circuit. To estimate the resonance frequency we fitted the experimentally obtained absolute squares of the impedance to the ones of an RCL circuit with a single inductivtiy, corresponding to a single slow delayed rectifier in the neuron model, given by the equation

$$
|Z(\nu)|^{2}=\frac{a+b \nu^{2}}{\nu^{4}+c \nu^{2}+d}
$$

with fit parameters $(a, b, c, d)$. Low frequency components of $0.5 \mathrm{~Hz}$ and less were dropped for the fits to exclude slow drifts [97]. The resonance frequency $v_{0}$ is then given by the maximum of the impedance fit at

$$
\nu_{\max }=\sqrt{-\frac{a+\sqrt{a^{2}-a b c+b^{2} d}}{b}}
$$

or $\nu_{\max }=0$ it the expression above is imaginary.

\subsection{Leak Induced Neuronal Excitability Switches in DNLL and CA3 Neurons}

In section 10.2.1 we have seen that an increase in leak conductance induces as transition from type I to type II neuronal excitability which is organized by a degenerate BogdanovTakens bifurcation. Let us first discuss some implications of this bifurcation structure that can be observed experimentally.

First, the creation of the limit cycles for small leak values $g_{\mathrm{L}}$ is via the SNIC and homoclinic bifurcation that both involve a fixed point which in its proximity slows down the dynamics so that the spiking frequency $\nu$ rises continuously form zero as a function of the input current $i_{\mathrm{e}}$ for small leak conductances. Starting from the NSL point the fixed point is no longer involved in the creation of the stable limit cycle and the neuron shows type II excitability with a non-zero onset spiking frequency. This is shown in the $\nu-i_{\mathrm{e}}$-curves for different leak values in the Wang-Buszaki neuron in fig. 12.3a. Moreover at the NSL point this offset frequency is zero and then increases along the DC line when the leak is increased as depicted in fig. 12.3b.

The dBT scenario also predicts that the transition in neuronal excitability is accompanied by a transition from integration to resonance at peri-threshold. This is organized by the the ordinary BT point from which the resonance frequency starts to become non-zero at threshold and increases for larger values of the leak conductance (cf. fig. 12.3b). The frequency becomes identical with the spiking onset frequency at the DH point.

A third feature of the transition is the creation of a region of bistability starting at the NSL point and terminating in the DH point. For the WB model the width in $i_{\mathrm{e}}$ direction 
(a)

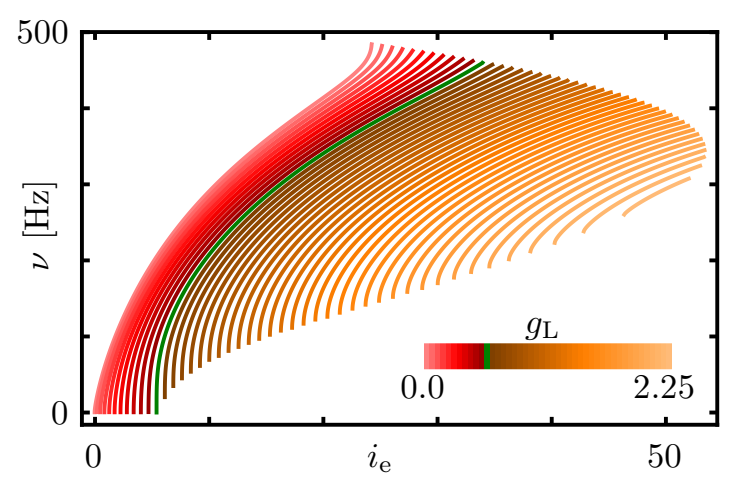

(b)

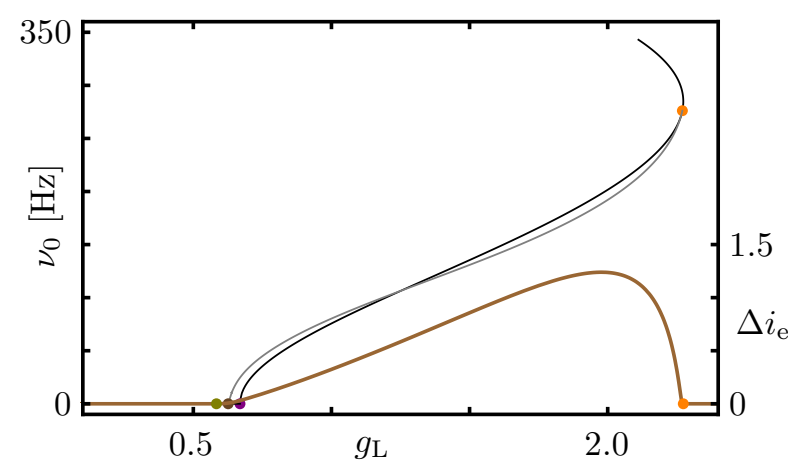

Figure 12.3. Frequencies and bistability in the WB neuron model. (a) spiking frequency $\nu_{0}$ of stable periodic orbits as a function of external input current $i_{\mathrm{e}}$ and leak conductance $g_{\mathrm{L}}$. Colors indicate the underlying bifurcation: SNIC (red), homoclinic (green) and DC (orange). (b) spike (gray) and resonance frequency (black) at threshold and width $\Delta i_{\mathrm{e}}$ (brown) of the region of coexisting stable fixed point and limit cycle. The onset spiking frequency becomes non-zero at the NSL point $(\bullet)$ reflecting the transition from type I to type II excitability. The resonance frequency starts to grow at the BT point $(\bullet)$. The region of bistability begins at the SNL point $(\bullet)$ and ends at the the DH point (•) (cf. also the bifurcation diagram in fig. 10.9).

of the region of bistability is shown in fig. 12.3. It increases over a broad range of leak values and then rapidly decreases to zero for large leak.

To study the spike frequency - input current $\nu$ - $i_{\mathrm{e}}$-relationship experimentally we induced step currents into the neuron with varying amplitudes. The effect of these step currents is illustrated for the Wang-Buzsaki model in fig. 12.5. For small leak conduc-

(a)

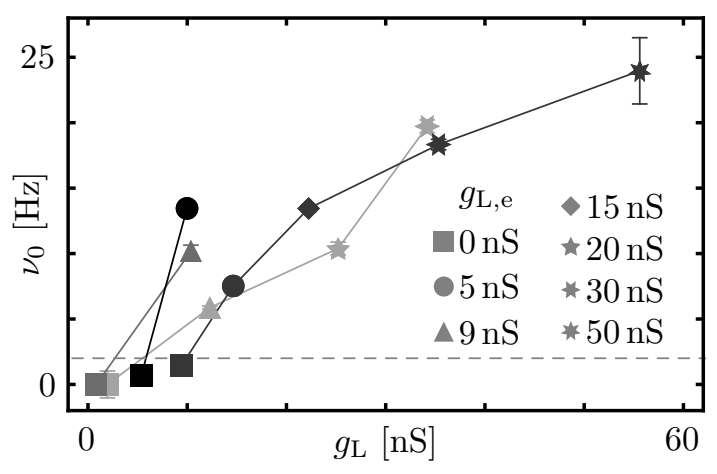

(b)

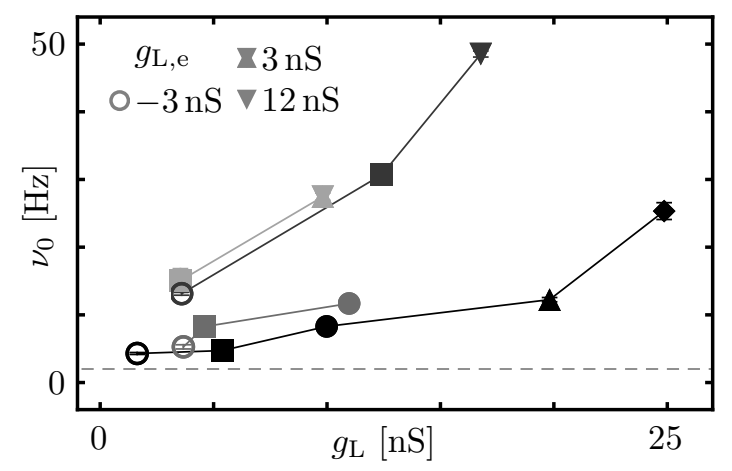

Figure 12.4. Leak conductance controls onset spiking frequency in DNLL neurons. Each plot shows the onset frequencies $\nu_{0}$ as a function of the measured leak conductance $g_{\mathrm{L}}$ for $n=4$ neurons (gray level, lines) each and different values of applied leak conductance $g_{\mathrm{L}, e}$ (symbols) (a) Intrinsic type I neurons with vanishing onset frequency $\nu_{0}<2 \mathrm{~Hz}$ (dashed line) at $g_{\mathrm{L}, \mathrm{e}}=0 \mathrm{nS}$ (ם) switch to type II neurons when the leak is increased. This finding is consistent with theorem 10.3.2 and the shape of the curves qualitatively matches the curves in fig. 12.3b. Lightest gray line is for neuron shown in fig. 12.6. (b) For intrinsic type II neurons the frequency increases with increasing $g_{\mathrm{L}}$. Leak subtraction by $g_{\mathrm{L}, \mathrm{e}}=-3 \mathrm{nS}(\mathrm{o})$ reduces the frequency but does not induce a switch to type I. Further leak reduction leads to unstable membrane potential dynamics (not shown). Both facts are consistent with the findings in section 10.2.5. 
(a)
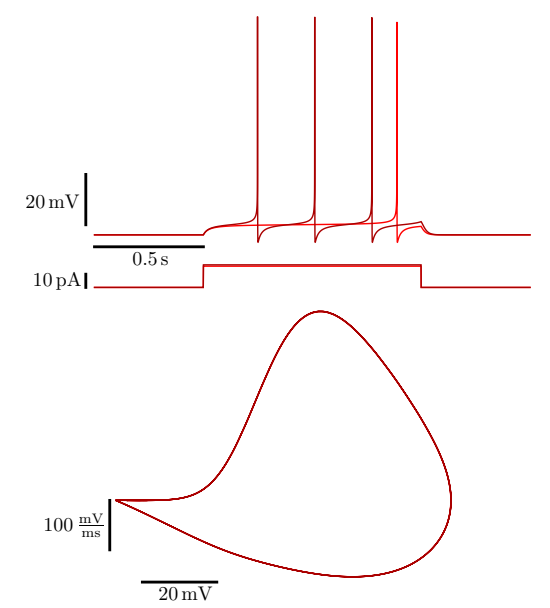

(b)
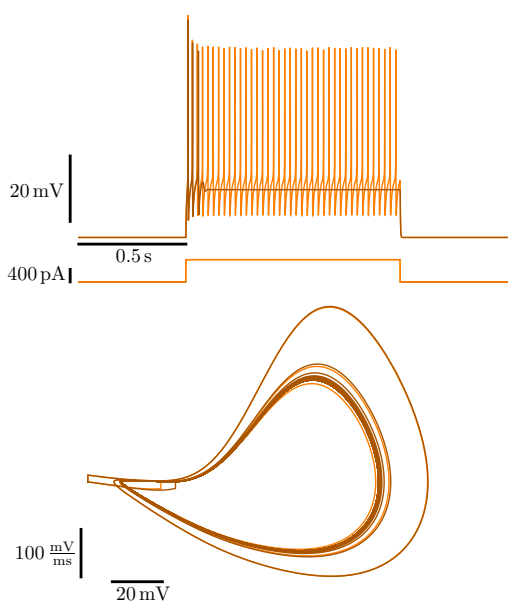

(e)

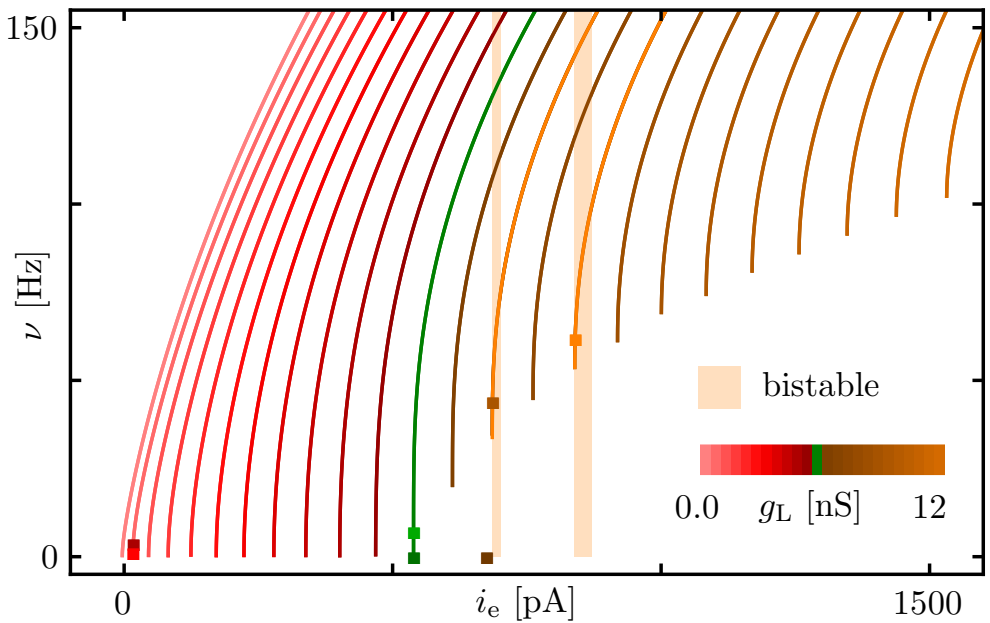

(c)

(d)

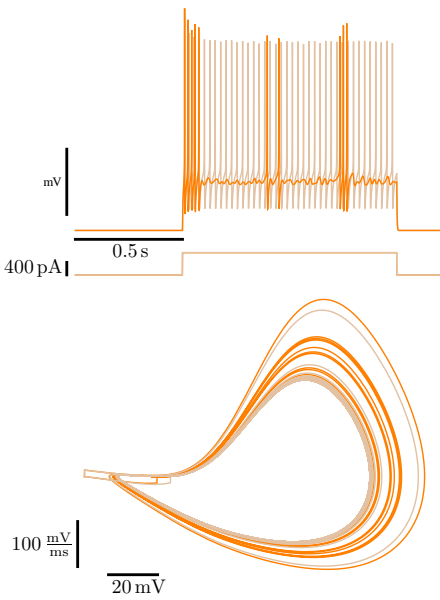

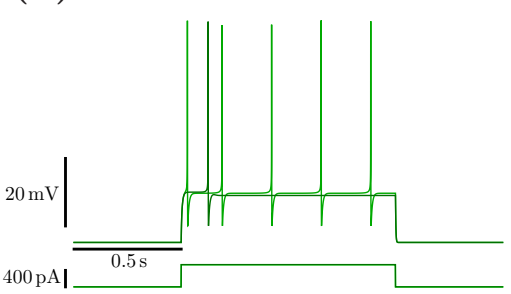

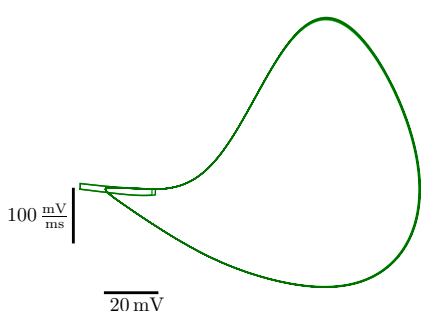

Figure 12.5. Leak induced neuronal excitability transition in the WB neuron predicts all features observed experimentally in fig. 12.6. Parameters for the WB model as in appendix D.3 with a cell surface area $A=10^{-4} \mathrm{~cm}^{2}$ to match the units in fig. 12.6 and capacitance in fig. 12.2. (a-d) each panel shows traces of membrane voltage $v$ (top) and spiking dynamics in the $v-\frac{d v}{d t}$ plane (bottom) for different input currents $i_{\mathrm{e}}$ (middle) and different values of externally applied leak conductance $g_{\mathrm{L}}(\mathrm{a}) 1 \mathrm{nS}(\mathrm{b}) 7 \mathrm{nS}$ and (c) $8 \mathrm{nS}$ and (d) $6 \mathrm{nS}$ corresponding to a SNIC (a), DC (b,d) and homoclinic (d) bifurcation (cf. fig. 10.9). In (c) we added small fluctuations to demonstrate the dynamics in the bistable region. (e) periodic spike frequency $\nu$ vs input current $i_{\mathrm{e}}$ for leak conductances. ( $\square$ ) indicate the traces shown in (a-d). For $g_{\mathrm{L}}=7 \mathrm{nS}$ and $g_{\mathrm{L}}=8 \mathrm{nS}$ the regions of bistability are also shown.

tances we see the typical features of type I behavior: Arbitrary large inter spike intervals with integrative properties reflected in a monotonic increase of the membrane potential to the spike. The spike shape and amplitude is stereotypical for all spikes and shows the same trajectory in the phase space like $v-\frac{d v}{d t}$-representation (fig. 12.5a).

For higher leak values periodic spiking is only possible starting from a non-zero frequency. For input currents that are to weak to give rise to periodic firing the traces show damped oscillations after the initial spikes indicating the resonance character of the dynamics. The initial spike amplitudes decay to some steady state value as can be seen in 
(a)

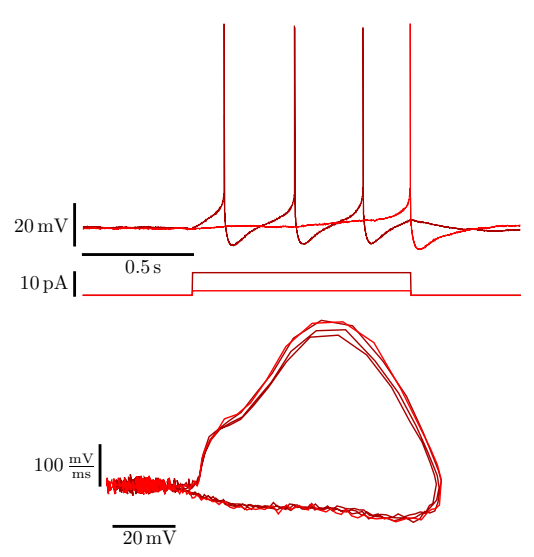

(b)
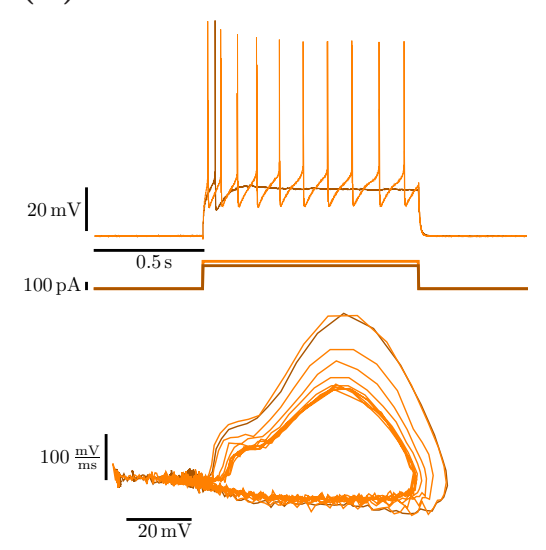

(e)

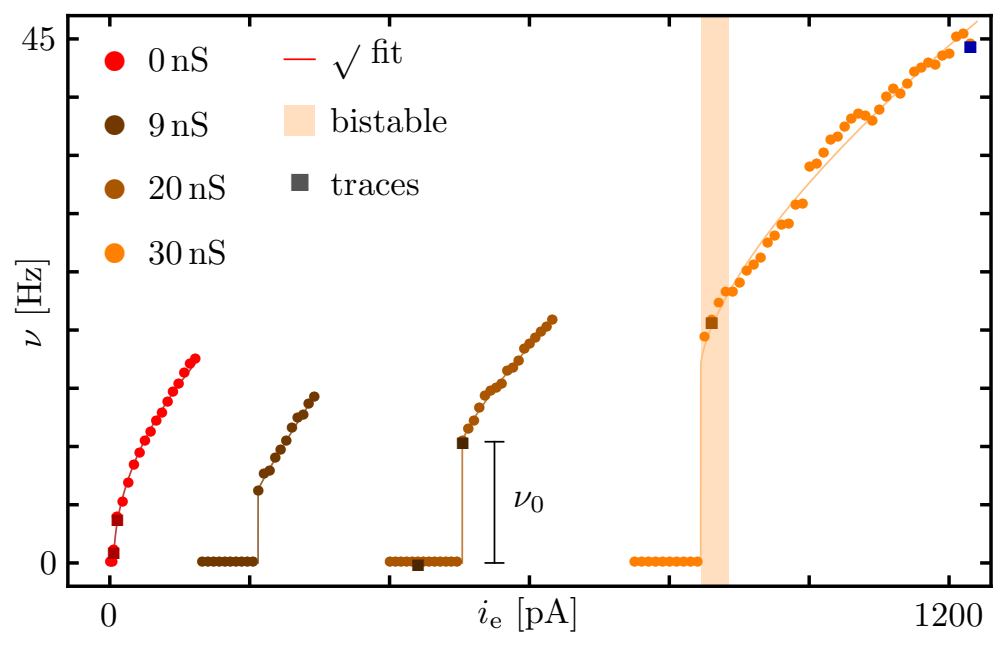

(c)

(d)
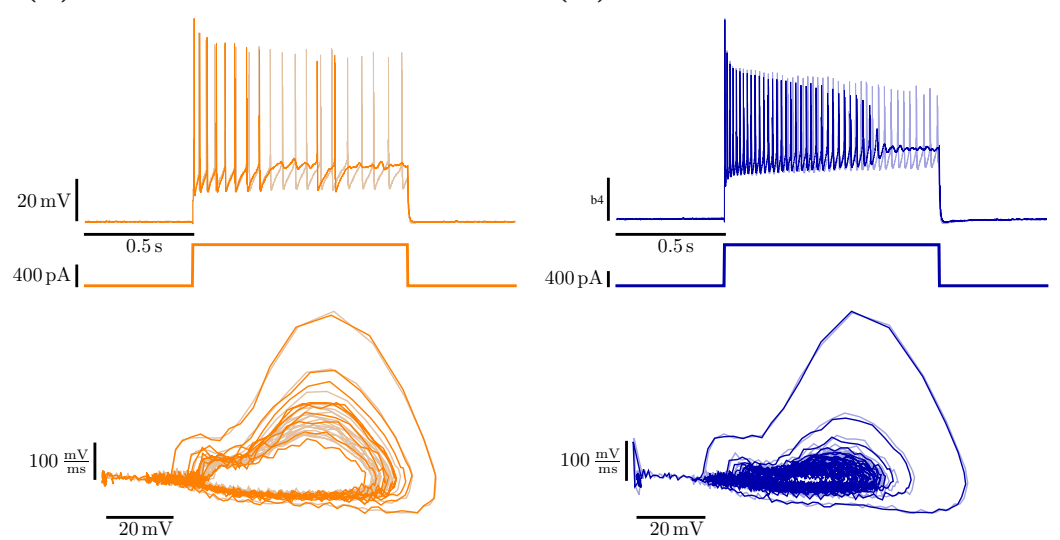

Figure 12.6. Leak induced neuronal excitability transition in a DNLL neuron. (a-d) each panel shows traces of membrane voltage $v$ (top) and spiking dynamics in the $v$ - $\frac{d v}{d t}$ plane (bottom) for different input currents $i_{\mathrm{e}}$ (middle) and different values of externally applied leak conductance $g_{\mathrm{L}}$ (a) $0 \mathrm{nS}$ (b) $20 \mathrm{nS}$ and (c,d) $30 \mathrm{nS}$. (e) periodic spike frequency $\nu$ vs input current $i_{\mathrm{e}}$ for the four different externally applied leak conductances. The traces in (a-d) are indicated by squares. Increase in leak conductance is reflected by the onset of periodic spiking at larger $i_{e}$ values and an increase of onset frequencies $\nu_{0}$ from zero to non-zero values, indicating a neuronal excitability transition from type I to type II. For $g_{\mathrm{L}, \mathrm{e}}=30 \mathrm{nS}$ the neuron exhibits bistable dynamics as shown in (c). For very large input currents spiking stops via the conductance block as shown in (d).

the $v-\frac{d v}{d t}$-representation (fig. 12.5b). Adding small noise fluctuations to the input current in the region of bistability shows switching between periodic spiking and non-spiking oscillatory sub-threshold dynamics. According to fig. 12.3 the spiking and the sub-threshold oscillations have similar frequencies (fig. 12.5c).

The analysis of the response of a DNLL neuron to such step currents is shown in figures $12.2,(12.6)$ and 12.4. The results in fig. 12.2 show the dependence of the measured leak $g_{\mathrm{L}}$ and capacity $c_{\mathrm{m}}$ for different artificially imposed leak conductances $g_{\mathrm{L}, e}$ confirming the validity of the dynamic clamp method. Figure 12.6 shows traces and $\nu-i_{\mathrm{e}}$-curves for a type I DNNL neuron subject to four different values of the leak conductance. The data very well resembles the model predictions in fig. 12.5. In particular, for no external leak $g_{\mathrm{L}, \mathrm{e}}=0 \mathrm{nS}$ the neuron exhibits type I behavior, with arbitrary slow firing close to the 
detection threshold of $1 \mathrm{~Hz}$ and weak spike-amplitude variability for periodic spiking (cf. the $v-\frac{d v}{d t}$ plot in fig. 12.5a).

When increasing the leak conductance using $g_{\mathrm{L}, \mathrm{e}}=9 \mathrm{nS}$ or $g_{\mathrm{L}, \mathrm{e}}=20 \mathrm{nS}$ the neuron starts spiking with non-zero frequency, it shows decaying spike amplitudes and oscillations after the initial spikes before the onset of periodic spiking (cf. fig. 12.5b) indicating type II neuronal excitability. Furthermore, at $g_{\mathrm{L}, \mathrm{e}}=20 \mathrm{nS}$ we detect a region of bistability (cf. fig. $12.5 \mathrm{c}$ and e). Comparison with fig. 12.5 shows that these experimental findings are fully consistent with a leak induced type I to type II transition as found analytically in chap. 10.

Note that the region to detect a homoclinic bifurcation is rather small (cf. fig. 12.5 and 12.3) and the steep frequency scaling $\nu_{0} \propto \frac{1}{\log \left(i_{\mathrm{e}}-i_{\mathrm{e}}^{*}\right)}$ makes it hard to discriminate it from a type II situation. We therefore conclude that the neuron is already type II for $g_{\mathrm{L}, \mathrm{e}}=9 \mathrm{nS}$ and the transition appeared before this point. Furthermore, near the transition the region of bistability is small but grows for larger values of the leak conductance (cf. fig. 12.3) which makes it hard to detect it initially.

We find similar transitions in all type I neurons $(n=4)$ measured in the DNLL. With natural leak conductance these neurons show an onset frequency below the detection threshold of $1 \mathrm{~Hz}$ and all the features of type I excitability while increasing the leak artificially switches these neurons to type II excitability. Figure 12.4a shows the relation between the onset periodic spiking frequency $\nu_{0}$ as a function of the measured leak $g_{\mathrm{L}}$ for all neurons. It is consistent with the theoretical finding in fig. 12.3b.

For type II neurons the onset frequency increases with increasing leak. Subtraction of leak lead to smaller onset frequencies but a switch to type I was not observed as the neuronal dynamics became unstable for strong leak subtraction (cf. fig. 12.4b). This finding fully agrees with the finding that type II model neurons cannot be switched back to type I neurons in general (cf. sec. 10.2.5 and theorem 10.3.2).

We also tested the effect of an increase in leak conductance in pyramidal neurons in the CA3 area of the hippocampus. The results are shown in fig. 12.7. Intrinsically these neurons showed type I excitability (cf. fig. 12.7a,c). An increase in leak conductance switched these neurons in all cases to type II (cf. fig. 12.7b,c) consistent with the theoretical predictions.

The region of bistability in these neurons is much larger compared to the DNLL neurons. We attribute this enlargement of the region of bistability to adaption currents that reduce firing rate and keep the neuron dynamics in the bistable regime. Preliminary bifurcation studies for neurons with additional adapting M-currents show that this is indeed the case. Note also that in contrast the DNLL neurons, the curves almost fall on top of each other. We attribute this to a broader diversity of cell properties in the DNLL [414] while in the CA3 region we only studied pyramidal cells.

To summarize, dynamic clamp recordings of DNLL and pyramidal neurons show a leak induced transition from type I to type II neuronal excitability. These experimental findings are fully consistent with an underlying excitability transition organized by a degenerate Bogdanov-Takens point as studied theoretically in sec. 10.2.1.2. We give further evidence for this in the next section. 
(a)
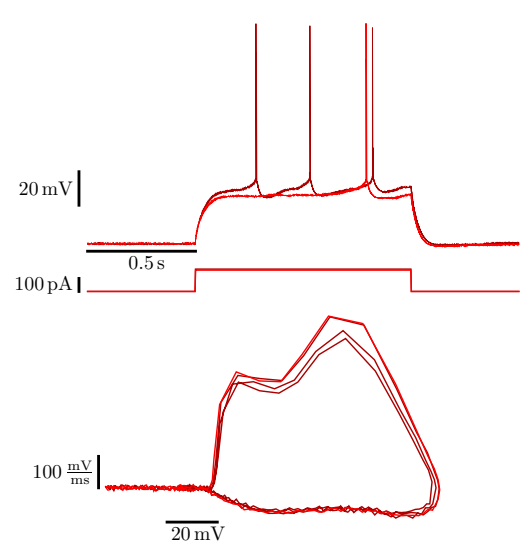

(b)

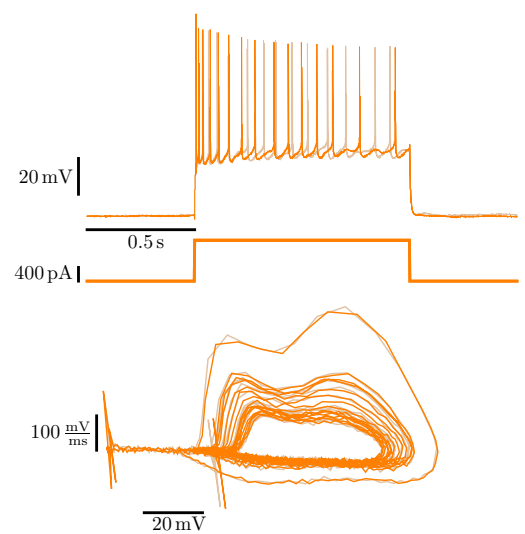

(c)

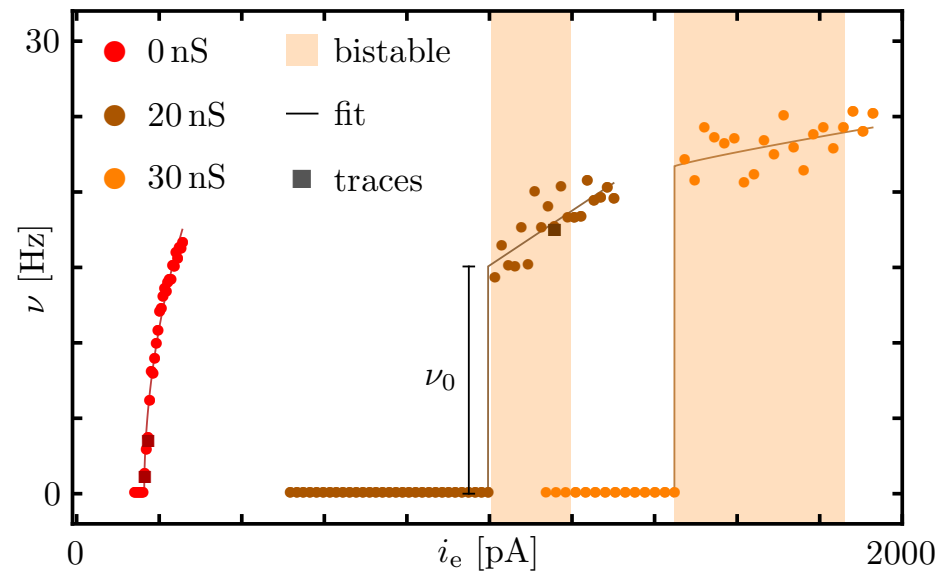

(d)

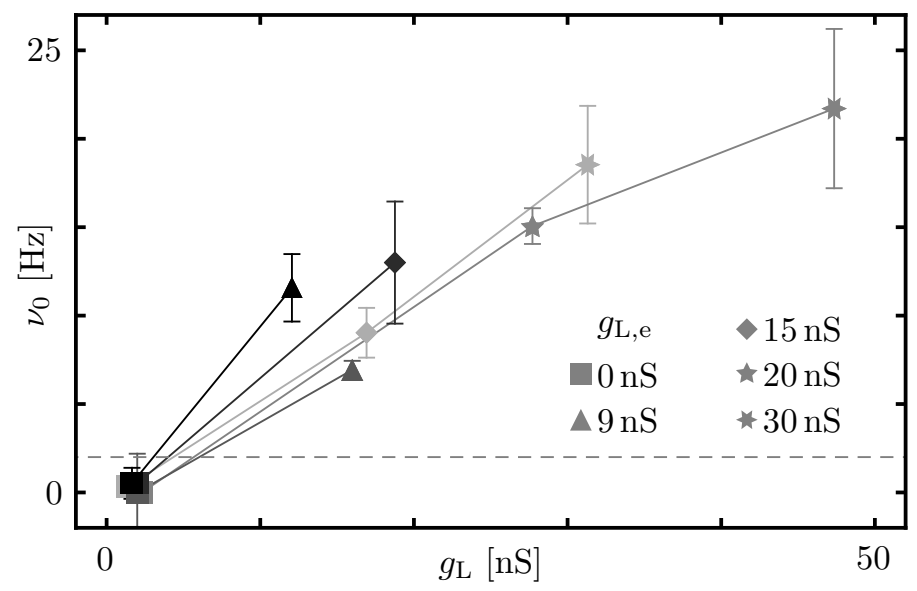

Figure 12.7. Leak conductance switches hippocampal CA3 pyramidal neurons from type I to type II. (a) neuron membrane potential $v$ (top) and $v$ vs. $\frac{d}{d t} v$ (bottom) in response to a step like input current (middle) with no external leak shows type I dynamics. (b) as in (a) but for externally applied leak conductance $g_{\mathrm{L}, \mathrm{e}}=20 \mathrm{nS}$ shows bistable type II dynamics. (c) frequency $\nu$ vs. input current $i_{\mathrm{e}}$ at different external leak conductances. The $\mathbf{\square}$ 's show the data for traces in (a),(b). (d) Onset frequencies $\nu_{0}$ as a function of the measured leak conductance $g_{\mathrm{L}}$ for $n=5$ neurons (gray level, lines) and different values of externally applied leak conductance $g_{\mathrm{L}, e}$ (symbols). All neurons show type I dynamics with vanishing onset frequency $\nu_{0}<2 \mathrm{~Hz}$ (dashed line) at $g_{\mathrm{L}, \mathrm{e}}=0 \mathrm{nS}(\boldsymbol{\square})$ and switch to type II neurons when the leak is increased. This finding is consistent with theorem 10.3.2. The shapes of the curves are very similar for all neurons and qualitatively match the curves in fig. $12.3 \mathrm{~b}$. Lightest gray line is for the neuron shown in (a-c).

\subsection{Leak Induced Transition From Integration to Res- onance at Peri-Threshold in CA3 Pyramidal Cells}

The transition from type I to type II neuronal excitability organized by a dBT point is always accompanied by a transition from integrative to resonance properties at perithreshold. Here we confirm this prediction experimentally.

The resonance frequency for the WB neuron model is shown in fig. 12.3b and shows a similar shape as the onset spiking frequency. In particular, the resonance frequency increases from zero starting at the NSL point and becomes identical to the onset frequency 
(a)

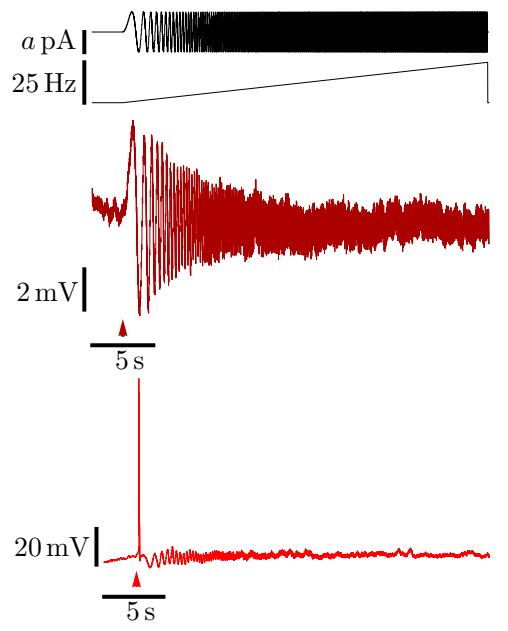

(c)

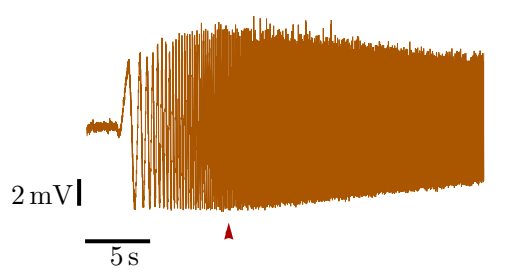

$20 \mathrm{mV}$

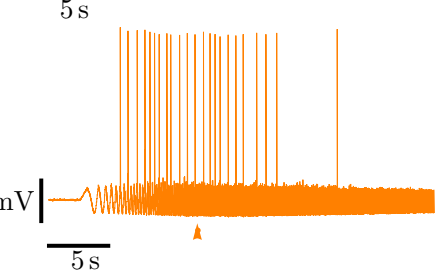

(d) (b)
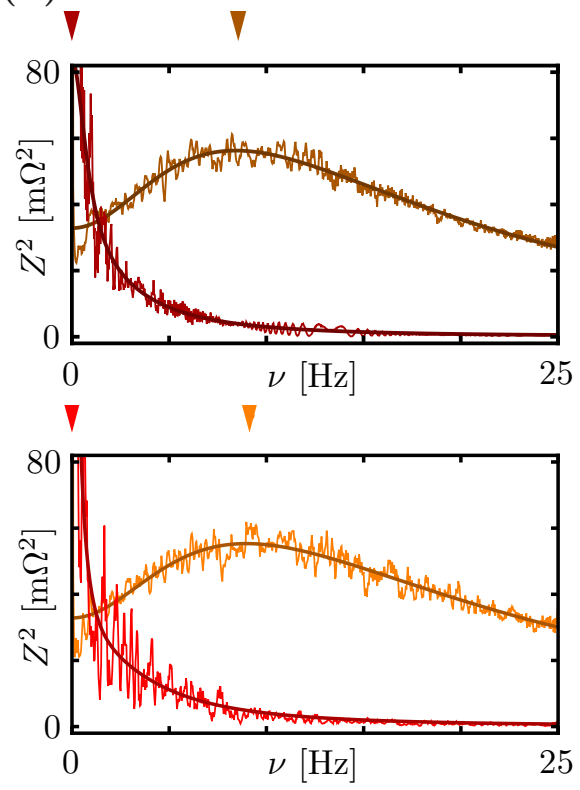
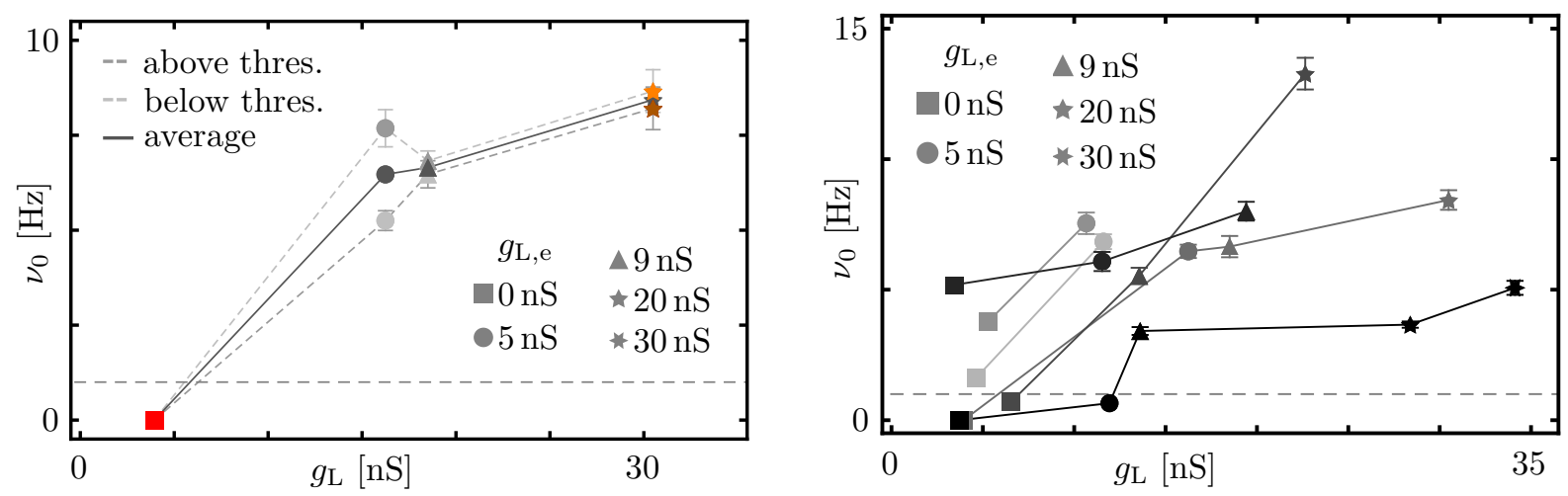

Figure 12.8. Leak induced integration to resonance transition at peri-threshold in CA3 pyramidal cells. (a) voltage traces near to but below (middle row) and above threshold (bottom row) in response to a zap stimulus (top) with current amplitude $a$ adjusted to achieve a $4-5 \mathrm{mV}$ voltage amplitude for $g_{\mathrm{L}, \mathrm{e}}=0 \mathrm{nS}$ (first column, red) and $g_{\mathrm{L}, \mathrm{e}}=20 \mathrm{nS}$ (second column, orange). Arrows indicate the time in which the instantaneous frequency of the zap current attains the resonance frequency determined via the impedance. (b) impedance curves for the traces in (a) below (top) and above threshold (bottom) showing that for zero external leak there is no resonance (red) whereas for $g_{\mathrm{L}, \mathrm{e}}=20 \mathrm{nS}$ (orange) the maxima (arrows) have shifted to non-zero values. (c) resonance frequencies $\nu_{0}$ determined in (b) for all applied leak conductances of this experiment plotted as a function of the measured leak $g_{\mathrm{L}}$. The graph shows that the resonance frequency above threshold (light gray, dashed) is always slightly larger than the frequency below (gray, dashed). To obtain the resonance frequency at peri-threshold we average both frequencies (dark gray, solid). The curve shows a transition from integration with zero resonance frequency to resonance for non-zero leak values above the detection threshold of $1 \mathrm{~Hz}$ (dashed horizontal line). (d) average resonance frequencies $\nu_{0}$ vs measured leak $g_{\mathrm{L}}$ as in (c) for $n=6$ CA3 neurons, three of which show a switch from integration to resonance while the other three starting already with non-zero frequency only increase their resonance frequency.

at the DH point.

We access the resonance frequency experimentally by measuring the impedance curve of CA3 pyramidal cells from the response of the neuron to injection of zap currents cover- 
(a)

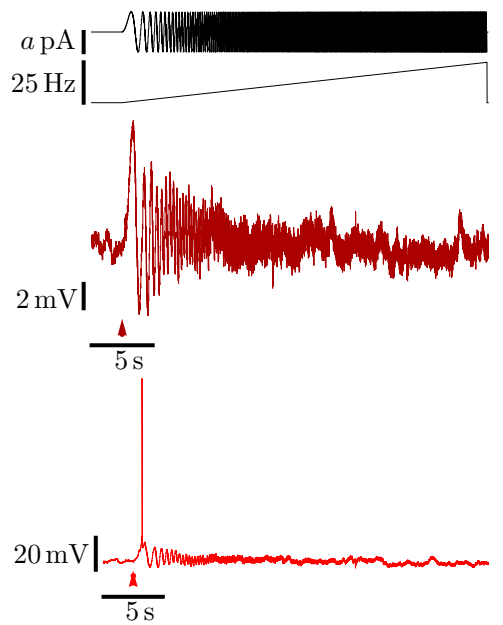

(c)

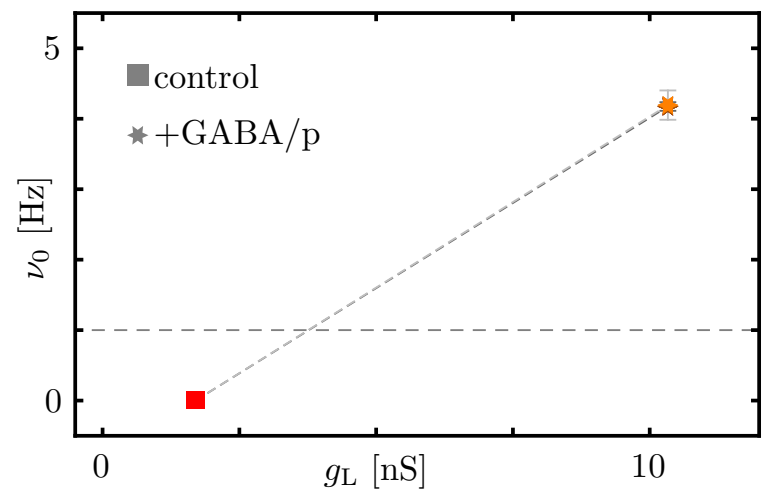

(b)
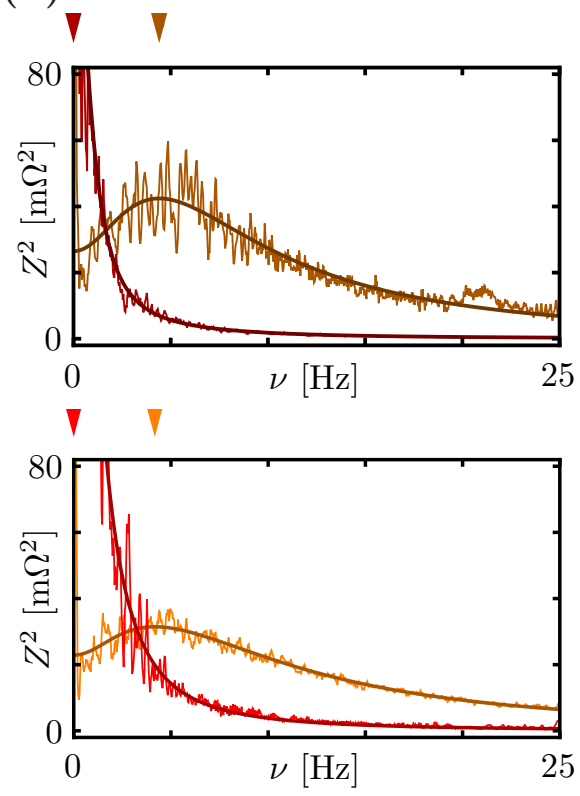

(d)

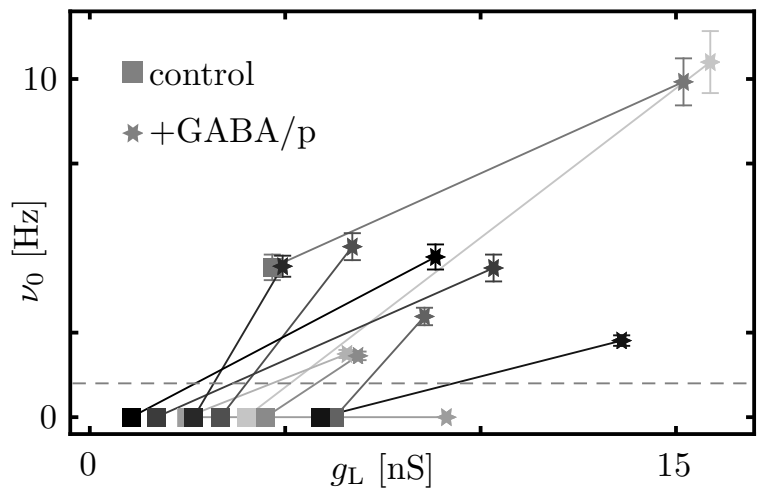

Figure 12.9. GABA induced integration to resonance transition at peri-threshold in CA3 pyramidal cells. (a) voltage traces near to but maximally $1 \mathrm{mV}$ below (middle row) and above threshold (bottom row) in response to a zap stimulus (top) for control (first column, red) and with GABA puffs close to the cell to activate inhibitory receptors (second column, orange). Arrows indicate the time at which the zap stimulus attains the resonance frequency determined in (b). (b) impedance curves for the traces in (a) for the control (red) and with GABA (orange), below (top) and above threshold (bottom). The maxima (arrows) shift from zero to non-zero values. (c) Resonance frequencies $\nu_{0}$ determined in (b) for control (red) and GABA (orange). The curve shows a leak-induced transition from integration with zero resonance frequency to resonance with a frequency above the detection threshold of $1 \mathrm{~Hz}$ (dashed horizontal line). (d) resonance frequencies $\nu_{0}$ vs measured leak $g_{\mathrm{L}}$ for $n=9 \mathrm{CA} 3$ neurons with and without GABA. For 7 cells we observe a switch from integration to resonance, one cell has a high leak conductance already in the control and GABA increases it's resonance frequency, one cell stays non-resonant.

ing a frequency range form $0-25 \mathrm{~Hz}$ (cf. sec. 12.1). The maxima of this curve determine the resonance frequency. To determine the resonance at peri-threshold a constant offset current is added to the zap stimulus and adjusted to make the stimulus slightly below and above spike threshold. The results are shown in fig. 12.8. For zero externally applied leak the voltage amplitudes in response to the zap stimuli decay monotonically for offset currents below threshold. Above threshold a spike is generated on the first peak of the 
stimulus. Due to an increase in the leak conductance the voltage amplitudes now attain a maximum below threshold and spiking starts not at the first but at later peaks. This change is reflected in the change of the impedance curves from an integrative characteristic with a zero resonance frequency to a non-monotonic shape with non-zero resonance frequency. In particular, non-resonant neurons become resonate with a frequency - leak relation ship qualitatively similar to the prediction in fig. 12.3b. Moreover the resonance frequencies are similar to the spiking frequencies at the same leak values and around $5-10 \mathrm{~Hz}$. For neurons that already showed a resonance an increase in the leak conductance increased the resonance frequency. Leak subtraction lead to unstable dynamics in these cases.

To summarize, for type I CA3 pyramidal cells we found a switch from integration to resonance by increasing the leak conductance as predicted and fully consistent with the findings for a transition organized by a dBT point.

\subsection{GABA Induced Transition From Integration to Resonance at Peri-Threshold in CA3 Pyramidal Cells}

One question that arises form the above results is whether the change in leak conductance needed to induce a neuronal excitability switch can be provided by shunting synapses. To access this question we activated inhibitory GABA receptors by applying GABA puffs close to the cell during the recordings. Applying GABA lead to an increase in leak conductance by $5-10 \mathrm{nS}$ which by comparison with the dynamic clamp results above should be sufficient to induce a switch in neuronal excitability. Indeed, fig. 12.9 shows that the application of GABA is sufficient to induce a switch from integration to resonance in full analogy to the dynamic clamp results in fig. 12.8. Moreover, the resonance frequencies for the induced leak change correspond to those found when artificially inducing the leak, indicating that GABA acts via shunting only. We attribute the stronger inter-neuronal variability in the resonance frequency vs leak curves to variations in effective leak caused by different distances of the GABA puff electrodes to the measured neurons.

In summary, this shows that the transition in neuronal excitability and from integration to resonance at peri-threshold can be controlled by shunting synapses alone. In particular, this opens the possibility to control the synchronization properties of neuronal cell groups dynamically. Inhibition (mediated by GABA) could thus increase the propensity of neuronal circuits to synchronize, supporting the role of GABAergic interneurons in generating rhythms in the $2-12 \mathrm{~Hz}$ range. We will study further consequences for the collective network dynamics in sec. 13.

\subsection{Summary and Discussion}

In this chapter we experimentally confirmed the theory for leak-induced neuronal excitability transitions developed in chapter 10. In particular, in CA3 pyramidal cells and neurons of the DNLL we found a type I to type II transitions by artificially imposing a leak conductance via dynamic patch clamp experiments. As predicted by the theory the experimentally observed transitions also showed a region of bistability and a transition in peri-threshold resonance. We further showed that the transition can be induced by 
the application of GABA, providing evidence that neuronal excitability can be controlled dynamically by synaptic shunting inhibition.

In hippocampal pyramidal neurons, both, type I [149] and type II neuronal excitability $[241,240,179]$ have been reported. Also switches between between the neuronal excitability types due the application of neuro-modulators been identified [352, 415]. In [306, 307] a switch from integration to resonance in these cells is shown when changing between in vivo to in vitro conditions. This switch is attributed to an interplay between shunting due to background synaptic activity [27, 74, 75, 293, 348] and adaption. Our findings are consistent with these finding in that both effectively contribute to an increase in the leak conductance at peri-threshold. Moreover, our bifurcation analysis gives an explanation for the bistability observed in the pyramidal cells. Preliminary numerical studies also indicate that the adaptation current may even increase the region of bistability. Our results further indicate that shunting alone is sufficient to induce a switch from type I to type II via activation of GABA-ergic synapses and therefore their excitability type can be controlled dynamically.

In [359] an increase in onset frequency of fast spiking interneurons in type II neurons due to an increased shunting conductance has been reported. This is in accordance with our experimental results for type II neurons that show an increase in their onset spiking and resonance frequency when increasing the leak conductance. It is also consistent with the predictions from the $\mathrm{dBT}$ bifurcation diagram where the onset frequency increases above the NSL point.

We found neuronal excitability transitions in hippocampal CA3 pyramidal cells as well as DNLL neurons, indicating that this is a general mechanism. In chapter 10 we found prevalence of excitability switches in a large number of neuron models further supporting the generality of this mechanism. Theoretically we showed that type II neurons cannot always be switched to type I excitability by leak subtraction because this destabilizes the dynamics. We exactly find this behavior in the experiments for both the CA3 region as well in the DNLL, further supporting the generality of our theory.

Interestingly, voltage independent Potassium leak channels exist in neurons [131] and are also found to regulate spiking activity [40]. Our results indicate that such channels in principle can provide a mechanism for neurons to regulate their neuronal excitability type.

We observed that application of GABA induces a switch to type II excitability at peri-threshold in CA3 pyramidal cells with a resulting resonance frequencies of $5-10 \mathrm{~Hz}$. This lies withing the frequency range of the theta rhythm $2-10 \mathrm{~Hz}$ in the hippocampus $[52,53]$. An intrinsic resonance is not required for entrainment of the pyramidal cells to this rhythm but strongly influences the responsiveness of the cells to these oscillations [159]. Switching the resonance properties of the pyramidal cells via shunting inhibition may thus provide a mechanism to dynamically control their participation in this rhythmic activity with further consequences for dynamic grouping [162, 158], sensimotor processing [392], navigation [288] and learning and memory [394] associated with theta oscillations.

Our findings provide evidence that neuronal excitability can be controlled dynamically via shunting inhibition. As neuronal excitability is connected to synchronization properties of networks $[157,100,177]$ (cf. also the next sec. 13), to the selective response to different stimulus features [151, 256, 318, 359, 360, 361], information coding [329, 166] and memory recall and storage [239] this mechanism may provide an efficient way to dynamically control these collective properties. 


\section{Chapter 13}

\section{Dynamic Neuronal Excitability and Collective Network Dynamics}

this chapter we study how dynamic neuronal excitability switches induced by changes in leak conductance are capable of controlling collective network dynamics. We use wellestablished results $[100,335]$ connecting neuronal excitability of neurons to their phase response curves and infer a transition from synchronization to desynchronization. We further study novel collective effects for stronger coupling arising from the bistable region encountered in the neuronal excitability transition. In networks of type I and II neurons we further show that only the type II neurons synchronize. By changing the excitability type via shunting inhibition dynamically this gives rise to a dynamic grouping mechanism. Finally we present a small circuit in which the neuronal excitability type and synchronization properties of excitatory neurons are controlled by a shunting inhibitory interneuron.

\subsection{Leak-Induced Neuronal Excitability Transition and Phase Response}

The neuronal excitability type of a neuron strongly influences their phase response $[100$, 335] (cf. also section 3.4). In the neuronal excitability transition of section 10.2.1.2 we encountered four different co-dimension one bifurcations to periodic spiking. The shapes of the phase response curves $z(\phi)$ (PRC) associated with these bifurcations are derived in $[100,335]$ : Close to a SNIC or a homoclinic bifurcation the phase response is always positive with approximate shapes given by z $(\phi)=c_{1}(1-\cos (\phi))$ or $\mathrm{z}(\phi)=c_{1} \exp \left(-c_{2} \phi\right)$, respectively. For double limit cycle and Hopf bifurcations the phase response attains negative parts and is of the form $c_{1} \sin \left(\phi-c_{2}\right)$. Thus, it follows that the PRC changes from a purely positive to a sinodial shape during the neuronal excitability transition. This is illustrated for the ML model in fig. 13.1. In weakly homogeneously and excitatory coupled neurons [177] these results imply that for small leak conductances the network will tend to desynchronize, while for larger leak conductances a transition to synchrony occurs. This is shown in fig. 13.2a,b.

Interestingly, in weakly coupled networks with both, type I neurons (due to a mall leak) and type II neurons (due to a large leak), we observe that the type II neurons synchronize their dynamics to form a single cluster, while the type I neurons show asynchronous dynamics (cf. fig. 13.2d). Thus, the (de)synchronization properties of a subgroup 
(a)

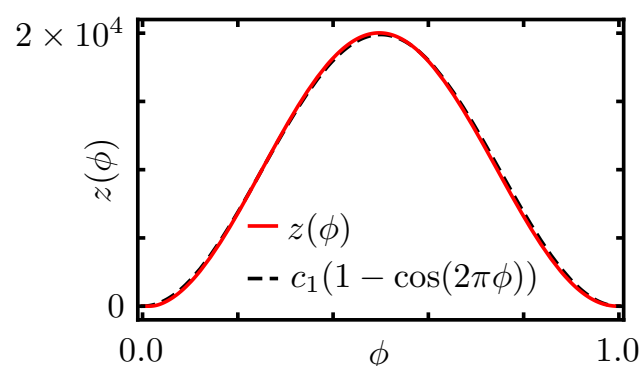

(c)

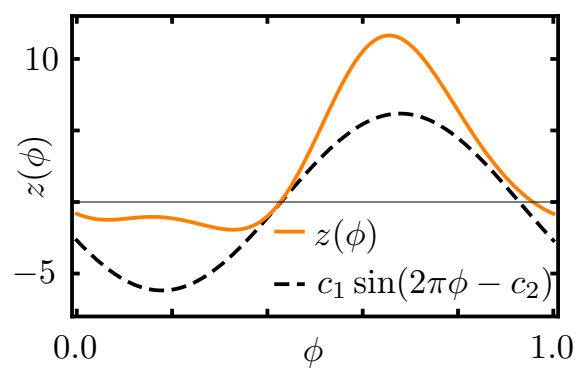

(b)

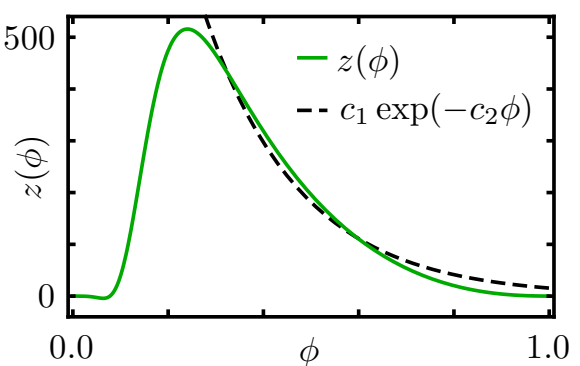

(d)

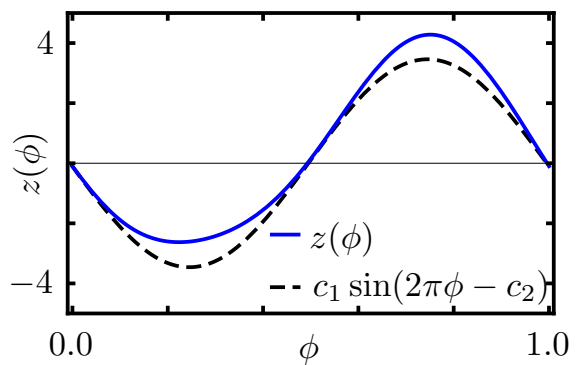

Figure 13.1. Phase response curves and neuronal excitability transition induced by an increasing leak conductance. Shown are the phase response curves $z(\phi)$ (solid) calculated via the adjoint method [228] (cf. eq. (3.4.7)) at different stages of the neuronal excitability transition form type I to II in the Morris-Lecar model together with the fits (dashed) predicted by underlying bifurcation to periodic spiking $[100,335]$ for (a) a SNIC bifurcation at $g_{\mathrm{L}}=2.0$, (b) a homoclinic bifurcation at $g_{\mathrm{L}}=4.2(\mathbf{c})$ a DC bifurcating at $g_{\mathrm{L}}=5.5$ and (b) Hopf bifurcation at $g_{\mathrm{L}}=8.7$. The transition form type I to type II excitability is present in the PRC attaining parts with negative values in the type II regime.

of neurons within a larger network can be controlled, by dynamically changing their leak conductance. In particular a dynamically controlled leak conductance (e.g. by slow shunting inhibition) can render a neuron to become synchronized to other neurons with similar leak values and thus may provide a mechanism for dynamic grouping of neurons [162, 341] with further consequences for neuronal coding [140].

Note that there are some limitations to this reasoning. First, the PRC may change if the input current $i_{\mathrm{e}}$ attains large values far away form the bifurcation point $i_{\mathrm{e}}^{*}$ for the onset of spiking. In particular the bifurcation diagram in fig. 10.6 shows that the termination of spiking due to the conductance block at large input currents is via a double limit cycle bifurcation. This implies a change from type I neurons with purely positive PRCs to a sinodial shape found in type II.

Second, the synchronization properties also depend on the precise relation between the synaptic time constants and the spike frequency of the neurons. For slow synapses and short inter-spike-intervals positive and negative parts of the PRC may be smeared out due to averaging. Thus type II neurons coupled via slow synapses will not always synchronize. On the contrary small negative parts of the PRC using fast synapses can be very effective in stabilizing synchronous states.

Third, neurons are not always coupled weakly. Effects of stronger coupling are shown in fig. $13.2 \mathrm{~b}, \mathrm{c}$. In particular, in the $\left(g_{\mathrm{L}}, i_{\mathrm{e}}\right)$-bifurcation diagrams for the different neuron models there are regions of bistability when the limit cycles are created via the homoclinic or DC bifurcations. Stronger coupling in the DC bifurcation still leads to synchronization. However, this synchronization results in an even stronger synchronized synaptic input 
(a)

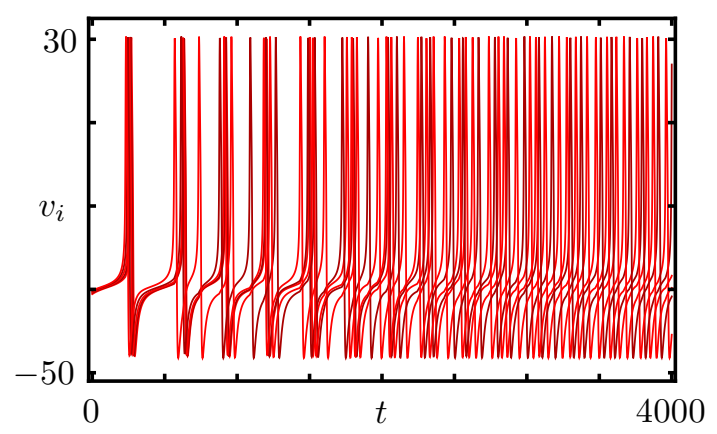

(c)

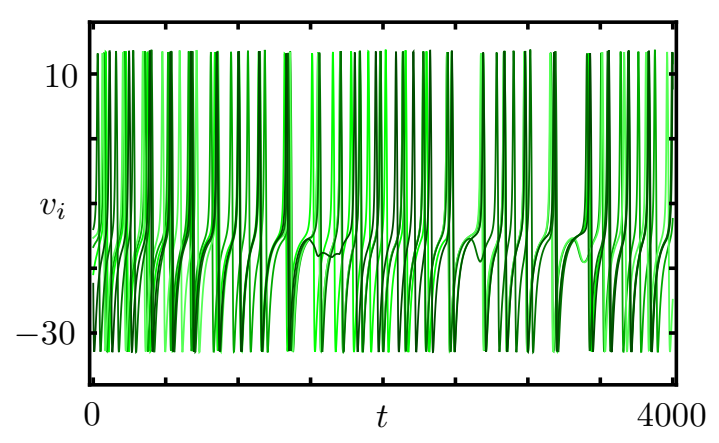

(b)

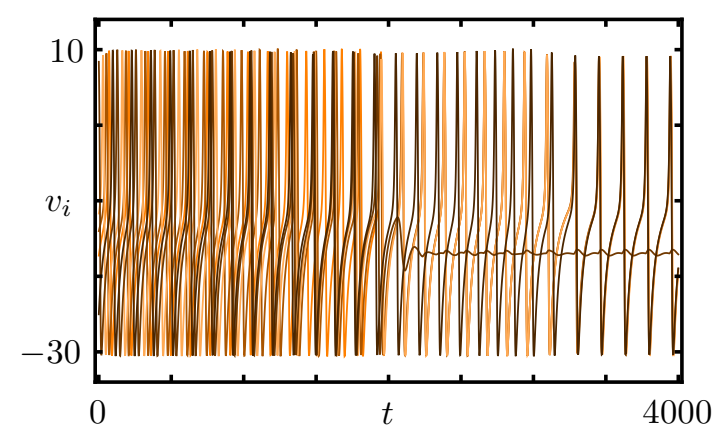

(d)

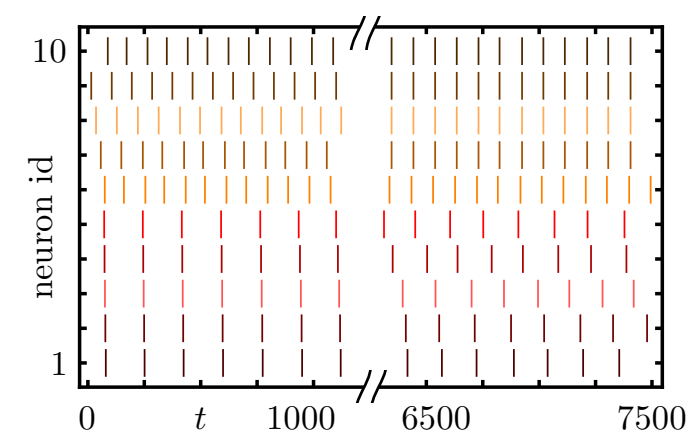

Figure 13.2. Neuronal excitability transition and collective network dynamics. (a-c) dynamics of homogeneously coupled $\varepsilon_{i j}=\left(1-\delta_{i, j}\right) \varepsilon$ networks of $N=5$ Morris-Lecar neurons with standard parameter as in appendix D.2 coupled via excitatory alpha synapses (2.7.2) with $\tau_{\mathrm{r}}=0.5$, $\tau_{\mathrm{d}}=20$ and $v_{\mathrm{syn}}=0$. (a) at a SNIC bifurcation for small leak $g_{\mathrm{L}}=2.0, i_{\mathrm{e}}=40$ and weak coupling $\varepsilon=0.15$ the network desynchronizes as predicted by an non-negative phase response curve in fig. 13.1. (b) At a DC bifurcation for leak $g_{\mathrm{L}}=5.5, i_{\mathrm{e}}=196$ and stronger coupling $\varepsilon=1$ the network synchronizes as predicted by the sinodial shape of the phase response. In addition, at some degree of synchronization the combined pulse strength is strong enough to kick several neurons into the basin of attraction of the coexisting fixed point. (b) for smaller leak $g_{\mathrm{L}}=5, i_{\mathrm{e}}=173$, close to a homoclinic bifurcation the system desynchronizes. Due to the strong coupling neurons are 'turned off' an 'on' by pulses that arrive when the neuron is close to the boundary of the basin of attraction. This leads to very irregular, possibly chaotic dynamics. (d) raster plot of a weakly $(\varepsilon=0.075)$ coupled inhomogeneous network of $N=10$ neurons. Neurons 1-5 have a leak as in (a), neurons 6-10 as in (b). Starting the system from random initial conditions, the type II neurons synchronize into a single group while the type I neurons get asynchronous.

pulse which can set some of the neurons into the basin of attraction of the stable fixed point and make them silent. This process continues until the combined pulse strength of the active neurons becomes to weak to further "turn off" neurons. For the homoclinic regime the neurons desynchronize, but are also pushed into the basin of the fixed point. This is due to the fact that in some parts of the state space the spiking dynamics are close to the basin boundary of the fixed point (cf. fig. 3.4). Due to non-synchronous inputs the neurons can additionally be kicked out of the fixed point basin. In total this leads to very irregular and possibly chaotic dynamics. An example is shown fig. 13.2. 


\subsection{A Synchronization-Desynchronization Circuit}

An increase in leak conductance may arise via inhibitory interneurons due to shunting. Thus using our results in chapter 10 and 12 neuronal excitability may be controlled dynamically via synapses. In fig. 13.3 we show that this is indeed possible already in small synchronization-desynchronization circuit consisting of two type I excitatory neurons that receive inhibition form an inhibitory interneuron. For weak external input the excitatory neurons being type I desynchronize. By increasing the external input the inhibition of the interneuron with a larger synaptic time constant effectively increases the leak conductance on the excitatory neurons that then switch their excitability type form I to II and start to synchronize. By releasing the external drive the neurons switch back to type I and start desynchronizing again. We also find this mechanism for inhibitory neurons only driven by the excitatory neurons and for larger populations of both excitatory and inhibitory neurons.

(a)

(b)

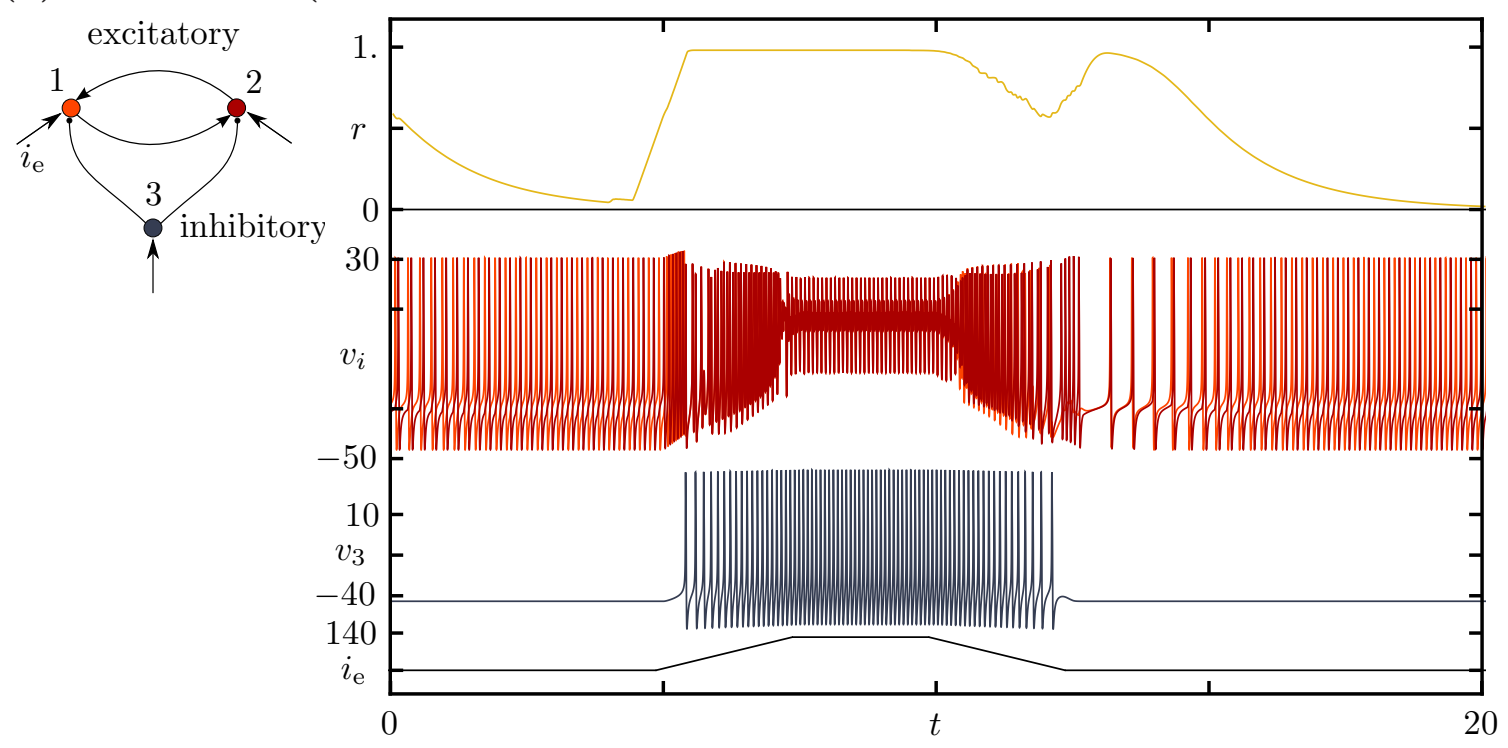

Figure 13.3. Dynamic control of synchronization and desynchronization via dynamic neural excitability switching in a neuronal circuit. (a) two mutually coupled excitatory neurons receive shunting inputs from an inhibitory interneuron. All neuron receive common inputs $i_{\mathrm{e}}$ from external sources. (b) dynamics of the sync-desync circuit in (a) using Morris-Lecar neurons with standard parameter as in D.2. Input currents are $i_{\mathrm{e}}=0$ for the inhibitory and $i_{\mathrm{e}}=40$ for the excitatory neurons. Synapses for excitation are as in fig. 13.2, for inhibition the synaptic decay time was larger $\left(\tau_{\mathrm{d}}=200 \mathrm{~ms}\right)$ with reversal potential $v_{\mathrm{syn}, \mathrm{inh}}=-60$. Shown are the potential traces $v_{i}, i=1,2$ for the excitatory neurons (red) and the inhibitory neuron $v_{3}$ (blue). The input current $i_{\mathrm{e}}$ (black) to all neurons is increased transiently. Top trace shows the synchrony measure $r=\frac{1}{2}\left|\sum_{j=1}^{2} \exp \left(2 \pi i \phi_{j}\right)\right|$ for the excitatory neurons with phases $\phi_{i}$ estimated by linear interpolation between the spike times. Thus $r$ is 1 for the phase synchronized state and 0 for the asynchronous state. Starting form random initial conditions the two excitatory neurons being in the type I regime desynchronize their spiking. When the external current is turned on the inhibition due to the activity of the interneuron increases the effective leak conductance and shifts the excitatory neurons into the type II regime which makes them synchronize. Turning the input off again decreases the shunting inhibition again and the almost synchronized system starts to desynchronize again. We note the synchronization for larger external inputs is not due to the transition. In fact it is stable even against strong perturbations (not shown). 


\subsection{Summary}

To conclude, we have shown that an increase in leak conductance of excitatory coupled neurons can change the dynamics from desynchronization for small to synchronization for larger values of the leak conductance. We used this mechanism to construct a circuit whose synchronization properties can be controlled by the strength of the external input currents. Moreover, increasing the leak conductance only for a subgroup of neurons leads to synchronization of this subgroup while the other neurons remained asynchronous. Thus, changing the leak of individual neurons dynamically, e.g. due to shunting inhibition, provides a mechanism for dynamic grouping of these neurons within a larger network. 


\section{Chapter 14}

\section{Conclusion and Outlook}

In this part of the thesis we theoretically and experimentally studied the impact of leak currents onto the excitability type of neurons. We found that an increase in leak conductance induces a switch from type I to type II neuronal excitability. In a large number of conductance-based neuron models, including those presented in refs. [273, 319, 320, $168,400,99,61,109,315,103,406]$, we showed numerically and analytically using a combination of multiple bifurcation theory and normal form theory that this transition consists of three intermediate steps via a saddle node loop, a neutral saddle node and a degenerate Hopf bifurcation. We further showed that this transitions is accompanied by a transition from integration to resonance at peri-threshold organized by an ordinary Bogdanov-Takens point and a region of bistable coexistence of a fixed point and a limit cycle. We explained this structure of the transition by a degenerate Bogdanov-Takens bifurcation of codimension-3 acting as an organizing center and proved the existence of such a point for all type I conductance based neuron models. We further showed that type II neurons cannot be switched back to type I by leak subtraction in general as the dynamics may lose stability.

We used normal form theory and the structure of the unfolding of the dBT point to transform a class of neuron models, including the Morris-Lecar and the Fitzhugh-Nagumo model, into a Liénard equation which gave rise to a novel interpretation of neuronal spiking dynamics in terms of an acceleration force and a nonlinear damping. This also facilitated an intuitive explanation for the switch in neuronal excitability. Using further transformations we determined the unfolding of the dBT point for the Fitzhugh-Nagumo model and proved the existence of an even higher degenerate Bogdanov-Takens point of co-dimension-4.

We applied these theoretical findings to study the effects of neuronal morphology on neuronal excitability. We explained a switch from type I to type II neuronal excitability which we found by varying the neuronal morphological from linearly extended to stellar like dendritic shapes in compartmental model neurons. Depending on the active dendritic ion channel properties these changes induce a effective change in leak conductance at the soma and cause the transition. We confirmed these theoretical results by analyzing data from experiments where the dendritic tree can be pinched temporarily [25].

Using dynamic patch clamp recordings we further confirmed the main predictions of our general theory in hippocampal CA3 pyramidal neurons and neurons of the dorsal lateral lemniscus. We find leak-induced neuronal excitability switches, a region of bistability and a transition at peri-threshold resonance. Application of an inhibitory synaptic transmitter likewise induced this transition. We therefore concluded that in principle neuronal 
excitability can be controlled dynamically via shunting inhibition.

We then studied several consequences of these findings for the control of collective network dynamics. Well-established results show that type I neuronal excitability leads to desynchronization while type II to synchronization in weakly coupled neurons [316, $100,184,177]$. We therefore concluded that regulating neuronal excitability via changes in leak conductance provides an effective mechanism to dynamically control the collective synchronization properties of neurons. We demonstrated this in a small circuit and further showed in larger networks of neurons with different neuronal excitability that the subgroup of type II neurons synchronizes while the type I neurons remain asynchronous. We concluded, that dynamic switching of neuronal excitability may thus serve as a mechanism for dynamic grouping of neurons with a wide range of applications in neuronal coding $[162,398,138,111]$. We further showed that in the region of bistability complex dynamics emerge for stronger couplings in homogeneous networks: For smaller leak conductances, stronger pulses arising via synchronization induce silencing of neurons, while for larger leak values the desynchronization causes irregular dynamics due to switching between resting and spiking of the individual neurons.

Neuronal networks in vivo can be highly active, resulting in a large fraction of open ion channels. In these high conductance states $[27,74,75,293,348]$ both, the excitatory inputs as well as the leak conductance are strongly increased. Our results then imply, that the neuronal excitability of the neurons may switch form type I at low activity, i.e. in low conductance network states, to type II in the high conductance state with the same consequences for the synchronization properties.

For future work it will be interesting to also investigate the impact of a change in leak conductance on bursting neurons. For these neurons, several underlying bifurcations to bursting activity are known [315, 189], but what are possible transitions between different bursting types and to tonic spiking and how are they organized by higher degenerate bifurcations? More generally, one can ask what higher degenerate bifurcations organize neuronal excitability. This not only provides simple normal forms for the mathematical analysis and efficient simulation of neuronal networks but may provide further insight into the existence of possible complex dynamical properties in single neurons. For example, we showed that an abstract neuron model $[108,278]$ is the unfolding of a degenerate Bogdanov-Takens bifurcation of co-dimension-4 [202] which predicts parameter regions with coexisting stable periodic orbits.

For future work our theoretical and experimental results provide a promising starting point for studying the role of dynamic neuronal excitability switches in brain function. In a first step a detailed study how dynamic neuronal excitability transitions influence the collective network dynamics of excitatory and inhibitory neurons and how these dynamics then act back in controlling the individual excitability types of the neurons may provide useful insights into collective control mechanisms for neuronal synchronization and neuronal dynamics in general. As the neuronal excitability type controls the phase response of a neuron, a better understanding of such complex dynamics may be gained by studying simpler pulse-coupled phase oscillator models (as used in part III of this thesis) with a variable phase response that depends on the inhibitory inputs.

Furthermore, the neuronal excitability type influences the selective response of neurons to different stimulus features [151, 256,318, 359, 360,361], the encoding of information $[329,166]$ and the recall and storage of memory [239]. It would therefore be interesting to see how dynamic transitions in neuronal excitability provide an efficient or even optimal mechanism for the control of these functions. 
For future experimental research, it would be interesting to confirm a dynamic control of neuronal excitability directly by stimulating inhibitory interneurons and simultaneously measuring a change in excitability type in a postsynaptic neuron. Moreover, by employing optical stimulation methods, it would be interesting to investigate how excitation of a larger number of inhibitory neurons is capable of controlling the neuronal excitability type of neurons in larger networks. 


\section{Part V}

\section{Local Control of Non-Local Information Flow}




\section{Chapter 15}

\section{Introduction}

Function of the brain is flexible. On a behavioral time scale dynamic routing of information is important to ensure an appropriate combination of perceived signals, contextual processing and activated memories of different entities and abstraction levels. Therefore the information flow between different neuronal areas has to be organized dynamically, it has to be controlled and learned.

Neuronal network connectivity is multi-scale. On the largest scale, the brain is organized into anatomically different modular regions each responsible for a certain information processing task [196]. On smaller scales neuronal structure is found to be organized in cortical columns [180], i.e. vertical aggregates of cells in the layered structure of cerebral cortex with similar response properties or receptive fields and sharing long range connections. On a cellular scale, experimental studies [344] show that the statistics of synaptic connectivity may be viewed as a skeleton of strong connections with an over representation of recurrently connected motives embedded in a sea of weaker connections. In ref. [294] it was shown that in neocortex, pyramidal neurons cluster into several interlaced groups of a few dozen neurons. Given this organization of neurons into groups, a question that arises in this context is how communication between the different components is organized and controlled.

Several mechanisms for communication and information transmission between subgroups of neurons have been proposed. For example, there are studies showing that synchronized neuronal spiking within a group of neurons can lead to stable propagation of this activity through a sequence of subgroups along embedded stronger feed-forward structures [77]. In neuronal networks with balanced inhibition and excitation [391], neuronal communication using firing rates can be changed by altering the balance between the long-range excitatory and inhibitory connections among two subgroups of neurons [395]. Both mechanisms rely on non-local adjustments of the long-range connections between the subgroups along which the signals are transmitted.

A different mechanism [326, 393, 111] uses excitability fluctuations during neuronal oscillations as the substrate for neuronal communication through coherence (CTC) [111]: Neurons in oscillatory groups not only synchronize their outgoing spikes which then become more effective in evoking postsynaptic responses [161, 300], but also periodically change their sensitivity to inputs $[396,49]$. Thus oscillations rhythmically open and close the neurons group's windows for communication and may provide a flexible mechanism for neuronal communication. Only coherently oscillating, e.g phase-locked neuronal groups can then communicate effectively if their communication windows for input and output are open at the same time $[111,112]$. 
This hypothesis emphasizes the role of oscillations in neuronal coding and information transmission [50, 324, 411, 169, 341] and is underpinned by several experimental studies $[113,330,410,412,142,408,28,56]$. First evidence was found in [113] where neurons in V4 activated by behaviorally relevant stimuli showed increased gamma frequency synchronization compared to neurons activated by distractors at nearby V4 sites. In [330] it was shown that neuronal coherence even facilitates long distance interactions between the spinal cord and the cortex.

The impact of the phase shift among two neuronal groups on their mutual influence was studied in [412]. There it was shown that the Spearman rank correlation coefficient of two multi unit activities varied as a function of the phase shift in $60 \mathrm{~Hz}$ gamma oscillations. At a preferred phase shift this correlation was highest and low otherwise. Thus the effective connectivity between two groups can be systematically varied by synchronization into different phase locking patterns. This result was confirmed recently in a simple model of two linearly coupled oscillators [98] and in a more biophysically plausible large scale model [48]. The simulations also showed that the speed of information exchange increases as a function of spectral power in specific frequency bands. In [365] it was further shown that in a numerical model of two unidirectional coupled groups of neurons undergoing gamma oscillations synchronous activation within the first group only spreads to the second for certain phase relations between the oscillations.

In [56] the influence of phase locking distributions among different local field potentials onto the spike generation of individual neurons was investigated. An inverse model based on a symmetrically coupled network of oscillators [59] was used to predict the underlying phase coupling network from the statistics of the measured phase differences [54]. It was shown that these phase couplings influenced the spike timing of individual neurons and that disperse neurons with a common preference for a certain phase coupling pattern tended to synchronize. This may be viewed as an extension of the CTC hypothesis originally proposed for two oscillating groups to many interlaced clusters.

To summarize, oscillations induce fluctuations in excitability and modulation of synchronized activity in neuronal groups and thereby provide a substrate for neuronal communication. In particular coherent oscillations and corresponding phase relations control the interaction between the groups. But what are the underlying mechanisms that control these phase relations and ultimately information flow in these oscillatory networks? How are they related to the underlying neuronal connectivity structure? Given that regulatory mechanisms in the brain act predominantly locally on the dynamical properties of individual neurons and their synaptic connections, one may further ask how local changes can contribute to the control of phase relations and information transfer on a larger scale.

This part of the thesis is devoted to these questions. We first study information flow in a general network model of coupled phase oscillators $[228,407,5]$, that not only serves as model for coupled oscillators in neuronal systems [251, 302, 177] and biology [407] but also in physics and chemistry. We derive an analytical expression for the information flow in these networks with arbitrary network topology. We then concentrate on hierarchical networks consisting of more strongly connected clusters with weaker inter-cluster couplings. We analytically derive the non-local information flow between the clusters as a function of all structural and dynamical network parameters. We use our results to reveal how information transfer is controlled by the underlying physical connectivity and find that local changes of links or frequencies within a group can control the non-local information flow from and to this cluster. Via this mechanism a group may thus "tune" to sources of relevant information and specify receivers of its own information output. Interestingly, 
our analytics unravel that local changes in the strength of a single link can also remotely control the information transmission between two other distant oscillators that are both physically unchanged. We then link our theoretical findings to more realistic hierarchical networks of spiking neurons exhibiting gamma oscillations (cf. also [23, 408]) and extend the study to information flow based on spike patterns.

\subsection{Neuronal Oscillations}

Collective neuronal oscillations are frequently observed in many parts of the nervous system, ranging from primary sensory circuits, through local cortical networks to larger inter areal formations $[26,138,374,53,128]$. Neurons in these systems share strong timevarying correlations that arise form external sources or synaptic interactions within the network which then lead to the macroscopic oscillations observable in electroencephalography recordings [26] or on a smaller scales in local field potentials [140] and multi-unit activity [113]. A broad range of oscillation frequencies is observed. Slow and delta rhythms $(1-3 \mathrm{~Hz})$ that are measured during slow wave sleep in the thalamus as well as in cortical areas and are associated to synaptic plasticity and memory formation [350, 181]. Theta oscillations $(4-8 \mathrm{~Hz})$ arise in the hippocampus for example in parallel to motor activity and spatial navigation [51]. Alpha band activity $(8-14 \mathrm{~Hz})$ observed in the occipital lobe [26] is thought to represent an idle state of the brain [7] and to serve as a mechanism for top-down inhibition [216]. Fast oscillations such as beta $(20-30 \mathrm{~Hz})$ and gamma $(30-100 \mathrm{~Hz})$ are widely observed in hippocampal [35], thalamic [349, 348] and cortical networks [140, 95, 374, 113, 94, 410, 412] and associated with learning, information binding and communication.

Different mechanisms can give rise to fast oscillations in recurrent networks of spiking neurons. Gamma oscillations can arise due to the interaction of inhibitory neurons (ING) with slow synaptic time constants that receive tonic excitatory inputs [403, 400, 21]: A strong inhibitory pulse then synchronizes these neurons and periods of silencing inhibition alternate with synchronous firing. A second mechanism [191, 404, 252, 31] is based on a feedback loop between mutually coupled excitatory pyramidal cells and inhibitory inter neurons (PING): Excitation of the inhibitory interneurons silences the excitatory population. In turn the inhibitory neurons do not receive sufficient excitation to sustain firing and inhibition decays. Remaining low excitatory activity then re-excites the pyramidal cells and the cycle repeats. Both mechanism are not mutually exclusive [366]. Further ING and PING generalize to sparsely synchronous oscillations in which only a small subset of neurons spike in a single cycle of the collective oscillation [45].

\subsection{Measuring Information Flow}

There exist several measures to quantify information flow between two or more time series, including bidirectional information [254], directed information [255, 223] and transfer entropy [331] and its extensions [346]. In this work we mainly focus on delayed mutual information (sec. 15.2.1) mainly because it is analytical tractable and has numerical advantages. However our study can be extended to a version of the transfer entropy measure (sec. 15.2.2) with similar results. 
(a)

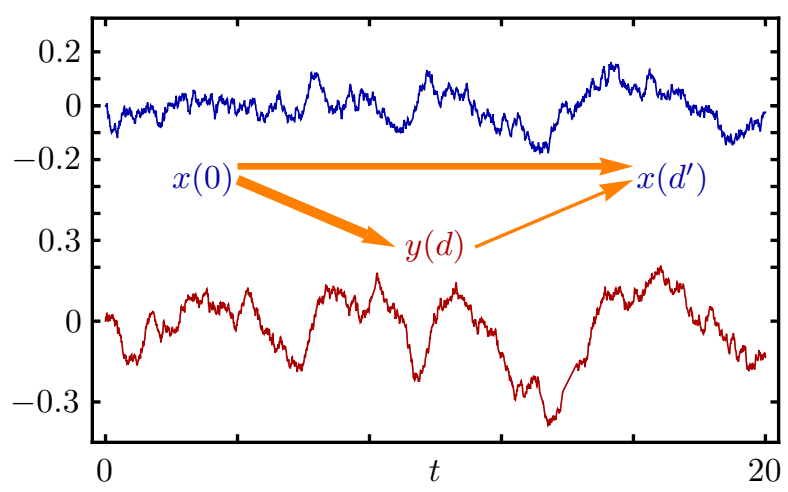

(b)

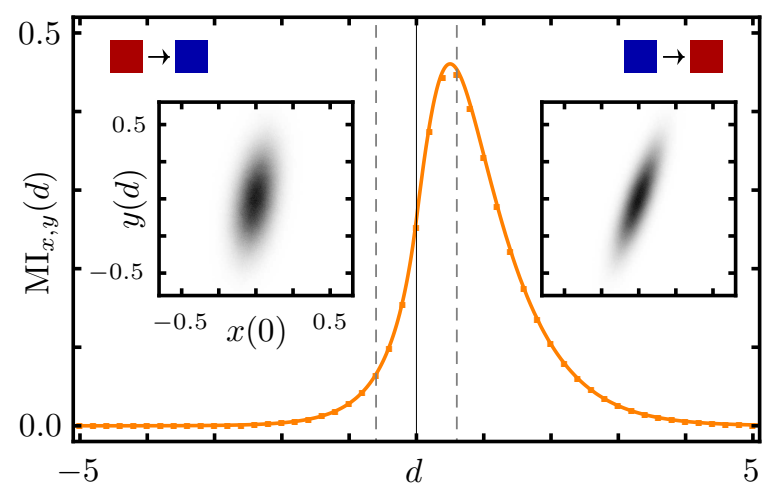

Figure 15.1. Delayed mutual information in the Ornstein-Uhlenbeck process. Shown are time series and the delayed mutual information (dMI) for the system $\dot{x}=-x+\zeta_{x}, \dot{y}=-y+3 x+\zeta_{y}$ with uncorrelated white noise sources $\zeta_{i}$ with $\left\langle\zeta_{i}, \zeta_{j}\right\rangle=0.1 \delta_{i j}$. Note that there is only a physical connection from $x \rightarrow y$. (a) sample of the time series $x_{t}$ (blue) and $y_{t}$ (red). Arrows indicate correlations between and within the processes leading to non-zero dMI for negative delays despite the absence of a physical coupling from $y \rightarrow x$ (cf. also text). (b) dMI between $x_{t}$ and $y_{t+d}$ (orange) obtained numerically (dots) (time series of length $t=50000$ with step size $d t=0.1$, estimation of the joint probability distributions uses a histogram with 1000 equally spaced bins in the interval $[-0.5,0.5])$ and theoretically (line) using the result (??). The dMI shows a strong asymmetry towards positive delays indicating effective information flow from $x \rightarrow y$ consistent with the underlying physical connectivity. Insets show joint probability distributions of $x(t)$ and $y(t+d)$ for $d=-0.6$ (left) and $d=0.6$ (right) indicated by the gray dotted lines.

\subsubsection{Delayed Mutual Information}

Mutual information [MI], characterizes the information that two stochastic processes share [249]. It is defined as the Kullback-Leibler divergence [227] between the joint probability distribution of the two signals and the product of their marginals, i.e. the joint distribution obtained if the two processes were independent. This quantity per se is fully symmetric in the two signals. To obtain a measure of directionality between the signal $x_{t}$ and $y_{t}$ one introduces a delay time $d$ into one of the signals and calculates the joint distribution $p\left(x_{t}, y_{t+d}\right)$ of $x_{t}$ and the time shifted version of $y_{t}$ denoted by $y_{t+d}$. Then the delayed mutual information $[\mathrm{dMI}]$ is given by

$$
\mathrm{MI}_{x, y}(d)=\iint p\left(x_{t}, y_{t+d}\right) \log \left(\frac{p\left(x_{t}, y_{t+d}\right)}{p\left(x_{t}\right) p\left(y_{t}\right)}\right) d x_{t} d y_{t+d}
$$

Assuming a causal world where signals can only flow forward in time, high values of $\mathrm{MI}_{x, y}(d)$ for positive delays $d>0$ and small values for $d<0$, i.e. an asymmetry of the functional form of $\mathrm{MI}_{x, y}(d)$ towards positive $d$ then indicates an effective information flowing from $x \rightarrow y$.

Figure 15.1 illustrates this for a multivariate Ornstein-Uhlenbeck process for which the dMI is calculated analytically in appendix G. We note that this example also shows that a single particular value of the dMI for a particular delay has little explanatory power in general and only the full shape of the dMI, and in particular its asymmetry characterizes aspects of the information flow. For example, consider the two processes $x_{t}$ and $y_{t}$ in fig. 15.1 where there is only a physical connection from $x_{t}$ to $y_{t}$ but the $\mathrm{MI}_{x, y}(d)$ for small negative delays $d<0$ yields non-zero values. The reason is a combination of 
the real physical influence of $x$ on $y$ and time correlations within the signal $x$ itself that give rise to non-zero correlations between $y_{t}$ and later $x_{t+d}$ (cf. fig. 15.1a). This in turn gives rise to positive values for the dMI even for negative delays (cf. also eqs. (G.2.1) and (??)). However the overall shape of the dMI in this example clearly shows a strong asymmetry towards positive delays (cf. fig. 15.1a) and may therefore be used as a measure of information flow.

Numerically we determine the delayed mutual information between two time series by estimating the joint probability $p\left(x_{t}, y_{t+d}\right)$ using a joint histogram of normalized counts of occurrences of the pairs $\left(x_{t}, y_{t+d}\right)$ within equally spaced bins in the $x_{t}$ - $y_{t}$-space. In each simulation the length of the time series was increased and the bin size was decreased until convergence of the dMI curve was obtained. In each figure the length of the time series and the binning is indicated. For the numerical integration of the stochastic differential equations we use a stochastic Runge-Kutta algorithm of order 2 [173].

In contrast to other information transfer measures, the dMI has the advantage of depending only on the joint probability $p\left(x_{t}, y_{t+d}\right)$ of two variables only. This speeds up numerical calculations as the amount of data needed to sample the probability distribution accurately is much less than for joint distributions of three or more variables. At the same time this fact also makes it a useful tool in analytical calculations and avoids lengthy expressions.

\subsubsection{Transfer Entropy}

Our study below focuses on delayed mutual information to quantify information flow. However, the analysis with some modifications but similar results can also be performed for a delayed transfer entropy measure of the form

$$
\mathrm{TE}_{x \rightarrow y}(d)=\iiint p\left(y_{t+d}, x_{t}, y_{t}\right) \log \left(\frac{p\left(y_{t+d} \mid x_{t}, y_{t}\right)}{p\left(y_{t+d} \mid y_{t}\right)}\right) d x_{t} d y_{t} d y_{t+d}
$$

where $p\left(y_{t+d}, x_{t}, y_{t}\right)$ is the joint distribution of the signal $x_{t}$ and $y_{t}$ and its time shifted version $y_{t+d}$. For a fixed $d>0$ this is the transfer entropy for a time discrete signal [331] with time resolution $d$. Calculating this quantity for all $d$ gives more information about the temporal structure of the signals and may serve as an alternative measure of transfer entropy in systems with continuous time [193]. In appendix G.3.1 the transfer entropy for the Ornstein-Uhlenbeck process is derived. 


\section{Chapter 16}

\section{Local Control of Non-Local Information Flow in Networks of Coupled Phase Oscillators}

In this section we study information flows in networks of coupled phase oscillators. For dynamics near a phase locked state we first derive an analytic expression for the information flow between any pair of oscillators and arbitrary network topologies. For systems close to synchrony we also derive an analytical expression for the phase locking pattern.

We then focus on hierarchical network structures of more strongly coupled groups of oscillators with weaker inter-cluster connections. We treat the clusters as individual meta-oscillators and by performing a second phase reduction step we obtain the collective phase response curves for each cluster as a function of the intra-cluster connectivity and individual frequencies of the cluster's oscillators. By performing a second averaging step we then deduce an analytical expression for the phase information flow between the different clusters as a function of their collective phase response curves. This enables us to study how local intra-cluster properties affect the global inter-cluster information flow. In particular, we find that the information flow between two clusters can be controlled via local network changes within the clusters only or even remotely by changes within a third cluster only.

\subsection{Model}

To study control mechanisms for information flow analytically we use networks of coupled phase oscillators. Such models arise in the phase reduction and averaging (cf. sec. 3.4) of weakly coupled oscillators with a strong attraction towards their limit cycle. We model external signals to the oscillators as white noise processes to keep analytical tractability but keep in mind that they originate from physical signals that always have small but nonzero time correlations $[386]^{1}$. The resulting model is then given by a Langevin equation

\footnotetext{
${ }^{1}$ We note that the phase reduction for stochastic oscillators depends on the ratio of the correlation time constants for the noise sources and the time constants associated with the attraction towards the limit cycle in the limit where both tend to zero [363]. For real white noise sources extra terms arise in the technically correct phase equation [417] while the deterministic reduction is valid in the limiting case of colored noise sources. Interestingly, to our knowledge, there is no general theory for the averaging of weakly coupled stochastic oscillators developed so far. See also section 18.3 for a detailed discussion.
} 
for $N$ oscillators with phases $\phi=\left(\phi_{1}, \ldots, \phi_{N}\right)$, with $\phi_{i} \in S^{1}=[0,2 \pi] / 0 \sim 2 \pi$ of the form

$$
d \phi_{i}=\left[\omega_{i}+\sum_{j} \gamma_{i j}\left(\phi_{i}-\phi_{j}\right)\right] d t+\xi_{i} d w_{i}
$$

with intrinsic oscillation frequencies $\omega_{i}$, coupling functions $\gamma_{i j}$, and white noise sources $w_{i}$ that obey

$$
\left\langle w_{i}(t), w_{j}(s)\right\rangle=\delta_{i j} \delta(t-s)
$$

and have strength $\xi=\left(\xi_{1}, \ldots, \xi_{N}\right)$. The real function $\gamma_{i j}$ is $2 \pi$ periodic such that there are no difficulties in defining the phase difference appearing in its argument. Moreover, we thus can treat the $\phi_{i}$ as variables in $\mathbb{R}$ keeping in mind that points modulo $2 \pi$ have the same physical meaning. For later convenience we also define the $N \times N$ matrix

$$
\Xi=\operatorname{diag}\left(\xi_{1}, \ldots, \xi_{N}\right) \text {. }
$$

We assume that for the noiseless deterministic model, $\xi_{i}=0$ in eq. (16.1.1), there is a stable phase-locked state with phase differences $\Delta \phi_{i j}=\phi_{i}-\phi_{j}=$ const. and collective oscillation frequency $\Omega$ solving the equation

$$
\Omega=\omega_{i}+\sum_{j} \gamma_{i j}\left(\Delta \phi_{i j}\right)
$$

We further assume that the noise strengths $\xi_{i}$ are small compared to the strength of attraction towards the phase locked solution.

\subsection{Information Flow in Networks of Coupled Phase Oscillators}

In this section we derive the information flow in networks of coupled phase oscillators. In particular, we analytically calculate the delayed mutual information between phase signals of two oscillators in the network (16.1.1) close to a phase locked state.

We denote the probability distribution of the phase signals $\phi(t)$ of all oscillators in the network (16.1.1) by $p\left(\phi_{t}\right)$ and the joint distribution of these signals with the phase signals $\phi(t+d)$ shifted by a time $d$ by $p\left(\phi_{t}, \phi_{t+d}\right)$. For positive delay times $d>0$ this distribution may be calculated via the identity

$$
p\left(\phi_{t}, \phi_{t+d}\right)=p\left(\phi_{t+d} \mid \phi_{t}\right) p\left(\phi_{t}\right)
$$

and for negative delays by reversing the roles of $\phi_{t}$ and $\phi_{t+d}$. The marginal distribution for the phase signal $\phi_{i}(t)$ of oscillator $i$ and the time shifted version $\phi_{j}(t+d)$ of oscillator $j$ is obtained from this distribution by integration:

$$
p\left(\phi_{i, t}, \phi_{j, t+d}\right)=\iint p\left(\phi_{t}, \phi_{t+d}\right) \prod_{k \neq i} d \phi_{t, k} \prod_{l \neq j} d \phi_{l, t+d}
$$

The delayed mutual information (15.2.1) between these two oscillators is then given by

$$
\mathrm{MI}_{i, j}(d)=\iint p\left(\phi_{i, t}, \phi_{j, t+d}\right) \log \left(\frac{p\left(\phi_{i, t}, \phi_{j, t+d}\right)}{p\left(\phi_{i, t}\right) p\left(\phi_{j, t}\right)}\right) d \phi_{i, t} d \phi_{j, t+d}
$$


Our analysis proceeds in three steps: We first introduce new average phase and phase deviation like coordinates suitable for linearization of the system and convenient for the following analysis. We then calculate the joint distribution $p\left(\phi_{t}, \phi_{t+d}\right)$ by deriving expressions for $p\left(\phi_{t}\right)$ and $p\left(\phi_{t+d} \mid \phi_{t}\right)$ and preform the integration (16.2.2) over all but two variables. Finally, we use this result to calculate the delayed mutual information as in (16.2.3). The main result of this analysis is summarized in theorem 16.2.1.

\subsubsection{Transformation to Average Phase and Phase Deviation Variables}

In this sub-section we transform the system (16.1.1) into a form suitable for linearization and the following derivation of the dMI.

We first introduce shifted coordinates $\psi_{i}=\phi_{i}-\Delta \phi_{i}$ where the constant $\Delta \phi_{i}$ measures the deviation of oscillator $i$ from the average phase in the deterministic phase-locked state, i.e. $\Delta \phi_{i j}=\Delta \phi_{i}-\Delta \phi_{j}$ and $\psi_{i}-\psi_{j}=\phi_{i}-\phi_{j}-\Delta \phi_{i j}$. Equation (16.1.1) then becomes ${ }^{2}$

$$
\begin{aligned}
d \psi_{i} & =\left[\omega_{i}+\sum_{j} \gamma_{i j}\left(\psi_{i}-\psi_{j}+\Delta \phi_{i j}\right)\right] d t+\xi_{i} d w_{i} \\
& :=f_{i}(\psi) d t+\xi_{i} d w_{i}
\end{aligned}
$$

Note that the right hand side and in particular the vector field $f$ of this equation only depends on the phase differences $\psi_{i}-\psi_{j}$. In the noiseless case, these differences vanish in the phase locked state and (16.2.4) reduces to (16.1.4). It is convenient to also transform to a rotating frame via

$$
\psi=\Omega t+\varphi
$$

such that (16.2.4) becomes

$$
d \varphi=[f(\varphi)-\Omega] d t+\Xi d w
$$

We then introduce coordinates $\tilde{\varphi}=\left(\bar{\varphi}, \delta \varphi_{2}, \ldots, \delta \varphi_{N}\right)=(\bar{\varphi}, \delta \varphi)$ with an average like phase $^{3}$

$$
\bar{\varphi}=\frac{1}{N} \sum_{i} \varphi_{i}
$$

and phase difference like variables $\delta \varphi_{i}$ given by any orthonormal matrix $O$ with $O_{1 k}=\frac{1}{\sqrt{N}}$ for all $k \in\{1, \ldots, N\}$ such that $O^{T} O=O O^{T}=\mathbb{1}$ and

$$
\tilde{\varphi}=\frac{1}{\sqrt{N}} O \varphi, \quad \varphi=\sqrt{N} O^{T} \tilde{\varphi}
$$

\footnotetext{
${ }^{2}$ Note that here and in the following we use a Stratonovich interpretation [120] of the stochastic differential equation (16.1.1) as we model physical input signals by the white noise sources. However, all applied transformations in this section are linear affine and therefore the results remain valid also using Ito's stochastic calculus.

${ }^{3}$ We note that the average of phase variables on the circle is given by $\bar{\varphi}=\arg \sum_{k} \exp \left(i \varphi_{k}\right)$. As we treat phases as variables on the real line (cf. sec. 16.1) this definition gives a good approximation to the average phase if the $\varphi_{k}$ are concentrated on a small interval. Generally, we treat (16.2.7) as a formal definition of a coordinate transformation.
} 
Then any phase difference can be written as a linear combination of the $\delta \varphi_{i}$, i.e.

$$
\psi_{i}-\psi_{j}=\varphi_{i}-\varphi_{j}=\sum_{k=2}^{N} o_{k}^{i, j} \delta \varphi_{k}
$$

where

$$
o_{k}^{i, j}=\sqrt{N}\left(O_{k i}-O_{k j}\right)
$$

Applying the transformation (16.2.8) the evolution equation for the new coordinates $\tilde{\varphi}$ is given by

$$
\begin{aligned}
d \tilde{\varphi} & =\frac{1}{\sqrt{N}}\left[O f\left(O^{T} \sqrt{N} \tilde{\varphi}\right)-\Omega\right] d t+\frac{1}{\sqrt{N}} O \Xi d w \\
& =: \tilde{f}(\delta \varphi) d t+B d w
\end{aligned}
$$

where we defined the matrix

$$
B=\frac{1}{\sqrt{N}} O \Xi
$$

and $\tilde{f}$ via the second equation. The vector field $\tilde{f}$ depends only on the $\delta \varphi$ because $f$ defined in (16.2.4) depends only on the phase differences $\psi_{i}-\psi_{j}$ which via (16.2.9) may be expressed in terms of the $\delta \varphi$ only.

Note that in this representation the equations for $\delta \varphi$ decouple from the average phase $\bar{\varphi}$. The equation for $\bar{\varphi}$ itself depends only on the processes $\delta \varphi$ and the white noise processes $w_{i}$ and therefore can be solved formally as

$$
\bar{\varphi}(t)=\bar{\varphi}(0)+\int_{0}^{t} \tilde{f}_{\bar{\varphi}}(\delta \varphi) d t+\sum_{j} \frac{\xi_{j}}{N} w_{j}(t)
$$

By transforming back to the original coordinates this shows that the collective rotation of the system is driven by three purely additive parts, the deterministic rotation $\Omega$ of the phase locked state, a rotation due to the deviations of the oscillators from the phase locked state and a noise term being the average of the input noises to each oscillator.

\subsubsection{Calculation of the Probability Distributions}

In this section we derive the joint probability distribution $p\left(\phi_{t}, \phi_{t+d}\right)$ for the system (16.1.1) close to a phase locked state. Therefore we utilize eq. (16.2.1) to calculate $p\left(\phi_{t+d} \mid \phi_{t}\right)$ and $p\left(\phi_{t}\right)$ separately. In both derivations we use the more convenient coordinates $\varphi$. We further employ the small noise assumption and first derive suitable linearizations of the system around the phase locked state from which the distributions can then be calculated analytically. We finally merge our results and perform the integration (16.2.2).

\subsubsection{Transition Probability}

In this section we derive the transition probability $p\left(\phi_{t+d} \mid \phi_{t}\right)$ using the $\varphi$ coordinates (16.2.5), i.e. $p\left(\varphi_{t+d} \mid \varphi_{t}\right)$. To calculate this transition probability we employ the assumption that the noise amplitudes $\xi_{i}$ are small compared to the strength of attraction towards the stable phase locked state. Then the phase differences $\varphi_{i}-\varphi_{j}$ (which are zero when the system is in the phase locked state) will stay small and we therefore may expand the 
right hand side of (16.2.6) in these phase differences. Truncation at linear order in $\varphi_{i}-\varphi_{j}$ then gives

$$
d \varphi=G \varphi d t+\Xi d W
$$

with the $N \times N$ matrix $G$ given by

$$
G_{i j}= \begin{cases}-\gamma_{i j}^{\prime}\left(\Delta \phi_{i j}\right) & i \neq j \\ \sum_{k} \gamma_{i k}^{\prime}\left(\Delta \phi_{i k}\right) & i=j\end{cases}
$$

Note that $G$ has a zero eigenvalue $\lambda_{0}$ with eigenvector $e_{0}=(1,1, \ldots, 1)$ which reflects the fact that (16.1.1) and (16.2.4) are invariant under simultaneous uniform shifts of all phases.

Despite of the zero eigenvalue of $G$, a solution of the Ornstein-Uhlenbeck process (16.2.14) for all finite times exists and is given formally by [120]

$$
\varphi(t)=\exp (G t) \varphi(0)+\eta \int_{0}^{t} \exp \left(G\left(t-t^{\prime}\right)\right) \Xi d w\left(t^{\prime}\right)
$$

Starting at $\varphi(0)=\varphi_{0}$ it follows that the mean $\langle\varphi(t)\rangle$ evolves according to

$$
\langle\varphi(t)\rangle=M_{t} \varphi_{0} \quad \text { where } \quad M_{t}:=\exp (G t)
$$

and the covariance matrix $\Sigma_{t}=\left\langle\varphi(t) \varphi(t)^{T}\right\rangle$ is given by

$$
\Sigma_{t}:=\int_{0}^{t} \exp \left(G\left(t-t^{\prime}\right)\right) \Xi \Xi^{T} \exp \left(G^{T}\left(t-t^{\prime}\right)\right) d t^{\prime}
$$

Denoting the multivariate Gaussian distribution with mean $\mu$ and covariances $\Sigma$ by $\mathcal{N}_{\mu, \Sigma}$, the transition probability is thus given by [120]

$$
p\left(\varphi_{t+d} \mid \varphi_{t}\right)=\mathcal{N}_{M_{d} \varphi_{t}, \Sigma_{d}}\left(\varphi_{t+d}\right)
$$

In terms of the $\phi$ coordinates this becomes

$$
p\left(\phi_{t+d} \mid \phi_{t}\right)=\mathcal{N}_{M_{d} \phi_{t}, \Sigma_{d}}\left(\phi_{t+d}-\Delta \phi-\Omega d\right)
$$

\subsubsection{Stationary Phase Distributions}

In this section we derive the distribution $p\left(\phi_{t}\right)$ using the $\tilde{\varphi}=(\bar{\varphi}, \delta \varphi)$ coordinates in (16.2.8). As before, the small noise amplitudes ensure that the phase differences $\delta \varphi$ (16.2.9) are small and linearizing the $\delta \varphi$-subsystem in (16.2.11) gives

$$
d \delta \varphi=\delta G \delta \varphi d t+\delta B d w
$$

where the $(N-1) \times(N-1)$ matrix $\delta G$ is defined via the equality

$$
O G O^{T}=\left(\begin{array}{cc}
0 & \bar{g}^{T} \\
0 & \delta G
\end{array}\right)
$$

and the $N-1 \times N$ matrix $\delta B$ has entries $\delta B_{i j}=B_{i+1, j}$. Note that in (16.2.18) $\bar{g}$ is some vector describing the influence of the phase deviations on the average phase of the system in linear approximation. 
As we assumed stability of the phase locked state, $\delta G$ only has negative eigenvalues, so there is a stationary solution to the Ornstein-Uhlenbeck process eq. (16.2.17) of the form

$$
\delta \varphi(t)=\tilde{\eta} \int_{-\infty}^{t} \exp \left(-\delta G\left(t-t^{\prime}\right)\right) \delta B d w\left(t^{\prime}\right)
$$

Thus the phase deviations $\delta \varphi$ are Gaussian distributed with zero mean and covariances

$$
\Sigma_{\delta}=\int_{-\infty}^{0} \exp (-\delta G t) \delta B \delta B^{T} \exp \left(-\delta G^{T} t\right) d t
$$

i.e.

$$
p_{\mathrm{st}}(\delta \varphi)=\mathcal{N}_{0, \Sigma_{\delta}}(\delta \varphi)
$$

Now eq. (16.2.13) shows, that for each realization of $\delta \varphi(t), \bar{\varphi}(t)$ has a Gaussian distribution with variance $\propto t$ when starting with deterministic initial conditions. In particular in the limit $t \rightarrow \infty$ this distribution on $\mathbb{R}$ becomes flat. However, we can now employ the fact that the oscillator network is in the same state if the $\varphi_{i}$ differ by a multiple of $2 \pi$ and hence $\bar{\varphi}$ defined by (16.2.7) has the same physical meaning for values that differ by $\frac{2 \pi}{N}$. Thus, regarding $\bar{\varphi}$ as a variable on a circle with $\bar{\varphi} \in\left[0, \frac{2 \pi}{N}\right)$, it follows that its stationary distribution is uniform with

$$
p_{\text {st }}(\bar{\varphi})=\frac{N}{2 \pi}
$$

This is a consequence of the invariance of the evolution equation (16.1.1) under uniform global phase shifts.

We are interested in the full stationary distribution $p_{\mathrm{st}}(\tilde{\varphi})$. As the $\delta \varphi$ equations do not involve $\bar{\varphi}$ and using (16.2.19) we conclude that

$$
p_{\text {st }}(\tilde{\varphi})=p_{\text {st }}(\delta \varphi) p_{\text {st }}(\bar{\varphi})=\frac{N}{2 \pi} \mathcal{N}_{0, \Sigma_{\delta}}(\delta \varphi)
$$

\subsubsection{Joint Probability Distribution}

We now merge our results form the previous two sections to obtain the joint distribution as

$$
\begin{aligned}
p\left(\varphi_{t}, \varphi_{t+d}\right) & =p\left(\varphi_{t+d} \mid \varphi_{t}\right) p_{\mathrm{st}}\left(\varphi_{t}\right) \\
& \propto \mathcal{N}_{M_{d} \varphi_{t}, \Sigma_{d}}\left(\varphi_{t+d}\right) \mathcal{N}_{0, \Sigma_{\delta}}\left(\delta \varphi_{t}\right)
\end{aligned}
$$

or more explicitly

$$
p_{s}\left(\varphi_{t}, \varphi_{t+d}\right) \propto \exp \left(-\frac{1}{2} \delta \varphi_{t}^{T} \Sigma_{\delta}^{-1} \delta \varphi_{t}-\frac{1}{2}\left(\varphi_{t+d}-M_{d} \varphi_{t}\right)^{T} \Sigma_{d}^{-1}\left(\varphi_{t+d}-M_{d} \varphi_{t}\right)\right) .
$$

\subsubsection{Integration}

We are interested in the marginal distribution $p_{s}\left(\varphi_{i, t}, \varphi_{j, t+d}\right)$ and thus have to integrate out the remaining coordinates in the full joint probability distribution (16.2.20). The Gaussian integrals over the $\varphi_{k, t+d}, k \neq j$ yield

$$
p\left(\varphi_{t}, \varphi_{j, t+d}\right) \propto \exp \left(-\frac{1}{2} \delta \varphi_{t}^{T} \Sigma_{\delta}^{-1} \delta \varphi_{t}-\frac{1}{2}\left(\varphi_{j, t+d}-\left(M_{d} \varphi_{t}\right)_{j}\right)^{2}\left(\Sigma_{d}\right)_{j j}\right)
$$


Now, as we can express any phase $\varphi_{j}$ via the phase $\varphi_{i}$ plus a linear combination of phase differences $\delta \varphi$ using (16.2.9) we have

$$
\varphi_{j, t+d}-\left(M_{d} \varphi_{t}\right)_{j}=\varphi_{j, t+d}-\varphi_{i, t}-\sum_{k, l}\left(M_{d}\right)_{j k} o_{l}^{k, i} \delta \varphi_{t, l}=\varphi_{j, t+d}-\varphi_{i, t}-\sum_{l} a_{d, l}^{j, i} \delta \varphi_{l, t}
$$

with

$$
a_{d, l}^{j, i}:=\sum_{k}\left(M_{d}\right)_{j k} o_{l}^{k, i}
$$

We may therefore insert (16.2.22) into (16.2.21) and integrate over the $\delta \varphi_{t}$. The integration can be performed by first making an orthogonal transformation to a basis in which $\Sigma_{\delta}$ is diagonal and then performing the individual Gaussian integrals. The result is

$$
p\left(\varphi_{i, t}, \varphi_{j, t+d}\right)=\frac{1}{2 \pi} \mathcal{N}_{0, \sigma_{i, j, d}^{2}}\left(\varphi_{j, t+d}-\varphi_{i, t}\right)
$$

where

$$
\sigma_{i, j, d}^{2}=\left(\Sigma_{d}\right)_{j j}+a_{d}^{j, i T} \cdot \Sigma_{\delta} \cdot a_{d}^{j, i}
$$

Via the second term this expression still depends on the coordinate transformations $O$ which we can get rid of. Therefore we define $[[A]]_{i, j}$ to be the matrix obtained from the matrix $A$ by deleting its $i^{\text {th }}$ row and $j^{\text {th }}$ column. With this notation straightforward algebra shows for $\delta B$ and $\delta G$ defined in (16.2.17) and (16.2.18) that

$$
\begin{aligned}
\delta B \delta B^{T} & =\left[\left[O \Xi^{2} O^{T}\right]\right]_{1,1} \\
\delta G^{n} & =\left[\left[O G^{n} O^{T}\right]\right]_{1,1}
\end{aligned}
$$

and thus also

$$
\exp (\delta G t)=\left[\left[O \exp (G t) O^{T}\right]\right]_{1,1}
$$

For any $N \times N$ matrices $A$ and $B$ we further have

$$
\left[\left[O A O^{T}\right]\right]_{1,1}\left[\left[O B O^{T}\right]\right]_{1,1}=\left[\left[O\left(A B-\frac{1}{N} A J B\right) O^{T}\right]\right]_{1,1}
$$

where $J$ is the $N \times N$ matrix of ones, i.e $J_{i j}=1$. As $G$ has an eigenvector $e_{0}=(1,1, \ldots, 1)$ with eigenvalue $\lambda_{0}=0$ we have $G J=J G^{T}=0$. Thus for any integers $n, m \geq 0$

$$
\begin{aligned}
\delta G^{n} \delta B \delta B^{T}\left(\delta G^{T}\right)^{m} & =\left[\left[O G^{n} O^{T}\right]\right]_{1,1}\left[\left[O \Xi \Xi^{T} O^{T}\right]\right]_{1,1}\left[\left[O\left(G^{T}\right)^{n} O^{T}\right]\right]_{1,1} \\
& =\left[\left[O G^{n} \Xi \Xi^{T}\left(G^{T}\right)^{m} O^{T}\right]\right]_{1,1}
\end{aligned}
$$

and it follows that

$$
\exp (\delta G t) \delta B \delta B^{T} \exp \left(\delta G^{T} s\right)=\left[\left[O \exp (G t) \Xi \Xi^{T} \exp \left(G^{T} s\right) O^{T}\right]\right]_{1,1} \quad .
$$

Hence

$$
\Sigma_{\delta}=\int_{-\infty}^{0}\left[\left[O \exp (-G t) \Xi \Xi^{T} \exp \left(-G^{T} t\right) O^{T}\right]\right]_{1,1} d t
$$

where it is essential to delete the first row and column before performing the integration to ensure convergence of the integral. 
Using again that $e_{0}=(1,1, \ldots, 1)$ is eigenvector of $G$ with zero eigenvalue we have

$$
\sum_{j}\left(M_{t}\right)_{i j}=\left(\exp (G t)\left(\begin{array}{c}
1 \\
\vdots \\
1
\end{array}\right)\right)_{i}=1
$$

for all $i \in\{1, \ldots N\}$ and setting

$$
H_{t}=\exp (G t) \Xi \Xi^{T} \exp \left(G^{T} t\right)=M_{t} \Xi \Xi^{T} M_{t}^{T}
$$

together with $(16.2 .23),(16.2 .10)$ and $o_{1}^{i, k}=0$ yields

$$
\begin{aligned}
\frac{1}{\eta^{2}} a_{d}^{j, i T} \cdot \Sigma_{\delta} \cdot a_{d}^{j, i} & =\frac{1}{N} \int_{-\infty}^{0} \sum_{k, m} \sum_{l, p \neq 1}\left(M_{d}\right)_{j k} o_{l}^{k, i}\left[\left[O H_{-t} O^{T}\right]\right]_{l p}\left(M_{d}\right)_{j m} o_{p}^{m, i} d t \\
& =\frac{1}{N} \int_{-\infty}^{0} \sum_{k, l, m, p}\left(M_{d}\right)_{j k} o_{l}^{k, i}\left(O H_{-t} O^{T}\right)_{l p}\left(M_{d}\right)_{j m} o_{p}^{m, i} d t \\
& =\int_{-\infty}^{0} \sum_{k, l, m, p, s, r}\left(M_{d}\right)_{j k}\left(O_{l k}-O_{l i}\right) O_{l r} H_{-t, r s} O_{m s}\left(O_{m p}-O_{m i}\right)\left(M_{d}\right)_{j m} d t \\
& =\int_{-\infty}^{0} \sum_{k, p}\left(M_{d}\right)_{j k}\left(H_{-t, k p}-H_{-t, k i}-H_{-t, i p}+H_{-t, i i}\right)\left(M_{d}\right)_{j p} d t \\
& =\int_{-\infty}^{0}\left(M_{d} H_{-t} M_{d}^{T}\right)_{j j}-2\left(M_{d} H_{-t}\right)_{j i}+H_{-t, i i} d t
\end{aligned}
$$

Note here, as before, that the sum has to be performed before integration to ensure convergence of the integral. Using (16.2.15) we obtain the basis free expression

$$
\sigma_{i, j, d}^{2}=\int_{0}^{d}\left(H_{t}\right)_{j j} d t+\int_{0}^{\infty}\left(H_{t+d}\right)_{j j}+\left(H_{t}\right)_{i i}-2\left(M_{d} H_{t}\right)_{j i} d t
$$

In the original coordinates the distribution (16.2.24) is given by

$$
p\left(\phi_{i, t}, \phi_{j, t+d}\right)=\mathcal{N}_{0, \sigma_{d, i, j}^{2}}\left(\phi_{j, t+d}-\phi_{i, t}-\Delta \phi_{j i}-\Omega d\right) \quad .
$$

Finally, note that the above results are for the pseudo phases on the real line. If we identify the phases at all points modulo $2 \pi$ the Gaussian distribution (16.2.24) becomes a wrapped Gaussian distribution which for small standard deviations

$$
\sigma=\sqrt{\frac{1}{k}} \ll 2 \pi
$$

is well approximated by a van Mises distribution for circular variables [44]. It is of the form

$$
\mathcal{M}_{\mu, k}(\phi)=\frac{1}{2 \pi I_{0}(k)} \exp (k \cos (\phi-\mu))
$$

where $I_{n}(k)$ denotes the $n^{\text {th }}$ modified Bessel function of the first kind [44], $\mu$ is the average phase and $k$ a concentration parameter. Thus, as the final result, we obtain

$$
\begin{aligned}
p\left(\phi_{i, t}, \phi_{j, t+d}\right) & =\mathcal{M}_{0, \sigma_{d, i, j}^{2}}\left(\phi_{j, t+d}-\phi_{i, t}-\Delta \phi_{j i}-\Omega d\right) \\
& =\mathcal{M}_{\Delta \phi_{j i}+\Omega d, \sigma_{d, i, j}^{2}}\left(\phi_{j, t+d}-\phi_{i, t}\right)
\end{aligned}
$$

with $\sigma_{d, i, j}^{2}$ given in (16.2.26). 


\subsubsection{Delayed Mutual Information}

For a van Mises probability distribution (16.2.28) of the from

$$
p\left(\phi_{1}, \phi_{2}\right)=\frac{1}{2 \pi} \mathcal{M}_{\mu, k}\left(\phi_{1}-\phi_{2}\right)
$$

we calculate

$$
\begin{aligned}
& \mathrm{MI}_{\mathrm{vM}}(k)=\iint p\left(\phi_{1}, \phi_{2}\right) \log \left(\frac{p\left(\phi_{1}, \phi_{2}\right)}{p\left(\phi_{1}\right) p\left(\phi_{2}\right)}\right) d \phi_{1} d \phi_{2} \\
& =\frac{1}{(2 \pi)^{2} I_{0}(k)} \iint \exp \left(k \cos \left(\phi_{1}-\phi_{2}+m\right)\right)\left[k \cos \left(\phi_{1}-\phi_{2}+m\right)-\log \left(I_{0}(k)\right)\right] d \phi_{1} d \phi_{2} \\
& =\frac{k I_{1}(k)}{I_{0}(k)}-\log \left(I_{0}(k)\right)
\end{aligned}
$$

Combining the results from the previous derivations and using (16.2.27) we obtain the main result of this chapter for the information flow in phase oscillator networks:

Theorem 16.2.1. The delayed mutual information $\mathrm{MI}_{i, j}(d)$ between oscillator $i$ and oscillator $j$ in system (16.1.1) close to a phase locked state ${ }^{4}$ with phase differences $\Delta \phi_{i j}$ and small noise fluctuations $\xi$ is given by

$$
\mathrm{MI}_{i, j}(d)= \begin{cases}\operatorname{MI}_{\mathrm{vM}}\left(\sigma_{d, i, j}^{-2}\right) & d \geq 0 \\ \operatorname{MI}_{\mathrm{vM}}\left(\sigma_{-d, j, i}^{-2}\right) & d<0\end{cases}
$$

with $\sigma_{d, i, j}^{2}$ as defined in (16.2.26) and $\mathrm{MI}_{\mathrm{vM}}$ given by (16.2.30).

Under the assumptions of theorem 16.2.1, the analytic solution (16.2.31) is in good agreement with numerical simulations as shown for example oscillator networks in fig. 16.1 and 16.2. For larger values of the noise the numerical values show qualitatively the same shapes as the theoretical prediction, but with systematically smaller values. This blurring is due to the fact that the large noise frequently pushes the system out of the phase locked regime.

There strong asymmetries in the shape of the dMI curves observed correspond to anisotropic directed information flow patterns within the network. What is the mechanism underlying this asymmetry in phase information flow in the networks? The next paragraph answers this question by considering a simple example.

\subsubsection{Example and Mechanism}

In this section we consider networks of $N=2$ oscillators to illustrate the mechanism leading to a directed phase information transfer. For simplicity we assume equal noise strength $\xi_{i}=\xi$. We obtain

$$
G=\left(\begin{array}{cc}
g_{1} & -g_{1} \\
-g_{2} & g_{2}
\end{array}\right)
$$

\footnotetext{
${ }^{4}$ Formally, if there are other stable dynamics in the deterministic systems the noise will set the stochastic system into the basin of attraction of one of these states with probability one. We therefore here implicitly restrict to time periods where the system is close to the phase-locked state under consideration.
} 
(a)

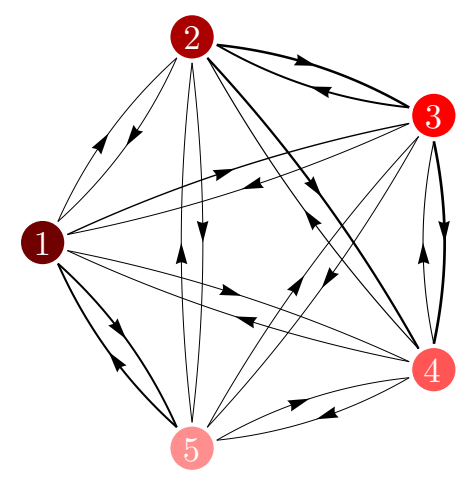

(b)

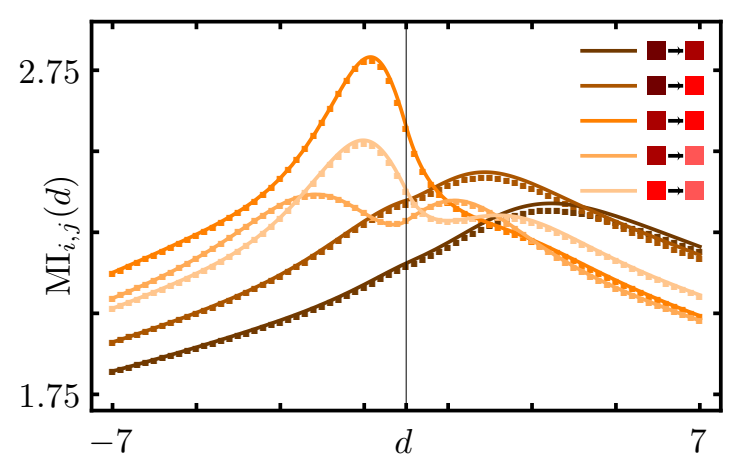

(b)

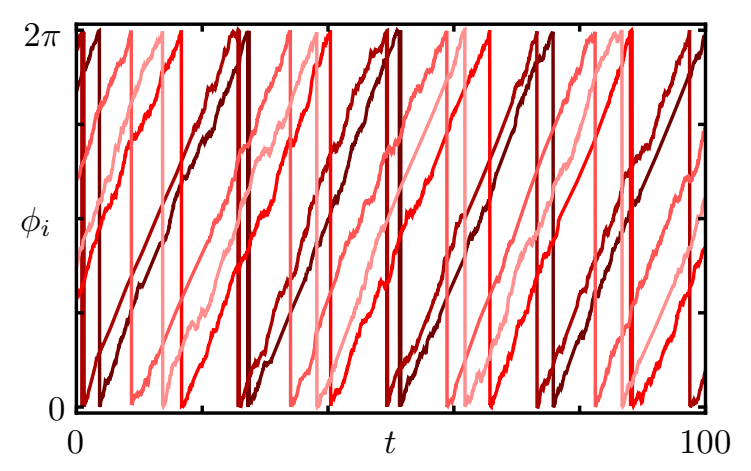

(d)

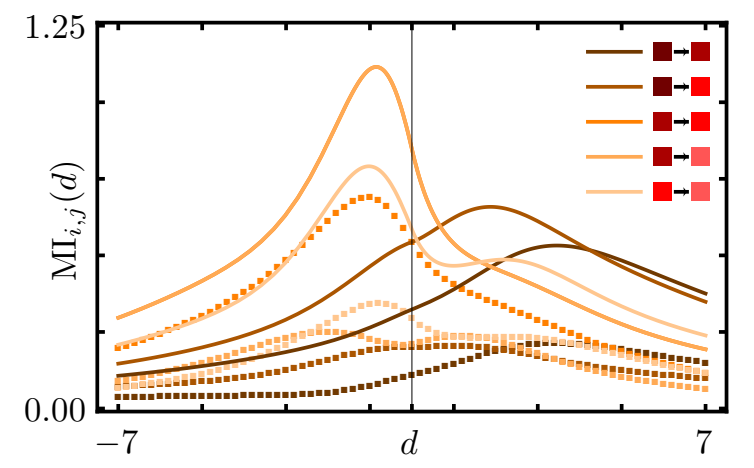

Figure 16.1. Delayed mutual information in networks of coupled phase oscillators close to a phase locked state. (a) network structure of $N=5$ oscillators (edge thickness proportional to connection weight $k_{i j}$ ) (b) traces of stochastic phase evolution according to (16.1.1) with $\xi_{i}=0.1$ and $\gamma_{i j}=k_{i j} \sin (1.2+\phi)$. (c) delayed mutual information $\mathrm{MI}_{i, j}(d)$. Dots indicate numerical values, calculated from traces of duration $t=10^{5}$ and $d t=0.02$ from which the probability distribution $p\left(\phi_{i, t}, \phi_{j, t+d}\right),\left(\phi_{i, t}, \phi_{j, t+d}\right) \in[0,2 \pi]^{2}$ was estimated using a histogram with equally spaced bins of size $2 \pi / 1000$. Lines show theoretical prediction eq. (16.2.31) and (16.2.26) and are in good agreement with the numerical values. (d) same as in (c) but for large noise $\xi=1$. The numerical values show qualitatively the same shapes as the theoretical prediction, but with systematically smaller values. This is due to the fact that the large noise frequently pushes the system out of the phase locked regime and the transients back blur the dMI.

where $g_{1}=\gamma_{12}^{\prime}\left(\Delta \phi_{12}\right)$ and $g_{2}=\gamma_{21}^{\prime}\left(\Delta \phi_{21}\right)=\gamma_{21}^{\prime}\left(-\Delta \phi_{12}\right)$. $G$ has eigenvalues $\lambda_{0}=0$ and $\lambda=g_{1}+g_{2}<0$ as we assume a stable phase-locked state. One calculates

$$
M_{t}=\exp (G t)=\frac{1}{\lambda}\left(\begin{array}{cc}
e^{\lambda t} g_{1}+g_{2} & \left(1-e^{\lambda t}\right) g_{1} \\
\left(1-e^{\lambda t}\right) g_{2} & e^{\lambda t} g_{2}+g_{1}
\end{array}\right)
$$

from which $H_{t}=\xi^{2} \exp (G t) \exp \left(G^{T} t\right)$ follows by a straightforward calculation. Using (16.2.26) we obtain for $i \neq j$

$$
\sigma_{i, j, d}^{2}=\frac{\xi^{2}}{\lambda^{3}} \begin{cases}d \lambda\left(g_{1}^{2}+g_{2}^{2}\right)-\lambda^{2}-2 g_{j}^{2}\left(e^{\lambda d}-1\right) & d \geq 0 \\ |d| \lambda\left(g_{1}^{2}+g_{2}^{2}\right)-\lambda^{2}-2 g_{i}^{2}\left(e^{\lambda|d|}-1\right) & d \leq 0\end{cases}
$$

and

$$
\sigma_{i, i, d}^{2}=\frac{\xi^{2}}{\lambda^{3}}\left(|d| \lambda\left(g_{1}^{2}+g_{2}^{2}\right)-2 g_{1} g_{2}\left(e^{\lambda|d|}-1\right)\right)
$$


(a)

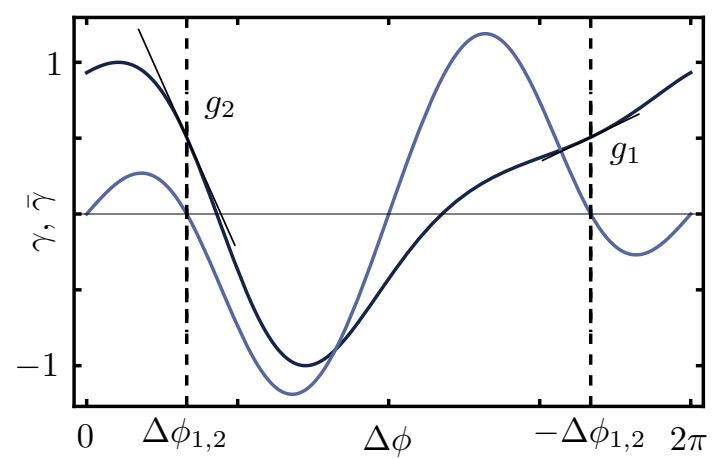

(c)

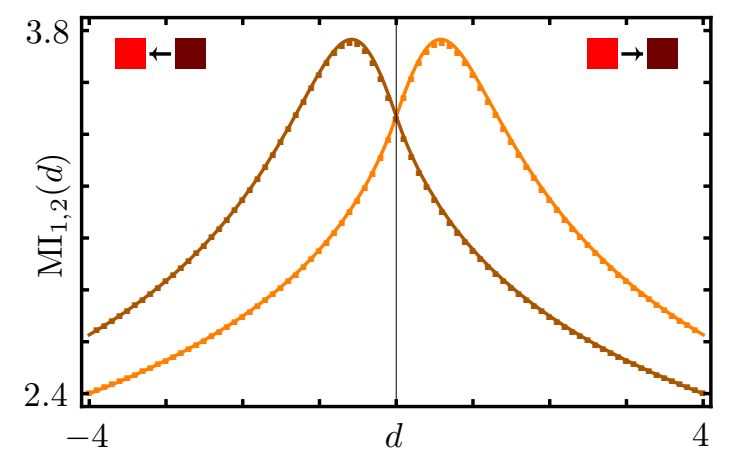

(b)

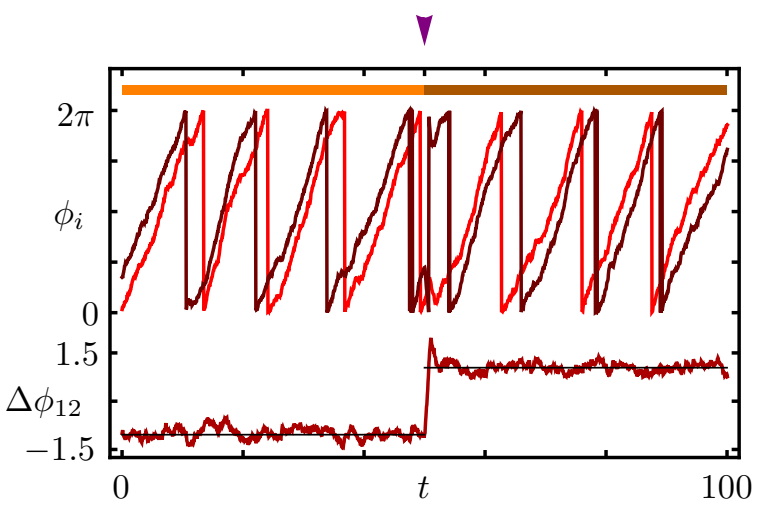

(d)

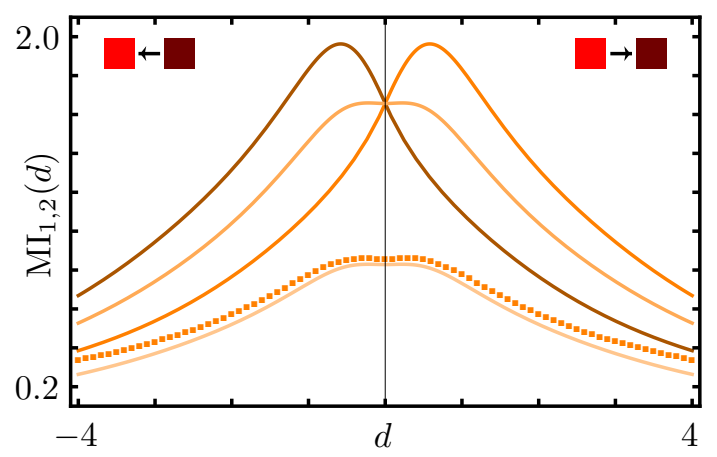

Figure 16.2. Delayed mutual information in coupled phase oscillators: Mechanism and dynamic information flow switching. (a) network of $N=2$ phase oscillators symmetrically coupled via the coupling function $\gamma(\Delta \phi)=\sin \left(1.2+\Delta \phi+1.5 \sin \left(\frac{\Delta \phi}{2}\right)^{2}\right)$ (dark blue) and its antisymmetric part $\bar{\gamma}(\Delta \phi)=\gamma(\Delta \phi)-\gamma(-\Delta \phi)$ (light blue). The zeros of $\bar{\gamma}$ are phase locked states that are stable if $\bar{\gamma}^{\prime}(\Delta \phi)<0$ indicated by $\Delta \phi_{1,2}$ and $-\Delta \phi_{12} . g_{1}$ and $g_{2}$ indicate the slopes of the the coupling function, $\gamma^{\prime}\left( \pm \Delta \phi_{12}\right)$. (b) stochastic time $t$ evolution of the oscillator phases $\phi_{i}$ (upper part) of system (16.1.1) with $\xi_{i}=0.1$ and phase difference $\Delta \phi_{12}(t)=\phi_{1}(t)-\phi_{2}(t)$ (lower part). Phase differences $\pm \Delta \phi_{12}$ predicted for the deterministic systems in (a) are indicated by black solid lines. At $t=50$ (purple arrow) a strong perturbation was applied to switch the system to the opposite phase locked state. (c) delayed mutual information $\mathrm{MI}_{i, j}(d)$. Dots indicate numerical values, calculated from traces of duration $t=10^{5}$ and $d t=0.05$ from which the probability distribution $p\left(\phi_{i, t}, \phi_{j, t+d}\right),\left(\phi_{i, t}, \phi_{j, t+d}\right) \in[0,2 \pi]^{2}$ was estimated using a bin size of $2 \pi / 1000$. Data for the system in the initial phase locked state in (a) (orange) and the final state in (a) (dark orange) are shown. Lines show theoretical predictions eqs. (16.2.31) and (16.2.33) for each phase locked state. (d) dMI as in (c) with $\xi=0.3$. The larger noise level induces switches between the two phase locked states resulting in blurred superposition of the individual dMI's for the phase locked states (orange darker orange lines). The average dMI (lighter orange) and half of the average lightest orange) are shown for reference.

from which we obtain the delayed mutual information via (16.2.31). Under the assumptions that lead to (16.2.31), the analytical prediction is in good agreement with the numerical simulations as shown in fig. 16.2.

The mechanism underlying a shift of the peak of the dMI into one direction, indicating a directionality in the effective connectivity can be understood in this simple example: Consider the interaction function $\gamma$ and its antisymmetric part $\bar{\gamma}$ in fig. 16.2a. The zeros 
$\pm \Delta \phi$ of $\bar{\gamma}$ with $\bar{\gamma}^{\prime}( \pm \Delta \phi)<0$ indicate stable phase locked states (cf. sec. 3.4). Lets assume we are in the state where oscillator 2 leads oscillator 1, i.e. the initial state in fig. $16.2 \mathrm{~b}$ with $\Delta \phi_{12}=\phi_{1}-\phi_{2}=-\Delta \phi<0$. Figure 16.2a then shows that the slopes of the coupling function, $g_{1}=\gamma^{\prime}\left(\Delta \phi_{12}\right)=\gamma^{\prime}(-\Delta \phi)$ and $g_{2}=\gamma^{\prime}\left(\Delta \phi_{21}\right)=\gamma^{\prime}(\Delta \phi)$ are different, giving rise to different linearized dynamics (16.2.14) and (16.2.32). In our example $g_{1}$ is small and thus the dynamics of $\phi_{1}$ is mainly determined by the driving noise $w_{1}$. On the other hand, the dynamics for $\phi_{2}$ is coupled to $\phi_{1}$ via the large $g_{2}$ and compensates for deviations from the phase locked state by pulling $\phi_{2}$ to be at the preferred phase difference $\phi_{1}+\Delta \phi_{21}$ with a relaxation time constant $\frac{1}{g_{2}}$. Thus the overall rotation of the system is more strongly aligned to $\phi_{1}$ than to $\phi_{2}$ and, as a result of this, an effective information flow from $1 \rightarrow 2$ is established.

Figure 16.2 also illustrates that this non-symmetric information transfer can arise in an fully symmetric network. This is a result of symmetry breaking in a phase locked state that is neither the synchronous nor the anti-synchronous state, $0 \neq \Delta \phi_{12} \neq \pi$. Of course, due to the overall symmetry the reversed phase locked state exists with a reversed direction of information flow. This leads to the possibility of dynamic switching of the information direction by a strong perturbation that moves the system from one phase locked state to the basin of attraction of the other (cf. also [23, 408]).

\subsubsection{Discussion}

Our approach is limited by the assumption of small noise. For large noise amplitudes the theoretical prediction typically shows deviations form the numerically obtained delayed mutual information: In systems where the phase-locked state is a global attractor stronger noise only reduces the amplitude of the numerically obtained dMI curves but their shape stays qualitatively the same as predicted theoretically (cf. fig. 16.1d). This is due to the fact that the strong noise makes it likely to push the system very far away from the phase locked dynamics such that the linear approximations (16.2.14) and (16.2.17) no longer remain valid and the transients back to the phase-locked state blur the dMI. In systems with multiple phase-locked attractors strong noise pushes the system into a different attractor from time to time and the dMI becomes a superposition of the dMIs in each phase locked state weighted by the probability to be (or average time spend) in each state. Additionally the transients between the phase-locked states may blur this superposition (cf. fig. 16.2d).

The derivation that lead to theorem 16.2.1 also assumed the existence of a stable phase locked solution in the absence of noise. However, even in the absence of a phase locked solution some insights from our analysis can be gained by conditioning the time series of the phases on a certain phase pattern that frequently appears in the dynamics, e.g. due an underlying periodic or quasi-periodic motion in the noiseless case. For time delays $d$ smaller than the time scale in which the phase differences change in the underlying deterministic system and further assuming small noise amplitudes our derivation remains valid and may shed some light on information flow patterns in more irregular than phaselocked dynamics. In particular, our method may then be used to detect time windows of efficient information transfer. We leave a detailed study of this aspect to future work and concentrate on the information flow in hierarchical networks in the next section. 


\subsection{Local Control of Information Flow in Hierarchical Networks of Phase Oscillators}

results from the previous section apply to phase oscillators coupled via any network structure. Motivated by the facts that neuronal connectivity has a hierarchical structure and neuronal oscillations typically occur synchronously in groups of neurons we apply our general results to networks of phase oscillators that are clustered into different groups. In particular, we derive the collective phase response of each group as a function of its underlying network structure and intrinsic oscillation frequencies of the individual oscillators. We show that the averaging method for these types of stochastic systems is applicable and use the result to predict the delayed mutual information between the clusters as a function of the cluster properties. We find that local changes within a single cluster, e.g. by a changes in a local link, can control the non-local information transfer between two clusters and even remotely, between two other physically unchanged clusters.

\subsubsection{Information Flow in Hierarchical Networks of Phase Oscillators}

this sub-section we derive the information flow between weakly coupled clusters in hierarchical networks of phase oscillators. We first reduce each individual cluster to a metaoscillator described by a collective phase and response function following refs. [200, 221]. We show that the resulting stochastic model is suited for the standard averaging method which results in a stochastic phase oscillator model that has the same functional form as the model we started with. We can therefore apply our results from section 16.2 to obtain the delayed mutual information between the clusters as a function of the cluster properties.

\subsubsection{Hierarchical Networks of Phase Oscillators}

Throughout this section we consider a hierarchical network of $N$ phase oscillators as described in (16.1). We assume that the oscillators are clustered into $M$ different groups such that each cluster $X \in\{1, \ldots, M\}$ consists of $N_{X}$ oscillators so that $\sum_{X} N_{X}=N$. We denote the $i^{\text {th }}$-oscillator in cluster $X$ by $i_{X}$. The network evolves according to

$$
d \phi_{i_{X}}(t)=\left[\omega_{i_{X}}+\sum_{j_{X}} \gamma_{i_{X} j_{X}}\left(\phi_{i_{X}}-\phi_{j_{X}}\right)+\sum_{Y} \sum_{j_{Y}} \gamma_{i_{X} j_{Y}}\left(\phi_{i_{X}}-\phi_{j_{Y}}\right)\right] d t+\xi_{i} d w_{i}
$$

where the first sum on the right hand side represents the stronger intra-cluster couplings and the second sum the weaker inter-cluster couplings.

For the noiseless model $\xi_{i}=0$, and uncoupled clusters, $\gamma_{i_{X} j_{Y}}=0$, we assume the existence of a stable phase locked state $\phi_{i_{X}, 0}(t)=\Phi_{X}(t)+\Delta \phi_{i_{X}}$ for each individual cluster $X$ where $\Phi_{X}$ is the collective cluster phase and the temporally constant phase offsets $\Delta \phi_{i_{X}}$ and phase differences $\Delta \phi_{i_{X} j_{X}}=\Delta \phi_{i_{X}}-\Delta \phi_{j_{X}}$ obey the equation

$$
\omega_{i_{X}}+\sum_{j_{X}} \gamma_{i_{X} j_{X}}\left(\Delta_{i_{X} j_{X}}\right)=\Omega_{X}=\text { const. }
$$

where $\Omega_{X}$ is the collective cluster frequency. We further assume that the $\Omega_{X}$ differ only by a small amount so that in the noiseless fully coupled model the clusters themselves 
show a stable phase-locked pattern. Finally, we assume that noise strengths $\xi_{i}$ are small in comparison to the strength of attraction towards the phase locking.

\subsubsection{Collective Phase Reduction}

In this section we make use of the hierarchical network structure by first neglecting the inter-cluster couplings and consider each cluster separately. Using the assumption that in the noiseless system each cluster has a stable phase locked state, eq. (16.3.2), we may regard each group as a single meta-oscillator, for each of which we can perform a further phase reduction step (cf. sec. 3.4). A cluster $X$ is then described by its collective phase $\Phi_{X}$ and its collective phase response curve $Z_{X}[200,221]$. We solve the adjoint equation (3.4.7) together with the initial condition (3.4.8) which both arising from the theory of phase reduction in order to determine $Z_{X}$. Using (16.3.1) and (16.3.2) the adjoint equation (3.4.7) in our notation becomes

$$
\frac{d}{d t} Z_{X}=-L_{X}^{T} Z_{X}
$$

with

$$
L_{X}= \begin{cases}-\gamma_{i_{X} j_{X}}^{\prime}\left(\phi_{i_{X}, 0}-\phi_{j_{X}, 0}\right) & i_{X} \neq j_{X} \\ \sum_{k_{X}} \gamma_{i_{X} k_{X}}^{\prime}\left(\phi_{i_{X}, 0}-\phi_{k_{X}, 0}\right) & i_{X}=j_{X}\end{cases}
$$

Note that $L_{X}$ is time independent and a Laplacian matrix. Thus, to solve (16.3.3) we can choose $Z_{X}$ to be the constant left eigenvector of $L_{X}$ with eigenvalue $\lambda_{X, 0}=0$. The initial condition (3.4.8) then reduces to $\sum_{i_{X}} Z_{X, i_{X}}=1$ and we thus obtain

$$
Z_{X, i_{X}}=\frac{\operatorname{det}\left(\left[\left[L_{X}\right]\right]_{i_{X}, i_{X}}\right)}{\sum_{i_{X}} \operatorname{det}\left(\left[\left[L_{X}\right]\right]_{i_{X}, i_{X}}\right)} .
$$

Note that $Z_{X}$ is a constant vector independent of the phase $\Phi_{X}$. As the white noise sources $w_{i}$ in our model represent physical input signals that always have correlations in time [386] and we assumed strong, that is fast, attraction towards the phase locked state the above phase reduction analysis for the deterministic situation $\xi_{i}=0$ remains valid for the stochastic system $\xi_{i}>0$ as shown in [363].

The fully coupled stochastic system (16.3.1) in the reduced form then becomes

$$
d \Phi_{X}=\Omega_{X}+\sum_{Y} Z_{X}^{T} G_{X, Y}\left(\Phi_{X}, \Phi_{Y}\right)+\sum_{i_{X}} Z_{X, i_{X}} \xi_{i_{X}} d w_{i_{X}}
$$

where

$$
G_{X, Y}\left(\Phi_{X}, \Phi_{Y}\right)_{i_{X}}=\sum_{i_{Y}} \gamma_{i_{X} j_{Y}}\left(\Phi_{X}-\Phi_{Y}-\Delta \phi_{i_{X}, j_{Y}}\right)
$$

For a deterministic system the standard approach at this stage is to further exploit the weak coupling by performing an averaging step (cf. section 3.4). However, similarly to modifications encountered in the phase reduction method for stochastic oscillators [200] the averaging method for a stochastic system may also change. Interestingly, to our knowledge, there is no general theory of stochastic averaging for weakly coupled oscillators developed so far (cf. also the discussion in sec. 18.3 where we propose steps towards the development of such a theory). 
For eq. (16.3.5), however, the $Z_{X}$ are constant vectors and $G_{X, Y}$ is already in a averaged like form as it only depends on the cluster phase differences and we therefore immediately obtain

$$
\frac{d}{d t} \Phi_{X}=\Omega_{X}+\sum_{Y} \Gamma_{X Y}\left(\Phi_{X}-\Phi_{Y}\right)+\varsigma_{X} d W_{X}
$$

where the inter-cluster coupling function $\Gamma_{X Y}$ is given by

$$
\Gamma_{X Y}\left(\Phi_{X}-\Phi_{Y}\right)=\sum_{i_{X}, j_{Y}} Z_{X, i_{X}} \gamma_{i_{X} j_{Y}}\left(\Phi_{X}+\phi_{i_{X}, 0}-\Phi_{Y}-\phi_{j_{Y}, 0}\right)
$$

with white noise processes $W_{X}$ obeying $\left\langle W_{X}(t), W_{Y}(s)\right\rangle=\delta_{X Y} \delta(t-s)$ and noise levels

$$
\varsigma_{X}^{2}=\sum_{i_{X}} Z_{X, i_{X}}^{2} \xi_{i_{X}}^{2}
$$

\subsubsection{Delayed Mutual Information between Collective Cluster Phases}

Note that eq. (16.3.6) has the same functional form as eq. (16.1.1) thus implying equivalent functional behaviour. Further using the assumption of phase-locking among the clusters in the deterministic system we may write $\Phi_{X}(t)=\Omega t+\Delta \Phi_{X}$ with $\Omega$ the collective rotation frequency of the system and phase locking offsets $\Delta \Phi_{X}$ satisfying $\Delta \Phi_{X Y}=\Delta \Phi_{X}-\Delta \Phi_{Y}$ the analog of eq. (16.1.4):

$$
\Omega=\Omega_{X}+\sum_{Y} \Gamma_{X Y}\left(\Delta \Phi_{X Y}\right)=\text { const. }
$$

Now we are in a situation completely analogous to section 16.2. We thus immediately obtain an expression for the delayed mutual information between the clusters via theorem 16.2.1:

Corollary 16.3.1. The delayed mutual information $\mathrm{MI}_{X, Y}(d)$ between the time series of the collective phases of cluster $X$ and cluster $Y$ in system (16.3.1) close to a phase locked state with phase and small noises $\xi_{i}$ is given by (16.2.31) when substituting $\phi_{i}$ with $\Phi_{X}$, $\Delta \phi_{i j}$ by $\Delta \Phi_{X Y}, \omega_{i}$ with $\Omega_{X}, \gamma_{i j}$ by $\Gamma_{X Y}$, and $\xi_{i}$ by $\varsigma_{X}$.

In fig. 16.3 this result is illustrated for a network of $N=12$ oscillators and $M=2$ clusters. Under the assumptions of phase locking and small noise the theoretical predictions are in good agreement with the numerically obtained delayed mutual information. Similar limitations apply as discussed for the general result, theorem 16.2.1, in sec. 16.2.5. In appendix $\mathrm{H}$ we use corollary 16.3.1 to derive an explicit analytical expression for the dMI between two collective cluster phases, when the phase locking patterns defined by equations (16.3.2) and (16.3.8) are close to the synchronous state. In particular for couplings of the form $\gamma_{i_{X} j_{Y}}(\phi)=K_{i_{X} j_{Y}} \gamma(\phi)$ this gives an explicit expression of the dMI as a function of the connectivity matrix $K_{i j}$ and in particular the local inter cluster connection strengths $K_{i_{X} j_{X}}$. These theoretical predictions are also in good agreement with numerical simulations as shown in fig. 16.3.

To summarize, we have calculated the delayed mutual information between the time series of the collective phases of each cluster in a hierarchical network of weakly coupled oscillators. The result implies, that the delayed mutual information between the clusters is a function of the local properties of each clusters. In particular, the local network 
(a)

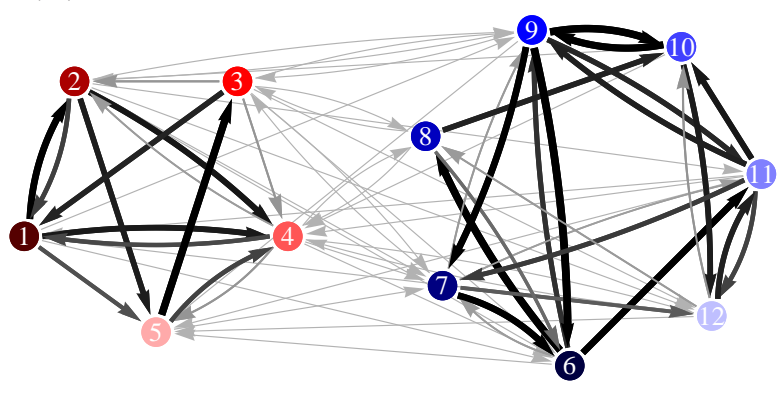

(c)

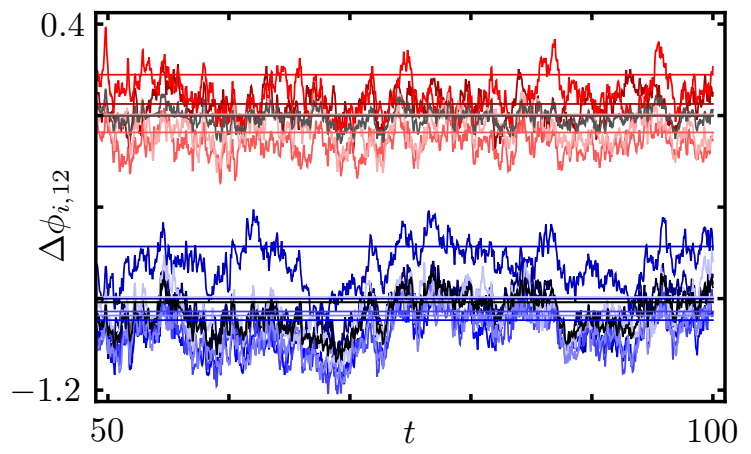

(e)

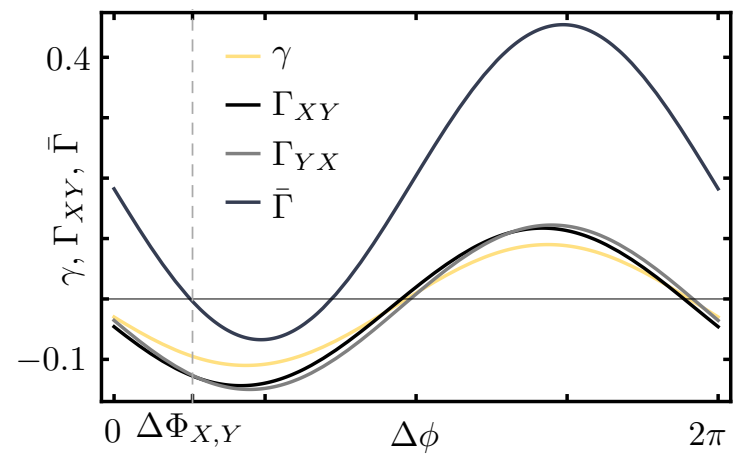

(b)

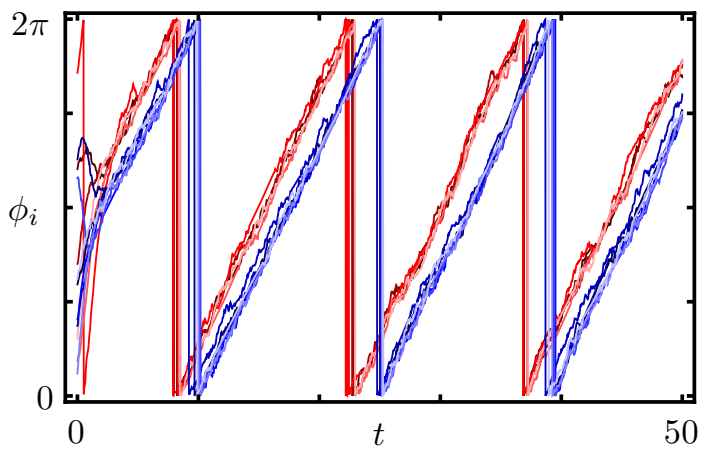

(d)

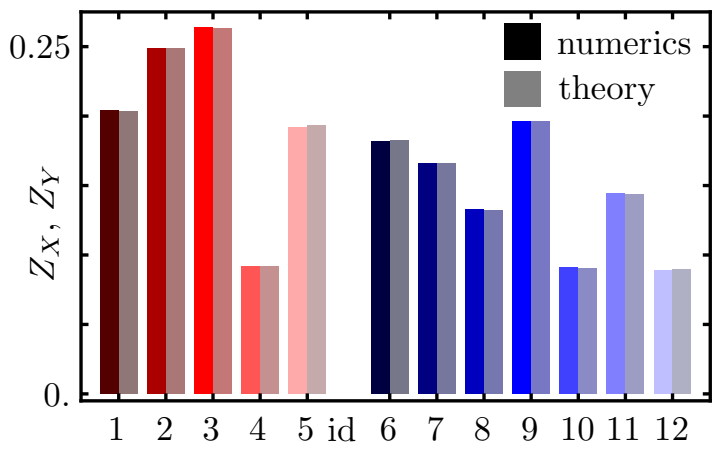

(f)

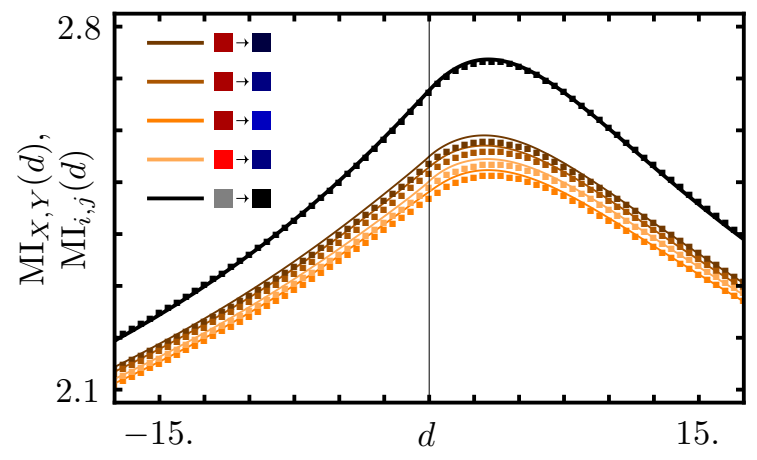

Figure 16.3. Delayed mutual information in hierarchical networks. (a) hierarchical network (16.3.1) with clusters $X$ (red) and $Y$ (blue), coupling function $\gamma_{i j}(\Delta \phi)=g_{i j} \gamma(\Delta \phi)$ with $\gamma(\Delta \phi)=-\sin (\Delta \phi+0.2)-0.1$ (cf. (e)) and $\omega_{i}=1$ and $\xi_{i}=0.1$. (b) phase traces starting from random initial conditions. (c) phase differences $\Delta \phi_{i, 12}=\phi_{i}-\phi_{12}$ fluctuate around the phase locked state (straight lines). (d) $Z_{X}$ and $Z_{Y}$ calculated numerically (dark) and theoretically (light) using (16.3.4). (e) coupling functions $0.1 \gamma, \Gamma_{X Y}$ and $\bar{\Gamma}(\Delta \Phi)=\Gamma_{X Y}(\Delta \Phi)-\Gamma_{Y X}(\Delta \Phi)+\Omega_{X}-\Omega_{Y} \cdot \Delta \Phi_{X Y}$ indicates prediction for the cluster phase difference (cf. (H.4)). (f) dMI between phase traces of a subset of individual oscillators obtained numerically (orange dots, duration $t=3 \cdot 10^{5}$ with step size $d t=0.05$ and bin size $2 \pi / 1000$ for the estimation of the probability distribution) and analytically via theorem 16.2.1 (orange lines). Also shown is the dMI between the collective cluster phase signals obtained numerically (black dots) and using corollary 16.3.1. As the noise is large compared to the weak inter cluster couplings the dMIs for the individual oscillators are slightly over estimated.

connectivity $\gamma_{i_{X} j_{X}}$ and the intrinsic frequencies of the oscillators $\omega_{i_{X}}$ determine the phase locking patterns $\Delta \phi_{i_{X} j_{X}}$ within each clusters. These in turn via eq. (16.3.8) determine 
the collective rotation frequency $\Omega_{X}$ of each cluster and via (16.3.4) the collective phase response $Z_{X}$. The collective phase response in turn together with the inter cluster couplings $\gamma_{i_{X} i_{Y}}$ determine the effective inter cluster coupling $\Gamma_{X Y}$ via (16.3.7). Thus, corollary 16.3.1 then gives the delayed mutual information between two clusters as a function of the local network conductivities $\gamma_{i_{X} j_{X}}$ and intrinsic frequencies of the oscillators $\omega_{i_{X}}$. As a consequence we obtain that local changes within a single cluster can control the non-local phase information flow between different clusters.

The remainder of this chapter is devoted to this local control of non-local information flow.

\subsubsection{Local Control of Non-Local Information Flow}

connectivity between different clusters may be changed. It is clear that by changing the connectivity between two clusters the effective connectivity between them can be changed (cf. theorem 16.2.1). More interestingly, the effective connectivity between different clusters can also be changed by local changes within the cluster, i.e. without changing the inter cluster structural connectivity. This is a consequence of the general result corollary 16.3.1. Here we investigate three mechanisms for a local control of non-local information flow: First, changes in local frequencies of oscillators within a cluster, e.g. mediated by a change in the local inputs. Second, changes of the local network structure within a cluster. Intriguingly, we also show that changes within a cluster can remotely control the information flow between two other clusters. Third, multistable states within each cluster.

\subsubsection{Control of Non-Local Information Flow via Local Intrinsic Oscilla- tion Frequencies: Information Flow Tuning}

The information flow measured by the delayed mutual information depends via corollary 16.3.1 strongly on the phase locking pattern between the clusters. One way of controlling these inter-cluster phase differences is by changing the intrinsic oscillation frequencies of oscillators within a single cluster. In particular, when changing all frequencies $\omega_{i_{X}}$ uniformly within one group $X$, the phase differences $\Delta \phi_{i_{X} j_{X}}$ of the phase locked state for this cluster do not change. Thus via (16.3.8) the collective cluster frequency $\Omega_{X}$ is changed while the effective cluster couplings (16.3.7) stay unchanged. Hence via (16.3.6) the phase locking of cluster $X$ to the others will change and by corollary 16.3.1 this entails a change in the delayed mutual information between cluster $X$ and the other clusters. This mechanism is illustrated in fig. 16.4a-e.

Changing the frequency of only a subset of oscillators within a clusters not only affects the collective frequency of the cluster but also its phase locking pattern. Hence also its collective phase response is changed. This effect is similar to the one caused by local connectivity changes that will be discussed in sec. 16.3.2.2.

Following the arguments in sec. 16.2.4, the effective change of non-local information flow via local frequency changes will be strongest if the slope of the collective coupling function $\Gamma_{X Y}$ varies strongly at locations where the phase differences between the clusters change, i.e. the curvature of the coupling function is large. For systems close to the synchronous phase-locked state this implies that the control of non-local information flow is most effective if the $\Gamma_{X Y}(\Phi)$ show a strong variation in slope near $\phi=0$. Equation (16.3.7) shows that in the near synchronous state $\Gamma_{X Y}(\Phi)$ is almost a superposition of the single oscillator coupling functions $\gamma_{i_{X} j_{Y}}(\phi)$. Thus a strong asymmetry in the slope 
of the single oscillator coupling functions near the origin will entail a strong asymmetry in the slope of the collective cluster couplings and therefore will provide a basis for an efficient local control mechanism of non-local information flow in these networks.

In numerical studies we find that locally increased frequencies in a single cluster mainly affect its own phase relation to the other clusters and only has a weak effect on the phase differences between the other clusters. This implies that for coupling functions with smaller asymmetry in their slopes the information flow between the other clusters is hardly altered. Thus, by changing its local frequency the cluster can tune its phase relation to other clusters and hence can tune its information flow to and from others without affecting the flow between the other clusters. In particular, this provides a mechanism for each cluster to tune to information sources from other clusters or to provide information to other clusters dynamically.

A change in the intrinsic frequencies in one cluster will not only change its phase relation to the others but, depending on the network structure, may also induce small changes in the phase locking between the other physically unchanged clusters. For coupling functions with large curvature around the phase locking regime a small phase shift already is sufficient to induce a stronger change in the delayed mutual information. Thus in this situation a change of the intrinsic frequencies in one cluster can remotely control the non-local information flow between two (or more) other clusters that are not directly affected by the physical change. An example for this remote control of information flow is shown in fig. 16.4f.

\subsubsection{Control of Non-Local Information Flow via Local Structural Con- nectivity: Information Flow Plasticity}

A different mechanism to control the delayed mutual information between clusters is by local modifications of the network structure. Such changes in a cluster $X$ entail changes in the phase-locking pattern $\Delta_{i_{X} j_{X}}$ of the cluster's oscillators via eq. (16.3.2) (cf. also fig. 16.5a-c). Both the change in phase locking as well as the link modification itself change the collective phase response $Z_{X}$ of the cluster as can be seen form (16.3.4). Both $Z_{X}$ and the phase differences $\Delta_{i_{X} j_{X}}$ enter the expression (16.3.7) for the effective inter cluster coupling functions $\Gamma_{X Y}$. As a consequence the phase locking pattern $\Delta \Phi_{X Y}$ between the clusters via (16.3.8) and also the shape of the coupling function itself changes. By corollary 16.3.1 these changes then entail a change in the delayed mutual information curve between the clusters.

An example of this mechanism by which local links control the non-local information flow is shown in fig. 16.5. Here the change of a single link in cluster $A$ (cf. fig. 16.5a,c) induces a change of the phase locking pattern of this cluster (cf. fig. 16.5b,d,f) and as a consequence also the cluster phase response changes (cf. fig. 16.5g). Both effects produce a change in the inter cluster phase-locking (cf. fig. $16.5 \mathrm{~b}, \mathrm{~d}, \mathrm{f}$ ) which in turn results in a change of the delayed mutual information curves (cf. fig. 16.5e).

Note that in fig. 16.5e the delayed mutual information between the unchanged clusters $B$ and $C$ is plotted. Thus a local change within cluster $A$ is capable to remotely control the information flow between the other clusters. Figure 16.5h shows how the switch in the direction of the information flow between clusters $B$ and $C$ is controlled by a single link strength within cluster $A$.

The control of non-local information flow in the phase oscillator models via local link modifications is most effective if the phase locking pattern and the cluster phase response 
(a)

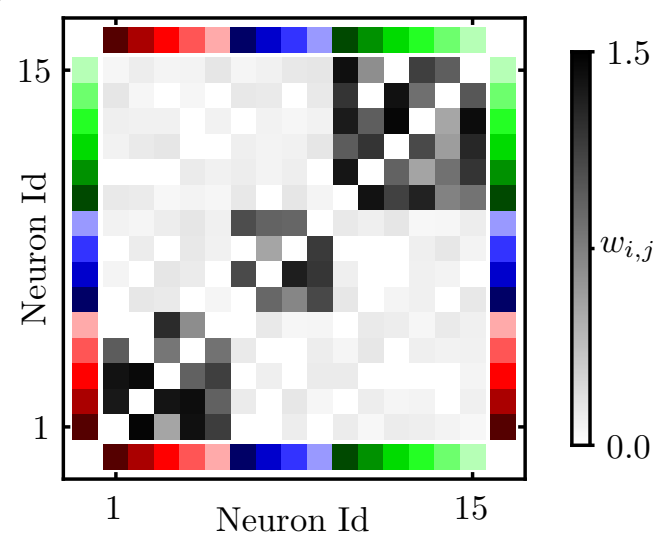

(c)

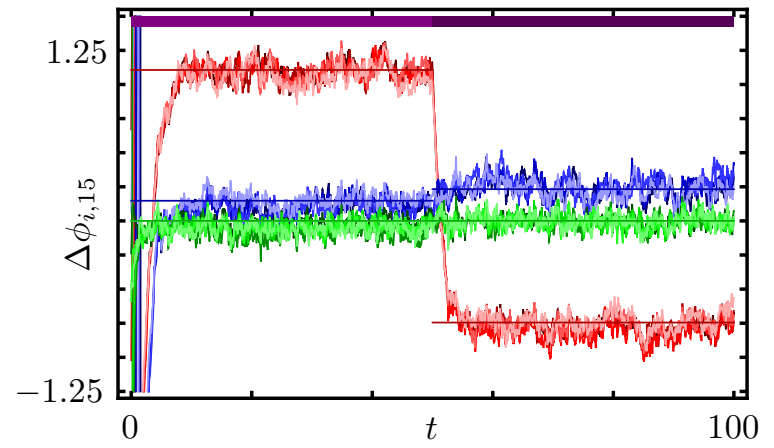

(e)

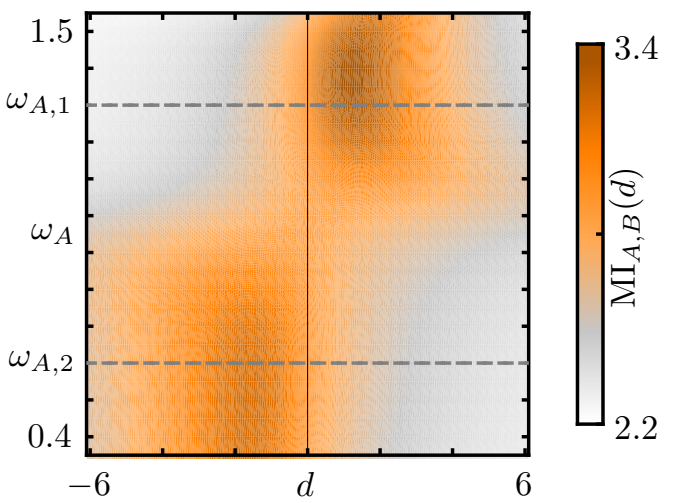

(b)

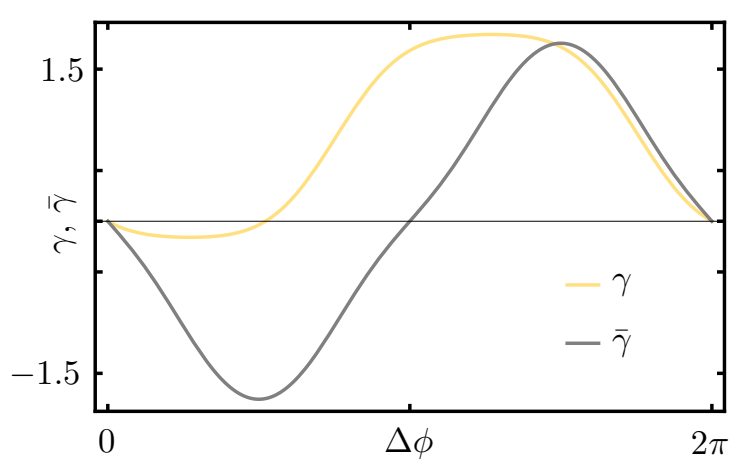

(d)

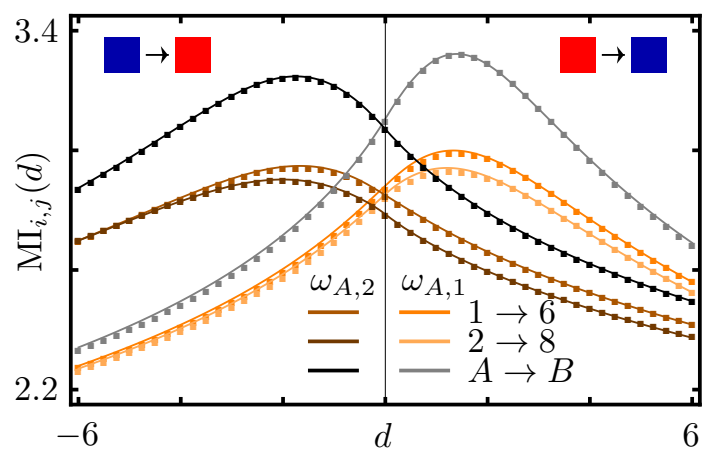

(f)

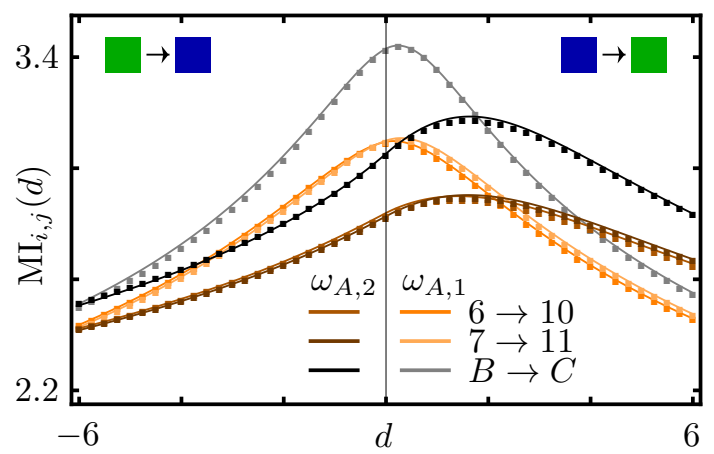

Figure 16.4. Remote control of non-local asymmetric information flow via local intrinsic oscillation frequencies. (a) connectivity matrix $g_{i j}$ for a network of $N=15$ oscillators, eq. (16.3.1), with $M=3$ clusters: $A$ (red), $B$ (blue) and $C$ (green) and $\xi_{i}=0.1, \omega_{i_{A}}=\omega_{A}$, $\omega_{i_{B}}=1, \omega_{i_{C}}=0.9, \gamma_{i j}(\Delta \phi)=g_{i j} \gamma(\Delta \phi)$. (b) coupling functions $\gamma(\Delta \phi)$ (yellow) and $\bar{\gamma}(\Delta \phi)=\gamma(\Delta \phi)-\gamma(-\Delta \phi)$ (gray) showing stability of the synchronous state. (c) phase differences $\Delta \phi_{i, 15}(t)=\phi_{i}(t)-\phi_{15}(t)$. At $t=50$ the intrinsic frequencies of cluster $A$ (red) are switched form $\omega_{A}=\omega_{A, 1}=1.3$ (purple bar) to $\omega_{A}=\omega_{A, 2}=0.6$ (dark purple bar). Cluster phase differences in the noiseless case are indicated by lines. (d) dMI between oscillators $1 \rightarrow 6,2 \rightarrow 8$ and between clusters $A \rightarrow B$ for $\omega_{A}=0.6$ (orange, gray) and $\omega_{A}=1.3$ (dark orange, black). Dots indicate numerical values obtained as in fig. 16.3. Lines show theoretical predictions using (16.2.31) and (16.2.26). The local frequency change within cluster $A$ shifts the maximum of the dMI from positive to negative $d$ effectively reversing the non-local information flow between clusters $A$ and $B$. (e) dMI between cluster $A$ and $B$ as a function of $\omega_{A}$ using (16.2.31) and (16.2.26). (f) dMI as in (d) but between oscillators $6 \rightarrow 10,7 \rightarrow 11$ and clusters $B \rightarrow C$. See text for details. 
(a)

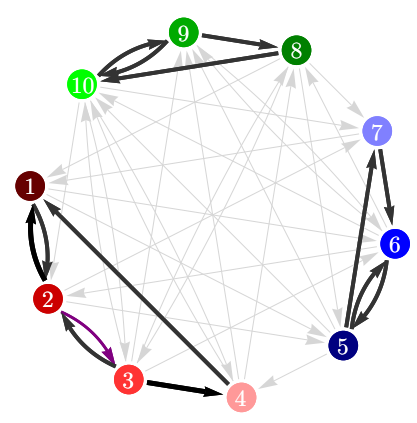

(d)

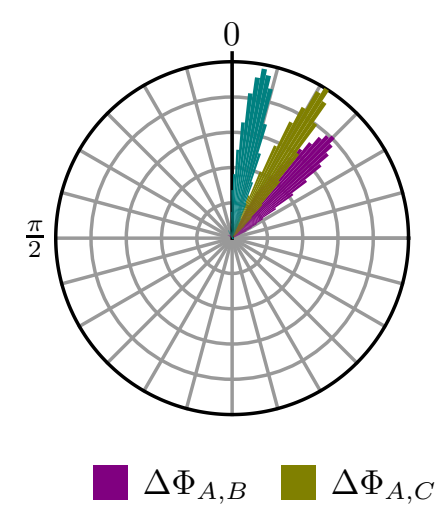

(g) (b)

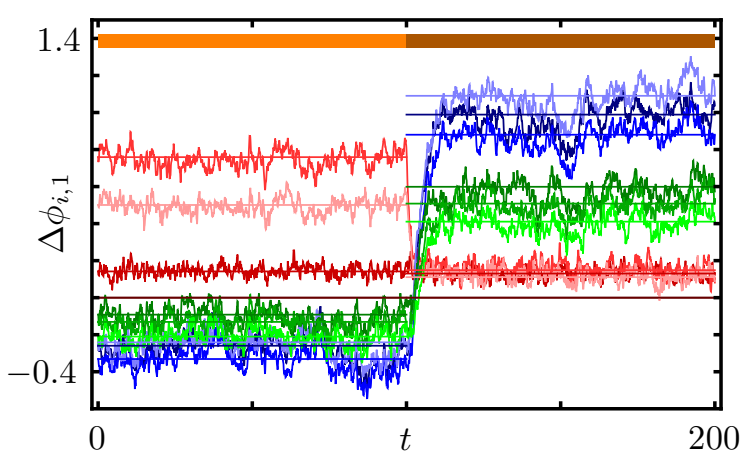

(e)

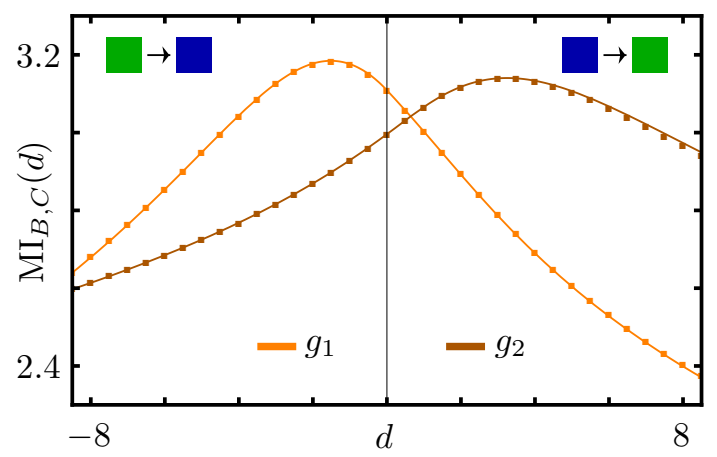

(c)

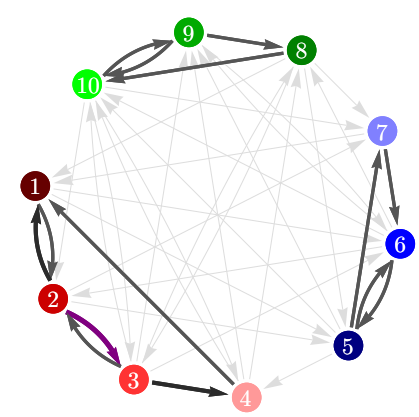

(f)

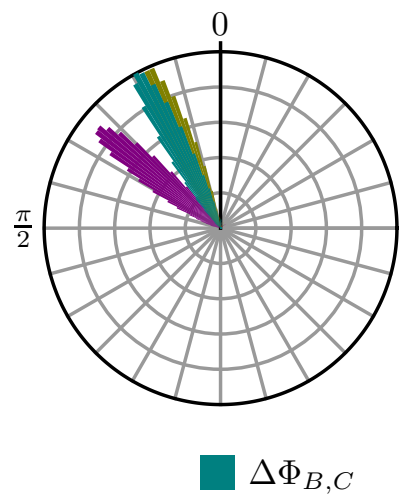

(h)

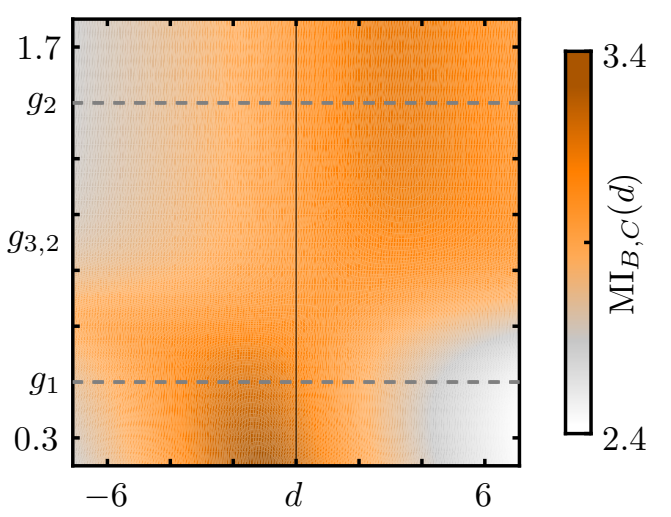

Figure 16.5. Remote control of non-local information flow via local connectivity. (a,c) connectivity $g_{i j}$ of $N=10$ oscillators and three clusters $A$ (red), $B$ (blue), $C$ (green) in network eq. (16.1.1), with $\xi_{i}=0.05, \omega_{i}=1, \gamma_{i j}(\Delta \phi)=g_{i j} \gamma(\Delta \phi), \gamma$ as in fig. 16.4(b). Local network connectivity of cluster $A$ is changed from $g_{32}=g_{1}=0.5$ in (a) to $g_{32}=g_{2}=1.5$ in (c) (purple link). (b) phase differences $\Delta \phi_{i, 1}(t)=\phi_{i}(t)-\phi_{1}(t)$. At $t=100$ the link is changed. Lines show phase differences in the noiseless case. (d,f) polar histograms of the cluster phase differences $\Delta \Phi_{A B}$ (purple), $\Delta \Phi_{A C}$ (brown) and $\Delta \Phi_{B C}$ (cyan) before and after the link change. (e) dMI between clusters $B \rightarrow C$ for network (a) (orange) and (b) (dark orange). Dots indicate numerical values obtained as in fig. 16.3. Lines show theoretical predictions using corollary 16.3.1. The local link change within cluster $A$ shifts the maximum of the dMI from negative to positive $d$ effectively reversing the remote information flow between clusters $B$ and $C$. (g) phase response vectors $Z_{X}$ of the clusters $X$ as a function of the link strength $g_{32}$. (h) dMI between clusters $B$ and $C$ as a function of $g_{32}$ using corollary 16.3.1. See text for details. 
(a)

(b)

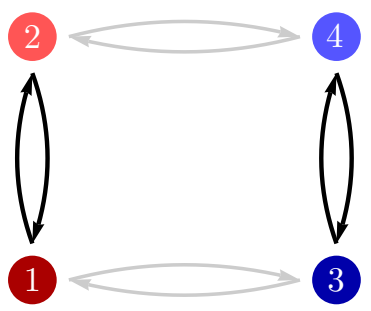

(c)

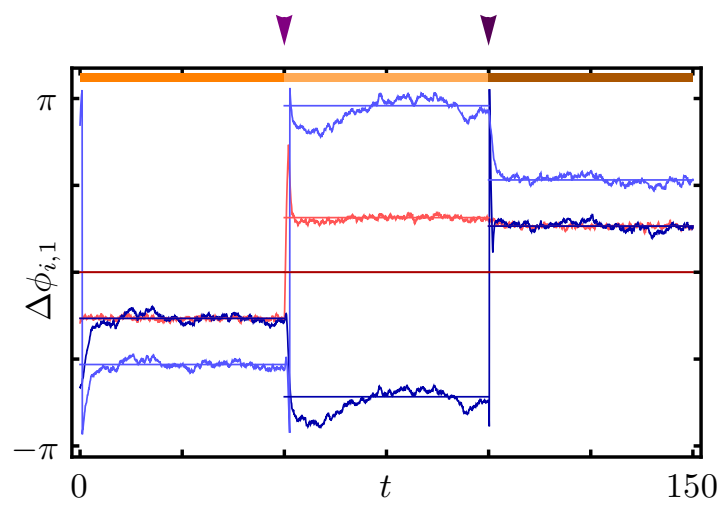

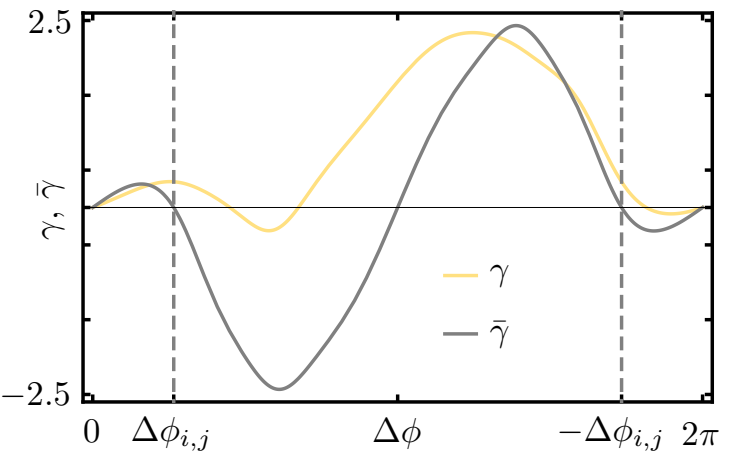

(d)

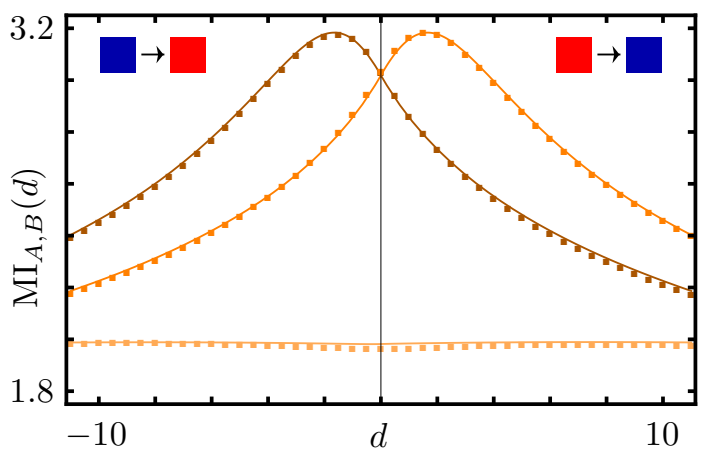

Figure 16.6. Information flow combinatorics: Local control of non-local information flow via multiple local phase locked states. (a) graph of a $N=4$ oscillator network, eq. (16.3.1), with two clusters $A$ (red) and $B$ (blue) with parameter $\xi_{i}=0.05, \omega_{i}=1, \gamma_{i j}(\Delta \phi)=g_{i j} \gamma(\Delta \phi)$, $g_{i_{X} j_{X}}=1$ within clusters and $g_{i_{Y} j_{Y}}=0.1$ between clusters. (b) coupling function $\gamma(\Delta \phi)$ (yellow) and anti-symmetric part $\bar{\gamma}(\Delta \phi)=\gamma(\Delta \phi)-\gamma(-\Delta \phi)$ (gray) that shows the existence of two stable phase locked states $\pm \Delta \phi_{i, j}$ within each cluster. (c) time $t$ evolution of phase differences $\Delta \phi_{i, 1}(t)=\phi_{i}(t)-\phi_{1}(t)$. At $t=50$ the a pulse is inserted into cluster $A$ (purple arrow) which switches its internal phase locking and as a result the inter cluster phase differences. At $t=100$ a pulse is applied to cluster $B$ (dark purple arrow) changing its internal phase locking and again the overall phase pattern. (d) Delayed mutual information between clusters $A \rightarrow B$ for the three different states in (c). Dots indicate numerical values obtained as in fig. 16.3, lines show theoretical predictions using corollary 16.3.1. For the initial state (orange) there is an effective information flow from $A$ to $B$. Switching the internal phase locking pattern of cluster $A$ essentially removes the directionality (lighter orange). Further switching the phase locking pattern in cluster $B$ turns the flow on again now in the opposite direction (darker orange).

are sensitive to these changes. We find that this sensitivity is increased if both the intracluster connectivity is sparse and the coupling functions are heterogeneous. Also, similarly to supporting the control mechanism via local frequency changes (sec. 16.3.2.1), strong asymmetric slopes support the effectiveness of local structural changes for the control of non-local information flow. Again, all these properties have been found in real neuronal networks making them a possible candidate for local control mechanisms of non-local information flow. 


\subsubsection{Information Flow Combinatorics}

A third mechanism for the local control of non-local information flow is an extension of the dynamic switching mechanism between multi-stable phase locked states observed in fig. 16.2 to hierarchical networks. As analyzed in the previous section, changes of phaselocking patterns within a single cluster are capable of controlling non-local information flows between clusters. Thus, local switching between phase locked states present in a single cluster will induce changes in the non-local information flow between the clusters in the full weakly coupled network. Moreover, if the different clusters in the network all posses multistable phase locked solutions, different combinations of the local phase locking patterns of the individual clusters will lead to different information flow patterns in the full network. In particular, if each cluster $X$ possess $m_{X}$ stable phase-locked solutions there are $\prod_{X} m_{X}$ information flow patterns in the full network (assuming all combinations result in phase locking among the clusters and give rise to different information flow patterns). This local control of combinatorial information flow is illustrated in fig. 16.6.

\subsection{Summary and Discussion}

In this section we analytically derived the delayed mutual information between times series of phase signals in networks of phase oscillators close to a phase locked state (theorem 16.2.1). The result is very general in that it applies to any coupling function and any network topology that lead to a phase locked state. We found that delayed mutual information curves arise that are asymmetric around the zero delay indicating an effective directionality in the information flow. We showed that the underlying mechanism for this directionality is an unbalance in the influence of the oscillators onto the overall rotation of the whole system combined with an asymmetric relaxation towards the phase locked state. We found that this mechanism is supported by a large curvature of the coupling functions at the phase differences of the phase locking pattern. We then focused on hierarchical networks consisting of stronger connected groups of phase oscillators with weaker inter cluster connections and derived the delayed mutual information between the collective cluster phases as a function of the underlying cluster properties (corollary 16.3.1).

Using this theoretical framework we found that local changes within a single cluster can control the non-local information transfer between the clusters. We identified three mechanism for the local control of non-local information flow:

\section{Local Oscillation Frequencies - Information Flow Tuning}

Uniform frequency changes of the oscillators within a single cluster cause changes of its phase relations to the others which as a consequence result in changes of the information flow to and from this cluster. In this way the cluster is able to tune to information sources from or make its information available to other clusters.

\section{Local Network Structure - Information Flow Plasticity}

Local changes within the network structure of a single cluster alter the collective cluster phase response and the effective inter-cluster couplings resulting in concert in a change in the information flow even remotely between two distant, physically unchanged groups.

3. Local Multistable Phase-Locking - Information Flow Combinatorics Switching between stable intra-cluster phase locking patterns induce changes of the 
collective cluster phase response and the effective inter-cluster couplings which in turn control the information flow between the clusters. Combinations of different phase locking patterns of the individual clusters then endow the full network with different information flow patterns.

We note that also a combination of the latter two or the first and the last are possible, in that local changes of the network structure may destabilize a phase locked state so that the clusters jumps into a different phase locked state. In this case already very small changes within a single link may give rise to large changes in the information flow.

All local control mechanism are supported by a strong curvature of the collective clusters coupling functions at values corresponding to the phase locking pattern. This can be seen using the example in 16.2.4. A large curvature implies a large change of the slopes of the interaction function for small changes of the phase-locking pattern. These slopes in turn effectively determine the information flow. Thus a small change in the phase-locking pattern gives rise to a stronger change in information flow if the curvature of the coupling function is large. In particular, for phase locking patterns close to the synchronous state such an asymmetry is induced by an asymmetry in the individual oscillator coupling functions. Interestingly, type II neurons that synchronize their dynamics, have a phase responses curve that is strongly asymmetric near the origin (cf. fig. 3.11 and [314, 361] for experimental measured curves). The coupling functions obtained via averaging, i.e. folding with a short synaptic pulse, preserves this property. Thus real neurons possess a basic property required for effective local control of non-local information flow.

We note further that in chapter 13 we have shown that the shape of the phase response curve of individual neurons can be changed dynamically via synaptic shunting (cf. fig. 13.1) and thus in this way not only the synchronization properties of the collective dynamics may be regulated (cf. sec. 13.1) but also the information flow between groups of neurons. Control of information flow via local network structure is additionally supported by sparse and heterogeneous inter-cluster connectivities also present in real neuronal networks.

The derivation of theorem 16.2.1 and corollary 16.3.1 relied on the existence of a stable phase locked state. However, in the absence of such a solution our analysis remains valid also for transient locking, by conditioning the time series on a certain initial phase difference and restricting the time delay in the mutual information to values smaller than the time scale on which these phase differences change in the underlying deterministic system. Thus our analysis may even shed some light on information flow patterns in more irregular collective dynamics and may find applications in detecting time windows of efficient information transfer in phase oscillator networks. We propose a detailed study of this aspect for future work.

Our analysis also relied on the assumption of noise levels that are small compared to the strength of attraction towards the phase locked state. Larger noise amplitudes resulted in stronger deviations from the phase locking pattern. In systems where this state was a global attractor the transients back to the neighbourhood of the phase-locked state only blurred the information flow by essentially shifting the delayed mutual information curve to smaller values. In systems with multiple stable phase locked states the dMI curve became a superposition of the dMI curves of each state due to noise-induced switching between the attractors.

In our analysis we modeled external signals by independent white noise sources. In real world applications however, correlations in space and time are ubiquitous. Our analysis can be immediately extended to correlations among the white noise sources by substituting the diagonal matrix $\Xi$ (eq. (16.1.3)), representing the variances of the noise sources, with a 
non-diagonal correlation matrix. We also hypothesize that extensions of theorem 16.2.1 to signals with correlations in time such as Ornstein-Uhlenbeck processes should be possible and could provide further insight into the information flow in coupled phase oscillator networks.

We observed that changes in certain links are more effective in controlling the nonlocal information flow than others. An interesting application of our theory thus might be to identify "high sensitivity" links and underlying network structures that are highly optimal in controlling the information flow of the full network. This might not only have applications to neuronal systems but also in regulatory networks of oscillatory chemical reactions and gene expression.

The above theory deals with networks of phase oscillators and thus with information flow between the phase signals of the oscillators. A natural extension of our work dealing with coupled phase oscillators would therefore be to include amplitude variables for the oscillators. Assuming symmetric couplings, amplitude oscillators near a Hopf bifurcation are analytically tractable [59] and show relaxation towards phase locked states. This property has been used in [175] to propose learning rules for phase locking patterns, and in [54] to solve the inverse problem of estimating phase-coupling strengths from the phase statistics assuming a maximum entropy model. We believe, supported by preliminary studies, that our derivation of the delayed mutual information for phase oscillators in a modified form may be applicable to these symmetrically coupled amplitude oscillators. Generally, such models would have the advantage of a separation of the control of the information flow (mediated by the phase) from the information flow itself (carried in the amplitude channels).

An interesting question that arises in the context of our study is how the mechanisms for local control of information flow found here may provide an efficient way in controlling and coordinating information processing in the brain, given the omnipresent oscillatory neuronal activity. In the next chapter we investigate this question in a network model of spiking neurons that shows collective gamma oscillations. There we not only consider information flows within phases but also its relation to amplitude-like channels associated with spike timing. 


\section{Chapter 17}

\section{Local Control of Non-Local Information Flow in Spiking Neuronal Networks}

Motivated by the findings of local control mechanisms for non-local information flows in coupled phase-oscillators in the previous section, we here study such mechanism for networks of spiking model neurons. We propose a hierarchical network model consisting of clusters of neurons which each undergo a collective gamma oscillation mediated by pyramidal inter-neuron interactions as modeled in [31]. We show that phase locking patterns between the clusters arise in this model. In analogy to chapter 16 we study the phase information flow between the clusters and find similar mechanisms for the local control of non-local phase information flow. We then extend our study to the the information flow in spike patterns. In particular, we show and explain that the information flow in the ordering of the spikes has the same directionality as the phase information flow and thus the same control mechanisms apply.

\section{$17.1 \quad$ Model}

We consider hierarchical model networks of $N$ spiking theta (QIF) neurons (cf. sec. 2.6.4) coupled via conductance based synapses (cf. sec. 2.7.1). The network consists of $M$ different clusters $X \in\{1, \ldots, M\}$ each consisting of $N_{X}^{\mathrm{e}}$ excitatory and $N_{X}^{\mathrm{i}}$ inhibitory neurons such that $N_{X}=N_{X}^{\mathrm{e}}+N_{X}^{\mathrm{i}}$ and $N=\sum_{X} N_{X}$. We denote the indices of a neuron $i \in\{1, \ldots, N\}$ in the network that belongs to cluster $X$ by $i_{X}$. Each individual cluster is based on the model in [31] and undergoes pyramidal interneuron gamma [PING] oscillations [404]. The clusters are coupled together by long range excitatory connections.

We next describe the structure and dynamics of a single cluster and then introduce the full hierarchical network model.

\subsubsection{Pyramidal Inter-Neuron Gamma Cluster}

In this section, we describe the model for an individual PING cluster as introduced in [31]. A single neuron $i_{X}$ in such a cluster $X$ is described by its state variable $\theta_{i_{X}}$ from which the membrane potential $v_{i_{X}}$ is deduced via $v_{i_{X}}=\frac{1}{2}\left(1+\tan \left(\frac{\theta_{i_{X}}}{2}\right)\right)$ (cf. sec. 2.6.4) so that the neuron spikes at $\theta_{i_{X}}=\pi$. To each neuron $i_{X}$ we associate a synaptic activation variable $s_{i_{X}}$ that gives rise to conductance-based synaptic currents at the postsynaptic neurons $j_{X}$ with maximal conductance $g_{i_{X} j_{X}}$ and reversal potentials $v_{\mathrm{syn}, \mathrm{i}_{X}}$ (cf. sec. 2.7.1). 
The time evolution of cluster $X$ is then given by the equations:

$$
\begin{aligned}
& \frac{d}{d t} \theta_{i_{X}}= 1-\cos \left(\theta_{i_{X}}\right)+i_{\mathrm{e}, i_{X}}\left(1+\cos \left(\theta_{i_{X}}\right)\right) \\
&+\sum_{j_{X}} g_{i_{X} j_{X}} s_{j_{X}}\left[\left(2 v_{\mathrm{syn}, \mathrm{j}_{\mathrm{X}}}-1\right)\left(1+\cos \left(\theta_{i_{X}}\right)-\sin \left(\theta_{i_{X}}\right)\right)\right] \\
&:=f_{i_{X}}\left(\theta_{i_{X}}, s_{X}\right)
\end{aligned}
$$

and

$$
\frac{d}{d t} s_{i_{X}}=-\frac{s_{i_{X}}}{\tau_{\text {decay }, i_{X}}}+e^{-\eta\left(1+\cos \left(\theta_{i_{X}}\right)\right)} \frac{1-s_{i_{X}}}{\tau_{\text {rise }}} .
$$

Time is measured in ms. In the equation $\tau_{\text {rise }}=0.1$ is the synaptic rise time and the parameter $\eta=5$ controls the activation of the synaptic conductances $s_{X}=\left(s_{i_{X}}\right)_{i=1}^{N_{X}}$. Thus $s_{i_{X}}$ rises quickly toward 1 at $\theta_{i_{X}} \approx \pi$ and decays exponentially with time constant $\tau_{\text {decay }, i_{X}}$. For excitatory synapses $\tau_{\text {decay }, i}=2$ and $v_{\text {syn }, i}=6.5$, for inhibition $\tau_{\text {decay }, i}=10$ and $v_{\mathrm{syn}, i}=-0.25$.

If not stated otherwise the constant input currents $i_{\mathrm{e}, i_{X}}$ for the excitatory neurons are $i_{\mathrm{e}, X}^{\mathrm{ex}}=0.05$ and for the inhibitory neurons $i_{\mathrm{e}, X}^{\text {in }}=0.002$. The synaptic conductances from excitatory to excitatory neurons are $g_{i_{X} j_{X}}=g^{\text {ex,ex }} / N_{X}^{\text {ex }}$ with $g^{\text {ex,ex }}=0.0$, from the excitatory to inhibitory neurons $g_{i_{X} j_{X}}=g^{\text {ex,in }} / N_{X}^{\text {ex }}$ with $g^{\text {ex,in }}=0.05$, from inhibitory to excitatory neurons $g_{i_{X} j_{X}}=g^{\text {in,ex }} / N_{X}^{\text {in }}$ with $g^{\text {ex,in }}=0.2$ and from the inhibitory to inhibitory neurons $g_{i_{X} j_{X}}=g^{\text {in,in }} / N_{X}^{\text {in }}$ with $g^{\text {in,in }}=0.0$ All parameter values are justified in detail from physiological considerations in [31].

The dynamics for a cluster evolving according to (17.1.1a) is illustrated in fig. 17.1. The collective oscillation in the gamma frequency range $(\approx 30-60 \mathrm{~Hz})$ arise due to the interplay of excitation and inhibition: The excitatory neurons spike and force the inhibitory neurons to also generate an action potential via the strong excitatory inhibitory projections. Their spiking in turn inhibits the excitatory neurons and keeps them from firing for a time window controlled by the inhibitory synaptic decay time constant $\tau_{\text {decay }}$. After inhibition has decayed the excitatory neurons can fire again and the cycle repeats. The period of this oscillation thus strongly depends on $\tau_{\mathrm{d}}$ and only weakly on $g^{\text {in,ex }}$ and $i_{\mathrm{e}}^{\mathrm{ex}}[31]$.

\subsubsection{Hierarchical Networks of Pyramidal Inter-Neuron Gamma Clusters}

To study information flow between the oscillatory PING clusters we introduce synaptic connections between the excitatory neurons of different clusters. The full system evolves according to the equations

$$
\begin{gathered}
\frac{d}{d t} \theta_{i_{X}}=f_{i_{X}}\left(\theta_{i_{X}}, s_{X}\right)+\sum_{Y} \sum_{j_{Y}} g_{i_{X} j_{Y}} s_{j_{Y}}\left(\left(2 v_{\mathrm{syn}, j_{Y}}-1\right)\left(1+\cos \left(\theta_{i_{X}}\right)-\sin \left(\theta_{i_{X}}\right)\right)\right) \\
+\sum_{\mathrm{a} \in\{\mathrm{ex}, i n\}} \xi_{i_{X}}^{\mathrm{a}} s_{\mathrm{e}, i_{X}}^{\mathrm{a}}\left(2 v_{\mathrm{syn}, \mathrm{e}}^{\mathrm{a}}-1\right)\left(1+\cos \left(\theta_{i_{X}}\right)-\sin \left(\theta_{i_{X}}\right)\right)
\end{gathered}
$$

together with (17.1.1b) for the synaptic conductances. Here, if $i_{X}$ and $j_{Y}$ label excitatory neurons in cluster $X$ and $Y$ respectively, there is a synaptic conductance $g_{i_{X} j_{Y}}=g_{i_{X} j_{Y}}^{\text {ex } e x} / N_{X}$ 
(a)

(b)
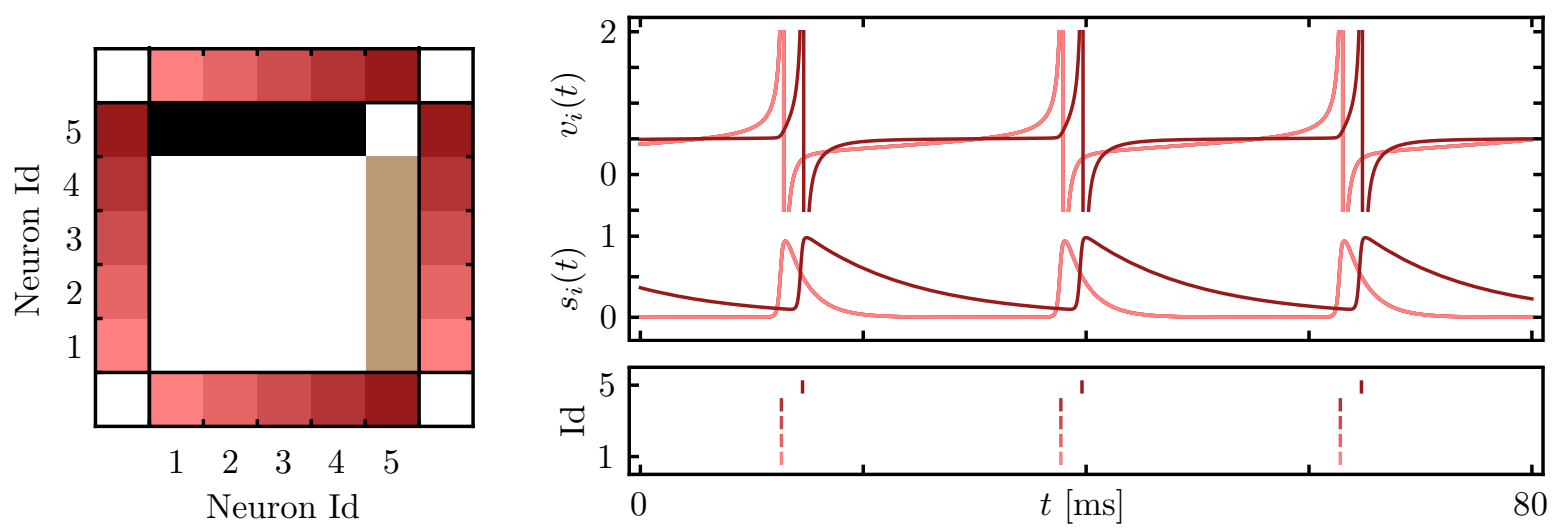

Figure 17.1. Pyramidal inter neuron gamma (PING) oscillation for a single cluster. (a) network connectivity matrix representing the maximal synaptic conductances $g_{i j}$ between neuron $i$ and $j$ in a network of $N^{\text {ex }}=4$ excitatory and $N^{\text {in }}=1$ inhibitory theta neurons eq. (17.1.1) with standard parameter values as described in sec. 17.1.1. Neurons $i=1, \ldots, 4$ are excitatory and project (black) onto the inhibitory neuron $i=5$ that projects back (brown ) onto the excitatory neurons. . (b) dynamics of the cluster showing PING oscillations. Time evolution of membrane potentials $v_{i}$ (top row), the synaptic conductances $s_{i}$ (middle row) and spikes (raster plot, bottom row). The gamma oscillation is generated by the excitatory neurons that spike and force the inhibitory neurons to also generate a spike. This in turn inhibits the excitatory neurons and keeps them from firing for a time window controlled by the inhibitory synaptic decay time constant $\tau_{\text {decay }}$. After inhibition has decayed the excitatory neurons can fire again and the cycle repeats.

from $j_{Y}$ to $i_{X}$. For all other combinations of long range connections between excitatory and inhibitory neurons we set $g_{i_{X} j_{Y}}=0$.

To account for synaptic input from external neurons we added excitatory and inhibitory synapses to the neurons with maximal conductances $\xi_{i_{X}}^{a}$, a $\in\{$ ex, in $\}$, reversal potentials $v_{\mathrm{syn}, \mathrm{e}}^{\mathrm{ex}}=6.5$ and $v_{\mathrm{syn}, \mathrm{e}}^{\mathrm{in}}=-0.25$ and synaptic conductances $s_{\mathrm{e}, i_{X}}^{\mathrm{a}}$ that evolve according to

$$
\frac{d}{d t} s_{\mathrm{e}, i_{X}}^{\mathrm{a}}=-\frac{s_{\mathrm{e}, i_{X}}^{\mathrm{a}}}{\tau_{\mathrm{d}, \mathrm{e}}^{\mathrm{a}}}+\sum_{k} \delta\left(t-t_{i_{X}, k}^{a}\right)
$$

where the spike times $t_{i_{X}, k}^{\mathrm{a}}$ are drawn from independent Poisson processes with rate $\lambda_{i_{X}}^{\mathrm{a}}=$ $\lambda^{\mathrm{a}}$ and the synaptic decay time constants are as before $\tau_{\mathrm{d}, \mathrm{e}}^{\mathrm{ex}}=2$ and $\tau_{\mathrm{d}, \mathrm{e}}^{\mathrm{in}}=10$.

If not stated otherwise we use $g_{i_{X} j_{Y}}^{\mathrm{ex}, \mathrm{ex}}=0.005$ for the long range connections and input noise $\xi_{i_{X}}^{\mathrm{a}}=0.001$ with rates $\lambda_{i_{X}}^{\mathrm{a}}=0.3$ corresponding to $300 \mathrm{~Hz}$ as time is measured in ms. ${ }^{1}$

\subsection{Local Control of Non-Local Phase Information Flow in Spiking Networks}

In this section we employ the fact that each individual cluster undergoes a stable collective oscillation which we describe by a collective phase and study local control mechanisms for information flow between these phase signals.

\footnotetext{
${ }^{1}$ Numerical integration of these networks was performed with our network simulation software [209, 207] using a discrete time second order Runge-Kutta integration.
} 
Similar to the analysis in section 16.3 we first perform a phase reduction of the cluster dynamics to a collective phase and phase response curve semi-analytically and use the result to predict the shape of the delayed mutual information curve. We find good agreement of this result with the numerical simulations and in analogy to section 16.3 we use it to study local control mechanisms for non-local phase information flow.

\subsubsection{Phase Reduction Analysis}

As illustrated in fig. 17.1 a single PING cluster without external noise sources exhibits dynamics on a stable limit cycle in form of a gamma oscillation. Thus in analogy to section 16.3 we may perform a collective phase reduction of a whole cluster $X$ and describe it by its collective phase $\Phi_{X} \in S^{1}$ and phase response curve $Z_{X}$. Denote the stable limit cycle solution of (17.1.1) parametrized by a collective phase $\Phi_{X}$ by $\left(\theta_{i_{X}}\left(\Phi_{X}\right), s_{i_{X}}^{\circlearrowleft}\left(\Phi_{X}\right)\right)_{i_{X}=1}^{N_{X}}$ which can only be calculated numerically. To obtain the collective phase response $Z_{X}\left(\Phi_{X}\right)$ we have to solve the adjoint equation (3.4.7). Again, this has to be done numerically. As the equation is a linear, non-autonomous differential equation with boundary conditions we used the chasing method [277] to find its solution ${ }^{2}$. Coupling of the the phase reduced clusters then results in the system

$$
\frac{d}{d t} \Phi_{X}=\Omega_{X}+Z_{X}\left(\Phi_{X}\right) \cdot\left[\sum_{Y} G_{X Y}\left(\Phi_{X}, \Phi_{Y}\right)+G_{\xi}^{\mathrm{ex}}\left(\Phi_{X}, s_{\mathrm{e}}^{\mathrm{ex}}\right)+G_{\xi}^{\mathrm{in}}\left(\Phi_{X}, s_{\mathrm{e}}^{\mathrm{in}}\right)\right]
$$

where $\Omega_{X}$ is the collective cluster rotation frequency,

$$
\left(G_{X Y}\left(\Phi_{X}, \Phi_{Y}\right)\right)_{i_{X}}=\sum_{Y} \sum_{j_{Y}} g_{i_{X} j_{Y}} h_{j_{Y}}\left(\theta_{i_{X}}^{\circlearrowleft}\left(\Phi_{X}\right), s_{j_{Y}}^{\circlearrowleft}\left(\Phi_{Y}\right)\right)
$$

with

$$
h_{j_{Y}}(\theta, s)=s\left(\left(2 v_{\mathrm{syn}, j_{Y}}-1\right)(1+\cos (\theta)-\sin (\theta))\right)
$$

and for $\mathrm{a} \in\{$ in, ex $\}$

$$
\left(G_{\xi}^{\mathrm{a}}\left(\Phi_{X}, s_{\mathrm{e}}^{\mathrm{a}}\right)\right)_{i_{X}}=h_{\mathrm{e}}\left(\theta_{i_{X}}^{\circlearrowleft}\left(\Phi_{X}\right), s_{\mathrm{e}, i_{X}}^{\mathrm{a}}\right)
$$

The robustness of the PING frequency [31] for each cluster ensures that the $\Omega_{X}$ differ only slightly due to possible heterogeneities in input currents and local network structure between the clusters.

For the deterministic system, $\xi_{i}^{\mathrm{a}}=0$, and under the assumption of weak inter cluster couplings we can then perform an averaging step according to eq. (3.4.13) to obtain

$$
\frac{d}{d t} \Phi_{X}=\Omega_{X}+\sum_{Y} \Gamma_{X Y}\left(\Phi_{X}-\Phi_{Y}\right)
$$

with coupling function

$$
\Gamma_{X Y}\left(\Phi_{X}-\Phi_{Y}\right)=\frac{1}{2 \pi} \int_{0}^{2 \pi} Z_{X}(\Phi) \cdot G_{X Y}\left(\Phi, \Phi_{Y}-\Phi_{X}+\Phi\right) d \Phi
$$

Assuming a phase locked solution between the clusters the phase differences $\Delta \Phi_{X Y}$ can be obtained via eq. (16.3.8).

\footnotetext{
${ }^{2}$ Calculations where done with our dynamical system package [206] for Mathematica which supports the detection of limit cycle solutions and the calculation of phase response curves.
} 


\subsubsection{Phase Locking of Coupled PING Clusters and Phase Information Flow}

Identical PING clusters that are symmetrically coupled in a hierarchical network that evolves according to eq. (17.1.2) synchronize their oscillations. This can be understood using the semi-analytical phase reduction of the previous section. For a network of two clusters $A$ and $B$ (fig. 17.2a) the result of this analysis is shown in fig. 17.2c. Note that in the collective phase response vector $Z_{X}$ the coordinates for the excitatory neurons differ form the inhibitory ones and obtain their maxima near the spike times of the neurons. The anti-symmetric part $\bar{\Gamma}(\Delta \Phi)=\Gamma_{A B}(\Delta \Phi)-\Gamma_{A B}(-\Delta \Phi)$ of the cluster coupling functions $\Gamma_{A B}$ has a zero crossing with negative slope at zero phase lags $\Delta \Phi_{A B}=0$ confirming the existence of stable synchronous state.

Introducing a difference in the input currents to the excitatory neurons in the clusters, $i_{\mathrm{e}, \mathrm{A}}^{\mathrm{ex}}<i_{\mathrm{e}, B}^{\mathrm{ex}}$, leads to a difference $\Delta \Omega_{A B}=\Omega_{A}-\Omega_{B}<0$ in the rotation frequencies of the uncoupled clusters. Via eq. (16.3.8) the zero crossings of $\bar{\Gamma}(\Delta \Phi)+\Delta \Omega_{A B}$ with negative slope then predict stable non-zero phase locking $\Delta \Phi_{A B}<0$ in good agreement with the numerically observed spiking pattern in fig. $17.2 \mathrm{~b}$ (cf. also inset in c).

We also calculated the dMI between the cluster phase signals $\Phi_{X}(t)$ estimated by average phases of the cluster's neurons, i.e. by

$$
\hat{\Phi}_{X}(t)=\arg \sum_{k_{X}} e^{i \theta_{k_{X}}(t)}
$$

The result for the above example is shown in fig. 17.2d. We find that the delayed mutual information $\mathrm{MI}_{A, B}(d)$ shows systematic fluctuations in the delay time $d$ within time windows equal to the collective oscillation period of the system. The reason for these oscillations in the dMI is the underlying interaction dynamics between the clusters: The clusters are coupled via synapses between the excitatory neurons that have a synaptic decay time constant $\tau_{\mathrm{d}}^{\text {ex }}$ that is small compared to the period of the PING cycle (cf. fig. (17.1)b). Further, the excitatory neurons within a single cluster generate their spikes only within a small time window with a length in the order of $\tau_{\mathrm{d}}^{\mathrm{ex}}$. Thus the clusters effectively interact only in a small fraction of the PING cycle, namely at times when the excitatory neurons generate their spikes. Only at these times information about the phase of the cluster can be transmitted to the others. Thus at time delays $d$ corresponding to these interaction times the $\mathrm{dMI} \mathrm{MI}_{X, Y}(d)$ increases, while in the interaction free periods the independent noise sources to the clusters causes a decrease in $\mathrm{MI}_{X, Y}(d)$.

Besides these fast oscillations the dMI curve also shows variations on larger time scales of the delay time $d$. This is highlighted in fig. $17.2 \mathrm{~d}$ by plotting the moving average of the dMI $\left\langle\mathrm{MI}_{X, Y}(d)\right\rangle$ using the fast oscillation period for the time window over which the mean is taken. In symmetric networks with synchronized clusters these dMI curves are symmetric about $d=0$, indicating that there is no direction in the phase information flow between the clusters. On the contrary, for systems with non-zero phase locking, these curves are asymmetric, as shown for the example in fig. $17.2 \mathrm{~d}$, indicating a preferred information flow directionality. We explain these findings in the next section by relating them to the results for phase oscillators obtained in section 16.3 and giving an approximation for the averaged dMI curves. 
(a)

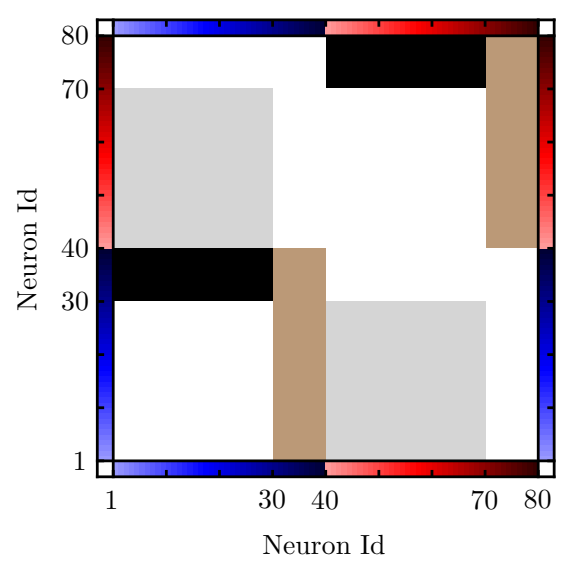

(c)

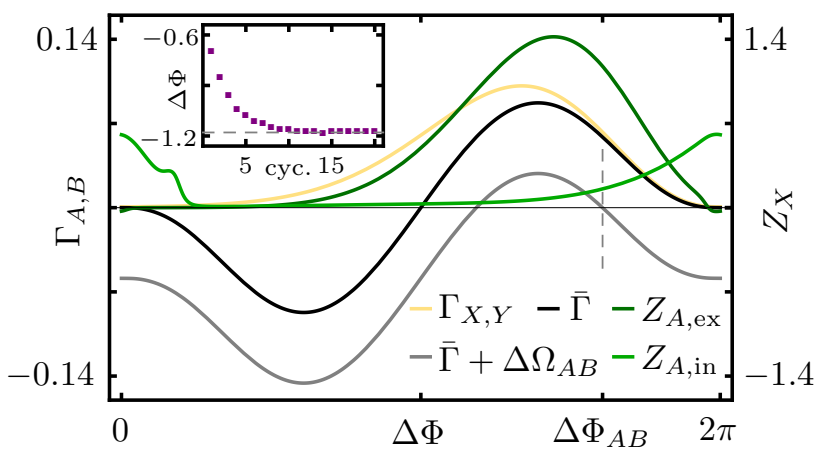

(b)

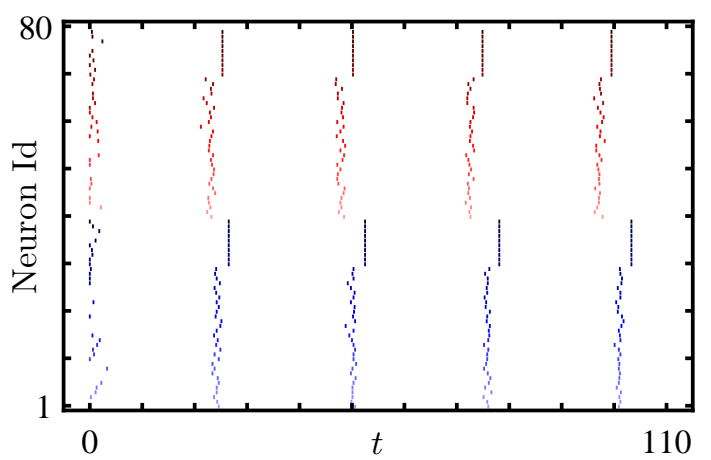

(d)

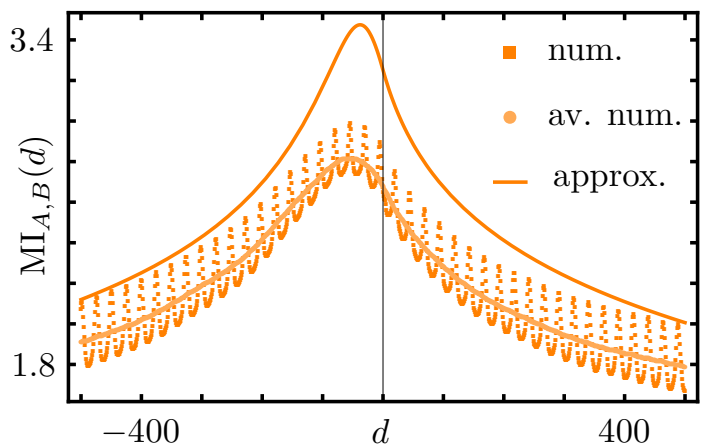

Figure 17.2. Phase information flow in hierarchical networks of PING clusters. (a) coupling matrix $g_{i j}$ representing the synaptic conductances between the neurons (black - local excitation, brown local inhibition, gray long range excitation) in a network of $N=80$ theta neurons with $M=2$ clusters $A$ (blue) and $B$ (red) evolving according to eq. (17.1.2). All parameters as described in section 17.1 except for $g^{\text {in,in }}=0.2$ and input currents $i_{\mathrm{e}, A}^{\text {ex }}=0.09$ to cluster $A$ and $i_{\mathrm{e}, B}^{\mathrm{ex}}=0.1$ to cluster $B$. The input current difference leads to a difference $\Delta \Omega_{A B}$ in the rotation frequencies of the uncoupled two clusters. (b) raster plot showing the spikes when starting the system from random initial conditions. After a transient phase locking between the two clusters is observed. (c) cluster phase response $Z_{X}, X \in(A, B)$ for the inhibitory (green) and excitatory neurons (darker green), averaged coupling function $\Gamma_{A B}(\Delta \Phi)=\Gamma_{B A}(\Delta \Phi)$ (yellow) and antisymmetric part $\bar{\Gamma}(\Delta \Phi)=\Gamma_{A B}(\Delta \Phi)-\Gamma_{B A}(-\Delta \Phi)+\Delta \Omega_{A B}$ (gray). The predicted phase shift $\Delta \Phi_{A B}$ (dashed line) is in good agreement with the shift of the spike timings between the clusters (inset). Note the strong asymmetry of the coupling function $\Gamma_{A B}(\Delta \Phi)$ around the synchronous state $\Delta \Phi=0$. (d) delayed mutual information [dMI] (squares) between the phase signals $\Phi_{X}(t)$ of the clusters of duration $t=10^{6}$, time step $d t=0.1$, and bin size $2500 / 2 \pi$ for the estimation of the probability distribution. Also averaged dMI (dots) over one oscillation cycle and the approximation obtained in sec. 17.2.1, eq. (17.2.4) (solid line) are shown. See text for details.

\subsubsection{Approximation for the Delayed Mutual Information Curve}

In section 16.3.1.2 we already discussed the lack of an averaging theory for stochastic systems and referred to the discussion in section 18.3 where we propose steps towards the development of such a theory. In contrast to section 16.3.1.2, where the phase reduced system was already in the form of an averaged system, here it is not clear how to formally 
including the noise terms $G_{\xi}^{\mathrm{a}}$ in (17.2.1) into the averaged system (17.2.2).

However, we here argue, supported by numerical simulations, that for the systems above an approximation for the delayed mutual information flow between the collective phase signals of the clusters may be obtained by the following steps: First add white noise sources to the system (17.2.2) with variances estimated from the variances of the input signals when integrated over the mean oscillation period $T=\Omega / 2 \pi$ of the full system. Then use this system in corollary 16.3.1 with phase differences $\Delta \Phi_{X Y}$ derived form the average inter spike intervals between the clusters to obtain an expression for the delayed mutual information curve $\mathrm{MI}_{\mathrm{XY}}(\mathrm{d})$. Finally, rescale the delay time $d$ in this expression by $\tau_{\mathrm{d}}^{\mathrm{ex}} / T$, to obtain the approximation for the delayed mutual information as

$$
\hat{\mathrm{MI}} \mathrm{X}, Y_{(}(d)=\mathrm{MI}_{X, Y}\left(\frac{\tau_{\mathrm{d}}^{\mathrm{ex}}}{T} d\right)
$$

These steps are justified by the fact that clusters interact only shortly during the oscillation period as explained in the previous section. As the clusters exhibit phaselocked states near the synchronous state this interaction is concentrated on a small time interval of the full period. At these interaction periods the clusters are on average in a phase locking pattern given by the average inter spike intervals. This justifies our choice of $\Delta \Phi_{X Y}$. Further, in the rest of the oscillation cycle the interaction between the clusters are effectively turned off. In contrast, in the system used for the derivation of the delayed mutual information $\mathrm{MI}_{X Y}(d)$ the interaction is present permanently. To correct for this, we have to rescale the delay time $d$ by the fraction of the interaction time in the oscillation period which is in the order of $\tau_{\mathrm{d}}^{\mathrm{ex}} / T$. This justifies the last step in the approximation. Finally, during the interaction free time window the external noisy inputs are basically integrated by the neurons membrane potential justifying the estimation of the variances of the white noise processes by the variances of these integrated signals.

The resulting delayed mutual information curves obtained via this procedure are shown in figs. 17.5, 17.6 and 17.7 together with the numerically obtained curves which show good qualitative agreement of the shape of the dMI curve. In particular, the positions of the peaks in both curves match well. The semi-analytical obtained curves are typically slightly shifted in the vertical direction in comparison with the numerical results. We attribute this shift to the crude approximation of the noise levels, in particular taking into account that the theta neuron has a varying phase response curve and therefore does not simply integrate the input noise. Changing the input noise levels basically shifts the dMI curve in the vertical direction. This is supported by the fact that when we fitted the noise levels much better agreement between the numerical data and our approximation was obtained.

We conclude, that the underlying mechanism for the phase information flow in the spiking networks is similar to the one found in chapter 16 for coupled phase oscillator models. In particular, a directionality in the phase information flow is generated as revealed in section 16.2.4.

\subsubsection{Local Control of Non-Local Phase Information Flow}

Due to the analogy between the phase information flow in the coupled oscillator systems of chapter 16 and in networks of coupled PING clusters as derived in the previous section, we find the same mechanisms for the local control of non-local phase information flow as in section 16.3.2. We here discuss two examples.

First, reconsider the two cluster network in fig. 17.2, discussed in section 17.2.2. There we increased the local input currents to the excitatory neurons, $i_{\mathrm{e}, X}^{\mathrm{ex}}$, within one cluster 
(a)

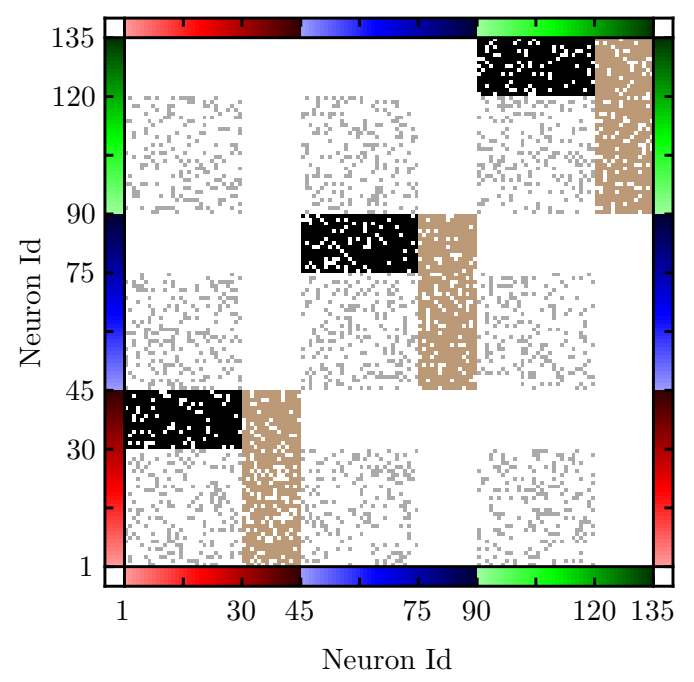

(c)

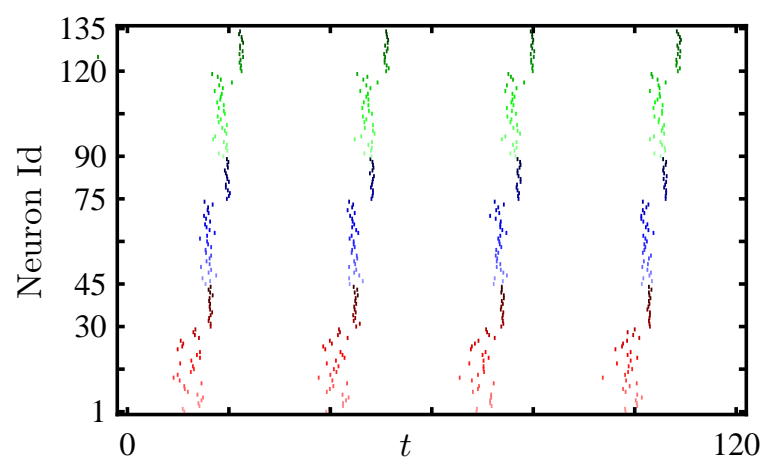

(e)

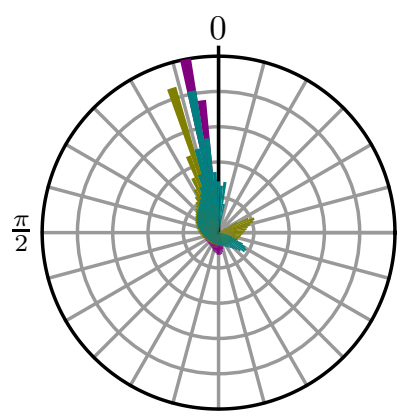

(f) (b)

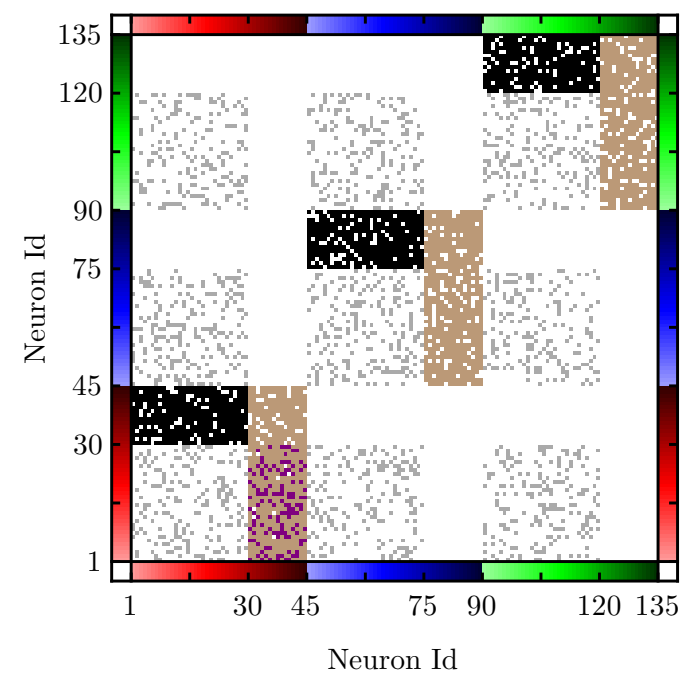

(d)

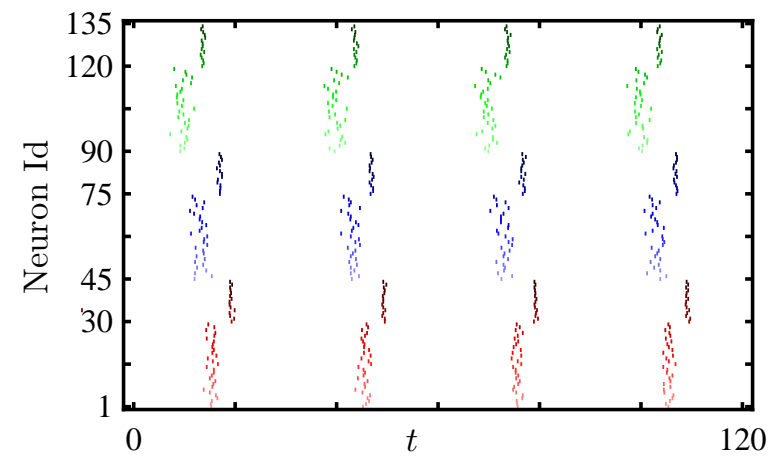

(g)
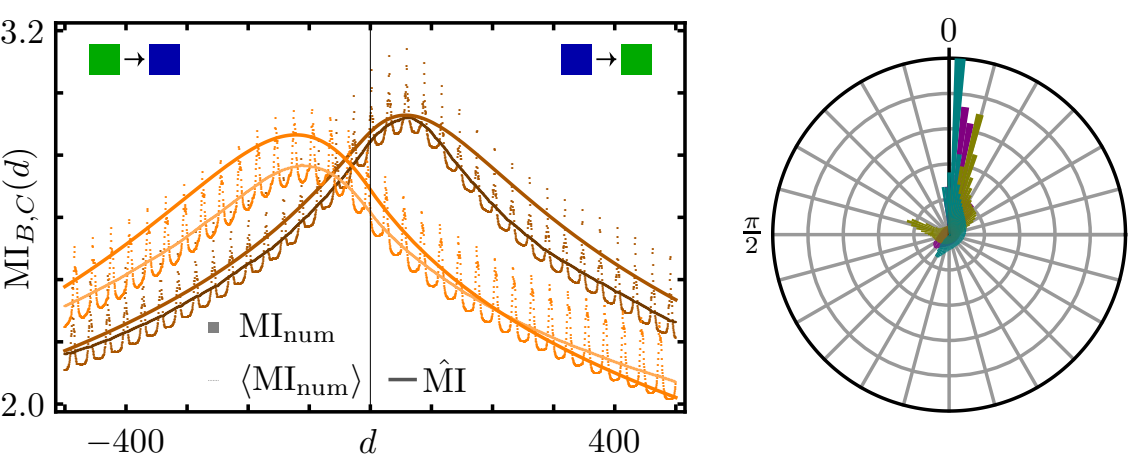

Figure 17.3. Remote control of non-local phase information flow in a hierarchical networks of PING clusters via local network structure. (a) coupling matrix representing synaptic conductances $g_{i j}$ (black excitation, brown inhibition) of $N=135$ neurons and three clusters $A$ (red), $B$ (blue) and $C$ (green) evolving according to eq. (17.1.2). Parameter as described in sec. 17.1. (b) as (a) with synaptic links flipped from off to on or vice versa at purple entries in cluster $A$. $(\mathbf{c}, \mathbf{d})$ raster plots showing phase locking patterns for the networks in (a) and (b). (e,g) polar histograms of the cluster phase differences $\Delta \Phi_{A B}$ (purple), $\Delta \Phi_{A C}$ (brown) and $\Delta \Phi_{B C}$ (cyan) for the networks in (a) and (b). (f) delayed mutual information between phase signals of clusters $B$ and $C$ in network (a) (orange) and (b) (dark orange). Squares: numerical values measured as in fig. 17.2; disks: moving average; lines: approximation (17.2.4). The change of the local network structure within cluster $A$ shifts the maximum of the dMI from negative to positive $d$ effectively reversing the information flow between clusters $B$ and $C$. 
(a)

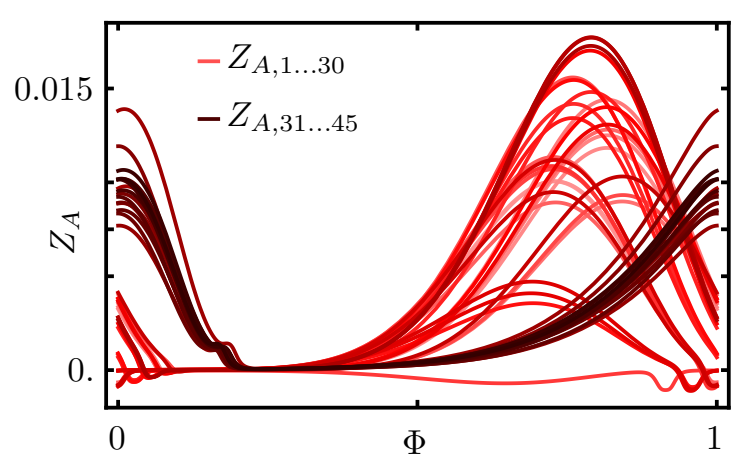

(b)

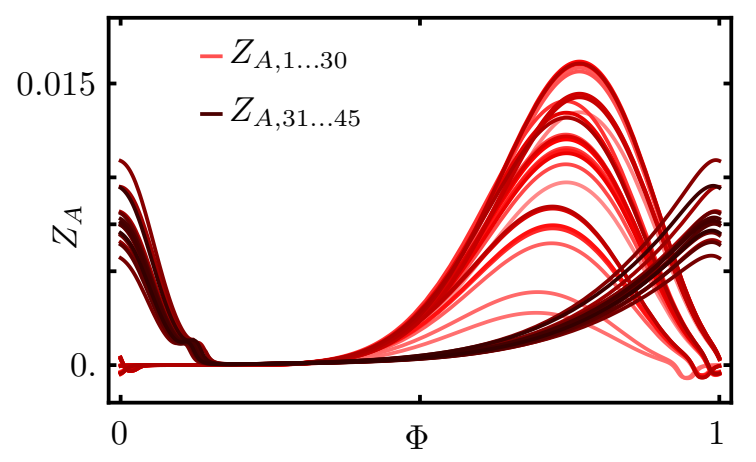

Figure 17.4. Remote control of phase information flow in a hierarchical networks of PING clusters via local network structure: phase response vectors. (a) phase response vector $Z_{A}$ of the cluster $A$ of the network in fig. 17.3a. (b) $Z_{A}$ for network in fig. 17.3b. The change in the phase response vector causes changes in the cluster interactions which in turn cause a change in the phase locking pattern (cf. fig. 17.3c,d). As a consequence also the phase information flow between the clusters is changed (cf. fig. 17.3f).

to obtain a phase locking between the clusters with non-zero phase lags $\Delta \Phi_{A B}$. This caused an asymmetry in the dMI curve indicating a directed phase information flow from the leading cluster to the lagging one. This mechanism is similar to the information flow tuning discussed in section 16.3.2.1.

An analog example to the remote control of phase information flow via the local network structure as observed in fig. 16.5 is shown for a clustered PING network in fig. 17.3. The mechanism is similar to the information flow plasticity discussed in section 16.3.2.2. In particular, changes in the local network structure induce changes in the collective phase response vector $Z_{X}$ as shown in fig. 17.4 which results in changes in the coupling functions $\Gamma_{X Y}$ between the clusters resulting in a change of the global phase locking pattern $\Delta \Phi_{X Y}$ as shown in fig. 17.3c,d,e,g. This in turn causes changes in the direction of the information flow even remotely between unchanged clusters as shown in fig. 17.3f.

We conclude that the local control mechanisms found in section 16.3.2 are also effective in clustered networks of spiking neurons undergoing collective oscillations.

\subsection{Information Flow In Spike Patterns}

In the previous section we studied information flow between the collective phase signals of the clusters. Theoretically, as the phases are continuous time signals, the information in these signals can be arbitrarily high and is only bounded by the resolution with which the signals are measured. In real systems the maximal information content is limited by the time scales of the underlying physiological processes an their potential noise sources. Further, in the oscillatory system under consideration, the phases evolve close to periodic dynamics and thus there is a limited potential for encoding large amounts of information within the phases itself. In this section we therefore investigate how this limitation in the phase information channel may be overcome by additional information channels that make use of the precise timing of the action potentials of the neurones within each clusters. We focus on the ordering of the spikes of the excitatory cells within a gamma cycle and show 
that there is an information flow in this spike permutation channel. We further find that its direction is parallel to the phase information flow and explain this result.

\subsubsection{Delayed Mutual Information in Permutations of Spike Times}

During one oscillation cycle of a single PING cluster the excitatory neurons fire once if the network heterogeneity is not to large [31]. Adding external Poisson inputs to the neurons the ordering of these neurons changes from cycle to cycle. Coupling different PING clusters together in a hierarchical network, a natural question that arises is how the spike pattern in one cluster influences the pattern of a second connected cluster? To quantify this influence we here propose a delayed mutual information measure based on the ordering of spikes in a single cluster.

Given the spike times $\left\{t_{i_{X}}^{(c)}\right\}$ of the $N_{X}^{\mathrm{ex}}$ excitatory neurons of a cluster $X$ during a single gamma oscillation cycle $c$, we associate to it the permutation $\pi^{(c)} \in S_{N_{X}^{\text {ex }}}$ in the permutation group $S_{N_{X}^{\text {ex }}}$ of the indices $i_{X} \in\left\{1, \ldots, N_{X}^{\text {ex }}\right\}$ that yields the time ordering of the spikes, i.e.

$$
t_{\pi^{(c)}(1)}^{(c)}<t_{\pi^{(c)}(2)}^{(c)}<\cdots<t_{\pi^{(c)}\left(N_{X}^{\text {ex }}\right)}^{(c)}
$$

For simultaneous spikes we chose a random ordering of these spikes and for cycles in which not all excitatory neurons spike or spike twice we associate a symbol \#. The result is a mapping from the spike times $\left\{t_{i_{X}}^{(c)}\right\}$ in each oscillation cycle $c$ to the discrete set $\mathcal{P}=S_{N_{X}^{\text {ex }}} \cup\{\#\}$ with $N_{X}^{\text {ex }} !+1$ elements.

A cluster of neurons undergoing PING oscillations in this way produces a sequence of symbols $\pi^{(c)} \in \mathcal{P}$. In a network of coupled PING clusters each cluster $X$ produces such a sequence $\left\{\pi_{X}^{(c)}\right\}_{c}$. For these sequences we denote by $p\left(\pi_{X}^{(c)}, \pi_{Y}^{(c+d)}\right)$ the joint probability distribution of the symbols in a sequence $\left\{\pi_{X}^{(c)}\right\}$ with the symbols of the time $d$ delayed sequence $\left\{\pi_{Y}^{(c+d)}\right\}$ and define the delayed mutual information between these sequences as

$$
\mathrm{MI}_{X, Y}^{\pi}(d)=\sum_{\pi_{X}^{(c)}, \pi_{Y}^{(c+d)} \in \mathcal{P}} p\left(\pi_{X}^{(c)}, \pi_{Y}^{(c+d)}\right) \log \left(\frac{p\left(\pi_{X}^{(c)}, \pi_{Y}^{(c+d)}\right)}{p\left(\pi_{X}^{(c)}\right) p\left(\pi_{Y}^{(c)}\right)}\right)
$$

As the amount of information transmitted through a channel is limited by the entropy [249] of the source we normalize the $\mathrm{MI}_{X, Y}^{\pi}(d)$ by the entropy

$$
H_{X}=\sum_{\pi_{X}^{(c)} \in \mathcal{P}} p\left(\pi_{X}^{(c)}\right) \log \left(p\left(\pi_{X}^{(c)}\right)\right)
$$

for $d>0$, by $H_{Y}$ for $d<0$ and by $\min \left(H_{X}, H_{Y}\right)$ for $d=0$.

This is illustrated in fig. 17.5 for a network of two clusters. Figure $17.5 \mathrm{~b}$ shows the association of the spike patterns to a permutation $\pi_{X}^{(c)}$ for each group and fig. $17.5 \mathrm{c}$ the delayed mutual information $\mathrm{MI}_{X, Y}^{\pi}(d)$ for the times series of these permutations. In this example the dMI peaks at $d=0$ due to a change in the underlying probability distributions (fig. 17.5d insets).

The definition (17.3.1) is based on spike time orderings and therefore only captures parts of the precise spike timing pattern. However, it has the advantage of a much smaller space of possible symbols than encountered for the full spike timing patterns and does 
(a)

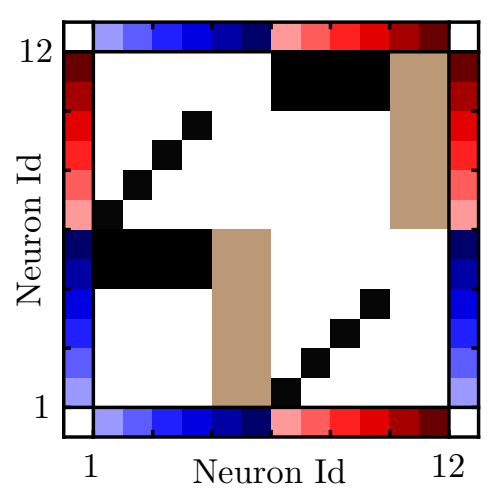

(c)

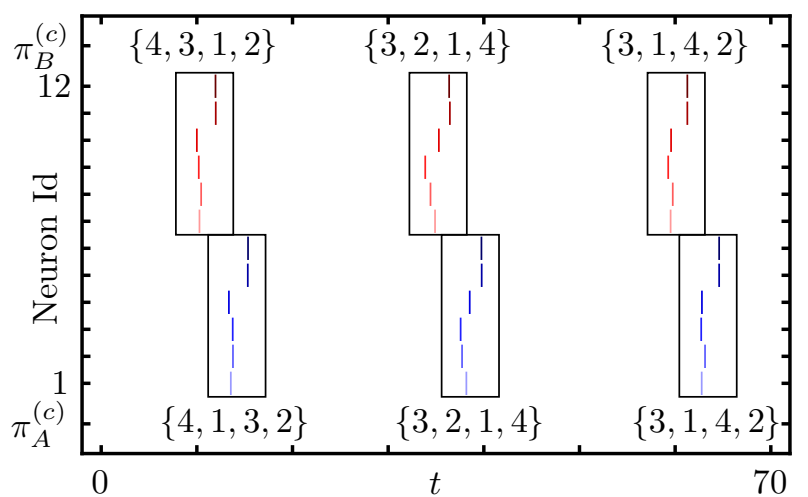

(b)

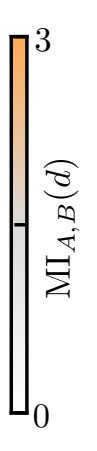

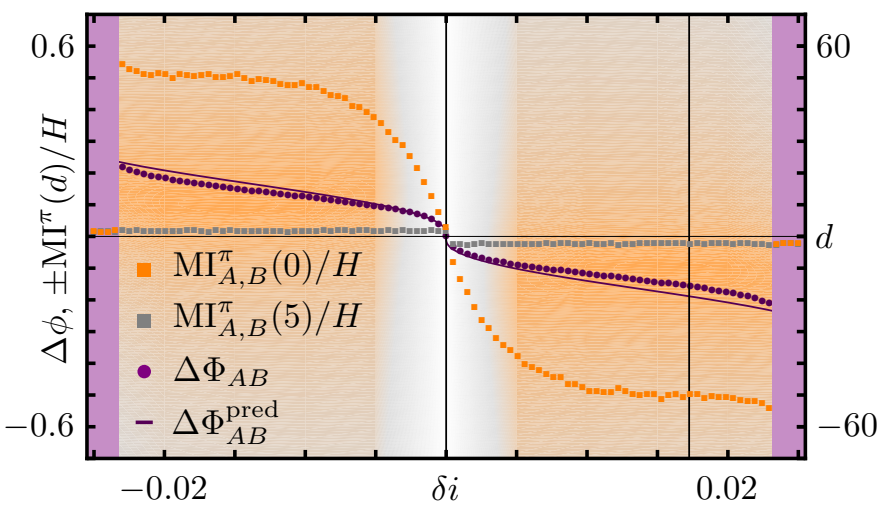

(d)

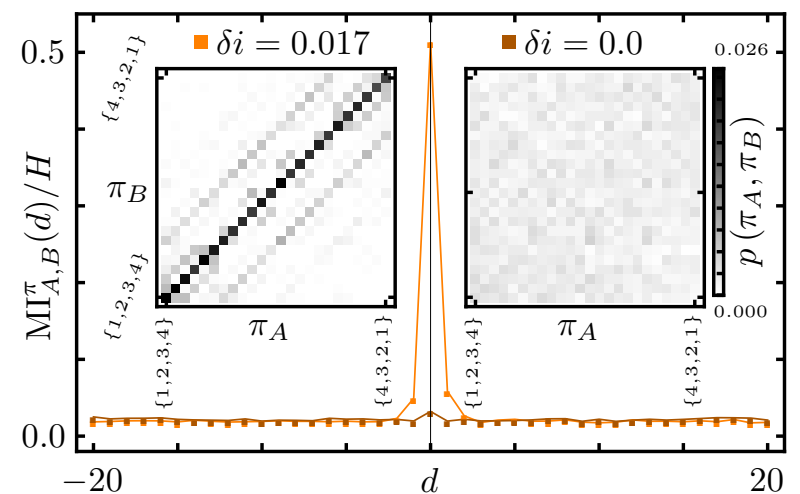

Figure 17.5. Control of information flow in spike time permutations in hierarchical networks of PING clusters. (a) connectivity matrix representing synaptic conductances $g_{i j}$ in a network of $N=12$ neurons with two PING cluster $A$ (blue) and $B$ (red). Parameter as described in sec. 17.1 with input currents $i_{\mathrm{e}, A}^{\mathrm{ex}}=0.1-\delta i$ and $i_{\mathrm{e}, B}^{\mathrm{ex}}=0.1+\delta i$ to the excitatory neurons which depend on $\delta i$ to control the phase difference between the clusters and smaller external spike rates $\lambda_{\mathrm{e}}^{\mathrm{a}}=100 \mathrm{~Hz}$ but larger amplitudes $\xi_{i}^{\mathrm{a}}=0.002$ to increase the variability in the spike patterns. (b) raster plot for $\delta i=0.017$ showing spike patterns and associated permutation code sequences $\pi_{X}^{(c)}$. (c) delayed mutual information $\mathrm{MI}_{A, B}^{\pi}(d)$ between spike permutation sequences of the clusters for $\delta i=0.017$ (orange) and $\delta i=0.0$ (darker orange). Insets show joint probability distributions $p\left(\pi_{A}^{(c)}, \pi_{B}^{,(d)}\right)$ of the permutations sequences for $\delta i=0.017$ (left) and $\delta i=0.0$ (right) and $d=0$. (d) phase differences $\Delta \Phi_{A B}$ between clusters as a function of $\delta i$ determined numerically (purple dots) via the average spike time differences and semi-analytically (purple line) using (16.3.8) for the averaged system (17.2.2). Orange squares show $\mathrm{MI}_{A, B}^{\pi}(d) / H$ multiplied by $\operatorname{sign}\left(\Delta \Phi_{A B}\right)$ to highlight the directionality of the information flow (see text). Orange shading shows the dMI $\mathrm{MI}_{A, B}(d)$ between the cluster phases (traces of duration $t=10^{7}$, step size $d t=0.01$ and bin size $2000 / 2 \pi$ for each $\delta i$ ). Note that when the phase locking breaks down (purple shaded area) the $\mathrm{MI}_{A, B}^{\pi}(0) / H$ decays to the base level $\mathrm{MI}_{A, B}^{\pi}(5) / H$ (gray). Note further the large slope in $\mathrm{MI}_{A, B}^{\pi}(0) / H$ near the synchronous state $\delta i=0$.

not rely on a certain binning that may introduce further complications [356]. Further, the fact that neurons are highly sensitive to inputs just before spike generation and only weakly sensitive afterwards (cf. the phase response curves in fig. 3.11) shows, that the spike ordering already captures some important aspects of neuronal communication.

We study the delayed mutual information $\mathrm{MI}_{X, Y}^{\pi}(d)$ and its control in the next section. 


\subsubsection{Local Control of Non-Local Information Flow in Permutations of Spike Times}

In this section we numerically study the delayed mutual information $\mathrm{MI}_{X, Y}^{\pi}(d)$ in networks of PING clusters.

We generally find that the $\mathrm{MI}_{X, Y}^{\pi}(d)$ is close to zero for non-zero cycle delays (cf. fig. 17.5c). This is also true for the auto mutual information $\mathrm{MI}_{X, X}^{\pi}(d)$. Thus across PING cycles there is almost no information flow between the spike patterns (i.e. there are no 'echo' effects due to a strong coherence across cycles as pointed out in fig. 15.1).

On the other hand, at $d=0$, there is a peak with a value $\mathrm{MI}_{X, Y}^{\pi}(0)$ that strongly depends on the precise phase relations between the clusters (fig. 17.5d). For synchronized clusters the peak value $\mathrm{MI}_{X, Y}^{\pi}(0)$ is close to zero but shows a large increase for small deviations in the phase difference controlled by the input currents to the clusters. Similar to the argument for the directionally of the delayed information between the phase signals, we assume that signals can only flow forward in time and therefore a large value of $\mathrm{MI}_{X, Y}^{\pi}(0)$ for a positive phase difference $\Delta \Phi_{X Y}=\Phi_{X}-\Phi_{Y}>0$ indicates a information flow from cluster $X$ to $Y$ and vice versa. We conclude that within a single oscillation cycle the information flow for the spike permutations is strongly controlled by the phase difference with a directionally from the leading cluster to the lagging one and across cycles the information in the spike ordering is lost.

We further find that the information flow in the cluster phase signals has always the same direction as the information flow in the spike patterns (cf. fig. 17.5c). The reason for this is the asymmetry of the cluster's phase response around the spike times of the excitatory neurons (cf. fig. 17.4 and 17.6). Just before spiking the phase response is high and if spikes from a different cluster arrive at this moment in time the cluster is rotated strongly while it is only influenced weakly if the spikes arrive after spiking within the cluster as then the phase response is small. Thus the same local mechanisms that

(a)

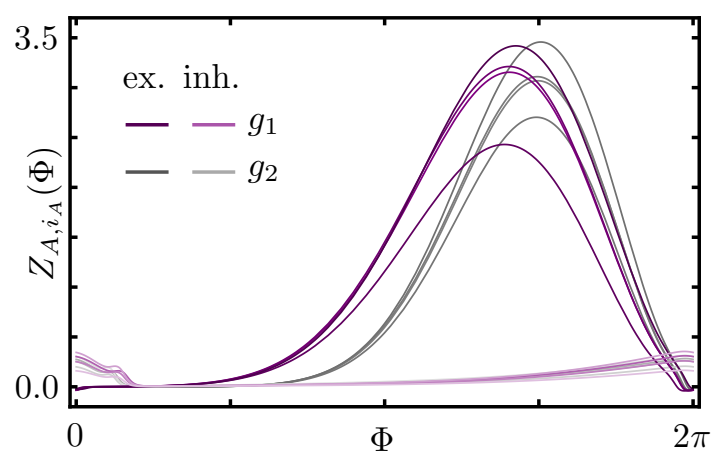

(b)

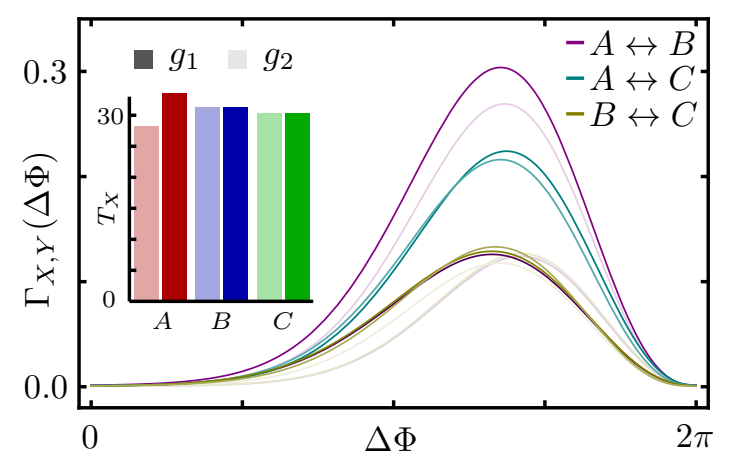

Figure 17.6. Control of non-local spike information flow in hierarchical PING networks via local network structure: phase response and average coupling functions. (a) phase response $Z_{A}$ for the network in fig. 17.7 change when the cluster's network structure is changed from the synaptic conductance $g_{1}$ (gray) to $g_{2}$ (purple). (b) averaged coupling functions $\Gamma_{A B}, \Gamma_{B A}$ (purple), $\Gamma_{A C}$, $\Gamma_{C A}$ (brown) $\Gamma_{B C}, \Gamma_{C B}$ (cyan) also change ( $g_{1}$ lighter colors, $g_{2}$ darker colors). Inset shows periods for the oscillations of the uncoupled clusters. Due to the synaptic changes in cluster $A$ also its oscillation period $T_{X}$ is slightly changed. Combined with the change in the coupling function this results in the different phase locking patterns in fig. $17.7 \mathrm{~b}, \mathrm{c}$ and in turn in the differences in information flow shown in fig. 17.7d,e. 
(a)

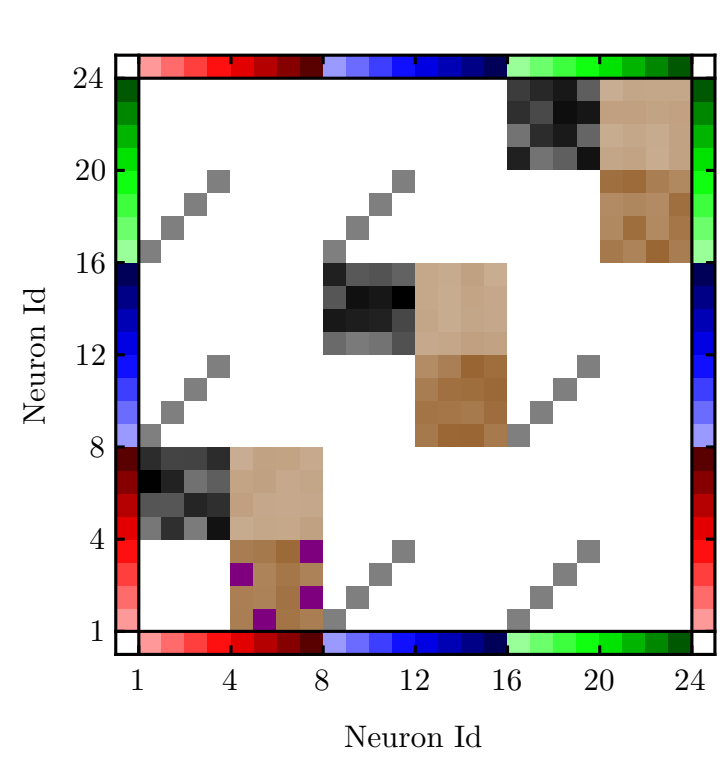

(d)

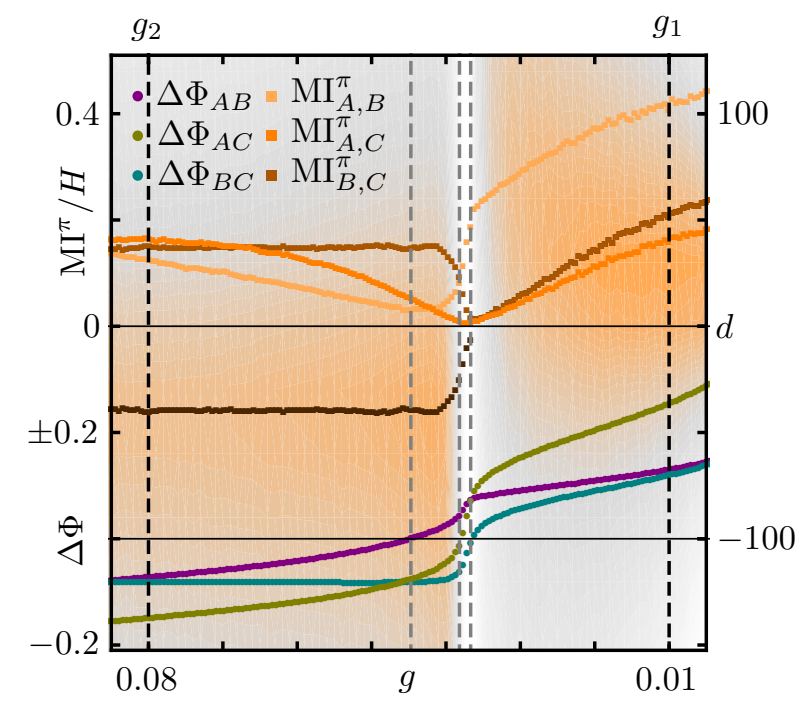

(b)

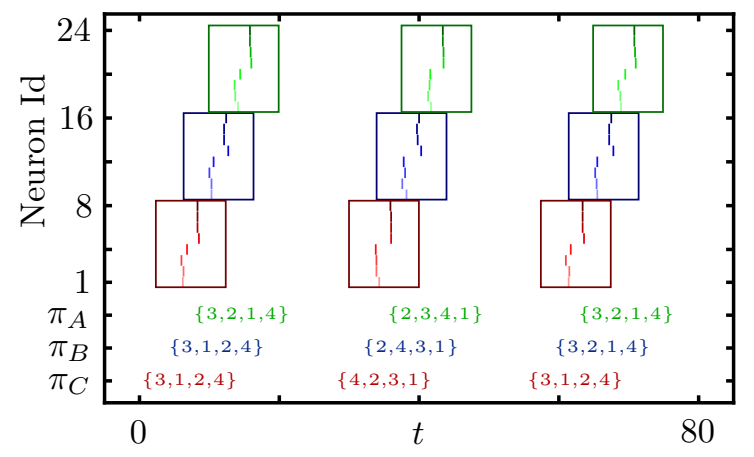

(c)

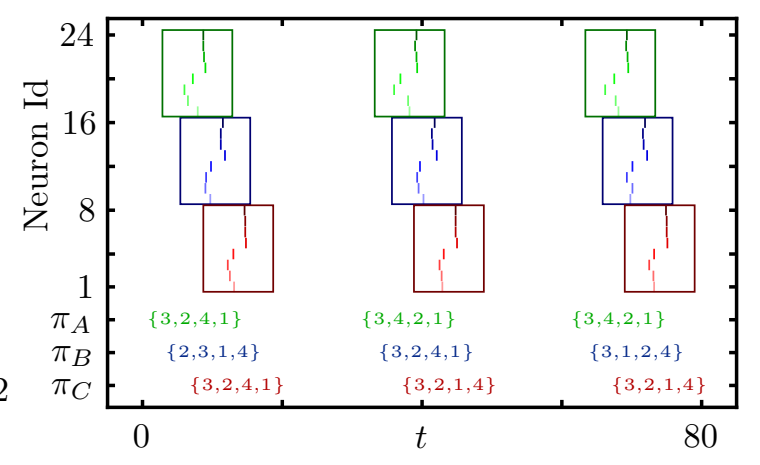

(e)

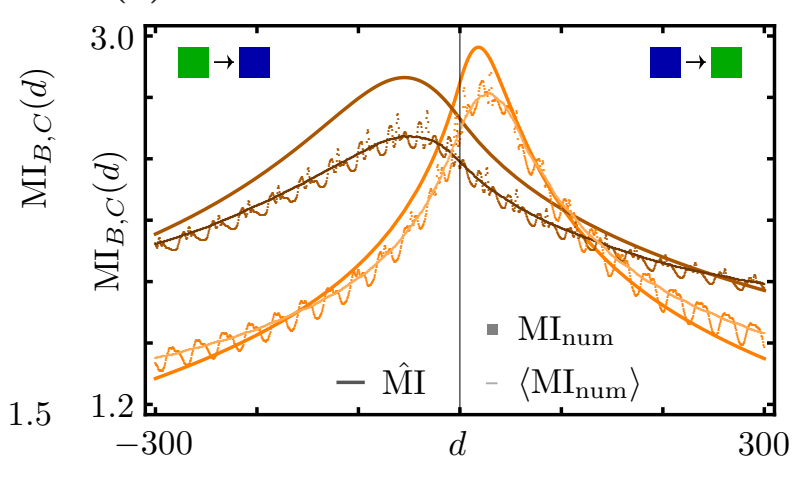

Figure 17.7. Local and remote control of non-local spike information flow in hierarchical PING networks via local network structure. (a) connectivity matrix representing synaptic conductances $g_{i j}$ in a network of $N=24$ neurons with three PING clusters $A$ (red), $B$ (blue) and $C$ (green) evolving according to (17.1.2) (parameter as described in sec. 17.1 and $i_{\mathrm{e}, A}^{\mathrm{ex}}=0.063, i_{\mathrm{e}, B}^{\mathrm{ex}}=0.062$, $\left.i_{\mathrm{e}, C}^{\mathrm{ex}}=0.065\right)$. Purple matrix entries indicate variable local inhibitory conductances with $g_{i j}=g$. (b,c) raster plots for $g=g_{1}=0.01$ (a) and $g=g_{2}=0.08$ (b) showing spike patterns and associated permutation code sequences $\pi_{X}^{(c)}$. (d) dots: phase differences $\Delta \Phi_{A B}$ (purple), $\Delta \Phi_{A C}$ (brown), $\Delta \Phi_{B C}$ (cyan) as a function of $g$ determined by the average spike time differences. squares: $\mathrm{MI}_{A, B}^{\pi}(d) / H$ (lighter orange), $\mathrm{MI}_{A, C}^{\pi}(d) / H$ (orange) and $\mathrm{MI}_{B, C}^{\pi}(d) / H$ (dark orange). orange shading: $\mathrm{MI}_{B, C}(d)$ for cluster phase signals (measured as in fig. 17.5). To highlight the parallel direction of the phase and spike information flows the points $\operatorname{sign}\left(\Delta \Phi_{B C}\right) \mathrm{MI}_{B, C}^{\pi}(d) / H$ where added (dark orange). (e) $\mathrm{MI}_{B, C}(d)$ as in fig. $17.2 \mathrm{~d}$ for $g=g_{1}$ (orange) and $g=g_{2}$ (dark orange). Note that the local changes within cluster $A$ caused a flip in the direction of information flow between the remote clusters $B$ and $C$. 
control the non-local phase information flow can be used to control the directionality of the information flow in the spike ordering.

In particular, changes of the local oscillation frequencies can revert the non-local information flow associated with the spike ordering as demonstrated in fig. 17.5. Figures 17.7 and 17.6 show that also modifications of the local network structure can control non-local information flow in spike patterns even remotely between unchanged clusters in analogy to the information flow plasticity observed in fig. 16.5 and 17.3.

We note that high mutual information in the spike patterns within the same cycle is favored by sparse, one-to-one and inhomogeneous long-range connections between the clusters. This is clear when considering fully homogeneous inter-cluster couplings. Then the effect of presynaptic spikes arriving from a distant cluster does not depend on the ordering of these spikes and therefore no information about the ordering can be transmitted.

Finally, we stress that the PING clusters show phase locking patterns close to the synchronous state consistent with experimental findings $[141,113]$. Interestingly, exactly in this region the peak value $\mathrm{MI}_{X, Y}^{\pi}(0)$ is most sensitive to variations in the phase locking pattern (cf. fig. 17.5b) and thus the control of information flow is most effective. Thus synchrony may provide the basis for a high ability of neuronal systems to control information flow.

We summarize and discuss our findings in the next chapter. 


\section{Chapter 18}

\section{Summary, Discussion and Outlook}

\subsection{Summary}

To summarize, in this part of the thesis we have studied local control mechanism for information flow in oscillatory neuronal networks. We showed how directionality in information flow can be measured via the delayed mutual information (dMI) curve. We then derived a general analytic expression for the dMI between two phase signals in networks of phase oscillators near a phase locked state (theorem 16.2.1). We explained how a direction in information flow arises due to an imbalance in the influence of the oscillators onto the collective rotation of the whole system combined with an asymmetric relaxation towards the phase locked state. We then focused on hierarchical networks consisting of strongly connected groups (clusters) of phase oscillators with weaker inter-cluster connections. For such networks using a collective phase reduction of the cluster's dynamics led to an equation of the same functional form as in theorem 16.2.1. We deduced the delayed mutual information between the collective cluster phases as a function of the underlying cluster properties (corollary 16.3.1).

Using this theoretical frame work we found that local changes within a single cluster can control the non-local information transfer between the clusters, even remotely between two other distant and physically unchanged groups. We identified three mechanism for the local control of non-local information flow:

- Local Oscillation Frequencies - Information Flow Tuning

Uniform frequency changes of the oscillators within a single cluster cause changes of its phase relations to the others and as a consequence the information flow to and from this cluster is changed. In this way the cluster is capable of tuning to information sources from or make its information available to other clusters.

\section{- Local Network Structure - Information Flow Plasticity}

Local changes within the network structure of a single cluster alter the collective response of the cluster phases and the effective inter-cluster couplings resulting in concert in a change of the information flow, even remotely between two distant physically unchanged groups.

\section{- Local Multistable Phase-Locking - Information Flow Combinatorics}

Switching between different stable intra-cluster phase locking patterns induce changes of the collective response of the cluster and of the effective inter-cluster couplings which in turn control the information flow between the clusters. Combinations of 
different phase locking patterns of the individual clusters then endow the full network with different information flow patterns.

We then developed a biophysical more detailed hierarchical network model of interconnected clusters of excitatory and inhibitory spiking neurons where each individual cluster is based on the model in [31] and undergoes collective gamma oscillations. We observed that in this model the clusters arrange in phase-locking patterns close to a synchronous state. Using a collective phase reduction for these clusters we semi-analytically derived an expression for the average phase dynamics and used this result to derive an approximation for the delayed mutual information that is based on theorem 16.2.1. We showed that the same local control mechanisms found for the phase oscillator model are found in these networks of spiking neurons.

We finally extended our study from information flow in phase signals to information transmission in spike patterns. More specifically, we introduced a delayed mutual information measure based on the ordering of the neurons' spike times in each cluster and in each oscillation cycle. We showed that the information flow in these channels for spike timing has the same directionality as the flow in the phase signals and therefore can also be controlled by the same mechanisms found for the phase oscillator model.

\subsection{Discussion and Outlook}

We already gave a detailed discussion of our theoretical results for the phase oscillator network in sections 16.2.5 and 16.4. The same limitations on the noise levels and the assumption of a stable phase locked state for the deterministic system apply to the results in the spiking neuron model. If the clusters do not phase lock but still show intrinsic oscillations, insight from our work can still be gained by conditioning the signals on a certain phase relation and studying the information flow in time windows around this phase pattern. This might lead to insights into dynamical information flow patterns with time-varying direction and efficiency (cf. [325] for an interesting experimental study). We propose a detailed study of this aspect for future work.

Strong heterogeneous input noise to a cluster typically destroys the PING oscillation $[30,31]$. Hence also the phase locking for this cluster breaks down and the delayed mutual information between this and other clusters becomes zero. In this way incoherent stimuli can effectively turn off communication. On the other hand, coherent input stimuli to a cluster only affect its relative phase to others and as a consequence the information flow (cf. fig. 17.2). This suggests mechanistic connections between neuronal communication though coherence [111] and binding by synchrony due to coherent stimuli [398, 342] - an interesting direction for future work in both theory and experiment.

We studied coupled groups of oscillatory neurons without interaction delays. Fast coherent gamma oscillations are mostly observed between neuronal groups within one cortical area $[139,114]$. Conduction delays on these spatial scales are around $1-4 \mathrm{~ms}$ [127] and thus an order of magnitude smaller than the period of the gamma cycles of $10-30$ ms which may justify our approach of neglecting the delays. Longer conduction delays of $5-8 \mathrm{~ms}$ are observed between cortical areas with a separation of $1 \mathrm{~cm}$ and larger [127]. This will not only affect the phase-locking pattern but also the information flow structure. In particular, due to the strong variation of the phase response near the firing of the excitatory neurons (cf. fig. 17.6), and especially due to the highly reduced sensitivity after spiking, the transmission of spikes will be effective only if the phase differences are 
large enough to compensate for the interaction delay. A starting point for a detailed future analytical investigation of this point could be the Kuramoto model with delay [416].

Here we studied small hierarchical networks of spiking neurons that facilitated the identification of mechanisms for locally controlling non-local information flows. Interestingly, experimentally such small networks of interlaced groups of only a few dozen neurons have been identified in neocortex [294]. However it is not clear whether they undergo coherent oscillations. Results in [56] point is such a direction. That study reports strong correlations in the activity between dispersed neurons if they show a similar preference to phase coupling patterns of surrounding local field potentials. However, future experiments are needed to clarify this point.

In larger networks the gamma oscillations in each cluster become more robust against noise and heterogeneities in the synaptic couplings [31]. As the local control of non-local information flow is made more effective by structural heterogeneity (cf. sections 16.3.2.2 and 17.3.2), larger networks should even better support these mechanisms. Additionally, in larger clusters, a division of the neurons into two specialized subgroups - one for the control of information flow and the other for the information transmission itself - may provide even more efficient ways for local control mechanisms.

We observed that changes in certain single links are more effective in controlling the information flow than in others. An interesting application of our theory thus would be to identify "high sensitivity" links and underlying network structures that are optimal for an efficient control of non-local information flow. This might not only lead to the identification of potential neuronal systems that strongly support a local control of non-local information flow but may be also useful in other systems, such as oscillating regulatory gene networks [383, 354].

Sparse local heterogeneous connections within a cluster (as long as they do not destroy the PING oscillations [31]), increase the sensitivity of the collective phase response to local structural changes. This is due to the fact that in homogeneous networks the excitatory neurons synchronize if external sources are turned off. Changes of a few internal links then only lead to small deviations in the spike times of the individual neurons in an isolated cluster. Thus also the change of the cluster's collective phase response, which determines the modification of the phase locking and ultimately the information flow, stays small. On the other hand in more sparsely connected clusters the spike times of the neurons are temporally more dispersed. Here changing a small number of links has a larger effect on the internal dynamics and thus on the cluster's phase response. Hence also the phase locking and the information flow is changed more strongly (cf. fig. 17.3 and 17.4).

Moreover, sparse heterogeneous long-range connections between the clusters improve the information flow in spike ordering patterns: For homogeneous inter-cluster connections each neuron receives all spikes generated in a different cluster and for identical synaptic strengths their effect on the neuron is independent on the time ordering of these spikes. Hence no information about the spike ordering is transmitted between the clusters. In contrast, if each neuron projects only to a single neuron in a connected cluster (cf. fig. 17.3) an earlier spike in the presynaptic neurons only affect the spike time of the single postsynaptic neuron and thus information about the ordering is transmitted. Hence we conclude that heterogeneity and sparseness in local as well as long-range connectivity support local control mechanism of non-local information flow.

We observed phase locking of the PING clusters near synchrony. Interestingly, exactly in this region the peak value in the delayed mutual information in both the phase signals as well as the spike ordering is highly sensitive to variations in the phase locking pattern 
(cf. fig. 17.5b). One reason for this is the strong variation and asymmetry of the cluster's response to inputs to excitatory neurons near their spike times (cf. fig. 17.4 and 17.6). In particular this excitability strongly decreases from large values to zero shortly before spiking and stays close to zero afterwards. This implies that the control of information flow is most effective near the synchronous state and thus synchronization of oscillatory neuronal clusters may provide the basis for a high capability of neuronal systems to locally control their information flow.

Figures 17.4 and 17.6 also show that a cluster's sensitivity to inputs to the inhibitory neurons is maximized at the spike times of the excitatory neurons. This suggests that long-range excitatory-to-inhibitory connections, also found experimentally [121], may substantially influence the phase synchronization properties of the clusters and thereby provide another way to enhance the effectiveness of local control mechanisms for non-local information flow. Preliminary numerical studies show that this is indeed the case.

We considered a model for gamma oscillations in which the firing frequency of the neuron is similar to the frequency of the oscillation cycle. Such oscillations are observed for example in the hippocampus [53]. In contrast, for collective oscillations in the cortex, sparse neuronal activity with frequencies much lower than the rhythm frequency are observed [246, 113]. It would therefore be interesting to see what local control mechanism exists when several of such groups (e.g modeled as in [45]) are coupled together in a hierarchical network. One promising step in this direction is [23] where multi-stable phase locked states between the collective oscillations of the groups are found. Switching between these phase-locked states then induces changes in direction of effective connectivity between the clusters. But how does the local network structure of each cluster affect the non-local information flow? Are there different local dynamical states of such networks that could lead to information flow combinatorics (section 16.3.2.3)? Future work will answer these questions.

We studied interactions between groups of neurons undergoing oscillations with a single frequency. Experimentally, also several simultaneous oscillations within a group are observed $[292,55]$ that even show phase-locking between the different rhythms, e.g. between high frequency gamma and low frequency theta [292]. Therefore studying how multiple oscillations among different interacting groups could lead to more complex e.g. parallel, information flow patterns and whether and what kind of local control mechanisms exist in such systems could lead to further insights into neuronal communication.

We have shown that changes in synaptic links within the local network structure of a group can control the information flow between groups. Synaptic connections are changed via synaptic plasticity $[196,2]$. Thus our study reveals a mechanistic link between plastic changes of local synapses and the control of non-local information flows. Given that effective connectivities systematically change during learning $[22,46]$ this may provide a first step to attack the fascinating problem of how information flows in the brain can be learned. But what are corresponding learning rules? Are the local learning rules particularly suited to learn information transfer between groups? Or do "population synaptic plasticity" rules exist by which a group of neurons can learn to receive relevant information form other groups or to influence them most? Exciting questions for future work. 


\subsection{Theoretical Challenges}

Our analysis revealed further theoretically challenges. We already discussed extension to amplitude oscillators in section 16.4 and outlined a possible route to an analytical treatment in symmetrically coupled networks of oscillators close to a Hopf bifurcation [59].

We considered networks of phase-oscillator models with coupling functions that depend on the phase difference of the connected units. Such models typically arise via averaging (cf. sec. 3.4) from networks of phase-reduced weakly coupled oscillators with an explicit dependence on their rise function. In part IV, chapter 13 of this thesis we have shown that these phase response curves can be changed dynamically via synapses. It would therefore be interesting to see how this mechanism could be employed, to not only control synchronization properties in networks (chapter 13) but also to provide a way for a local control of non-local information flow. We therefore propose to study networks of oscillators with tunable phase response curves [329] and to examine how their shape controls the information flow and which shapes are optimal for local control mechanisms.

Our analysis of information flow in hierarchical networks of clusters of phase oscillators was facilitated by the fact that the system of the phase-reduced clusters had the same functional form as the system we started with. In particular we where able to apply theorem 16.2.1 originally derived for phase-oscillator systems in averaged form. In section 17.2.2 the phase-reduced system of the spiking network no longer had this property. We where able to deduce an approximate expression for the delayed mutual information in this system assuming that the neurons interacted only for a short time within the oscillation cycle. For general systems, these special properties cannot be expected and a natural step to proceed with the analysis would be to average these systems. Interestingly, to our knowledge, there is no general analog of such a theory for stochastic oscillator systems. Given that the phase reduction of stochastic oscillators agrees with the one for deterministic oscillators only in certain cases $[417,363]$ we expect also differences in a stochastic averaging theory from the deterministic one. In [186] averaging for two stochastic oscillators is undertaken by reducing the system to two interacting probability distributions for the phases neglecting their actual correlations. The result is a deterministic system of coupled phase oscillators. This is in contrast to our analysis where we obtained a stochastic system and observed strong correlations between the phase variables. Therefore a more rigorous approach is needed for the formulation of a stochastic averaging theory. The connection between normal form theory and averaging for deterministic systems [276, 327] transferred to stochastic normal form theory [12] might be a promising ansatz. 


\section{Part VI}

\section{Conclusion and Outlook}




\section{Chapter 19}

\section{Conclusion and Outlook}

In this thesis on mathematical, computational and experimental neurophysics we studied how properties of single units affect the collective dynamics of neuronal networks. For that we took three different approaches.

First, we proposed and motivated a general model of pulse-coupled neuronal threshold units with partial reset. We showed that this partial reset, being an intrinsic response property of the local units, acts as a desynchronization mechanism for the collective network dynamics. We further found that it induces a transition from networks of unstable attractors to heteroclinic switching dynamics and gave a complete mathematical treatment of the underlying novel type of global bifurcation.

Second, we showed theoretically for biologically more detailed conductance-based neuron models as well as experimentally for real neurons that their neuronal excitability type is influenced by their morphology and can be dynamically controlled via a change in leak conductance or by shunting inhibition. We theoretically studied consequences of this transition for the control of synchronization and dynamic grouping in the collective network dynamics.

Third, we moved from the single neuron level to clustered hierarchical networks of groups of neurons undergoing neuronal oscillations and revealed that the local network properties within each group are capable of controlling the non-local information flow and the collective dynamics of the entire network.

In part III of this thesis (chapters 4 - 8) we introduced a partial reset mechanism into the general class of pulsed-coupled threshold units that keeps the collective network dynamics analytically tractable and at the same time describes additional, physically or biologically relevant dynamical features of local units. We motivated the partial reset neurobiologically by relating it to the spike time responses of conductance based neuron models that receive supra-threshold excitation. In homogeneous networks of neuronal oscillators with fast interactions we numerically described and mathematically proved that the partial reset causes an extensive sequence of desynchronizing bifurcations of cluster states. This transition is robust against structural perturbations in the coupling strength and variations of the local sub-threshold dynamics [210, 213].

Previous studies of pulse-coupled threshold units have not particularly focused on the implications of the reset onto the collective dynamics. They either considered full conservation of supra-threshold inputs $[378,389,154,37,39,174]$ or, the opposite extreme, a fully dissipative reset $[269,124,33,333,370,369]$. Here we closed this gap and showed that in fact the reset mechanism strongly influences the synchronization processes $[210$, $213,211]$. 
The desynchronization due to the partial reset, i.e. due to local processing of suprathreshold input, differs strongly from that induced by previously known mechanisms based on, e.g. heterogeneity, noise, or delayed feedback [389, 388, 251, 214, 301, 71]. Possibly, this desynchronization mechanism may also be helpful in modified form to prevent pathological synchronization in neuronal activity such as in Parkinson tremor or in epileptic seizures $[362,160]$. Our analysis may thus serve as a starting point for interesting future theoretical, neurobiological and medical research.

To study the impact of the partial reset on the collective dynamics we mainly considered homogeneous networks. Besides regular asynchronous splay states, we also found complex irregular spike patterns in homogeneous globally coupled networks when two opposing dynamical effects were in competition: The desynchronization of neurons in larger clusters caused by the partial reset and the synchronization of smaller clusters due to the sub-threshold dynamics. Complex, irregular and even chaotic dynamics are also observed in systems with inhomogeneous network structure [190, 272]. This raises the question to what extend complex spike patterns, also observed in real neuronal networks [187], are caused by the dynamical properties of the individual neurons or have their origin in the complex network structure. Our analytically tractable partial reset model might therefore serve as a candidate for future investigations of the role of single unit dynamics in the generation of distributed spike patterns in complex networks.

In chapters 7 and 8 we studied the impact of a partial reset in pulse-coupled systems with interaction delays. We found that in homogeneous networks the partial reset induces a robust transition from networks of unstable attractors $[371,372,16]$ to heteroclinic switching. For the strict mathematical study of this phenomenon we developed general mathematical methods applicable to pulse-coupled units to determine the regions in state space that give rise to a predefined sequence of pulse generation and reception events. We used this nonlinear non-local analysis to identify the state space structure underlying the transition in a small network and showed that it is the result of an interplay between a local instability and a contraction of state space volume that depends on the degree of the partial reset [211].

The developed mathematical methods may find future applications in the study of pulse-coupled systems: Estimation of the domain size of predefined event sequences could be used to predict their probability of occurrence as well as their stability against noise. Applied inversely, they can be used to find properties of networks that give rise to a certain state space structure (cf. also [263, 262]). For example to design predefined heteroclinic structures or even hybrid networks of stable and unstable attractors. This could become very useful for finding optimized networks that compute via switching dynamics [17, $282,15,214,290]$, as proposed for example in neuronal coding in the olfactory system $[238,237]$. A further potentially interesting application could be to facilitate a better understanding of the flux-tube state space structure found in the balanced state of pulsecoupled leaky integrate-and-fire neurons [271]. Here small perturbations decay while larger perturbations lead to an exponential separation of trajectories akin to chaotic dynamics. A detailed analysis of the state space structure using the developed event-sequence-based domain analysis for different trajectories might help to unravel the underlying state space structure and mechanisms.

The transition from networks of unstable attractors to heteroclinic switching establishes the first known bifurcation involving non-trivial unstable attractors. We provided a complete rigorous mathematical treatment of this novel type of global bifurcation in hybrid dynamical systems. This can be considered as a first step in the field of bifurcation 
theory for unstable attractors. In figs. 8.1 and 8.2 we sketched further possible bifurcation scenarios involving unstable attractors. A good understanding of these bifurcations may facilitate the analytical investigation of system exhibiting heteroclinic switching by studying their limiting counterpart with unstable attractors. This novel field offers many promising opportunities for future research, not only mathematically interesting but also with applications to complex physical systems and neuronal networks performing computational tasks.

In part IV (chapters 9 - 14) of this thesis we theoretically and experimentally studied the impact of leak currents onto the excitability type of neurons. We found that an increase in leak conductance induces a switch from type I to type II neuronal excitability. In a large number of conductance-based neuron models, including those presented in refs. $[273,319,320,168,400,99,61,109,315,103,406]$, we showed numerically and analytically, using a combination of multiple bifurcation theory and normal form theory, that this transition consists of three intermediate steps that are organized by a degenerate Bogdanov-Takens-cusp bifurcation [87]. We further showed that this implies a region of a bistable coexistence of resting and periodic firing and also a transition from integration to resonance in the sub-threshold dynamics. We explained the transition in neuronal excitability by conjugating a class of neuronal models to a Liénard type equation which gives an interpretation of the dynamics in terms of accelerating and dampening forces of a non-linear oscillator.

The results explain a switch from type I to type II neuronal excitability which we found by varying the neuronal morphological from linearly extended to stellar like dendritic shapes in compartmental model neurons. Depending on the active dendritic ion channel properties these changes induce a effective change in leak conductance at the soma and cause the transition. We confirmed these theoretical results by analyzing data from experiments [25] where the dendritic tree can be pinched temporarily.

Using dynamic patch clamp recordings we further confirmed the main predictions of our general theory in hippocampal CA3 pyramidal neurons and neurons of the dorsal lateral lemniscus. We found leak-induced neuronal excitability switches, a region of bistability and a transition at peri-threshold resonance. Application of an inhibitory synaptic transmitter likewise induced this transition. We therefore concluded that in principle neuronal excitability can be controlled dynamically via shunting inhibition.

We then studied several consequences of these findings for the control of collective network dynamics. Well-established results show that type I neuronal excitability leads to desynchronization while type II to synchronization in weakly coupled neurons [316, $100,184,177]$. We therefore concluded that regulating neuronal excitability via changes in leak conductance provides an effective mechanism to dynamically control the collective synchronization properties of neurons. We demonstrated this in a small circuit and further showed in larger networks of neurons with different neuronal excitability that the subgroup of type II neurons synchronizes while the type I neurons remain asynchronous. We concluded, that dynamic switching of neuronal excitability may thus serve as a mechanism for dynamic grouping of neurons with a wide range of applications in neuronal coding $[162,398,138,111]$. We further showed that in the region of bistability complex dynamics emerge for stronger couplings in homogeneous networks: For smaller leak conductances, stronger pulses arising via synchronization induce silencing of neurons, while for larger leak values the desynchronization causes irregular dynamics due to switching between resting and spiking of the individual neurons.

For future research it will be interesting to also investigate the impact of a change in 
leak conductance on bursting neurons. For these neurons, several underlying bifurcations to bursting activity are known $[315,189]$. But what are possible transitions between different bursting types or even to tonic spiking and how are they organized by higher degenerate bifurcations? More generally, one can ask what higher degenerate bifurcations organize neuronal excitability. This would not only provide simple normal forms for the mathematical analysis and efficient simulation of neuronal networks but may also give further insights into the existence of possible complex dynamical properties in single neurons. For example, we proved for an abstract neuron model $[108,278]$ that it is the unfolding of a degenerate Bogdanov-Takens bifurcation of codimension four [202 $]^{1}$ which predicts parameter regions with coexisting stable periodic orbits.

Our theoretical and experimental results provide a promising starting point for future research on the role of dynamic neuronal excitability switches in brain function. In a first step it would be interesting to investiage how dynamic neuronal excitability transitions influence the collective network dynamics of excitatory and inhibitory neurons and how these dynamics then act back in controlling the individual excitability types of the neurons. This may give useful insights into collective control mechanisms for neuronal synchronization and neuronal dynamics in general. As the neuronal excitability type controls the phase response of a neuron, a better understanding of such complex dynamics may be gained by studying simpler pulse-coupled phase oscillator models (as used in part III of this thesis) with a variable phase response that depends on the inhibitory inputs.

Furthermore, the neuronal excitability type influences the selective response of neurons to different stimulus features [151, 256,318, 359, 360,361], the encoding of information $[329,166]$ and the recall and storage of memory [239]. It would therefore be interesting to see how dynamic transitions in neuronal excitability provide an efficient or even optimal mechanism for the control of these functions.

In part IV of this thesis (chapters 15 - 18) we moved from single neurons to clustered hierarchical networks of interacting groups of neurons and studied how local network properties can control non-local information flows between the different clusters. To measure information flow we focused on the delayed mutual information curve between two time series. Motivated by recent experimental and theoretical findings that coherent phase locked collective oscillations in different groups influence the communication between these groups $[412,411,111,48,56,23,408]$, we first concentrated on general networks of coupled phase oscillators in a phase-locked state and analytically derived the delayed mutual information between any pair of oscillators. We applied this general result to hierarchical network structures to predict the information flow between groups of neurons as a function of the underlying network properties within a local group. We identified three different mechanism for the local control of non-local information flow: Tuning of information flows from and to other groups via changes in the intrinsic collective group's rotation frequency (information flow tuning), via changes in the local network structure (information flow plasticity) and changes in a single group's dynamical state of collective oscillation (information flow combinatorics). All mechanisms are based on an interaction between a change in the phase response properties of the local group and a change in the non-local phase-locking patterns between the groups.

We extended our work to biologically more realistic networks of coupled groups of spiking neurons each undergoing collective pyramidal interneuron gamma oscillations [31].

\footnotetext{
${ }^{1}$ We note that such higher degenerate bifurcations are only partly understood mathematically and subject to current research [148, 276, 230, 86, 87]
} 
We found the same three mechanisms for local control of non-local information flow. To further account for information flow in the precise spike pattern [212] we proposed a parameter-free mutual information measure based on the ordering of the spikes. Using this measure, we showed that the information flow in the spike patterns between the groups is weak across different oscillation cycles while for patterns generated in the same cycle it is high and controlled by the phase-locking patterns between the clusters. Moreover, its direction is then in parallel to the phase information flow. We discussed our results in the context of experimental observations on coherent neuronal oscillations and gave predictions on neuronal network properties that support local control mechanisms of nonlocal information flow.

Intriguingly, our theoretical analysis in hierarchical networks was facilitated by the fact that the functional form of the equations for the phase coupled oscillator system stayed invariant under the collective stochastic phase reduction of the individual clusters. For general systems this property cannot be expected and a natural step in the analysis would be to perform a further averaging step. Interestingly, to our knowledge, no general averaging theory for stochastic systems has been developed so far that results in a nondeterministic system. In analogy to the differences encountered between the deterministic and stochastic phase reduction $[417,363]$ we also expect changes for a stochastic averaging theory. The connection between averaging and normal form theory in deterministic systems $[276,327]$ transferred to results on stochastic normal form theory [12] might serve as a promising ansatz for the development of such a general theory.

Future research concerning the control of information flows in neuronal networks should include to consider more complex systems and dynamics. In particular, in networks of coupled amplitude oscillators the control of information flow via the phases could be separated from the actual flow in the amplitudes. Our results on the control of information flow in spike patterns is a step into this direction. We considered neuronal networks in which each group showed collective gamma oscillations generated by interactions between excitatory pyramidal and inhibitory interneurons. Here the collective oscillation frequency is similar to the individual neuron firing frequencies akin to oscillations found in the hippocampus [53]. Collective oscillations in the cortex often show sparse neuronal activity with individual neuronal frequencies that are much lower than the frequency of the collective rhythm [246, 113]. Also other mechanisms for the generation of oscillations such as purely inhibitory interactions and mixtures between both exist [21, 374]. Furthermore, several simultaneous oscillations within a group are observed that even show phase-locking between the different rhythms $[292,55]$. It therefore would be interesting to identify control mechanisms for the information flow in these types of neuronal dynamics. More generally, one could ask what control mechanisms for information flow exists in systems with irregular spiking activity and how time windows during which efficient directed information transfer takes place can be created (cf. [325] for an experimental study in this direction).

Our theoretical analysis in part IV showed that the information flow is strongly influenced by the phase response of the individual neurons. The phase response of a neuron is determined by its excitability type $[100,316,188]$. In part IV of this thesis we demonstrated that the neuronal excitability type can be controlled dynamically via slow inhibitory synapses. Therefore, an interesting task for future research is to combine these findings and to study how slow shunting inhibition provides an efficient local control mechanism for non-local information flows in hierarchical networks. Moreover, this study might be facilitated by using pulse-coupled phase oscillator models studied in part III of 
this thesis.

Interestingly, we found that the control of information flow is most effective near the synchronous state. Thus synchronization of oscillatory neuronal clusters may provide the basis for a high capability of neuronal systems to locally control their information flow. We further found that changes in the collective oscillation frequency of a group of neurons control its information flow from and to other clusters. Coherent stimulation of a group increases its frequency and therefore can be used to control the information flow. On the other hand we found that incoherent stimuli break the phase locking and shut off the nonlocal information flow. This suggests mechanistic connections between the two theories of binding by synchrony due to coherent stimulation [398, 342] and neuronal communication through coherence [111] - an interesting area for future research.

Our study shows that changes of synaptic links within a neuronal cluster can control the information flow between groups. As synaptic connections are changed via synaptic plasticity our results reveal a mechanistic link between plastic changes of local synapses and the control of non-local information flows. Given that effective connectivities systematically change during learning $[22,46]$ this may provide a first step to attack the fascinating problem of how information flows in the brain can be learned. In particular, what are synaptic plasticity rules among single neurons that are suited to learn information transfer between groups? Do population-plasticity-rules exist that act in order to change the synaptic structures of a whole group collectively in order to learn information flows? What kind of mechanisms facilitate learning of appropriate dynamic routing of information in general? Exciting questions for future research.

Understanding brain function is an extremely hard task. A possible systematic way towards a better insight into neuronal dynamics and complex systems in general would be to find a chain or a network of interlinked models ranging from highly detailed and close to experimental observations to highly abstract and mathematically tractable models from which general dynamical mechanisms can be uncovered, disentangled and explained. In this thesis we have studied aspects of how local properties influence the collective dynamics. In principle this direction of study can also be reversed and one may ask how collective dynamics impinge on single unit properties. Our hope is that we have contributed a small part to the intricate and puzzling challenge of understanding the fascinating collective phenomena in the brain and in complex systems in general. Adopting a constructivist point of view, these emergent phenomena include science and the language in which this thesis was formulated. 
Immer ängstlicher im Niederschreiben. Es ist begreiflich. Jedes Wort, gewendet in der Hand der Geister - dieser Schwung der Hand ist ihre charakteristische Bewegung - wird zum Spieß, gekehrt gegen den Sprecher. Eine Bemerkung wie diese ganz besonders. Und so ins Unendliche. Der Trost wäre nur: es geschieht, ob du willst oder nicht. Und was du willst, hilft nur unmerklich wenig.

Franz Kafka 
Appendix 


\section{Appendix A}

\section{The Eneström-Kakeya Theorem}

\section{A.1 Spectral-Radius and Matrix-Norm}

Let $\mathbf{A}=a_{i j}$ be a $n \times n$ matrix. The spectral radius $\rho$ of a $\mathbf{A}$ is defined as [178]

$$
\rho(\mathbf{A})=\max _{\|\mathbf{x}\|=1}\|\mathbf{A} \mathbf{x}\|=\max _{i=1, . ., n}\left|\lambda_{i}\right|
$$

where \|\| denotes a norm and $\left\{\lambda_{i}\right\}_{i=1}^{n}$ are the complex eigenvalues of A.If $\|-\|$ is any matrix norm (see [260]) the inequality

$$
\rho(A) \leq\|A\|
$$

is valid and in fact $\rho(A)=\inf \|A\|$ where the infimum is taken over all matrix norms [178]. Here we only need the maximum-absolute-column-sum norm of $\mathbf{A}$ defined as

$$
\|\mathbf{A}\|=\max _{j=1, \ldots, n} \sum_{i=1}^{n}\left|a_{i j}\right|
$$

\section{A.2 Companion Matrices}

A $(n+1) \times(n+1)$ companion matrix $\mathbf{C}$ has the standard form

$$
\mathbf{C}=\left(\begin{array}{cccc}
0 & \ldots & 0 & -\tilde{c}_{0} \\
1 & & 0 & -\tilde{c}_{1} \\
& \ddots & & \vdots \\
0 & & 1 & -\tilde{c}_{n}
\end{array}\right)
$$

with characteristic polynomial

$$
\tilde{p}_{n+1}(z)=\operatorname{det}(z-\mathbf{C})=\tilde{c}_{0}+\tilde{c}_{1} z+\ldots+\tilde{c}_{n} z^{n}+z^{n+1}
$$

\section{A.3 The Eneström-Kakeya Theorem}

The Eneström-Kakeya theorem ${ }^{1}[93,194,182,11,178]$ can be stated in the following form:

\footnotetext{
${ }^{1}$ In 1893 the Swedish actuary and mathematics historian Gustaf Eneström published this result of roots of certain polynomials with real coefficients in a paper on pension insurance (in Swedish) [93]. This result is now often called the Eneström-Kakeya theorem, since S. Kakeya published a similar result in 1912-1913 [194]. But Kakeya's theorem contained a mistake, which was corrected by A. Hurwitz in 1913 [182].
} 
Theorem A.3.1. Let $p_{n}(z)=\sum_{j=0}^{n} c_{j} z^{j}$ with $c_{j}>0$ then for all $\lambda$ with $p_{n}(\lambda)=0$

$$
|\lambda| \leq \max _{0 \leq i<n}\left\{\frac{c_{i}}{c_{i+1}}\right\}=: \beta
$$

Proof. Note first that $\beta>0$. We set

$$
\tilde{p}_{n+1}(z):=\frac{(z-1) p_{n}(\beta z)}{c_{n} \beta^{n}}=z^{n+1}+\sum_{i=0}^{n} \tilde{c}_{i} z^{i}
$$

where

$$
\tilde{c}_{i}= \begin{cases}\frac{c_{i-1}-\beta c_{i}}{c_{n} \beta^{n-i+1}} & 1 \leq i \leq n \\ \frac{-c_{0}}{c_{n} \beta^{n}} & i=0\end{cases}
$$

Using the definition of $\beta$ one observes that $\tilde{c}_{j} \leq 0$. Comparing (A.2.2) with (A.3.1) the companion matrix of $\tilde{p}_{n+1}$ is given by (A.2.1). Since $1+\sum_{j=1}^{n+1} \tilde{c}_{j}=\tilde{p}_{n+1}(1)=0$ if follows that $\|\mathbf{C}\|=\sum_{j=1}^{n+1}\left|\tilde{c}_{j}\right|=-\sum_{j=1}^{n+1} \tilde{c}_{j}=1$ when using the maximum-absolute-column-sum norm (A.1.3) and hence from (A.1.2)

$$
\rho(\mathbf{C}) \leq 1
$$

Thus for all $\tilde{\lambda}$ with $p_{n+1}(\tilde{\lambda})=0$ we have $|\tilde{\lambda}| \leq \rho(\mathbf{C}) \leq 1$. For a $\lambda$ with $p_{n}(\lambda)=0$ it follows from the definition of $\tilde{p}_{n+1}$ that $\tilde{p}_{n+1}(\tilde{\lambda})=0$ for $\tilde{\lambda}=\frac{\lambda}{\beta}$ and thus $|\lambda| \leq \beta$.

Corollary A.3.2. Let A be a matrix of the form (cf. (6.4.5))

$$
\mathbf{A}=\left(\begin{array}{ccccc}
-a_{n} & a_{1} & 0 & \ldots & 0 \\
-a_{n} & 0 & a_{2} & \ddots & \vdots \\
\vdots & \vdots & \ddots & \ddots & 0 \\
-a_{n} & 0 & \ldots & 0 & a_{n-1} \\
-a_{n} & 0 & \ldots & 0 & 0
\end{array}\right)
$$

with $a_{i}>0$ then

$$
\rho(\mathbf{A}) \leq \max \left\{a_{i}\right\}_{i=1}^{n}
$$

Proof. By a permutation of rows and columns we can cast $\mathbf{A}$ into a matrix $\mathbf{B}=b_{i, j}$ with non-zero entries $b_{i,(i+1)}=a_{i}, i \in\{1, \ldots, n-1\}$ and $b_{i, n}=-a_{n}, i \in\{1, \ldots, n\}$. This does not change the spectral radius. The similarity transformation to $\mathbf{C}=\mathbf{Q}^{-1} \mathbf{B Q}$ with $\mathbf{Q}=\operatorname{diag}\left(q_{1}, \ldots, q_{N-1}\right)$ and $q_{1}=1, q_{i}=\prod_{j=1}^{i-1} a_{j}, i \in\{2, \ldots, n\}$ also preserves the spectral radius and $\mathbf{C}$ has the form of a companion matrix (A.2.1) with $c_{i}=\prod_{j=i+1}^{n} a_{i}>0$, $i \in\{0, \ldots, n-1\}$. Thus $\rho(\mathbf{A})=\rho(\mathbf{C}) \leq \max _{0 \leq i<n}\left\{\frac{c_{i}}{c_{i+1}}\right\}=\max _{1 \leq i \leq n}\left\{a_{i}\right\}$ 


\section{Appendix B}

\section{Rise Functions}

In this section we derive rise functions for several integrate and fire type models as introduced in sec. 2.6 and some of their properties.

\section{B.1 Rise Functions for Integrate-and-Fire Models}

In this section we derive the rise functions for single variable models of the form (2.6.2). We distinguish between current based inputs $i_{\text {syn }}(t)=P(t)$ with $P(t)=\sum_{s} \varepsilon_{s} \delta\left(t-t_{s}\right)$ and the conductance based approach $i_{\mathrm{syn}}(t)=g_{\mathrm{syn}} P(t)\left(v_{\mathrm{syn}}-v(t)\right), v_{\mathrm{syn}}>1$. If the rise function $U$ for current based inputs is known the conductance based rise function $U^{\mathrm{CB}}$ is calculated with the help of (5.2.19).

For the leaky-integrate-and-fire (LIF) model (2.6.4) $U$ is given by (5.2.18) which yields

$$
U_{\mathrm{LIF}}^{\mathrm{CB}}(\phi)=\frac{\ln \left(1-v_{\mathrm{syn}}^{-1} U_{\mathrm{LIF}}(\phi)\right)}{\ln \left(1-v_{\mathrm{syn}}^{-1}\right)}
$$

For the quadratic-integrate-and-fire (QIF) model (2.6.3) one obtains

$$
U_{\mathrm{QIF}}(\phi)=\frac{\alpha-\tan (\arctan (\alpha)-\phi(\arctan (\alpha)-\arctan (\beta)))}{\alpha-\beta}
$$

where $\alpha=\frac{v_{r}+v_{t}}{\gamma}, \quad \beta=\alpha-\frac{2}{\gamma}, \quad \gamma=\sqrt{\frac{4 i_{\mathrm{e}}}{g_{2}}-\left(v_{\mathrm{t}}-v_{\mathrm{r}}\right)^{2}}>0$. Hence

$$
U_{\mathrm{QIF}}^{\mathrm{CB}}(\phi)=\frac{\ln \left(1-v_{\mathrm{syn}}^{-1} U_{\mathrm{QIF}}(\phi)\right)}{\ln \left(1-v_{\mathrm{syn}}^{-1}\right)}
$$

Note that depending on the IF model and coupling type convex, concave and sigmoidal shapes are possible (cf. tab. B.1). We remark that as $v_{\text {syn }} \rightarrow \infty$ we recover the potential independent model from the conductance based version, i.e. $U^{\mathrm{CB}} \rightarrow U$ and the conditions for the different properties of $U^{\mathrm{CB}}$ become the conditions for $U$ in tab. B.1.

\section{B.2 Icpd and Dcpd Rise Functions}

Usually it is difficult to verify the icpd or depd property (6.5.3) of a rise function. Here we show that it is closely related to the third derivative of $U$. 


\begin{tabular}{|c|c|c|c|c|c|c|}
\hline$U$ & $\begin{array}{c}\text { parameter } \\
\text { domain }\end{array}$ & concave & convex & sigmoidal & icpd & dcpd \\
\hline$U_{\mathrm{LIF}}$ & $v_{\text {eq }}>1$ & $\sqrt{ }$ & - & - & $\sqrt{ }$ & - \\
\hline$U_{\mathrm{LIF}}^{\mathrm{CB}}$ & $\begin{array}{c}v_{\mathrm{syn}}>1 \\
v_{\mathrm{eq}}>1\end{array}$ & $v_{\text {syn }}>v_{\text {eq }}$ & $v_{\text {syn }}<v_{\text {eq }}$ & - & $v_{\mathrm{syn}} \geq v_{\mathrm{eq}}$ & $v_{\text {syn }} \leq v_{\text {eq }}$ \\
\hline$U_{\mathrm{QIF}}$ & $\begin{array}{c}0 \leq \alpha<\infty \\
-\infty<\beta \leq 0 \\
\alpha>\beta\end{array}$ & $\beta=0$ & $\alpha=0$ & $\beta<0<\alpha$ & - & $\begin{array}{c}\alpha \leq 1 \\
-1 \leq \beta\end{array}$ \\
\hline$U_{\mathrm{QIF}}^{\mathrm{CB}}$ & $\begin{array}{c}v_{\text {syn }}>1 \\
0 \leq \alpha<\infty \\
-\infty<\beta \leq 0\end{array}$ & - & $\begin{array}{c}0 \leq 1+ \\
\alpha(\alpha-2 \eta)\end{array}$ & $\begin{array}{c}0>1+ \\
\alpha(\alpha-2 \eta)\end{array}$ & - & $\begin{aligned} \alpha^{2} & \leq \frac{\eta}{\eta-\alpha-\alpha^{-1}} \\
\beta^{2} & \leq \frac{\eta-\alpha+\beta}{\eta-\alpha-\beta^{-1}}\end{aligned}$ \\
\hline$U_{b}$ & $b \in \mathbb{R} \backslash\{0\}$ & $b<0$ & $b>0$ & - & $\sqrt{ }$ & $\sqrt{ }$ \\
\hline
\end{tabular}

Table B.1: Properties of different rise functions. $\eta=v_{\text {syn }}(\alpha-\beta)$.

We first note that $\Delta H$ obeys the relations $\Delta H(\phi, 0, \varepsilon) \equiv 0$ and $\Delta H(\phi, \Delta \phi, 0) \equiv \Delta \phi$ and hence $\frac{\partial}{\partial \phi} \Delta H(\phi, \Delta \phi, 0)=0$ and

$$
\frac{\partial}{\partial \phi} \Delta H(\phi, \Delta \phi, \varepsilon)=\int_{0}^{\varepsilon} \int_{0}^{\Delta \phi} \frac{\partial}{\partial \phi} \frac{\partial}{\partial \varepsilon} \frac{\partial}{\partial \Delta \phi} \Delta H(\phi, \tilde{\Delta \phi}, \tilde{\varepsilon}) \mathrm{d} \tilde{\Delta} \phi \mathrm{d} \tilde{\varepsilon}
$$

Thus $U$ is icpd if

$$
\frac{\partial^{3}}{\partial \phi \partial \varepsilon \partial \Delta \phi} \Delta H(\phi, \Delta \phi, \varepsilon) \geq 0 \quad \text { for all }(\phi, \Delta \phi, \varepsilon) \in \mathcal{D}
$$

Using $\leq$ instead of $\geq$ yields an analogous condition for dcpd $U$. By definition of $\Delta H$ eq. (B.2.1) yields the condition

$$
\begin{aligned}
& \frac{\partial^{3}}{\partial \phi \partial \varepsilon \partial \Delta \phi} \Delta H(\phi, \Delta \phi, \varepsilon)= 3 \frac{U^{\prime \prime}(H(\phi+\Delta \phi, \varepsilon))^{2} U^{\prime}(\phi+\Delta \phi)^{2}}{U^{\prime}(H(\phi+\Delta \phi, \varepsilon))^{5}} \\
&-\frac{U^{\prime \prime}(\phi+\Delta \phi) U^{\prime \prime}(H(\phi+\Delta \phi, \varepsilon))}{U^{\prime}(H(\phi+\Delta \phi, \varepsilon))^{3}} \\
&-\frac{U^{\prime}(\phi+\Delta \phi)^{2} U^{\prime \prime \prime}(H(\phi+\Delta \phi, \varepsilon))}{U^{\prime}(H(\phi+\Delta \phi, \varepsilon))^{4}} \\
& \geq 0 \quad \forall(\phi, \Delta \phi, \varepsilon) \in \mathcal{D} .
\end{aligned}
$$

Substituting $H(\phi+\Delta \phi, \varepsilon) \rightarrow \phi$ and $\phi+\Delta \phi \rightarrow \psi$ one obtains

$$
U^{\prime \prime \prime}(\phi) \leq 3 \frac{U^{\prime \prime}(\phi)^{2}}{U^{\prime}(\phi)}-\frac{U^{\prime \prime}(\psi) U^{\prime \prime}(\phi) U^{\prime}(\phi)}{U^{\prime}(\psi)^{2}} \quad \forall 0 \leq \psi \leq \phi \leq 1
$$

as a non-local sufficient condition for a rise function to be icpd. The condition for dcpd $U$ is given when replacing $\leq$ by $\geq$.

Now note that if (B.2.2) is satisfied locally for $\phi=\psi$ the sign of the derivative

$$
\frac{\partial}{\partial \psi}\left(3 \frac{U^{\prime \prime}(\phi)^{2}}{U^{\prime}(\phi)}-\frac{U^{\prime \prime}(\psi) U^{\prime \prime}(\phi) U^{\prime}(\phi)}{U^{\prime}(\psi)^{2}}\right)=U^{\prime \prime}(\phi) U^{\prime}(\phi)\left(2 \frac{U^{\prime \prime}(\psi)^{2}}{U^{\prime}(\psi)^{3}}-\frac{U^{\prime \prime \prime}(\psi)}{U^{\prime}(\psi)^{2}}\right)
$$


is determined by $U^{\prime \prime}(\phi)$ since the term in brackets on the right hand side at $\phi=\psi$ is positive using inequality (B.2.2) and $U^{\prime}>0$. Hence, if $U$ is concave, a sufficient local condition for a rise function to be icpd is

$$
U^{\prime \prime}(\phi) \leq 0 \quad \text { and } \quad U^{\prime \prime \prime}(\phi) \leq 2 \frac{U^{\prime \prime}(\phi)^{2}}{U^{\prime}(\phi)} \quad \forall 0 \leq \phi \leq 1
$$

Conversely a local condition for a convex rise functions to be dcpd is given by

$$
U^{\prime \prime}(\phi) \geq 0 \quad \text { and } \quad U^{\prime \prime \prime}(\phi) \geq 2 \frac{U^{\prime \prime}(\phi)^{2}}{U^{\prime}(\phi)} \quad \forall 0 \leq \phi \leq 1 .
$$

Different properties of commonly used rise functions are summarized in table B.1. 


\section{Appendix C}

\section{Event-Sequence-Based Domain Analysis for $\mathcal{N}^{4}$}

In this section we state and proof technical results for the pulse coupled oscillator network $\mathcal{N}^{4}$ defined in section 7.2.3.1 using event-sequence-based domain analysis as described in section 7.2.2.

\section{C.1 Evolution of States in $\mathcal{U}_{i}^{\eta}$ to $\mathcal{P}_{i}^{4}$}

Here we consider the evolution of states in the neighbourhood $\mathcal{U}_{i}^{\eta}$, eq. (7.2.18), of the periodic orbits $\mathcal{O}_{i}$. As the results for $\mathcal{U}_{2}^{\eta}$ can be obtained from the ones of $\mathcal{U}_{1}^{\eta}$ by exchanging indices $(1,2) \leftrightarrow(3,4)$ we concentrate on $\mathcal{U}_{1}^{\eta}$. Note that via tab. 7.1 the orbit $\mathcal{O}_{1}$ consists of three straight lines between the different events in state space and therefore $\mathcal{U}_{1}^{\eta}$ consists of three components. We consider states in each component. We note that for $\eta$ small enough, the definition of $\mathcal{U}_{1}^{\eta}$ in (7.2.18) ensures that in each component the number of not received pules is the same as in the orbit segment. Therefore it is sufficient to only consider the deviations from the periodic orbit in the phases and in the spike times of not received pulses.

We first observe that states in the component where all spikes have been received lead to a state in $\mathcal{P}_{1}^{4}$ via the uniform phase shift (7.2.1). The evolution of states in the other two components is then described by the event tables C.1 and C.2.

Tab. C.1 is valid if

$$
\begin{aligned}
H_{2 \varepsilon, \tau} \circ H_{\varepsilon, \zeta_{2}-\zeta_{1}} \circ H_{0, \tau-\zeta_{1}}\left(\delta_{1}\right) & <1 \\
H_{2 \varepsilon, \tau} \circ H_{0, \zeta_{2}-\zeta_{1}} \circ H_{\varepsilon, \tau-\zeta_{1}}\left(\delta_{2}\right) & <1 \\
S_{\zeta_{2}-\zeta_{1}} \circ H_{\varepsilon, \tau-\zeta_{1}}\left(\alpha+\delta_{3,4}\right) & <1 \\
H_{\varepsilon, \zeta_{2}-\zeta_{1}} \circ H_{\varepsilon, \tau-\zeta_{1}}\left(\alpha+\delta_{3,4}\right) & >1
\end{aligned}
$$

and tab. C.2 if

$$
\begin{aligned}
H_{\varepsilon, \zeta_{3}-\zeta_{4}} \circ H_{\varepsilon, \tau-\zeta_{3}+\delta_{1,2}} \circ H_{\varepsilon, \tau}(0) & <1 \\
H_{\varepsilon, \zeta_{3}-\zeta_{4}} \circ H_{0, \tau-\zeta_{3}+\delta_{3}} \circ J_{2 \varepsilon, \tau}(\alpha) & <1 \\
H_{0, \zeta_{3}-\zeta_{4}} \circ H_{\varepsilon, \tau-\zeta_{3}+\delta_{3}} \circ J_{2 \varepsilon, \tau}(\alpha) & <1
\end{aligned}
$$

For $\delta_{i}=0$ and $\zeta_{i}=0$ the conditions are all fulfilled via the parameter conditions (7.2.13) and hence by continuity of the maps $H, J$ and $S$ there is a $\eta>0$ such that these conditions 
Table C.1: Evolution of states in $\mathcal{U}_{1}^{\eta}$ after the event $\left(s_{1}, s_{2}\right)$ for $\zeta_{1}>\zeta_{2}$

\begin{tabular}{|c|c|c|c|c|c|c|c|c|c|}
\hline event & time & $\phi_{1}$ & $\sigma_{1}$ & $\phi_{2}$ & $\sigma_{2}$ & $\phi_{3}$ & $\sigma_{3}$ & $\phi_{4}$ & $\sigma_{4}$ \\
\hline start & 0 & $\delta_{1}$ & $\zeta_{1}$ & $\delta_{2}$ & $\zeta_{2}$ & $\alpha+\delta_{3}$ & - & $\alpha+\delta_{4}$ & - \\
\hline$r_{1}$ & $t_{1}:=\tau-\zeta_{1}$ & $\phi_{1,2}:=H_{0}\left(t_{1}+\delta_{1}\right)$ & $\tau \rightarrow-$ & $\phi_{2,2}:=H_{\varepsilon}\left(\delta_{2}+t_{1}\right)$ & $t_{1}+\zeta_{2}$ & $\phi_{3,2}:=H_{\varepsilon}\left(\alpha+\delta_{3}+t_{1}\right)$ & & $\phi_{4,2}:=H_{\varepsilon}\left(\alpha+\delta_{4}+t_{1}\right)$ & - \\
\hline$r_{2}, s_{3}, s$ & $t_{2}:=\tau-\zeta_{2}$ & $1,3:=H_{\varepsilon}\left(\phi_{1,2}-\zeta_{2}+\zeta_{1}\right)$ & - & $\phi_{2,3}:=H_{0}\left(\phi_{2,2}-\zeta_{2}+\zeta_{1}\right.$ & $\tau \rightarrow-c$ & $\phi_{3,3}:=J_{\varepsilon}\left(\phi_{3,2}-\zeta_{2}+\zeta_{1}\right)$ & 0 & $\phi_{4,3}:=J_{\varepsilon}\left(\phi_{3,2}-\zeta_{2}+\zeta_{1}\right)$ & 0 \\
\hline$r_{3}, r_{4}$ & $t_{3}:=t_{2}+\tau$ & $\phi_{1,4}:=H_{2 \varepsilon}\left(\phi_{1,3}+\tau\right)$ & - & $\phi_{2,4}:=H_{2 \varepsilon}\left(\phi_{2,3}+\tau\right)$ & - & $\phi_{3,4}:=H_{\varepsilon}\left(\phi_{3,3}+\tau\right)$ & $\tau \rightarrow-$ & $\phi_{4,4}:=H_{\varepsilon}\left(\phi_{4,3}+\tau\right)$ & $\tau \rightarrow-$ \\
\hline$s_{1}^{\prime}$ & $t_{3}+1-\phi_{1,4}$ & 0 & 0 & $\phi_{2,4}+1-\phi_{1,4}$ & - & $\phi_{3,4}+1-\phi_{1,4}$ & - & $\phi_{4,4}+1-\phi_{1,4}$ & - \\
\hline
\end{tabular}

Table C.2: Evolution of states in $\mathcal{U}_{1}^{\eta}$ after the event $\left(r_{1}, r_{2}, s_{3}, s_{4}\right)$ for $\zeta_{3}>\zeta_{3}$

\begin{tabular}{|c|c|c|c|c|c|c|c|c|c|}
\hline event & time & $\phi_{1}$ & $\sigma_{1}$ & $\phi_{2}$ & $\sigma_{2}$ & $\phi_{3}$ & $\sigma_{3}$ & $\phi_{4}$ & $\sigma_{4}$ \\
\hline start & 0 & $\phi_{1,0}:=H_{\varepsilon, \tau}(0)+\delta_{1}$ & - & $\phi_{2,0}:=H_{\varepsilon, \tau}(0)+\delta_{2}$ & - & $\phi_{3,0}:=J_{2 \varepsilon, \tau}(\alpha)+\delta_{3}$ & $\zeta_{3}$ & $\phi_{3,0}:=J_{2 \varepsilon, \tau}(\alpha)+\delta_{4}$ & $\zeta_{4}$ \\
\hline$r_{3}$ & $t_{1}:=\tau-\zeta_{3}$ & $\phi_{1,1}:=H_{\varepsilon, t_{1}}\left(\phi_{1,0}\right)$ & - & $\phi_{2,1}:=H_{\varepsilon, t_{1}}\left(\phi_{2,0}\right)$ & - & $\phi_{3,1}:=H_{0, t_{1}}\left(\phi_{3,0}\right)$ & $\tau \rightarrow-$ & $\phi_{4,1}:=H_{\varepsilon, t_{1}}\left(\phi_{3,0}\right)$ & $\zeta_{4}+t_{1}$ \\
\hline$r_{4}$ & $t_{2}:=\tau-\zeta_{4}$ & $\phi_{1,2}:=H_{\varepsilon, \zeta_{3}-\zeta_{4}}\left(\phi_{1,1}\right)$ & - & $\phi_{2,2}:=H_{\varepsilon, \zeta_{3}-\zeta_{4}}\left(\phi_{2,1}\right)$ & - & $\phi_{3,2}:=H_{\varepsilon, \zeta_{3}-\zeta_{4}}\left(\phi_{23,1}\right)$ & - & $\phi_{4,2}:=H_{\varepsilon, \zeta_{3}-\zeta_{4}}\left(\phi_{4,1}\right)$ & $\tau \rightarrow-$ \\
\hline$s_{1}^{\prime}$ & $t_{2}+1-\phi_{1,2}$ & 0 & 0 & $\phi_{2,3}+1-\phi_{1,2}$ & - & $\phi_{3,3}+1-\phi_{1,2}$ & - & $\phi_{4,3}+1-\phi_{1,2}$ & - \\
\hline
\end{tabular}


also hold for $\left|\delta_{i}\right|<\eta$ and $\left|\zeta_{i}\right|<\eta$. For $\zeta_{1}<\zeta_{2}$ exchange indices $1 \leftrightarrow 2$ and for $\zeta_{1}=\zeta_{2}$ the third condition becomes the weaker condition $S_{\tau-\zeta_{1}}\left(\alpha+\delta_{3,4}\right)<1$. For $\zeta_{3}<\zeta_{4}$ exchange indices $3 \leftrightarrow 4$ and for $\zeta_{3}=\zeta_{4}$ the form of the conditions does not change.

\section{C.2 Event-Sequence-Based Domain and Parameter Anal- ysis for $\mathcal{N}^{4}$}

In this section we derive several properties of maps withing and between the local Poincare representations $\mathcal{P}_{i}$, eq. (7.2.19).

\section{C.2.1 Return Maps}

\section{C.2.1.1 Expanding Maps $R_{i}^{\uparrow}$}

Lemma C.2.1. The return map $R_{1}^{\uparrow}: \mathcal{D}\left(R_{1}^{\uparrow}\right) \subset \mathcal{P}_{1} \rightarrow \mathcal{P}_{1}, \delta^{(0)} \mapsto \delta^{(1)}$ with event sequence

$$
E\left(R_{1}^{\uparrow}\right)=\left(s_{1}\right)\left(s_{2}\right)\left(r_{1}\right)\left(r_{2}, s_{3}, s_{4}\right)\left(r_{3}, r_{4}\right)
$$

is given by

$$
\begin{aligned}
& \delta_{1}^{(1)}=0 \\
& \delta_{2}^{(1)}=\operatorname{sign}\left(\delta_{2}^{(0)}\right)\left[H_{2 \varepsilon, \tau} \circ H_{\varepsilon, \tau}\left(\left|\delta_{2}^{(0)}\right|\right)-H_{2 \varepsilon, \tau+\left|\delta_{2}^{(0)}\right|} \circ H_{\varepsilon, \tau}\left(-\left|\delta_{2}^{(0)}\right|\right)\right] \\
& \delta_{3}^{(1)}=H_{\varepsilon, \tau} \circ J_{\varepsilon, \mid \delta_{2}^{(0)}} \circ H_{\varepsilon, \tau}\left(\alpha+\delta_{3}^{(0)}\right)+1-H_{2 \varepsilon, \tau} \circ H_{\varepsilon, \tau}\left(\left|\delta_{2}^{(0)}\right|\right)-\alpha \\
& \delta_{4}^{(1)}=H_{\varepsilon, \tau} \circ J_{\varepsilon, \mid \delta_{2}^{(0)}} \circ H_{\varepsilon, \tau}\left(\alpha+\delta_{4}^{(0)}\right)+1-H_{2 \varepsilon, \tau} \circ H_{\varepsilon, \tau}\left(\left|\delta_{2}^{(0)}\right|\right)-\alpha
\end{aligned}
$$

with domain $\mathcal{D}\left(R_{1}^{\uparrow}\right)$ defined by the inequalities

$$
\begin{aligned}
\left|\delta_{2}\right| & >0 \\
H_{2 \varepsilon, \tau} \circ H_{\varepsilon, \tau}\left(\left|\delta_{2}\right|\right) & <1 \\
S_{\left|\delta_{2}\right|} \circ H_{\varepsilon, \tau}\left(\alpha+\delta_{3,4}\right) & \leq 1 \\
H_{\varepsilon,\left|\delta_{2}\right|} \circ H_{\varepsilon, \tau}\left(\alpha+\delta_{3,4}\right) & \geq 1
\end{aligned}
$$

The map $R_{2}^{\uparrow}$ is obtained via exchange of indices $(1,2) \leftrightarrow(3,4)$.

Proof. The analytical form follows from the event based analysis is given in tab. C.3 for states $\left(\delta_{2}, \delta_{3}, \delta_{4}\right) \in \mathcal{P}_{1}$ assuming $\delta_{2}<0$. For $\delta_{2}>0$ the event sequence is given by relabeling $1 \leftrightarrow 2$. The event table C.3 is valid if

$$
\begin{aligned}
0<\left|\delta_{2}\right| & <\tau \\
0<\alpha+\delta_{3,4} & <1-\left|\delta_{2}\right| \\
H_{2 \varepsilon, \tau} \circ H_{\varepsilon, \tau}\left(\left|\delta_{2}\right|\right) & <1 \\
H_{2 \varepsilon, \tau+\left|\delta_{2}\right|} \circ H_{\varepsilon, \tau}\left(-\left|\delta_{2}\right|\right)+\left|\delta_{2}\right| & <1 \\
H_{\varepsilon, \tau}\left(\alpha+\delta_{3,4}\right)+\left|\delta_{2}\right| & \leq 1 \\
H_{\varepsilon}\left(H_{\varepsilon, \tau}\left(\alpha+\delta_{3,4}\right)+\left|\delta_{2}\right|\right) & \geq 1 \\
H_{\varepsilon}\left(J_{\varepsilon}\left(H_{\varepsilon}\left(\alpha+\delta_{3,4}+\tau\right)+\left|\delta_{2}\right|\right)+\tau\right) & <1
\end{aligned}
$$


which using the properties (7.2.8)-(7.2.9) reduces to the conditions (C.2.3). In particular, the first inequality follows from (C.2.3) together with $H_{2 \varepsilon, \tau} \circ H_{\varepsilon, \tau}(\tau)>1$. The second inequality follows from the third and fourth inequalities in (C.2.3). The third inequality follows from property $(7.2 .9)$ and using $S_{\tau} \circ H_{2 \varepsilon, \tau} \circ H_{\varepsilon, \tau}(0)<1$. To obtain $H_{\varepsilon, \tau} \circ J_{\varepsilon,\left|\delta_{2}\right|} \circ$ $H_{\varepsilon, \tau}\left(\alpha+\delta_{3,4}+\tau\right)<1$, consider $H_{\varepsilon, \tau} \circ H_{\varepsilon}(0)<1$.

\section{C.2.1.2 The Return Map $R_{1}^{a}$}

Lemma C.2.2. The return map $R_{1}^{a}: \mathcal{D}\left(R_{1}^{a}\right) \subset \mathcal{P}_{1} \rightarrow \mathcal{P}_{1}, \delta^{(0)} \mapsto \delta^{(1)}$ with event sequence

$$
E\left(R_{1}^{a}\right)=\left(s_{1}\right)\left(s_{2}\right)\left(r_{1}\right)\left(r_{2}, s_{3}\right)\left(s_{4}\right)\left(r_{3}\right)\left(r_{4}\right)
$$

is given by

$$
\begin{aligned}
& \delta_{1}^{(1)}=0 \\
& \delta_{2}^{(1)}=\operatorname{sign}\left(\delta_{2}^{(0)}\right)\left[\phi_{1,6}-H_{\varepsilon, 1-\phi_{4,3}} \circ H_{\varepsilon, \tau} \circ H_{0,\left|\delta_{2}^{(0)}\right|} \circ H_{\varepsilon, \tau}\left(-\left|\delta_{2}^{(0)}\right|\right)\right] \\
& \delta_{3}^{(1)}=H_{\varepsilon, 1-\phi_{4,3}} \circ H_{0, \tau} \circ J_{\varepsilon,\left|\delta_{2}^{(0)}\right|} \circ H_{\varepsilon, \tau}\left(\alpha+\delta_{3}^{(0)}\right)+1-\phi_{1,6}-\alpha \\
& \delta_{4}^{(1)}=H_{0,1-\phi_{4,3}} \circ H_{\varepsilon, \tau}\left(-1+\phi_{4,3}\right)+1-\phi_{1,6}-\alpha
\end{aligned}
$$

with

$$
\begin{aligned}
& \phi_{1,6}=H_{\varepsilon, 1-\phi_{4,3}} \circ H_{\varepsilon, \tau} \circ H_{\varepsilon,\left|\delta_{2}^{(0)}\right|} \circ H_{0, \tau}(0) \\
& \phi_{4,3}=H_{\varepsilon,\left|\delta_{2}^{(0)}\right|} \circ H_{\varepsilon, \tau}\left(\alpha+\delta_{4}^{(0)}\right)
\end{aligned}
$$

with domain $\mathcal{D}\left(R_{1}^{a}\right)$ defined by the inequalities

$$
\begin{aligned}
0<\left|\delta_{2}\right| & <\tau \\
S_{\left|\delta_{2}\right|} \circ H_{\varepsilon, \tau}\left(\alpha+\delta_{3}\right) & \leq 1 \\
H_{\varepsilon,\left|\delta_{2}\right|} \circ H_{\varepsilon, \tau}\left(\alpha+\delta_{3}\right) & \geq 1 \\
1-\tau<H_{\varepsilon,\left|\delta_{2}\right|} \circ H_{\varepsilon, \tau}\left(\alpha+\delta_{4}\right) & <1
\end{aligned}
$$

By exchanging indices $3 \leftrightarrow 4$ we obtain a second map $R_{i}^{\bar{a}}$ with an additional domain, for simplicity we will denote the map form the union of both domains $R_{1}^{a}$. The map $R_{2}^{a}$ is obtained via exchange of indices $(1,2) \leftrightarrow(3,4)$.

Proof. The analytical form follows from the event based analysis is given in tab. C.4 for states $\left(\delta_{2}, \delta_{3}, \delta_{4}\right) \in \mathcal{P}_{1}$ assuming $\delta_{2}<0$. For $\delta_{2}>0$ the event sequence is given by relabeling $1 \leftrightarrow 2$. The event table C.4 is valid if

$$
H_{\varepsilon, 1-\phi_{4,3}} \circ H_{\varepsilon, \tau} \circ H_{\varepsilon, \mid \delta_{2}^{(0)}} \circ H_{0, \tau}(0)<1
$$


Table C.3: $\delta_{2}$-expansive map $R_{1}^{\uparrow}$ for $\delta_{2}<0$

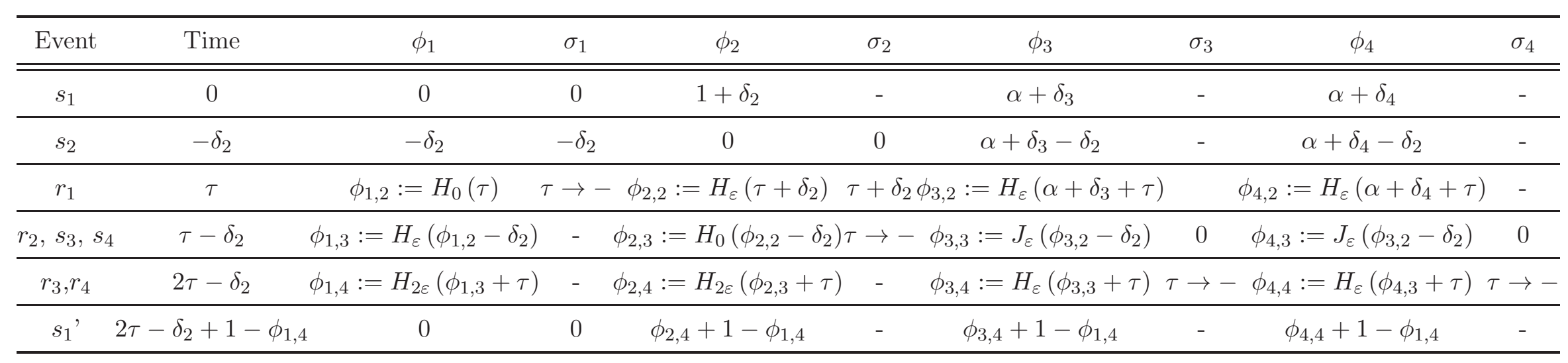

Table C.4: Return map $R_{1}^{a}$ for $\delta_{2}<0$ and $\delta_{3}>\delta_{4}$

\begin{tabular}{|c|c|c|c|c|c|c|c|c|c|}
\hline event & time & $\phi_{1}$ & $\sigma_{1}$ & $\phi_{2}$ & $\sigma_{2}$ & $\phi_{3}$ & $\sigma_{3}$ & $\phi_{4}$ & $\sigma_{4}$ \\
\hline$s_{1}$ & 0 & 0 & 0 & $1+\delta_{2}$ & - & $\alpha+\delta_{3}$ & - & $\alpha+\delta_{4}$ & - \\
\hline$s_{2}$ & $-\delta_{2}$ & $-\delta_{2}$ & $-\delta_{2}$ & 0 & 0 & $\alpha+\delta_{3}-\delta_{2}$ & - & $\alpha+\delta_{4}-\delta_{2}$ & - \\
\hline$r_{1}$ & $\tau$ & $\phi_{1,2}:=H_{0}(\tau)$ & $\tau \rightarrow-$ & $\phi_{2,2}:=H_{\varepsilon}\left(\tau+\delta_{2}\right)$ & $\tau+\delta_{2}$ & $\phi_{3,2}:=H_{\varepsilon}\left(\alpha+\delta_{3}+\tau\right)$ & - & $\phi_{4,2}:=H_{\varepsilon}\left(\alpha+\delta_{4}+\tau\right)$ & - \\
\hline$r_{2}, s_{3}$ & $\tau-\delta_{2}$ & $\phi_{1,3}:=H_{\varepsilon}\left(\phi_{1,2}-\delta_{2}\right)$ & - & $\phi_{2,3}:=H_{0}\left(\phi_{2,2}-\delta_{2}\right) \quad \tau$ & $\tau \rightarrow-$ & $\phi_{3,3}:=J_{\varepsilon}\left(\phi_{3,2}-\delta_{2}\right)$ & 0 & $\phi_{4,3}:=H_{\varepsilon}\left(\phi_{4,2}-\delta_{2}\right)$ & - \\
\hline$s_{4} t$ & $t_{4}:=\tau-\delta_{2}+1-\phi_{4,3}$ & $\phi_{1,4}:=\phi_{1,3}+1-\phi_{4,3}$ & - & $\phi_{2,4}:=\phi_{2,3}+1-\phi_{4,3}$ & - & $\phi_{3,4}:=\phi_{3,3}+1-\phi_{4,3}$ & $1-\phi_{4,3}$ & 0 & 0 \\
\hline$r_{3}$ & $2 \tau-\delta_{2}$ & $\phi_{1,5}:=H_{\varepsilon}\left(\phi_{1,3}+\tau\right)$ & - & $\phi_{2,5}:=H_{\varepsilon}\left(\phi_{2,3}+\tau\right)$ & - & $\phi_{3,5}:=H_{0}\left(\phi_{3,3}+\tau\right)$ & \multicolumn{3}{|c|}{$\tau \rightarrow-\phi_{4,5}:=H_{\varepsilon}\left(-1+\phi_{4,3}+\tau\right) \tau-1+\phi_{4,3}$} \\
\hline$r_{4}$ & $t_{6}:=t_{4}+\tau$ & $\phi_{1,6}:=H_{\varepsilon}\left(\phi_{1,5}+1-\phi_{4,3}\right)$ & )$-c$ & $\phi_{2,6}:=H_{\varepsilon}\left(\phi_{2,5}+1-\phi_{4,3}\right)$ & - & $\phi_{3,6}:=H_{\varepsilon}\left(\phi_{3,5}+1-\phi_{4,3}\right)$ & - & $\phi_{4,6}:=H_{0}\left(\phi_{4,5}+1-\phi_{4,3}\right)$ & $\tau \rightarrow-$ \\
\hline$s_{1}$ & $t_{6}+1-\phi_{1,6}$ & 0 & 0 & $\phi_{2,7}:=\phi_{2,6}+1-\phi_{1,6}$ & - & $\phi_{3,7}:=\phi_{3,6}+1-\phi_{1,6}$ & - & $\phi_{4,7}:=\phi_{4,6}+1-\phi_{1,6}$ & - \\
\hline
\end{tabular}


and

$$
\begin{aligned}
0<\left|\delta_{2}\right| & <\tau \\
H_{\varepsilon, 1-\phi_{4,3}} \circ H_{\varepsilon, \tau} \circ H_{0,\left|\delta_{2}\right|} \circ H_{\varepsilon, \tau}\left(-\left|\delta_{2}\right|\right) & <1
\end{aligned}
$$

and

$$
\begin{aligned}
S_{\left|\delta_{2}\right|} \circ H_{\varepsilon, \tau}\left(\alpha+\delta_{3}\right) & \leq 1 \\
H_{\varepsilon,\left|\delta_{2}\right|} \circ H_{\varepsilon, \tau}\left(\alpha+\delta_{3}\right) & \geq 1 \\
H_{\varepsilon, 1-\phi_{4,3}} \circ H_{0, \tau} \circ J_{\varepsilon,\left|\delta_{2}\right|} \circ H_{\varepsilon, \tau}\left(\alpha+\delta_{3}\right) & <1
\end{aligned}
$$

and

$$
\begin{aligned}
0<1-\phi_{4,3}=1-H_{\varepsilon,\left|\delta_{2}\right|} \circ H_{\varepsilon, \tau}\left(\alpha+\delta_{4}\right) & \leq \tau \\
H_{0,1-\phi_{4,3}} \circ H_{\varepsilon, \tau}\left(-1+\phi_{4,3}\right) & <1
\end{aligned}
$$

which are satisfied if the domain conditions (C.2.6), the smallness condition 7.2.23 and the parameter condition

$$
H_{\varepsilon, \tau} \circ H_{\varepsilon, \tau} \circ H_{\varepsilon, \tau}(0)<1
$$

hold. The parameter condition holds via (7.2.13).

\section{C.2.1.3 The Return Map $R_{1}^{b}$}

Lemma C.2.3. The return map $R_{1}^{b}: \mathcal{D}\left(R_{1}^{b}\right) \subset \mathcal{P}_{1} \rightarrow \mathcal{P}_{1}, \delta^{(0)} \mapsto \delta^{(1)}$ with event sequence

$$
E\left(R_{1}^{b}\right)=\left(s_{1}\right)\left(s_{2}\right)\left(r_{1}\right)\left(r_{2}, s_{3}\right)\left(r_{3}, s_{4}\right)\left(r_{4}\right)
$$

is given by

$$
\begin{aligned}
\delta_{1}^{(1)} & =0 \\
\delta_{2}^{(1)} & =\operatorname{sign}\left(\delta_{2}^{(0)}\right)\left[\phi_{1,5}-H_{\varepsilon, \tau} \circ H_{\varepsilon, \tau} \circ H_{0,\left|\delta_{2}^{(0)}\right|} \circ H_{\varepsilon, \tau}\left(-\left|\delta_{2}^{(0)}\right|\right)\right] \\
\delta_{3}^{(1)} & =H_{\varepsilon, \tau} \circ H_{0, \tau} \circ J_{\varepsilon, \mid \delta_{2}^{(0)}} \circ H_{\varepsilon, \tau}\left(\alpha+\delta_{3}^{(0)}\right)+1-\phi_{1,5}-\alpha \\
\delta_{4}^{(1)} & =H_{0, \tau} \circ J_{\varepsilon, \tau} \circ H_{\varepsilon,\left|\delta_{2}^{(0)}\right|} \circ H_{\varepsilon, \tau}\left(\alpha+\delta_{4}^{(0)}\right)+1-\phi_{1,5}-\alpha
\end{aligned}
$$

with

$$
\phi_{1,5}=H_{\varepsilon, \tau} \circ H_{\varepsilon, \tau} \circ H_{\varepsilon,\left|\delta_{2}^{(0)}\right|} \circ H_{0, \tau}(0)
$$

with domain $\mathcal{D}\left(R_{1}^{b}\right)$ defined by the inequalities 


$$
\begin{aligned}
\left|\delta_{2}\right| & >0 \\
H_{\varepsilon, \tau} \circ H_{\varepsilon, \tau} \circ H_{\varepsilon, \tau}\left(\left|\delta_{2}\right|\right) & <1 \\
S_{\left|\delta_{2}\right|} \circ H_{\varepsilon, \tau}\left(\alpha+\delta_{3}\right) & \leq 1 \\
H_{\varepsilon,\left|\delta_{2}\right|} \circ H_{\varepsilon, \tau}\left(\alpha+\delta_{3}\right) & \geq 1 \\
S_{\tau} \circ H_{\varepsilon,\left|\delta_{2}\right|} \circ H_{\varepsilon, \tau}\left(\alpha+\delta_{4}\right) & \leq 1 \\
H_{\varepsilon, \tau} \circ H_{\varepsilon,\left|\delta_{2}\right|} \circ H_{\varepsilon, \tau}\left(\alpha+\delta_{4}\right) & \geq 1
\end{aligned}
$$

The map $R_{2}^{b}$ is obtained via exchange of indices $(1,2) \leftrightarrow(3,4)$.

Proof. The analytical form follows from the event based analysis is given in tab. C.5 for states $\left(\delta_{2}, \delta_{3}, \delta_{4}\right) \in \mathcal{P}_{1}$ assuming $\delta_{2}<0$. For $\delta_{2}>0$ the event sequence is given by relabeling $1 \leftrightarrow 2$. The event table C.5 is valid if

$$
H_{\varepsilon, \tau} \circ H_{\varepsilon, \tau} \circ H_{\varepsilon,\left|\delta_{2}^{(0)}\right|} \circ H_{0, \tau}(0)<1
$$

and

$$
\begin{aligned}
& 0<\left|\delta_{2}\right|<\tau \\
& H_{\varepsilon, \tau} \circ H_{\varepsilon, \tau} \circ H_{0,\left|\delta_{2}\right|} \circ H_{\varepsilon, \tau}\left(-\left|\delta_{2}\right|\right)<1
\end{aligned}
$$

and

$$
\begin{aligned}
S_{\left|\delta_{2}\right|} \circ H_{\varepsilon, \tau}\left(\alpha+\delta_{3}\right) & \leq 1 \\
H_{\varepsilon,\left|\delta_{2}\right|} \circ H_{\varepsilon, \tau}\left(\alpha+\delta_{3}\right) & \geq 1 \\
H_{\varepsilon, \tau} \circ H_{0, \tau} \circ J_{\varepsilon,\left|\delta_{2}\right|} \circ H_{\varepsilon, \tau}\left(\alpha+\delta_{3}\right) & <1
\end{aligned}
$$

and

$$
\begin{aligned}
S_{\tau} \circ H_{\varepsilon,\left|\delta_{2}\right|} \circ H_{\varepsilon, \tau}\left(\alpha+\delta_{4}\right) & \leq 1 \\
H_{\varepsilon, \tau} \circ H_{\varepsilon,\left|\delta_{2}\right|} \circ H_{\varepsilon, \tau}\left(\alpha+\delta_{4}\right) & \geq 1 \\
H_{0, \tau} \circ J_{\varepsilon, \tau} \circ H_{\varepsilon,\left|\delta_{2}\right|} \circ H_{\varepsilon, \tau}\left(\alpha+\delta_{4}\right) & <1
\end{aligned}
$$

which are satisfied if the domain conditions (C.2.9), the smallness condition 7.2.23 and the parameter condition

$$
H_{\varepsilon, \tau} \circ H_{\varepsilon, \tau} \circ H_{\varepsilon, \tau}(0)<1
$$

hold. The parameter condition holds via (7.2.13).

\section{C.2.2 Maps on the Synchronization Manifolds}

In this section we consider several return maps of the synchronization manifolds $\mathcal{S}_{1}=$ $\left\{\delta \in \mathcal{P}_{1} \mid \delta_{2}=0\right\}$ and $\mathcal{S}_{2}=\left\{\delta \in \mathcal{P}_{2} \mid \delta_{4}=0\right\}$.

The maps $Q_{i}^{z}, z \in\{\uparrow, a, b, c\}$ on the synchronization manifold are obtained by taking the limits of $\delta_{2} \rightarrow 0$ in sections C.2.1.1-C.2.1.3. This can be verified by direct calculation of the event tables. 
Table C.5: Return map: $R_{1}^{b}$ for $\delta_{2}<0$

\begin{tabular}{cccccccccc}
\hline Event & Time & $\phi_{1}$ & $d_{1}$ & $\phi_{2}$ & $d_{2}$ & $\phi_{3}$ & $d_{3}$ & $\phi_{4}$ \\
\hline \hline$s_{1}$ & 0 & 0 & 0 & $1+\delta_{2}$ & - & $\alpha+\delta_{3}$ & - & $\alpha+\delta_{4}$ \\
\hline$s_{2}$ & $-\delta_{2}$ & $-\delta_{2}$ & $-\delta_{2}$ & 0 & 0 & $\phi_{3,1}:=\alpha+\delta_{3}-\delta_{2}$ & - & $\phi_{4,1}:=\alpha+\delta_{4}-\delta_{2}$ & - \\
\hline$r_{1}$ & $\tau$ & $\phi_{1,2}:=H_{0}(\tau)$ & $\tau \rightarrow-$ & $\phi_{2,2}:=H_{\varepsilon}\left(\tau+\delta_{2}\right)$ & $\tau+\delta_{2} \phi_{3,2}:=H_{\varepsilon}\left(\alpha+\delta_{3}+\tau\right)$ & - & $\phi_{4,2}:=H_{\varepsilon}\left(\alpha+\delta_{4}+\tau\right)$ & - \\
\hline$r_{2}, s_{3}$ & $\tau-\delta_{2}$ & $\phi_{1,3}:=H_{\varepsilon}\left(\phi_{1,2}-\delta_{2}\right)$ & - & $\phi_{2,3}:=H_{0}\left(\phi_{2,2}-\delta_{2}\right)$ & $\tau \rightarrow-$ & $\phi_{3,3}:=J_{\varepsilon}\left(\phi_{3,2}-\delta_{2}\right)$ & 0 & $\phi_{4,3}:=H_{\varepsilon}\left(\phi_{4,2}-\delta_{2}\right)$ & - \\
\hline$r_{3}, s_{4}$ & $2 \tau-\delta_{2}$ & $\phi_{1,4}:=H_{\varepsilon}\left(\phi_{1,3}+\tau\right)$ & - & $\phi_{2,4}:=H_{\varepsilon}\left(\phi_{2,3}+\tau\right)$ & - & $\phi_{3,4}:=H_{0}\left(\phi_{3,3}+\tau\right)$ & $\tau \rightarrow-\phi_{4,4}:=J_{\varepsilon}\left(\phi_{4,3}+\tau\right)$ & 0 \\
\hline$r_{4}$ & $3 \tau-\delta_{2}$ & $\phi_{1,5}:=H_{\varepsilon}\left(\phi_{1,4}+\tau\right)$ & - & $\phi_{2,5}:=H_{\varepsilon}\left(\phi_{2,4}+\tau\right)$ & - & $\phi_{3,5}:=H_{\varepsilon}\left(\phi_{3,4}+\tau\right)$ & - & $\phi_{4,5}:=H_{0}\left(\phi_{4,4}+\tau\right)$ & $\tau \rightarrow-$ \\
\hline$s_{1}$ & $t_{5}+1-\phi_{1,6}$ & 0 & 0 & $\phi_{2,6}:=\phi_{2,5}+1-\phi_{1,5}$ & - & $\phi_{3,6}:=\phi_{3,5}+1-\phi_{1,5}$ & - & $\phi_{4,6}:=\phi_{4,5}+1-\phi_{1,5}$ & - \\
\hline
\end{tabular}

Table C.6: Transient map $T_{12}^{a}$ for $\delta_{2}<0$

\begin{tabular}{|c|c|c|c|c|c|c|c|c|c|}
\hline event & time & $\phi_{1}$ & $\sigma_{1}$ & $\phi_{2}$ & $\sigma_{2}$ & $\phi_{3}$ & $\sigma_{3}$ & $\phi_{4}$ & $\sigma_{4}$ \\
\hline$s_{1}$ & 0 & 0 & 0 & $1+\delta_{2}$ & - & $\alpha+\delta_{3}$ & - & $\alpha+\delta_{4}$ & - \\
\hline$s_{2}$ & $-\delta_{2}$ & $-\delta_{2}$ & $-\delta_{2}$ & 0 & 0 & $\alpha+\delta_{3}-\delta_{2}$ & - & $\alpha+\delta_{4}-\delta_{2}$ & - \\
\hline$r_{1}$ & $\tau$ & $\phi_{1,2}:=H_{0}(\tau)$ & $\tau \rightarrow-$ & $\phi_{2,2}:=H_{\varepsilon}\left(\tau+\delta_{2}\right)$ & $\tau+\delta_{2}$ & $\phi_{3,2}:=H_{\varepsilon}\left(\alpha+\delta_{3}+\tau\right)$ & - & $\phi_{4,2}:=H_{\varepsilon}\left(\alpha+\delta_{4}+\tau\right)$ & - \\
\hline$r_{2}, s_{3}, s_{4}$ & $\tau-\delta_{2}$ & $\phi_{1,3}:=H_{\varepsilon}\left(\phi_{1,2}-\delta_{2}\right)$ & - & $\phi_{2,3}:=H_{0}\left(\phi_{2,1}-\delta_{2}\right)$ & $\tau \rightarrow-$ & $\phi_{3,3}:=J_{\varepsilon}\left(\phi_{3,2}-\delta_{2}\right)$ & 0 & $\phi_{4,3}:=J_{\varepsilon}\left(\phi_{4,2}-\delta_{2}\right)$ & 0 \\
\hline$r_{3}, r_{4}, s_{1}$ & $2 \tau-\delta_{2}$ & $\phi_{1,4}:=J_{2 \varepsilon}\left(\phi_{1,3}+\tau\right)$ & 0 & $\phi_{2,4}:=H_{2 \varepsilon}\left(\phi_{2,3}+\tau\right)$ & - & $\phi_{3,4}:=H_{\varepsilon}\left(\phi_{3,3}+\tau\right)$ & $\tau \rightarrow-$ & $\phi_{4,4}:=H_{\varepsilon}\left(\phi_{4,3}+\tau\right)$ & $\tau \rightarrow-$ \\
\hline$r_{1}, s_{2}$ & $3 \tau-\delta_{2}$ & $\phi_{1,5}:=H_{0}\left(\phi_{1,4}+\tau\right)$ & $\tau \rightarrow-$ & $\phi_{2,5}:=J_{\varepsilon}\left(\phi_{2,4}+\tau\right)$ & 0 & $\phi_{3,5}:=H_{\varepsilon}\left(\phi_{3,4}+\tau\right)$ & - & $\phi_{4,5}:=H_{\varepsilon}\left(\phi_{4,4}+\tau\right)$ & - \\
\hline$r_{2}$ & $4 \tau-\delta_{2}$ & $\phi_{1,6}:=H_{\varepsilon}\left(\phi_{1,5}+\tau\right)$ & - & $\phi_{2,6}:=H_{0}\left(\phi_{2,5}+\tau\right)$ & $\tau \rightarrow-$ & $\phi_{3,6}:=H_{\varepsilon}\left(\phi_{3,5}+\tau\right)$ & - & $\phi_{4,6}:=H_{\varepsilon}\left(\phi_{4,5}+\tau\right)$ & - \\
\hline$s_{3}$ & $4 \tau-\delta_{2}+1-$ & $\phi_{1,7}:=\phi_{1,6}+1-\phi_{3,6}$ & - & $\phi_{2,7}:=\phi_{2,6}+1-\phi_{3,6}$ & - & 0 & 0 & $\phi_{4,7}:=\phi_{4,6}+1-\phi_{3,6}$ & \\
\hline
\end{tabular}


Lemma C.2.4. $Q_{i}^{a}\left(\mathcal{D}\left(Q_{i}^{a}\right)\right) \subset \mathcal{D}\left(Q_{i}^{\uparrow}\right)$

Proof. The image $\delta^{(1)}$ of $\delta^{(0)}$ under $Q_{1}^{a}$ of a state in $\mathcal{D}\left(Q_{1}^{a}\right)$ is given by

$$
\begin{aligned}
& \delta_{2}^{(1)}=0 \\
& \delta_{3}^{(1)}=H_{\varepsilon, 1-\phi_{4,1}} \circ H_{0, \tau} \circ J_{2 \varepsilon, \tau}\left(\alpha+\delta_{3}^{(0)}\right)+1-\phi_{1,4}-\alpha \\
& \delta_{4}^{(1)}=H_{0,1-\phi_{4,1}} \circ H_{\varepsilon, \tau-\phi_{4,1}+1}+1-\phi_{1,4}-\alpha
\end{aligned}
$$

where

$$
\begin{aligned}
& \phi_{1,4}=H_{\varepsilon, 1-\phi_{4,1}} \circ H_{\varepsilon, \tau}^{2}(0) \\
& \phi_{4,1}=H_{2 \varepsilon, \tau}\left(\alpha+\delta_{4}^{(0)}\right)
\end{aligned}
$$

Via the smallness assumption on $J_{\varepsilon}$ we only have to check if this image is in the interior of the domain $\mathcal{D}\left(Q_{1}^{\uparrow}\right)$, i.e. we must have via (C.2.3)

$$
\begin{aligned}
H_{\varepsilon, \tau}\left(H_{\varepsilon, 1-\phi_{4,1}} \circ H_{0, \tau}(0)+1-H_{\varepsilon, 1-\phi_{4,1}} \circ H_{\varepsilon, \tau}^{2}(0)\right) & <1 \\
H_{2 \varepsilon, \tau}\left(H_{\varepsilon, 1-\phi_{4,1}} \circ H_{0, \tau}(0)+1-H_{\varepsilon, 1-\phi_{4,1}} \circ H_{\varepsilon, \tau}^{2}(0)\right) & >1 \\
H_{\varepsilon, \tau}\left(H_{0,1-\phi_{4,1}} \circ H_{\varepsilon, \tau+\phi_{4,1}-1}(0)+1-H_{\varepsilon, 1-\phi_{4,1}} \circ H_{\varepsilon, \tau}^{2}(0)\right) & <1 \\
H_{2 \varepsilon, \tau}\left(H_{0,1-\phi_{4,1}} \circ H_{\varepsilon, \tau+\phi_{4,1}-1}(0)+1-H_{\varepsilon, 1-\phi_{4,1}} \circ H_{\varepsilon, \tau}^{2}(0)\right) & >1
\end{aligned}
$$

which all follow as $1-\phi_{4,1}<\tau$ and using the parameter conditions

$$
\begin{aligned}
H_{\varepsilon, \tau}\left(H_{\varepsilon, 0} \circ H_{0, \tau}(0)+1-H_{\varepsilon, 0} \circ H_{\varepsilon, \tau}^{2}(0)\right) & <1 \\
H_{2 \varepsilon, \tau}\left(H_{0, \tau} \circ H_{\varepsilon, 0}(0)+1-H_{\varepsilon, \tau}^{3}(0)\right) & >1
\end{aligned}
$$

By an appropriate exchange of indices we obtain the result for $Q_{2}^{b}$.

Lemma C.2.5. $Q_{i}^{b}\left(\mathcal{D}\left(Q_{i}^{b}\right)\right) \subset \mathcal{D}\left(Q_{i}^{\uparrow}\right)$

Proof. The image $\delta^{(1)}$ of $\delta^{(0)}$ under $Q_{1}^{b}$ of a state in $\mathcal{D}\left(Q_{1}^{b}\right)$ is given by

$$
\begin{aligned}
& \delta_{2}^{(1)}=0 \\
& \delta_{3}^{(1)}=H_{\varepsilon, \tau} \circ H_{0, \tau} \circ J_{\varepsilon, \tau}\left(\alpha+\delta_{3}\right)+1-H_{\varepsilon, \tau}^{3}(0)-\alpha \\
& \delta_{4}^{(1)}=H_{0, \tau} \circ J_{\varepsilon, \tau} \circ H_{\varepsilon, \tau}\left(\alpha+\delta_{3}\right)+1-H_{\varepsilon, \tau}^{3}(0)-\alpha
\end{aligned}
$$

which analog to the previous lemma gives the two strongest conditions

$$
\begin{array}{r}
H_{\varepsilon, \tau}\left(H_{\varepsilon, \tau} \circ H_{0, \tau}(0)+1-H_{\varepsilon, \tau}^{3}(0)\right)<1 \\
H_{2 \varepsilon, \tau}\left(H_{0, \tau}(0)+1-H_{\varepsilon, \tau}^{3}(0)\right)>1
\end{array}
$$

which follow form the parameter conditions. By an appropriate exchange of indices we obtain the result for $Q_{2}^{b}$. 


\section{C.2.3 Transient Maps}

In this section we derive all maps between the Poincare spaces $\mathcal{P}_{i} \rightarrow \mathcal{P}_{j}$ with $i \neq j$.

\section{C.2.3.1 Transient Maps $T_{i j}^{a}$}

Lemma C.2.6. The transient map $T_{12}^{a}: \mathcal{D}\left(T_{12}^{a}\right) \subset \mathcal{P}_{1} \rightarrow \mathcal{P}_{2} \delta^{(0)} \mapsto \delta^{(1)}$ with event sequence

$$
E\left(T_{12}^{a}\right) \doteq\left(s_{1}\right)\left(s_{2}\right)\left(r_{1}\right)\left(r_{2}, s_{3}, s_{4}\right)\left(r_{3}, r_{4}, s_{1}\right)\left(r_{1}, s_{2}\right)\left(r_{2}\right)
$$

for $\delta_{2}^{(0)}<0$ is given by

$$
\begin{aligned}
& \delta_{1}^{(1)}=H_{\varepsilon, \tau} \circ H_{0, \tau} \circ J_{2 \varepsilon, \tau} \circ H_{\varepsilon,\left|\delta_{2}^{(0)}\right|} \circ H_{0, \tau}(0)+1-\phi_{3,6}-\alpha \\
& \delta_{2}^{(1)}=H_{0, \tau} \circ J_{\varepsilon, \tau} \circ H_{2 \varepsilon, \tau} \circ H_{0, \mid \delta_{2}^{(0)}} \circ H_{\varepsilon, \tau}\left(-\left|\delta_{2}^{(0)}\right|\right)+1-\phi_{3,6}-\alpha \\
& \delta_{3}^{(1)}=0 \\
& \delta_{4}^{(1)}=H_{\varepsilon, \tau} \circ H_{\varepsilon, \tau} \circ H_{\varepsilon, \tau} \circ J_{\varepsilon,\left|\delta_{2}^{(0)}\right|} \circ H_{\varepsilon, \tau}\left(\alpha+\delta_{4}^{(0)}\right)-\phi_{3,6}
\end{aligned}
$$

where

$$
\phi_{3,6}=H_{\varepsilon, \tau} \circ H_{\varepsilon, \tau} \circ H_{\varepsilon, \tau} \circ J_{\varepsilon,\left|\delta_{2}^{(0)}\right|} H_{\varepsilon, \tau}\left(\alpha+\delta_{3}^{(0)}\right)
$$

For $\delta_{2}^{(0)}>0$ exchange indices $1 \leftrightarrow 2$. The domain $\mathcal{D}\left(T_{12}^{a}\right)$ is given by the inequalities

$$
\begin{aligned}
\left|\delta_{2}\right| & <\tau \\
H_{2 \varepsilon, \tau} \circ H_{\varepsilon, \tau}\left(\left|\delta_{2}\right|\right) & \geq 1 \\
S_{\left|\delta_{2}\right|} \circ H_{\varepsilon, \tau}\left(\alpha+\delta_{3,4}\right) & <1 \\
H_{\varepsilon,\left|\delta_{2}\right|} \circ H_{\varepsilon, \tau}\left(\alpha+\delta_{3,4}\right) & \geq 1
\end{aligned}
$$

The map $T_{21}^{a}$ is obtained by exchanging $(1,2) \leftrightarrow(3,4)$.

Proof. The analytical form follows from the event based analysis is given in tab. C.6 for states $\left(\delta_{2}, \delta_{3}, \delta_{4}\right) \in \mathcal{P}_{1}$ assuming $\delta_{2}<0$. For $\delta_{2}>0$ the event sequence is given by relabeling $1 \leftrightarrow 2$. The event table C.6 is valid if

$$
\begin{array}{r}
S_{\tau} \circ H_{\varepsilon,\left|\delta_{2}\right|} \circ H_{0, \tau}(0)<1 \\
H_{2 \varepsilon, \tau} \circ H_{\varepsilon,\left|\delta_{2}\right|} \circ H_{0, \tau}(0) \geq 1
\end{array}
$$

and

$$
\begin{aligned}
\left|\delta_{2}\right| & <\tau \\
S_{\tau} \circ H_{2 \varepsilon, \tau} \circ H_{0,\left|\delta_{2}\right|} \circ H_{\varepsilon, \tau}\left(-\left|\delta_{2}\right|\right) & <1 \\
H_{\varepsilon, \tau} \circ H_{2 \varepsilon, \tau} \circ H_{0,\left|\delta_{2}\right|} \circ H_{\varepsilon, \tau}\left(-\left|\delta_{2}\right|\right) & \geq 1
\end{aligned}
$$

and 


$$
\begin{aligned}
S_{\left|\delta_{2}\right|} \circ H_{\varepsilon, \tau}\left(\alpha+\delta_{3,4}\right) & <1 \\
H_{\varepsilon,\left|\delta_{2}\right|} \circ H_{\varepsilon, \tau}\left(\alpha+\delta_{3,4}\right) & \geq 1 \\
H_{\varepsilon, \tau} \circ H_{\varepsilon, \tau} \circ H_{\varepsilon, \tau} \circ J_{\varepsilon,\left|\delta_{2}\right|} \circ H_{\varepsilon, \tau}\left(\alpha+\delta_{3,4}\right) & <1
\end{aligned}
$$

These conditions are all satisfied if (C.2.12), the smallness condition on $J_{\varepsilon}$ and the parameter conditions

$$
\begin{aligned}
S_{\tau} \circ H_{2 \varepsilon, \tau} \circ H_{\varepsilon, \tau}(0) & <1 \\
H_{\varepsilon, \tau} \circ H_{2 \varepsilon, \tau} \circ H_{\varepsilon, \tau}(0) & >1 \\
H_{\varepsilon, \tau} \circ H_{\varepsilon, \tau} \circ H_{\varepsilon, \tau}(0) & <1
\end{aligned}
$$

We used property (7.2.9) for $S_{\tau} \circ H_{2 \varepsilon, \tau} \circ H_{0,\left|\delta_{2}\right|} \circ H_{\varepsilon, \tau}\left(-\left|\delta_{2}\right|\right) \leq S_{\tau} \circ H_{2 \varepsilon, \tau} \circ H_{\varepsilon, \tau}(0)<1$. The parameter conditions follow from (7.2.13).

Note that for $J_{\varepsilon}=0, \delta_{3}^{(1)}=\delta_{4}^{(1)}$.

\section{C.2.3.2 Transient Maps $T_{i j}^{b}$}

Lemma C.2.7. The transient map $T_{12}^{b}: \mathcal{D}\left(T_{12}^{b}\right) \subset \mathcal{P}_{1} \rightarrow \mathcal{P}_{2} \delta^{(0)} \mapsto \delta^{(1)}$ with event sequence

$$
E\left(T_{12}^{b}\right)=\left(s_{1}\right)\left(r_{1}, s_{2}\right)\left(r_{2}, s_{3}, s_{4}\right)\left(r_{3}, r_{4}, s_{1}\right)\left(r_{1}, s_{2}\right)\left(r_{2}\right)
$$

is for $\delta_{2}^{(0)}<0$ given by

$$
\begin{aligned}
\delta_{1}^{(1)} & =H_{\varepsilon, \tau} \circ H_{\varepsilon, \tau} \circ J_{2 \varepsilon, \tau} \circ H_{\varepsilon, \tau} \circ H_{0, \tau}(0)+1-\phi_{3,5}-\alpha \\
\delta_{2}^{(1)} & =H_{0, \tau} \circ J_{\varepsilon, \tau} \circ H_{2 \varepsilon, \tau} \circ H_{0, \tau} \circ J_{\varepsilon, \tau}\left(1-\left|\delta_{2}^{(0)}\right|\right)+1-\phi_{3,5}-\alpha \\
\delta_{3}^{(1)} & =0 \\
\delta_{4}^{(1)} & =H_{\varepsilon, \tau} \circ H_{\varepsilon, \tau} \circ H_{\varepsilon, \tau} \circ J_{\varepsilon, \tau} \circ H_{\varepsilon, \tau}\left(\alpha+\delta_{4}^{(0)}\right)-\phi_{3,5}
\end{aligned}
$$

where

$$
\phi_{3,5}=H_{\varepsilon, \tau} \circ H_{\varepsilon, \tau} \circ H_{\varepsilon, \tau} \circ J_{\varepsilon, \tau} \circ H_{\varepsilon, \tau}\left(\alpha+\delta_{3}^{(0)}\right)
$$

For $\delta_{2}^{(0)}>0$ exchange indices $1 \leftrightarrow 2$. The domain $\mathcal{D}\left(T_{12}^{b}\right)$ is given by the inequalities

$$
\begin{aligned}
\left|\delta_{2}\right| & \geq \tau \\
H_{\varepsilon, \tau}\left(1-\left|\delta_{2}\right|\right) & \geq 1 \\
S_{\tau} \circ H_{\varepsilon, \tau}\left(\alpha+\delta_{3,4}\right) & <1 \\
H_{\varepsilon, \tau} \circ H_{\varepsilon, \tau}\left(\alpha+\delta_{3,4}\right) & \geq 1
\end{aligned}
$$

The map $T_{21}^{b}$ is obtained by exchanging indices $(1,2) \leftrightarrow(3,4)$. 
Proof. The analytical form follows from the event based analysis is given in tab. C.7 for states $\left(\delta_{2}, \delta_{3}, \delta_{4}\right) \in \mathcal{P}_{1}$ assuming $\delta_{2}<0$. For $\delta_{2}>0$ the event sequence is given by relabeling $1 \leftrightarrow 2$. The event table C.7 is valid if

$$
\begin{array}{r}
S_{\tau} \circ H_{\varepsilon, \tau}(\tau) \leq 1 \\
H_{2 \varepsilon, \tau} \circ H_{\varepsilon, \tau}(\tau) \geq 1
\end{array}
$$

and

$$
\begin{aligned}
S_{\tau}\left(1+\delta_{2}\right) & \leq 1 \\
H_{\varepsilon, \tau}\left(1+\delta_{2}\right) & \geq 1 \\
S_{\tau} \circ H_{2 \varepsilon, \tau} \circ H_{0, \tau} \circ J_{\varepsilon, \tau}\left(1+\delta_{2}\right) & <1 \\
H_{\varepsilon, \tau} \circ H_{2 \varepsilon, \tau} \circ H_{0, \tau} \circ J_{\varepsilon, \tau}\left(1+\delta_{2}\right) & \geq 1
\end{aligned}
$$

and

$$
\begin{aligned}
S_{\tau} \circ H_{\varepsilon, \tau}\left(\alpha+\delta_{3,4}\right) & <1 \\
H_{\varepsilon, \tau} \circ H_{\varepsilon, \tau}\left(\alpha+\delta_{3,4}\right) & \geq 1 \\
H_{\varepsilon, \tau} \circ H_{\varepsilon, \tau} \circ H_{\varepsilon, \tau} \circ J_{\varepsilon, \tau} \circ H_{\varepsilon, \tau}\left(\alpha+\delta_{3,4}\right) & <1
\end{aligned}
$$

The first conditions are parameter conditions satisfied by (7.2.13). The second and third conditions follow from (C.2.15), the smallness assumption of $J_{\varepsilon}(7.2 .23)$ and the pure parameter conditions

$$
\begin{aligned}
S_{\tau} \circ H_{2 \varepsilon, \tau} \circ H_{0, \tau}(0) & <1 \\
H_{\varepsilon, \tau} \circ H_{2 \varepsilon, \tau} \circ H_{0, \tau}(0) & \geq 1 \\
H_{\varepsilon, \tau} \circ H_{\varepsilon, \tau} \circ H_{\varepsilon, \tau}(0) & <1
\end{aligned}
$$

which follow from (7.2.13).

\section{C.2.3.3 Transient Map $T_{12}^{c}$}

Lemma C.2.8. The transient map $T_{12}^{c}: \mathcal{D}\left(T_{12}^{c}\right) \subset \mathcal{P}_{1} \rightarrow \mathcal{P}_{2} \delta^{(0)} \mapsto \delta^{(1)}$ with event sequence

$$
E\left(T_{12}^{c}\right) \doteq\left(s_{1}\right)\left(r_{1}, s_{2}\right)\left(r_{2}\right)
$$

for $\delta_{2}^{(0)}<0$ is given by

$$
\begin{aligned}
\delta_{1}^{(1)} & =H_{\varepsilon, \tau} \circ H_{0, \tau}(\tau)+1-\phi_{3,2}-\alpha \\
\delta_{2}^{(1)} & =H_{0, \tau} \circ J_{\varepsilon, \tau}\left(1+\delta_{2}^{(0)}\right)+1-\phi_{3,2}-\alpha \\
\delta_{3}^{(1)} & =0 \\
\delta_{4}^{(1)} & =H_{\varepsilon, \tau} \circ H_{\varepsilon, \tau}\left(\alpha+\delta_{4}^{(0)}\right)-\phi_{3,2}
\end{aligned}
$$

where

$$
\phi_{3,2}=H_{\varepsilon, \tau} \circ H_{\varepsilon, \tau}\left(\alpha+\delta_{3}^{(0)}\right)
$$


For $\delta_{2}^{(0)}>0$ exchange indices $1 \leftrightarrow 2$. The domain $\mathcal{D}\left(T_{12}^{c}\right)$ is given by the inequalities

$$
\begin{aligned}
\left|\delta_{2}\right| & \geq \tau \\
H_{\varepsilon, \tau}\left(1-\left|\delta_{2}\right|\right) & \geq 1 \\
\alpha+\delta_{3,4} & >0 \\
H_{\varepsilon, \tau} \circ H_{\varepsilon, \tau}\left(\alpha+\delta_{3,4}\right) & <1
\end{aligned}
$$

The map $T_{21}^{c}$ is obtained by exchanging $(1,2) \leftrightarrow(3,4)$.

Proof. The analytical form follows from the event based analysis is given in tab. C.8 for states $\left(\delta_{2}, \delta_{3}, \delta_{4}\right) \in \mathcal{P}_{1}$ assuming $\delta_{2}<0$. For $\delta_{2}>0$ the event sequence is given by relabeling $1 \leftrightarrow 2$. The event table C. 8 is valid if

$$
\begin{aligned}
S_{\tau}\left(1+\delta_{2}\right) & \leq 1 \\
H_{\varepsilon, \tau}\left(1+\delta_{2}\right) & \geq 1 \\
H_{\varepsilon, \tau} \circ J_{\varepsilon, \tau}\left(1+\delta_{2}\right) & <1
\end{aligned}
$$

and

$$
\begin{aligned}
\alpha+\delta_{3,4} & >0 \\
H_{\varepsilon, \tau} \circ H_{\varepsilon, \tau}\left(\alpha+\delta_{3,4}\right) & <1
\end{aligned}
$$

which are satisfied if (C.2.18), the smallness condition on $J_{\varepsilon} 7.2 .23$ and

$$
H_{\varepsilon, \tau} \circ H_{\varepsilon, \tau}(0)<1
$$

is satisfied. The last inequality follows from the parameter conditions (7.2.13).

\section{C.2.3.4 Transient Maps $T_{i j}^{d}$}

Lemma C.2.9. The transient map $T_{12}^{d}: \mathcal{D}\left(T_{12}^{d}\right) \subset \mathcal{P}_{1} \rightarrow \mathcal{P}_{2} \delta^{(0)} \mapsto \delta^{(1)}$ with event sequence

$$
E\left(T_{12}^{d}\right) \doteq\left(s_{1}\right)\left(r_{1}, s_{2}\right)\left(r_{2}, s_{3}\right)\left(s_{4}\right)\left(r_{3}\right)\left(r_{4}, s_{1}\right)\left(r_{1}, s_{2}\right)\left(r_{2}\right)
$$

for $\delta_{2}^{(0)}<0$ and $\delta_{3}^{(0)}>\delta_{4}^{(0)}$ is given by

$$
\begin{aligned}
\delta_{1}^{(0)} & =H_{\varepsilon, \tau} \circ H_{0, \tau} \circ J_{\varepsilon, 1-\phi_{4,2}} \circ H_{\varepsilon, \tau} \circ H_{\varepsilon, \tau} \circ H_{0, \tau}(0)+1-\phi_{3,7}-\alpha \\
\delta_{2}^{(0)} & =H_{0, \tau} \circ J_{\varepsilon, \tau} \circ H_{\varepsilon, 1-\phi_{4,2}} \circ H_{\varepsilon, \tau} \circ H_{0, \tau} \circ J_{\varepsilon, \tau}\left(1-\left|\delta_{2}^{(0)}\right|\right)+1-\phi_{3,7}-\alpha \\
\delta_{3}^{(1)} & =0 \\
\delta_{4}^{(1)} & =H_{\varepsilon, \tau} \circ H_{\varepsilon, \tau} \circ H_{0,1-\phi_{4,2}} \circ H_{\varepsilon, \tau}\left(-\left(1-\phi_{4,2}\right)\right)+1-\phi_{3,7}
\end{aligned}
$$

where

$$
\begin{aligned}
& \phi_{4,2}=H_{\varepsilon, \tau} \circ H_{\varepsilon, \tau}\left(\alpha+\delta_{4}\right) \\
& \phi_{3,7}=H_{\varepsilon, \tau} \circ H_{\varepsilon, \tau} \circ H_{\varepsilon, 1-\phi_{4,2}} \circ H_{\varepsilon, \tau}\left(-\left(1-\phi_{4,2}\right)\right)
\end{aligned}
$$


For $\delta_{2}^{(0)}>0$ exchange indices $1 \leftrightarrow 2$, for $\delta_{3}^{(0)}<\delta_{4}^{(0)}$ exchange indices $3 \leftrightarrow 4$. The domain $\mathcal{D}\left(T_{12}^{d}\right)$ for $\delta_{3}>\delta_{4}$ is given by the inequalities

$$
\begin{aligned}
\left|\delta_{2}\right| & >\tau \\
H_{\varepsilon, \tau}\left(1-\left|\delta_{2}\right|\right) & \geq 1 \\
S_{\tau} \circ H_{\varepsilon, \tau}\left(\alpha+\delta_{3}\right) & \leq 1 \\
H_{\varepsilon, \tau} \circ H_{\varepsilon, \tau}\left(\alpha+\delta_{3}\right) & \geq 1 \\
H_{\varepsilon, \tau} \circ H_{\varepsilon, \tau}\left(\alpha+\delta_{4}\right) & <1 \\
H_{\varepsilon, \tau} \circ H_{\varepsilon, \tau} \circ H_{\varepsilon, \tau}\left(1-\phi_{4,2}\right) & <1
\end{aligned}
$$

together with the part determined by exchanging $\delta_{3} \leftrightarrow \delta_{4}$. The map $T_{21}^{d}$ is obtained by exchanging indices $(1,2) \leftrightarrow(3,4)$.

Proof. The analytical form follows from the event based analysis is given in tab. C.9 for states $\left(\delta_{2}, \delta_{3}, \delta_{4}\right) \in \mathcal{P}_{1}$ assuming $\delta_{2}<0$ and $\delta_{3}>\delta_{4}$. For $\delta_{2}>0$ the event sequence is given by relabeling $1 \leftrightarrow 2$ and for $\delta_{3}<\delta_{4}$ by relabeling $3 \leftrightarrow 4$. The event table C.9 is valid if

$$
\begin{aligned}
S_{1-\phi_{4,2}} \circ H_{\varepsilon, \tau} \circ H_{\varepsilon, \tau} \circ H_{0, \tau}(0) & \leq 1 \\
H_{\varepsilon, 1-\phi_{4,2}} \circ H_{\varepsilon, \tau} \circ H_{\varepsilon, \tau} \circ H_{0, \tau}(0) & \geq 1 \\
H_{\varepsilon, \tau} \circ H_{0, \tau} \circ J_{\varepsilon, 1-\phi_{4,2}} \circ H_{\varepsilon, \tau} \circ H_{\varepsilon, \tau} \circ H_{0, \tau}(0) & <1
\end{aligned}
$$

and

$$
\begin{aligned}
S_{\tau}\left(1+\delta_{2}\right) & \leq 1 \\
H_{\varepsilon, \tau}\left(1+\delta_{2}\right) & \geq 1 \\
S_{\tau} \circ H_{\varepsilon, 1-\phi_{4,2}} \circ H_{\varepsilon, \tau} \circ H_{0, \tau} \circ J_{\varepsilon, \tau}\left(1+\delta_{2}\right) & \leq 1 \\
H_{\varepsilon, \tau} \circ H_{\varepsilon, 1-\phi_{4,2}} \circ H_{\varepsilon, \tau} \circ H_{0, \tau} \circ J_{\varepsilon, \tau}\left(1+\delta_{2}\right) & \geq 1
\end{aligned}
$$

and

$$
\begin{aligned}
S_{\tau} \circ H_{\varepsilon, \tau}\left(\alpha+\delta_{3}\right) & \leq 1 \\
H_{\varepsilon, \tau} \circ H_{\varepsilon, \tau}\left(\alpha+\delta_{3}\right) & \geq 1 \\
H_{\varepsilon, \tau} \circ H_{\varepsilon, \tau} \circ H_{\varepsilon, 1-\phi_{4,2}} \circ H_{0, \tau} \circ J_{\varepsilon, \tau} \circ H_{\varepsilon, \tau}\left(\alpha+\delta_{3}\right) & <1
\end{aligned}
$$

and

$$
\begin{aligned}
H_{\varepsilon, \tau} \circ H_{\varepsilon, \tau}\left(\alpha+\delta_{4}\right) & <1 \\
0<1-\phi_{4,2}=1-H_{\varepsilon, \tau} \circ H_{\varepsilon, \tau}\left(\alpha+\delta_{4}\right) & <\tau \\
H_{\varepsilon, \tau} \circ H_{\varepsilon, \tau} \circ H_{0,1-\phi_{4,2}} \circ H_{\varepsilon, \tau}\left(-1+\phi_{4,2}\right) & <1
\end{aligned}
$$

which are using properties (7.2.13) are all satisfied if (C.2.21), (7.2.23) and the parameter conditions (7.2.13) hold. 
Table C.7: Transient map $T_{12}^{b}$ for $\delta_{2}<0$

\begin{tabular}{|c|c|c|c|c|c|c|c|c|c|}
\hline event & time & $\phi_{1}$ & $\sigma_{1}$ & $\phi_{2}$ & $\sigma_{2}$ & $\phi_{3}$ & $\sigma_{3}$ & $\phi_{4}$ & $\sigma_{4}$ \\
\hline$s_{1}$ & 0 & 0 & 0 & $1+\delta_{2}$ & - & $\alpha+\delta_{3}$ & - & $\alpha+\delta_{4}$ & - \\
\hline$r_{1}, s_{2}$ & $\tau$ & $\phi_{1,1}:=H_{0}(\tau)$ & $\tau \rightarrow-$ & $\phi_{2,1}:=J_{\varepsilon}\left(1+\delta_{2}+\tau\right)$ & 0 & $\phi_{3,1}:=H_{\varepsilon}\left(\alpha+\delta_{3}+\tau\right)$ & - & $\phi_{4,1}:=H_{\varepsilon}\left(\alpha+\delta_{4}+\tau\right)$ & - \\
\hline$r_{2}, s_{3}, s_{4}$ & $2 \tau$ & $\phi_{1,2}:=H_{\varepsilon}\left(\phi_{1,1}+\tau\right)$ & - & $\phi_{2,2}:=H_{0}\left(\phi_{2,1}+\tau\right)$ & $\tau \rightarrow-$ & $\phi_{3,2}:=J_{\varepsilon}\left(\phi_{3,1}+\tau\right)$ & 0 & $\phi_{4,2}:=J_{\varepsilon}\left(\phi_{4,1}+\tau\right)$ & 0 \\
\hline$r_{3}, r_{4}, s_{1}$ & $3 \tau$ & $\phi_{1,3}:=J_{2 \varepsilon}\left(\phi_{1,2}+\tau\right)$ & 0 & $\phi_{2,3}:=H_{2 \varepsilon}\left(\phi_{2,2}+\tau\right)$ & - & $\phi_{3,3}:=H_{\varepsilon}\left(\phi_{3,2}+\tau\right)$ & $\tau \rightarrow-$ & $\phi_{4,3}:=H_{\varepsilon}\left(\phi_{4,2}+\tau\right)$ & $\tau \rightarrow-$ \\
\hline$r_{1}, s_{2}$ & $4 \tau$ & $\phi_{1,4}:=H_{0}\left(\phi_{1,3}+\tau\right)$ & $\tau \rightarrow-$ & $\phi_{2,4}:=J_{\varepsilon}\left(\phi_{2,3}+\tau\right)$ & 0 & $\phi_{3,4}:=H_{\varepsilon}\left(\phi_{3,3}+\tau\right)$ & - & $\phi_{4,4}:=H_{\varepsilon}\left(\phi_{4,3}+\tau\right)$ & - \\
\hline$r_{2}$ & $5 \tau$ & $\phi_{1,5}:=H_{\varepsilon}\left(\phi_{1,4}+\tau\right)$ & - & $\phi_{2,5}:=H_{0}\left(\phi_{2,4}+\tau\right)$ & $\tau \rightarrow-$ & $\phi_{3,5}:=H_{\varepsilon}\left(\phi_{3,4}+\tau\right)$ & - & $\phi_{4,5}:=H_{\varepsilon}\left(\phi_{4,4}+\tau\right)$ & - \\
\hline$s_{3}$ & $5 \tau+1-$ & $\phi_{1,6}:=\phi_{1,5}+1-\phi_{3,5}$ & - & $\phi_{2,6}:=\phi_{2,5}+1-\phi_{3,5}$ & - & 0 & 0 & $\phi_{4,6}:=\phi_{4,5}+1-\phi_{3,5}$ & \\
\hline
\end{tabular}

Table C.8: Transient map $T_{12}^{c}$ for $\delta_{2}<0$

\begin{tabular}{|c|c|c|c|c|c|c|c|c|c|}
\hline event & time & $\phi_{1}$ & $\sigma_{1}$ & $\phi_{2}$ & $\sigma_{2}$ & $\phi_{3}$ & $\sigma_{3}$ & $\phi_{4}$ & $\sigma_{4}$ \\
\hline$s_{1}$ & 0 & 0 & 0 & $1+\delta_{2}$ & - & $\alpha+\delta_{3}$ & - & $\alpha+\delta_{4}$ & - \\
\hline$r_{1}, s_{2}$ & $\tau$ & $\phi_{1,1}:=H_{0}(\tau)$ & $\tau \rightarrow$ & $-\phi_{2,1}:=J_{\varepsilon}\left(1+\delta_{2}+\tau\right)$ & 0 & $\phi_{3,1}:=H_{\varepsilon}\left(\alpha+\delta_{3}+\tau\right)$ & & $\phi_{4,1}:=H_{\varepsilon}\left(\alpha+\delta_{4}+\tau\right)$ & - \\
\hline$r_{2}$ & $2 \tau$ & $\phi_{1,2}:=H_{\varepsilon}\left(\phi_{1,1}+\tau\right)$ & - & $\phi_{2,2}:=H_{0}\left(\phi_{2,1}+\tau\right)$ & $\tau \rightarrow-$ & $\phi_{3,2}:=H_{\varepsilon}\left(\phi_{3,1}+\tau\right)$ & & $\phi_{4,2}:=H_{\varepsilon}\left(\phi_{4,1}+\tau\right)$ & - \\
\hline$s_{3}$ & $2 \tau+1-$ & $\phi_{1,3}:=\phi_{1,2}+1-\phi_{3,2}$ & - & $\phi_{2,3}:=\phi_{2,1}+1-\phi_{3,2}$ & - & 0 & 0 & $\phi_{4,3}:=\phi_{4,2}+1-\phi_{3,2}$ & - \\
\hline
\end{tabular}


Table C.9: Transient map $T_{12}^{d}$ for $\delta_{2}<0$ and $\delta_{3}>\delta_{4}$

\begin{tabular}{|c|c|c|c|c|c|c|c|c|c|}
\hline event & time & $\phi_{1}$ & $\sigma_{1}$ & $\phi_{2}$ & $\sigma_{2}$ & $\phi_{3}$ & $\sigma_{3}$ & $\phi_{4}$ & $\sigma_{4}$ \\
\hline$s_{1}$ & 0 & 0 & 0 & $1+\delta_{2}$ & - & $\alpha+\delta_{3}$ & - & $\alpha+\delta_{4}$ & - \\
\hline$r_{1}, s_{2}$ & $\tau$ & $\phi_{1,1}:=H_{0}(\tau)$ & $\tau \rightarrow-$ & $\phi_{2,1}:=J_{\varepsilon}\left(1+\delta_{2}+\tau\right)$ & 0 & $\phi_{3,1}:=H_{\varepsilon}\left(\alpha+\delta_{3}+\tau\right)$ & - & $\phi_{4,1}:=H_{\varepsilon}\left(\alpha+\delta_{4}+\tau\right)$ & - \\
\hline$r_{2}, s_{3}$ & $2 \tau$ & $\phi_{1,2}:=H_{\varepsilon}\left(\phi_{1,1}+\tau\right)$ & - & $\phi_{2,2}:=H_{0}\left(\phi_{2,1}+\tau\right)$ & $\tau \rightarrow-$ & $\phi_{3,2}:=J_{\varepsilon}\left(\phi_{3,1}+\tau\right)$ & 0 & $\phi_{4,2}:=H_{\varepsilon}\left(\phi_{4,1}+\tau\right)$ & - \\
\hline$s_{4}$ & $2 \tau+1-\phi_{4,2}$ & $\phi_{1,3}:=\phi_{1,2}+1-\phi_{4,2}$ & - & $\phi_{2,3}:=\phi_{2,2}+1-\phi_{4,2}$ & - & $\phi_{3,3}:=\phi_{3,2}+1-\phi_{4,2}$ & $1-\phi_{4,2}$ & 0 & 0 \\
\hline$r_{3}$ & $3 \tau$ & $\phi_{1,4}:=H_{\varepsilon}\left(\phi_{1,2}+\tau\right)$ & - & $\phi_{2,4}:=H_{\varepsilon}\left(\phi_{2,2}+\tau\right)$ & - & $\phi_{3,4}:=H_{0}\left(\phi_{3,2}+\tau\right)$ & $\tau \rightarrow-$ & $\phi_{4,4}:=H_{\varepsilon}\left(\tau-1+\phi_{4,2}\right)$ & $\tau-1+\phi_{4,2}$ \\
\hline$r_{4}, s_{1}$ & $3 \tau+1-\phi_{4,2}$ & $\phi_{1,5}:=J_{\varepsilon}\left(\phi_{1,4}+1-\phi_{4,2}\right)$ & 0 & $\phi_{2,5}:=H_{\varepsilon}\left(\phi_{2,4}+1-\phi_{4,2}\right)$ & - & $\phi_{3,5}:=H_{\varepsilon}\left(\phi_{3,4}+1-\phi_{4,2}\right)$ & - & $\phi_{4,5}:=H_{0}\left(\phi_{4,4}+1-\phi_{4,2}\right)$ & $\tau \rightarrow-$ \\
\hline$r_{1}, s_{2}$ & $4 \tau+1-\phi_{4,2}$ & $\phi_{1,6}:=H_{0}\left(\phi_{1,5}+\tau\right)$ & $\tau \rightarrow-$ & $\phi_{2,6}:=J_{\varepsilon}\left(\phi_{2,5}+\tau\right)$ & 0 & $\phi_{3,6}:=H_{\varepsilon}\left(\phi_{3,5}+\tau\right)$ & - & $\phi_{4,6}:=H_{\varepsilon}\left(\phi_{4,5}+\tau\right)$ & - \\
\hline$r_{2}$ & $t_{7}:=5 \tau+1-\phi_{4,2}$ & $\phi_{1,7}:=H_{\varepsilon}\left(\phi_{1,6}+\tau\right)$ & - & $\phi_{2,7}:=H_{0}\left(\phi_{2,6}+\tau\right)$ & $\tau \rightarrow-$ & $\phi_{3,7}:=H_{\varepsilon}\left(\phi_{3,6}+\tau\right)$ & - & $\phi_{4,7}:=H_{\varepsilon}\left(\phi_{4,6}+\tau\right)$ & - \\
\hline$s_{3}^{\prime}$ & $t_{7}+1-\phi_{3,7}$ & $\phi_{1,8}:=\phi_{1,7}+1-\phi_{3,7}$ & - & $\phi_{2,8}:=\phi_{2,7}+1-\phi_{3,7}$ & - & 0 & 0 & $\phi_{4,8}:=\phi_{4,7}+1-\phi_{3,7}$ & - \\
\hline
\end{tabular}




\section{Appendix D}

\section{Conductance-Based Neuron Models}

Here we list all the parameters and functions for the different neuron models used in this thesis. If no parameter values are specified explicitly the values listed here are used and referred to as standard parameter.

\section{D.1 The Hodgkin Huxley Model}

The Hodgkin Huxley model $[172,171]$ is defined by equation (2.4.1) and (2.3.2) with

$$
\begin{array}{ll}
\alpha_{m}(v)=\Phi(T) \frac{0.1(25-v)}{\exp (0.1(25-v))-1} & \beta_{m}(v)=\Phi(T) 4.0 \exp \left(-\frac{v}{18}\right) \\
\alpha_{h}(v)=\Phi(T) 0.07 \exp \left(-\frac{v}{20}\right) & \beta_{h}(v)=\Phi(T) \frac{1.0}{1+\exp (0.1(30-v))} \\
\alpha_{n}(v)=\Phi(T) \frac{0.01(10-v)}{\exp (0.1(10-v))-1} & \beta_{n}(v)=\Phi(T) 0.125 \exp \left(-\frac{v}{80}\right)
\end{array}
$$

and temperature dependence

$$
\Phi(T)=3^{\frac{T-6.3}{10}}
$$

Specific standard parameters are $c_{\mathrm{S}}=1 \frac{\mu \mathrm{F}}{\mathrm{cm}^{2}}, g_{\mathrm{Na}}=120 \frac{\mathrm{mS}}{\mathrm{cm}^{2}}, g_{\mathrm{K}}=36 \frac{\mathrm{mS}}{\mathrm{cm}^{2}}, g_{\mathrm{L}, \mathrm{HH}}=0.3 \frac{\mathrm{mS}}{\mathrm{cm}^{2}}$, $v_{\mathrm{Na}}=115 \mathrm{mV}, v_{\mathrm{K}}=-12.0 \mathrm{mV}, v_{\mathrm{L}, \mathrm{HH}}=-10.599 \mathrm{mV}$ and $T=6.3^{\mathrm{O}} \mathrm{C}$. For the bifurcation analysis we added an extra leak current $g_{\mathrm{L}}\left(v_{\mathrm{L}}-v\right)$ mimicking a shunting synapse with $v_{\mathrm{L}}=-60 \mathrm{mV}$ and $g_{\mathrm{L}}=0 \frac{\mathrm{mS}}{\mathrm{cm}^{2}}$. Time is measured in milliseconds.

\section{D.2 The Morris-Lecar Neuron Model}

The Morris-Lecar neuron model [273] is defined by equations (2.4.4).

The temperature constant $\Phi^{-1}=15$ equivalent to a room temperate of $22^{\circ} \mathrm{C}$ is used and specific standard parameters are $c_{\mathrm{s}}=20 \frac{\mu \mathrm{F}}{\mathrm{cm}^{2}}, g_{\mathrm{Na}}=4.0 \frac{\mathrm{mS}}{\mathrm{cm}^{2}}, g_{\mathrm{K}}=8.0 \frac{\mathrm{mS}}{\mathrm{cm}^{2}}, g_{\mathrm{L}}=$ $2.0 \frac{\mathrm{mS}}{\mathrm{cm}^{2}}, v_{\mathrm{Na}}=120 \mathrm{mV}, v_{\mathrm{K}}=-80.0 \mathrm{mV}, v_{\mathrm{L}}=-60 \mathrm{mV}$. Half-activation and slope of the activation curves are $v_{1}=-1.2 \mathrm{mV}, v_{2}=18 \mathrm{mV}, v_{3}=12 \mathrm{mV}$ and $v_{4}=17.4 \mathrm{mV}$. These parameters are from $[316,100]$. 


\section{D.3 The Wang-Buzsaki Neuron Model}

The Wang-Buzsaki neuron model [400] is defined by equation (2.4.1) with $m$ substituted by $m_{\infty}$ and (2.3.2) for the gating variables. The rate constants are given by:

$$
\begin{array}{rlrl}
\alpha_{m}(v) & =\frac{0.1(v+35)}{1-\exp (-0.1(v+35))} & \beta_{m}(v)=4.0 \exp \left(-\frac{(v+60)}{18}\right) \\
\alpha_{h}(v)=0.07 \exp \left(-\frac{(v+58)}{20}\right) & \beta_{h}(v)=\frac{1.0}{1+\exp (-0.1(v+28))} \\
\alpha_{n}(v)=\frac{0.01(v+34)}{1-\exp (-0.1(v+34))} & \beta_{n}(v)=0.125 \exp \left(-\frac{(v+44)}{80}\right)
\end{array}
$$

Specific standard parameters are $c_{\mathrm{s}}=1 \frac{\mu \mathrm{F}}{\mathrm{cm}^{2}}, g_{\mathrm{Na}}=35 \frac{\mathrm{mS}}{\mathrm{cm}^{2}}, g_{\mathrm{K}}=9 \frac{\mathrm{mS}}{\mathrm{cm}^{2}}, g_{\mathrm{L}}=0.1 \frac{\mathrm{mS}}{\mathrm{cm}^{2}}$, $v_{\mathrm{Na}}=35 \mathrm{mV}, v_{\mathrm{K}}=-90 \mathrm{mV}$ and $v_{\mathrm{L}}=-65 \mathrm{mV}$.

\section{D.4 The Fast Spiking Neuron Model by Erisir et al.}

The fast spiking model by Erisir et al. [99] is defined by equation (2.4.5) and (2.3.2) for the gating variables with

$$
\begin{array}{ll}
\alpha_{m}(v)=\frac{(40 v+3020)}{1-\exp (-(v-75.5) / 13.5)} & \beta_{m}(v)=1.2262 \exp \left(-\frac{v}{42.248}\right) \\
\alpha_{h}(v)=0.0035 \exp \left(-\frac{v}{24.186}\right) & \beta_{h}(v)=0.017 \frac{(51.25+v)}{1-\exp (-(51.25+v) / 5.2)} \\
\alpha_{n}(v)=\frac{(v-95)}{1-\exp (-(v-95) / 11.8)} & \beta_{n}(v)=0.025 \exp \left(-\frac{v}{22.2}\right) .
\end{array}
$$

Specific standard parameters are $c_{\mathrm{S}}=1 \frac{\mu \mathrm{F}}{\mathrm{cm}^{2}}, g_{\mathrm{Na}}=112.5 \frac{\mathrm{mS}}{\mathrm{cm}^{2}}, g_{\mathrm{K}}=225 \frac{\mathrm{mS}}{\mathrm{cm}^{2}}, g_{\mathrm{L}}=0.25 \frac{\mathrm{mS}}{\mathrm{cm}^{2}}$, $v_{\mathrm{Na}}=74 \mathrm{mV}, v_{\mathrm{K}}=-90 \mathrm{mV}$ and $v_{\mathrm{L}}=-70 \mathrm{mV}$.

\section{D.5 The Connor-Stevens and Rose-Hindmarsh Neuron Model}

The Connor-Stevens model $[61,62,63]$ is defined by 2.4.6 and (2.3.2) with rate functions

$$
\begin{array}{rlrl}
\alpha_{m}(v) & =\Phi_{m} \frac{0.1(v+29.7)}{1-\exp (-0.1(v+29.7))} & \beta_{m}(v)=\Phi_{m} 4.0 \exp \left(-\frac{(v+54.7)}{18}\right) \\
\alpha_{h}(v)=\Phi_{h} 0.07 \exp \left(-\frac{(v+48)}{20}\right) & \beta_{h}(v)=\Phi_{h} \frac{1.0}{1+\exp (-0.1(v+18))} \\
\alpha_{n}(v)=\Phi_{n} \frac{0.01(v+45.7)}{1-\exp (-0.1(v+45.7))} & \beta_{n}(v)=\Phi_{n} 0.125 \exp \left(-\frac{(v+55.7)}{80}\right)
\end{array}
$$


and steady state activation and time constants for the A-type channel given by

$$
\begin{array}{ll}
a_{\infty}(v)=0.0761 \exp \left(-\frac{(v+94.22)}{31.84}\right) & \tau_{a}(v)=0.3632+\frac{1.158}{1+\exp ((v+55.96) / 20.12)} \\
b_{\infty}(v)=\left[\frac{1}{1+\exp \left(\gamma_{b}(v+53.3)\right)}\right]^{4} & \tau_{b}(v)=1.24+\frac{2.678}{1+\exp ((v+50) / 16.027)} \\
\alpha_{n}(v)=\frac{0.01(v+45.7)}{1-\exp (-0.1(v+45.7))} & \beta_{n}(v)=0.125 \exp \left(-\frac{(v+55.7)}{80}\right)
\end{array}
$$

The Rose-Hindmarsh neuron model $[319,320]$ is a reduction of the Connor-Stevens model defined by equations (2.4.5) and (2.3.2) with effective activation $q$

$$
q_{\infty}(v)=n_{\infty}(v)^{4}+A b_{\infty}(v) \quad \text { and } \quad \tau_{q}(v)=\frac{1}{2}\left(\tau_{b}(v)+\tau_{n}(v)\right)
$$

Specific standard parameters for both models are $c_{\mathrm{s}}=1 \frac{\mu \mathrm{F}}{\mathrm{cm}^{2}}, g_{\mathrm{Na}}=120 \frac{\mathrm{mS}}{\mathrm{cm}^{2}}, g_{\mathrm{K}}=20 \frac{\mathrm{mS}}{\mathrm{cm}^{2}}$, $g_{\mathrm{A}}=47.7 \frac{\mathrm{mS}}{\mathrm{cm}^{2}}, g_{\mathrm{L}, \mathrm{CS}}=0.3 \frac{\mathrm{mS}}{\mathrm{cm}^{2}}, v_{\mathrm{Na}}=55 \mathrm{mV}, v_{\mathrm{K}}=v_{\mathrm{A}}=-72 \mathrm{mV}$ and $v_{\mathrm{L}, \mathrm{CS}}=-17 \mathrm{mV}$. As the reversal potential of the leak is to high for inhibitory shunting we added a second leak current with standard values $g_{\mathrm{L}}=0 \frac{\mathrm{mS}}{\mathrm{cm}^{2}}$ and $v_{\mathrm{L}}=-70 \frac{\mathrm{mS}}{\mathrm{cm}^{2}}$. The dimensionless parameter arising in the reduction in the Rose-Hindmarsh neuron model are $A=0.50085$ and $\gamma_{b}=0.069$. The temperature scalings are $\Phi_{m}=\Phi_{h}=0.26$ and $\Phi_{n}=0.52$.

\section{D.6 The Simplified Traub-Miles Pyramidal Neuron Model}

The simplified Traub-Miles [375, 103] model is defined by

$$
\begin{aligned}
\frac{d}{d t} v & =i_{\mathrm{e}}+i_{\mathrm{syn}}+g_{\mathrm{L}}\left(v_{\mathrm{L}}-v\right)+g_{\mathrm{Na}} m_{\infty}(v) h(n)\left(v_{\mathrm{Na}}-v\right)+g_{\mathrm{K}} n^{4}\left(v_{\mathrm{K}}-v\right) \\
\frac{d}{d t} n & =(1-n) \alpha_{n}(v)-n \beta_{n}(v)
\end{aligned}
$$

The steady state functions and time constants are inferred form the rates

$$
\begin{aligned}
\alpha_{m}(v) & =\frac{0.32(v+54)}{1-\exp (-(v+54) / 4)} & \beta_{m}(v) & =\frac{0.28(v+27)}{\exp ((v+27) / 5)-1} \\
\alpha_{n}(v) & =\frac{0.032(v+52)}{1-\exp (-(v+52) / 5)} & \beta_{n}(v) & =0.5 \exp \left(-\frac{(v+57)}{40}\right)
\end{aligned} .
$$

Specific standard parameters are $c_{\mathrm{s}}=1 \frac{\mu \mathrm{F}}{\mathrm{cm}^{2}}, g_{\mathrm{Na}}=100 \frac{\mathrm{mS}}{\mathrm{cm}^{2}}, g_{\mathrm{K}}=80 \frac{\mathrm{mS}}{\mathrm{cm}^{2}}, g_{\mathrm{L}}=0.1 \frac{\mathrm{mS}}{\mathrm{cm}^{2}}$, $v_{\mathrm{Na}}=50 \mathrm{mV}, v_{\mathrm{K}}=-100 \mathrm{mV}$ and $v_{\mathrm{L}}=-67 \mathrm{mV}$.

\section{D.7 The Rinzel Neuron Model}

The Rinzel neuron model [315] arises from a simplification of a Hodgkin-Huzley type neuron model (cf. 2.6) and is defined by equation (2.6.5). The steady state voltages and time constants are derived form the following transition rates 


$$
\begin{aligned}
\alpha_{m}(v) & =\frac{0.1(v+25)}{\exp (0.1(v-25))-1} & \beta_{m}(v) & =4.0 \exp \left(-\frac{v}{18}\right) \\
\alpha_{h}(v) & =0.07 \exp \left(-\frac{v}{20}\right) & \beta_{h}(v) & =\frac{1}{1+\exp (-(51.25+v) / 5.2)} \\
\alpha_{n}(v) & =\frac{0.01(v+10)}{\exp (-0.1(v+10))-1} & \beta_{n}(v) & =0.125 \exp \left(-\frac{v}{80}\right) .
\end{aligned}
$$

The dynamics of the effecitve activation $w$ is defined as

$$
w_{\infty}(v)=\frac{s}{1+s^{2}}\left(n_{\infty}(v)+s\left(1-h_{\infty}(v)\right)\right), \quad \tau_{w}(v)=1+5 \exp \left(-\left(\frac{v+60}{55}\right)^{2}\right) .
$$

Specific standard parameters are $c_{\mathrm{S}}=20 \frac{\mu \mathrm{F}}{\mathrm{cm}^{2}}, g_{\mathrm{Na}}=120 \frac{\mathrm{mS}}{\mathrm{cm}^{2}}, g_{\mathrm{K}}=36 \frac{\mathrm{mS}}{\mathrm{cm}^{2}}, g_{\mathrm{L}}=0.3 \frac{\mathrm{mS}}{\mathrm{cm}^{2}}$, $v_{\mathrm{Na}}=50 \mathrm{mV}, v_{\mathrm{K}}=-92 \mathrm{mV}$ and $v_{\mathrm{L}}=-50.528 \mathrm{mV}$ and effective parameter $s=1.271$. 


\section{Appendix E}

\section{Resonance and Impedance in Neuron Models}

To estimate the resonance frequency of neuron models experimentally we fitted the impedance to an RCL circuit. Here we derive the impedance for a general conductance based neuron model. Using this method near the resting potential leads to simplified quasi-linear neuron models [257] that are used to study resonant and fire dynamics as well dynamics on extended dendritic trees [65].

Consider a conductance based neuron model of the form (cf. sec. 2.3)

$$
\begin{aligned}
c_{M} \frac{d}{d t} v & =i_{\mathrm{e}}+g_{\mathrm{L}}\left(v_{\mathrm{L}}-v\right)+\sum_{j} g_{j} m_{j}^{p_{i}} h_{j}^{q_{i}}\left(v_{j}-v\right) \\
\frac{d}{d t} a_{j, k} & =\frac{1}{\tau_{j, k}(v)}\left(a_{j, k, \infty}(v)-a_{j, k}\right)
\end{aligned}
$$

with $m_{j}=a_{j, 1}$ and $h_{j}=a_{j, 2}$. We denote a steady state of the dynamics for an external current $i_{\mathrm{e}}=i_{0}$ by $x_{0}=\left(v_{0}, m_{1,0}, h_{1,0}, \ldots\right)$, set $i_{\mathrm{e}}=i_{0}+\delta i$ and define

$$
g_{j, w, 0}=g_{j, w}\left(v_{0}\right)=g_{j} a_{j, 1, \infty}\left(v_{0}\right)^{p_{i}} a_{j, 2, \infty}\left(v_{0}\right)^{q_{i}}=g_{j} m_{j, 0}^{p_{i}} h_{j, 0}^{q_{i}} .
$$

Linearization around the fixed point in the variables $\delta x=x-x_{0}$ yields

$$
\frac{d}{d t} \delta x=D f\left(x_{0}\right) \delta x
$$

or explicitly

$$
\begin{aligned}
c_{\mathrm{m}} \frac{d}{d t} \delta v & =-g_{\mathrm{L}} \delta v+\sum_{j} g_{j, w 0}\left(v_{j}-v_{0}\right)\left(p_{j} \frac{\delta m_{j}}{m_{j, 0}}+q_{j} \frac{\delta h_{j}}{h_{j, 0}}-\frac{\delta v}{v_{j}-v_{0}}\right)+\delta i(t) \\
\frac{d}{d t} \delta a_{j, k} & =-\frac{\delta a_{j, k}}{\tau_{m, k, 0}}+\left(\frac{a_{j, k, 0}^{\prime}}{\tau_{j, k, 0}}-\frac{a_{j, k, 0} \tau_{j, k, 0}^{\prime}}{\tau_{j, k, 0}^{2}}\right) \delta v=\frac{1}{\tau_{j, k, 0}}\left(-\delta a_{j, k}+\bar{a}_{j, k, 0}^{\prime} \delta v\right)
\end{aligned}
$$

For periodic inputs of the form $\delta i(t)=i_{\delta} e^{i \omega t}$ we take the ansatz for the solutions of the linearized system

$$
x(t)=y e^{i \omega t}
$$

where $y=\left(Z, Z_{m, 1}, Z_{h, 1}, \ldots\right)$. Inserting this into the linearized system yields

$$
Z_{m, j}=\frac{\bar{m}_{j}^{\prime} Z}{i \omega \tau_{m, j, 0}+1}
$$


and thus for the impedance $Z$ we get by setting $i_{\delta}=1$ and

$$
\begin{gathered}
b_{j}\left(v_{0}\right)=g_{j, w, 0}\left(v_{j}-v_{0}\right)\left(\left[p_{j} \frac{\bar{m}_{j, 0}^{\prime}}{m_{j, 0}}+q_{j} \frac{\bar{h}_{j, 0}^{\prime}}{h_{j, 0}}\right] \frac{1}{1+i \omega \tau_{m, j, 0}}-\frac{1}{v_{j}-v_{0}}\right) \\
Z\left(\omega, v_{0}\right)=\frac{1}{i \omega c_{\mathrm{m}}+g_{\mathrm{L}}+\sum_{j} b_{j}\left(v_{0}\right)}
\end{gathered}
$$

This is nothing else than the well know expression for a parallel RLC circuit:

$$
\frac{1}{Z}=\frac{1}{Z_{C}}+\frac{1}{Z_{R}}+\sum_{j} \frac{1}{Z_{j}}
$$

Thus the nonlinear parallel circuit in fig. 2.2 has become a RLC circuit due to linearization around a fixed holding potential. Note that for very fast currents one can approximate $\tau_{m, j} \rightarrow 0$ and obtains a pure real contribution to the impedance.

For a small time dependent inputs with Fourier representation

$$
\delta i(t)=\frac{1}{\sqrt{2 \pi}} \int \delta \tilde{i}(\omega) e^{2 \pi i \omega t} d \omega
$$

we get a linear response

$$
\delta v(t)=\frac{1}{\sqrt{2 \pi}} \int Z(\omega) \delta \tilde{i}(\omega) e^{2 \pi i \omega t} d \omega
$$

or

$$
\delta \tilde{v}(\omega)=Z(\omega) \delta \tilde{i}(\omega)
$$

and thus for the impedance

$$
Z(\omega)=\frac{\delta \tilde{v}(\omega)}{\delta \tilde{i}(\omega)}
$$




\section{Appendix F}

\section{Normal Form Coefficients for the Degenerate Bogdanov-Takens Bifurcation}

In this appendix we derive the normalform coefficients for the degenerate BogdanovTakens bifurcation as calculated in [231]. The technique combines the center manifold reduction with normal form reduction.

We start with a critical ODE with $x \in \mathbb{R}^{d}$

$$
\frac{d}{d t} x=f(x)=D f x+\frac{1}{2} D^{2} f\left(x_{0} ; x, x\right)+\mathcal{O}^{3}(x)
$$

Being at a Bogdanov-Takens point at $x_{0}$, the Jacobian $A=D f\left(x_{0}\right)$ has generalized eigenvectors

$$
A q_{0}=0 \quad A q_{1}=q_{0} \quad A^{T} p_{1}=0 \quad A^{T} p_{0}=p_{1}
$$

with $\left\langle p_{i}, q_{j}\right\rangle=\delta_{i j}$. Further there is a critical center manifold with coordinates $\left(w_{0}, w_{1}\right)$ given by

$$
x=H\left(w_{0}, w_{1}\right)=x_{0}+w_{0} q_{0}+w_{1} q_{1}+\sum_{j, k} \frac{1}{j ! k !} h_{j k} w_{0}^{j} w_{1}^{k}
$$

with $h_{j k} \in \mathbb{R}^{n}$. The critical normal form is then given by $[87,276]$

$$
\begin{aligned}
\frac{d}{d t} w_{0} & =w_{1} \\
\frac{d}{d t} w_{1} & =\beta w_{0} w_{1}+\gamma w_{0}^{2}+\delta w_{0}^{3}+\epsilon w_{0}^{2} w_{1}+\mathcal{O}^{4}\left(w_{0}, w_{1}\right)
\end{aligned}
$$

Differentiating (F.1) with respect to time we obtain

$$
\dot{w}_{0} \partial_{w_{0}} H\left(w_{0}, w_{1}\right)+\dot{w}_{1} \partial_{w_{1}} H\left(w_{0}, w_{1}\right)=f\left(H\left(w_{0}, w_{1}\right)\right)
$$

In normal form theory this is equivalent to the homological equations used to determine the terms that can be removed from the vector field [276]. Comparing coefficients of (10.3.35) and (F.3) give singular linear systems for the $h_{i j}$ from which the normal form coefficients can be calculated. Here we are only interested in $\gamma$ which can be derived by comparing coefficients of $w_{0}^{2}$ to give

$$
A h_{20}=2 \gamma q_{1}-D^{2} f\left(x_{0} ; q_{0}, q_{0}\right)
$$


Multiplying by $p_{1}^{T}$ from the right gives

$$
\gamma=\frac{1}{2} p_{1}^{T} D^{2} f\left(x_{0} ; q_{0}, q_{0}\right),
$$

the result used in theorem 10.3.2. 


\section{Appendix G}

\section{Information Flow in the Ornstein Uhlenbeck Process}

In this section we derive the delayed mutual information and transfer entropy for an multivariate Ornstein-Uhlenbeck (OU) process [384] of the form

$$
d x=G x d t+B d W
$$

with $x=\left(x_{1}, \ldots, x_{n}\right) \in \mathbb{R}^{n}$ and $G$ and $B$ real $n \times n$-matrices. We derive the delayed mutual information in two steps: In section G.1 we first calculate the joint stationary probability distribution for the process for all variables at two instances in time and integrate this distribution over all but two variables to obtain a marginal distribution. In section G.2 we use this distribution to derive the delayed mutual information in the OU process. The result is illustrated in fig. 15.1. The derivation of the delayed transfer entropy is very similar and we give a short outline in section G.3.

\section{G.1 Stationary Joint Distribution}

Here we assume that $G$ has eigenvalues with negative real parts and derive the stationary distribution of (G.1) of being in the state $x_{0}=x(0)$ at time $t=0$ and $x_{d}=x(d)$ at time $t=d$ given by

$$
p_{s}\left(x_{d}, x_{0}\right)=p\left(x_{d} \mid x_{0}\right) p_{s}(x)
$$

The solution of (G.1) is [120]

$$
x(t)=\exp (G t) x(0)+\int_{0}^{t} \exp \left(G\left(t-t^{\prime}\right)\right) B d W\left(t^{\prime}\right)
$$

For deterministic initial conditions $x(0)=x_{0}$ the mean is

$$
\langle x(t)\rangle=\exp (G t) x_{0}=M_{t} x_{0} \quad \text { where } \quad M_{t}:=\exp (G t)
$$

and the variance is given by

$$
\begin{aligned}
\left\langle x(t), x^{T}(t)\right\rangle & =\int_{0}^{t} \exp \left(G\left(t-t^{\prime}\right)\right) B B^{T} \exp \left(G^{T}\left(t-t^{\prime}\right)\right) d t^{\prime} \\
\Sigma_{t} & =\int_{0}^{t} \exp \left(G t^{\prime}\right) B B^{T} \exp \left(G^{T} t^{\prime}\right) d t^{\prime}
\end{aligned}
$$


Thus the probability of being at $x_{t}=x(t)$ starting at $x_{0}=x(0)$ is given by by the normal distribution

$$
\begin{aligned}
p\left(x_{t} \mid x_{0}\right) & =\mathcal{N}_{M_{t} x_{0}, \Sigma_{t}}\left(x_{t}\right) \\
& =N_{M_{t}, \Sigma_{t}} \exp \left(-\frac{1}{2}\left(x_{t}-M_{t} x_{0}\right)^{T} \Sigma_{t}^{-1}\left(x_{t}-M_{t} x_{0}\right)\right)
\end{aligned}
$$

with some normalization constant $N_{M_{t}, \Sigma_{t}}$.

As $G$ has eigenvalues with negative real parts only there is a stationary solution

$$
x_{s}(t)=\int_{-\infty}^{t} \exp \left(G\left(t-t^{\prime}\right)\right) B d W\left(t^{\prime}\right)
$$

with stationary variance matrix given by

$$
\begin{aligned}
\Sigma_{\infty} & =\left\langle x_{s}(t), x_{s}^{T}(t)\right\rangle \\
& =\int_{-\infty}^{0} \exp \left(-G t^{\prime}\right) B B^{T} \exp \left(-G^{T} t^{\prime}\right) d t^{\prime}
\end{aligned}
$$

Hence the stationary probability distribution is

$$
\begin{aligned}
p_{s}(x) & =\mathcal{N}_{0, \Sigma_{\infty}}(x) \\
& =N_{\Sigma_{\infty}} \exp \left(-\frac{1}{2} x^{T} \Sigma_{\infty}^{-1} x\right)
\end{aligned}
$$

Thus (G.1.1) explicitly reads

$$
\begin{aligned}
p_{s}\left(x_{0}, x_{d}\right) & =p\left(x_{d} \mid x_{0}\right) p_{s}\left(x_{0}\right) \\
& =N_{M_{d}, \Sigma_{d}, \Sigma_{\infty}} \exp \left(-\frac{1}{2} x_{0}^{T} \Sigma_{\infty}^{-1} x_{0}-\frac{1}{2}\left(x_{d}-M_{d} x_{0}\right)^{T} \Sigma_{d}^{-1}\left(x_{d}-M_{d} x_{0}\right)\right) \\
& =N_{M_{d}, \Sigma_{d}, \Sigma_{\infty}} \exp \left(-\frac{1}{2}\left(x_{0}^{T}, x_{d}^{T}\right) C_{0, d}^{-1}\left(\begin{array}{c}
x_{0} \\
x_{d}
\end{array}\right)\right)=\mathcal{N}_{0, C_{0, d}}\left(x_{0}, x_{d}\right)
\end{aligned}
$$

with covariance matrix

$$
\begin{aligned}
C_{0, d} & =\left(\begin{array}{cc}
\Sigma_{\infty}^{-1}+M_{d}^{T} \Sigma_{d}^{-1} M_{d} & -M_{d}^{T} \Sigma_{d}^{-1} \\
-\Sigma_{d}^{-1} M_{d} & \Sigma_{d}^{-1}
\end{array}\right)^{-1} \\
& =\left(\begin{array}{cc}
\Sigma_{\infty} & \Sigma_{\infty} M_{d}^{T} \\
M_{d} \Sigma_{\infty} & \Sigma_{d}+M_{d} \Sigma_{\infty} M_{d}^{T}
\end{array}\right)=\left(\begin{array}{cc}
\Sigma_{\infty} & \Sigma_{\infty} M_{d}^{T} \\
M_{d} \Sigma_{\infty} & \Sigma_{\infty}
\end{array}\right)
\end{aligned}
$$

where we used that variances add, i.e. $\Sigma_{d}+M_{d} \Sigma_{\infty} M_{d}^{T}=\Sigma_{\infty}$, in the last equation. Performing the Gaussian integral over all variables except $x_{0, i}$ and $x_{d, j}$ we obtain

$$
p\left(x_{0, i}, x_{d, j}\right)=N_{d, i, j} \exp \left(\left(x_{0, i}, x_{d, j}\right) C_{i, j, d}^{-1}\left(\begin{array}{c}
x_{0, i} \\
x_{d, j}
\end{array}\right)\right)
$$

with covariance matrix

$$
C_{i, j, d}=\left(\begin{array}{cc}
\left(\Sigma_{\infty}\right)_{i i} & \left(\Sigma_{\infty} M_{d}^{T}\right)_{i j} \\
\left(M_{d} \Sigma_{\infty}\right)_{j i} & \left(\Sigma_{\infty}\right)_{j j}
\end{array}\right)
$$




\section{G.2 Delayed Mutual Information}

Using (15.2.1) the mutual information for a two dimensional Gaussian distribution centered around zero with covariance matrix $C$

$$
p\left(x_{1}, x_{2}\right)=\frac{1}{\sqrt{(2 \pi)^{2} \operatorname{det}(C)}} \exp \left(-\frac{1}{2} x C^{-1} x^{T}\right)
$$

and marginal distributions

$$
p\left(x_{i}\right)=\frac{1}{\sqrt{(2 \pi) C_{i i}}} \exp \left(-\frac{1}{2 C_{i i}} x_{i}^{2}\right)
$$

is given by

$$
\begin{aligned}
\mathrm{MI}_{C} & =\iint p\left(x_{1}, x_{2}\right) \log \left(\frac{p\left(x_{1}, x_{2}\right)}{p\left(x_{1}\right) p\left(x_{2}\right)}\right) d x_{1} d x_{2} \\
& =\log \left(\sqrt{\frac{C_{11} C_{22}}{\operatorname{det}(C)}}\right) \\
& +\iint \frac{1}{\sqrt{(2 \pi)^{2} \operatorname{det}(C)}} \exp \left(-\frac{1}{2} x C^{-1} x^{T}\right)\left(-\frac{1}{2} x C^{-1} x^{T}+\frac{1}{2 C_{11}} x_{1}^{2}+\frac{1}{2 C_{22}} x_{2}^{2}\right) d x_{1} d x_{2} \\
& =\log \left(\sqrt{\frac{C_{11} C_{22}}{\operatorname{det}(C)}}\right)-1+\frac{1}{2}+\frac{1}{2} \\
& =-\frac{1}{2} \log \left(1-\frac{C_{12}^{2}}{C_{11} C_{22}}\right)
\end{aligned}
$$

Using the above expression (G.1.3) for the covariance matrix for the OU process we obtain the delayed mutual information

$$
\mathrm{MI}_{i, j}(d)=-\frac{1}{2} \log \left(1-\frac{\left(\Sigma_{\infty} M_{d}^{T}\right)_{i j}^{2}}{\left(\Sigma_{\infty}\right)_{i i}\left(\Sigma_{\infty}\right)_{j j}}\right)
$$

In terms of the coupling matrix $G$ and mixtures of the white noise processes $B$ we obtain the expression

$$
\begin{aligned}
& \mathrm{MI}_{i, j}(d)= \\
& -\frac{1}{2} \log \left(1-\frac{\left(\int_{0}^{\infty}\left(\exp (G t) B B^{T} \exp \left(G^{T}(t+d)\right)\right) d t\right)_{i j}^{2}}{\left(\int_{0}^{\infty}\left(\exp (G t) B B^{T} \exp \left(G^{T} t\right)\right) d t\right)_{i i}\left(\int_{0}^{\infty}\left(\exp (G t) B B^{T} \exp \left(G^{T} t\right)\right) d t\right)_{j j}}\right)
\end{aligned}
$$

This is the main result of this section. An example is shown in fig. 15.1.

\section{G.3 Delayed Transfer Entropy}

In analogy to the derivation of the delayed mutual information we can calculate the delayed transfer entropy. For the conditional probabilities appearing in the definition of the transfer entropy in (15.2.2) we can write

$$
p\left(x_{d, j} \mid x_{0, j}, x_{0, i}\right)=\frac{p\left(x_{d, j}, x_{0, j}, x_{0, i}\right)}{p\left(x_{0, j}, x_{0, i}\right)} \quad p\left(x_{d, j} \mid x_{0, j}\right)=\frac{p\left(x_{d, j}, x_{0, j}\right)}{p\left(x_{0, j}\right)}
$$


to obtain

$$
\mathrm{TE}_{i \rightarrow j}(d)=\int p\left(x_{d, j}, x_{0, j}, x_{0, i}\right) \log \left(\frac{p\left(x_{d, j}, x_{0, j}, x_{0, i}\right) p\left(x_{0, j}\right)}{p\left(x_{0, j}, x_{0, i}\right) p\left(x_{d, j}, x_{0, j}\right)}\right) d x_{d, j} d x_{0, j} d x_{0, i}
$$

Thus we have to calculate the distribution $p\left(x_{d, j}, x_{0, j}, x_{0, i}\right)$ and some of its marginals. Using (G.1.2) and performing all Gaussian integrals except for the coordinates $x=$ $\left(x_{d, j}, x_{0, j}, x_{0, i}\right)$ we obtain for the Gaussian distribution

$$
p\left(x_{d, j}, x_{0, j}, x_{0, i}\right)=\mathcal{N}_{0, C_{i \rightarrow j, d}}
$$

with covariances

$$
C=C_{i \rightarrow j, d}=\left(\begin{array}{ccc}
\left(\Sigma_{\infty}\right)_{j j} & \left(\Sigma_{\infty} M_{d}^{T}\right)_{j j} & \left(\Sigma_{\infty} M_{d}^{T}\right)_{i j} \\
\left(M_{d} \Sigma_{\infty}\right)_{j j} & \left(\Sigma_{\infty}\right)_{j j} & \left(\Sigma_{\infty}\right)_{i j} \\
\left(M_{d} \Sigma_{\infty}\right)_{j i} & \left(\Sigma_{\infty}\right)_{j i} & \left(\Sigma_{\infty}\right)_{i i}
\end{array}\right)
$$

For such a Gaussian distribution calculation of the marginals and Gaussian integrals in analogy to sec. G.2 yields

$$
\mathrm{TE}_{i \rightarrow j}(d)=-\frac{1}{2} \log \left(1-\frac{\left(C_{12} C_{23}-C_{13} C_{22}\right)^{2}}{\left(C_{11} C_{22}-C_{12}^{2}\right)\left(C_{22} C_{33}-C_{23}^{2}\right)}\right)
$$

and thus explicitly for the OU process

$$
\mathrm{TE}_{i \rightarrow j}(d)=-\frac{1}{2} \log \left(1-\frac{\left(\left(\Sigma_{\infty} M_{d}^{T}\right)_{j j}\left(\Sigma_{\infty}\right)_{i j}-\left(\Sigma_{\infty} M_{d}^{T}\right)_{i j}\left(\Sigma_{\infty}\right)_{j j}\right)^{2}}{\left(\left(\Sigma_{\infty}\right)_{j j}^{2}-\left(\Sigma_{\infty} M_{d}^{T}\right)_{j j}^{2}\right)\left(\left(\Sigma_{\infty}\right)_{j j}\left(\Sigma_{\infty}\right)_{i i}-\left(\Sigma_{\infty}\right)_{i j}^{2}\right)}\right)
$$




\section{Appendix $\mathrm{H}$}

\section{Information Flow in Hierarchical Networks of Phase Oscillators Near Synchronous Phase-Locked States}

In this section we derive an explicit analytical expression for the delayed mutual information between the cluster phases in a hierarchical network of the form (16.3.1) under the assumption that the phase locked states (16.3.2) and (16.3.8) are close to the synchronous state.

In this situation the phase differences $\Delta \phi_{i_{X}, j_{Y}}=\Delta \phi_{i_{X}}-\Delta \phi_{i_{Y}}$ in (16.3.2) are small and neglecting terms of $\mathcal{O}\left(\Delta \phi_{i_{x}, j_{X}}^{2}\right)$ we may linearize it to obtain

$$
\omega+\delta \omega_{i_{X}}+\sum_{j_{X}}\left(\gamma_{i_{X} j_{X}}(0)+\gamma_{i_{x} j_{X}}^{\prime}(0)\left(\Delta \phi_{i_{X}, j_{Y}}\right)\right)=\Omega_{X}
$$

where we have used $\omega_{i_{X}}=\omega+\delta \omega_{i_{X}}$. If the group consists of $N_{X}$ oscillators, there are $N_{X}-1$ unknown stationary phase differences $\delta \phi_{i_{X}}=\Delta \phi_{i_{X} 1_{X}}$ and the unknown collective oscillation frequency $\Omega_{X}=\omega+\delta \Omega_{X}$ in this expression. In writing $\delta_{X}=\left(\delta \Omega_{X}, \delta \phi_{2_{X}}, \ldots, \delta \phi_{N_{X}}\right)$ one can rearrange terms to obtain

$$
P_{X} \delta_{X}=I_{X}
$$

where the $N_{X} \times N_{X}$ matrix $P_{X}$ and the vector $I_{X}$ are given by

$$
P_{i_{X} j_{X}}=\left\{\begin{array}{ll}
1 & j_{X}=1 \\
-\sum_{k_{X} \neq i_{X}} \gamma_{i_{X} k_{X}}^{\prime}(0) & i_{X}=j_{X} \\
\gamma_{i_{X} j_{X}}^{\prime}(0) & \text { else }
\end{array} \quad I_{i_{X}}=\sum_{j_{X}} \gamma_{i_{X} j_{X}}^{\prime}(0)+\delta \omega_{i_{X}}\right.
$$

The phase differences are then obtained by inverting this equation to obtain

$$
\delta_{X}=P_{X}^{-1} I_{X}
$$

in each group $X$. Note that there are explicit formulas for the inverse of a matrix [260].

Further by setting $\delta \phi_{1_{X}}=0$ the matrix $L_{X}$ in (16.3.3) becomes

$$
L_{X}= \begin{cases}-\left(\gamma_{i_{X} j_{X}}^{\prime}(0)+\gamma_{i_{X} j_{X}}^{\prime \prime}(0) \Delta \phi_{i_{X} j_{X}}\right) & i_{X} \neq j_{X} \\ \sum_{k_{X}}\left(\gamma_{i_{X} k_{X}}^{\prime}(0)+\gamma_{i_{X} k_{X}}^{\prime \prime}(0) \Delta \phi_{i_{X} j_{X}}\right) & i_{X}=j_{X}\end{cases}
$$

form which we obtain an explicit expression for $Z_{X}$ via equation (16.3.4). 
For the inter cluster phase coupling function we have assuming that also that the clusters are close to synchrony, i.e. $\Delta \Phi_{X Y}$ small and keeping only the linear terms

$$
\begin{aligned}
\Gamma_{X Y}\left(\Phi_{X}-\Phi_{Y}\right) & =\sum_{i_{X}, j_{Y}} Z_{X, i_{X}}\left(\gamma_{i_{X} j_{Y}}(0)+\gamma_{i_{X} j_{Y}}^{\prime}(0)\left(\Phi_{X}-\Phi_{Y}+\Delta \phi_{i_{X} j_{Y}}\right)\right) \\
& =I_{X Y}+\left(\Phi_{X}-\Phi_{Y}\right) J_{X Y}
\end{aligned}
$$

where

$$
\begin{aligned}
I_{X Y} & =\sum_{i_{X}, j_{Y}} Z_{X, i_{X}}\left(\gamma_{i_{X} j_{Y}}(0)+\gamma_{i_{X} j_{Y}}^{\prime}(0) \Delta \phi_{i_{X} j_{Y}}\right) \\
J_{X Y} & =\sum_{i_{X}} \sum_{j_{Y}} Z_{X, i_{X}}\left[\gamma_{i_{X} j_{Y}}^{\prime}(0)+\gamma_{i_{X} j_{Y}}^{\prime \prime}(0) \Delta \phi_{i_{X} j_{Y}}\right]
\end{aligned}
$$

As for the individual clusters, we also linearize the collective phase locking equation (16.3.8), and solve for $\Delta=\left(\Delta \Omega, \Delta \Phi_{2}, \ldots \Delta \Phi_{N}\right)$ where $\Delta \Phi_{X}=\Delta \Phi_{X A}$ and $\Delta \Omega=\Omega-\omega$ to obtain

$$
\Delta=Q^{-1} O
$$

where the $M \times M$ matrix $Q$ and the vector $O$ are given by

$$
Q_{X Y}=\left\{\begin{array}{ll}
1 & Y=1 \\
-\sum_{Z \neq X} J_{X Z} & X=Y \\
J_{X Y} & \text { else }
\end{array} \quad O_{X}=\sum_{Y} I_{X Y}+\delta \Omega_{X}\right.
$$

Eqs. (H.2), (H.3) and (H.4) give explicit expressions needed to determine the delayed mutual information between the phase signals of two clusters via corollary 16.3.1. In particular, we obtain a long but explicit formula for the delayed mutual information as a function of the network parameter $\gamma_{i_{X} j_{Y}}(0), \gamma_{i_{X} j_{Y}}^{\prime}(0), \gamma_{i_{X} j_{Y}}^{\prime \prime}(0), \delta \omega_{i_{X}}$ and $\xi_{i_{X}}$.

For couplings of the form $\gamma_{i_{X} j_{Y}}(\phi)=g_{i_{X} j_{Y}} \gamma(\phi)$ this gives an explicit expression of the dMI as a function of the connectivity matrix $g_{i j}$ and in particular the local inter cluster connection strengths $g_{i_{X} j_{X}}$. Under the above assumptions of a close to synchronous collective oscillation these theoretical predictions are in good agreement with numerical simulations as shown in fig. 16.3. 


\section{Bibliography}

[1] L. F. Abbott and T. B. Kepler. From Hodgkin-Huxley to Hopfield. In L. Garrido, editor, Statistical Mechanics of Neural Networks, pages 5-18. Springer, Berlin, 1990.

[2] L. F. Abbott and S. B. Nelson. Synaptic plasticity: taming the beast. Neural Networks, 3:1178-1182, 2000.

[3] L. F. Abbott and C. van Vreeswijk. Asynchronous states in networks of pulsecoupled oscillators. Phys. Rev. E, 48:1483-1490, 1993.

[4] M. Abeles. Time is Precious. Science, 304:523-524, 2004.

[5] J. A. Acebron, L. L. Bonilla, C. J. P. Vicente, F. Ritort, and R. Spigler. The Kuramoto model: a simple paradigm for synchronization phenomena. Rev. Mod. Phys., 77:137-185, 2005.

[6] T. B. Achacoso and W. S. Yamamoto. AY's Neuroanatomy of C. elegans for Computation. CRC Press, Boca Raton, Florida, 1992.

[7] E. D. Adrian and B. H. C. Matthews. The Berger rhythm, potential changes from the occipital lobe in man. Brain, 57:345-359, 1934.

[8] H. Agmon-Snir, C. E. Carr, and J. Rinzel. The role of dendrites in auditory coincidence detection. Nature, 393:268-272, 1998.

[9] B. E. Alger and R. A. Nicoll. GABA-mediated biphasic inhibitory responses in hippocampus. Nature, 281:315-317, 1979.

[10] A. Alonso and R. R. Llinás. Subthreshold $\mathrm{Na}^{+}$-dependent theta-like rhythmicity in entorhinal cortex layer II stellate cells. Nature, 342:175-177, 1989.

[11] N. Anderson, E. B. Saff, and R. S. Varga. On the Eneström-Kakeya Theorem and its Sharpness. Lin. Algeb. Appl., 28:5-16, 1979.

[12] L. Arnold. Random Dynamical Systems. Springer Monographs in Mathematics. Springer, Berlin, Germany, 1998.

[13] V. I. Arnol'd. Lectures on Bifurcations in Versal Families. Russ. Math. Surv., 27:54-119, 1972.

[14] A. Arvanitaki. Recherches sur la reponse oscillatoire locale de l'axone geant isole de sepia. Arch. Int. Physiol. 49, 49:209-256, 1936.

[15] P. Ashwin and J. Borresen. Encoding via conjugate symmetries of slow oscillations for globally coupled oscillators. Phys. Rev. E, 70:026203, 2004. 
[16] P. Ashwin and M. Timme. Unstable Attractors: existence and robustness in networks of oscillators with delayed pulse coupling. Nonlinearity, 18:2035-2060, 2005.

[17] P. Ashwin and M. Timme. When instability makes sense. Nature, 436:36-37, 2005.

[18] R. Badii and A. Politi. Complexity. Cambridge University Press, Cambridge, England, 1997.

[19] V. M. Bajo, M. A. Merchan, D. E. Lopez, and E. M. Rouiller. Neuronal morphology and efferent projections of the dorsal nucleus of the lateral lemniscus in the rat. $J$. Comput. Neurosci., 334:241-262, 1993.

[20] M. Banaji. Clustering in globally coupled oscillators. Dynamical Systems, 17:263$285,2002$.

[21] M. Bartos, I. Vida, and P. Jonas. Synaptic mechanisms of synchronized gamma oscillations in inhibitory interneuron networks. Nat. Rev. Neurosci., 8:45-56, 2007.

[22] D. S. Bassett, N. F. Wymbs, M. A. Porter, P. J. Mucha, J. M. Carlson, and S. T. Grafton. Dynamic reconfiguration of human brain networks during learning. Proc. Natl. Acad. Sci. U. S. A., 108:7641-6446, 2011.

[23] D. Battaglia, A. Witt, T. Geisel, and F. Wolf. Dynamic transitions in the effective connectivity of interacting cortical areas. FENS 2010 abstract.

[24] J. M. . Beggs and D. Plenz. Neuronal Avalanches in Neocortical Circuits. J. Neurosci., 23(35):11167-11177, 2003.

[25] J. M. Bekkers and M. Häusser. Targeted dendrotomy reveals active and passive contributions of the dendritic tree to synaptic integration and neuronal output. Proc. Natl. Acad. Sci. U. S. A., 104:11447-11452, 2007.

[26] H. Berger. Über das Elektroenkephalogramm des Menschen. Arch. Psychiat. Nervenkr., 87:527-570, 1929.

[27] Ö. Bernander, R. J. Douglas, K. A. C. Martin, and C. Koch. Synaptic background activity influences spatiotemporal integration in single pyramidal cells. Proc. Natl. Acad. Sci. U. S. A., 88:11569-11573, 1991.

[28] M. Besserve, Y. Murayma, B. Schölkopf, N. Logothetis, and S. Panyeri. High frequency phase-spike synchronization of extracellular signals modulates causal interactions in monkey primary visual cortex. Neuroscience Meeting Planner. Society for Neuroscience, 2010. Online.

[29] R. I. Bogdanov. Versal deformations of a singular point of a vector field in the plane in the case of zero eigenvalues. Funktsional'nyi Anliz $i$ Ego Prilozheniya, 9(2):63, 1975 .

[30] C. Börgers and N. Kopell. Synchronization in Networks of Excitatory and Inhibitory Neurons with Sparse, Random Connectivity. Neural Comput., 15:509-538, 2003.

[31] C. Börgers and N. Kopell. Effects of Noisy Drive on Rhythms in Networks of Excitatory and Inhibitory Neurons. Neural Comput., 17:557-608, 2005. 
[32] S. Bornholdt and H. G. Schuster. Handbook on Graphs and Networks. Wiley-VCH, Weinheim, Germany, 2002.

[33] S. Bottani. Pulse-Coupled Relaxation Oscillators: From Biological Synchronization to Self-Organized Criticality. Phys. Rev. Lett., 74:4189-4192, 1995.

[34] J. M. Bower and D. Beerman. The Book of Genesis: Exploring Realistic Neural Models with the GEneral NEural SImulation System. Springer, Berlin, 1997.

[35] A. Bragin, G. Jando, Z. Nadasdy, J. Hetke, K. Wise, and G. Buzsaki. Gamma (40$100 \mathrm{~Hz}$ ) oscillation in the hippocampus of the behaving rat. J. Neurosci., 15:47-60, 1995.

[36] V. Braitenberg and A. Schüz. Cortex: Statistics and Geometry of Neuronal Connectivity. Springer, Berlin, 1998.

[37] P. Bressloff, S. Coombes, and B. de Souza. Dynamics of a Ring of Pulse-Coupled Oscillators: Group Theoretic Approach. Phys. Rev. Lett., 79(15):2791-2794, 1997.

[38] P. C. Bressloff. Dynamics of a compartmental model integrate-and-fire neuron with somatic potential reset. Physica D, 90:399, 1995.

[39] P. C. Bressloff and S. Coombes. Dynamics of Strongly Coupled Spiking Neurons. Neural Comput., 12:91-129, 2000.

[40] S. G. Brickley, V. Revilla, S. G. Cull-Candy, W. Wisden, and M. Farrant. Adaptive regulation of neuronal excitability by a voltage-independent potassium conductance. Nature, 409:88-92, 2001.

[41] F. Brink, D. W. Bronk, and M. G. Larrabee. Chemical excitation of nerve. Ann. N.Y. Acad. Sci., 47:457-485, 1946.

[42] H. W. Broer, B. Krauskopf, and G. Vegter, editors. Global Analysis of Dynamical Systems. Institute of Physics Publishing Bristol and Philadelphia, 2001.

[43] B. Brogliato. Nonsmooth Mechanics. Springer, London, 1999.

[44] I. N. Bronstein, K. A. Semendjaev, G. Musiol, and H. Mühlig. Taschenbuch der Mathematik. Verlag Harry Deutsch, Frankfurt am Main, Germany, 1999.

[45] N. Brunel and X. J. Wang. What determines the frequency of fast network oscillations with irregular neural discharges? I. Synaptic dynamics and excitationinhibition balance. J. Neurophysiol., 90:415-430, 2003.

[46] C. Büchel, J. T. Coull, and K. J. Friston. The predictive value of changes in effective connectivity for human learning. Science, 283:1538-1541, 1999.

[47] J. Buck and E. Buck. Synchronous Fireflies. Sc. American, 234:74-85, 1976.

[48] A. Buelmann and G. Deco. Optimal Information Transfer in the Cortex through Synchronization. PLoS Comput. Biol., 6:e1000934, 2010.

[49] T. R. Burchell, H. J. Faulkner, and M. A. Whittington. Gamma frequency oscillations gate temporally coded afferent inputs in the rat hippocampal slice. Neuroscience Letters, 255(3):151 - 154, 1998. ISSN 0304-3940. 
[50] T. J. Buschman and E. K. Miller. Top-down versus bottom-up control of attention in the prefrontal and posterior parietal cortices. Science, 315:1860-1862, 2007.

[51] G. Buszaki. Theta rhythm of navigation: link between path integration and landmark navigation, episodic and semantic memory. Hippocampus, 15:827-840, 2005.

[52] G. Buzsaki. Theta oscillations in the hippocampus. Neuron, 33:325-340, 2002.

[53] G. Buzsaki. Rythms of the Brain. Oxford University Press, 2006.

[54] C. F. Cadieu and K. Koepsell. Phase Coupling Estimation from Multivariate Phase Statistics. Neural Comput., 22:3107-3126, 2010.

[55] R. T. Canolty, E. Edwards, S. S. Dalal, M. Soltani, S. S. Nagarajan, H. E. Kirsch, M. S. Berger, N. M. Barbaro, and R. T. Knight. High Gamma Power Is PhaseLocked to Theta Oscillations in Human Neocortex. Science, 313:1626-1628, 2007.

[56] R. T. Canolty, K. Ganguly, S. W. Kennerley, C. F. Cadieu, K. Koepsell, J. D. Wallisb, and J. M. Carmenaa. Oscillatory phase coupling coordinates anatomically dispersed functional cell assemblies. Proc. Natl. Acad. Sci. U. S. A., 107:1735617361, 2010.

[57] A. Champneys and Y. Kuznetsov. Numerical detection and continuation of codimension two homoclinic bifurcations. Int. J. Bif. Chaos, 4:795-822, 1994.

[58] G. Chartrand and L. Lesniak. Graphs and Digraphs. Chapman and Hall, Boca Raton, Florida, 3 edition, 1996.

[59] M. A. Cohen and S. Grossberg. Absolute stability and global pattern formation and parallel memory storage by competitive neural networks. IEEE Trans. Systems, Man and Cybernetics, 13:815-821, 1983.

[60] R. Cohen and S. Havlin. Complex Networks: Structure, Robustness and Function. Cambridge University Press, 2010.

[61] J. A. Connor and C. F. Stevens. Prediction of repetitive firing behaviour from voltage clamp data on an isolated neurone soma. J. Physiol., 213:31-53, 1971.

[62] J. A. Connor and C. F. Stevens. Voltage clamp studies of a transient outward membrane current in gastropod neural somata. J. Physiol., 213:11-30, 1971.

[63] J. A. Connor, D. Walter, and R. McKown. Neural Repetitive Firing; Modifications of the Hodgkin-Huxley Axon Suggested by Experimental Results form Crustacean Axons. Biophys. J., 18:81-102, 1977.

[64] B. W. Connors and M. J. Gutnick. Intrinsic firing patterns of diverse neocortical neurons. Trends Neurosci., 13:99-104, 1990.

[65] S. Coombes, Y. Timofeeva, C.-M. Svennson, G. Lord, K. Josic, S. J. Cox, and C. Colbert. Branching dendrites with resonant membrane: a "sum-over-trips" approach. Biol. Cybern., 97:137-149, 2007.

[66] A. Corral, C. J. Perez, A. Diaz-Guilera, and A. Arenas. Synchronization in a Lattice Model of Pulse-Coupled Oscillators. Phys. Rev. Lett., 75(20):3697-3700, 1995. 
[67] J. H. P. Dawes and T. L. Tsai. Frequency locking and complex dynamics near a periodically forced robust heteroclinic cycle. Phys. Rev. E, 74:055201(R), 2006.

[68] P. Dayan and L. F. Abbott. Theoretical Neuroscience. MIT Press, Cambridge, Massachusetts, 2001.

[69] J. Deane and D. Hamill. Instability, Subharmonics, and Chaos in Power Electronic Systems. IEEE Trans. Power Electron., 5:260, 1990.

[70] M. Denker. Complex networks of spiking neurons with a generalized rise function. Diploma thesis, Department of Physics, University of Göttingen, 2002.

[71] M. Denker, M. Timme, M. Diesmann, F. Wolf, and T. Geisel. Breaking Synchrony by Heterogeneity in Complex Networks. Phys. Rev. Lett., 92:074103, 2004.

[72] A. Destexhe and T. Bal. Dynamic Clamp: from principles to applications. Springer, 2009.

[73] A. Destexhe, Z. F. Mainen, and T. J. Sejnowski. An Efficient Method for Computing Synaptic Conductances Based on a Kinetic Model of Receptor Binding. Neural Comput., 6:14, 1994.

[74] A. Destexhe and D. Pare. Impact of Network Activity on the Integrative Properties of Neocortical Pyramidal Neurons In Vivo. J. Neurophysiol., 81:1531-1547, 1999.

[75] A. Destexhe, M. Rudolph, and D. Paré. The high-conductance state of neocortical neurons in vivo. Nat. Rev. Neurosci., 4:739-751, 2003.

[76] M. di Bernado. Piecewise-smooth Dynamical Systems. Springer, 2007.

[77] M. Diesmann, M.-O. Gewaltig, and A. Aertsen. Stable propagation of synchronous spiking in cortical neural networks. Nature, 402:529-533, 1999.

[78] R. Diestel. Graph Theory. Graduate Texts in Mathematics. Springer, Berlin, 2nd edition, 2000.

[79] E. J. Doedel, H. B. Keller, and J. P. Kernèvez. Numerical analysis and control of bifurcation problems: (I) Bifurcation in finite dimensions. Int. J. Bif. Chaos, 4:493-520, 1991.

[80] E. J. Doedel, H. B. Keller, and J. P. Kernèvez. Numerical analysis and control of bifurcation problems: (II) Bifurcation in infinite dimensions. Int. J. Bif. Chaos, 4:745-772, 1991.

[81] E. J. Doedel and B. E. Oldeman. AUTO-0\%p: Continuation and Bifurcation software for ordinary differentail equations. Concordia University, Montreal, Canada, 2009. http://sourceforge.net/projects/auto2000.

[82] E. J. Doedel, R. C. Paffenroth, A. R. Champneys, T. F. Faigrieve, Y. A. Kuznetsov, B. E. Oldeman, B. Sandstede, and X. J. Wang. AUTO2000: Software for continuation and bifurcaton problems in ordinary differential equations. Technical report, California Institute of Tchnology, Pasadena CA 91125, 2000. 
[83] B. Doiron, A.-M. Oswald, and L. Maler. Interval Coding II. Dendrite-Dependent Mechanisms. J. Neurophysiol., 97:2744-2757, 2007.

[84] F. Dumortier and C. Li. On the uniqueness of limit cycles surrounding one or more singularities in Liénard equations. Nonlinearity, 9:1489-1500, 1996.

[85] F. Dumortier, R. Roussarie, and J. Sotomayor. Generic 3-parameter families of vector fields on the plane, unfolding a singularity with nilpotent linear part. The cusp case of codimension 3. Ergodic Theory and Dynamical Systems, 7(3):375-413, 1987.

[86] F. Dumortier, R. Roussarie, and J. Sotomayor. Bifurcations of cupsidal loops. Nonlinearity, 10:1369-1408, 1997.

[87] F. Dumortier, R. Roussarie, J. Sotomayor, and H. Zoladek. Bifurcations of Planar Vector Fields: Nilpotent Singularities and Abelian Integrals, volume 1480 of Lecture Notes in Mathematics. Springer, 1991.

[88] J. C. Eccles. The Physiology of Synapses. Springer, Berlin, 1964.

[89] J. C. Eccles, B. Libet, and R. R. Young. The behaviour of chromatolyes motoneurons studied by intracellular recording. J. Physiol., 143:11-40, 1958.

[90] R. Eckhorn, R. Bauer, W. Jordan, M. Brosch, W. Kruse, M. Munk, and H. J. Reitboeck. Coherent oscillations: A mechanism of feature linking in the visual cortex? Biol. Cybern., 60:121-130, 1988.

[91] H. Eichenbaum and N. J. Cohen. Memory, Amnesia, and the Hippocampal System. MIT Press, 1993.

[92] R. J. Elble and W. C. Koller. Tremor. John Hopkins University Press, Baltimore, USA, 1990.

[93] G. Eneström. Härledning af en allmän formel för antalet pesionärer. Öfv. af. Kungl. Vetenskaps-Akademiens Förhandlingen, Stockholm, (6), 1893.

[94] A. K. Engel, P. Fries, and W. Singer. Dynamic Predictions: Oscillations and Synchrony in Top-Down Processing. Nature Reviews, 2:704-716, 2001.

[95] A. K. Engel, P. König, A. K. Kreiter, and W. Singer. Interhemispheric synchronization of oscillatory neuronal responses in cat visual cortex. Science, 252:1177-1179, 1991.

[96] J. Epsztein, A. K. Lee, E. Chorev, and M. Brecht. Impact of Spikelets on Hippocampal CA1 Pyramidal Cell Activity During Spatial Exploration. Science, 327:474-, 2010 .

[97] I. Erchova, G. Kreck, U. Heinemann, and A. V. M. Herz. Dynamics of rat enthohinal cortex layer I and II cells: characteristics of membrane potential resonance at rest predict oscillation properties near threshold. J. Physiol., 560:89-110, 2004.

[98] D. Eriksson, R. Vicente, and K. Schmidt. A linear model of phase-dependent power correlations in neuronal oscillations. Frontiers in Computational Neuroscience, 5:114, 2011. ISSN 1662-5188. 
[99] A. Erisir, D. Lau, B. Rudy, and C. S. Leonard. Function of Specific K ${ }^{+}$Channels in Sustained High-Frequency Firing of Fast-Spiking Neocortical Interneurons. $J$. Neurophysiol., 82:2476-2489, 1999.

[100] G. B. Ermentrout. Type I Membranes, Phase Resetting Curves, and Synchrony. Neural Comput., 8:979-1001, 1996.

[101] G. B. Ermentrout and N. Kopell. Parabolic bursting in an excitable system couled with a slow oscillation. SIAM J. Appl. Math., 46:233-253, 1986.

[102] G. B. Ermentrout and N. Kopell. Multiple pulse interactions and averaging in systems of coupled neural oscillators. J. Math. Biol., 29:195-217, 1991.

[103] G. B. Ermentrout and N. Kopell. Fine Structure of Neural spiking and synchronization in the presence of conduction delays. Proc. Natl. Acad. Sci. U. S. A., 95:1259-1264, 1998.

[104] U. Ernst, K. Pawelzik, and T. Geisel. Synchronization Induced by Temporal Delays in Pulse-Coupled Oscillators. Phys. Rev. Lett., 74(9):1570-1573, 1995.

[105] U. Ernst, K. Pawelzik, and T. Geisel. Delay-Induced multistable synchronization of biological oscillators. Phys. Rev. E, 57(2):2150-2162, 1998.

[106] C. W. Eurich, J. M. Herrmann, and U. A. Ernst. Finite-size effects of avalanche dynamics. Phys. Rev. E, 66:066137, 2002.

[107] J. Feng. Is the integrate-and-fire model good enough ? - a review. Neural Networks, 14:955-957, 2001.

[108] R. Fitzhugh. Impulses and physiological states in theoretical models of nerve membrane. Biophys. J., 1:445-466, 1961.

[109] R. FitzHugh. Mathematicla models of excitation and propagation in nerve. In H. P. Schwan, editor, Biological Engineering. McGraw-Hill Book Company, Toronto, Canada, 1969.

[110] N. Fourcaud-Trocme, D. Hansel, C. van Vreeswijk, and N. Brunel. How Spike Generation Mechanisms Determine the Neuronal Response to Fluctuating Inputs. J. Neurosci., 23(37):11628-11640, 2003.

[111] P. Fries. A mechanism for cognitive dynamics: neuronal communication through neuronal coherence. Trends in Cognitive Sciences, 9:474-480, 2005.

[112] P. Fries. Neuronal Gamma-Band Synchronization as a Fundamental Process in Cortical Computation. Ann. Rev. Neurosci., 32:209-224, 2009.

[113] P. Fries, J. H. Reynolds, A. E. Rorie, and R. Desimone. Modulation of Oscillatory Neuronal Synchronization by Selective Visual Attention. Science, 291:1560-1563, 2001.

[114] P. Fries, P. R. Roelfsema, A. K. Engel, P. König, and W. Singer. Synchronization of oscillatory responses in visual cortex correlates with perception in interocular rivalry. Proc. Natl. Acad. Sci. U. S. A., 94:12699-12704, 1997. 
[115] P. M. Fuller, J. J. Gooley, and C. B. Saper. Neurobiology of the Sleep-Wake Cycle: Sleep Architecture, Circadian Regulation, and Regulatory Feedback. J. Biol. Rhythms, 21:482-492, 2006.

[116] R. F. Galan, G. B. Ermentrout, and N. N. Urban. Efficient Estimation of PhaseResetting Curves in Real Neurons and its Significance for Neural-Network Modeling. Phys. Rev. Lett., 94:158101, 2005.

[117] W. G. Gall and Y. Zhou. An organizing center for planar neural excitabiltiy. Neurocomputing, 32-33:757-765, 2000.

[118] R. Gallagher and T. Appenzeller, editors. Sciene: Special Issue: Complex Systems, volume 284. 1999.

[119] D. L. F. Garden, P. D. Dodson, C. O'Donnell, M. D. White, and M. F. Nolan. Tuning of Synaptic Integration in the Medial Entorhinal Cortex to the Organization of Grid Cell Firing Fields. Neuron, 60:875-889, 2008.

[120] C. W. Gardiner. Handbook of Stochastic Methods. Springer, Berlin, Heidelberg, Germany, 1985.

[121] M. Germuska, S. Saha, J. Fiala, and H. Barbas. Synaptic distinction of laminarspecifc prefrontal-temporal pathways in primates. Cereb. Cortex, 16:865-875, 2006.

[122] G. L. Gerstein and B. Mandelbrot. Random walk models for the spike activity of a single neuron. Biophys. J., 4:41-68, 1964.

[123] W. Gerstner and W. M. Kistler. Spiking Neural Models. Cambridge University Press, Cambridge, England, 2002.

[124] W. Gerstner and J. L. van Hemmen. Coherence and Incoherence in a Globally Coupled Ensemble of Pulse-Emitting Units. Phys. Rev. Lett., 71:312-315, 1993.

[125] V. L. Ginzburg and L. D. Landau. On the Theory of Superconductivity. Zh. Eksp. Teor. Fiz., 20:1064, 1950. English version in L. D. Landau, Collected Papers. Pergamon Press Oxford, 1965.

[126] L. M. Giocomo, E. A. Zilli, E. Fransen, and M. E. Hasselmo. Temporal frequency of subthreshold oscillations scales with enthorinal grid cell field spacing. Science, 315:1719-1722, 2007.

[127] P. Girard, J. M. Hupe, and J. Bullier. Feedforward and feedback connections between areas V1 and V2 of the monkey have similar rapid conduction velocities. $J$. Neurophysiol., 85:1328-1331, 2001.

[128] L. Glass. Synchronization and rhythmic processes in physiology. Nature, 410:277$284,2001$.

[129] R. Goebel, R. G. Sanfelice, and A. R. Teel. Hybrid dynamical systems. IEEE Control Systems, 29:29-93, 2009.

[130] J. A. Goldberg, C. A. Deister, and C. J. Wilson. Response Properties and Synchronization of Ryhtmically Firing Dendritic Neurons. J. Neurophysiol., 97:208-219, 2007. 
[131] S. A. Goldstein, D. Bockenhauer, I. O'Kelly, and N. Zilberberg. Potassium leak channels and the KCNK family of two-P-domain subunits. Nat. Rev. Neurosci., $2: 175-184,2001$.

[132] M. Golubitsky and I. I. Stewart. Nonlinear Dynamics of Networks: The Groupoid Formalism. Bull. Amer. Math. Soc., 43:305-364, 2006.

[133] M. Golubitsky and D. G. Schaeffer. Singularities and Groups in Bifurcation Theory: Vol I. Springer, New York, 1985.

[134] M. Golubitsky and D. G. Schaeffer. Singularities and Groups in Bifurcation Theory: Vol II. Springer, New York, 1988.

[135] A. Gorji. Spreading depression: a review of the clinical relevance. Brain Research Reviews, 38:33-60, 2001.

[136] W. J. F. Govaerts. Numerical Methods for Bifurcations of Dynamical Equilibria. Society for Industrial Mathematics, 1987.

[137] W. J. F. Govaerts and B. Sautois. The onset and extinction of neural spiking: a numerical bifurcation approach. J. Comput. Neurosci., 18:273-282, 2005.

[138] C. M. Gray. Synchronous Oscillations in Neuronal Systems: Mechanisms and Functions. J. Comput. Neurosci., 1:11-38, 1994.

[139] C. M. Gray, A. K. Engel, P. König, and W. Singer. Synchronization of oscillatory neuronal responses in cat striate cortex: temporal properties. Vis. Neurosci., 8:337347, 1992.

[140] C. M. Gray, P. König, A. K. Engel, and W. Singer. Oscillatory responses in cat visual cortex exhibit inter-columnar synchronization which reflects global stimulus properties. Nature, 338:334-337, 1989.

[141] C. M. Gray, P. König, A. K. Engel, and W. Singer. Stimulus-Specific Neuronal Oscillations in Cat Visual Cortex Exhibit Inter-Columnar Synchronization Which Reflects Global Stimulus Properties. Proc. Natl. Acad. Sci. U. S. A., 86:1698-1702, 1989.

[142] G. G. Gregoriou, S. J. Gotts, H. Zhou, and R. Desimone. High-frequency, long-range coupling between prefrontal and visual cortex during attention. Science, 324:12071210, 2009.

[143] T. J. Grudt and E. R. Perl. Correlations between neuronal morphology and electrophysiological features in the rodent superficial dorsal horn. J. Physiol., 540:189-207, 2002.

[144] J. Guckenheimer. Multiple Bifurcation Problems for Chemical Reactors. Physica $D, 20: 1-20,1986$.

[145] J. Guckenheimer and P. Holmes. Nonlinear Oscillations, Dynamical Systems, and Bifurcations of Vector Fields. Springer, Berlin, 1983.

[146] J. Guckenheimer and P. Holmes. Structurally stable heteroclinic cycles. Math. Proc. Camb. Phil. Soc., 103:189-192, 1988. 
[147] J. Guckenheimer and J. Labouriau. Bifurcation of the Hodgkin and Huxley equations: A new twist. Bulletin of Mathematical Biology, 55:937-952, 1993. ISSN 0092-8240. 10.1007/BF02460693.

[148] J. Guckenheimer and P. Meerkamp. Unfoldings of singular Hopf bifurcation. arXiv:110\%.3185v1, 2011.

[149] B. Gustafsson and H. Wigstrom. Shape of frequency-current curves in CAI pyramidal celss in the hippocampus. Brain Res., 223:417-421, 1981.

[150] Y. Gutfreund, Y. Yarom, and I. Segev. Subthreshold oscillations and resonant frequency in guinea pig cortical neurons: physiology and modelling. J. Physiol., 483:621-640, 1995.

[151] B. S. Gutkin and G. B. Ermentrout. Dynamics of Membrane Excitability Determine Interspike Interval Variability: A Link Between Spike Generation Mechanisms and Cortical Spike Trian Statistics. Neural Comput., 10:1947-1065, 1998.

[152] H. Haken. Advanced Synergetics. Springer, Berlin, 1983.

[153] H. Haken. Synergetics, an Introduction. Springer, Berlin, 1983.

[154] D. Hansel and G. Mato. Asynchronous States and the Emergence of Synchrony in Large Networks of Interacting Excitatory and Inhibitory Neurons. Neural Comput., $15: 1-56,2003$.

[155] D. Hansel, G. Mato, and C. Meunier. Clustering and slow switching in globally coupled phase oscillators. Phys. Rev. E, 48:3470-3477, 1993.

[156] D. Hansel, G. Mato, and C. Meunier. Phase Dynamics for Weakly Coupled HodgkinHuxley Neurons. Europhys. Lett., 23(5):367-372, 1993.

[157] D. Hansel, G. Mato, and C. Meunier. Synchrony in Excitatory Neural Networks. Neural Comput., 7:307-337, 1995.

[158] K. D. Harris, J. Csicsvari, H. Hirase, G. Dragoi, and G. Buzsáki. Organization of cell assemblies in the hippocampus. Nature, 424:552-556, 2003.

[159] K. D. Harris, D. A. Henze, H. Hirase, X. Leinekugel, G. Dragoi, A. Czurko, and G. Buzsàki. Spike train dynamics predicts theta-related pase precession in hippocampal pyramidal cells. Nature, 417:738-741, 2002.

[160] C. Hauptmann, J.-C. Roulet, J. J. Niederhauser, W. Döll, M. E. K. dn B. Lysyansky, V. Krachkovskyi, M. A. Bhatti, U. B. Barnikol, L. Sasse, C. P. Bührle, E.-J. Speckmann, M. Götz, V. Sturm, H.-J. Freund, U. Schnell, and P. A. Tass. External trial deep brain stimulation device for the application of desynchronizing stimulation techniques. J. Neural Engineering, 6:1-12, 2009.

[161] M. Häusser, N. Spruston, and G. Stuart. Diversity and dynamics of dendritic signaling. Science, 290:739-744, 2000.

[162] D. O. Hebb. The organization of behaviour. Wiley, New York, 1949. 
[163] J. Hertz, A. Krogh, and R. G. Palmer. Introduction to the Theory of Neural Computation. Addison-Wesley, Redwood City CA, USA, 1991.

[164] A. V. M. Herz and J. J. Hopfield. Earthquake Cycles and Neural Revertebrations: Collective Oscillations in Systems with Pulse-Coupled Threshold Elements. Phys. Rev. Lett., 75:1222-1225, 1995.

[165] P. Heyward, E. Matthew, A. Keller, and M. T. Shipley. Membrane Bistability in Olfactory Bulb Mitral Cells. J. Neurosci., 21:5311-5320, 2001.

[166] M. S. Hilaire and A. Longtin. Comparison of coding capabilities of type I and type II neurons. J. Comput. Neurosci., 16:299-313, 2004.

[167] B. Hille. Ion Channels of Excitable Membranes. Sinauer, 1992.

[168] J. L. Hindmarsh and R. M. Rose. A model of the nerve impulse using two first-order differential equations. Nature, 296:162-164, 1982.

[169] J. F. Hipp, A. K. Engel, and M. Siegel. Oscillatory synchronization in large-scale cortical networks predicts perception. Neuron, 69:387-396, 2011.

[170] A. L. Hodgkin. The local electric changes associated with repetitive action in a non-medullated axon. J. Physiol., 107:165-191, 1948.

[171] A. L. Hodgkin and A. F. Huxley. A quantitative description of membrane current and is application to conduction and excitation in nerve. J. Physiol., 117:500-544, 1952.

[172] A. L. Hodkin and A. F. Huxley. Current carried by sodium and potassium ions through the membrane of the giant axon of Loligo. J. Physiol., 116:449-473, 1952.

[173] R. L. Honeycutt. Stochastic runge-kutta algorithms. I. White noise. Phys. Rev. A, 45:600-603, 1992.

[174] J. J. Hopfield and A. V. M. Herz. Rapid local synchronization of action potentials: Toward computation with coupled integrate-and-fire neurons. Proc. Natl. Acad. Sci. U. S. A., 92:6655-6662, 1995.

[175] F. C. Hoppensteadt and E. M. Izhikevich. Synaptic organizations and dynamical properties of weakly connected neural oscillators. Biol. Cybern., 75:129-135, 1996.

[176] F. C. Hoppensteadt and E. M. Izhikevich. Canonical Neural Models. In M. A. Arbib, editor, Brain Theory and Neural Networks. MIT Press, 2001.

[177] F. C. Hoppensteatd and E. M. Izhikevich. Weakly Connected Neural Networks, volume 126 of Applied Mathematical Sciences. Springer, New York, 1997.

[178] R. A. Horn and C. R. Johnson. Matrix Analysis. Cambridge University Press, Cambridge, England, 1996.

[179] H. Hu, K. Vervaeke, and J. F. Storm. Two forms of electrical responance at theta frequencies generated by M-current, h-current and persistent $\mathrm{Na}+$ current in rat hippocampal pyramidal cells. J. Physiol., 545:783-805, 2002. 
[180] D. H. Hubel and T. N. Wiesel. Receptive fields, binocular and functional architecture in the cat's visual cortex. J. Physiol. London, 160:106-154, 1962.

[181] R. Huber, M. F. Ghilardi, M. Massimini, and G. Tononi. Local sleep and learning. Nature, 430:78-81, 2004.

[182] A. Hurwitz. Mathematische Werke. Birkhäuser, Basel, Stuttgart, 1933.

[183] B. Hutcheon, R. M. Miura, and E. Puil. Models for Subthreshold Membrane Resonance in Neocortical Neurons. J. Neurophysiol., 76:698-714, 1996.

[184] B. Hutcheon, R. M. Miura, and E. Puil. Subthreshold membrane resonance in neocortical neurons. J. Neurophysiol., 76:683-697, 1996.

[185] B. Hutcheon and Y. Yarom. Resonance, oscillation and the intrinsic frequency preferences of neurons. Trends Neurosci., 23:216-222, 2000.

[186] A. Ichiki and Y. Sato. A Phase Reduction Method for Weakly Coupled Stochastic Oscillator Systems. In D. Liu, H. Zhang, M. Polycarpou, C. Alippi, and H. He, editors, Advances in Neural Networks - ISNN 2011, volume 6675 of Lecture Notes in Computer Science, pages 251-259. Springer Berlin / Heidelberg, 2011.

[187] Y. Ikegaya, G. Aaron, R. Cossart, D. Arnonov, I. Lampl, D. Ferster, and R. Yuste. Synfire Chains and Cortical Songs: Temporal Modules of Cortical Activity. Science, 304:559-564, 2004.

[188] E. M. Izhikevic. Dynamical Systems in Neuroscience. MIT Press, Cambridge, Massachusetts, 2007.

[189] E. M. Izhikevich. Neural excitability, spiking and bursting. Int. J. Bif. Chaos, 10:1171-1266, 2000.

[190] S. Jahnke, R. Memmesheimer, and M. Timme. Stable Irregular Dynamics in Complex Neural Networks. Phys. Rev. Lett., 100:048102, 2008.

[191] J. G. Jefferys, R. D. Traub, and M. A. Whittington. Neuronal networks for induced $40 \mathrm{~Hz}$ rhythms. Trends Neurosci., 19:202-208, 1996.

[192] P. X. Joris, P. H. Smith, , and T. C. T. Yin. Coincidence Detection in the Auditory System: 50 Years after Jeffress. Neuron, 21:1235-1238, 1998.

[193] A. Kaiser and T. Schreiber. Information transfer in continuous processes. Physica $D, 166: 43-62,2002$.

[194] S. Kakeya. On the limits of the roots of an algebraic equation with positive coefficients. Tohoku Math. J., 2:140-142, 1912.

[195] N. Kalisman, G. Silberberg, and H. Makram. The neocortical microcircuit as a tabula rasa. Proc. Natl. Acad. Sci. U. S. A., 102:880-885, 2005.

[196] E. R. Kandel, J. H. Schwartz, and T. M. Jessell. Principles of Neuroscience. Mc Graw-Hill / Appleton \& Lange, 2000. 
[197] E. R. Kandel and W. A. Spencer. Electrophysiology of Hippocampal Neurons: IV. Fase Prepotnetials. J. Neurophysiol., 24:272-285, 1961.

[198] K. Kaneko. Dominance of Milnor Attractors and Noise-Induced Selection in a Multiattractor System. Phys. Rev. Lett., 78:2736-2739, 1997.

[199] A. Katok and B. Hasselblatt. Introduction to the Modern Theory of Dynamical Systems. Cambridge University Press, Cambridge, England, 1995.

[200] Y. Kawamura, H. N. andK. Arai, H. Kori, and Y. Kuramoto. Collective Phase Sensitivity. Phys. Rev. Lett., 101:024101, 2008.

[201] P. Kenning and H. Plassmann. NeuroEconomics: An overview from an economic perspective. Brain Research Bulletin, 67:343-354, 2005.

[202] A. I. Khibnik, B. Krauskopf, and C. Rousseau. Global study of a family of cubic Lienard equations. Nonlinearity, 11:1505-1519, 1998.

[203] H. Kielblock, C. Kirst, and M. Timme. Breakdown of Oder preservation in Networks of Pulse-Coupled Oscialltors. Chaos, 21:025113, 2011.

[204] C. Kirst. Partial Reset in Pulse-Coupled Oscillators. Master's thesis, University of Göttingen, Germany, 2006.

[205] C. Kirst. AutoMath - a AUTO bifurcation software - Mathematica interface. http://www.nld.ds.mpg.de. Max Planck Institute for Dynamics and Selforganization, Göttingen, Germany, 2011.

[206] C. Kirst. DynamicMath - A dynamical systems package for Mathematica. http://www.nld.ds.mpg.de. Max Planck Institute for Dynamics and Selforganization, Göttingen, Germany, 2011.

[207] C. Kirst. NNSim. A neuronal network simulation tool. http://www.nld.ds.mpg.de. Max Planck Institute for Dynamics and Selforganization, Göttingen, Germany, 2011.

[208] C. Kirst. PatchMath - a patch clamp data analysis package for Mathematica. http://www.nld.ds.mpg.de. Max Planck Institute for Dynamics and Selforganization, Göttingen, Germany, 2011.

[209] C. Kirst. SimKernel. A parallel $C++$ symbolic calculation and parameter scaning tool. http://simkernel.svn.sourceforge.net/. Max Planck Institute for Dynamics and Selforganization, Göttingen, Germany, 2011.

[210] C. Kirst, T. Geisel, and M. Timme. Sequential Desynchronization in Networks of Spiking Neurons with Partial Reset. Phys. Rev. Lett., 102:068101, 2009.

[211] C. Kirst and M. Timme. From networks of unstable attractors to heteroclinic switching. Phys. Rev. E, 78:065210(R), 2008.

[212] C. Kirst and M. Timme. How precise is the timing of action potentials? Frontiers in Neurosci., 3:2, 2009. 
[213] C. Kirst and M. Timme. Partial Reset in Pulse-Coupled Oscillators. SIAM J. Appl. Math., 70:2119-2149, 2010.

[214] I. Z. Kiss, C. G. Rusin, H. Kori, and J. L. Hudson. Engineering Complex Dynamical Structures: Sequential Patterns and Desynchronization. Science, 316:1886-1889, 2007.

[215] W. M. Kistler, W. Gerstner, and J. L. van Hemmen. Reduction of the HodgkinHuxley Equations to a Single-Variable Threshold Model. Neural Comput., 9:10151045, 1997.

[216] W. Klimesch, P. Sauseng, and S. Hanslmayr. EEG alpha oscillations: The inhibition-timing hypothesis. Brain Res. Rev., 53:63-88, 2007.

[217] B. W. Knight. Dynamics of encoding in a population of neurons. J. Gen. Physiol., 59:734-766, 1972.

[218] C. Koch. Biophysics of computation. Oxford University Press, New York, 1999.

[219] C. Koch and G. Laurent. Complexity and the Nervous System. Science, 284:96-98, 1999 .

[220] Y. M. Kokoz and V. I. Krinskii. Analysis of equations of excitable membranes - II. Method of analysing the electrophysiological charactersitics of the Hodgkin-Huxley membrane from the graphs of the zero-isoclines of a second order system. Biofizika, 18:937-944, 1973.

[221] H. Kori, Y. Kawamura, H. Nakao, K. Arai, and Y. Kuramoto. Collective-phase description of coupled oscillators with general network structure. Phys. Rev. E, 80:036207, 2009.

[222] H. Kori and Y. Kuramoto. Slow switching in globally coupled oscillators: robustness and occurrence through delayed coupling. Phys. Rev. E, 63:046214-10, 2001.

[223] G. Kramer. Directed information for channels with feedback. Ph.D. thesis, Swiss Federal Institute of Technology, Zurich., 1998.

[224] B. Krauskopf, H. M. Osinga, and J. Galan-Vioque. Numerical continuation methods for dynamical systems. Understanding Complex Systems. Springer, 1 edition, 2007.

[225] M. Krupa. Robust Heteroclinic Cycles. J. Nonlinear Sci., 7:129-176, 1997.

[226] S. W. Kuffler and C. Edwards. Mechanism of gamma aminobutyric acid (GABA) action and its relation to synaptic inhibition. J. Neurophysiol., 21:589-610, 1958.

[227] S. Kullback and R. A. Leibler. On information and sufficiency. Ann. Math. Statist., 22:79-86, 1951.

[228] Y. Kuramoto. Chemical Oscillations, Waves and Turbulence. Springer, Berlin, 1984.

[229] Y. Kuramoto. Collective Synchronization of pulse-coupled oscillators and excitable units. Physica D, 50:15-30, 1991.

[230] Y. A. Kuznetsov. Elements of Applied Bifurcation Theory. Springer, 3 edition, 2004. 
[231] Y. A. Kuznetsov. Practical Computation of Normal Forms on Center Manifolds at Degenerate Bogdanov-Takens Bifurcation. Int. J. Bif. Chaos, 15:3535-2546, 2004.

[232] L. D. Landau and E. Lifschitz. Lehrbuch der Theoretischen Physik, Band V und IX Statistische Physik, Teil I und II. Akademie Verlag, Berlin, 1986.

[233] W. F. Langford and K. Zhan. Interactions of Andronov-Hopf and Bogdanov-Takens bifurcations. In B. Bierstone, B. Khesin, A. Khovanskii, and J. E. Marsden, editors, The Arnoldfest: Proceedings of a Conference in Honour of V. I. Arnold for his Sixtieth Birthday, volume 24, pages 365-383. Fields Institute, Fields Institute Communications, 1999.

[234] L. Lapicque. Recherches quantitatives Sur l'excitation electrique des nerfs traitee comme une polarisation. Journal de physiologie, 9:620-635, 1907.

[235] P. E. Latham, B. J. Richmond, P. G. Nelson, and S. Nirenberg. Intrinsic Dynamics in Neuronal networks. I Theory. J. Neurophysiol., 83:808-827, 2000.

[236] P. E. Latham, B. J. Richmond, P. G. Nelson, and S. Nirenberg. Intrinsic Dynamics in Neuronal networks. II. Experiment. J. Neurophysiol., 83:828-835, 2000.

[237] G. Laurent. Olfactory network dynamics and the coding of multidimensional signals. Nat. Rev. Neurosci., 3:884-895, 2002.

[238] G. Laurent, M. Stopfer, R. W. Friedrich, M. I. Rabinovich, A. Volkovskii, and H. D. I. Abarbanel. Odor Encoding as an Active, Dynamical Process: Experiments, Computation, and Theory. Annu. Rev. Neurosci., 24:263-297, 2001.

[239] M. Lengyel, J. Kwag, O. Paulsen, and P. Dayan. Matching storage and recall: hippocampal spike timing-dependent plasticity and phase-response curves. Nature Neurosci., 8:1677-1683, 2005.

[240] L. S. Leung and H. W. Yu. Theta-frequency resonance inhippocampal CA1 neurons in vitro demonstrated by sinusodial current injection. J. Neurophysiol., 79:325-341, 1998.

[241] L. W. Leung and C. Y. Yim. Intrinsic membrane potential oscillations in hippocampal neurons in vitro. Brain Res., 553:261-274, 1991.

[242] A. Levina, J. M. Herrmann, and T. Geisel. Dynamical synapses causing selforganized criticality in neural networks. Nature Physics, 3:857-860, 2007.

[243] A. Liénard. Etude des oscillations entretenues. Revue générale de l'électricité, 23:901-912 and 946-954, 1928.

[244] Z. Liu, Y.-C. Lai, and F. C. Hoppenstaedt. Phase clustering and transition to phase synchronization in a large number of coupled nonlinear oscillators. Phys. Rev. E, 63:055201-4, 2001.

[245] R. R. Llinás. The intrinsic electrophysiological properties of mammalian neurons: insights into central nervous stystem function. Science, 242:1654-1664, 1988. 
[246] N. K. Logothetis, J. Pauls, M. A. Augath, T. Trinath, and A. Oeltermann. Neurophysiological investigation of the basis of the fMRI signal. Nature, 412:150-157, 2001.

[247] M. London and M. Häusser. Dendritic computation. Ann. Rev. Neurosci., 28:503$532,2005$.

[248] W. Maass and C. M. Bishop. Pulsed Neural Networks. MIT Press, Cambridge, Massachusetts, 1999.

[249] D. J. C. MacKay. Information theory, inference, and learning algorithms. Cambridge University Press, 2003. ISBN 978-0-521-64298-9.

[250] Z. F. Mainen and T. J. Sejnowski. Influence of dendritic structure on firing pattern in model neocortical neurons. Nature, 382:363-366, 1996.

[251] Y. Maistrenko, O. Popovych, O. Burylko, and P. A. Tass. Mechanism of Desynchronization in the finite-Dimensional Kuramoto Model. Phys. Rev. Lett., 93(8):084102, 2004

[252] E. O. Mann, J.-M. Suckling, N. Hajos, S. A. Greenfield, and O. Paulsen. Perisomatic feedback inhibition underlies cholinergically induced fast network oscillations in the rat hippocampus in vitro. Neuron, 45:105-117, 2005.

[253] E. Marder and R. L. Calabrese. Principles of rhythmic motor pattern generation. Physiol. Rev., 76:687-717, 1996.

[254] H. Marko. The bidirectional communication theory - a generalization of information theory. IEEE Trans. Communication, 21:1345-1351, 1973.

[255] J. L. Massey. Causality, feedback and directed information. In Proc. Intl. Symp. on Info. th. and its Applications. Waikiki, Hawai, USA, 1990.

[256] G. Mato and I. Samengo. Type I and Type II Neuron Models Are Selectively Driven by Differential Stimulus Features. Neural Comput., 20:2418-2440, 2008.

[257] A. Mauro, F. Conti, F. Dodge, and R. Schor. Subthreshold behavior and phenomenological impedance of the squid giant axon. J. Gen. Physiol., 55:497-523, 1970 .

[258] R. M. May and W. J. Leonard. Nonlinear Aspects of Competition Between Three Species. SIAM J. Appl. Math., 29:243-253, 1975.

[259] D. A. McCormick and D. Contreras. On the Cellular and Network Bases of Epileptic Seizures. Ann. Rev. Physiol., 63:815-46, 2001.

[260] M. L. Mehta. Matrix Theory. Hindustan Publishing, Delhi, India, 1989.

[261] B. W. Mel. Information Processing in Dendritic Trees. Neural Comput., 6:10311085, 1994.

[262] R.-M. Memmesheimer and M. Timme. Designing complex networks. Physica D, 224:181-201, 2006. 
[263] R.-M. Memmesheimer and M. Timme. Designing the Dynamics of Spiking Neural Networks. Phys. Rev. Lett., 97:188101, 2006.

[264] R.-M. Memmesheimer and M. Timme. Synchrony and Precise Timing in Complex Neural Networks. In Y. Moreno, V. Latora, and S. Boccaletti, editors, Handbook on Biological Networks. World Scientific, London, 2009.

[265] R.-M. Memmesheimer and M. Timme. Stable and unstable periodic orbits in complex networks of spiking neurons with delays. Discr. Cont. Dyn. Syst., 28:1555-1588, 2010 .

[266] T. Metzinger. Einleitung: Das problem des Bewusstseins. In Bewusstsein. Beiträge aus der Gegenwartsphilosohie. Mentis, Paderborn, Germany, 1996.

[267] J. Milnor. On the Concept of Attractor. Comm. Math. Phys., 99:177-195, 1985.

[268] J. Milnor. On the Concept of Attractor: Correction and Remarks. Comm. Math. Phys., 102:517-519, 1985.

[269] R. E. Mirollo and S. H. Strogatz. Synchronization of Pulse-Coupled Biological Oscillators. SIAM J. Appl. Math., 50(6):1645-1662, 1990.

[270] A. Miyawaki, J. Llopis, R. Heim, J. M. MacCaffery, J. A. Adams, M. Ikura, and R. Y. Tsien. Flourescent indicators for $\mathrm{Ca}^{2+}$ based on green flourescent proteins and calmodulin. Nature, 388:882-887, 1997.

[271] M. Monteforte. Chaotic Dynamics in Networks of Spiking Neurons in the Balanced State. Ph.D. thesis, University of Göttingen, 2010.

[272] M. Monteforte and F. Wolf. Dynamical Entropy Production in Spiking Neuron Networks in the Balanced State. Phys. Rev. Lett., 105:268104, 2010.

[273] C. Morris and H. Lecar. Voltage Oscillations in the Barnacle Giant Muscle Fiber. Biophys. J., 35:193-213, 1981.

[274] R. Morris, L. Tarassenko, and M. Kenward. Cognitive Systems - Information Processing Meets Brain Science. Academic Press, New York, 2005.

[275] E. Moser, E. Kropff, and M. Moser. Place cells, grid cells, and the brain's spatial representation system. Annual review of neuroscience, 31:69-89, 2008.

[276] J. Murdock. Normal Forms and Unfoldings for Local Dynamical Systems. Springer, New York, 2003.

[277] T. Y. Na. Computational Methods in Engineering: Boundary Value Problems. Mathematics in Science and Engeneering. Academic Press, New York, USA, 1979.

[278] J. Nagumo, S. Arimoto, and S. Yoshizawa. An active pulse transmission line simulating nerve axon. Proc IRE., 50:2061-2070, 1962.

[279] B. Naundorf, F. Wolf, and M. Volgushev. Unique features of action potential initiation in cortical neurons. Nature, 440:1060-1063, 2006. 
[280] E. Neher. Two fast transient current components during voltage clamp on snail neurons. J. gen. Physiol., 58:36-53, 1971.

[281] E. Neher and B. Sakmann. Single-channel currents recorded from membrane of denervated frog muscle fibers. Nature, 260:799-801, 1976.

[282] F. S. Neves and M. Timme. Controlled perturbation-induced switching in pulsecoupled oscillator networks. J. Phys. A: Math. Theor., 42:345103, 2009.

[283] M. Newman, A.-L. Barabàsi, and D. J. Watts. The Structure and Dynamics of Networks. Princeton University Press, Princeton, USA, 2006.

[284] J. G. Nicholls, A. R. Martin, and B. G. Wallace. From Neuron to Brain. Sinauer Associactes, Inc. - Publishers, Sunderland, Massachusetts, USA, 1992.

[285] G. Nicolis and C. Nicolis. Foundations of Complex Systems. World Scientific, Sigapore, 2007.

[286] H. F. Nijhout, L. Nadel, and D. Stein, editors. Pattern Formation in the Physical and Biological Sciences. Addison Wesley Longman, 1997.

[287] M. Numberger and A. Draguhn. Patch-clamp Technik. Spektrum Akademischer Verlag, Heidelberg, Berlin, Germany, 1995.

[288] J. O'Keefe and L. Nadel. The Hippocampus as a Cognitive Map. Oxford University Press, 1978.

[289] Z. Olami, H. J. S. Feder, and K. Christensen. Self-Organized Criticality in a Continuous, Nonconservative Cellular Automaton Modeling Earthquakes. Phys. Rev. Lett., 68:1244-1247, 1992.

[290] G. Orosz, P. Ashwin, and S. Townley. Learning of spatiotemporal codes in a coupled oscillator system. IEEE Trans. Neural Networks, 20:1135-1147, 2009.

[291] A. M. Oswald, B. Dorion, and L. Maler. Interval Coding. II. Dendrite Dependent Mechanisms. J. Neurophysiol., 97:2744-2757, 2007.

[292] J. M. Palva, S. Palva, and K. Kaila. Phase Synchrony among Neuronal Oscillations in the Human Cortex. J. Neurosci., 25:3962-3972, 2005.

[293] D. Paré, E. Shink, H. Gaudreau, A. Destexhe, and E. J. Lang. Impact of spontaneous synaptic activity on the resting properties of cat neocortical pyramidal neurons in vivo. J. Neurophysiol., 79:1450-1460, 1998.

[294] R. Perin, T. K. Berger, and H. Markram. A synaptic organizing principle for cortical neuronal groups. Proc. Natl. Acad. Sci. U. S. A., 108:5419-5424, 2010.

[295] D. H. Perkel, J. H. Schulman, T. H. Bullock, G. P. Moore, and J. P. Segundo. Pacemaker Neurons: Effects of Regularly Spaced Synaptic Input. Science, 145(3627):6163, 1964.

[296] C. Peskin. Mathematical Aspects of Heart Physiology. Courant Institute of Mathematical Sciences, New York University, 1984. 
[297] A. Pikovsky, O. Popovych, and Y. Maistrenko. Resolving Clusters in Chaotic Ensembles of Globally Coupled Identical Oscillators. Phys. Rev. Lett., 87:044102, 2001.

[298] A. Pikovsky, M. Rosenblum, and J. Kurths. Synchronization. Cambridge University Press, Cambridge, England, 2001.

[299] P. F. Pinsky. Synchrony and Clustering in an Excitatory Neural Network Model with Intrinsic Relaxation Kinetics. SIAM J. Appl. Math., 55(1):220-241, 1995.

[300] A. Polsky, B. W. Mel, and J. Schiller. Computational subunits in thin dendrites of pyramidal cells. Neural Networks, 7:621-627, 2004.

[301] O. Popovych, V. Krachkovskyi, and P. A. Tass. Desynchronization transitions in coupled phase oscillator systems with delay. In R. Stoop, editor, Prooceedings of the 2003 Workshop on Nonlinear Dynamics of Electronic Systems, pages 197-200. 2003.

[302] O. V. Popovych, C. Hauptmann, and P. A. Tass. Effective desynchronization by nonlinear delayed feedback. Phys. Rev. Lett., 94:1614102, 2005.

[303] C. M. Postlethwaite and J. H. P. Dawes. Regular and irregular cycling near a heteroclinic network. Nonlinearity, 18:1477-1509, 2005.

[304] A. Pouget, P. Dayan, and R. Zemel. Information Processing with population codes. Nature Reviews Neuroscience, 1:125-132, 2000.

[305] S. A. Prescott, Y. D. Koninck, and T. J. Sejnowski. Biophysical basis for three distinct dynamical mechanims of action potential initiation. PLoS Comput. Biol., 4:31000198, 2008.

[306] S. A. Prescott, S. Ratté, Y. D. Koninck, and T. J. Sejnowski. Nonlinear interaction between shunting and adaptation controls a switch between integration and coincidence detection in pyramidal neurons. J. Neurosci., 26:9084-9097, 2006.

[307] S. A. Prescott, S. Ratté, Y. D. Koninck, and T. J. Sejnowski. Pyramidal Neurons Switch from Integrators In Vitro to Resonators Under In Vivo-Like Conditions. J. Neurophysiol., 100:3030-3042, 2008.

[308] E. Puil, B. Gimbarzevsky, and R. M. Miura. Quantification of membrane properties of trigeminal root ganglion neurons in guinea pigs. J. Neurophysiol., 55:995-1016, 1986.

[309] W. Rall. Theoretical significance of dendritic trees for neuronal input-ouput relations. In R. Reiss, editor, Neuronal Theory and Modeling, pages 73-97. Stanford University Press, Stanford, CA, USA, 1964.

[310] W. Rall, R. E. Burke, W. R. Holmes, J. J. B. Jack, S. J. Redman, and I. Segev. Matching Dendritic Neuron Models to Experimental Data. Physiological Reviews, 72(4):S159-S185, 1992.

[311] S. Ramón y Cajal. Texture of the Nervous System of Man and the Vertebrates. Springer, Berlin, 1899. 
[312] S. Ramón y Cajal. Histologie du Sysème Nerveux de l'Homme et des Vertébré. A. Maloine, Paris, 1909.

[313] A. D. Reyes and E. E. Fetz. Effects of Transient Depolarizing Potential on the Firing Rate of Cat Neocortical Neurons. J. Neurophysiol., 69(5):1673-1683, 1993.

[314] A. D. Reyes and E. E. Fetz. Two Modes of Interspike Interval Shortening by Brief Transient Depolarizations in Cat Neocortical Neurons. J. Neurophysiol., 69(5):16611672, 1993.

[315] J. Rinzel. Excitation dynamics: insights form simplified membrane models. Federation Proc., 44:653-675, 1985.

[316] J. Rinzel and B. Ermentrout. Analysis of neuronal excitability and oscillations. In Methods in Neuronal Modeling: From Synapes to Networks. MIT press, London, 1989 .

[317] J. Rinzel, D. Terman, X.-J. Wang, and B. Ermentrout. Propagating Activity Patterns in Large-Scale Inhibitory Neuronal Networks. Science, 279:1351-1355, 1998.

[318] H. P. C. Robinson and A. Harsch. Stages of spike time variability during neuronal response to transient inputs. Phys. Rev. E, 66:061902, 2002.

[319] R. M. Rose and J. L. Hindmarsh. The Assembly of Ionic Currents in a Thalamic Neuron I. The Three-Dimensional Model. Proc. R. Soc. Lond. B, 237:267-288, 1989.

[320] R. M. Rose and J. L. Hindmarsh. The Assembly of Ionic Currents in a Thalamic Neuron II. The Stability and State Diagrams. Proc. R. Soc. Lond. B, 237:289-312, 1989 .

[321] J. P. Rospars and P. Lansky. Stochastic model neuron without resetting of dendritic potential: application to the olfactory system. Biol. Cybern., 69:283, 1993.

[322] O. E. Rössler and L. Wegmann. Chaos in the Zhabotinskii reaction. Nature, 271:8990, 1978.

[323] M. E. Rush and J. Rinzel. The potassium A-current, low firing rates and rebound excitation in Hodgkin-Huxley models. Bull. Math. Biol., 57:899-929, 1995.

[324] Y. B. Saalmann, I. N. Pigarev, and T. R. Vidyasagar. Neural mechanisms of visual attention: how top-down feedback highlights relevant locations. Science, 215:15321534, 2007.

[325] N. T. Sahin, S. Pinker, S. S. Cash, D. Schomer, and E. Halgren. Sequential Processing of Lexical, Grammatical, and Phonological Information Within Broca's Area. Science, 326:445-449, 2009.

[326] E. Salinas and T. J. Sejnowski. Correlated neuronal activity and the flow of neural information. Nat. Rev. Neurosci., 2:539-550, 2001.

[327] J. Sanders, F. Verhulst, and J. Murdock. Averaging Methods for Nonlinear Dynamical Systems. Springer, New York, 2007. 
[328] S. Schecter. The saddle-node separatrix-loop bifurcation. SIAM J. Math. Analy., 18:1142-1156, 1987.

[329] J.-H. Schleimer and M. Stemmler. Coding of Information in Limit Cycle Oscillators. Phys. Rev. Lett., 103:248105, 2009.

[330] J.-M. Schoffelen, R. Oostenveld, and P. Fries. Neuronal Coherence as a Mechanism of Effective Corticospinal Interaction. Science, 308:111-113, 2005.

[331] T. Schreiber. Measuring Information Transfer. Phys. Rev. Lett., 85:461-464, 2000.

[332] I. Segev, J. W. Fleshman, and R. E. Bruke. Compartmental models of complex neurons. In C. Koch and I. Segev, editors, Methods in Neuronal Modeling, pages 63-96. MIT Press, Cambridge, Massachusetts.

[333] W. Senn and R. Urbanczik. Similar non-leaky integrate-and-fire neurons with instantaneous couplings always synchronize. SIAM J. Appl. Math., 61:1143-1155, 2001.

[334] A. A. Sharp, L. F. Abbott, and E. Marder. Artificial Electrical Synapses in Oscillatory Networks. J. Neurophysiol., 67:1691-1694, 1992.

[335] E. Shea-Brown, J. Moehlis, and P. Holmes. On the Phase Reduction and Response Dynamics of Neural Oscillator Populations. Neural Comput., 16:673-715, 2004.

[336] G. M. Shepard. Neurobiology. Oxford University Press, New York, 1994.

[337] S. Shoham, D. H. O'Connor, and R. Segev. How silent is the brain: is there a "dark matter" problem in neuroscience? J. Comp. Physiol. A, 192:777-7784, 2006.

[338] Y. Shu, A. Hasenstaub, and D. A. McCormick. Turning on and off recurrent balanced cortical activity. Nature, 423:288-293, 2003.

[339] A. G. Siapas, E. V. Lubenov, and M. A. Wilson. Prefrontal Phase Locking to Hippocampal Theta Oscillations. Neuron, 46(1):141 - 151, 2005. ISSN 0896-6273.

[340] W. Singer. Neuronal synchrony: a versatile code for the definition of relations? Neuron, 24:49-65, 1999.

[341] W. Singer. Dynamic formation of functional networks by synchronization. Neuron, 69:733-739, 2008.

[342] W. Singer and C. M. Gray. Visual Feature Integration and the temporal correlation hypothesis. Ann. Rev. Neurosci., 18:555-586, 1995.

[343] W. R. Softky, C. K. W. R. Softky, and C. Koch. The highly irregular firing of cortical cells is inconsistent with temporal integration of random EPSPs. J. Neurosci., 13:334-350, 1993.

[344] S. Song, P. J. Sjöström, M. Reigl, S. Nelson, and D. B. Chklovskii. Highly Nonrandom Features of Synaptic Connectivity in Local Cortical Circuits. PLoS Biol., 3:e68, 2005. 
[345] L. Späth. The Weirestrass preparation theorem. J f. d. reine und angewandte Mathematik, 161:95-100, 1929.

[346] M. Staniek and K. Lehnertz. Symbolic Transfer Entropy. Phys. Rev. Lett., 100:158101, 2008.

[347] A. Stepanyants and D. B. Chklovskii. Neurogeometry and potential synaptic connectivity. Trends in Neuroscience, 28:387-394, 2005.

[348] M. Steriade. Impact of network activities on neuronal properties in cortico-thalamic systems. J. Neurophysiol., 86:1-39, 2001.

[349] M. Steriade, D. Contreras, F. Amzica, and I. T. I. Synchronization of fast (30$40 \mathrm{~Hz}$ ) spontaneous oscillations in intrathalamic and thalamocortical networks. $J$. Neurosci., 16:2788-2808, 1996.

[350] M. Steriade and I. Timofeev. Neuronal plasticity in thalamocortical networks during sleep and waking oscillations. Neuron, 37:563-576, 2003.

[351] C. F. Stevens. Letters to the Editor. Biophys. J., 4:417-419, 1964.

[352] K. M. Stiefel, B. S. Gutkin, and T. J. Sejnowski. Cholinergic Neuromodulation Changes Phase Response Curve Shape and Type in Cortical Pyramidal Neurons. PLoS ONE, 3:e3947, 2008.

[353] M. Storace, D. Linaro, and E. de Lange. The Hindmarsh-Rose neuron model: Bifurcation analysis and piecewise-linear approximations. Chaos, 18:033128, 2008.

[354] J. Stricker, S. Cookson, M. R. Bennett, W. H. Mather, L. S. Tsimring, and J. Hasty. A fast, robust and tunable synthetic gene oscillator. Nature, 456:516-519, 2009.

[355] S. H. Strogatz. Nonlinear Dynamics and Chaos: With Applications to Physics, Biology, Chemistry and Engineering. Perseus Publ., Cambridge, Massachusetts, 2000 .

[356] S. P. Strong, R. Koberle, R. R. de Ruyter van Steveninck, and W. Bialek. Entropy and Information in Neural Spike Trains. Phys. Rev. Lett., 80:197-200, 1998.

[357] G. Stuart, N. Spruston, and M. Häusser, editors. Dendrites. Oxford University Press, 1999.

[358] F. Takens. Forced oscillations and bifurcations. Comm. Math. Inst. Rijksuniv. Utrecht, 2:1-111, 1994.

[359] T. Tanteno, A. Harsh, and H. P. Robinson. Threshold firing frequency-current relationships of neurons in rat somatosensory cortex: type 1 and type 2 dynamics. J. Neurophysiol., 92:2283-2294, 2004.

[360] T. Tanteno and H. P. Robinson. Rate coding and spike-time variability in cortical neurons with two types of threshold dynamics. J. Neurophysiol., 95:2650-2663, 2006 .

[361] T. Tanteno and H. P. Robinson. Phase resetting curves and oscillatory stability in interneurons of rat somatosensory cortex. Biophys. J., 92:683-695, 2007. 
[362] P. A. Tass. A model of desynchronizing deep brain stimulation with a demandcontrolled coordinated reset of neural subpopulations. Biol. Cybern., 89:81-88, 2003.

[363] J.-N. Teramae, H. Nakao, and G. B. Ermentrout. Stochastic Phase Reduction for a General Class of Noisy Limit Cycle Oscillators. Phys. Rev. Lett., 102(19):194102, May 2009.

[364] W. M. Thorburn. Occam's razor. Mind, 24:287-288, 1915.

[365] P. Tiesinga, J.-M. Fellous, and T. J. Sejnowski. Regulation of spike timing in visual cortical circuits. Nat. Rev. Neurosci., 9:97-107, 2008.

[366] P. Tiesinga and T. J. Sejnowski. Cortical enlightenment: are attentional gamma oscillations driven by ING or PING? Neuron, 63:727-732, 2009.

[367] P. H. E. Tiesinga, J.-M. Fellous, E. Salinas, J. V. Jose, and T. S. Sejnowski. Synchronization as a mechanism for attentional gain modulation. Neurocomputing, 58-60:641-646, 2004.

[368] A. N. Tikhonov. On systems of differential equations containing parameters (in Russian). Matematicheskii Sbornik, 27:147-156, 1950.

[369] M. Timme and F. Wolf. The simplest problem in the collective dynamics of neural networks: is synchrony stable? Nonlinearity, 7:1579-1599, 2008.

[370] M. Timme, F. Wolf, and T. Geisel. Coexistence of Regular and Irregular Dynamics in Complex Networks of Pulse-Coupled Oscillators. Phys. Rev. Lett., 89:258701, 2002.

[371] M. Timme, F. Wolf, and T. Geisel. Prevalence of Unstable Attractors in Networks of Pulse-Coupled Oscillators. Phys. Rev. Lett., 89:154105, 2002.

[372] M. Timme, F. Wolf, and T. Geisel. Unstable attractors induce perpetual synchronization and desynchronization. Chaos, 13(1):377-387, 2003.

[373] B. Todd and D. Andrews. The identification of peaks in physiological signals. Computers and Biomedical Research, 32:322-335, 1999.

[374] R. Traub, J. G. R. Jefferys, and M. A. Whittington. Fast Oscillations in Cortical Circuits. MIT Press, Cambridge MA, USA, 1999.

[375] R. D. Traub and R. Miles. Neuronal Networks of the Hippocampus. Cambridge University Press, Cambridge, England, 1991.

[376] R. D. Traub and R. K. S. Wong. Cellular mechnism of neuronal synchornization in epilepsy. Science, 216:745-747, 1982.

[377] R. D. Traub, R. K. S. Wong, R. Miles, and H. Michelson. A Model of CA3 Hippocampal Pyramidal Neuron Incorporating Voltage-Clamp Data on Intrinsic Conductances. J. Neurophysiol., 66:635-650, 1991.

[378] M. Tsodyks, I. Mitkov, and H. Sompolinsky. Pattern of Synchrony in Inhomogeneous Networks of Oscillators with Pulse Interactions. Phys. Rev. Lett., 71(8):1280-1283, 1993. 
[379] K. Tsumoto, H. Kitajima, T. Yoshinaga, K. Aihara, and H. Kawakami. Bifurcations in Morris-Lecar neuron model. Neurocomputing, 69:293-316, 2006.

[380] H. C. Tuckwell. Introduction to theoretical neurobiology, volume 1,2 of Cambridge Studies in Mathematical Biology. Cambridge University Press, Cambridge, England, 1988.

[381] D. L. Turcotte and J. B. Rundle. Self-organized complexity in the physical, bilogical and social sciences. Proc. Natl. Acad. Sci. U. S. A., 99:2463-2465, 2002.

[382] A. M. Turing. The chemical basis of morphogenesis. Phil. Trans. R. Soc. Lond. B, 237:37-72, 1952 .

[383] J. J. Tyson, K. C. Chen, and B. Novak. Sniffers, buzzers, toggles and blinkers: dynamics of regulatory and signaling pathways in the cell. Current Opinion in Cell Biology, 15:221-231, 2003.

[384] G. E. Uhlenbeck and L. S. Ornstein. On the theory of Brownian Motion. Phys. Rev., 36:823-841, 1930.

[385] B. van der Pol. A theory of the amplitude of free and forced triode vibrations. Radio Review, 1:701-710, 1920.

[386] N. G. van Kampen. Stochastic Processes in Physics and Chemistry. North-Holland, Amsterdam, 1981.

[387] A. van Ooyen, J. Duijnhouwer, M. W. H. Remme, and J. van Pelt. The effect of dendritic topology on firing patterns in model neurons. Network: Comput. Neural Systs., 13:311-325, 2002.

[388] C. van Vreeswijk. Analysis of the Asynchronous State in Networks of Strongly Coupled Oscillators. Phys. Rev. Lett., 84:5110-5113, 2000.

[389] C. van Vreeswijk, L. F. Abbott, and G. B. Ermentrout. When Inhibition not Excitation Synchronizes Neural Firing. J. Comp. Neurosci., 1:313-321, 1994.

[390] C. van Vreeswijk and H. Sompolinsky. Chaos in Neuronal Networks with Balanced Excitatory and Inhibitory Activity. Science, 274:1724-1726, 1996.

[391] C. van Vreeswijk and H. Sompolinsky. Chaos in neuronal networks with balanced excitatory and inhibitory activity. Science, 274:1724-1726, 1996.

[392] C. H. Vanderwolf. Hippocampal electrical activity and voluntary movement in the rat. EEG Clin. Neurophysiol., 26:407-418, 1969.

[393] F. Varela, J. P. Lachaux, E. Rodriguez, and J. Martinerie. The brainweb: phase synchronization and large-scale integration. Nat. Rev. Neurosci., 2:229-239, 2001.

[394] R. P. Vertes. Hippocampal theta rhythm: a tag for short-term memory. Hippocampus, 15:923-935, 2005.

[395] T. P. Vogels and L. F. Abbott. Gating multiple signals through detailed balance of excitation and inhibition in spiking networks. Neural Networks, 12:483-491, 2009. 
[396] M. Volgushev, M. Chistiakova, and W. Singer. Modification of discharge patterns of neocortical neurons by induced oscillations of the membrane potential. Neuroscience, 83:15-25, 1998.

[397] O. von Bohlen und Halbach and R. Dermietzel. Neurotransmitters and neuromodulators: handbook of receptors and biological effects. Wiley-VHC, Weinheim, Germany, 2006.

[398] C. von der Malsburg. The Correlation Theory of Brain Function. Internal Report 81-2, Dept. of Neurobiology, Max-Planck-Institute for Biophysical Chemistry, G/"ottingen, Germany, 1981.

[399] C. von der Malsburg and W. Schneider. A neural cocktail-party processor. Biol. Cybern., 54:29-40, 1986.

[400] X.-J. Wang and G. Buzsáki. Gamma Oscillation by Synaptic Inhibition in a Hippocampal Interneuronal network Model. J. Neurosci., 16(20):6402-6413, 1996.

[401] D. J. Watts. Small Worlds. Princeton University Press, Princeton, New Jersey, 1999.

[402] M. Wehr and A. M. Zador. Balanced inhibition underlies tuning and sharpens spike timing in auditory cortex. Nature, 426:442-446, 2003.

[403] M. A. Whittington, R. D. Traub, and J. G. Jefferys. Synchronized oscillations in interneuron networks driven by metabotropic glutamate receptor activation. Nature, 373:612-615, 1995.

[404] M. A. Whittington, R. D. Traub, N. Kopell, B. Ermentrout, and E. H. Buhl. Inhibition-based rhythms: experimental and mathematical observations on network dynamics. Int. J. Psychophysiol., 38:315-336, 2000.

[405] S. Wiggins. Introduction to Applied Nonlinear Dynamical Systems and Chaos. Springer, New York, 1990.

[406] H. R. Wilson and J. D. Cowan. Excitatory and Inhibitory Interactions in Localized Populations of Model Neurons. Biophys. J., 12:1-24, 1972.

[407] A. T. Winfree. The Geometry of Biological Time. Springer, Berlin, 1980.

[408] A. Witt, D. Battaglia, T. Geisel, and A. Gail. Dynamic effective connectivity analysis of goal directed reaching. Front Comput Neurosci 2010, BCCN conference abstract, doi: 10.3389/conf.fncom.2010.51.00021.

[409] D. Wolpert and C. Frith. The Neuroscience of Social Interactions: Decoding, Influencing, and Imitating the Actions of Others. Oxford University Press, New York, 2004.

[410] T. Womelsdorf and P. Fries. Neuronal coherence during selective attentional processing and sensory-motor integration. J. Physiol. Paris, 100:182-193, 2006.

[411] T. Womelsdorf and P. Fries. The Cognitive Neurosciences IV, chapter Selective attention through selective neuronal synchronization, pages 289-304. MIT Press, 2008. 
[412] T. Womelsdorf, J. M. Schoffelen, R. Oostenveld, W. Singer, R. Desimone, A. K. Engel, K. Andreas, and P. Fries. Modulation of Neuronal Interactions Through Neuronal Synchronization. Science, 316:1609-1612, 2007.

[413] R. O. L. Wong. Retinal waves and visual system development. Annu. Rev. Neurosci., 22:29-47, 1999.

[414] S. H. Wu and J. B. Kelly. In vitro brain slice studies of the rat's dorsal nucleus of the lateral lemniscus. II. Physiological properties of biocytin-labeled neurons. $J$. Neurophysiol., 73:794-805, 1995.

[415] J. Yang, J.-L. Xing, N.-P. Wu, Y.-H. Liu, C.-Z. Zhang, F. Kuang, V.-Z. Han, and S.J. Hu. Membrane Current-based mechanisms for excitability transitions in neurons of the rat mesencephalic trigeminal nuclei. Neuroscience, 163:799-810, 2009.

[416] M. Yeung and S. H. Strogatz. Time Delay in the Kuramoto Model of Coupled Oscillators. Phys. Rev. Lett., 82(3):648-651, 1999.

[417] K. Yoshimura and K. Arai. Phase Reduction of Stochastic Limit Cycle Oscillators. Phys. Rev. Lett., 101:154101, 2008.

[418] K. Ziemelis, editor. Nature Insight: Complex Systems, volume 410. Nature, London, 2001. 


\section{Nomenclature}

List of abbreviations.

Abbreviations used for all bifurcations are listed in tab. 3.1 on page 37 .

\begin{tabular}{|c|c|c|c|}
\hline abbreviation & description & reference & page \\
\hline dMI & delayed mutual information & 15.2 .1 & 180 \\
\hline DNLL & dorsal nucleus of the lateral lemniscus & 12.1 & 155 \\
\hline GABA & $\gamma$-amino-butyric-acid & 12 & 155 \\
\hline LIF & leaky integrate and fire neuron & 2.6.1, eq. $(2.6 .4)$ & 28 \\
\hline ML & Morris-Lecar neuron model & 2.4 .2 & 23 \\
\hline $\mathrm{PRC}$ & phase response curve & 3.4 , eq. $(3.4 .7)$ & 47 \\
\hline QIF & quadratic integrate fire neuron & 2.6 .1$, eq. $(2.6 .3)$ & 27 \\
\hline WB & Wang-Buzsaki neuron model & 2.4 .3 & 24 \\
\hline
\end{tabular}

List of symbols.

\begin{tabular}{llrc}
\hline symbol & description & reference & page \\
\hline \hline$[[A]]_{i, j}$ & matrix obtained from $A$ deleting row $i$ and column $j$ & 16.2 .2 .4 & 187 \\
\hline$\bigodot_{i} f_{i}$ & composition of maps $f_{i}$ & 6.3, eq. $(6.3 .8)$ & 73 \\
\hline$a_{i, \infty}$ & steady state activation / gating & 2.3, eq. $(2.3 .3)$ & 21 \\
\hline$a_{x, i}$ & $i^{\text {th }}$ gating variable of ion species $x$ & 2.3, eq. $(2.3 .1)$ & 21 \\
\hline $\mathcal{B}(\mathcal{A})$ & basin of attraction of invariant set $\mathcal{A}$ & 3.1, eq. $(3.1 .3)$ & 35 \\
\hline$c_{\mathrm{cr}}^{(n)}$ & critical reset strength for clusters size $n$ & 6.6, prop. 6.6 .1 & 83 \\
\hline$c_{\mathrm{m}}$ & membrane capacitance & 2.2, eq. $(2.2 .3)$ & 20 \\
\hline $\mathcal{D}(X)$ & domain of map $X$ & 7.2 .5 & 100 \\
\hline$\varepsilon_{i j}, \varepsilon$ & coupling strength from oscillator $j$ to $i$ & 7.2 .2$, eq. $(7.2 .7)$ & 95 \\
\hline$E$ & event sequence & 7.2 .2$, eq. $(7.2 .7)$ & 95 \\
\hline$e_{i}$ & $i^{\text {th }}$-event & 7.2 .1 & 92
\end{tabular}




\begin{tabular}{|c|c|c|c|}
\hline symbol & description & reference & page \\
\hline$F^{(l)}$ & event-based map & 7.2 .1 & 92 \\
\hline $\mathcal{F}^{t}$ & semi flow of a dynamical system & 3.1 & 34 \\
\hline $\mathcal{F}$ & firing sequence & 6.3, eq. $(6.3 .7)$ & 73 \\
\hline$g_{\mathrm{x}}$ & maximal conductance for ion channel species $x$ & 2.3, eq. $(2.3 .1)$ & 21 \\
\hline$g_{\mathrm{L}}$ & leak conductance & 2.2, eq. $(2.2 .4)$ & 20 \\
\hline$g_{\mathrm{DS}}, g_{\mathrm{SD}}$ & dendro-somatic intracellular conductance & 2.5, eq. $(2.5 .1)$ & 26 \\
\hline$g_{\text {syn }}$ & synaptic conductance & 2.6, eq. $(2.7 .1)$ & 29 \\
\hline$\gamma_{i j}$ & coupling function between oscillator $i$ and $j$ & 3.4, eq. $(3.4 .13)$ & 49 \\
\hline$\Gamma_{X Y}$ & collective coupling function between cluster $X$ and $Y$ & 16.3.1.2, eq. (16.3.7) & 196 \\
\hline$H, H_{\varepsilon}, H_{\varepsilon, \tau}$ & sub-threshold pulse interaction function & 5.2 .4$, eq. $(5.2 .14)$ & 61 \\
\hline$i_{X}$ & index of oscillator $i$ in cluster $X$ & 16.3 .1 & 194 \\
\hline$i_{x}$ & membrane current carried by ion species $x$ & 2.3, eq. $(2.3 .1)$ & 21 \\
\hline$i_{\infty}$ & steady state current & 10.3.1, eq. (10.3.1) & 133 \\
\hline$i_{\mathrm{e}}$ & external current & 2.2 , eq. $(2.2 .3)$ & 20 \\
\hline$i_{\mathrm{a}}, i_{\text {ion }}$ & total active membrane currents & 2.2 , eq. $(2.2 .3)$ & 20 \\
\hline$i_{\text {syn }}$ & synaptic membrane current & 2.7, eq. $(2.7 .1)$ & 29 \\
\hline$I_{0}(k), I_{1}(k)$ & modified Bessel functions of the first kind & 16 , eq. $(16.2 .28)$ & 189 \\
\hline$J, J_{\varepsilon}, J_{\varepsilon, \tau}$ & supra-threshold pulse interaction function & 5.2 .4$, eq. $(5.2 .15)$ & 61 \\
\hline $\mathbf{K}$ & firing map & 6 , eq. $(6.3 .4)$ & 72 \\
\hline$\lambda(\cdot)$ & Lebesgue measure & 3.1 & 34 \\
\hline $\mathcal{M}_{\mu, k}$ & $\begin{array}{l}\text { van Mises distribution with mean } \mu \text { and } \\
\text { concentration } k\end{array}$ & 16 , eq. $(16.2 .28)$ & 189 \\
\hline M & Poincaré return map & 6 , eq. $(6.3 .6)$ & 73 \\
\hline$M_{\mathcal{F}}$ & firing map with firing sequence $\mathcal{F}$ & 6.5, eq. $(6.5 .1)$ & 78 \\
\hline $\mathrm{MI}_{i, j}(d)$ & $\begin{array}{l}\text { delayed mutual information between unit } i \text { and } j \text { with } \\
\text { delay } d\end{array}$ & 15.2 .1 , eq. 15.2 .1 & 180 \\
\hline $\mathrm{MI}_{\mathrm{vM}}(k)$ & $\begin{array}{l}\text { mutual information for a multivariate van Mises } \\
\text { distribution with concentration } k\end{array}$ & 16.2 .2 .4 , eq. $(16.2 .28)$ & 189 \\
\hline $\mathrm{MI}_{X Y}^{\pi}(d)$ & $\begin{array}{l}\text { delayed mutual information in spike patterns between } \\
\text { clusters } X \text { and } Y\end{array}$ & 17.3 .1$, eq. $(17.3 .1)$ & 215 \\
\hline$\mu_{i}$ & unfolding parameter & 3.3 .3$, eq. $(3.3 .1)$ & 44 \\
\hline$N$ & number of units in a network & 6.3, eq. $(6.3 .1)$ & 72 \\
\hline $\mathcal{N}^{4}$ & specific network of $N=4$ oscillators & 7.2 .3 .1 & 96 \\
\hline $\mathcal{N}_{\mu, \Sigma}$ & Gaussian distribution with mean $\mu$ and covariances $\Sigma$ & 16.2.2.1, eq. (16.2.16) & 186 \\
\hline$\nu$ & firing rate & part IV & 110 \\
\hline
\end{tabular}




\begin{tabular}{|c|c|c|c|}
\hline symbol & description & reference & page \\
\hline $\mathcal{O}(x)$ & orbit of $x$ & 3.1 & 34 \\
\hline $\mathcal{O}_{i}$ & periodic orbit starting from $o_{i}$ & 7.2 .3 .2$, eq. $(7.2 .16)$ & 97 \\
\hline$p(\cdot), p(\cdot, \cdot)$ & (joint) probability distributions & part V & 176 \\
\hline$p_{\mathrm{st}}(\cdot), p_{\mathrm{st}}(\cdot, \cdot)$ & stationary (joint) probability distributions & part V & 176 \\
\hline $\mathcal{P}$ & Poincaré section & 6.3, eq. $(6.3 .2)$ & 72 \\
\hline $\mathcal{P}_{i}$ & local Poincaré section near periodic orbit $\mathcal{O}_{i}$ & 7.2 .4$, eq. $(7.2 .19)$ & 99 \\
\hline$\Phi$ & phase vector & 6.3, eq. $(6.3 .1)$ & 72 \\
\hline$\phi_{i}$ & phase of oscillator $i$ & 6.3, eq. $(6.3 .1)$ & 72 \\
\hline$\Phi_{X}$ & collective phase of cluster $X$ & 16.3 .1 .1 & 194 \\
\hline$Q_{i}^{z}$ & maps on the synchronization manifold & 7.2 .5$, C.2.2 & 100,245 \\
\hline$R$ & partial reset function & 5.2 .2$, eq. $(5.2 .6)$ & 58 \\
\hline$R_{c}$ & linear partial reset function & 5.2 .2$, eq. $(5.2 .7)$ & 58 \\
\hline$R_{i}^{\uparrow}, R_{i}^{z}$ & return maps & 7.2 .5$, C.2.3 & 100,248 \\
\hline$r_{i}, s_{i}$ & pulse reception and sending event of oscillator $i$ & 7.2 .1 & 92 \\
\hline$S_{\sigma}$ & phase shift by $\sigma$ & 6.3, eq. $(6.3 .5)$ & 73 \\
\hline$S^{1}$ & one sphere & 6.3, eq. $(6.3 .1)$ & 72 \\
\hline$S_{N}$ & permutation group of $N$ elements & 6.3, eq. $(6.3 .3)$ & 72 \\
\hline $\mathcal{S}_{i}$ & synchronization manifold near orbit $\mathcal{O}_{i}$ & 7.2 .5 & 100 \\
\hline$\Sigma_{t}$ & time dependent correlation matrix & 16.2 .2$, eq. $(16.2 .15)$ & 186 \\
\hline$\sigma_{i, k}$ & time since $k^{\text {th }}$-last pulse generation of oscillator $i$ & 7.2 .1$, eq. $(7.2 .1)$ & 93 \\
\hline$\zeta$ & supra-threshold input & 5.2 .2 & 58 \\
\hline$T$ & period of oscillation / limit cycle & 3.4 , eq. $(3.4 .2)$ & 47 \\
\hline$T_{i}^{z}$ & transient maps & 7.2 .5$, C.2.3 & 100,248 \\
\hline$t_{\mathrm{s}}, t_{\mathrm{s}, i}$ & $s^{\text {th }}$-spike time of oscillator $i$ & 5.1 & 56 \\
\hline$\Theta, \Theta^{(i)}$ & avalanche sets & 5.2 .3$, eq. $(5.2 .8)$ & 59 \\
\hline$\tau_{x}$ & time constant of ion species / neuronal process $x$ & $(2.3)$, eq. $(2.3 .1)$ & 21 \\
\hline$\tau, \tau_{i j}$ & pulse transmission delay & 5.2, eq. $(5.2 .1)$ & 57 \\
\hline $\mathbb{T}^{N}$ & $N$-torus & 6.3, eq. $(6.3 .1)$ & 72 \\
\hline$\theta_{i}$ & phase of theta neuron $i$ & 2.6 .4$, eq. $(2.6 .9)$ & 29 \\
\hline$U$ & rise function & 5.2 .4 .2 & 61 \\
\hline$U_{b}$ & Mirollo-Strogatz rise function with curvature $b$ & 5.2 .4 .2$, eq. $(5.2 .20)$ & 62 \\
\hline$u_{i}$ & potential of pulse-coupled unit $i$ & 5.1 , eq. $(5.1 .1)$ & 56 \\
\hline $\mathcal{U}_{i}$ & neighbourhood of orbit $\mathcal{O}_{i}$ & 7.2 .4$, eq. $(7.2 .18)$ & 99 \\
\hline
\end{tabular}




\begin{tabular}{llrl}
\hline symbol & description & reference & page \\
\hline \hline$v$ & membrane potential & 2.2, eq. $(2.2 .3)$ & 20 \\
\hline$v_{S}, v_{D}$ & somatic and dendritic membrane potential & 2.5, eq. $(2.5 .1)$ & 26 \\
\hline$v_{\text {syn }}$ & synaptic reversal potential & 2.7, eq. $(2.7 .1)$ & 29 \\
\hline$v_{\mathrm{L}}$ & leak reversal potential & 2.2, eq. $(2.2 .4)$ & 20 \\
\hline$v_{x}$ & reversal potential of ion species $x$ & 2.2, eq. $(2.2 .1)$ & 20 \\
\hline$\omega_{i}$ & oscillation frequency of oscillator $i$ & 3.4, eq. $(3.4 .3)$ & 47 \\
\hline$\Omega_{X}$ & collective frequency of cluster $X$ & $16.1,16.3 .1 .2$ & 182,195 \\
\hline$w_{,} W$ & white noise processes & $16.1,16.3 .1 .2$ & 182,195 \\
\hline$\zeta_{i}, \varsigma_{X}$ & noise amplitudes & 3.4, eq. $(3.4 .7)$ & 48 \\
\hline$z(\phi)$ & phase response curve / vector & 16.3 .1 .2$, eq. $(16.3 .4)$ & 195 \\
\hline$Z_{X}$ & collective phase response curve / vector & 3.4, eq. $(3.4 .2)$ & 47 \\
\hline$x_{\circlearrowleft}(t)$ & periodic solution & 3.1 & 34 \\
\hline $\mathcal{X}$ & state space & $16.3)$ & 194 \\
\hline
\end{tabular}


Chirstoph Kirst

Ausbildung

1998 Abitur, Gymnasium Großburgwedel

seit 1999 Studium der Physik und Mathematik, Universität Göttingen

2001 Vordiplom in Mathematik und Physik, Universität Göttingen

2002 Studium der Physik, University of Oxford (UK)

2004-2005 Certificate of Advanced Study in Mathematics (Distinction), University of Cambridge (UK)

2006-2007 Diplom in Physik, Universität Göttingen

seit 2006 Promotion am Max-Planck-Institut f. Dynamik

und Selbstorganisation, Göttingen und

Institut für nichtlineare Dynamik, Universität Göttingen

Wissenschaftliche Anstellungen

05-07 2003 Gastwissenschaftler, Clarendon Laboratory, Nuclear Orientation Group, University of Oxford (UK)

2008 Wissenschaftlicher Mitarbeiter, Institut f. Theoretische Biologie, Humboldt Universität und BCCN Berlin

2004, 06, Wissenschaftlicher Mitarbeiter am Max-Planck-Institut f. Dynamik seit 2007 und Selbstorganisation, Göttingen

Auszeichnungen

1997 Bundeswettbewerb 'Jugend Forscht', Bereich Technik

2004/05 Stipendium des Deutschen Akademischen Austausch Dienstes für das Mathematikstudium an der Cambridge University (UK)

2004/05 Stipendium des Kurt Hahn Trust, Cambridge University (UK)

2007 Dr. Berliner - Dr. Ungewitter Preis für herausragenden Diplomabschluss, Universität Göttingen

Publikationen

Partial Reset in Pulse-Coupled Oscillators.

C. Kirst and M. Timme. SIAM J. Appl. Math. 70, 2119-2149 (2010).

Sequential Desynchronization in Networks of Spiking Neurons with Partial Reset.

C. Kirst, T. Geisel, and M. Timme, Phys. Rev. Lett. 102, 068101 (2009).

From networks of unstable attractors to heteroclinic switching.

C. Kirst and M. Timme, Phys. Rev. E 78, 065210 R (2008). 


\section{Acknowledgements}

I am very grateful to Prof. Dr. Theo Geisel for creating a unique work environment with excellent conditions for interdisciplinary scientific research. It makes it a great pleasure to be part of this group. Thank you for this opportunity.

I would also like to express my thanks to Prof. Dr. Rainer Kree for kindly agreeing to be the second examiner of this thesis and Prof. Dr. Anja Sturm, Prof. Dr. Ulrich Parlitz and PD Dr. Ulf-Rainer Fiebig for being part of the examination board.

Then I would like to thank my supervisor Prof. Dr. Marc Timme for his motivating support in many respects. He gave me an excellent insight into scientific work ranging from actual research over composing cv's and applications, making posters and giving talks (following the WiM-rule!), writing and submitting papers, convincing referees to meeting deadlines. I also thank him for guiding me through the writing process of this thesis. Furthermore he patiently assisted me in all my scientific undertakings (including a year of absence for research in Berlin and Munich), supported my numerous visits to conferences and gave me great freedom to develop my own ideas and projects.

I would like to thank Prof. Dr. Andreas Herz (LMU Munich) for giving me the opportunity to work in his group, first in Berlin and later in Munich, and for his scientific enthusiasm and support.

Dr. Martin Stemmler (LMU Munich) has not only been an invaluable source of ideas, advice and knowledge but also motivated and energized me throughout our project which resulted in part IV of this thesis. I thank him for carefully proof-reading this part.

I thank Dr. Felix Felmy and Julian Ammer (LMU Munich) for the great collaboration and excellent experimental skills concerning chapter 'XXX' of this thesis. They introduced me to many aspects of experimental neuroscience as well as biological reasoning and let me experience the work with real neurons. I thank both for proof-reading part IV.

For the highly fruitful collaboration concerning part IV of this thesis I would like to thank Dr. Demian Battaglia. In particular, for many ideas and discussions, invaluable last-minute help in preparing the Stockholm-talk and for proof-reading part V. Thank you.

I am grateful to Hinnrich Kielblock for overtaking the oscillators in our chaos publication and Johannes Klingmayr for synchronizing mobile phones in our forthcoming publication.

With Judith Gabel, Collin Stonekin and Calden Wloka I had the opportunity to advise bachelor and internship students in doing research. I hope you enjoyed it just as much as I did. I learned a lot from this experience. Thank you.

I gratefully acknowledge helpful scientific discussions with Dr. Jan Benda (BCCN Munich), Prof. Gyorgy Buzsaki (Rutgers University), Prof. Tsampikos Kottos (Wesleyan University) (especially on topics in random matrix theory), Prof. in spe Matthias Kaschube (Princeton University) [also for inviting me to give a talk at Princeton], Prof. Christian Leibold (LMU Munich), Prof. Benjamin Lindner (HU Berlin), Prof. Raoul-Martin 
Memmesheimer (Radboud University Nijmegen), Prof. Michael Rosenblum (Potsdam University), Dr. Mikhail Rabinovich (University of California San Diego), Prof. John Rinzel (New York University), Prof. Jonathan Rubin (University of Pittsburgh), Dr. Jan Mattheis Schöfflen (Donders Institute), Prof. Paul Tiesinga (University of North Carolina), Dr. Thilo Womelsdorf (Donders Institute) and Dr. Magteld Zeitler (Radboud University Nijmegen) [also for inviting me to a talk to N.].

I thank Andreas Neef for discussions on patch clamp experiments and neuron models, Annette Witt for help concerning statistical tests, and Fred Wolf for useful comments mainly during all the Winter-Seminars.

I wish to acknowledge the constant support of Yorck-Fabian Beensen, Ragnar Fleischmann, Denny Fliegner, Achiem Hennies and Markus Schwammberger (even ill from home) in computing and software related matters.

I also like to thank Ayse Bolik, Regina Wunderlich and Viktoryia Novak for helpful assistance in all administrative concerns.

Oliver Bendix was a supportive companion in the $\mathrm{PhD}$-race to meet the final deadline.

People who provided valuable scientific and non-scientific support and which I like to thank are:

All members of the MPI DS including Philip Bittihn, Nikolai Chapochnikov, Kai Bröking, Stephan Eule, Harold Gutch, David Hofmann, Mirko Lukovic, Dominika Lyzwa, Anna und Georg Martius, Tatjana Tchumatchenko, Jan Nagler, Lars Reichl and all those I forgot.

The Berlin office mates, Bartosz Telenczuk, Jan-Hendrik Schleimer and Karin Fisch as well as Eric Reifenstein and Susanne Schreiber.

And the Munich community: Axel Kammerer, Alex Loebel, Alexander Mathis, Dinu Partirniche and Christian Porres. In particular, I am grateful to Alexander and Christian for providing hospitality during my several visits to Munich.

My recent office mates Hinrich Kielblock and Martin Rohden and all (former) members of the networks dynamics group, in particular, David Breuer, Carsten Grabow, Christoph Kolodziejski, Birgit Kriener, Fabio Schittler-Neves and Andreas Sorge.

Then I would like to thank all the members of the Institute's Jazzband, including Theo Geisel (also for providing us with a room for rehearsals) and my former office mates Wolfgang Keil and Jacob Metzger as well as our "visting performers" Michael Monteforte, Olav Stetter, Max Puelma Touzel and David Lamouroux, for great rehearsals and memorable performances.

With Christian Bick I had a lot of fun exploring conferences and national parks. Thanks for your great sense of humor!

Last, but not least, my very special thanks go to my brother, Henning, not only for his amazing hospitality during my stay in San Francisco and Berkeley.

I am also deeply indebted to my parents: There is my father who with his passion for science initially inspired me to do research in this field and my mother whose long-lasting support and encouragement was invaluable for the successful completion of this thesis. Thank you. 
It's not that I am so smart. It's just that I stay with problems longer.

Albert Einstein 
Für Jane 
